\title{
Oak Ridge National Laboratory Corrective Action Plan in Response to Tiger Team Assessment
}

\section{Volume 1}

Date Issued: August 23, 1991 (Revision 5)

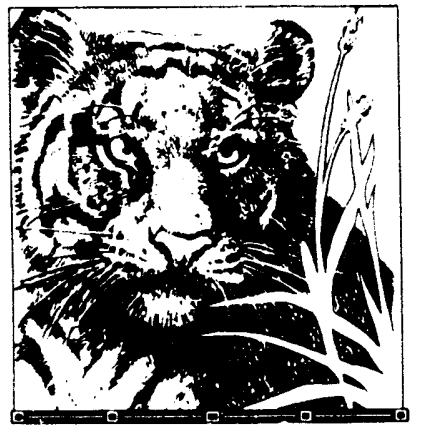

Prepared by

OAK RIDGE NATIONAL LABORATORY

Oak Ridge, Tennessee 37831-6285

managed by

MARTIN MARIETTA ENERGY SYSTEMS, INC.

for the

U.S. DEPARTMENT OF ENERGY

under contract DE-AC05-84OR21400

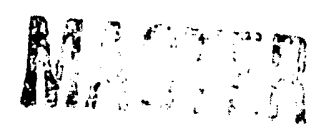




\section{CONTENTS VOLUME 1}

PREFACE $\ldots \ldots \ldots \ldots \ldots \ldots \ldots \ldots \ldots \ldots \ldots \ldots \ldots \ldots \ldots \ldots \ldots, \mathrm{v}$

LIST OF TABLES $\ldots \ldots \ldots \ldots \ldots \ldots \ldots \ldots \ldots \ldots \ldots \ldots \ldots \ldots \ldots \ldots \ldots \ldots$ vii

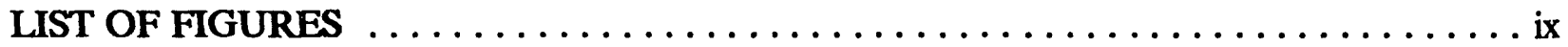

LIST OF ABBREVIATIONS $\ldots \ldots \ldots \ldots \ldots \ldots \ldots \ldots \ldots \ldots \ldots \ldots \ldots \ldots \ldots$

INDEX OF FINDINGS/CONCERNS $\ldots \ldots \ldots \ldots \ldots \ldots \ldots \ldots \ldots \ldots \ldots \ldots \ldots$ xix

EXECUTIVE SUMMARY $\ldots \ldots \ldots \ldots \ldots \ldots \ldots \ldots \ldots \ldots \ldots \ldots \ldots \ldots$ xxxiii

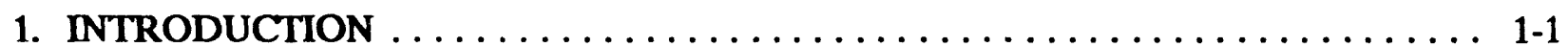

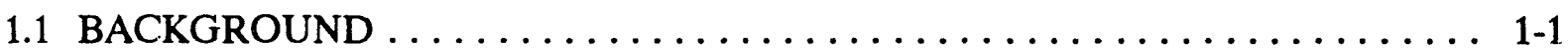

1.2 PURPOSE AND SCOPE OF THE ACTION PLAN .................. $1-1$

1.3 ORGANIZATION AND CONTENT OF THE ACTION PLAN $\ldots \ldots \ldots \ldots \ldots 1$.

1.4 ACTION PLAN METHODOLOGY $\ldots \ldots \ldots \ldots \ldots \ldots \ldots \ldots \ldots \ldots \ldots, 1-2$

1.5 SITE DESCRIPTION $\ldots \ldots \ldots \ldots \ldots \ldots \ldots \ldots \ldots \ldots \ldots \ldots \ldots \ldots \ldots, 1-3$

1.6 FINANCIAL ASSUMPTIONS $\ldots \ldots \ldots \ldots \ldots \ldots \ldots \ldots \ldots \ldots \ldots, 1-9$

1.7 PRIORITIZATION AND TRACKING $\ldots \ldots \ldots \ldots \ldots \ldots \ldots \ldots \ldots \ldots \ldots$

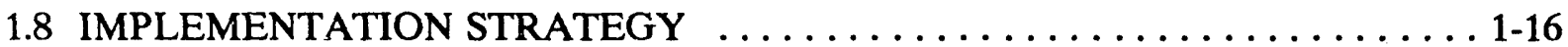

2. ORGANIZATION AND MANAGEMENT SCRUCTURE TO IMPLEMENT THE

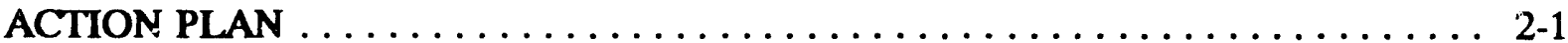

2.1 OVERVIEW $\ldots \ldots \ldots \ldots \ldots \ldots \ldots \ldots \ldots \ldots \ldots \ldots \ldots \ldots \ldots \ldots \ldots \ldots \ldots \ldots, 2-1$

2.2 DEPARTMENT OF ENERGY $\ldots \ldots \ldots \ldots \ldots \ldots \ldots \ldots \ldots \ldots \ldots \ldots, 2-1$

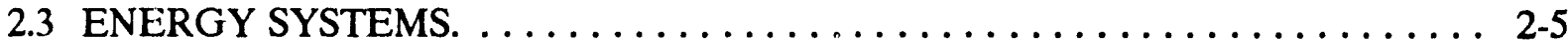

2.4 OAK RIDGE NATIONAL LABORATORY $\ldots \ldots \ldots \ldots \ldots \ldots \ldots \ldots \ldots \ldots \ldots \ldots$

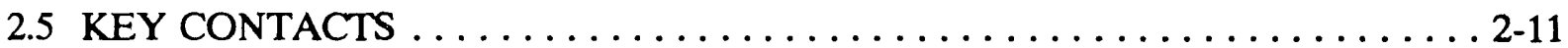

3. FINDINGS, RESPONSES, AND PLANNED ACTIONS $\ldots \ldots \ldots \ldots \ldots \ldots \ldots, 3-1$

3.1 ROOT CAUSES $\ldots \ldots \ldots \ldots \ldots \ldots \ldots \ldots \ldots \ldots \ldots \ldots \ldots \ldots \ldots \ldots \ldots \ldots \ldots \ldots \ldots, 1$

3.2 ENVIRONMENTAL FINDINGS, RESPONSES, AND

PLANNED ACTIONS $\ldots \ldots \ldots \ldots \ldots \ldots \ldots \ldots \ldots \ldots \ldots \ldots \ldots \ldots \ldots . . .2 .1$

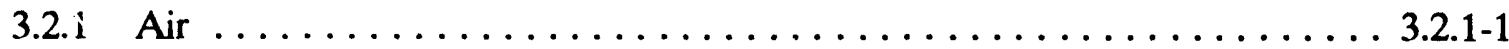

3.2.2 Soil/Sediments/Biota $\ldots \ldots \ldots \ldots \ldots \ldots \ldots \ldots \ldots \ldots \ldots \ldots \ldots . \ldots \ldots . \ldots \ldots .2 .2-1$

3.2.3 Surface Water/Drinking Water $\ldots \ldots \ldots \ldots \ldots \ldots \ldots \ldots \ldots \ldots . . \ldots \ldots .2 .3-1$

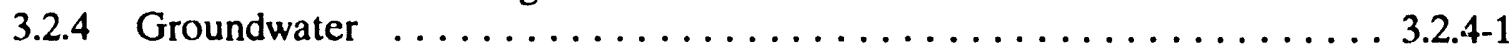

3.2.5 Waste Management $\ldots \ldots \ldots \ldots \ldots \ldots \ldots \ldots \ldots \ldots \ldots \ldots \ldots \ldots . .2 .5-1$

3.2.6 Toxic and Chemical Materials $\ldots \ldots \ldots \ldots \ldots \ldots \ldots \ldots \ldots \ldots \ldots \ldots \ldots \ldots \ldots \ldots .2 .6-1$

3.2.7 Quality Assurance $\ldots \ldots \ldots \ldots \ldots \ldots \ldots \ldots \ldots \ldots \ldots \ldots \ldots \ldots \ldots \ldots \ldots \ldots \ldots \ldots .2 .7-1$

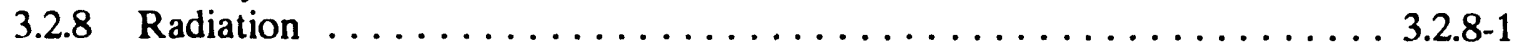


3.2.9 Inactive Waste Sites

3.2.10 National Environmental Policy Act

3.3 SITEWIDE SAFETY AND HEALTH FINDINGS, RESPONSES,

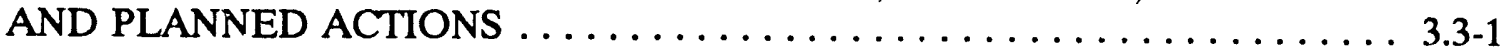

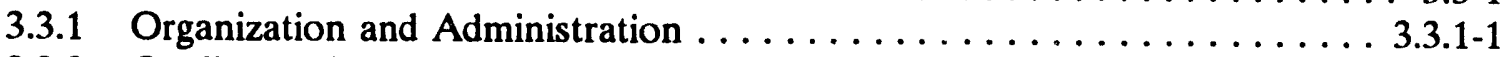

3.3.2 Quality Verification $\ldots \ldots \ldots \ldots \ldots \ldots \ldots \ldots \ldots \ldots \ldots \ldots \ldots \ldots \ldots \ldots \ldots \ldots \ldots \ldots . .3 .2 .1$

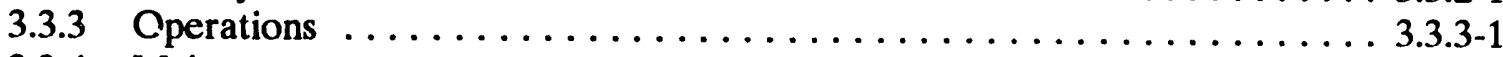

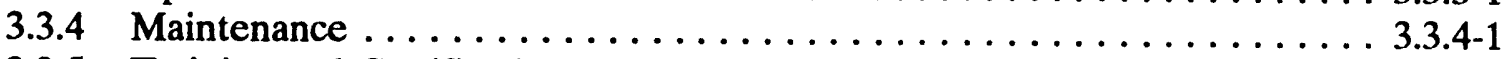

3.3.5 Training and Certification $\ldots \ldots \ldots \ldots \ldots \ldots \ldots \ldots \ldots \ldots \ldots \ldots \ldots \ldots \ldots \ldots \ldots \ldots .3 .5 .1$

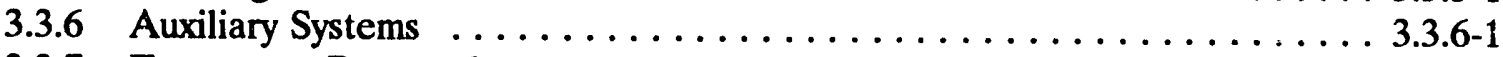

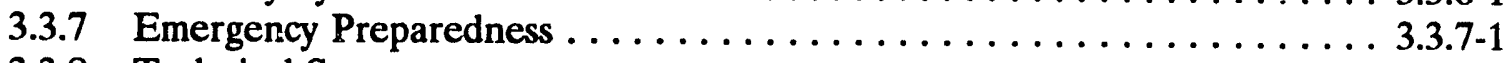

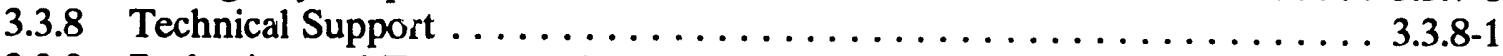

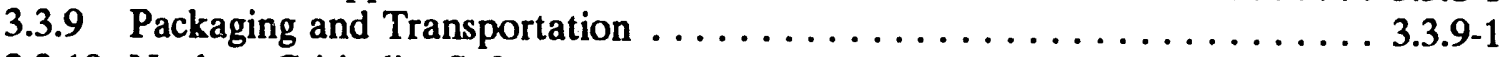

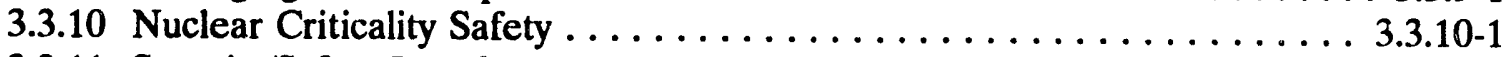

3.3.11 Security/Safety Interface $\ldots \ldots \ldots \ldots \ldots \ldots \ldots \ldots \ldots \ldots \ldots \ldots \ldots \ldots \ldots . . \ldots \ldots .11-1$

3.3.12 Experimental Activities $\ldots \ldots \ldots \ldots \ldots \ldots \ldots \ldots \ldots \ldots \ldots \ldots \ldots \ldots \ldots \ldots \ldots \ldots \ldots \ldots \ldots \ldots .3 .12-1$

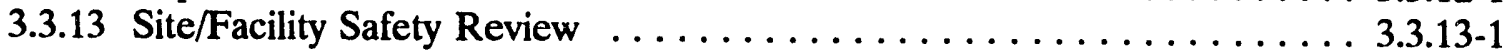

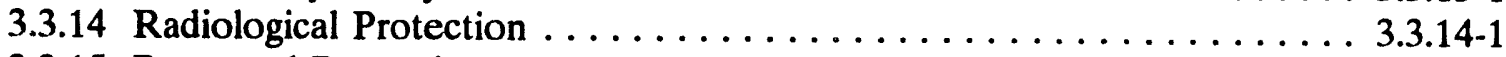

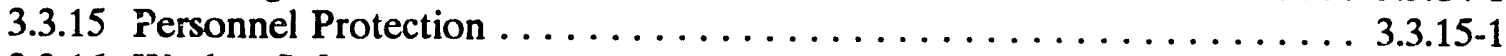

3.3.16 Worker Safety . . . . . . . . . . . . . . . . . . . . . . . . . . .

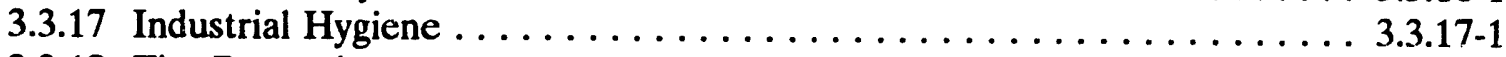

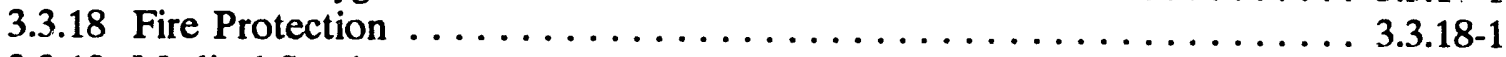

3.3.19 Medical Services $\ldots \ldots \ldots \ldots \ldots \ldots \ldots \ldots \ldots \ldots \ldots \ldots \ldots \ldots \ldots \ldots \ldots \ldots . . \ldots \ldots .19 .1$ 


\section{PREFACE TO REVISION 5}

The ORNL Corrective Action Plan in Response to Tiger Team Assessment presents a complete response to the Tiger Team assessment that was conducted at Oak Ridge National Laboratory (ORNL) and at the U.S. Department of Energy (DOE) Oak Ridge Operations Office (ORO) from October 22, 1990, through November 30,1990. The action plans have undergone both a discipline review and a cross-cutting review with respect to root cause. In addition, the action plans have been integrated with initiatives being pursued across Martin Marietta Energy Systems, Inc., in response to Tiger Team findings at other DOE facilities operated by Energy Systems. The root cause section is complete and describes how ORNL intends to address the root causes of the findings identified during the assessment.

The action plan has benefitted from a complete review by various offices at DOE Headquarters as well as review by the Tiger Team that conducted the assessment to ensure that the described actions are responsive to the observed problems.

All actions listed in this action plan are contingent upon suitable funding being provided. Action plan schedules for findings listed as "funded" are current best estimates of expected completion. The projected completion dates for actions listed as "requested" or "new" are technically feasible dates based on work scope and available or projected nonfinancial resources. Actual completion dates will depend on when work is authorized and funding is received.

This action plan has been a cooperative effort between ORNL and ORO and is the result of exceptional efforts by many individuals in both organizations to meet a very demanding schedule.

Michael A. Kuliasha

Action Plan Leader

A igust 23, 1991 


\section{LIST OF TABLES VOLUME 1}

S.1 Total ES\&H Cost Summary $\quad \ldots \ldots \ldots \ldots \ldots \ldots \ldots \ldots \ldots \ldots \ldots \ldots \ldots$

S.2 ORNL Corrective Action Plan Cost Summary $\ldots \ldots \ldots \ldots \ldots \ldots \ldots \ldots \ldots$ xli 


\section{LIST OF FIGURES VOLUME 1}

1.1 Oak Ridge National Laboratory $\ldots \ldots \ldots \ldots \ldots \ldots \ldots \ldots \ldots \ldots \ldots$

1.2 Oak Ridge $\mathrm{Y}-12$ Plant $\ldots \ldots \ldots \ldots \ldots \ldots \ldots \ldots \ldots \ldots \ldots \ldots \ldots \ldots \ldots$

1.3 ORNL Resource Management System $\ldots \ldots \ldots \ldots \ldots \ldots \ldots \ldots \ldots \ldots \ldots \ldots \ldots \ldots$

1.4 Distribution of weights for all Tiger Team findings $\ldots \ldots \ldots \ldots \ldots \ldots \ldots \ldots$

1.5 Organizations responsible for corrective action monitoring $\ldots \ldots \ldots \ldots \ldots \ldots \ldots$

2.1 DOE-ORO organization and management structure $\ldots \ldots \ldots \ldots \ldots \ldots \ldots \ldots$

2.2 Martin Marietta Energy Systems, Inc., organization and management structure $\ldots \ldots \ldots \ldots \ldots \ldots \ldots \ldots \ldots \ldots \ldots \ldots \ldots \ldots \ldots$

2.3 ORNL organization and management structure $\ldots \ldots \ldots \ldots \ldots \ldots \ldots$ 


\section{LIST OF ABBREVIATIONS}

AC

$\mathrm{ACD}$

$\mathrm{ACM}$

ADS

AERO

AFDP

AIP

ALARA

AMERD

AMERWM

AMESQ

ANS

ANSI

AOI

APRAS

ARIMS

ARSRC

ASME

AST

ATF

AVID

AWS

BMAP

BMP

BSF

BSR

BVLLW

CAP

CARTS

CAS

CE

CEF

CEMP

CEQ

CERCLA

CFL

CFR

CFS

$\mathrm{CH}$

$\mathrm{CM}$

$\mathrm{CME}$ air-conditioning

Analytical Chemistry Division

asbestos-containing materials

Activity Data Sheet

Association for Excellence in Reactor Operations

Award Fee Determination Plan

Agreement in Principle

as low as reasonably achievable

Assistant Manager for Energy Research and Development

Assistant Manager for Environmental Restoration and Waste Management

Assistant Manager for Environment, Safety, and Quality

American Nuclear Society

American National Standards Institute

abnormal operating instruction

Automated Procedures Requirements Accountability System

Accelerator and Reactor Improvements Modifications

Accelerators and Radiation Sources Review Committee

American Society of Mechanical Engineers

advanced systems technology

Advanced Toroidal Facility

Accelerated Vendor Inventory Delivery

American Welding Society

Biological Monitoring and Abatement Program

best management practice

Bulk Shielding Facility

Bulk Shielding Reactor

Bethel Valley low-level waste

corrective action plan

Critical Actions and Requirements Tracking System

criticality alarm system

Conservation and Renewable Energy

Critical Experiments Facility

Comprehensive Emergency Management Plan

Council on Environmental Quality

Comprehensive Environmental Response, Compensation, and Liability Act credible fire loss

Code of Federal Regulations

Comprehensive Facility Survey

contact-handled

construction manager

continuing medical education 


\begin{tabular}{|c|c|}
\hline CMS & corrective measures study \\
\hline COR & contracting officers representative \\
\hline CPAF & cost plus award fee \\
\hline CPX & command post exercises \\
\hline CRC & Criticality Review Committee \\
\hline CSA & criticality safety analysis \\
\hline CSAUP & Comprehensive Safety Appraisal and Upgrade Program \\
\hline $\mathrm{CSO}$ & criticality safety officer \\
\hline CTD & Chemical Technology Division \\
\hline CWMD & Central Waste Management Division \\
\hline CY & calendar year \\
\hline CYRTF & Coal Yard Run-off Treatment Facility \\
\hline CXD & categorical exclusion determinations \\
\hline D\&D & decontamination and decommissioning \\
\hline DASMA & Deputy Assistant Secretary for Military Applications \\
\hline $\mathrm{DCC}$ & Document Control Center \\
\hline DCG & derived concentration guide \\
\hline DCM & design change memorandum \\
\hline DCN & design change notice; drawing change notice \\
\hline DIMS & Data Information Management System \\
\hline DOE & Department of Energy \\
\hline DOELAP & Department of Energy Laboratory Accreditation Program \\
\hline DOP & dioctylphthalate \\
\hline DOT & Department of Transportation \\
\hline DP & Defense Program \\
\hline DRC & Director's Review Committee \\
\hline DRD & direct-reading dosimeter \\
\hline DSO & division safety officer \\
\hline E\&HPD & Environmental and Health Protection Division \\
\hline EAG & Exposure Assessment Group \\
\hline EAL & emergency action level \\
\hline EAM & environmental ALARA memorandum \\
\hline EDS & Evaluation Database System \\
\hline EHP & Office of Environmental and Health Protection \\
\hline EIS & environmental impact studies \\
\hline EM & Environmental Restoration and Waste Management \\
\hline EM\&C & environmental monitoring and compliance \\
\hline EMF & electromagnetic field \\
\hline EMP & Environmental Protection Manual \\
\hline EOC & Emergency Operations Center \\
\hline EOP & emergency operating procedure \\
\hline EPA & Environmental Protection Agency \\
\hline EPCRA & Emergency Planning and Community Right-to-Know Act \\
\hline EPO & environmental protection officer \\
\hline EPZ & emergency planning zone \\
\hline ER & Energy Research \\
\hline ERAI & Emergency Response Action Items \\
\hline
\end{tabular}




\begin{tabular}{|c|c|}
\hline ERD & Ecological Research Division \\
\hline ERDS & Environmental Review and Documentation Section \\
\hline ERP & Environmental Restoration Program \\
\hline ES\&H & environinent, safety, and health \\
\hline ES\&HC & envirormental, safety, and health compliance \\
\hline ESA & envitonmental and safety activity \\
\hline ESAMS & Energy Systems Action Management System: \\
\hline ESD & Environmental Sciences Division \\
\hline ESH & Environmental Safety and Health (Stanclard) \\
\hline ESI & environmental sampling and instrumentation \\
\hline ESP & Exploratory Studies Program \\
\hline ESPQCP & Environmental Surveillance Procedures Quality Control Program \\
\hline ESQIS & Energy Systems Quality Information System \\
\hline ETD & Engineering Technology Division \\
\hline F\&MD & Finance and Materials Division \\
\hline FFA & Federal Facility Agreement \\
\hline FFCA & Federal Facility Compliance Agreement \\
\hline FHA & fire hazard analysis \\
\hline FIP & Facility Inspection Program \\
\hline FP & fire protection \\
\hline FPE & fire protection engineering \\
\hline FR & Federal Register \\
\hline FS & feasibility study \\
\hline FSAR & final safety analysis report \\
\hline FSET & Facility Safety Evaluation Teams \\
\hline FTE & full-time equivalent \\
\hline FWP & Field Work Proposal \\
\hline FY & fiscal year \\
\hline G\&A & general and administrative \\
\hline GEAT & General Employee Access Training \\
\hline GET & General Employee Training \\
\hline GFCI & ground fault circuit interrupter \\
\hline GIS & geographic information system \\
\hline gpm & gallons per minute \\
\hline GM & Geiger-Muller \\
\hline GOCO & government-owned contractor-operated \\
\hline GPE & general plant equipment \\
\hline GPS & General Plant Services \\
\hline GPNAAF & General Purpose Neutron Activation Analysis Facility \\
\hline GPP & General Plant Project \\
\hline GQM & groundwater quality monitoring \\
\hline GWC & Groundwater Program Coordinator \\
\hline GWPC & Ground-Water Program Coordinator \\
\hline $\mathrm{H} \& \mathrm{R}$ & hoisting and rigging \\
\hline HAD & heat-activated device \\
\hline
\end{tabular}


HAZCOM

HAZWRAP

HEPA

HFIR

HHIRF

HHMS

HPIC

HMIS

HP

HPP

HPRR

HQ

HRD

HSWA

HVAC

HWOG

$I \& C$

ICM

IDLH

IEEE

IEF

IFSP

IFSTA

IHAS

IHP

INEL

INPO

IPA

IPDP

IRMS

ITSM

LANL

LASL

LDR

LEPC

LIP

LLLW

LLW

LMA

LMB

LOCA

LSA

$\mathrm{M} \& \mathrm{O}$

M\&TE

MAPS

MC\&A
Hazardous Communication

Hazardous Waste Remedial Action Program

high-efficiency particulate air

High Flux Isotope Reactor

Holifield Heavy Ion Research Facility

Hydraulic Head Monitoring Station

Health Physics Instrument Committee

Hazardous Materials Inventory System

health physics

Health Physics Procedure

Health Physics Research Ruactor

headquarters

Human Resource Development

Hazardous and Solid Waste Amendments

heating, ventilation, and air conditioning

Hazardous Waste Operations Group

Instrumentation and Controls Division

interim corrective measure

immediately dangerous to life and health

Institute of Electrical and Electronics Engineers, Inc.

Isotope Enrichment Facility

Isotopes Facilities Shutdown Program

International Fire Service Training Association

Industrial Hygiene Air Sampling

Industrial Hygiene Procedure

Idaho National Engineering Laboratory

Institute of Nuclear Power Operations

ionic and physical analysis

Isotope Production and Distribution Program

Integrated Resource Management Systems

Installation Transportation Safety Manager

Los Alamos National Laboratory

Los Alamos Scientific Laboratory

land disposal restricted

Local Emergency Planning Committee

Line Item Project

low-level liquid waste

low-level waste

laboratory monitor alpha

laboratory monitor beta

loss-of-coolant accident

low specific activity

manage and operate

measurement and test equipment

Manpower Analysis and Planning Survey

Materials Control and Accountability 


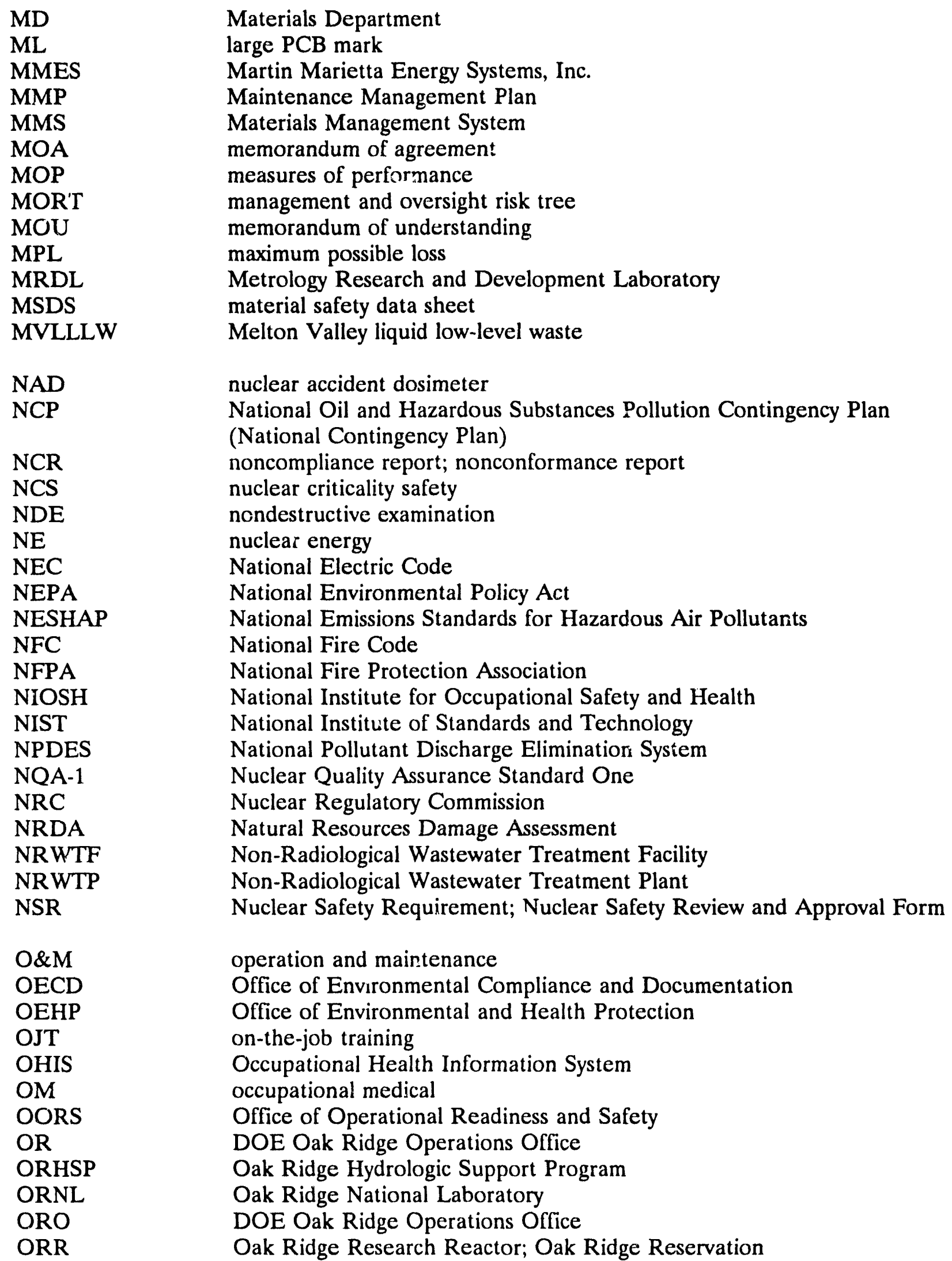




\section{ORRHAGS \\ ORS \\ OS\&Y \\ OSA \\ OSHA \\ OSR \\ OWMRA}

P\&.A

P\&E

P\&T

PAG

PC

PCA

PCB

PDM

PEC

PFT

PIP

PIV

PLC

PM

PNAD

PO\&C

POC

PORC

PPE

PPR

PR

IRA

PSO

PT

PVC

PWTP

\section{QA \\ QAC \\ QAP \\ QAS \\ QC \\ QE\&I \\ QER \\ QIR}

$R \& R$

RAMSPAC

RAP

RAP

RASCAL
Oak Ridge Reservation Hydrologic and Geologic Studies

Occurrence Reporting System

outside screw and yoke

Office of Safety Appraisals

Occupational Safety and Health Administration/Act

Operational Safety Requirement

Office of Waste Management and Remedial Action

plugging and abandonment

Plant and Equipment Division

packaging and transportation

protective action guideline

personal computer

pool critical assembly

polychlorinated bipheny!

Project Description Memorandum

Performance Evaluation Committee

pulmonary function test

Performance Improvement Process

post indicator valves

Professional Loss Control, Inc.

programmed maintenance

personnel nuclear accident dosimeter

performance objectives and criteria

particulate organic carbon

Plant Operations Review Committee

personal protective equipment

Performance Planning and Review

posted regulation

probabilistic risk assessment

program secretarial officer

packaging and transportation

polyvinyl chloride

Process Water Treatment Plant

quality assurance

quality assurance coordinator

quality assurance plan

quality assurance specialist

quality control

Quality Engineering and Inspection

quality event report

quality investigation report

roles and responsibilities

radioactive materials shipping and packaging

Remedial Action Program

Radiological Assistance Program

Radiation Standards and Calibration Laboratory 


\begin{tabular}{|c|c|}
\hline $\mathrm{RCO}$ & radiation control officer \\
\hline RCRA & Resource Conservation and Recovery Act \\
\hline REAJab & Radiation Exposure Assessment Laboratory \\
\hline REDC & Radiochemical Engineering Development Center \\
\hline RERC & Reactor Experiments Review Committee \\
\hline RFA & RCRA facility assessment \\
\hline RFI & RCRA facility investigation \\
\hline RFM & Request for Modification \\
\hline RH & remote-handled \\
\hline RH TRU & remote-handled transuranic \\
\hline $\mathrm{RI}$ & remedial investigation \\
\hline RMW & radioactive inixed waste \\
\hline RORC & Reactor Operations Review Committee \\
\hline RP & Radiation P: ection Section \\
\hline RPC & risk prioritization committee \\
\hline RPPA & Radiation Protection Program Assessment \\
\hline $\mathrm{RQ}$ & reportable quantity \\
\hline RRD & Research Reactors Division \\
\hline RSWO & Radioactive Solid Waste Operations \\
\hline RTP & Reactor Technoiogy Procedure \\
\hline RW & Civilian Radioactive Waste Management \\
\hline RWP & radiation work permit \\
\hline S\&H & safety and health \\
\hline SAIC & Science Applications International Corporation \\
\hline SAR & Safety Analysis Report \\
\hline SARA & Superfund Amendment Reauthorization Act \\
\hline SARPS & Safety Analysis Report for Packaging \\
\hline $\mathrm{SC}$ & special case \\
\hline SERC & State Emergency Response Committee \\
\hline SHD & Safety and Health Division \\
\hline SOI & special operating instruction \\
\hline SOP & standard operating procedure \\
\hline SNM & Special Nuclear Materials \\
\hline SPCC & Spill Prevention Control and Countermeasure \\
\hline SPP & standard practice procedure \\
\hline SRP & Savannah River Plant \\
\hline SSD & Solid State Division \\
\hline SSE & Systems Safety Engineering \\
\hline STEL & short-term exposure limit \\
\hline STP & Sewage Treatment Plant; Surveillance Test Procedure \\
\hline SWMU & Solid Waste Management Unit \\
\hline SWOD & Solid Waste Operations Department \\
\hline SWP & safe work permit \\
\hline SWSA. & Solid Waste Storage Area \\
\hline TBD & to be determined \\
\hline TCLP & Toxicity Characteristic Leaching Procedure \\
\hline
\end{tabular}




$\begin{array}{ll}\text { TDHE } & \text { Tennessee Department of Health and Environment } \\ \text { TEG } & \text { Technical Evaluation Group } \\ \text { TLV } & \text { threshold limit value } \\ \text { TORC } & \text { Technical Oversight and Review Committee } \\ \text { TOMS } & \text { Transportation Operation Management Section } \\ \text { TPAP } & \text { Training Progiam Accreditation Plan } \\ \text { TRU } & \text { transuranic } \\ \text { TRUST } & \text { Thorium Reactor Uranium Storage Tarik } \\ \text { TSA } & \text { Technical Safety Appraisal } \\ \text { TSCA } & \text { Toxic Substances Corttrol Act } \\ \text { TSF } & \text { Tower Shielding Facility } \\ \text { TSR } & \text { Tower Shielding Reactor } \\ \text { TSR-II } & \text { Tower Shielding Reactor II } \\ \text { TVA } & \text { Tennessee Valley Authority } \\ & \\ \text { UEPIP } & \text { Uranium Enrichment Performance Improvement Program } \\ \text { UL } & \text { Underwriters Laboratories } \\ \text { UOR } & \text { Unusual Occurrence Report } \\ \text { USQ } & \text { unreviewed safety question } \\ \text { USQD } & \text { unreviewed safety question determination } \\ \text { UST } & \text { underground storage tank } \\ \text { VOC } & \\ \text { VOG } & \text { volatile organic chcmical } \\ \text { WAC } & \text { vessel off-gas } \\ \text { WAG } & \text { waste acceptance criteria } \\ \text { WEAF } & \text { waste area grouping } \\ \text { WFO } & \text { Waste Examination Assay Facility } \\ \text { WHPP } & \text { Work for Others } \\ \text { XSO } & \text { Waste Handling and Packaging Plant } \\ & \text { ORNL (X-10) Site Office } \\ & \end{array}$




\section{INDEX OF FINDINGS/CONCERNS}

\subsection{ENVIRONMENTAL}

\subsubsection{Air}

$\begin{array}{ll}\text { A/CF-1 } & \text { Excursions Above Air Permit Limits } \\ \text { A/CF-2 } & \text { High Efficiency Particulate Air (HEPA) Testing Program Deficiencies } \\ \text { A/CF-3 } & \text { Absence of State Air Permits for P.adionuclide Sources } \\ \text { A/CF-4 } & \text { Ambient Air Monitoring Deficiencies } \\ \text { A/CF-5 } & \text { Effluent Stack Sampling and Monitoring Deficiencies } \\ \text { A/CF-6 } & \begin{array}{l}\text { Lack of Control Room Operator Training in Stack Release Emergency } \\ \text { Procedures }\end{array} \\ \text { A/BMPF-1 } & \begin{array}{l}\text { Inadequate Stack Emission Monitoring and Test Procedures for NESHAP } \\ \text { Compliance }\end{array} \\ \text { A/BMPF-2 } & \begin{array}{l}\text { Lack of Verifying Documentation to Der:onstrate Compliance with Air Permit } \\ \text { Conditions }\end{array} \\ \text { A/BMPF-3 } & \begin{array}{l}\text { Lack of Consistent Installation of Stack Sampling, Monitoring, and Alarm } \\ \text { S/BMPtems for Radioactive Releases }\end{array} \\ & \text { Deficient Asbestos Waste Disposal Management }\end{array}$

\subsubsection{Soil/Sediments/Biota}

SSB/CF-1 Inadequate Radioactive Contamination Control

SSB/BMPF-1 Delayed Benthic Data Analyses and Reporting

\subsubsection{Surface Water/Drinking Water}

SW/CF-1 $\mathrm{SW} / \mathrm{CF}-2$ SW/CF-3 SW/CF-4 SW/CF-5 SW/CF-6 SW/BMPF-1 SW/BMPF-2 SW/BMPF-3 SW/BMPF-4 SW/BMPF-5
Discharges Not Included on the ORNL NPDES Permit or Permit Renewal Inconsistent Labeling of ORNL Sinks and Drains Inaccurate Stream Flow Measurement Devices NPDES Permit Exceptions Lack of NPDES Best Management Practices Program Plan Deficiencies of Spill Prevention Control and Countermeasure Plan Inadequate ORNL Cross-Connections Study Liquid Radionuclide Releases from ORNL Facilities Lack of Backflow Prevention Devices Unrepaired Leaks from Wastewater Sewer Systems Lack of Certification of Treatment Plant Operators and Backflow Preventer Repairers 


\subsubsection{Groundwater}

GW/CF-1 Inadequate Hydrogeologic Characterization at SWSA 6

GW/CF 2 Inadequate Implementation of Well Purging Procedures at SWSA 6

GW/BMPF-1 Inadequate Well and Borehole Abandonment

GW/BMPF-2 Inadequate Monitoring Well and Borehole Inventory, Security, and Maintenance

GW/BMPF-3 Cross-Contamination Between Aquifers and Strata

GW/BMPF-4 No Custodian for Unused Wells

GW/BMPF-5 Inadequate Characterization of the Hydrogeologic Regime

\subsubsection{Waste Management}

WM/CF-1 Inadequate Operation of Mixed Waste Storage Facilities

WM/CF-2 Improper Operation of Satellite Accumulation Areas at ORNL Facilities at Y-12

WM/CF-3 Inadequate Hazardous Waste Accumulation and Minimization at ORNL Biology

Division at Y-12 Plant

WM/CF-4 Lack of Integrity Assessment of 7860A Hazardous Waste Storage Tank

WM/CF-5 Inadequate Training for Onsite Hazardous Waste Transporters

WM/CF-6 Storage of Land Disposal Restricted Mixed Waste

WM/CF 7 Inadequate Storage of Radioactively Contaminated Hazardous Waste Lead

WM/CF-8 Inadequate Training Documentation and Procedures for 7507 Hazardous Waste

Storage Facility

WM/CF-9 Inadequate Training Records and Inspection Records at the 3001 Storage Canal

WM/CF-10 Inadequate Leak Detection of Petroleum Underground Storage Tanks

WM/CF-11 Inadequate Hazardous Waste Determination of Sanitary Sewage Treatment Plant

Sludge

WM/CF-12 Inadequate Daily Inspections of RCRA Facilities on Weekends

WM/CF-13 Inadequate Characterization of Mixed Waste in Storage

WM/BMPF-1 Inadequate Assessments of Offsite Vendors Recycling Lead-Acid Batteries and Circuitboards

WM/BMPF-2 Inadequate Waste Minimization Program

\subsubsection{Toxic and Chemical Materials}

TCM/CF-1 PCB Wastes Stored Longer Than One Year

TCM/CF-2 Deficiencies with the TSCA Assumptions Requirements for Liquid Filled

Electrical Equipment

TCM/CF-3 Inadequate Labeling of Equipment Containing PCB Capacitors

TCM/CF-4 Deficiencies with TSCA Temporary Storage Facility Requirements

TCM/CF-5 Lack of Hazard Identification Labels for Some Chemical Storage Tanks

TCM/CF-6 Lack of Secondary Containment for Some Aboveground Hazardous Materials Chemical Storage Tanks/Containers

TCM/CF-7 Deficiency with TSCA Storage for Disposal Monitoring Policy and Storage for Disposal Policy

TCM/BMPF-1 Pesticides Program Deficiencies 


\subsubsection{Quality Assurance}

QA/CF-1 Standard Operating Procedures Deficiencies for Some MMES-ORNL Piojects

QA/CF-2 Deficiencies with the MMES Environmental Surveillance Procedures Quality Control Program Manual

QA/BMPF-1 QA/QC Deficiencies in MMES-ORNL Environmental Sampling Programs

\subsubsection{Radiation}

$\begin{array}{ll}\text { RAD/CF-1 } & \text { Inadequate Radiological Dose Assessment } \\ \text { RAD/CF-2 } & \text { Inadequate Radiological Postings } \\ \text { RAD/CF-3 } & \text { Unmonitored Decontamination Laundry Discharges } \\ \text { RAD/CF-4 } & \text { Inadequate Calibration of Radiological Monitors }\end{array}$

\subsubsection{Inactive Waste Sites}

IWS/CF-1 Inadequate Inventory and Identification of Inactive Waste Sites

IWS/CF-2 Lack of Formal Natural Resources Damage Assessment Notification

IWS/CF-3 Incomplete Distribution of Emergency Planning and Community Right-to-Know Act Reports

IWS/BMPF-1 Informality of Operations in the Environmental Restoration Program

IWS/BMPF-2 Proceeding Without Approved Plans

IWS/BMPF-3 Lack of Adequate Planning for Federal Facility Agreement Activities

IWS/BMPF-4 Incomplete Evaluation of Continuous Releases

\subsubsection{National Environmental Policy Act}

NEPA/CF-1 Inefficient DOE NEPA Implementation Procedures

NEPA/CF-2 Project Implementation Without Completed NEPA Process

NEPA/BMPF-1 Lack of Environmental Impact Assessment for ORNL Continuing Operations

\subsection{SITEWIDE SAFETY AND HEALTH}

\subsubsection{Organization and Administration}

OA.1-1 Flowdown of ES\&H Policies and Requirements

OA $1-2$

OA. $1-3$

OA. $1-4$

OA. $1-5$

Dissemination of DOE Orders and Other Requirements

ES\&H Issues for Construction Management

Acceptance of ES\&H Requirements at ORNL

Implementing ES\&H Activities

Consistency of Safety Kequirements 
OA.3-1

OA.6-1

OA 5-2

OA. $7-1$

OA. $7-2$

OA. 8 -1
ES\&H Goals for ORNL

Requirements for Job Descriptions

Employee Appraisal Plan Requirements

Centralized System for Safety Document Control

Records Storage Facilities

Supervisory Training in Behavior Observation

\subsubsection{Quality Verification}

QV.1-1

QV.1-2

QV.1-3

QV.1-4

QV.1-5

QV.1-6

QV.2-1

QV.3-1

QV.4-1

QV.4-2

QV.5-1

QV.6-1

QV.7-1
Resources for QA Functions

Implementation of the QA Program

Quality Department Audit Programi

Frequency of Internal Audits and Surveillances

QA Plans and Manuals

Effectiveness of Corrective Actions

Procurement Controls

Implementation of Receiving and Preinstallation Inspections

Calibration Facilities

Calibrations Program

Control of Safety-Related Materials

Quality-Related Inspections

Special Process Training

\subsubsection{Operations}

OP.3-1 ORNL Operating Procedures

OP.4-1 Facility Status Displays

OP.4-2 ORNL Lockout/Tagout Systems

OP.7-1 ORNL Shift Overlap

OP.8-1

OP. $8-2$

OP.8-3

Uniformity of Data Collection and Terminology

Alarm Panel Signal Configuration

Uniformity of Labels, Signs, and Signals

\subsubsection{Maintenance}

MA.1-1

MA.2-1

MA.2-2

MA.5-1

MA.5-2

MA.6-1

MA.8-1
Control of Removal of Equipment from Service

ORNL Lockout/Tagout System's Effectiveness

Conduct of Maintenance

General Facility and Post-Work Inspections

Identification of Energy Conservation Corrective Actions

P\&E Preventive Maintenance Program

Appropriate Maintenance Procedures 


\subsubsection{Training and Certification}

TC.1-1 Consistency and Administration of Training Programs

TC. $1-2$

TC.1-3

TC.5-1

TC. $7-1$

TC. $7-2$

Adequacy of Training Examinations

Training Staff

Miaintenance Personnel Training Program

Training Facilities

TC. $10-1$

Storage of Training Records

Consistency of Supervisory and Management Training

\subsubsection{Auxiliary Systems}

AX.1-1 Remedial Program for Auxiliary Systems

AX.1-2

AX.2-1

AX.3-1

AX.4-1

AX.4-2

AX.5-1

AX.6-1

Configuration Control System for Auxiliary Systems

No Implementation of the Waste Minimization Policy

Energy Systems Policy Procedure on Waste Management

Fissile Material Storage Handling Activities

Building $\mathbf{3 0 2 7}$ Storage Vault Operation

Gaseous Effluent Discharges

Backup Power Diesel Generators

\subsubsection{Emergency Preparedness}

EP.1-1

EP.1-2

EP.1-3

EP.1-4

EP. $2-1$

EP. 2-2

EP.3-1

EP.3-2

EP.4-1

EP.4-2

EP.5-1

EP.5-2

EP.6-1

EP.7-1

EP.7-2
Accident Consequence Assessment

Emergency Preparedness Recommendations

Facility Hazards Information

Analysis of Emergency Preparedness

Classification of Emergency Events

Adequacy of ORNL Emergency Plans

Training for Emergency Functions

Spill Response Training

Emergency Preparedness Exercises

Drill Planning

Emergency Monitoring of Releases

Regional Radiological Event

Protective Action Guides/Emergency Action Levels

Personnel Accountability Systems

Emergency Notification Systems

\subsubsection{Technical Support}

TS.2-1

TS. $3-1$
Safety Analysis Report Update Program

Procedures for Low-Cost Facility Modification 
TS.3-2

TS.4-1
Resource Allocation for Drawing Updates

Publication of Unusual Occurrence Reports

\subsubsection{Packaging and Transportation}

PT.1-1

PT.1-2

PT.1-3

PT.1-4

PT.1-5

PT.1-6

PT.2-1

PT.3-1

PT.3-2

PT.6-1

PT.6-2

PT.6-3

PT.8-1

PT.8-2

PT.8-3

PT.9-1

PT.9-2

PT.12-1

PT.12-2

PT.12-3

PT.12-4
Finance and Materials Division Staff

Packaging and Transportation Procedures

Packaging and Transportation Procedural Documents

ORNL Onsite Transportation Manual

Crossover of Packaging and Transportation Responsibilities

Transportation Program

Hazardous Materials Transportation Information

Divisional QA Packaging and Transportation Procedures

Transportation Program Audits

Onsite Transport of Waste

Low-Level Waste Bottle Testing

Inconsistency of Regulatory Terminolo gy

Safety Standards for Vehicle Identification

Unnecessary Transport of Hazardous Materials

Onsite Transfer of Hazardous Materials

Centra: File for Offsite Shipping Documents

Traffic Hazards on Bethel Valley Road

Handling and Storage of Hazardous Materials

Planning of Radioactive Materials Packaging Needs

Absence of Onsite Transfer Plan

Conflicting Contamination Limits

\subsubsection{Nuclear Criticality Safety}

CS.1-1 Nuclear Criticality Safety Training Program

CS.1-2 ORO Oversight and Support Functions

CS.1-3 ORNL Criticality Safety Program

CS.1-4 Nuclear Criticality Safety Program Documentation

CS.3-1 Safety Analysis Documentation Requirements

CS.4-1 Review of Nuclear Criticality Safety Analyses

CS.4-2 Dissemination of Nuclear Criticality Safety Guidance

CS.4-3 Nuclear Criticality Safety Remedial Action Plan

CS.5-1 Nuclear Criticality Safety Emergency Response Plan

CS.5-2 Criticality Alarm System Evacuation Drills

CS.5-3 Criticality Alarm Systems

\subsubsection{Security/Safety Interface}

SS.1-1 Analyses of Protective Force Equipment 


\subsubsection{Experimental Activities}

EA.2-1 ORNL Policy Regarding Independent Safety Reviews

EA.3-1

Guidance on Internal Safety Reviews

EA.3-2

Documentation of Safety Reviews of Experimental Plans

EA.4-1

Random Safety Surveillances

\subsubsection{Site/Facility Safety Review}

FR.1-1

FR.2-1

Organization and Implementation of Safety Review System

FR.3-1

Review of Safety Questions and Topics

FR.4-1

Shortcomings of the Safety Review System

FR.4-2

Management Response to Safety Committee Recommendations

FR.5-1

Annual Facility Appraisals

FR.6-1

Triennial Appraisal

Industry Lessons Learned

\subsubsection{Radiological Protection}

RP.1-1

RP.1-2

RP. 1-3

RP.3-1

RP.3-2

RP.3-3

RP.3-4

RP.3-5

RP.3-6

RP.3-7

RP.5-1

RP. $5-2$

RP.6-1

RP.6-2

RP.6-3

RP.7-1

RP.7-2

RP.8-1

RP.8-2

RP.8-3

RP.8-4

RP.8-5

RP.10-1

RP.10-2

RP.10-3

RP.10-4
Accomplishing ES\&H Compliance

ES\&H Compliance Staff

Management Oversight

ORNL Posting and Contamination Program

Contamination Control Program

Source Control Program

X-Ray Generating Machine Policy and Requirements

Accelerator Policy Requirements and Oversight

Material Clearance

Documentation of Radiation Hazards

Personnel Nuclear Accident Dosimeters

Direct-Reading Dosimeters

Air Samples

Timeliness of Air Sampling Program

Survaying Personnel for Contamination

Internal Radiation Dosimetry Program

In Vivo Calibrations

Radiation Protection Instrument Program

Approval of Radiation Protection Instruments

Testing of Safety-Related Instruments

High Range Radiation Protection Instrumentation

Radiation Protection Instrumentation Program

Positive Control of Contamination

Consistency of Radiation Protection Policies

Control of Laundry Wastewater

Requirements for Laundry Contamination Control 
RP.11-1

RP.12-1

RP.12-2

RP.12-3
Management Support for ALARA

Occupational Exposure Records Program

Reporting of Dosimetry Data

Control of Occupational Exposure Records

\subsubsection{Personnel Protection}

PP.1-1

PP.2-1

PP. 2-2

PP.3-1

PP. 3-2

PP. 5-1

PP. 5-2

PP. 5-3

PP.5-4
Resources for Workplace Maintenance

ORNL Health and Safety Program

Workplace Exposure Monitoring and Medical Records

Health and Safety Concerns

ORNL Construction Oversight Program

Hazard Communication Program Deficiencies

Health and Safety Program Deficiencies

Explosives Safety Program

Implementation of the Industrial Safety Program

\subsubsection{Worker Safety}

WS.3-1 Control of Asbestos

WS.4-1 Machine Guarding

WS.4-2 Noncompliances of Building Egress

WS.4-3 Machinery Inspection and Preventative Maintenance

WS.4-4 ORNL Electrical Compliance

WS.4-5 Equipment and Operations Areas

WS.4-6 ORNL Fire Protection

\subsubsection{Industrial Hygiene}

IH.2-1

IH.2-2

IH.3-1

IH.4-1

IH.5-1

IH.5-2

IH.5-3

IH.5-4

IH.5-5

IH.5-6

IH.6-1
Documentation of Procedures by Industrial Hygiene

Implementation of Industrial Hygiene Reviews

Personnel Protective Equipment

Surveillance of Industrial Hygiene Monitoring

Hearing Conservation Program

Chemical Carcinogen Program

Confined Space Entry

Respiratory Protection Program

Sanitation and Potable Water Program

Ergonomics Program

Handling, Storage, and Labeling of Chemicals 


\subsubsection{Fire Protection}

$\begin{array}{ll}\text { FP.1-1 } & \text { Resources of Fire Protection Engineering Section } \\ \text { FP.1-2 } & \text { Fire Department Resources and Work Load } \\ \text { FP.1-3 } & \text { Fire Protection of ORNL Facilities at the Y-12 Plant } \\ \text { FP.1-4 } & \text { Fire Protection Policies Regarding Improved Risk } \\ \text { FP.1-5 } & \text { Facility Reoccupancy Policy } \\ \text { FP.1-6 } & \text { Management's Role in Fire Protection } \\ \text { FP.2-1 } & \text { Egress from Bldg. 4500N, Machinery Space } \\ \text { FP.2-2 } & \text { Egress from Bldg. 4500N, Office Space } \\ \text { FP.2-3 } & \text { Life Safety Code Surveys } \\ \text { FP.2-4 } & \text { Action Plans Regarding Life Safety Code Surveys } \\ \text { FP.3-1 } & \text { ORNL Testing of Fire Equipment } \\ \text { FP.3-2 } & \text { Adequate Documentation of Fire Study } \\ \text { FP.3-3 } & \text { Design Basis Fires Review Program } \\ \text { FP.4-1 } & \text { Adequacy of Fire Protection } \\ \text { FP.5-1 } & \text { Fire Protection Systems in Bldg. 4500N } \\ \text { FP.6-1 } & \text { Physical Fitness Program for Fire Fighters } \\ \text { FP.6-2 } & \text { Fire Department Staffing Level } \\ \text { FP.6-3 } & \text { Prefire Plans for ORNL Facilities } \\ \text { FP.7-1 } & \text { Fire Protection of Main Computer Centers } \\ \text { FP.7-2 } & \text { Fire Hazard Analysis and Facility Protection Survey } \\ \text { FP.7-3 } & \text { Fire Protection Oversight } \\ \text { FP.7-4 } & \text { Review of Documents Affecting Fire Protection } \\ \text { FP.7-5 } & \text { Fire Water Supply System }\end{array}$

\subsubsection{Medical Services}

MS.1-1 Voluntary Health Examination Program

MS.2-1 Medical Division Administrative Assistance

MS.3-1 Backup Pulmonary Function Testing Personnel

MS.3-2 Medical Division Space Allocation

MS.3-3 Decontamination Facilities

\subsection{REACTORS}

\subsubsection{Orgénization and Administration}

$\begin{array}{ll}\text { ROA.1-1 } & \text { Funding for Maintenance of ORR } \\ \text { ROA.1-2 } & \text { Approval of ORR Shutdown Plans } \\ \text { ROA.1-3 } & \text { Decommissioning Plan for HPRR } \\ \text { ROA.1-4 } & \text { Funds for Maintenance of HPRR } \\ \text { ROA.1-5 } & \text { Funding for the Bulk Shielding Facility } \\ \text { ROA.1-6 } & \text { Status of the Bulk Shielding Facility }\end{array}$


ROA.1-7

RCA.1-8

ROA.1-9

ROA.3-1

ROA.6-1

ROA.7-1

ROA.7-2

ROA.7-3

ROA.7-4

ROA.7.5

ROA.8-1
Status of the CEF "W" Cell

Line Management Responsibilitics in RRD

Management Position Descriptions for RRD

Program to Increase Safety

Performance Evaluations for Safety

HFIR Final Safety Analysis Report

Updating of Safety Analysis Reports for BSF and PCA

Updating of Safety Analysis Report for TSF

Control of Documents

Procedures

Substance Abuse Program

\subsubsection{Quality Verification}

RQV.1-1

RQV.1-2

RQV.1-3

RQV.1-4

RQV.1-5

RQV.1-6

RQV.1-7

RQV.1-8

RQV.1-9

RQV.1-10

RQV.1-11

RQV.2-1

RQV.2-2

RQV.3-1

RQV.3-2

RQV.4-1

RQV.4-2

RQV.4-3

RQV.4-4

RQV.5-1

RQV.5-2

RQV.5-3

RQV.5-4

RQV.6-1

RQV.7-1

RQV.7-2
RRD Quality Assurance Program Manual

Management Assessment of RRD

Implementing RRD Procedures

Thoroughness of Procedures

Revision of Procedures

Frequency of RRD QA Audits

Method of Questioning in RRD QA Audits

RRD Corrective Action Program

Conflicting Goals in RRD

Data Base Trending System

Occurrence Reporting System

Justification of Procurement Deviations

Purchase Order and Quality Requirements

Adequacy of Inspections

Documentation of "Use-As-Is" Classification

Documentation of Acceptance Inspections

Measurement and Test Equipment Calibrations

Use of Uncontrolled and Uncalibrated Instruments

Determining Effects of Out-of-Tolerance Equipment

Justification of Deviations and Nonconformances

Trending of Nonconforming Items

Identification and Storage of Parts and Material

Evaluating Unreviewed Safety Questions

Inspection Reports

Control of Special Process Material

Special Process Procedures

\subsubsection{Operations}

ROP.2-1 Deficiencies in HFIR Operating Instructions

ROP.2-2 Technical Specifications at HFIR

ROP.2-3 Ambiguities in HFIR Operating Instructions 


$\begin{array}{ll}\text { ROP.2-4 } & \text { HFIR Shift Check Sheets } \\ \text { ROP.2-5 } & \text { HFIR Reactor Log } \\ \text { ROP.2-6 } & \text { HFIR Crew Communications } \\ \text { ROP.3-1 } & \text { HFIR Operating Manual } \\ \text { ROP.3-2 } & \text { Use of HFIR Procedures } \\ \text { ROP.3-3 } & \text { Preparation and Review of HFIR Operating Procedures } \\ \text { ROP.4-1 } & \text { Tagging Procedures } \\ \text { ROP.4-2 } & \text { Records of HFIR Equipment }\end{array}$

\subsubsection{Maintenance}

RMA.1-1 Control of Maintenance Support

RMA.2-1 Torquing of Equipment Bolting

RMA.2-2 Unsafe Conditions at the B Reactor Facilities

RMA.3-1 Maintenance of B Reactor Areas

RMA.3-2 Inspection of RRD Maintenance Activities

RMA.4-1 Oversight by Maintenance Supervisors

RMA.5-1 Maintenance at Shut Down Reactors

RMA.8-1 Deficient Procedural Information

\subsubsection{Training and Certification}

$\begin{array}{ll}\text { RTC.1-1 } & \text { Position Task Analyses } \\ \text { RTC.1-2 } & \text { Class B Reactor Training Plan } \\ \text { RTC.1-3 } & \text { Instructors for Maintenance Training } \\ \text { RTC. } 2-1 & \text { Examinations for Operator and Reactor Supervisor Training } \\ \text { RTC. } 4-1 & \text { General Employee Access Training } \\ \text { RTC.5-1 } & \text { Maintenance Personnel Training Program } \\ \text { RTC.5-2 } & \text { Training Facilities for Maintenance Personnel } \\ \text { RTC. } 10-1 & \text { Training for Managers, Supervisors, and Technical Staff }\end{array}$

\subsubsection{Auxiliary Systems}

RAX.2-1 Resin Carryover in Resin Regenerative System

RAX.3-1 Contamination of the HFIR Pool

RAX.4-1 HFIR Spent Fuel Cask Not Approved by DOE

\subsubsection{Emergency Preparedness}

REP.2-1 HFIR Emergency Preparedness Planning

REP.3-1

Training of Designated Emergency Responders

REP.5-1

HFIR Stack Radiological Effluent Monitors 
REP.5-2

Emeigency Resources

REP.6-1

Emergency Assessment and Notification Procedures

REP.?-1

HFIR Emergency Preparedness Planning

\subsubsection{Technical Support}

$\begin{array}{ll}\text { RTS.1-1 } & \text { Technical Support for RRD Tasks } \\ \text { RTS.1-2 } & \text { Backup for RRD Staff } \\ \text { RTS.2-1 } & \text { Consistency of Technical Specifications and Procedures } \\ \text { RTS.2-2 } & \text { Safety Analysis Reports for the HFIR and TSF } \\ \text { RTS.3-1 } & \text { "Facility Design Modifications" } \\ \text { RTS.3-2 } & \text { "Configuration Control of Plant Design Modification" } \\ \text { RTS.3-3 } & \text { Drawing Changes for the HFIR } \\ \text { RTS.4-1 } & \text { Equipment Performance Tracking } \\ \text { RTS.5-1 } & \text { Environmental Impact } \\ \text { RTS.7-1 } & \text { Reactor Engineering Function at the HFIR and TSF }\end{array}$

\subsubsection{Nuclear Criticality Safety}

RCS.1-1 Maragement of Criticality Safety Program

RCS.1-2 Tim.ly Resolution of Criticality Safety Issues

RCS.5-1 Criticality Alarm Systems

RCS.5-2 Distribution of Nuclear Accident Dosimeters

RCS.5-3 Performance of Criticality Drills

\subsubsection{Security/Safety Interface}

RSS.1-1 New Safeguards and Security Elements

RSS.3-1 Analyses to Determine Appropriate Weapons

RSS.4-1 Safety Appraisals and Audits for Firearms

\subsubsection{Experimental Activities}

REA.1-1 Safety Overview of Bldg. 7900

REA.3-1 Updating of Research Reactors Experimenters' Guide

REA.3-2 Verification of Reactor Experiment Calculations

REA.4-1 Control of Potential Personnel Exposure 


\title{
3.4.12 Site/Facility Safe:,y Review
}

RFR.1-1 Training for Unreviewed Safety Question Determinations

RFR.1-2 Review Board for Nuclear Safety Assessments

RFR.4-1 New Format for Written Reports

\subsubsection{Radiological Protection}

RRP.3-1

RRP.3-2

RR.P.4-1

RRP.4-2

RRP.8-1

RRP.10-1

RRP.10-2

RRP.11-1
Source Control

Radicactive Source Inventcries

Posting of Radiological Conditions

Radiological Controls in the Experiment Area

Instruments

Control of Low-Level Contamination

Waste Minimization

Addressing ALARA Issues

\subsubsection{Personnel Protection}

\author{
RPP.1-1 Review of RRD \\ RPP.2-1 \\ RPF. 2-2 \\ ORNL Safety Manual \\ RPP. 2-3 \\ Safety Personnel Involvement with Safety Work Permits \\ RPP.2-4 \\ Updating of RRD Manuals \\ RPP.3-1 \\ RPP.3-2 \\ RPP.3-3 \\ RPP.4-1 \\ RPP.5-1 \\ ORNL Hoisting and Rigging Equipment \\ Industrial Safety at TSF \\ Carcinogens in RRD Facilities \\ ORNL. Electrical Program \\ RRD Monitoring Program \\ HAZCOM Program at RRD
}

\subsubsection{Fire Protection}

RFP.1-1 Organization and Administration of Fire Protection Program

RFP.4-1

RFP.4-2

RFP.4-3

RFP.7-1

RFP.7-2

Sprinkler System in Bldg. 7902

Smoke Detection Systems at the HFIR

Use of Preaction-Type Sprinklers

Diking and Fire-Resistant Enclosures at the HFIR

Potential Fire Hazards at the HFIR 


\subsection{MANAGEMENT}

MF-1

MF-2

MF-3

MF-4

MF-5

MF-6

MF-7

MF-8

MF-9

MF-10

MF-11

MF-12
ES\&H Goals and Objectives

ES\&H Management Systems

Quality Assurance

Human Resources

Independent Oversight Systems

ORNL Tracking ES\&H Issues to Closure

ORNL ES\&H Interfaces with Onsite External Groups ES\&H Review of Work for Others

Contractual Matters

DOE Directive System

OR Oversight Systems

Contract Award Fee Process

\subsection{SELF-ASSESSMENT}

SA-1 The ORNL Self-Assessment Process 
EXECUTIVE SUMMARY 


\section{EXECUTIVE SUMMARY}

\section{ASSESSMENT RESULTS}

A Tiger Team assessment was conducted at Oak Ridge National Laboratory (ORNL) and the U.S. Department of Energy (DOE) Oak Ridge Operations Office (ORO) between October 22, 1990 , and November 30,1990 . The assessment was conducted by a team of over 80 specialists from various DOE offices, contractors, and consultants organized into four subteams: environmental, sitewide safety and health assessment, reactors safety and health assessment, and management.

Although the Tiger Team acknowledged that there has been significant improvement in ORNL's environmental, safety, and health (ES\&H) performance since the release of the Secretary of Energy's initiatives in June 1989, many deficiencies in the ES\&H program were identified during the review. The environmental subteam identified a total of 70 findings; 43 findings related to nonconformance with ORNL procedures, Martin Marietta Energy Systems, Inc., (Energy Systems) procedures, DOE orders, or federal or state laws and regulations, and 27 findings in which best management practices were not attained. The sitewide safety and health appraisal identified 202 findings. No Category I findings were identified, ten of the findings were Category II findings, and the balance were Category III findings. The reactors safety and health appraisal identified 128 findings of which 2 were Category II and the rest were Category III. The management subteam noted 12 management findings and 1 finding related to the self-assessment process.

Five noteworthy practices were highlighted during the Tiger Team assessment.

ORNL is using a risk-based prioritization system as an aid in scheduling and allocating resources. The system considers consequences in the areas of public health and safety, environmental protection, site personnel safety, regulatory compliance and external confidence, and business performance and economic concern, together with the probability of events occurring to derive a risk weight for each problem. A plot of the risk weights for all Tiger Team findings shows a sharp break in the curve of risk weights, with 75 of the 413 Tiger Team findings having risk weights greater than 200 . Six findings were determined to have risk weights greater than 900 , and these are considered serious safety hazards. Other factors besides risk weight are also considered in prioritization, with higher priority given to actions that relate to improving ORNL's ability to manage its ES\&H activities and to actions that directly aduiress root causes that contribute to other, more serious problems.

Each finding was also prioritized by using the Tiger Teáiı Action Prioritization System. This system assigns each activity a priority of $1,2,3$, or 4 according to the following definitions: Priority 1 -actions necessary to prevent significant risk to the public, worker health or safety, or the environment; Priority 2-actions necessary to meet statutes and DOE orders, although lack of action would not result in a significant risk to the public, worker health or safety, or the environment; Priority 3-actions consistent with best management practices; and Priority 4-actions not required by law, regulation, or agreement but that would be desirable to 
accomplish. When these definitions were applied to the Tiger Team findings at ORNL, 16 findings were determined to be Priority 1; 211, Priority 2; 158, Priority 3; and 28, Priority 4.

\section{ACTION PLAN PROCESS}

The action plan deals with three types of problems. The first type relates to the large legacy of conditions resulting from past practices dating back to the inception of ORNL in 1943. Many of these conditions require extensive and expensive remedial actions. The second type relates to deficiencies in current programs. In many cases, correcting these deficiencies will require a substantial reallocation of programmatic resources to meet all ES\&H expectations with an attendant impact on research missions. The third type relates to correcting management deficiencies and improving acceptance and responsibility for ES\&H requirements. Many of these changes can be implemented at little or no cost and offer the potential to make ORNL a better research institution.

The results of the Tiger Team assessment of ORNL, previous audits of ORNL, the results of Tiger Team assessments of other Energy Systems sites, Tiger Team assessments of other DOE installations, and the ORNL self-assessment were carefully considered to identify 11 root causes that appear to explain the findings identified during the ORNL Tiger Team assessment. These 11 root causes are inadequate policy, inadequate policy implementation, insufficient resources, inadequate management commitment, inadequate management approach, inadequate oversight, inadequate communications, ambiguous requirements or expectations, inadequate training, poorly defined roles and responsibilities, and regulatory barriers. The 11 root causes are being addressed by ORNL's adopting a comprehensive management approach consisting of seven key elements described below, by actions outlined in individual action plans, and by Energy Systems initiatives. Collectively, the action plan seeks to remedy these root causes and provide an infrastructure that not only meets all ES\&H requirements and expectations but can support ORNL's long-range goal of excellence.

The action plan used a matrix approach to identify common elements and to ensure a consistent approach in dealing with root causes. All action plans in a given assessment area were reviewed by discipline experts to ensure that the proposed actions were consistent in technical approach and to identify common elements. Individual action plans were also submitted to a cross-cutting review whereby all action plans attributable to a particular root cause were reviewed as a group by ORNL division directors to ensure that the root causes were being addressed in a consistent manner. Consequently, many action plans contain cross-references to other findings addressing similar problems. A special cross-cut review of other internal and external audit findings across Energy Systems was made to coordinate management and root cause issues with Energy Systems initiatives addressing similar probiems.

\section{KEY FINDINGS AND ACTIONS}

The Tiger Team identified 12 Category II findings and highlighted a number of key findings in management and environmental areas. These key issues were also amplified in the Secretary of Energy's memorandum transmitted with the draft Tiger Team report to various DOE offices and 
in his letter to Mr. Norman Augustine, Chairman and Chief Executive Officer of Martin Marietta Corporation, on the Tiger Team Assessment. ORNL was directed to pay special attention to self-assessment, containination spread, waste minimization, quality verification, safety programs, corporate oversight, and management issues. Key actions completed, under way, or planned to respond to these issues are summarized in this section.

The action plan outlines a series of aggressive actions, many of which have already been completed, to mitigate the present risks associated with each finding as well as long-term actions designed to address the root cause of the problem. Of the 12 category II concerns identified by the Tiger Team, actions to resolve 2 have already been completed, 5 more will be completed before the end of FY 1991, 3 will be completed in FY 1992, 1 in FY 1993, and 1 in FY 1994. In those cases that require extensive action over an evtended period of time, such as correcting serious electrical deficiencies in ORNL's 306 buildings, actions are being prioritized to address the most serious problems first.

The Tiger Team noted in Finding SA-1 that ORNL has not institutionalized its self-assessment process and that the current process lacks many of the elements of an effective self-assessment program. The action plan prepared to respond to that finding outlines a plan to fully implement a continuous self-assessment process combined with periodic independent external review that meets all the criteria for an effective self-assessment program.

Virtually all contamination spread from ORNL occurs through runoff from waste sites or permitted releases within the White Oak Creek drainage basin. To control the latter, the Process Waste Treatment Plant was modified in 1986 to reduce low-level liquid waste volume by $80 \%$. A zeolite ion exchange project is scheduled for FY 1992 to remove cesium-137 from process wastewater discharges. Removal of sediments that are impacting the hydraulic performance of flow-monitoring structures is a key concern in the assurance of quality of surface water-flow data, which are essential to monitoring liquid radioactive releases. Additional funding is being requested to complete time-critical removal of contaminated sediment to address this issue.

Another possible path is to deep aquifers along pathways created by abandoned, unplugged wells. Corrective actions focus is mainly on ensuring that plugging and abandonment is carried out in instances where potential exists for contaminant spread along boreholes and poorly constructed or poorly maintained wells. A groundwater program coordinator has been appointed to provide a central focus for all groundwater activities at ORNL. Funds are being requested to accelerate previously planned projects to maintain, characterize, and remediate potential paths for groundwater contamination. These actions, together with the other actions outlined in the action plan, provide an aggressive start to addressing this long-term problem.

With regard to waste minimization, nonhazardous scintillation cocktails are being used wherever possible. ORNL has instituted an aluminum recycle program, and efforts are under way to develop a comprehensive waste-minimization program.

Management is fully committed to an effective and comprehensive quality verification program. Energy Systems has instituted a total quality management program, led by senior management. A Quality Assurance Audit Program Manager was named effective December 1, 1990. A central tracking system called the Energy Systems Action Management System is currently under development to provide tracking of actions resulting from all audits and Energy Systems sites. An 
Integrated Resource Management System is also being developed to assist in prioritizing actions and managing resources.

The action plan describes numerous actions proposed to improve safety programs in industrial hygiene, fire protection, radiation protection, and hazards communication. The Tiger Team cited insufficient resources in a number of findings related to safety prograns, and discussions are continuing with DOE Headquarters to identify options for funding ihese activities to bring them into compliance. In the meantime, efforis are continuing to make the best use of available resources.

Martin Marietta Corporation is also playing an active role in improving ES\&H performance at Energy Systems facilities. The Energy Systems board of directors, chaired by Tom Young, the President of Martin Marietta Corporation, meets bimonthly and provides top management review of Energy Systems operations with special attention to performance deficiencies in ES\&H. Technical and management assistance is provided to Energy Systems by other parts of Martin Marietta Corporation through an interdivisional operating directive. Recent ES\&H-related assistance provided by Martin Marietta Corporation to Energy Systems includes planning assistance for the Y-12 technical audit; calibration standards and measuring; analysis, development, and implementation of plant performance objectives; and environmental task force assistance. In addition, ORNL is using the knowledge base and experience of Martin Marietta Corporation and other Energy Systems sites in developing its self-assessment process. Technical audits are performed by Martin Marietta Corporation for all Martin Marietta businesses. A pre-Tiger Team audit was conducted at ORNL during 1990. During 1991, eleven audits are scheduled, including three at Energy Systems installations. Finally, the Corporate Environmental Management group has established a local office in Oak Ridge, Tennessee, with two full-time staff members. The activities of this group include reviewing ongoing environmental programs relative to compliance with applicable federal, state, and local regulations.

ORNL's response to its acknowledged management deficiencies is to firmly establish ES\&H as an integral part of the mission of the Laboratory and to apply the same rigor to meeting ES\&H laws and requirements as is applied to scientific laws and requirements. Energy Systems and ORNL management have developed the following approach to establishing and maintaining excellence in ES\&H. This approach envisions seven key elements:

- Strategic plan for ES\&H: a strategic plan for ES\&H is under development to provide vision and coherence to ES\&H activities. It will integrate with the strategic plan being developed Energy Systems-wide.

- Goals and structure: institutional goals will be established and roles and responsibilities will be clearly defined and utilized in performance planning and review.

- Conduct of Operations: uniformity of management approach and formality of operations will be strengthened by the implementation of Conduct of Operations throughout ORNL. 
- Surveillance: effective and independent oversight of ES\&H performance will be established, and adequate technical assistance will be provided. The oversight and technical assistance roles will be managed to avoid conflicts of interest.

- Measurement: performance goals will be established, and tracking and trending systems will be implemented.

- Self-assessment: a continuous self-assessment process combined with periodic independent external review that meets all the criteria for an effective self-assessment program will be implemented.

- Total quality management: a philosophy of continuous improvement and dedication to excellence will serve as the umbrella under which elements are defined and implemented.

\section{FINANCIAL ASSUMPTIONS}

As a multiprogram laboratory, ORNL receives funding from many program sponsors, and each is responsible for providing adequate resources to ensure that its programs are conducted in a manner that protects health and safety and prevents environmental damage. Estimated project costs for corrective actions to address Tiger Team findings are allocated to various funding sources based upon the type of work to be completed and the expected beneficiaries. Each action plan includes a cost estimate by action and source for the corrective actions listed. Many actions required to address a finding establish new infrastructure that must be perpetuated to stay in compliance. One-time and annual ongoing costs are listed separately, with the understanding that ongoing costs must be continually supported with suitable escalation factors to maintain compliance with the requirement cited in the finding.

The cost estimates contained in this action plan are current best estimates and are generally planning-quality estimates rather than budget-quality estimates. DOE's Environmental Restoration and Waste Management Five-Year Plan is currently being revised. If there are discrepancies between the resource estimates for EM activities in this action plan and the validated cost estimates in the five-year plan, then the cost estimates in the five-year plan shall supersede those in this action plan.

All actions listed in this action plan are contingent upon suitable funding being provided. The schedules for actions listed as "funded" are current best estimates of expected completion. The projected completion dates for actions listed as "requested" or "new" are technically feasible dates based on work scope and available or projected resources. Actual completion dates will depend on when work is authorized and funding received.

The implementation strategy proposed for use at ORNL includes submitting to DOE a prioritized collection of proposed actions based on risk assessment, cost, and other factors each year as part of the normal DOE budget process. An annual operating plan will be prepared based upon the funding provided that allocates funds and assigns responsibility for each action to be undertaken. 
Expected results during the year and prioritization of remaining actions will be the basis for the development of proposals for subsequent year funding.

Actions to respond to the Tiger Team assessment represent only part of ORNL's ES\&H a tivities. ES\&H improvements started long before the Tiger Team arrived, and substantial resources have already been spent or committed to correct known problems. By definition, Tiger Team findings only cover deficiencies in the activities that were assessed. Underway activities that the Tiger Team found sufficient are not the subject of findings but must be continued until the problem is fully resolved. Also, most Tiger Team findings are narrowly drawn. For example, the majority of ORNL's facilities were constructed during a time when asbestos was a common material of construction. ORNL has committed substantial resources to its asbestos control program, and consequently there are no findings of a general nature that capture the cost of this long-recognized, expensive activity. Rather, there are two findings that relate to specific aspects of the asbestos program, one on controlling the use of new asbestos-containing materials (Finding WS.3-1) and one on designation of the asbestos disposal area (Finding A/BMPF-4). Consequently, Tiger Team costs must be considered in the context of ORNL's total ES\&H requirements.

\section{RESOURCE REQUIREMENTS}

ORNL is the oldest and largest of DOE's multiprogram research and development (R\&D) laboratories, with an estimated replacement cost in the range $\$ 7-10$ billion. About $63 \%$ of ORNL's facilities were constructed before 1960. DOE capital expenditures to upgrade and replace facilities have been a small fraction of normal industrial practice. The buildings, utilities, and equipment have now aged to the point where substantial increases in maintenance costs and decreases in reliability are being experienced for systems and facilities supporting R\&D efforts, and many facilities have not been upgraded to current health and safety standards. The low capital expenditure rate has been highlighted to DOE as a major institutional issue for many years. The Tiger Team concurred citing insufficient resources in two of its nine root causes. Combined with the unique environmental contamination problems at ORNL related to its original participation in the Manhattan Project, ORNL has accumulated a substantial ES\&H legacy.

Estimated project costs for corrective actions to address Tiger Team findings are allocated to various funding sources based upon two criteria: the type of work to be completed and the expected beneficiaries. Each action plan includes a cost estimate by action and source for the corrective actions listed. "Funded" project costs have already been spent or are currently available in an approved ORO financial plan. "Requested" project costs have been previously requested in a field work proposal (FWP), activity data sheet (ADS), or similar budget submission to DOE. "New" costs are not currently funded or have not been previously requested.

The total estimated cost of ES\&H requirements to meet DOE's zoal of full compliance with all ES\&H laws and regulations at ORNL is on the order of $\$ 1.5$ billion. It is highly unlikely that this large amount of money will be available over any near-term planning horizon. Consequently, prioritization is absolutely necessary to ensure that the most important problems are addressed first. Implementation will require careful allocation of available funding to achieve the best results with limited resources and to weigh Tiger Team actions against other ES\&H needs. 
Table S.1 shows a summary of the estimated cost to bring ORNL into compliance with current ES\&H laws, regulations, orders, and standards. Entries in the table listed as "to be determined" have not had work scope and cost estimates completed, but most are known to represent substantial additional costs. The planning effort to support this action plan has made a substantial contribution toward developing a comprehensive strategic plan for all ES\&H activities at ORNL.

Table S.2 shows a summary of the estimated costs for all actions required to fully address the findings of the ORNL Tiger Team assessment. The table shows a fiscal year breakdown of costs by type of cost and by funding source. The table also summarizes what portion of the funding listed is funded, requested, or new by year and funds category.

A number of key conclusions can be drawn from Table S.2. As stated above, the current estimate for bringing ORNL into compliance with current ES\&H laws, regulations, orders, and standards is on the order of $\$ 1.5$ billion. Actions related to the Tiger Team assessment total approximately $\$ 739$ million. The majority of action plan cost, $\$ 457$ million, is in three findings related to environmental restoration and waste management:

- SW/BMPF-4, Unrepaired Leaks from Wastewater Sewer Systems (\$229 million);

- GW/BMPF-5, Inadequate Characterization of the Hydrogeologic Regime (\$168 million); and

- GW/BMPF-1, Inadequate Well and Borehole Abandonment ( $\$ 60$ million).

All three findings had been identified previously and are included in the current Environmental Restoration and Waste Management 5-year plan. Next to the Office of Environmental Restoration and Waste Management, the Office of Energy Research, as the major funding sponsor of R\&D at ORNL, experiences the greatest programmatic cost associated with new ES\&H reçuirements.

Of the total estimated cost of $\$ 739$ million, only $\$ 78.9$ million is new costs that had not been previously identified and submitted to DOE for funding. As actions are completed, ongoing costs necessary to support the improvements in the future grow to around $\$ 16$ million per year.

Of the total estimated cost of $\$ 62.3$ million in FY 1991 needed to be fully responsive to the Tiger Team assessment, consisting of both one-time and ongoing costs, over $\$ 51$ million is already funded. These activities include Tiger Team-related activities that were already under way prior to the Tiger Team assessment as well as new tasks resulting from the Tiger Team assessment that have been funded in lieu of lower priority tasks and represent a substantial commitment of overhead and programmatic funds to ES\&H activities. Of the $\$ 11$ million shortfall for FY 1991, roughly $\$ 5$ million is needed to initiate high-priority activities to move toward compliance with ES\&H laws, regulations, orders, and standards. 


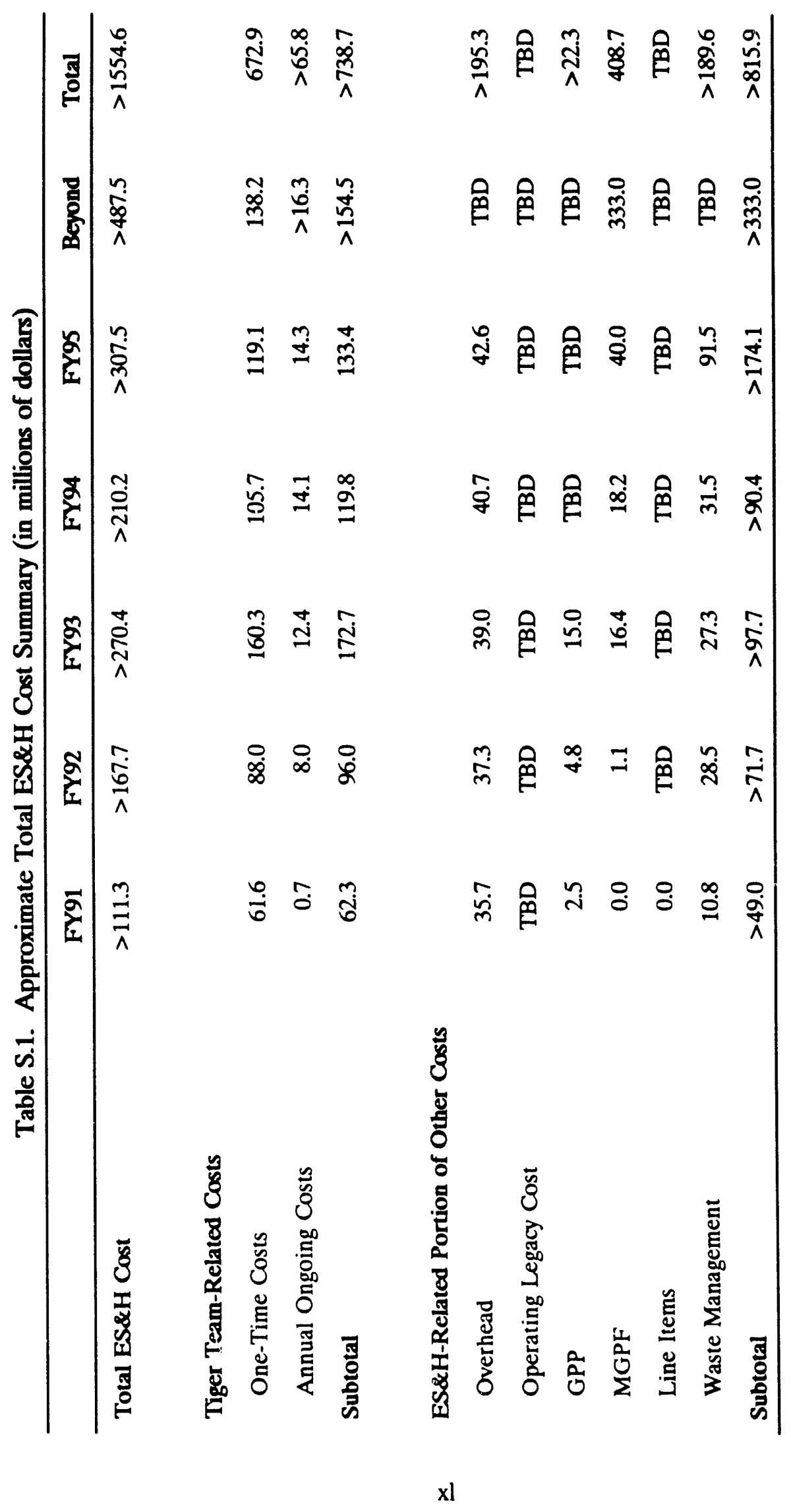




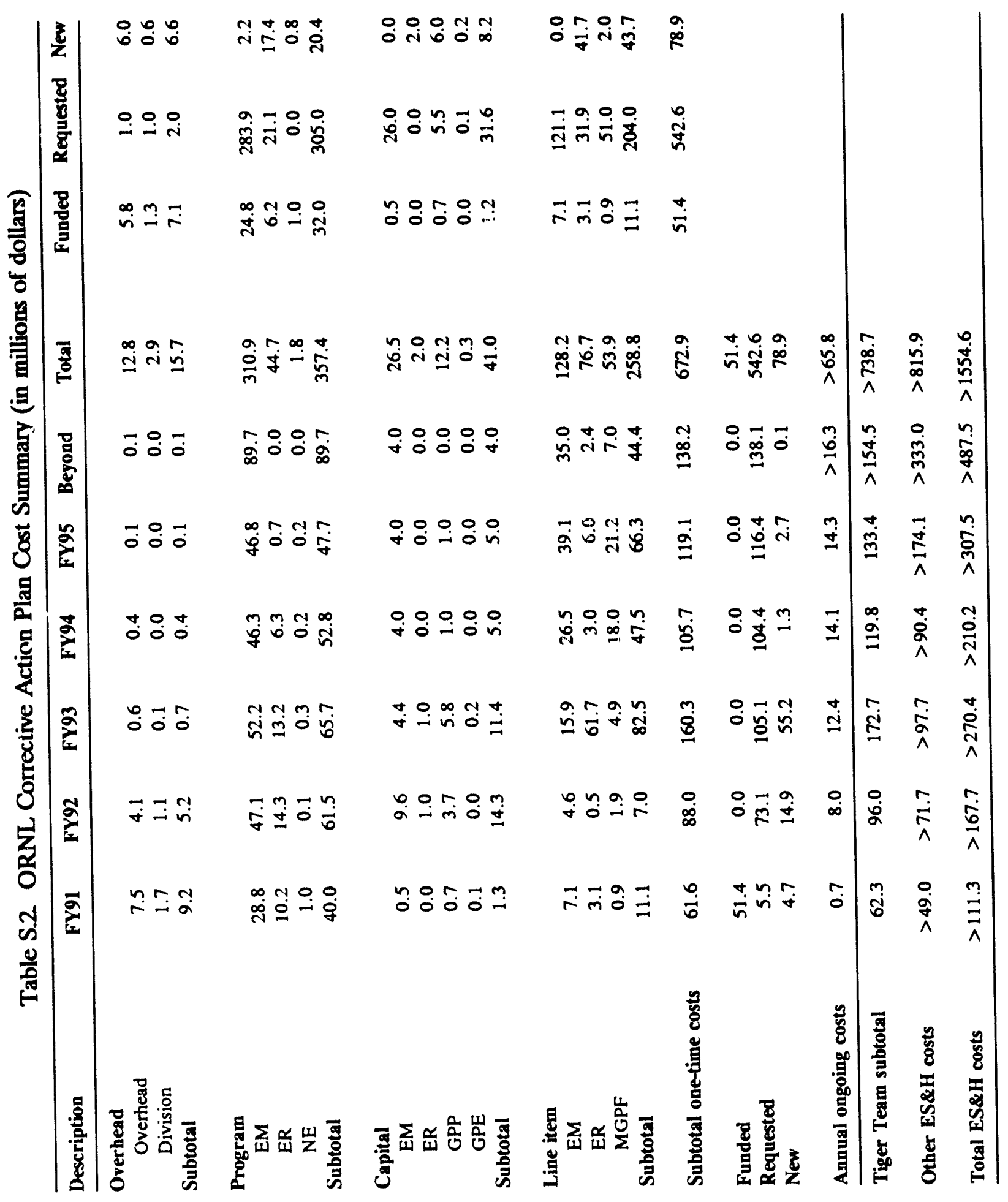




\section{THE CHALLENGE}

ORNL's involvement in radiochemical processing for the Manhattan Project led to historic environmental contamination problems, unique among the DOE multiprogram R\&D laboratories. Also, as the oldest and laigest of DOE's multiprogram laboratories and lacking adequate resources for capital improvements, ORNL not surprisingly has substantial problems in meeting current health and safety standards. However, one resource that ORNL is not lacking is a highquality, motivated staff. ORNL has a long and outstanding record of national R\&D leadership in the biomedical, health and safety, and environmental sciences and has DOE's largest single R\&D program in those areas. The Tiger Team acknowledged that ORNL has a wealth of talent from which to draw, that improvements have been significant, and that pockets of excellence exist in some areas. The challenge stated by the Tiger Team for ORNL is to prove that the improvement can be sustained-a challenge accepted with this action plan. 


\section{INTRODUCTION}




\section{INTRODUCTION}

\subsection{BACKGROUND}

On June 27, 1989, Secretary of Energy James D. Watkins announced a ten-point initiative to strengthen safety, environmental-protection, and waste-management activities at the U.S. Department of Energy's (DOE's) production, research, and testing facilities. In support of the ten-point initiative, the Secretary established independent Tiger Teams to conduct environmental compliance assessments at DOE facilities. The assessments are on-site, independent reviews of DOE environmental, safety, and health (ES\&H) programs to ensure compliance with applicable federal, state, and local regulations; permit requirements; agreements, orders, and consent decrees; and DOE orders. In addition, the Tiger Teams assess DOE operations for conformance with applicable "best" and "accepted" industry practices and for the adequacy of DOE and site contractor management programs.

A Tiger Team assessment was conducted at the Oak Ridge National Laboratory (ORNL) and the DOE Oak Ridge Operations Office (ORO) between October 22, 1990, and November 30, 1990. The assessment was conducted by a team of over 80 specialists from various DOE offices and subcontractor organizations. The team was directed by a senior DOE manager, John R. Patterson, Deputy Director of the Savannah River Special Projects Office. Four subteams comprised the Tiger Team: environmental, site-wide technical safety appraisal (TSA), reactors TSA, and management. A report, U.S. Department of Energy Environment, Safety, and Health Tiger Team Assessment, Oak Ridge National Laboratory, DOE/EH-0148 (Draft), November 1990, documents the findings of the Tiger Team.

Two separate TSA subteams worked on-site during the ORNL assessment, evaluating sitewide and reactor operations according to a standard set of TSA performance objectives and criteria. Consequently, many findings in the sitewide TSA portion of the Tiger Team report have duplicate finding numbers to findings in the reactors TSA portion of the report. To help minimize confusion, all reactors TSA findings are preceded by an " $R$ " to distinguish them from sitewide findings with the same number. For example, Finding EP.5-1 refers to a sitewide TSA finding, and Finding REP.5-1 refers to a reactors TSA finding. Also, the term "finding" designates the conclusion drawn from a number of observations, encompassing both the term "finding" used by the environmental and management subteams and the term "concern" used by the TSA subteams.

\subsection{PURPOSE AND SCOPE OF THE ACTION PLAN}

This action plan provides a formal response to each of the findings cited in the Tiger Team assessment report. The action plan describes the actions pianned to satisfy the findings, action schedules and milestones, and associated costs; it also identifies the parties responsible for implementation. The document also identifies actions and costs that are included, or planned for inclusion, in DOE's Environmental Restoration and Waste Management Five-Year Budget Plan Fiscal Years 1992-1996, DOE/S-0078P, June 1990. 


\subsection{ORGANIZATION AND CONTENT OF THE ACTION PLAN}

The action plan includes four sections. Section 1 provides background on the Tiger Team assessments, outlines the purpose and scope of the action plan prepared in response to the Tiger Team assessment report, describes the methodology used to produce the action plan, describes the ORNL site, outlines key financial assumptions, and presents the risk-based system used to prioritize findings and actions. Section 2 describes the principal parties and their roles in implementing the plan. Section 3 encompasses six subsections: (1) Root Causes;

(2) Environmental Findings, Responses, and Planned Actions; (3) Sitewide Safety and Health Findings, Responses, and Planned A.ctions; (4) Reactors Safety and Health Findings, Responses, and Planned Actions; (5) Management Findings, Responses, and Planned Actions; and (6) SelfAssessment Findings, Responses, and Planned Actions. Each of the last five subsections includes planned actions, schedules for implementing the actions, and associated costs for addressing those findings. Section 4 summarizes the action plan, including planned actions, schedules, and costs, and provides a 5-year plan of budgets for the planned actions.

\subsection{ACTION PLAN METHODOLOGY}

ORNL is the fourth DOE facility operated by Martin Marietta Energy Systems, Inc. (hereafter referred to as Energy Systems), that has undergone a Tiger Team assessment. Many of the findings identified during the Tiger Team assessment are similar to those identified at the other sites. As described in the ORNL Tiger Team assessment report, a Tiger Team assessment is a "snapshot in time." Improvements that are under way or planned but not completed are identified as findings to ensure that the assessment documents a complete and accurate status of the site's condition.

In response to previous audits and Tiger Team assessments, Energy Systems has instituted a number of initiatives across all its facilities to improve management and tracking systems, more clearly define roles and responsibilities, improve communications, clarify policies, and improve policy implementation. In many cases, the Tiger Team acknowledged that progress has been made but that implementation is not yet complete. The ORNL action plan must be considered in the context of these corporate-wide initiatives; every attempt is being made to ensure that actions executed at various levels in the organization are integrated and consistent (see Sects. 2.2 and 3.1).

A fundamental premise of the Tiger Team prociss is that action plans must address the root causes and not just the symptoms of a problem. It is very easy to lose sight of this objective when faced with the prospect of addressing 413 individual findings. The results of the ORNL Tiger Team assessment, previous audits of ORNL, and Tiger Team assessments of other Energy Systems sites and other DOE installations were carefully considered in identifying 11 root causes that appear to explain the findings identified during the ORNL Tiger Team assessment.

Section 3.1 describes the 11 root causes.

This action plan used a matrix approach to identify common elements and to ensure consistency in determining root causes. All action plans in a given assessment area were reviewed by discipline experts to ensure (1) that the proposed actions were consistent in technical approach and (2) to 
identify common elements. In addition, a crosscutting review scrutinized all action plans attributable to a particular root cause as a group to ensure consistency in root causes. Consequently, many action plans contain cross-references to other findings that address similar problems.

\subsection{SITE DESCRIPTION}

ORNL is a multiprogram energy research and development (R\&D) laboratory situated on a number of sites on the Oak Ridge Reservation. Figure 1.1 shows a map of the main ORNL site. The total land area used by ORNL approaches 26,680 acres. The Laboratory proper encompasses 330 acres, and outlying facilities and waste-management storage areas include another 1135 acres. A total of 19,500 acres is mianaged to protect renewable resources for programmatic requirements, and 13,590 acres are assigned for ecological study. Remaining areas are used for other special environmental studies.

ORNL possesses the oldest physical plant of any DOE laboratory. About one-third of ORNL's total existing building area is over 40 years old. Only $37 \%$ of ORNL's facilities have been constructed since 1960, compared with 45 to $61 \%$ for all other energy R\&D laboratories.

ORNL occupies 306 buildings, totaling approximately 3.8 million square feet of gross building area. Over two-thirds of this building space (about 2.6 million square feet) is located at the main Bethel Valley site and the adjacent Melton Valley site. More than 1 million square feet is located at the Oak Ridge Y-12 Plant, and the remaining 200,000 square feet is at the K-25 Site. A few buildings are located in the remote areas south of Melton Valley. The types of buildings range from accelerators and reactors to laboratories, offices, and support structures.

The Laboratory's Bethel Valley area can be divided into five distinct building groups: (1) the Life Sciences Complex (1000 and 1500 areas) at the west end of the site; (2) the Laboratory's Initial Development (2000, 2500, 3000, and 3500 areas) just east of the Life Sciences Complex; (3) the Central Research Complex (4000, 4500, 5000, and 5500 areas) in the center of the site; (4) the Physics Complex (6000 area) to the east of the Central Research Complex; and (5) Support Services (7000 area) at the far east end of the site. The Melton Valley area contains buildings clustered in several widely separated locations. The two major locations are the High Flux Isotope Reactor area (7900 area) and the Consolidated Fuel Reprocessing Program (7600 area). The remaining major structures in Melton Valley are inactive reactors (awaiting decontamination and decommissioning) and their support buildings. South of Melton Valley are two experimental reactor facilities: the Health Physics Research Reactor and the Tower Shielding Facility. Each of these facilities contains a cluster of smaller facilities.

Most ORNL buildings at the Y-12 Site were built during World War II to house uraniumenrichment processes or their support activities. Several smaller structures were added in the 1960s and 1970s to house offices, laboratories, and support equipment. Although the Laboratory's facilities are commingled with those at the Y-12 Site, four relatively distinct areas can be identified: (1) the Biology Complex, (2) the Engineering Technology facilities, (3) the Fusion Energy facilities, and (4) the Isotope Separation Facility. Figure 1.2 shows a map of the Y-12 Site. 


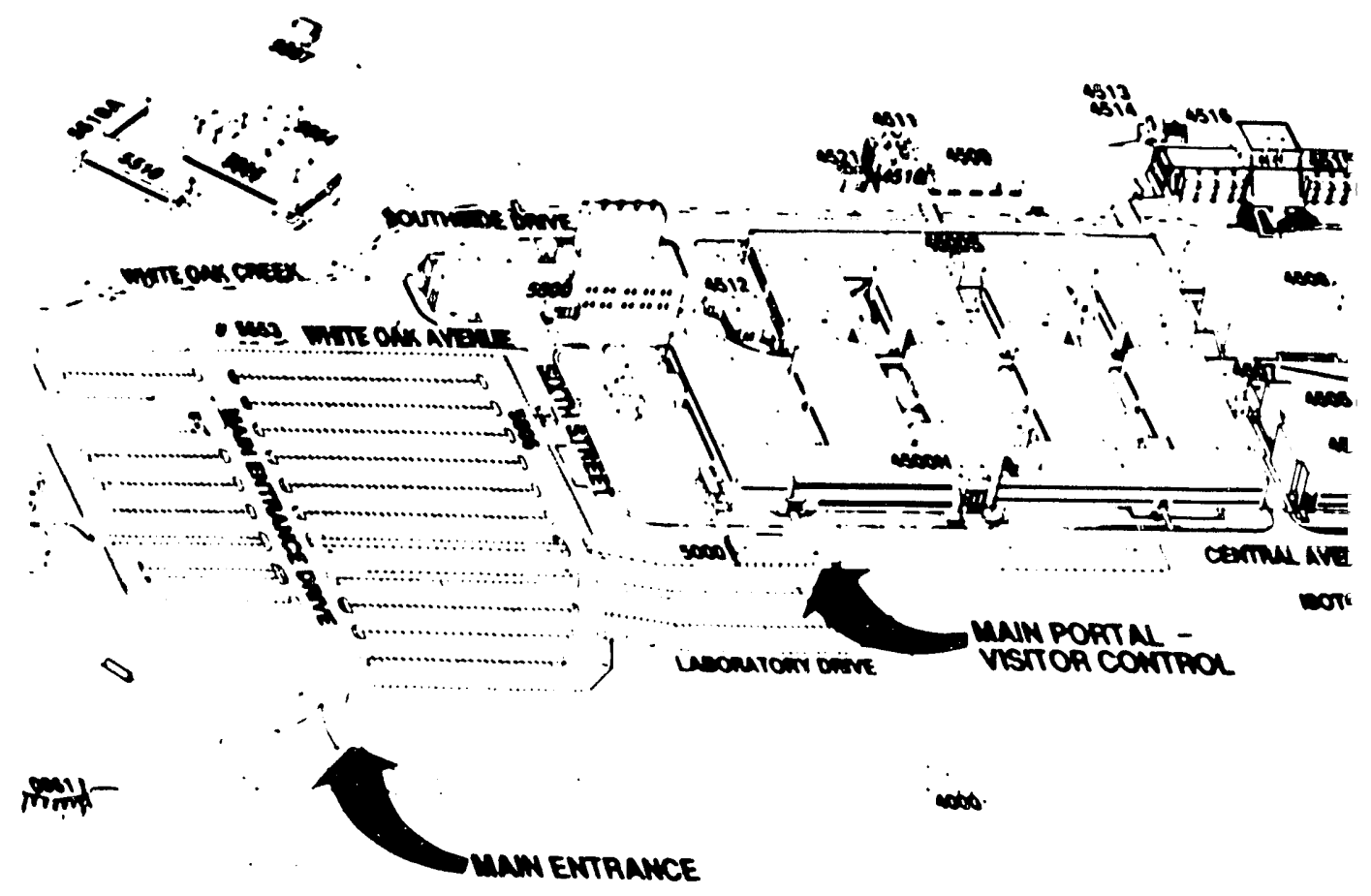

Fig. 1.1. Oak Ridge National Laboratory. 


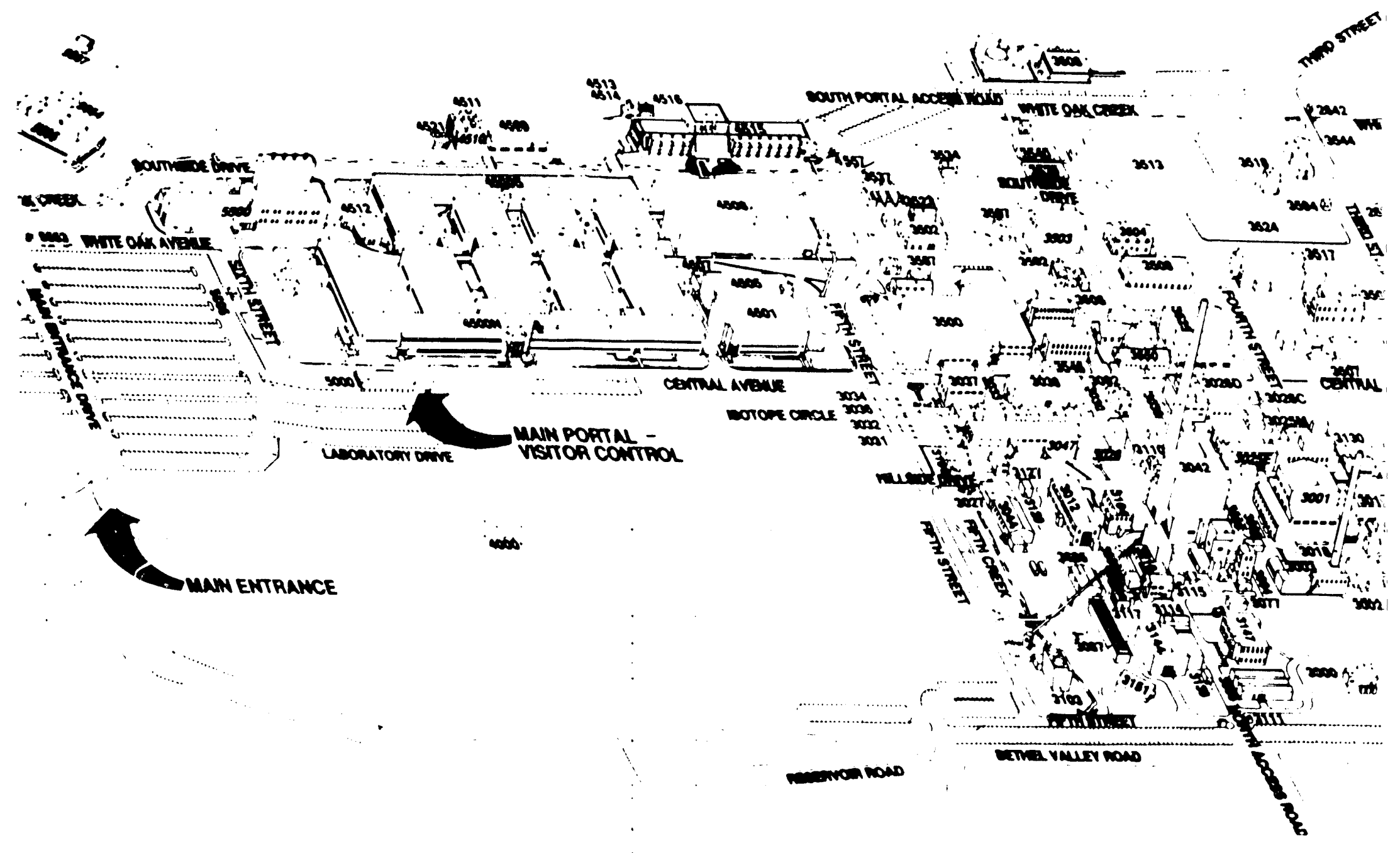

Fig. 1.1. Oak Ridge National Laboratory. 


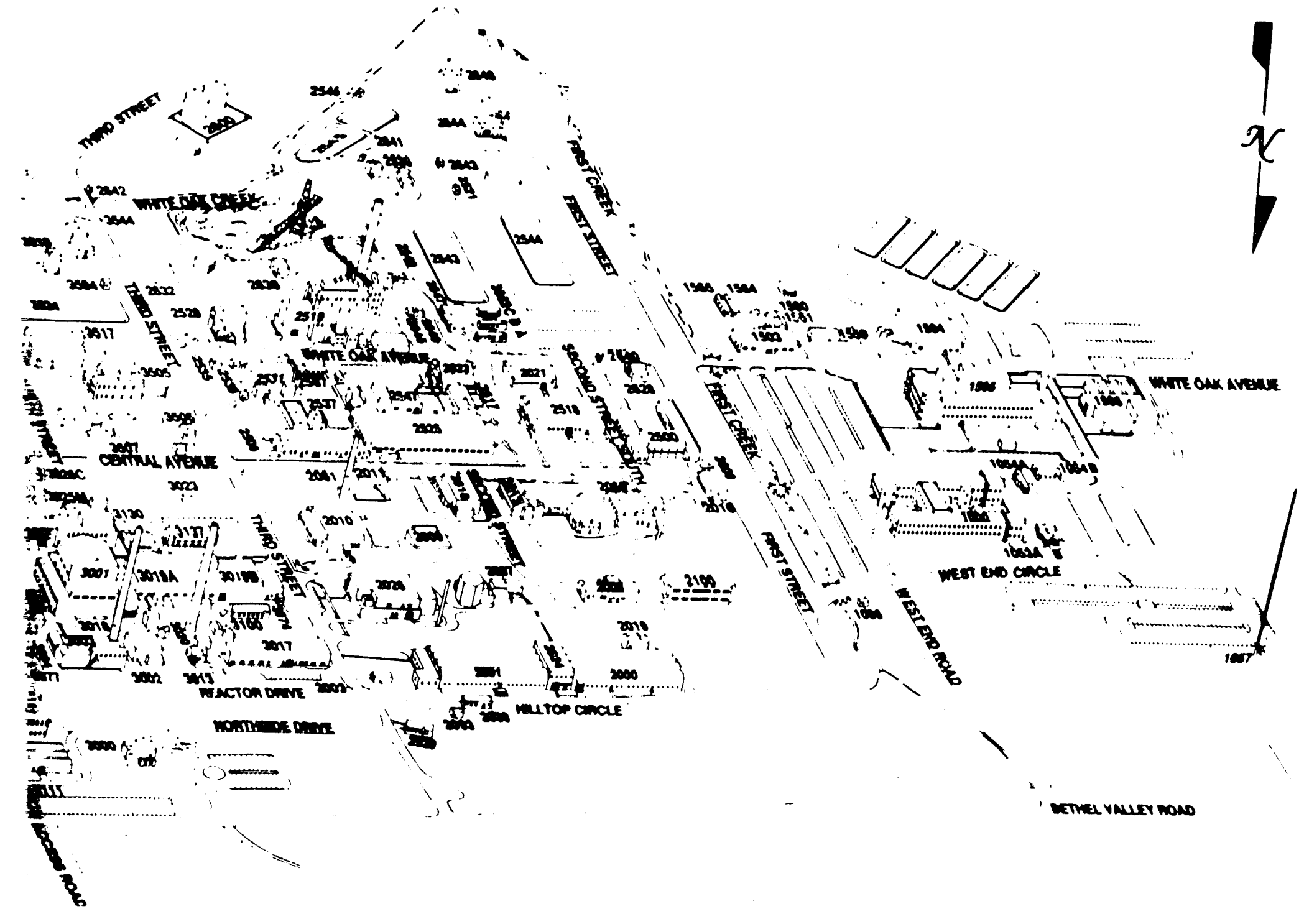




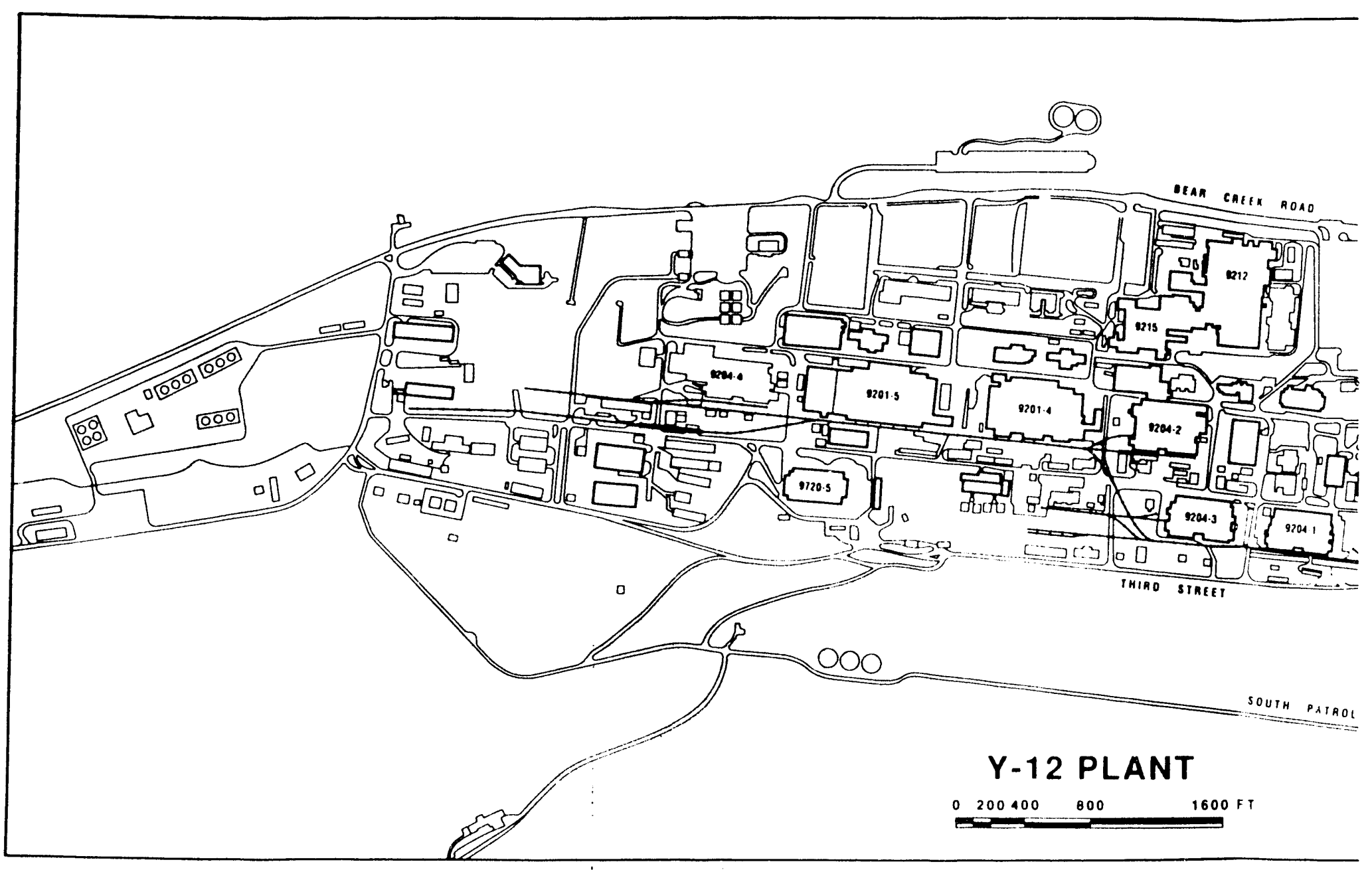

Fig. 1.2. Oak Ridge $Y-1 Z$-Plant. 


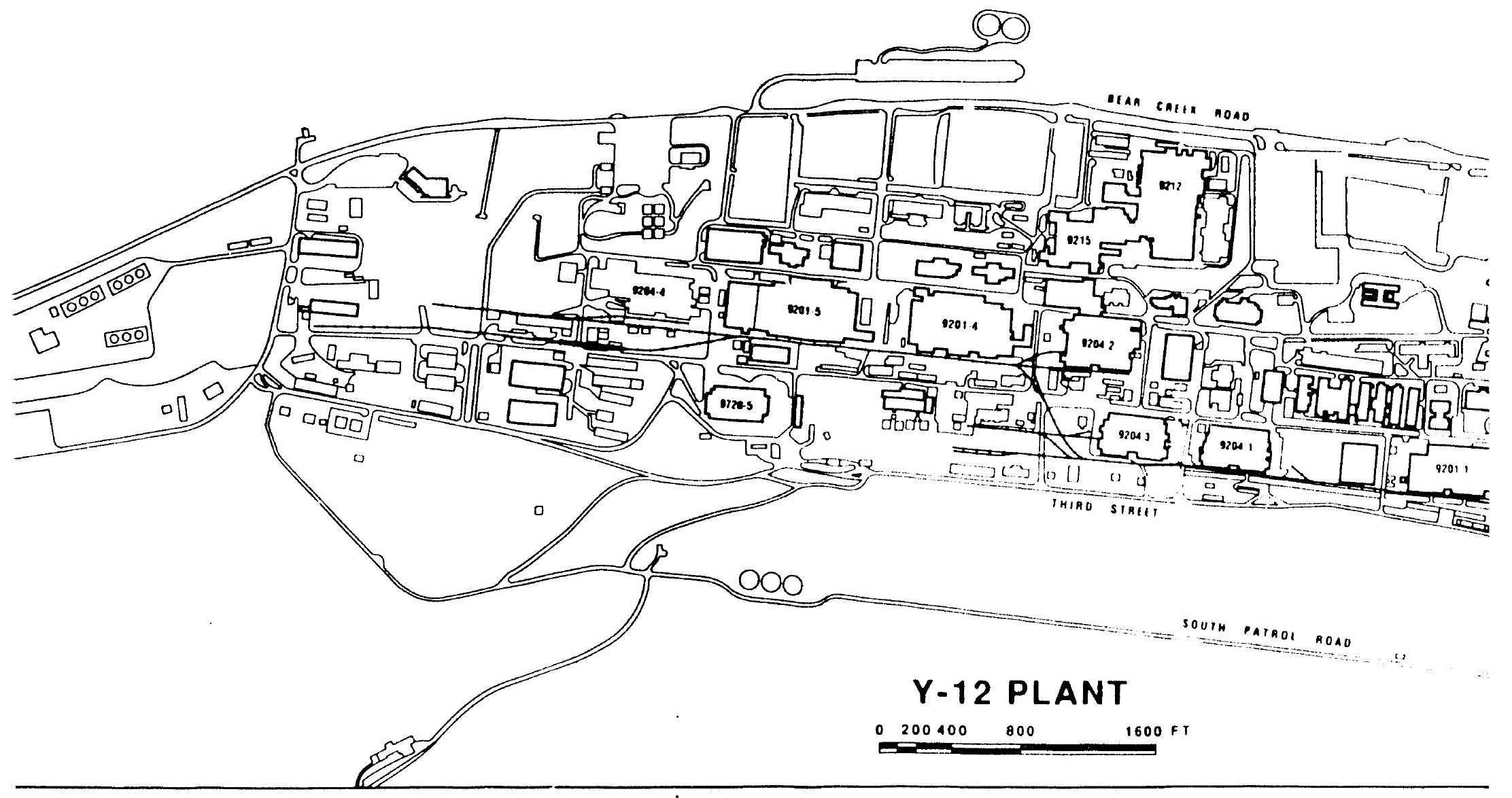

Fig. 1.2. Oak Ridge Y-12 Plant. 
ORNL's primary facility at the K-25 Site is the Applied Technology Division, engaged in the development of polymer composites and rotating mechanical systems. Several other ORNL activities of the Chemical Technology Division are housed at various locations within the K-25 Site. ORNL facilities at K-25 were not reviewed during the ORNL Tiger Team assessment but will be included in the Tiger Team assessment of the K-25 Site, currently scheduled for 1991.

\subsection{FINANCIAL ASSUMPTIONS}

As a multiprogram laboratory, ORNL receives funding from many program sponsors. Each sponsor is responsible for providing adequate resources to ensure that its programs are conducted in a manner that protects health and safety and prevents environmental damage.

Estimated project costs for corrective actions to address Tiger Team findings are allocated to various funding sources based upon two criteria: the type of work to be completed and the expected beneficiaries. Each action plan includes a cost estimate by action and source for the corrective actions listed.

The cost estimates contained in this action plan are current best estimates and are generally planning-quality estimates rather than budget-quality estimates. DOE's Environmental Restoration and Waste Management Five-Year Plan is currently being revised. If there are discrepancies between the resource estimates for EM activities in this action plan and the validated cost estimates in the five-year plan, then the cost estimates in five-year plan shall supersede those in this action plan.

"Funded" project costs have already been spent or are currently available in an approved ORO financial plan. "Requested" project costs have been previously requested in a field work proposal (FWP), activity data sheet (ADS), or similar budget submission to DOE. "New" costs are not currently funded or have not been previously requested.

A single action plan or individual action item may require funding from several sources. For example, procedures may be developed by overhead personnel and implemented by an individual program or division (via training, equipment purchase, and upgrade of facilities).

Proper consideration of ongoing costs poses a major dilemma: many required actions establish new infrastructures at ORNL that will require funding long after a Tiger Team action has been closed. For example, to fully address Finding EP.5-1, an emergency field monitoring team must be organized, equipped, and trained. Once the team is fully functional, the finding has been fully addressed. However, the emergency field-monitoring team must be perpetuated to stay in compliance. In this action plan, one-time and annual ongoing costs are listed separately, with the understanding that ongoing costs must be continually supported and suitable escalation factors must be included to maintain compliance with the requirement cited in the finding. A 4.5\%/year escalation factor has been used for cost estimates contained in this action plan.

Documentation relating to the calculation of estimates has been retained. Items such as personnel estimates and materials usage are documented with the ralculations and methodology used to 
arrive at the estimate, including rationales such as prior-year experience and professional judgement.

\subsubsection{Research Programmatic Funds}

Programmatic funding is the preferred category if a specific sponsor or program can be identified. Research programmatic funds include all project costs for which the benefit of the project accrues to one final cost objective or one program. Each activity is evaluated to determine which DOE program (i.e., FWP/Project) will receive the direct benefit if the Tiger Team issue is corrected, and costs are budgeted to that program as appropriate. Examples include development of safety analysis reports (SARs) for a single program facility and environmental compliance costs for a program's operation such as National Environmental Policy Act (NEPA) documentation and Resource Conservation and Recovery Act (RCRA) requirements. If the costs cannot be identified within an existing FWP, then the costs are added to the upcoming FWPs and scheduling is based on the funds being available in FY 1993. If an action is critical and requires funding in FY 1991 or FY 1992, funding needs are prioritized and funds are requested. As an example, the shutdown B-reactor funding should be programmatic, and direct funding has been requested from the previous sponsor(s) rather than using overhead funds. These and other legacy problems constitute a major funding dilemma. Progammatic sponsors see no value from money spent on legacies, and the use of overhead is inappropriate.

\subsubsection{ES\&H and OSHA Programmatic Funds}

FWPs are being submitted to cover the cost to upgrade programs and facilities to reach compliance. These FWPs are not intended to fund ongoing, routine ES\&H compliance activities, but rather to fund one-time upgrades to achieve compliance or to provide critical funding to facilities without an identifiable sponsor. In most cases, these costs represent fixes to multiprogram or sitewide compliance problems, and multiple FWPs will be prepared for these tasks. These follow-ons to the two compliance FWPs submitted for FY 1991 provide opportunities to request funding in critical cases for activities during FY 1991 through FY 1993, with FY 1993 having the highest probability of funding. An individual FWP will be prepared and submitted to the site landlord for each of the following tasks.

Safety Documentation. This task supports the development of safety documentation (SARs and Operational Safety Requirements) of ORNL facilities that do not have an identifiable program sponsor or that require critical funding.

Configuration Management. DOE Order 5481.1B stipulates that SARs include a detailed comparison of the current plant configuration against current DOE design criteria, highlighting and explaining any deviations. This task will meet this requirement as well as "as-built" drawings of all safety systems as required by DOE/OR-901 through development of the configuration management program and assistance for facilities that lack an identifiable program sponsor or that require critical funding. 
Naturai Phenomena Analysis. This task analyzes hazardous facilities to identify vulnerabilities to natural phenomena and disasters (earthquakes, tornadoes, and floods).

Emergency Preparedness. This task provides the required support to allay concerns involving personnel accountability, protection of response personnel, protective-action guides, predetermined emergency-response levels, and emergency alarm/notification systems. These tasks are one-time upgrades of ORNL's Emergency Preparedness Program. Examples include a design for the expansion of the Emergency Operations Center and the acquisition of emergency equipment.

Radiation Protection. This funds activities such as the ORNL as low as reasonably achievable (ALARA) program, including implemention of ORO's Contamination Control Policy and establishing a tracking ind trending program for radiation incidents; radiation characterization of areas (including buildings) adjacent to but not controlled by operations involving the presence of radioactive materials; surveys of roadways, walkways, and parking lots; establishing a program for the contamination survey of vehicles accessing/egressing ORNL; a metrology system; records management; and other radiation protection programs.

NEPA Regulations. This task provides ORNL staff support and information to outside parties in the preparation of reservation-wide environmental documentation as required by SEN-15-90.

Environmental Compliance. This task is necessary to ensure a comprehensive and rigorous environmental compliance program at the Laboratory to achieve full compliance with all applicable orders and state and federal regulations.

Industrial Hygiene. This task provides resources necessary to address ORNL Industrial Hygiene compliance with regulatory and DOE guidelines. Examples are procedures upgrades and other projects necessary to comply with regulatory and DOE requirements and a sitewide evaluation for compliance status. Also included are localized asbestos abatement, indoor air-pollution studies, development of protocols for monitoring and managing hazardous materials, and recordsmanagement/data-management methods development to enhance the Industrial Hygiene section's ability to track and ensure quality data.

Training Accreditation. This task provides resources that will concentrate on the accreditation of ES\&H-related positions and reactor-related technical positions as well as design of a comprehensive, accredited training program for ORNL.

OSHA. This task funds activities to bring ORNL into compliance with OSHA requirements. It supports program management; long-range planning; installation of equipment for walking/working surfaces; improvements in egress, health, and environmental signs and protective equipment; installation of hazardous material protective equipment; accident-prevention signs; upgrade of the fire extinguisher program; machine guarding; general electrical improvements; and air-contaminants evaluation and sampling (including asbestos). 


\subsubsection{Environmental Restoration and Waste Management Funds}

Under a Memorandum of Understanding (MOU) among DOE line programs, the Office of Environmental Restoration and Waste Management (EM) provides programmatic oversight, program direction, and significant funding for environmental restoration and waste management (ERWM) actions at ORNL. Activities that fall under the jurisdiction of EM include corrective actions; environmental restoration including remedial actions, decontamination, and decommissioning; waste operations; and technology development related to restoration and cleanup. DOE's plans for each of its sites are described in the report Environmental Restoration and Waste Management Five-Year Plan, Fiscal Years 1992-1996, DOE/S-0078P, June 1990. Specific projects are described in ADSs. If a required action is part of the current 5-year plan, the ADS number is cited. If the action falls within the scope of EM and is not contained in the current 5-year plan, it is listed as a new activity and will be submitted for inclusion in the next 5-year plan.

\subsubsection{Division Administration: Overhead Funds}

Division Administration includes project costs for which the benefit of the project accrues to one division but cannot be specifically linked to a single program within that division. Examples include additional personnel resources applied to division ES\&H activities (procedures writing, monitoring, assistance) and purchase of minor safety equipment for division personnel. This category is not applicable to nonprogrammatic divisions (i.e., divisions that are funded with overhead dollars).

\subsubsection{Laboratory Overhead Funds}

Overhead funding should not be used as an alternative funding source. Several tests must be met to qualify for overhead funding. Specifically, overhead-funded projects should be ongoing, base (i.e., routine) activities that benefit multiple programs and multiple divisions. Examples include funding for the ongoing activities of the ES\&H Compliance offices, Plant and Equipment, and Medical.

\subsubsection{Capital Equipment}

Capital equipment is defined as equipment having a cost exceeding $\$ 5000$ and a useful life of more than 2 years. Capital equipment includes all costs incurred in the acquisition or fabrication of capital equipment not related to construction projects for additions or replacements, including any necessary installation and transportation costs. It also includes the removal costs of demolishing, dismantling, tearing down, or otherwise removing equipment associated with an eq lipment project. Sources for these funds are programmatic with General Purpose Equipment (GPE) funds identified as Energy Research Budget and Reporting Numbers. 


\subsubsection{GPP and Line-Hem Projects}

General Purpose Projects (GPP) and line-item projects include all costs incurred on projects and subprojects involving the design, construction, installation, or other acquisition of land, property rights, buildings, structures, equipment, utility lines, roads, and other facilities. It also includes betterments, additions, and replacements of plant and equipment and the removal cost less salvage in the retirement of plant and equipment. GPP cost between $\$ 5000$ and $\$ 1.2$ million. Projects exceeding the $\$ 1.2$ million limit must be reviewed by Congress on a line-item basis.

\subsection{PRIORITIZATION AND TRACKING}

The efforts to respond to the 1990 Tiger Team findings build on a continuing initiative at ORNL to improve and formalize the methods that introduce new commitments into the system and allocate or plan for allocation of resources. This initiative, known as the Resource Management System, is depicted in Fig. 1.3. New findings and issues raised by audit and review teams are prioritized using a risk-based methodology developed by Energy Systems for use at all five Energy Systems sites (described in Appendix A of this report). This process stems from work pioneered by the ORNL Research Reactors Division and is a good example of implementing lessons learned.

A team of top-level ORNL managers and key ES\&H personnel, chaired by the Director of the Office of Environmental and Health Protection, prioritized the Tiger Team findings. The findings reported by the Reactor TSA team were prioritized separately by the Research Reactors Division Technical Evaluation Group, chaired by the division director. Figure 1.4 shows the distribution of weights for all 413 Tiger Team TSA, environmental, and management findings. Section 4 discusses the consequences of this risk distribution. Following prioritization, the findings were distributed to the appropriate managers for development of the actions plans.

The Tiger Team process specifies that each activity be prioritized using the Tiger Team Action Prioritization System. This system assigns a priority of $1,2,3$, or 4 to each activity using the following definitions.

Priority 1. Actions necessary to prevent significant risk to the public, worker health or safety, or the environment, whether or not they are required by statute or DOE orders. This priority also includes all compliance agreements and corrective activities necessary to prevent near-term adverse impacts to the public, worker health or safety, or the environment.

Priority 2. Actions necessary to meet statutes and DOE orders, although lack of action would not result in a significant risk to the public, worker health or safety, or the environment. This priority also includes those activities required to meet the terms of compliance agreements (in place or in negotiation) between DOE and local, state, or federal agencies, although lack of action would not result in near-term adverse impacts to the public, worker health or safety, or the environment. 


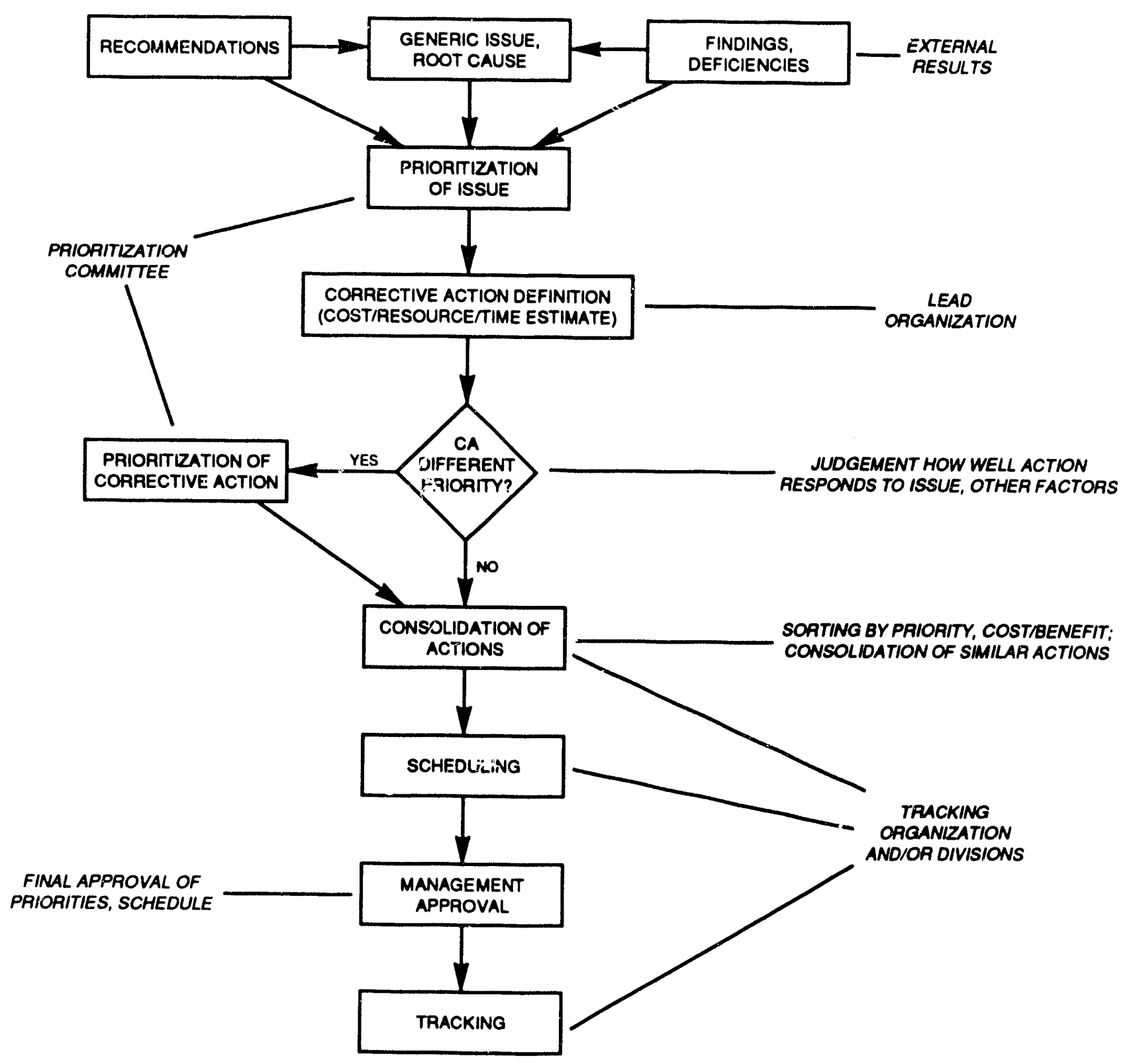

Fig. 1.3. ORNL Resource Management System. 


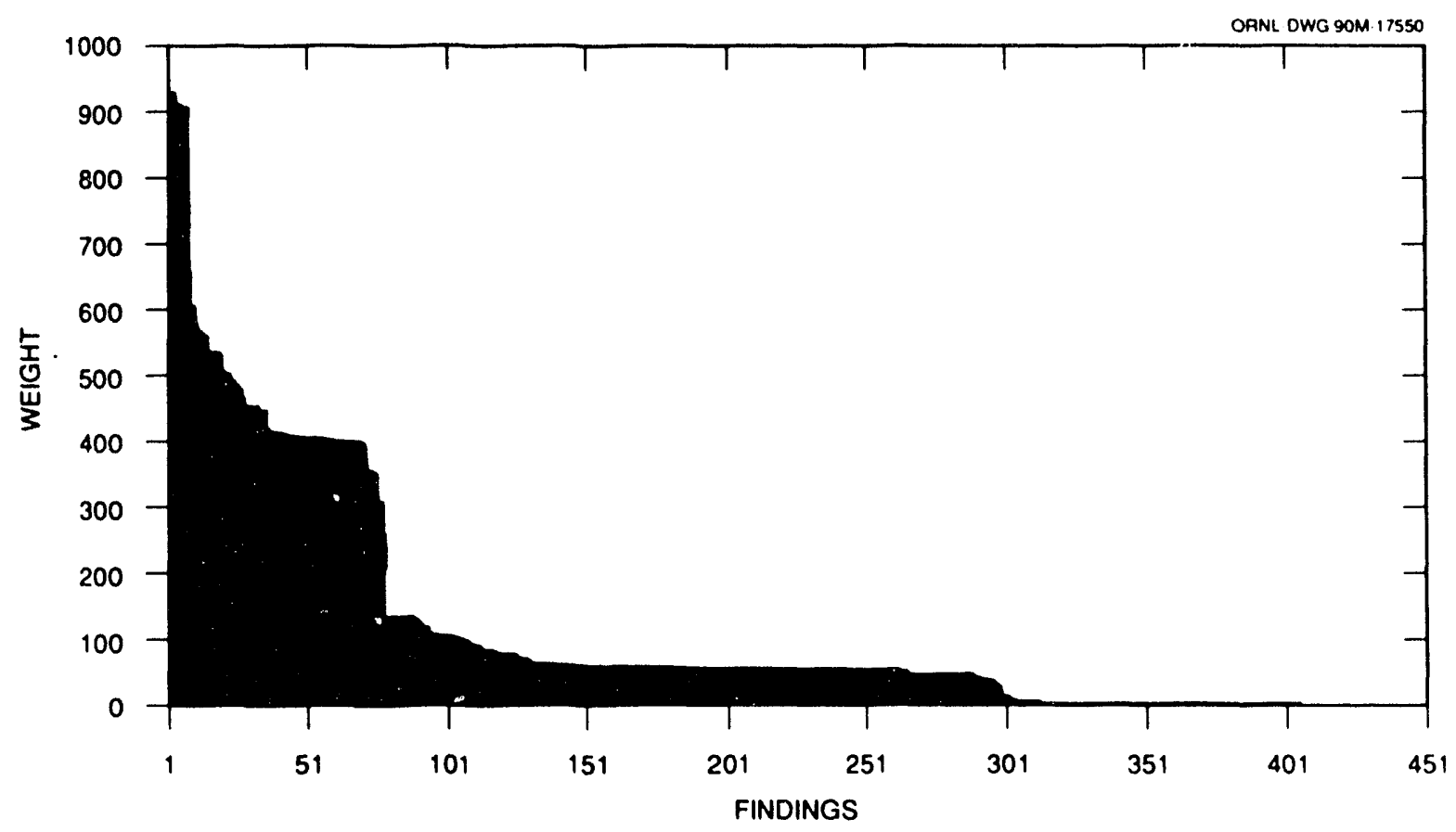

Fig. 1.4. Distribution of weights for all Tiger Team findings.

Priority 3. Actions consistent with Best Management Practices. This priority also includes regulatory activities not captured under priorities 1 or 2 including compliance with DOE orders that implement external regulations or that set specific DOE regulatory standards, actions that would reduce risks or costs, or actions that would prevent disruption of the DOE production mission.

Priority 4. Actions not required by law, regulation, or agreement but that would be desirable to accomplish.

When these definitions were applied to the Tiger Team findings at ORNL, 7 findings were determined to be Priority 1; 219 Priority 2; 159 Priority 3; and 28 Priority 4.

Consequently, each action plan listed in Sect. 3 has been prioritized using both the Energy Systems Resource Management System and the Tiger Team Action Prioritization System. The Energy Systems risk weight and Tiger Team action plan priority are listed on each finding. The two prioritization systems produce substantially similar results. For example, the 7 Priority 1 findings all have risk weights greater than 600 . The only other finding with a risk weight greater than 600 was Finding NEPA/CF-1, Inefficient DOE NEPA Implementation Procedures, with a risk weight of 658 which derives from a combination of being a compliance with law issue together with having a substantial impact on business performance. Consequently, the Energy Systems risk weight can be viewed as a means to rank actions within the 4 major priorities of the Tiger Team Action Prioritization System. 
The findings and related corrective actions will be tracked via a PC-based system, the Evaluation Database System. The ORNL Quality Department is responsible for monitoring progress and reporting monthly to line and ES\&H managers on the status of Tiger Team items as well as other key ES\&H findings. In November 1990, ORNL began meeting with the Performance Evaluation Committee (PEC) of DOE-ORO to discuss significant accomplishments during the month, key issues, and corrective-action status. In addition, the ORNL Quality Department prepares a quarterly status report on ES\&H/QA audits, reviews, and appraisals and related corrective actions. These reports cover internal as well as external audits. The quarterly reports are previewed by the ORNL ES\&H Coordinating Committee prior to presentation to the Energy Systems Technical Evaluation Committee, which is chaired by the vice president for Technical Operations.

Tracking and reporting efforts should be significantly facilitated in the summer of 1991 with the introduction of a new mainframe system, Energy Systems Action Management System (ESAMS). This system will be made available to all five Energy Systems sites to track key issues, findings, and corrective actions and to share information and lessons learned. Representatives from ORNL's ES\&H organization and the Research Reactors Division have played a key role in the design development and critical design reviews of ESAMS.

Closure of all corrective actions is independently verified by a professional QA specialist. Documentation of closure is maintained in the Central Quality Department audit tiles for external audits including the DOE Tiger Team. The corrective action management process is described in ORNL QA procedure, QA-L-16-102, "Corrective Actions."

This revision of the action plan lists many actions that have been completed. Because the action plan has not yet been approved and is still subject to change, efforts have not yet been initiated to verify completion. Consequently, no actions in the plan are considered closed.

ORNL strongly believes that the responsibility for corrective-action implementation lies with line and support organizations. The Quality Department provides the independent monitoring, verification, and reporting that are essential elements of a strong, effective, auditable system. The corrective-action management system is routinely audited by Energy Systems and external audit teams, as well as the independent safety appraisal system at ORNL. The organizations responsible for corrective-action monitoring of all items including Tiger Team findings are shown in Fig. 1.5. This process is one of the important ingredients of the overall self-assessment program currently being upgraded at ORNL to fully meet the intent of the recent guidance provided by the Secretary of Energy.

\subsection{IMPLEMENTATION STRATEGY}

As discussed in Sect. 4 of the report, the total estimated cost of ES\&H requirements to mee: DOE's goal of full compliance with all environmental and safety laws and regulations at ORNL is on the order of $\$ 2$ billion. It is highly unlikei, that this large amount of money will be available over any near-term planning horizon. Consequently, prioritization is absolutely necessary to ensure that the most important problems are addressed first. Implementation will require careful allocation of available funding to achieve the best results "rith limited resources and to weigh Tiger Team actions against other ES\&H needs. The pricritization process is not static, and priorities will change as previous problems are solved or new problems arise. 
ORNL-OWG 90M-1755

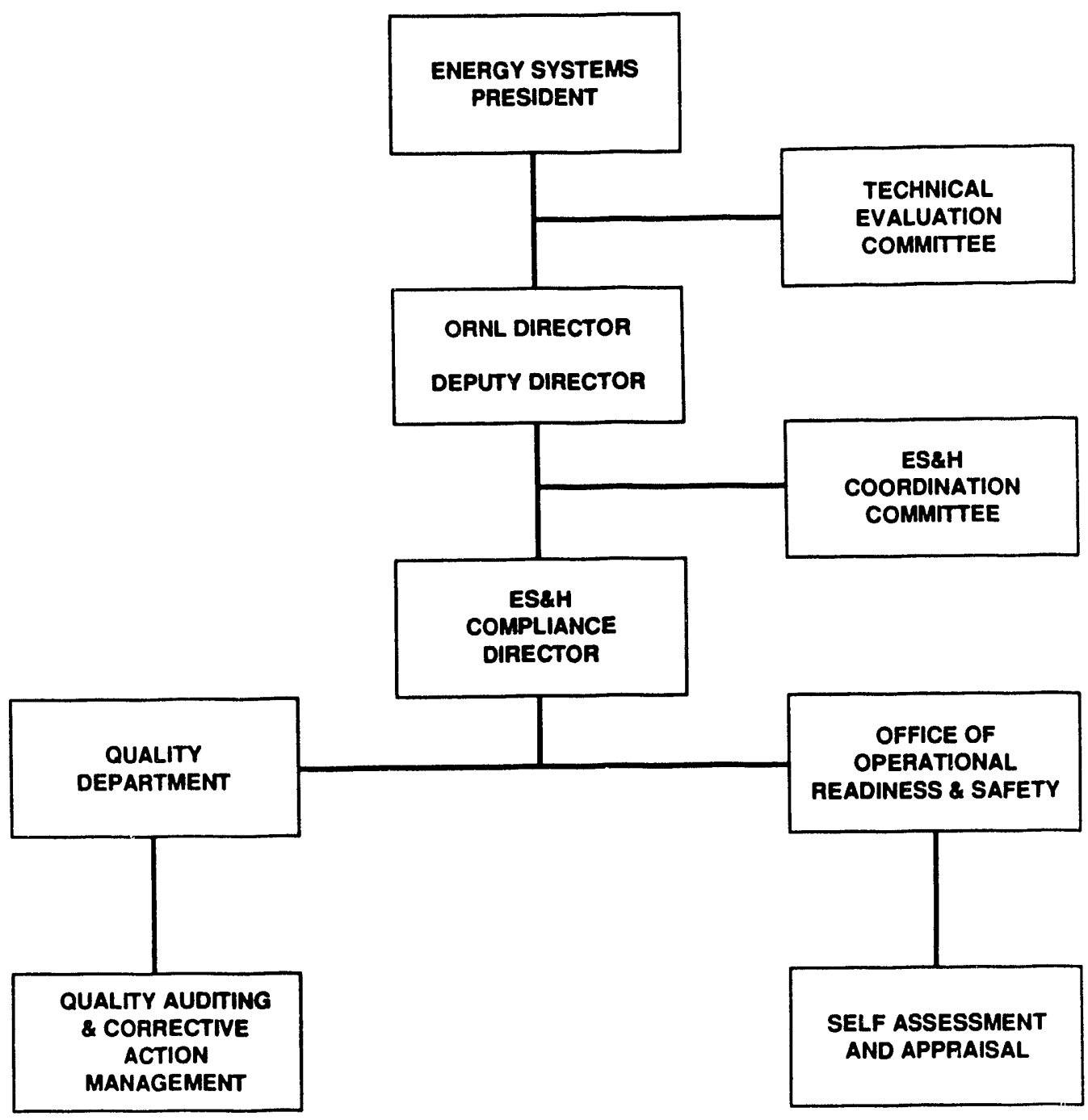

Fig. 1.5. Organizations responsible for corrective action monitoring. 
All actions listed in this action plan are contingent upon suitable funding being provided. The implementation strategy proposed for use at ORNL is tied to the DOE annual funding cycle. ES\&H proposals in the various categories outlined in Sect. 1.6 will be submitted as part of the normal DOE budget process. The proposals will contain a prioritized collection of proposed actions based on risk assessment, cost, and other factors as described in Sect. 1.7.

The funding authorized for the subsequent fiscal year will provide the basis for actions taken during that year. An annual operating plan will be prepared based on the available funding. The plan will be prepared by the Environmental Health and Safety Compliance organization, approved by the ORNL ES\&H Coordinating Committee, and submitted to DOE for concurrence. Based on this plan, funds will be allocated and responsibilities will be assigned for each action to be undertaken. The assignments, budgets, and expected milestones will be entered into the Evaluation Database System described in Sect. 1.7. The Quality Department will be responsible for tracking the status of actions and providing periodic reports as required by DOE. The reports will be evaluated by the ES\&H Coordinating Committee along with information provided by ongoing self-assessment and oversight activities to ensure that appropriate corrective steps are taken in the event of problems.

Documentation of action closure will be made by the organization assigned implementation responsibility, and verification will be accomplished by the Quality Department and DOE as appropriate.

Expected results during the year and prioritization of remaining actions will be the basis for the development of proposals for subsequent year funding. The preparation of these proposals will be organized and reviewed by the ES\&H Coordinating Committee. 


\section{ORGANIZATION AND MANAGEMENT STRUCTURE TO IMPLEMENT THE ACTION PLAN}




\section{ORGANIZATION AND MANAGEMENT STRUCTURE TO IMPLEMENT THE ACTION PLAN}

\subsection{OVERVIEW}

Management and staff from the oversight and line organizations of DOE, Energy Systems, and ORNL worked as a team to respond to the Tiger Team assessment of ORNL. This section identifies the management structure in these organizations for implementing the Action Plan and outlines their relationships and responsibilities. This structure includes changes already implemented in response to independent audits and the Tiger Team findings that became effective January 1991.

ORNL is a multiprogram energy research and development laboratory managed by Energy Systems for DOE. Sections 2.2 through 2.4 describe the management structures of DOE, Energy Systems, and ORNL. The names and addresses of key contacts are listed in Section 2.5.

\subsection{DEPARTMENT OF ENERGY}

\subsubsection{DOE Headquarters}

The Secretary of Energy, Admiral James D. Watkins, has clearly identified his expectations for Headquarters line management responsibility and accountability in operating DOE facilities. While the DOE Office of Energy Research (ER) exercises landlord responsibility for ORNL, many other program sponsors share the responsibility for ensuring that their programs are conducted in a manner that protects the health and safety of the employees and prevents environmental insult. Other major DOE sponsors include the Assistant Secretary for Nuclear Energy, the Assistant Secretary for Conservation and Renewable Energy, the Assistant Secretary for Defense Programs, and the Office of New Production Reactors.

ER provides Headquarters oversight and program direction; it is also the major funding sponsor of R\&D at ORNL. Under an MOU among DOE line programs, the Office of Environmental Restoration and Waste Management (EM) provides programmatic oversight, program direction, and significant funding for waste management and environmental corrective actions at ORNL. In accordance with a management agreement between DOE-Nuclear Energy (NE) and ER, NE has line management responsibility for the operation of the ER-funded reactors at ORNL. One exception is the Tower Shielding Facility, funded and operated by NE.

For this action plan, ER will take responsibility for overall Headquarters coordination of activities. Headquarters' responsibilities include the following:

- requesting the appropriate funding from Congress to implement this action plan, 
- concurring in the prioritization of activities established by ORO and ORNL,

- providing ORO guidance identifying which items to delay if funding is not available,

- assessing the status and quality of the action plan implementation, and

- ensuring that the contractor's performance is adequately reflected through the contractor performance evaluation process.

\subsubsection{DOE-Oak Ridge Operations}

ORO provides day-to-day management oversight of ORNL operations. Joe La Grone, the ORO manager, is the contracting officer and fee-determining official responsible for the management and administration of the contract. Funding for Energy Systems-managed facilities is allocated from DOE and other government agency program offices and controlled through ORO.

Within ORO, line management responsibility is assigned to the Assistant Manager for Energy Research and Development (AMERD), R. L. Egli. The Deputy AMERD, J. A. Reafsnyder, is the Contracting Officers Representative (COR) for ORNL; he is located at the ORNL site. The Assistant Manager for Environmental Restoration and Waste Management (AMERWM), W. D. Adams, has COR authority for Environmental Restoration and Waste Management Activities at ORNL. Several ORO organizations provide support to these two groups. Figure 2.1 shows the ORO organization.

Within ORO, lead responsibility for action plan implementation rests with AMERD and will be directed by James A. Reafsnyder, Deputy Assistant Manager for Energy Research and Development, in accordance with AMERD policies and procedures. AMERD responsibilities include the following:

- ensuring that adequate funding is requested from Headquarters,

- implementing Headquarters guidance,

- providing the contractor with formal guidance for implementation of Headquarters directives,

- coordinating activities with ORO support organizations,

- identifying problems and barriers to implementation,

- evaluating contractor performance,

- verifying, on a prioritized basis, the completion of action items, and

- providing periodic status reports to Headquarters on the progress of implementation. 


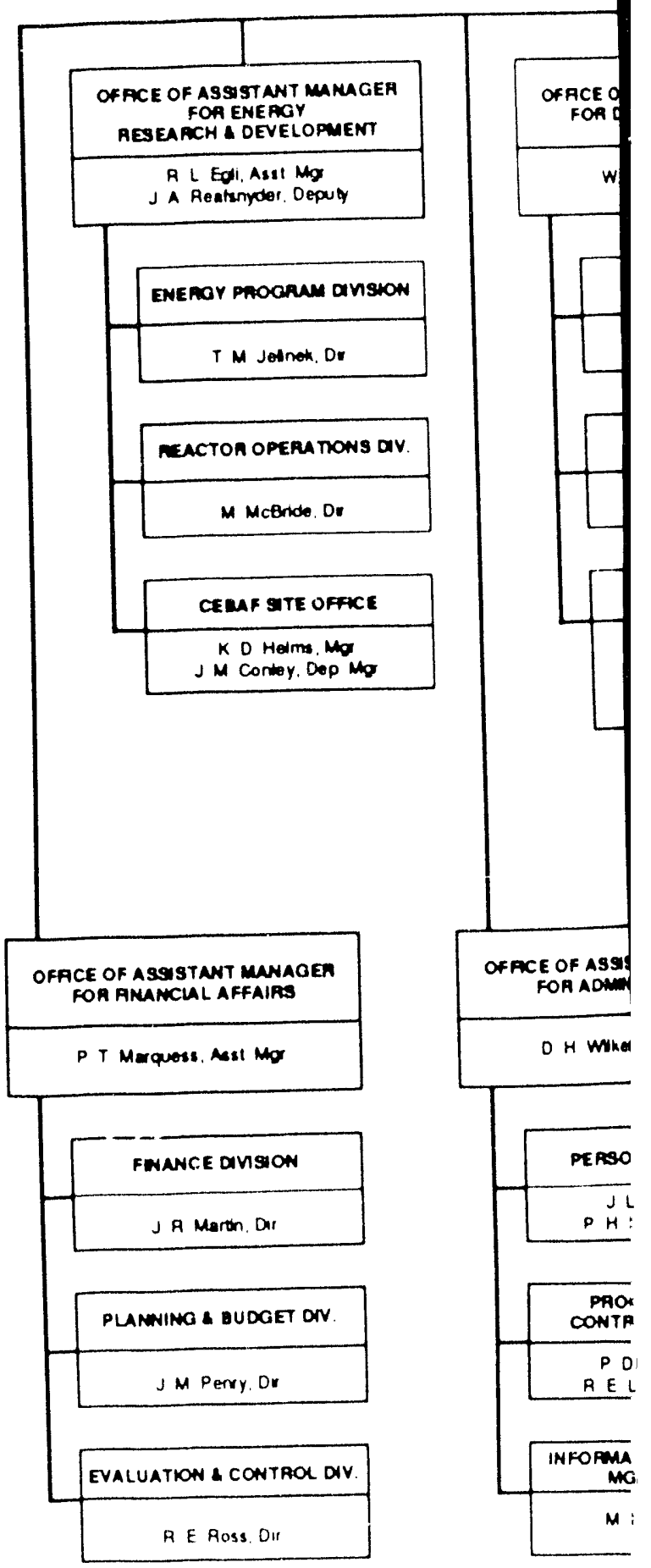

Fig. 21. DOE-ORO organiza 


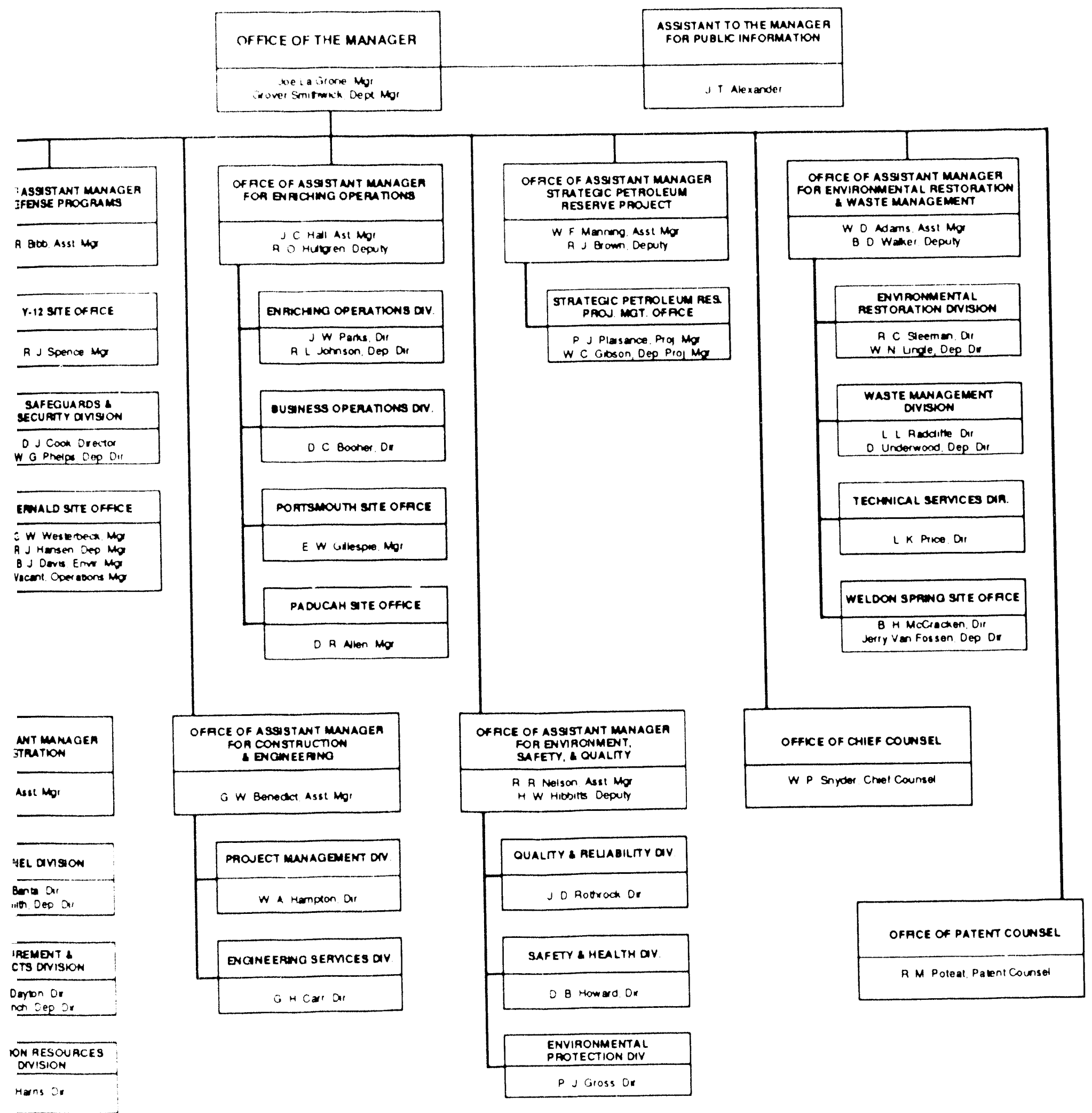

iion and management structure. 
As explained above, AMERWM has responsibility for Environmental Restoration and Waste Management activities according to the memorandum of agreement with AMERD. These responsibilities extend to items identified in this action plan. AMERWM is responsible for:

- ensuring that adequate funding is identified and requested for ERWM activities;

- ensuring that Headquarters concurs in any prioritizations that are established;

- assessing that the contractor's performance is adequately reflected in the performance evaluation process;

- verifying, on a prioritized basis, the completion of action items; and

- providing periodic status reports on action plan implementation to AMERD for inclusion in reports to Headquarters.

These ORO responsibilities add to the reporting requirements currently established for individual programs and other requirements formally established in MOUs and other formal management plans. Headquarters sponsors are encouraged to seek information on action plan progress through existing reporting systems.

The ORO support organizations will assist AMERD and AMERWM in providing independent verification of action completion, evaluation of contractor performance, identification of problem areas, and technical assistance, as needed. These organizations will provide periodic status reports to AMERD on the action plan implementation. In addition, these groups will serve an important function in ensuring that lessons learned are shared across ORO organizations and contractors. As necessary, ORO will utilize support contractors to aid in performing several of these roles.

\subsection{ENERGY SYSTEMS}

Energy Systems is an operating entity of Martin Marietta Corporation responsible to DOE for managing ORNL, the Y-12 Plant, and the K-25 Site in Oak Ridge; the Paducah Gaseous Diffusion Plant in Kentucky; and the Portsmouth Gaseous Diffusion Plant in Ohio. Energy Systems is contracted under a cost-plus-award-fee contract using a "government-owned, contractor-operated" agreement. Energy Systems provides centralized ES\&H policy, compliance oversight, QA, and lessons learned support to its five facilities. Martin Marietta Corporation management has established a matrix relationship between Energy Systems staff members and the corresponding functions at the Mar+in Marietta Corporation level to facilitate effective communications and oversight.

The president of Energy Systems is Clyde Hopkins, responsible to the Martin Marietta Corporation president and chief operating officer. Management oversight is provided by a board of directors that meets bimonthly and is chaired by the president of Martin Marietta Corporation. Hopkins has delegated responsibility for management of the Energy Systems production facilities to the senior vice president who, in turn, has delegated operational responsibility to the three vice 
presidents responsible for applied technology, uranium enrichment, and the Y-12 Plant. ORNL reports directly to the president. Six other vice presidents provide support to Energy Systems in administration; compliance, evaluation, and policy; human resources; technical operations; procurement; and technology applications. Additionally, the staff of the president of Energy Systems includes a general counsel, public relations director, and financial audit manager. Figure 2.2 shows the top-level Energy Systems organization.

Policies established by the president and vice presidents are implemented through standards and procedures that define requirements and responsibilities and provide guidance to the operating organizations in Energy Systems. Top-level standards and procedures are prepared by staffs of Energy Systems vice presidents. These documents are periodically reviewed to ensure that Energy Systems standards and procedures fully respond to federal laws, rules, and DOE orders. Chartered formal committee structures meet regularly to review and guide the development of policies, standards, procedures, progress in improvement performance, technical audit schedules, findings and corrective action status, and key personnel development and assignments.

The Vice President for Compliance, Evaluation, and Policy has delegated responsibility for environmental, safety, and health and quality oversight to the Environmental and Safety Activities Director, the Corporate Medical Director, the Quality Director, and the Evaluations Program Manager. The Evaluations Program Manager and the Quality Director each direct independent Energy Systems-level reviews of environmental, health, safety, quality, and operations performance at each site each year.

The Evaluations' effort is a bottom-to-top, field-observation-based process that uses a core group of INPO-trained observers in a 3 to 5 week annual review of each site. The Quality Director organizes and leads a top-down integrated technical audit composed of several subteams to review ES\&H and quality performance across all Energy Systems functions. These combined reviews are conducted with a frequency that meets or exceeds DOE independent oversight requirements. Martin Marietta Corporation conducts periodic audits of Energy Systems and the respective sites in technical audits led by the corporate Vice President of Quality and integrated with the Energy Systems technical audit process. The results of these technical audits and evaluations and those of site audits and surveillance are reviewed quarterly by the Energy Systems Technical Review Committee.

\subsection{OAK RIDGE NATIONAL LABORATORY}

ORNL is DOE's largest multiprogram laboratory, with strong programs in energy technology development and applied and basic research. The Director of ORNL is Alvin Trivelpiece, and the Deputy Director is Murray Rosenthal. ORNL has a large and experienced environmental and health R\&D program that matrixes its technical capabilities to provide supplemental scientific support for ES\&H compliance to ORNL and other Energy Systems facilities.

Teamwork and cooperation form the management philosophy of ORNL with thorough commitment to excellence in science and ES\&H. Although the delegation of responsibilities in a large and complex organization clearly compartmentalizes the organizational structure, open communications are encouraged while ensuring accountabilities of line management in all organizational elements. 


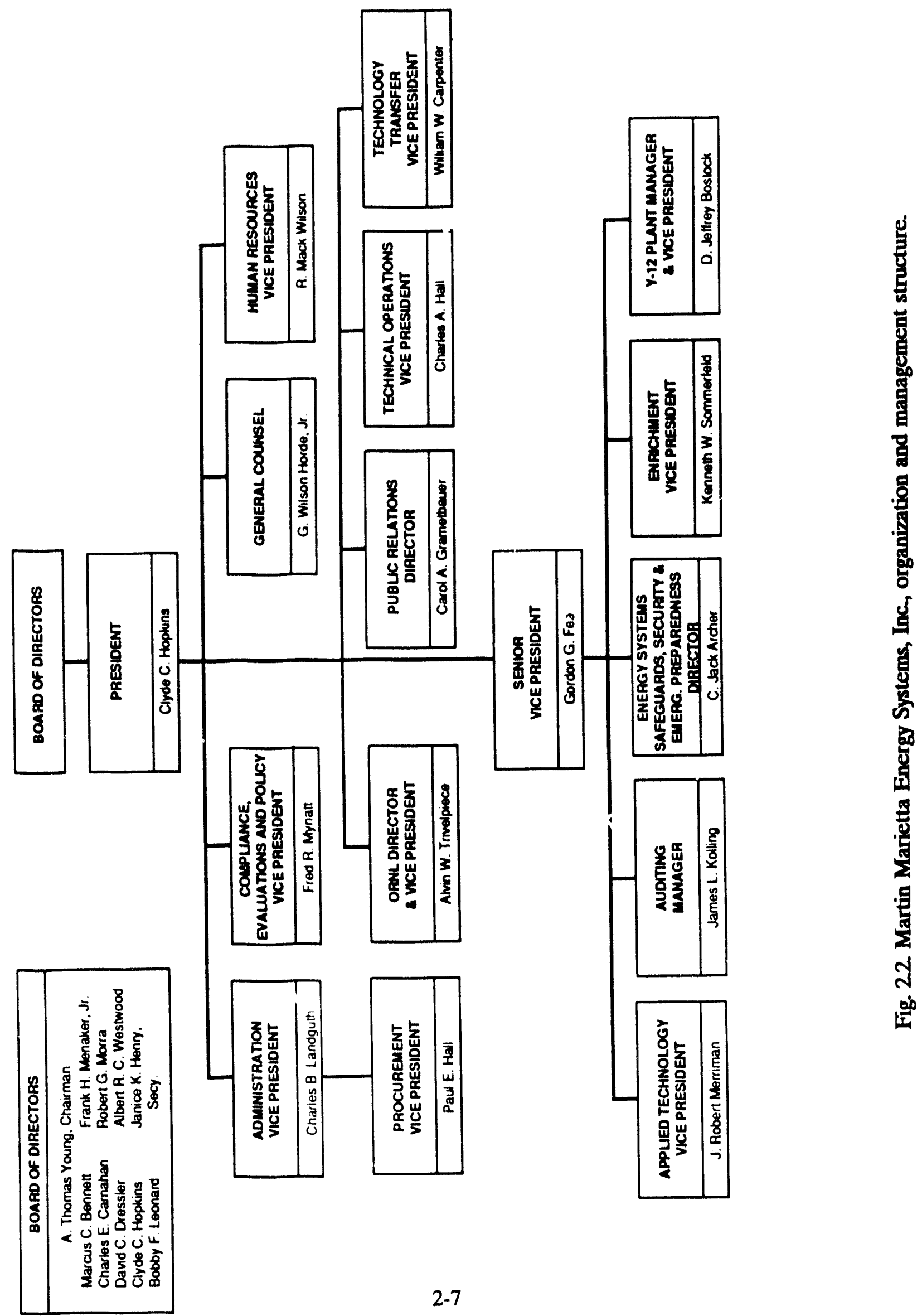


Management duties are divided along divisional and program lines. In this structure, four technical associate directors manage both divisions (generally organized by discipline or technology) and programs (using the expertise within multiple divisions to accomplish programmatic objectives): Advanced Energy Systems, Biomedical Sciences, Environmental Sciences, Nuclear Technologies, and Physical Sciences and Advanced Materials. Another associate director is responsible for Operations; separate organizations are responsible for Research Reactors and ES\&H Compliance. The ORNL Executive Committee comprises all seven of these entities with the Laboratory Director and Deputy Director. Consequently, multidivisional efforts are characteristic of the Laboratory's R\&D projects. The majority of the Laboratory divisions are located at the X-10 site, with approximately $25 \%$ of the technical divisions at the Y-12 and K-25 sites. Figure 2.3 shows the organization chart for ORNL.

The ORNL central ES\&H Compliance organization consists of four offices: Environmental Health and Protection, Environmental Compliance and Documentation, Operational Readiness and Safety, and Quality Assurance (called the Quality Department). Each office directs the oversight and support for a different facet of compliance and improvement in the operations of ORNL. This directorate was formed recently by reorganizing one division into separate offices and transferring the responsibilities of two of the offices from the Operations Directorate. These actions better coordinate the ES\&H and QA functions and bring a unified and higher-level focus of attention on its activities. A number of strategic goals have been identified:

- provide a compliance program that protects staff and allows efficient conduct of operations,

- provide guidance for changes in the operational philosophy needed to achieve excellence,

- provide an operational environment that reduces the need for extensive ES\&H and QA audits,

- anticipate regulatory changes in sufficient time to plan cost-effective compliance,

- maintain current levels of trust by state and federal regulations, and

- improve communications with DOE.

This organization will play an important role in the success of ORNL as the emphasis of DOE and its contractors on ES\&H and QA continues.

An ES\&H Coordinating Committee was created in 1989 to provide better communication and coordination of tasks among the central support, management, and line organizations involved in ES\&H facilities. The committee is chaired by the Deputy Director of the Laboratory and consists of senior ES\&H managers and four members of the Executive Committee, providing top-level overview of this vital area. 

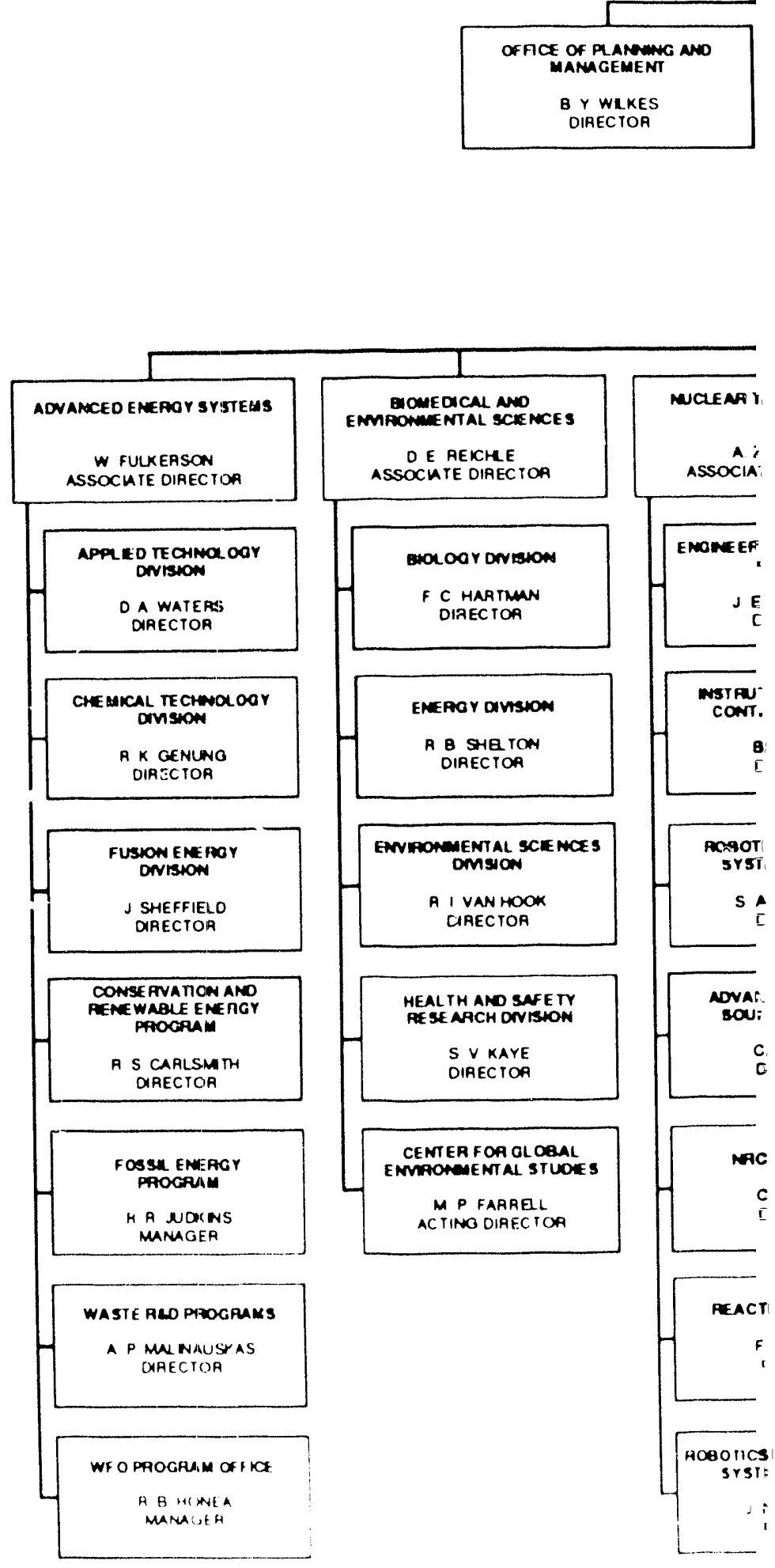

Fig. 23. ORNL, organization and man: 


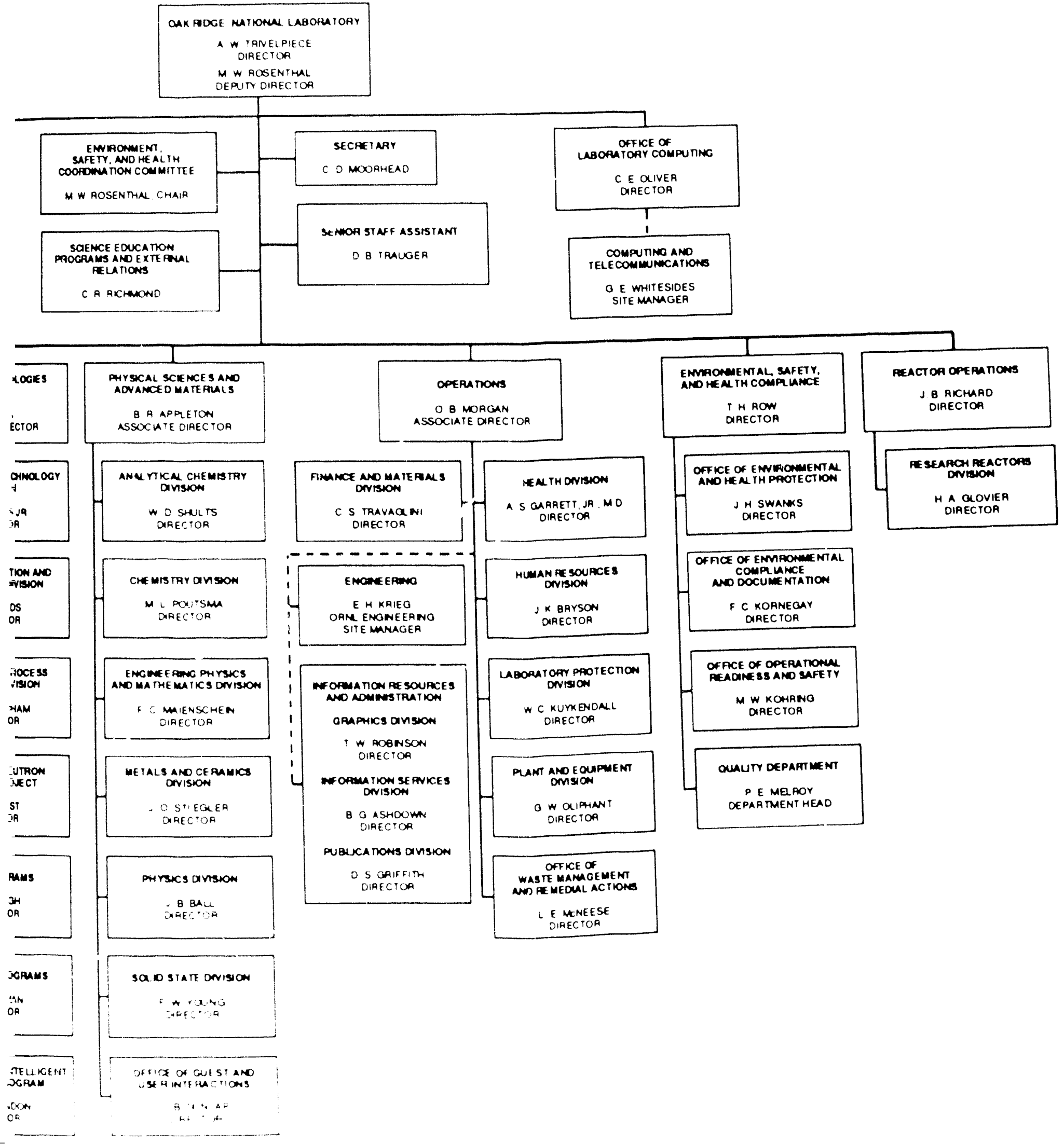

ent structure 


\subsection{KEY CONTACTS}

\section{DOE-ORO}

William D. Aams, Acting Assistant Manager

Environmental Restoration and

Waste Management

U.S. Department of Energy

Post Office Box 2001

Oak Ridge, TN 37831-8546

(615) 576-0742 (FTS) 626-0742

Richard L Egli, Assistant Manager

Energy Research and Development

U.S. Department of Energy

Oak Ridge Operations

Post Office Box 2001

Oak Ridge, TN 37831-8600

(615) 576-0723 (FTS) 626-0723

Joe La Grone, Manager

Oak Ridge Operations Office

U.S. Department of Energy

Post Office Box 2001

Oak Ridge, TN 37831-8651

(615) 576-4444 (FTS) 626-4444

James A. Reafsnyder, Deputy Assistant Manager

Energy Research and Development

U.S. Department of Energy

Oak Ridge Operations

ORNL Site Office

Post Office Box 2008

Oak Ridge, TN 37831-8269

(615) 576-4523 (FTS) 626-4523

\section{DOE-Headquarters}

J. William Bennett, Director of Operations and Facility Reliability

U. S. Department of Energy

19901 Germantown Road

Germantown, MD 20874

(301) $353-5832$ (FTS) 233-5832 
James F. Decker, Acting Director

Office of Energy Research

U.S. Department of Energy

1000 Independence Avenue SW

Forrestal Building

Washington, DC 20505

(202) 586-5430 (FTS) 896-5430

Clyde Frank, Acting Associate Director

Office of Technology Development

U.S. Department of Energy

1000 Independence Avenue SW

Forrestal Building

Washington, DC 20585

(202) 586-7709 (FTS) 896-7709

William H. Young, Assistant Secretary for Nuclear Energy

U.S. Department of Energy

1000 Independence Avenue SW

Forrestal Building

Washington, DC 20585

(202) $586-6450$ (FTS) $896-6450$

Paul L. Ziemer, Assistant Secretary

Environment, Safety, and Health

U.S. Department of Energy

1000 Independence Avenue SW

Forrestal Building

Washington, DC 20505

(202) 586-6151 (FTS) 896-6151

Martin Marietta Energy Systems, Inc.

Clyde C. Hopkins, President

Martin Marietta Energy Systems, Inc.

Post Office Box 2009

Oak Ridge, TN 37831-8001

(615) 576-5663 (FTS) 626-5663

M. E Mitchell, Director

Environmental and Safety Activities

Martin Marietta Energy Systems, Inc.

Post Office Box 2003

Oak Ridge, TN 37831-7155

(615) 476-8006 (FTS) 626-8006 
Fred E. Mynatt, Vice President

Compliance, Evaluations, and Policy

Martin Marietta Energy Systems, Inc.

Post Office Box 2008

Oak Ridge, TN 37831-6275

(615) 574-4182 (FTS) 624-4182

\section{Oak Ridge National Laboratory}

R. E. Fenstermaker, Manager

Quality Department

Oak Ridge National Laboratory

Post Office Box 2008

Oak Ridge, TN 37831-6052

(615) 574-7234 (FTS) 624-7234

M. W. Kohring, Director

Office of Operational Readiness and Safety

Oak Ridge National Laboratory

Post Office Box 2008

Oak Ridge, TN 37831-6256

(615) 574-4337 (FTS) 624-4337

F. C. Korncgay, Director

Office of Environmental Compliance and Dousmentation

Oak Ridge National Laboratory

Post Office Box 2008

Oak Ridge, TN 37831-6198

(615) 574-5776 (FTS) 624-5776

Murray W. Rosenthal, Deputy Director

Oak Ridge National Laboratory

Post Office Box 2008

Oak Ridge, TN 37831-6241

(615) $574-4322$ (FTS) 624-4322

Tom H. Row, Director

Environmental, Safety, and Health Compliance

Martin Marietta Energy Systems, Inc.

Oak Ridge National Laboratory

Post Office Box 2008

Oak Ridge, TN 37831-6198

(615) 574-5974 (FTS) 624-5974 
Jerry H. Swanks, Director

Office of Environmental and Health Protection

Martin Marietta Energy Systems, Inc.

Oak Ridge National Laboratory

Post Office Box 2008

Oak Ridge, TN 37831-6396

(615) 574-6688 (FTS) 624-6688

Alvin W. Trivelpiece, Director

Oak Ridge National Laboratory

Post Office Box 2008

Oak Ridge, TN 37831-6255

(615) 576-2900 (FTS) 6-2900 


\section{FINDINGS, RESPONSES, AND PLANNED ACTIONS}




\section{FINDINGS, RESPONSES, AND PLANNED ACTIONS}

\subsection{ROOT CAUSES}

\subsubsection{Root Cause Determination}

The results of the Tiger Team assessment of ORNL, previous audits of ORNL, Tiger Team assessments of other Energy Systems sites, Tiger Team assessments of other DOE installations, and the ORNL self-assessment were carefully considered to identify 11 root causes that appear to explain the findings and concerns identified during the ORNL Tiger Team assessment. The following definitions are used for the 11 root causes.

Inadequate policy-Policy statements do not exist or are incomplete regarding all requirements or expectations.

Inadequate policy implementation-Policy statements exist but are not being used because of unclear or incompletely documented and controlled procedures or instructions.

Insufficient resources-Funding, personnel, equipment, or facilities are insufficient to comply with requirements or expectations.

Inadequate management commitment-Management has not accepted the need to meet the requirement or expectation or has not devoted sufficient attention to ensuring that adequate policies are defined and implemented.

Inadequate management approach-Management systems and organization are not effective in implementing, tracking, and reviewing requirements that have been committed to.

Inadequate oversight-Independent evaluation and review systems are inadequate to ensure that all operations are meeting requirements and expectations, including auditing, tracking, trending, and feedback functions.

Inadequate communications-Communications are not effective in conveying requirements or expectations down to the level of implementation, or do not provide adequate information to management to properly determine the state of affairs.

Ambiguous requirements or expectations-Requirements or expectations for performance have not been clearly defined, leading to interpretation and disagreements as to whether all requirements have been met.

Inadequate training-Training has not been sufficient to ensure that personnel have adequate understanding of all requirements or the necessary skills to implement required procedures. 
Poorly defined roles and responsibilities-Roles, responsibilities, and accountability for requirements have not been adequately documented and understood, leading to confusion as to who is responsible for implementation and oversight of requirements.

Regulatory barriers-Laws, regulations, orders, or standards are conflicting or prohibit required actions necessary to meet all requirements or expectations.

Each finding or concern is attributable to one or more of these 11 root causes. Collectively, the action plan seeks to remedy these root causes and provide an infrastructure that not only meets all ES\&H requirements and expectations but can support ORNL's long-range goal of excellence.

\subsubsection{Relationship to Tiger Team Assessment Root Causes}

The ORNL Tiger Team cited nine root causes in their report for the findings and concerns noted during the assessment. The 11 ORNL root causes listed previously are in many cases an attempt to break down into key elements the root cause statements made by the Tiger Team. There is a well-defined relationship between the 9 root causes listed by the Tiger Team and 11 root causes used in this action plan.

The environmental subteam of the Tiger Team cited 2 root causes for the 70 environmental findings: policy and policy implementation. Inadequate policy and inadequate policy implementation are also elemental root causes as described in this action plan.

The TSA teams cited three root causes for both the sitewide and the reactors TSA concerns identified during the assessment. The root cause statement by the Tiger Team is followed in parentheses by the ORNL root causes that correspond to the statement.

"Management has not effectively identified and implemented ES\&H requirements throughout ORNL." (inadequate policy and inadequate policy implementation)

"Implementation of ES\&H requirements and management attention to the details of meeting those requirements is not done with the same rigor as that applied to scientific research." (inadequate policy implementation and inadequate management commitment)

"There are insufficient resources to support essential functions in key safety and health programs." (insufficient resources)

The management subteam cited 4 root causes for the 12 management and 1 self-assessment finding. The root causes identified by the Tiger Team and their relationship to the ORNL element root causes follow.

"ORNL management has not accepted ES\&H as an integral part of their scientific program responsibilities and, accordingly, has not demanded those astions which are necessary to accomplish ES\&H excellence." (inadequate management commitment and inadequate policy implementation) 
"ORNL's collegial, consensus, and informal management style is not well-suited to the demands of ES\&H compliance." (inadequate management approach)

"MMES has not responded to the DOE ES\&H initiatives with the imaginative, aggressive management action necessary to bring about definitive improvements in ORNL's management of the ES\&H program." (inadequate management commitment and inadequate policy implementation)

"DOE has not accompanied its mandate for vigorous DOE oversight of ORNL's ES\&H program, with planning, guidance, and resources necessary to successfully accomplish that mandate." (inadequate policy, inadequate policy implementation, and insufficient resources)

\subsubsection{ORNL Actions to Address Root Causes}

There is a strong consensus that a collegial management style is an essential part of ORNL's scientific culture. It is the exchange of ideas, the give and take, and the consensus identification of scientific certainty that sets the scientific method apart from speculation. However, there needs to be a clear demarcation between consensus in deciding on a course of action and uniform adherence to the consensus decision. The Tiger Team touched on this distinction in their discussion of root causes. One root cause states that "ORNL's collegial, consensus, and informal management style is not well-suited to the demands of ES\&H compliance." However, in another root cause, the Tiger Team cites ORNL's distinguished record of scientific achievement and concludes that some ES\&H deficiencies can be traced to the fact that "Implementation of ES\&H requirements and management attention to the details of meeting those requirements is not done with the same rigor as that applied to scientific research." ORNL's response to its acknowledged management deficiencies is to firmly establish ES\&H as an integral part of the mission of the Laboratory and to apply the same rigor to meeting ES\&H laws and requirements as is applied to scientific laws and requirements. At the same time, initiatives are being pursued that will hopefully strengthen both research and ES\&H performance.

Energy Systems and ORNL management have developed the following approach to establishing and maintaining excellence in ES\&H. This approach envisions seven key elements:

- Strategic plan for ES\&H: a strategic plan for ES\&H is under development to provide vision and coherence to ES\&H activities. It will integrate with the strategic plan being developed Energy Systems-wide.

- Goals and structure: institutional goals will be established and roles and responsibilities will be clearly defined and utilized in performance planning and review.

- Conduct of Operations: uniformity of management approach and formality of operations will be strengthened by the implementation of Conduct of Operations throughout ORNL. 
- Surveillance: effective and independent oversight of ES\&H performance will be established, and adequate technical assistance will be provided. The oversight and technical assistance roles will be managed to avoid conflicts of interest.

- Measurement: performance goals will be established, and tracking and trending systems will be implemented.

- Self-assessment: a continuous self-assessment process combined with periodic independent external review that meets all the criteria for an effective self-assessment program will be implemented.

- Total quality management: a philosophy of continuous improvement and dedication to excellence will serve as the umbrella under which elements are defined and implemented.

ORNL intends to strengthen its management approach by using a framework modeled after the Institute of Nuclear Power Operations (INPO) Guidelines for Conduct of Operations and as required in DOE Order 5480.19. Conduct of Operations is a philosophy of doing work in a formal, disciplined manner to enhance safe and efficient management of activities and to achieve excellence. Key elements of the Conduct of Operations philosophy are that individuals take "ownership" for their activities and that management be routinely visible in the workplace to observe and assist where feasible.

Because of their wide acceptance, Conduct of Operations guidelines will be used as the starting point for the ORNL system. The guidelines fail to address many of the issues of importance to the Laboratory and must be supplemented in many areas, including conduct of research, definition of research directions, resource allocation, hiring practices, reporting procedures, evaluation of performance of both individuals and operating units, and recognition of performance through awards and salary administration. Furthermore, the guidelines are intended to cover the operation of nuclear power plants and are written in the jargon specific to that operation, so they must be translated into language appropriate for a research organization.

Implementation of Conduct of Operations will utilize a graded approach commensurate with the level of operational risk involved. Energy Systems is currently training managers in Conduct of Operations principles in preparation for implementing a program throughout the organization. The implementation at ORNL will be within the context of the Energy Systems-wide initiative.

Other root causes are being addressed by Energy Systems actions that have been initiated to strengthen ES\&H management as a result of Tiger Team visits and other audits at other Energy Systems sites. Energy Systems management has recognized the need and accepted the challenge to improve its ES\&H performance. As described in Sect. 2, this recognition led to the establishment of the position of Vice President for Compliance, Evaluations, and Policy, a function charged with identifying the need for improvements and implementing these improvements within Energy Systems.

The Energy Systems Senior Vice President has chartered Management Issue Teams to develop solutions to generic issues. Fourteen generic issues are being addressed by Energy Systems at this time: roles and responsibilities, policies and procedures, resources, self-assessment, ES\&H 
training, records management, Conduct of Operations, ES\&H strategic planning, management, safety analysis reports, emergency preparedness, environmental compliance, OSHA compliance, and radiation protection. Comparing the list of generic issues being addressed by Energy Systems with the root causes determined from the ORNL Tiger Team Assessment, one notes that all but two of the root causes, regulatory barriers (a regulatory agency issue) and ambiguous requirements or expectations (primarily a DOE issue), are being addressed either wholly or partially at an Energy Systems level. In many cases, actions listed in response to concerns describe ORNL implementation plans for Energy Systems initiatives.

Martin Marietta Corporation is also playing an active role in improving ES\&H performance at Energy Systems facilitics. The Energy Systems board of directors, chaired by Tom Young, the President of Martin Marietta Corporation, mects bimonthly and provides top management review of Energy Systems operations with special attention to performance deficiencies in ES\&H. Technical and management assistance is provided to Energy Systems by other parts of Martin Marietta Corporation through an interdivisional operating directive. Recent ES\&H-related assistance provided by Martin Marietta Corporation to Energy Systems includes planning assistance for the Y-12 technical audit; calibration standards and measuring; analysis, development, and implementation of plant performance objectives; and environmental task force assistance. In addition, ORNL is using the knowledge base and experience of Martin Marietta Corporation and other Energy Systems sites in developing its self-assessment process. Technical audits are performed by Martin Marictta Corporation for all Martin Marietta businesses. A pre-Tiger Team audit was conducted at ORNL during 1990. During 1991, cleven audits are scheduled, including three at Energy Systems installations. Finally, the Corporate Environmental Management group has established a local office in Oak Ridge, Tennessee, with two full-time staff members. The activities of this group include reviewing ongoing environmental programs relative to compliance with applicable federal, state, and local regulations.

Substantive organizational transformations do not occur instantaneously. Energy Systems recognizes that some findings traceable to generic root causes will likely continue to be identified during subsequent assessments. Achievement of lasting and continuous improvement will require persistence and the continued dedication of resources.

The 11 root causes identified during the ORNL Tiger Team assessment are being addressed by ORNL's implementation of Conduct of Operations, by actions outlined in individual action plans, and by Energy Systems initiatives. The Energy Systems initiatives are described more fully in the report Environmental Safety and Health Program Action Plan for Martin Marietta Eneryy Systems, Y/MS-0001, January 1991. The key actions addressing each root cause are summarized in the following sections.

\section{Inadequate Policy}

Energy Systems is pursuing an initiative to create a management system for policies and procedures that will provide the formality, discipline, and accountability needed for adequate document control and to ensure compliance with regulations. The initiative includes central policy direction; interpretation; integration of requirements; and controlled flowdown of policies, standards, guidelines, and procedures. For each major functional area, a Central Policy Manager has been assigned, reporting directly to the executive manager of that functional area. Overall 
integration of policy initiatives will be the responsibility of a Policy Review Board composed of Energy Systems executives and chaired by the Vice President of Compliance, Evaluations, and Policy. A phased approach to implementing the management system is planned with early emphasis on those functional areas most likely to be addressed by DOE's rule-making process.

Organizational policies are also the subject of Chapter 1 of Conduct of Operations and will be addressed by ORNL's implementation of Conduct of Operations. Under Conduct of Operations, policy is to be established in support of regulatory guidance with clearly documented responsibility, authority, and interface. Measures to ensure that policy is understood will be taken as required.

Inadequate policy guidance was also the subject of ORNL Tiger Team Finding MF-10 (DOE Directive System). The finding states that "DOE's communication of ES\&H directives (Orders, Secretary of Energy Notices, and Memoranda of Understanding) does not provide guidance or specific instructions to the contractor or provides differing instructions depending on the program sponsor." The action plan in response to Finding MF-10 outlines a series of actions to clarify DOE policy including ORO establishing a Compliance Guidance Coordination Team to serve as a focal point for preparing and controlling ES\&H policy guidance, and improving procedures for distribution and control of DOE directives.

\section{Inadequate Policy Implementation}

Effective policy implementation depends not only on clear and adequate procedures or instructions but also on effective communications, adequate resources to implement the policy, and measuring expected performance against actual performance. Consequently, this ront cause is closely related to several other root causes.

There are several elements to effective policy implementation, and these elements are outlincd in action plans prepared in response to various findings. The first element is to identify what procedures and instructions are needed. The action plan in response to Finding MF-2 (ES\&H Management Systems) describes the development of a strategic plan for ES\&H so that individual policies and procedures can be viewed in the context of a cohesive ES\&H program. The second element is to establish clear authority for developing procedures. This authority has been assigned to functional managers under the Energy Systems initiative described previously under the response to the inadequate policy root cause. The third element is to ensure that the procedures developed under this authority are adequate. The response to Finding OP.3-1 (ORNL Operating Procedures) describes a plan to develop an ORNL standarci practice procedure to provide guidance on format, content, and approval of operating procedures and requirements. When adequate procedures have been defined, they must flow down to the appropriate levels of the organization for implementation. The response to Finding OA.1-1 (Flowdown of ES\&H Policies and Requirements) describes implementation of the Automated Procedures and Requirements Accountability System that provides flowdown of requirements, orders, policies, and procedures and that identifies the individual responsible for implementation. Once procedures and instructions have been implemented, documents must be adequately controlled to ensure that the appropriate version is being used. Document control is the subject of one of the elements of the NQA-1 quality assurance program being implemented at ORNL as well as a new standard practice procedures on controlled document tracking and auditing as described in the action plans 
in response to Findings OA.7-1 (Centralized System for Safety Document Control) and ROA.7-4 (Control of Documents).

\section{Insufficient Resources}

Insufficient resources for implementing ES\&H requirements is cited in two of the nine root causes identified by the ORNL Tiger Team. Some aspects of the problem of insufficient resources are within ORNL's or ORO's control, whereas others are completely dependent on external factors and decisions. ORNL is responsible for identifying ES\&H funding requirements and requesting funding. The ES\&H strategic plan described in the response to Finding MF-2 will aid the identification of funding needs and provide a cohesive framework for prioritizing ES\&H needs.

Many cases are cited by the Tiger Team where ES\&H funding has been requested but adequate funds have not been provided to meet all requirements. The entire DOE complex is struggling with the problem of the huge costs associated with environmental restoration and waste management and with bringing a very old physical plant up to modern standards. In such an environment, ORNL must prioritize and apply the limited resources in the most cost-effective manner. However, achieving DOE's goal of full compliance with all applicable laws, orders, and standards will require a substantial and sustained commitment to increased funding for ES\&H.

In a few cases, funding is available but staffing and facilitics are the critical constraint on the pace of ES\&H improvements. Energy Systems has conducted a systematic and thorough assessment of personnel availability and recruitment initiatives to ensure that adequate staffing levels are established, that appropriate budget levels are requested, and that the staffing organizations are provided with timely information regarding staffing needs. With regard to facilities, DOE attention to correcting the problems with the NEPA process cited by the Tiger Team in Finding NEPA/CF-1 will remove what has proven to be a major obstacle to the timely completion of new facilities.

\section{Inadequate Management Commitment}

Management commitment is a difficult concept to quantify and is likely to result in various answers depending on what criteria are used to measure it. The ORNL Tiger Team concluded that "It is apparent that Laboratory personnel have heard DOE's message relative to ES\&H performance and are working to achieve it, but ownership and commitment to the values associated with ES\&H performance have not yet developed." In its self-assessment report, ORNL stated that "We do believe that the required change in culture is now spreading through the Laboratory, but we know that some people accept it with reluctance. The upper management is convinced of the need and is trying to convert everyone through example, exhortation, and mandate." A number of initiatives are being pursued to strengthen management commitment to and acceptance of ES\&H goals.

Energy Systems is pursuing a number of actions to reinforce, management commitment and to promote leadership in ES\&H areas. A values program has been developed to communicate corporate values to all levels of management. A total quality nianagement program, led by senior 
management, has been instituted to . riveve a leadership role in performance that meets all ES\&H requirements.

Setting comprehensive, quantifiable goals for ES\&H performance is another tool for fostering management commitment. The determination of ES\&H goals is the subject of several Tiger Team findings. The responses to Findings MF-1 (ES\&H Goals and Objectives) and OA.1-4 (Acceptance of ES\&H Requirements at ORNL) outline steps being taken to develop a consistent, ORNL-wide set of ES\&H goals that stress specific, positive, attainable, and measurable ES\&H goals. ES\&H goals will also be incorporated into position de criptions and the ORNL Performance Planning and Review System.

The award fee process for providing appropriate incentives for enbanced ES\&H performance is the subject of Tiger Team Finding MF-12 (Contract Award Fee Prccess). A properly structured incentive system is one tool to help foster management commitment. In its action plan in response to this Tiger Team finding, ORO states that additional measurable performance criteria will be identified and incorporated into the Award Fee Determination Plan and that it will continue to seek Headauarters participation in the process.

\section{Inadequate Management Approach}

As described above, ORNL's response to an inadequate management approach is to firmly establish ES\&H as an integral part of the mission of the Laboratory and to apply the same rigor to meeting ES\&H laws and requirements as is applied to scientific laws and requirements. A Conduct $\delta\{$ Operations framework is being used to strengthen its management approach, with the desired outcome being improved performance in both ES\&H and research.

Also as described above, Energy Systems has established the position of Vice President for Compliance, Evaluations, and Policy to identify and implemeni improvements within Energy Systems. Management Issue Teams have been chartered to address a total of 14 generic issues, and further management changes can be expected as recommendations are implemented.

\section{Inadequate Oversight}

The inadequate oversight root cause encompasses a number of related aspects of independent evaluation and review systems including auditing, tracking, trending, and feedback functions. The primary function of an oversight system is to provide management with accurate information regarding the true status of the organization. Oversight includes both internal and external reviews. ORNL has undergone a near-continuous series of external audits and reviews leading up to and including the Tiger Team assessme it. In addition, ORNL conducted a comprehensive selfassessment activity in preparation for the Tiger Team. The cha'lenge is to institutionalize an effective oversight process that can provide accurate feedback on an ongoing basis.

A key componcnt of the Energy Secretary's ten-point initiative is a robust self-assessment process whereby ES\&H cieficiencies are identified, reported, and corrected. The Tiger Team noted in Finding SA-1 (The ORNL Self-Assessment Process) that ORNL has not institutionalized its selfassessment process and that the current process lacks many of the elements of an effective self- 
assessment program. The action plan prepared to respond to that finding outlines a plan in fully implement a continuous self-assessment process combined with periodic independent externat review that meets all the criteria for an effective self-assessment program.

The Tiger Team also cited inadequate oversight of ORNL ES\&H activities by OPOO in Finding MF-11 (OR Oversight Systems). Tre action plan in response to that finding describes a staffing plan to fully implement ES\&H surveillance procedures.

Adequate oversight is also a basic element of the NOA-1 quality assurance program. Inadequate oversight was cited in a number of quality verification concerns including Findings QV.1-3 (Quality Department Audit Program). QV.1-4 (Frequency of Internal Audits and Surveillances). and RQV.1-6 (Frequency of RDD QA Audits). As described in the action plans to those concerns, a Quality Assurance Audit Program Manager was named effective December 1, 1990. Minimum requirements for audits and surveiliances have been established and will be tracked to ensure that they are performed as scheduled.

Another aspect of oversight is tracking and trending systems for corrective actions. ORNL is participating in an Energy Systems initiative to develop an Energy Systems-wide corrective action prioritization and tracking system that will help direct management attention and resources to the most critical is.ues. A central tracking system called the Energy Systems Action Management System is currently being developed to track actions resulting from all audits at Energy Systems sites. Energy Systems is also developing the Integrated Resource Management System that will assist in prioritizing actions through a risk-based apf ioach.

In addition, Martin Marietta Corporation has assignc d full-time advisory personnel to Energy Systems to strengthen corporate oversight.

\section{Inadequate Communicaticns}

The root cause of inadequate communications encompasses a variety of communication needs, including conveying requirements or expectations down to the level of implementation, feedback required to provide a clear understanding of the actual state of the organization, and communications needed to properly coordinate complex or multiple tasks.

Several of the Tiger Team findings relate to communications issues. Findings OA.1-1 (Flowdown of ES\&H Policies and Requirements) and OA.1-2 (Dissemination of DOE Orders and Other Requirements) deal with the issue of top-down communications used to convey requirements or expectations down to the level of implementation. The response to these concerns describes implementation of the Automated Procedures and Requirements Accountability System that provides flowdown of requirements, orders, policies, and procedures and identifies the individual responsible for implementation. Finding OP.7-1 deals with shift overlap to permit effective turnover of duties, while facility status displays are the topic of Finding OP.4-1. Both shift overlap in critical facilities and facility status displays are elemerts of Conduct of Operations, which is being implemented at ORNL and will be applied to operations where deemed necessary. The need for an improved lockout/tagout procedure was identified in the ORNL self-assessment and was the topic of Findings OP.4-2 and MA.2-1. A draft procedure consistent with the requirements of DOE Order 5480.19 hâs becñ completed and will be implemented. 
The bottom-up aspect of communication in providing management with timely information on the true status of the organization is closely related to the root cause of inadequate oversight. The responses to Findings SA-1 (The ORNL Self-Assessment Process), QV.1-3 (Quality Department Audit Program), QV.1-4 (Frequency of Internal Audits and Surveillances), and RQV.1-6 (Frequency of RDD QA Audits) describe a set of actions being taken to ensure that the frequency and quality of communications regarding facility status are adequate to kecp management fully informed.

\section{Ambiguous Requirements or Expectations}

Ambiguous requirements or expectations arise when policies are unclear, incomplete, or conflicting. leading to interpretation and disagreements as to whether all requirements have been met. Given the explosive growth in the number and scope of ES\&H policies, it is inevitable that some confusion exists with regard to requirements or expectations.

Inadequate policy guidance was the subject of ORNL Tiger Team Finding MF-10 (DOE Directive System). The action plan in response to this finding outlines a series of actions to clarify DOE policy, including the establishment by ORO of a Compliance Guidance Coordination Team to serve as a focal point for preparing and controlling ES\&H policy guidance and the improvement of procedures for distributing and controlling DOE directives.

Conflicting policy guidance from different program secretarial officers was the subject of Tiger Team Finding NEPA/CF-1 (Inefficient DOE NEPA Implementation Procedures). With a multiprogram laboratory such as ORNL, DOE must make some effort to coordinate requirements and resolve conflicts between various cognizant officials.

\section{Inadequate Training}

Training is one means used to communicate new requirements, as well as a mechanism to assist in developing the skills necessary to respond to requirements. Inadequate training is cited in numerous Tiger Team findings. Insufficient resources is cited in a number of training findings including TC.1-3 (Training Staff), TC.7-1 (Training Facilities), and TC.'/-2 (Storage of Training Records).

Prior to the Tiger Team visit, ORNL formed a Training and Development Department under the Human Resources Division with the objective of providing a central focal point for coordinating training activities to ensure consistency, eliminate redundancy, and achieve programs that meet compliance requirements. ORNL is currently conducting a needs assessment of training to determine critical needs within the context of currently available limited resources.

An Energy Systems Central Training organization has been established to manage the development and delivery of compliance, technical, and management training applicable to all of Energy Systems. Training will be implemented by using a lead site concept, with training being developed to a consistent standard. 


\section{Poorly Defined Roles and Responsibilities}

Energy Systems is developing and implementing a roles and responsibilities policy applicable to all of Energy Systems. The objective of this policy is to provi ; a rigorous process for the definition and delineation of the roles and responsibilities of organizations, individuals, and key committees. The system will provide a consistent process and format and will be regularly maintained. It will also be used as a factor in performance planning and review. The system has been implemented on a pilot basis at both the K-25 Site and ORNL, with sitewide implementation to be initiated by December 1991.

The responses to Findings MF-4 (Human Resources) and OA.1-5 (Implementing ES\&H Activities) describe ORNL's implementation of this Energy Systems initiative. Position, organization, and committee charters will be developed to include ES\&H roles, responsibility, and authority. ES\&H performance goals will be included in training for managers and supervisors and emphasized in employee performance evaluations.

\section{Regulatory Barriers}

Regulatory barriers arise when laws, regulations, orders, or standards are conflicting or prohibit required actions necessary to meet all requirements or expectations. By definition, regulatory barrier issues must be negotiated with the cognizant regulatory agency. When a potential regulatory barrier is identified, clarification must be sought from the regulatory body. If there is indeed a regulatory barrier, ORNL will work with DOE to reach agreement with the appropriate regulatory authority. 


\subsection{ENVIRONMENTAL FINDINGS, RESPONSES, AND PLANNED ACTIONS}




\subsection{ENVIRONMENTAL FINDINGS, RESPONSES, AND PLANNED} ACTIONS

\begin{tabular}{||l|c|c||}
\hline \multicolumn{1}{|c|}{ Finding discipline } & $\begin{array}{c}\text { Finding number } \\
\text { prefix }\end{array}$ & $\begin{array}{c}\text { Section } \\
\text { number }\end{array}$ \\
\hline \hline Air & A & 3.2 .1 \\
Soil/Sediments/Biota & SSB & 3.2 .2 \\
Surface Water/Drinking Water & SW & 3.2 .3 \\
Groundwater & GW & 3.2 .4 \\
Waste Management & WM & 3.2 .5 \\
Toxic and Chemical Materials & TCM & 3.2 .6 \\
Quality Assurance & QA & 3.2 .7 \\
Radiation & RAD & 3.2 .8 \\
Inacti /e Waste Sites & IWS & 3.2 .9 \\
National Environmental Policy Act & NEPA & 3.2 .10 \\
\hline
\end{tabular}




\subsubsection{Air}

Finding No.: A/CF-1 Excursions Above Air Permit Limits

Finding

Description:

Code: $\quad$ Compliance

Compliance

Protocol:

Priority: $\quad$ Energy Systems Risk Weight 448

Tiger Team Action Plan Priority 2

Response: It is the policy of the Environmental Compliance staff to transmit a copy of air permits to facility operators with instructions to ensure that they review the permit conditions and ensure compliance with all permit conditions.

The concern of policy implementation to ensure compliance with all air permit conditions was addressed in two letters to facility operators on April 12, 1990, and on June 19, 1990. As a result of increased awareness by facility operators of their responsibilities regarding compliance with air permit conditions, three exceedances of permit conditions were identified.

These exceedances of specific air permit conditions were previously identified by ORNL staff in the Self-Assessment Report for the Nonradiological Wastewater Treatment Plant and the Process Water Treatment Plant. In the case of Tank 2519A exceedances were identified by the Facility Manager. Revised permit applications have been prepared and submitted to the TDHE. As of this writing, two of the revised air permits have been received from TDHE. Routine follow-up to ensure compliance with permit conditions has been implemented.

These policies will be formalized in a plant procedure to be included in the Environmental Protection Manual under the procedure for Air Permits, procedure number EPM-9.0.

Root Causes:

Inadequate policy implementation, inadequate procedures, and inadequate training 
Planned Actions and Schedules:

Item/Description

1. ORNL submit revised permit applications to DOE-ORO for sources that can not meet permit conditions.

2. DOE-ORO submit revised permit applications to TDHE.

3. Initiate source-specific compliance surveillance.

4. Issue Revised EPM-9.0 to reflect responsibility of facility operators for ensuring continued compliance with permit conditions.
Completion Date

Complete

Complete

Complete

$7 / 91$

Costs: $\quad$ No significant costs associated with action listed.

References: Letter from C. E. Nix to Distribution, "Compliance with Air Pollution Control Permits", dated April 12, 1990

Letter from C. E. Nix to Distribution, "Compliance with Air Pollution Control Permits", dated June 19, 1990 
Finding No.: A/CF-2 High Efficiency Particulate Air (HEPA) Testing Program Deficiencies

Finding

Description:

Code: $\quad$ Compliance

Compliance Protocol:

Priority:

Response: ACP 14.
Scheduled MMES-ORNL HEPA filter testing deadlines imposed by the MMESORNL Health Physics Procedure RP-1.3 are routinely missed, and tests are currently being conducted by an MMES-ORNL employee who is not properly trained and certified in accordance with ACP 14.

Inspectors performing HEPA filter tests were not certified per the requirements of

Energy Systems Risk Weight 5

Tiger Team Action Plan Priority 3

Filter tests are sometimes missed in the month that they are originally scheduled. Approved Operational Safety Requirements documents do, however, allow a 2-month extension on semiannual inspections and three months on arinual inspections. These extensions are adhered to with the exception of a very small number of inspections that are delayed due to extenuating circumstances. If an extension must be exceeded, this fact and the reason for the extension is agreed to by both Inspection and the filter owner. The inspection is ccmpleted as soon as possible.

The particular filter inspection observed was conducted by one qualified filter inspector and another inspector who was being given on-the-job instruction as required under ACP 14. The inspector being trained was functioning as an assistant and all pertinent data were being taisen by the qualified inspector. No filters are inspected without the presence of at lease one qualified filter inspector.

ACP 14 was recently revised to provide an extensive written examination for certification in addition to previously required classroom instruction and on-the-job training. Filter inspection procedures SSI 150-155 are currently being upgraded. Five filter inspectors will be trained on SSI 150-155 and given an examination in accordance with ACP 14.

\section{Root Causes:}

Inadequate policy implementation and inadequate training; training of filter inspectors was not sufficiently documented with a written examination as required by policy. 
Planned Actions and Schedules:

Item/Description

1. Revise and issue QE\&I Procedures SSI 150-155.

2. Train filter inspectors to procedures SSI $150-155$ in accordance with ACP 14.

\section{Comuletion Date}

Complete

$10 / 91$

\section{Costs:}

Type of funds: Research Programmatic

Source of funds: ER-KC

\begin{tabular}{crrrrrrr} 
& \multicolumn{5}{c}{ Estimated costs per fiscal year (\$K) } \\
\cline { 2 - 6 } Action item & 1991 & 1992 & 1993 & 1994 & 1995 & Beyond & Total \\
\hline 1 & 2 & $*$ & & & & & 2 \\
2 & 2 & $*$ & & & & & 2
\end{tabular}

Status:

Funded 4

Requested

New

*Estimated annual ongoing cost: $\$ 2 \mathrm{~K}$.

References: $\quad$ ORNL Health Physics Procedure RP-1.3 and ORNL Quality Engineering and Inspection Procedure ACP 14 
Finding No.: A/CF-3 Absence of State Air Permits for Radionuclide Sources

Finding

Description. XSO has not obtained air permits for ORNL radionuclide sources, in accordance with TDHE Air Pollution Control Rule 1200-3-9-.04(4).

Code: $\quad$ Compliance

Compliance

Protocol:

TDHE Rule 1200-3-9-.04(4) states, in part, that "...no facility processing materials containing lead, beryllium, asbestos, mercury, or any other pollutant named in rule 1200-3-11 shall be exempt." TDHE Rule 1200-3-11-.01(1) states, in part, that radionuclides are designated as hazardous air contaminants.

Priority: $\quad$ Energy Systems Risk Weight 400

Tiger Team Action Plan Priority 2

Response: The TDHE presently has no specific permit requirements for radioactive air emissions. The NESHAPS regulations establish emission and ambient radioactive limits. The Energy Systems NESHAPS compliance strategy will be provided to TDHE, and written concurrence that this strategy finally satisfies TDHE permitting requirements will be requested.

Root Caws:

Lack of clearly defined TDHE, DOE-HQ and/or DOE-ORO policy

Planned Actions and Schedules:

\section{$\underline{\text { Item/Description }}$}

1. Submit NESHAPS compliance strategy to and request concurrence from TDHE.

Costs: $\quad$ The submission will be accomplished with existing resources.
Completion Date

Complete

References: TDHE Rules 1200-3-9-.04(4) and 1200-3-11-.01 
Finding No.: A/CF-4 Ambient Air Monitoring Deficiencies

Finding

Description: The MMES-ORNL siting and design configuration of at least 7 of 18 ORR ambient air sampling stations (Stations 7, 22, 23, 34, 41, 44, and 46) may not be providing representative measurements to assess radiation dose to members of the public, in accordance with the Draft DOE Regulatory Guide for 10 CFR 834.

Code: $\quad$ Compliance

Compliance Protocol.

10 CFR 834 states "unless documented site-specific evidence exists to justify otherwise, the sample(s) at each air sampling station should be collected at a height of $2.0 \mathrm{~m}$ above ground level, in a location free from unusual localized effects or other conditions (proximity to a large building, vehicular traffic, or trees) that could result in artificially high or low concentrations." In addition, flow uniformity should be ensured, leak tests conducted, and adequacy of exchange of samples documented.

Prionity: $\quad$ Energy Systems Risk Weight 55

Tiger Team Action Plan Priority 2

Response: Improvements in siting and design configuration are presently under review as part of the overall Oak Ridge Reservation Environmental Monitoring Plan. Compliance inspections will be completed and documented at all stations to ensure proper intake height, proximity to buildings or trees, and potential perturbations of air flow. Correspondence will be initiated with DOE-ORO discussing fencing at Station 46 since present direction from ORO stipulates that a slated fence be erected.

Maintaining $+/-20 \%$ flow rate is already being addressed. The need for leak testing was recognized, and implementation is planned. Documentation for tritium sampling frequency is being prepared.

Standard Operating Procedures and protocols addressing these subjects, which will be an integral part of the Environmental Monitoring Plan, will include new requirements.

Root Causes:

Inadequate policy implementation, inadequate oversight, and inadequate communications 
Planned Actions and Schedules:

\section{Item/Description}

1. Perform and document a survey to evaluate air stations in the ORNL environmental surveillance system for compliance with Draft DOE Regulatory Guide for 10 CFR 834 siting and/or design configuration requirements. Results of the evaluation will be compiled and a status report issued.

2. Determine, from survey, tasks to be completed in order to meet the draft regulatory guide to $10 \mathrm{CFR} 834$.

3. Develop an action plan to carry out needed tasks to bring stations into compliance. This plan will include necessary work orders and associated permits.

4. Complete items listed on the action plan.

5. Submit a summary to DOE-ORO of siting and design configuration noncompliances and related recommendations at stations 41 and 46 .

6. Implement further actions based upon written guidance from DOE/ORO regarding the recommended modifications and include the rationale applied to these changes.
Completion Date

Complete 


\section{Costs:}

Type of funds: Overhead

Source of funds: Overhead

\begin{tabular}{|c|c|c|c|c|c|c|c|}
\hline \multirow[b]{2}{*}{ Action item } & \multicolumn{6}{|c|}{ Estimated costs per fiscal year ( $\$ \mathrm{~K}$ ) } & \multirow[b]{2}{*}{ Total } \\
\hline & 1991 & 1992 & 1993 & 1994 & 1995 & Beyond & \\
\hline 1 & 10 & & & & & & 10 \\
\hline 2 & - & & & & & & \\
\hline 3 & 15 & & & & & & 15 \\
\hline 4 & & 40 & & & & & 40 \\
\hline 5 & - & & & & & & \\
\hline 6 & - & & & & & & \\
\hline \multicolumn{8}{|l|}{ Status: } \\
\hline Funded & 25 & & & & & & \\
\hline \multicolumn{8}{|l|}{ Requested } \\
\hline New & & 40 & & & & & $\$ 65$ \\
\hline
\end{tabular}

References: Draft Regulatory Guide for 10 CFR 834 
Finding No.: $\quad$ A/CF-5 Effluent Stack Sampling and Monitoring Deficiencies

Finding

Description: Six of the 11 radionuclide stack monitoring systems examined at ORNL have some air monitoring deficiencies with respect to the draft Regulatory Guide for 10 CFR 834, including poor sample extraction sites, unsuitable sample transport line configurations, absence of air-florw measurements, an inappropriately designed sampling probe, and a non-operational strip chart.

Code: $\quad$ Compliance

Compliance Protocol:

DOE's Draft Radiological Effluent Monitoring and Environmental Surveillance Regulatory Guide for 10 CFR 834 (July 1990) requires effluent monitoring to provide representative measurements of the quantities and concentrations of airborne discharges.

Priority: $\quad$ Energy Systems Risk Weight 55

Tiger Team Action Plan Priority 2

Response: The items listed in this finding were listed in self assessment with the exception of the strip chart. The nonoperational strip chart recorder is located at the Y-12 Site. The roles and responsibilities between Y-12 and ORNL for ORNL facilities at Y-12 have not been fully established aná documented.

Effluent monitoring to comply with National Emission Standards for Hazardous Air Pollutants (NESHAP) will be conducted. Modification to the system will be conducted in accordance with a NSHAP compliance plan, which will be approved by EPA.

\section{Root Causes:}

Inadequate policy implementation, poorly defined roles and responsibilities, and insufficient resources

Planned Actions and Schedules:

\section{$\underline{\text { Item/Description }}$}

1. Document roles and responsibilities for $\mathrm{Y}-12$ and ORNL management for stack sampling of ORNL facilities located at Y-12.

2. Survey all ORNL radionuclide stack systems to determine compliance with draft DOE Regulatory Guide for 10 CFR 834 and with NESHAP compliance plan.
Completion Date

Complete

Complete 
3. Review and document status of funding of ORNL stack

Complete upgrade projects.

4. Review and document status of project criteria to ensure

$05 / 91$ that the design configuration is in accordance with Draft DOE Regulatory Guide for 10 CFR 834.

5. Initiate required upgrade projects and funding for radionuclide stacks which are not currently included in an upgrade program.

6. Complete required stack upgrades.

Casts: $\quad$ Stack upgrades will require additional capital funding. In addition, the upgrades will require expense funding to complete study, estimates, and maintenance.

Type of funds: Overhead

Source of funds: Overhead

Estimated costs per fiscal year $(\$ \mathrm{~K})$

\begin{tabular}{crrrrrrr}
\cline { 2 - 5 } Action item & 1991 & 1992 & 1993 & 1994 & 1995 & Beyond & Total \\
\hline 1 & - & & & & & \\
2 & - & & & & & & \\
3 & 15 & & & & & \\
4 & & 200 & & & & \\
5 & - & & & & & \\
6 & & & & $*$ & &
\end{tabular}

Status

Funded

15

Requested

200

New

*Estimated annual ongoing cost: $\$ 200 \mathrm{~K}$. 
Rev. 5

Type of funds: Capital

Source of funds: GPP

Estimated costs per fiscal year (\$K)

\begin{tabular}{llllllll}
\cline { 2 - 5 } Action item & 1991 & 1992 & 1993 & 1994 & 1995 & Beyond & Total \\
\hline 4 & & & 3000 & & & & 3000 \\
Status: & & & & & & &
\end{tabular}

Funded

Requested

New

3000

$\$ 3000$

References: Draft Regulatory Guide 10 CFR 834 
Finding No.: A/CF-6 Lack of Control Room Operator Training in Stack Release Emergency Procedures

Finding

Description: At least one control room technician in the High Radiation Level Analytical Laboratory is not trained in the stack emergency operating procedures required by Section 9 of the Emergency Operating Procedure.

Code: $\quad$ Compliance

Compliance

Protocol: Section 9 of the Emergency Operating Procedure for Building 2026 requires the facility manager to make sure the procedure is understood, adhered to, and current at all times.

Priority: $\quad$ Energy Systems Risk Weight 5

Tiger Team Action Plan Priority 3

Response: A formal training program is in place within the Ionic and Physical Analysis (IPA) Group which contains a two-hour session on each of the operating procedures related to the safe operation of the facility. Each requirement of these procedures is discussed to make sure that personnel working within the facility understands the procedure and their responsibility relative to the procedure. These training sessions are documented by an examination taken by attending personnel on the material covered.

These training requirements and training sessions are part of the facility training program outlined in SOP AC-OP-104-0203, "Training for the IPA Laboratory." Retaining requirements will be specified.

\section{Root Cause:}

Inadequate policy implementation and inadequate training

\section{Planned Actions and Schedules:}

\section{Item/Description}

1. Issue the Emergency Operating Procedure.

2. Complete Training Session on the Emergency Operating Procedure.

3. Document understanding of responsibilities within the procedure by facility personnel.
Completion Date

Complete

Complete

Complete 


\section{Casts:}

Type of funds: Research Programmatic

Source of funds: ER-KC

\begin{tabular}{|c|c|c|c|c|c|c|c|}
\hline \multirow[b]{2}{*}{ Action item } & \multicolumn{6}{|c|}{ Estimated costs per fiscal year (\$K) } & \multirow[b]{2}{*}{ Total } \\
\hline & 1991 & 1992 & 1993 & 1994 & 1995 & Beyond & \\
\hline 2 & 2 & & & & & & 2 \\
\hline \multicolumn{8}{|l|}{ Status: } \\
\hline Funded & 2 & & & & & & \\
\hline \multicolumn{8}{|l|}{ Requested } \\
\hline New & & & & & & & $\$ 2$ \\
\hline
\end{tabular}

References: ORNL SOP AC-OP-104-0203 
Finding No.: A/BMPF-1 Inadequate Stack Emission Monitoring and Test Procedures for NESHAP Compliance

Finding

Description: ORNLMMES has not conducted a formal evaluation of its radionuclide emission points, stacks, or vents in accordance with best management practice, to implement monitoring requirements as defined in of 40 CFR 61.93(b), Subpart H.

Code: Best Management Practice

Compliance

Protocol:

Priority:

Response: In accordance with the requirements for stack monitoring in 40 CFR 61, a study of all ORNL radioactive emission sources will be completed to determine which sources require monitoring. Details of this study will be described in a NESHAP Compliance Plan, which will be submitted to EPA-IV and will form the basis for a Federal Facilities Compliance Plan. The NESHAP regulations provide guidance for determining which sources require monitoring. The TDHE regulatory requirements are very similar to the NESHAP criteria for evaluation of sources which require monitoring, and therefore a single study, with appropriate modifications, will be used to provide documentation for both requirements. Emission sources to be evaluated will include, but will not be limited to, laboratory hoods, laboratory equipment, and LLLW tanks.

\section{Root Cause:}

Inadequate policy implementation

\section{Item/Description}

1. Complete radionuclide NESHAP Compliance Plan and submit to EPA.
Completion Date

$5 / 91$

Costs: Preparation is part of ongoing scheduled activities and therefore requires no additional costs or manpower.

References: None 
Finding No.: A/BMPF-2 Lack of Verifying Documentation to Demonstrate Compliance with Air Permit Conditions

\section{Finding}

Description: ORNL compliance with opacity and emission limit conditions in TDHE air operating permits is not being documented by MMES-ORNL in accordance with best management practice.

Code:

Best Management Practice

Compliance Protocol:

Priority:

Response:
Facility operators should have in place an auditable system that documents compliance with air permit conditions.

\section{Energy Systems Risk Weight 1}

Tiger Team Action Plan Priority 4

Permit conditions for some permitted air emission sources limit the opacity of the stack discharge. In a letter dated October 18, 1990, the Environmental Protection Officers were notified that a representative from each facility should be certified by the TDHE to read stack opacity. Facility operators will be required to provide documentation of annual opacity readings to demonstrate continued compliance with this condition, however based on an evaluation of the potential for emissions, some sources may be evaluated more frequently. As an immediate resolution of this deficiency a subcontract will be placed with a consulting firm to obtain an opacity reading for sources which contain this limiting condition and have not been evaluated by the operator.

Emissions from five permitted emission sources are limited to .02 grains per cubic foot at the stack. An evaluation of these sources will be completed to determine the potential emissions and if necessary, an appropriate stack monitoring schedule will be initiated to prove compliance with this permit condition.

These deficiencies were identified by ORNL in the Self-Assessment Report and corrective measures were planned.

The procedure for Air Permits, EPM-9.0, in the Environmental Protection Manual will be modified to indicate that facility operators are responsible for demonstrating compliance with these, and all conditions listed on the air permits for their permitted emission sources.

Permit conditions for some permitted emission sources limit the opacity from the source. However, due to the nature of the activity conducted at some sources, emissions resulting in opacity above the permitted level are extremely unlikely or in some cases impossible. In these cases, an annual opacity reading is adequate. Compliance with all permit conditions, including opacity limitations, is discussed in response to Compliance Finding A/CF-1. Specifically, EPM-9.0 will be revised to reflect responsibility of facility operators for ensuring continued compliance with 
permit conditions, and an annual inspection program to discuss appropriate compliance documentation with facility operators has been initiated.

\section{Root Causes:}

Inadequate policy implementation

Planned Actions and Schedules:

\section{Item/Description}

1. Evaluate sources with grain loading limitations to determine the potential emissions and if necessary initiate appropriate stack monitoring schedule to prove compliance with this permit condition.

2. Obtain certification for facility operators for future demonstration of compliance status for affected facilities.

3. Document by Environmental Compliance personnel opacity readings conducted by facility operators.

4. Issue revised EPM-90.
Completion Date

Complete

$10 / 91$

$12 / 91$

$10 / 91$

Costs: $\quad$ Estimated annual ongoing cost: $\$ 20 \mathrm{~K}$

References: Tennessee Department of Health and Environment, Air Pollution Control Rules, 1200-3 
Finding No.: A/BMPF-3 Lack of Consistent Installation of Stack Sampling, Monitoring, and Alarm Systems for Radioactive Releases

Finding

Descrip̧ion: MMES-ORNL surveillanre of some atmospheric radionuclide emissions from existing sources at ORNI, is inconsistent and is not in accordance with best management practice.

Code: $\quad$ Best Management Practice

Compliance

Protocol:

Similar air releases of radionuclides should have similar surveillance systems, in accordance with best management practices.

Priority: $\quad$ Energy Systems Risk Weight 1

Tiger Team Action Plan Priority 4

Response: ORNL is submitting to the EPA a description of all ORNL stack sampling systems (see Finding A/BMPF-1). A stack and vent survey is also being conducted which will provide additional information. Once these actions are completed an analysis will be done to determine the level of consistency necessary for best management practice at ORNL.

Root Causes:

Inadequate policy and ambiguous requirements or expectations

Planned Actions and Schedules:

\section{$\underline{\text { Item/Description }}$}

1. Determine the criteria for stack real-time monitoring at ORNL based on an ORNL needs assessment and requirements of DOE orders.

2. Determine the criteria for stack sampling at ORNL.

3. Determine which systems meet the above criteria.

4. Establish policy and necessary proceciures to assure stack surveillance for atmospheric radionuclide emissions is uniform and consistent with the established criteria.

5. Submit funding request as required to upgrade stack monitoring systems.
Completion Date

Complete

Complete

$08 / 91$

$01 / 92$

$06 / 92$ 
Costs: $\quad$ Costs for items listed are funded under Finding A/CF-5.

References: $\quad$ A/BMPF-1 Inadequate Stack Emission Monitoring and Test Procedures for NESHAP Compliance 
Finding No.: A/BMPF-4 Deficient Asbestos Waste Disposal Management

Finding

Description: MMES-ORNL is sending asbestos wastes to the $Y-12$ landfill, where the asbestos disposal area is not designated, which is not in accordance with best management practices.

Code: $\quad$ Best Management Practice

Compliance

Protocol:

Priority: $\quad$ Energy Systems Risk Weight 1

Tiger Team Action Plan Priority 4

Response: The asbestos disposal operations are conducted in accordance with the operation plan for the $\mathrm{Y}-12$ sanitary landfill as approved by the TDHE. The areas used for the disposal of asbestos are designated with appropriate signs as required by the TDHE regulations 1200-3-11-.02 during the periods in which asbestos is being removed from the transport vehicle, placed in the prepared cell, and covered with soil. Additional signs to identify the landfill as an asbestos disposal facility will be placed at the entrance to the landfill.

\section{Root Causes:}

Inadequate policy implementation

Planned Actions and Schedules:

\section{Item/Description}

1. Install signs to identify the $\mathrm{Y}-12$ landfill as an asbestosdisposal facility.
Completion Date

Complete 


\section{Costs:}

Type of funds: Overhead

Source of funds: Y-12 Plant Overhead

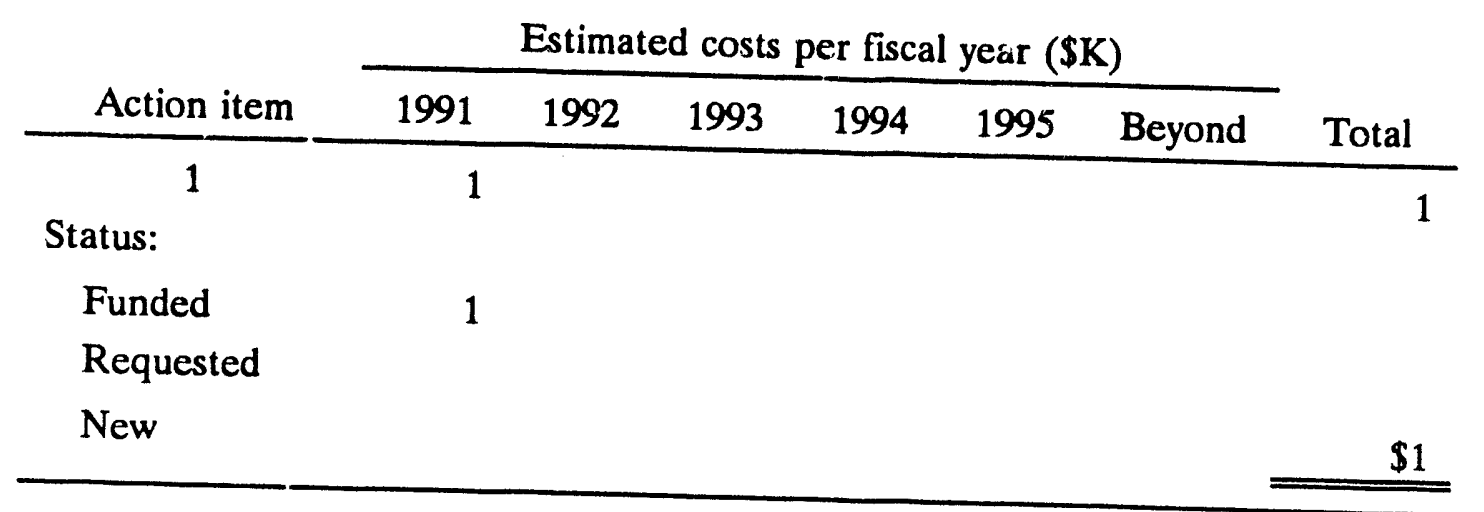

References: None 


\subsubsection{Soil/Sediments/Biota}

Finding No.: SSB/CF-1 Inadequate Radioactive Con iamination Control

Finding

Description: MMES-ORNL is not adequately surveying and containing surface and subsurface radioactive contamination in accordance with DOE 5400.1 and 5400.5.

Code: $\quad$ Compliance

Compliance

Protocol:

None

Priority: $\quad$ Energy Systems Risk Weight 108

Tiger Team Action Plan Priority 2

Response: Health Physics procedures, including the frequency of local surveys and " 'ministrative controls, will be reviewed for the SWSAs. The vehicle monitors at SWSA 6 and at Post 24 (pits and trench areas) will continue to be used. The general SWSA areas and roadways have been surveyed and are not contaminated. The primary control of personnel and vehicle contamination will continue to be entry and egress controls at the actual contaminated sites within the SWSA boundaries. Frisking is required upon exiting Contamination Areas and Regulated Areas; these requirements are well $u_{2}$ derstood by the persons who work at or enter the SWSAs. General access to the SWSA should not present a risk of contamination and, therefore, does not require a frisk upon exiting.

All areas of access to the SWSA 4 have signs that state "Health Physics Coverage Required." The Hea!th Physics staff is required for access to these areas to provide advisement as well as monitoring equipment for personnel and vehicle frisking. Postings of the SWSA 4 perimeter shall be verified and corrected to clearly identify the area with "Radiation Hazard Keep Out" signs.

Office of Environmental and Health Protection field personnel will be instructed to be alert for evicience of animal intrusions into contaminated areas. Reported intrusions will be investigated, and if a potential for contamination "trackout" is verified, appropriate measures (trapping, exterminations, etc.) will be taken.

Problems with birds and wasps transporting radioactive material from 3524 pond are well understood by the Radiation Protection staff in that area. Buildings surrounding 3524 have had an extensive survey to determine the extent of the problems caused by the birds and wasps. Closure of the 3524 pond is part of a scheduled remedial action and will eliminate the source of contamination.

Sediment sampling to determine the spread of material will cortinue in the Clinch River. 


\section{Root Causes:}

Insufficient resources, inadequate oversight, ambiguous requirements or expectations, and inadequate training

Planned Actions and Schedules:

\section{Item/Description}

1. Assess and document the re $\mathrm{C}_{2}$ uirements of $\mathrm{DOE}$ Order 5400.1 and DOE Order 5400.5 against the monitoring and posting practices at the SWSAs.

2. Submit corrective action plans to EM for review and request of funds to implement approved corrective actions.

3. Review Health Physics procedures, including the frequency of local surveys and administraive controls.

4. Assess regulatory requirements for the SWSAs and bordering areas.

5. Complete time critical removal to colstrol contaminated sediment transport into the Clinch River.
Completion Date

$6 / 91$

$8 / 91$

$8 / 91$

$12 / 91$

Casts:

\section{Type of funds: Overhead}

Source of funds: Overhead

\begin{tabular}{crrrrrrr} 
& \multicolumn{5}{c}{ Estimated costs per fiscal year (\$K) } & \\
\cline { 2 - 6 } Action item & 1991 & 1992 & 1993 & 1994 & 1995 & Beyond & Total \\
\hline 1 & 24 & & & & & & 24
\end{tabular}

Status:

Funded

Requested

New 
Type of funds: ERWM Programmatic

Source of funds: $\mathrm{EM}$

Estimated costs per fiscal year (\$K)

\begin{tabular}{lrrrrrrr}
\cline { 2 - 5 } Action item & 1991 & 1992 & 1993 & 1994 & 1995 & Beyond & Total \\
\hline 4 & & 1500 & & & & 1500 \\
$\begin{array}{l}\text { Status: } \\
\text { Funded } \\
\text { Requested } \\
\text { New }\end{array}$ & & & & & & \\
\hline
\end{tabular}

References: $\quad$ DOE Order 5400.1

DOE Order 5400.5 
Finding No.: SSB/BMPF-1 Delayed Benthic Data Analyses and Reporting

Finding

Description: Benthic samples collected as part of the ORNL Biological Monitoring and Abatement Program are not being analyzed and, therefore, the resulting data are not being reported in a timcly manner, as is expected using best management practices.

Code: $\quad$ Best Management Practice

Compliance Protocol:

Pricrity: $\quad$ Energy Systems Risk Weight 5

Tiger Team Action Plan Priority 3

Response: Currently, there is a 2.5-year backlog for the analysis of benthic invertebrate samples collected as part of the ORNL BMAP. Although this is a new finding and has not been identified in previous audits;appraisals of the BMAP, the backlog was recognized as a concern in July 1990, and a plan has been developed to address the issue. More laboratory space has been obtained, and the hiring of additional personnel to work on the sample backlog is currently in progress. An off-site subcontractor will also be selected to assist with the reduction of the backlog of samples from uncontaminated reference sites and a plan will be developed to monitor progress and to establish milestones.

Root Cause:

Insufficient resources

Planned Actions and Schedules:

\section{$\underline{\text { Item/Description }}$}

1. Obtain additional on-site staff for the benthic invertebrate laboratory.

2. Complete data analyses and procedures to support modifications in sampling program (e.g., reduced sampling frequency or number of sampling sites).

3. Establish capability for off-site analysis of benthic samples from uncontaminated reference streams.

4. Eliminate benthic sample backlog.
Completion Date

Complete

$7 / 91$

$3 / 92$

$2 / 93$ 
Costs: $\quad$ Sufficient funding is available in the current FY 1991 BMAP budget to support Items 1 and 2; the source of this funding is Laboratory overhead. An estimate of $\$ 150 \mathrm{~K}$ is necessary to facilitate and fast track an off-site contract in FY 1992. Completion of the backlog will require approval of the FY 1992 and FY 1993 budgets, to accommodate the accelerated rate of sample analysis.

Type of funds: Overhead

Source of funds: Overhead

Estimated costs per fiscal year (\$K)

\begin{tabular}{|c|c|c|c|c|c|c|c|}
\hline Action item & 1991 & 1992 & 1993 & 1994 & 1995 & Beyond & Total \\
\hline 1 & $*$ & & & & & & \\
\hline 2 & - & & & & & & \\
\hline \multicolumn{8}{|l|}{ Status: } \\
\hline \multicolumn{8}{|l|}{ Funded } \\
\hline \multicolumn{8}{|l|}{ Requested } \\
\hline New & & & & & & & $\$$ \\
\hline
\end{tabular}

*Estimated annual ongoing cost: \$50K starting in FY 1991.

Type of funds: ESH Programmatic

Source of funds: ER-AT EC

\begin{tabular}{crrrrrrr} 
& \multicolumn{5}{c}{ Estimated costs per fiscal year (\$K) } & \\
\cline { 2 - 6 } Action item & 1991 & 1992 & 1993 & 1994 & 1995 & Ecyond & Total \\
\hline 3 & & 150 & & & & & 150 \\
4 & & & 75 & & & & 75
\end{tabular}

Status:

Funded

Requested

$150 \quad 75$

New

References: None 


\subsubsection{Surface Water/Drinking Water}

Finding No.: SW/CF-1 Discharges Not Included on the ORNL NPDES Pcrmit or Permit Renewal

\author{
Finding \\ Description:

\section{Compliance} \\ Protocol:
}

Code: Compliance

Priority:

Response: waters. outfall.

\begin{abstract}
At least eight liquid waste discharges at ORNL are not listed in the existing NPDES Permit No. TNO002941, and the XSO has not listed at least six of them in the application for permit renewal as required by the performance objective.
\end{abstract}

The Tennessee Code Annotated Section 69-3-108(b)(6) requires a valid permit for the discharge of sewage, industrial wastes, or other wastes, into surface waters or a location from which it is likely that the discharged substance will move into surface

The instructions for Item II-B on EPA Form 3510-2C used in applying for an NPDES Permit require that the applicant list all sources of wastewater to each

Energy Systems Risk Weight 448

Tiger Team Action Plan Priority 2

ORNL had previously identified additional point source outfalls that are not listed on the current permit. Efforts had been initiated to characterize the discharges and to prepare the EPA Form 3510-2C applications. Some outfalls (Well Drilling Steam Cleaning Area, Hill Cut Disposal Demonstration) were considered as nonpoint source discharges, i.e., the discharged substance does not pass discernably into a surface stream. Therefore they had been listed on the NPDES permit application with the recommendation that the discharges be handled by Best Management Practices plans. The interpretation of the Tiger Team differed from that of ORNL and Energy Systems ESA environmental compliance staff. The Tiger Team recommended the listing of diffuse sources as discrete discharges. In order to achieve resolution on this portion of the finding, ORNL will enter into discussion with Energy Systems Central Staff ES\&A, DOE, and TDHE personnel to ensure that the Tiger Team interpretation of what constitutes a permittable point source discharge conforms to regulatory expectations. Final decisions on permitting strategy for those outfalls in this category will be based on the results of such discussions with TDHE.

For those point source outfalls previously identified by ORNL and the new ones identified in this finding, the wastewater discharges are being characterized, and EPA Form 3510-2C applications will be prepared and submitted as an amendment to the permit renewal application. 
A program to conduct surveillances of wastewater discharges will be developed in concert with the Division Environmental Protection Officers.

\section{Root Causes:}

Inadequate evaluation systems to ensure that all outfalls are verified, characterized, and permitted; and inadequate policy and procedures implementation. (1) Energy Systems interpretation of requirements of the Tennessee Code Annotated was not as conservative as the Tiger Team's. (2) Lack of formal documentation of regulatory requirements necessitates some interpretation by the regulated community.

Planned Actions and Schedules:

\section{Item/Description}

1. Request a formal interpretation by the TDHE of the regulatory requirements and/or interpretation of what constitutes a permittable point source discharge under NPDES.

2. As required, eliminate the outfall or prepare and submit NPDES applications.

3. Initiate comprehensive ORNL outfall and drain system survey to identify and characterize all contributions to ORNL outfalls (see Finding SW/BMPF-1).

4. Prepare NPDES application for all sources identified by comprehensive study.

\section{Completion Date}

$06 / 91$

$10 / 91$

$09 / 94$

\section{Costs:}

Type of funds: Overhead

Source of funds: Overhead

\begin{tabular}{|c|c|c|c|c|c|c|c|}
\hline \multirow[b]{2}{*}{ Action item } & \multicolumn{6}{|c|}{ Estimated costs per fiscal year (\$K) } & \multirow[b]{2}{*}{ Total } \\
\hline & 1991 & 1992 & 1993 & 1994 & 1995 & Beyond & \\
\hline All & 12 & & & 90 & & & 102 \\
\hline \multicolumn{8}{|l|}{ Status: } \\
\hline Funded & 12 & & & & & & \\
\hline \multicolumn{8}{|l|}{ Requested } \\
\hline New & & & & 90 & & & $\$ 102$ \\
\hline
\end{tabular}


Type of funds: Overhead

Source of funds: Division Administration

Estimated costs per fiscal year (\$K)

\begin{tabular}{lrrrrrrr}
\cline { 2 - 5 } Action item & 1991 & 1992 & 1993 & 1994 & 1995 & Beyond & Total \\
\hline All & 90 & & & & & & 90 \\
Status: & & & & & & & \\
\end{tabular}

Funded

Requested

New

90

$\$ 90$

References: Tennessee Code Annotated 69-3-108(b)(6); 40 CFR 122 
Finding No.: SW/CF-2 Inconsistent Labeling of ORNL Sinks anci Drains

Finding

Description: MMES-ORNL administrative controls on labeling of sinks and drains are applied inconsistently and are not in accordance with EPM 18.0, part 6.2.3.

Code: $\quad$ Compliance

Compliance Protocol:

Priority:

Response:
ORNL Environmental Protection Manual (EPM) Section 18.0, Liquid Waste Disposal, part 6.2.3 lists under responsibilities of a supervisor in charge of waste generators: "Ensures that laboratory drains and sinks are labelled properly and that waste generators use the appropriate drainage systems for the disposal of liquid waste."

\section{Energy Systems Risk Weight 20}

Tiger Team Action Plan Priority 3

Although a program exists to label sinks and drains with labels indicating (a) the system to which that drain is tied (e.g., sanitary sewer, process waste, etc.) and (b) any materials not appropriate for disposal in that particular system, this policy is inconsistently and incompletely applied across various Divisions and in various ORNL buildings. In addition, the policy does not provide sufficient instructions for drain labeling.

During the next scheduled update of EPM-18.0, additionai instructions concerning appropriate labeling of the various laboratory liquid drainage systems will be included. Consistent and complete implementation of the policy will be accomplished through training provided to all ORNL Division Environmental Protection Officers who are responsible for implementing and coordinating EPM policy requirements. In order to verify that EPM-18.0 policy requirements are properly implemented, Environmental Compliance staff members will initiate a surveillance program to document that drains are appropriately labelled.

\section{Root Causes:}

(1) Inadequate policy implementation; existing procedure requiring drain labeling is not sufficiently detailed, and the existing procedure has been incompletely implemented. (2) Inadequate training and supervision; the importance of administrative controls has not been adequately and completely communicated to all ORNL personnel. 
Planned Actions and Schedules:

Item/Description

1. Issue revised Environmental Protection Manual Chapter 18.0.

2. Develop and implement an EPO training module that incorporates the revised policy requirements.

3. Implement periodic drain system inspection and documentation.

\section{Completion Date}

$06 / 91$

$09 / 91$

$12 / 91$

Costs:

Type of funds: Overhead

Source of funds: Overhead

Estimated costs per fiscal year (\$K)

\begin{tabular}{crrrrrrr}
\cline { 2 - 6 } Action item & 1991 & 1992 & 1993 & 1994 & 1995 & Beyond & Total \\
\hline $1-2$ & 12 & & & & & & 12 \\
3 & 53 & $*$ & & & & & 53
\end{tabular}

Status:

Funded

12

Requested

New

*Estimated annual ongoing cost: $\$ 8 \mathrm{~K}$.

References: $\quad$ ORNL Environmental Protection Manual Section 18.0 
Finding No.: $\quad$ SW/CF-3 Inaccurate Stream Flow Measurement Devices

Finding Description:

Code:

\section{Compliance} Protocol:

Priority:

Response:
Weirs at Outfalls X13 and X14 are not being maintained by MMES-ORNL to provide accurate stream flow measurements, as required by the ORNL NPDES Permit, due to sediment accumulation behind both weirs and obstructions downstream of the weir at Outfall X13.

\section{Compliance}

Part II, Section C, Paragraph 2 of ORNL's NPDES Permit requires that stream flow measurement devices be maintained to ensure the accuracy of these measurements.

\section{Energy Systems Risk Weight 405}

Tiger Team Action Plan Priority 2

The NPDES standards and guidelines call for an accuracy tolerance on measured flow rate of $+/-10 \%$ of actual flow. Standard references for primary device configuration call for the approach to a sharp crested weir to have a clear depth below the notch of twice the maximum head of the weir. Effluent conditions should not cause submergence of the primary device.

The sites listed do not meet the requirements for approach and effluent configuration, primarily due to sediment and vegetation accumulation, and were identified in self-assessment. One site suffers from original design deficiencies and limitations on the natural channel. Preliminary investigations indicate that the sediment to be removed will be classified as mixed waste, which will significantly influence remediation.

Several steps toward resolution have been initiated. Sediment and vegetation data have been collected. An Engineering Service Order is active for a study and estimate to define the work to be done along with the method of accomplishment and associated costs. The results of the study may significantly affect the completion date. Erosion controi has been improved through the efforts and practices of the environmental field interface staff. Hydraulic studies are collecting data on flow parameters and investigating methods of management. Funding, is being requested through the ESP expense budget as well as through Environmental Restoration Projects.

\section{Root Causes:}

Inadequate policy implementation, inadequate policy, and poorly defined roles and responsibilities 
Planned Actions and Schedules:

\section{Item/Description}

1. Perform engineering study and estimate for method, scope, requirements, and costs for a project to remove and dispose of the materials and bring the sites to proper conditions.

2. Develop a procedure for weir, flume, and channel inspection and routine maintenance.

3. Integrate the defined maintenance tasks into a maintenance schedule.

4. Initiate engineering design.

5. Complete construction phase.
$01 / 92$

\section{Completion Date}

$06 / 91$

$06 / 91$

$12 / 91$

$09 / 93$

Costs:

Type of funds: Overhead

Source of funds: Overhead

Estimated costs per fiscal year (\$K)

\begin{tabular}{rrrrrrrr}
\cline { 2 - 6 } Action item & 1991 & 1992 & 1993 & 1994 & 1995 & Beyond & Total \\
\hline 1 & 40 & & & & 40 \\
2 & 2 & & & & & \\
3 & & & & $*$ & &
\end{tabular}

Status:

Funded

42

Requested

New

*Estimated annual ongoing cost: \$100K starting in FY 1994. 
Type of funds: Capital

Source of funds: ER

\begin{tabular}{|c|c|c|c|c|c|c|c|}
\hline \multirow[b]{2}{*}{ Action item } & \multicolumn{6}{|c|}{ Estimated costs per fiscal year (\$K) } & \multirow[b]{2}{*}{ Total } \\
\hline & 1991 & 1992 & 1993 & 1994 & 1995 & Beyond & \\
\hline 4 & & 50 & & & & & 50 \\
\hline 5 & & & 950 & & & & 950 \\
\hline \multicolumn{8}{|l|}{ Status: } \\
\hline \multicolumn{8}{|l|}{ Funded } \\
\hline \multicolumn{8}{|l|}{ Requested } \\
\hline New & & 50 & 950 & & & & $\$ 1000$ \\
\hline
\end{tabular}

Reference: $\quad$ NPDES Permit No. TN0002941 
Finding No.: SW/CF-4 NPDES Permit Exceptions

Finding

Description: Surface water discharges from ORNL periodically exceed the discharge limits as established in the NPDES Pcrmit.

Code: $\quad$ Compliance

Compliance

Protocol:

As described in Chapter 1200-4-1-.05 of the Rules and Regulations of the State of Tennessee and under ORNL's NPDES Permit No. TN0002941, ORNL is required to comply with the limitations and standards established in its discharge permit.

Priority: $\quad$ Energy Systems Risk Weight 448

Tiger Team Action Plan Priority 2

Response: As indicated in the discussion of this finding, except for storm water outfalls, Building 7002 drains, the single event at the ORNL Sewage Treatment Plant (STP), and chlorine in cooling water discharges, NPDES limit exceptions are infrequent and have no identifiable trends. ORNL has addressed or is addressing each NPDES Permit exceedence. Actions concerning resolution of each of these exceedences are taken immediately or as soon as practicable, and they have been documented in the Monthly Environmental Complianc: Report since June 1989. ORNL has recognized that several of the exceedences are of a recurring nature and actions have been initiated to address each. A corrective action has been implemented or proposed for each type of exceedence. On April 11, 1990, ORNL requested an NPDES Permit modification to revise Permit limitations on total suspended solids, oil, and grease in ORNL storm water runoff, based on knowledge to date indicating no significant impacts on ORNL surface waters from these pollutants. In addition, ORNL is concucting preliminary studies and cost estimates on storm water treatment strategies and methods and will submit a line item funding request for the event that the Permit modification request is refused by TDHE.

In May 1990 STP clarifier operating procedures were revised to preclude recurrence of the carry-over event mentioned in the Tiger Team finding. In addition, an experimental high-sludge-level alarm was purchased for the clarifier and is currently being installed. This alarm should provide further assurance against carry-over. A GPP proposal to de-chlorinate several of ORNL's most significant chlorinated cooling water discharges has been submitted to DOE-ORO. Activities to minimize discharge of pollutants from ORNL cooling towers include replacement of metal components with PVC; minimizing chlorine usage, and modifying maintenance and operating procedures. The 7002 discharge was discontinued in March 1990. 


\section{Root Cause:}

Inadequate management commitment; perceived low risk by ORNL and DOE of exceedances of NPDES Permit limits at storm water outfalls and cooling water/cooling tower outfalls, pending TDHE resolution of permit modification requests on storm water outfall limits, and completion of GPP project(s) to remove chlorine from cooling water discharges; this perception is tempered by the fact that ORNL is not unique among DOE facilities in discharges of chlorinated water and conventional storm water pollutants.

\section{Planned Actions and Schedules:}

\section{Item/Description}

1. Complete installation of experimental STP sludge blanket level alarm.

2. Complete a Conceptual Design Report for treatment systems(s) for storm water runoff.

3. Submit line item funding request for storm drain remediation for FY 1994.

4. Complete FY 1991 Chlorine Removal GPP Project.

\section{Completion Date}

$06 / 91$

$09 / 91$

$12 / 91$

$04 / 93$

Costs:

Type of funds: Overhead

Source of funds: Overhead

Estimated costs per fiscal year (\$K)

\begin{tabular}{crrrrrrr}
\cline { 2 - 5 } Action item & 1991 & 1992 & 1993 & 1994 & 1995 & Beyond & Total \\
\hline 1 & 4 & & & & & & 4 \\
2 & 48 & & & & & & 48
\end{tabular}

Status:

Funded 52

Requester

New 
Type of funds: Capital

Source of funds: EM-ADS399 GPP

Estimated costs per fiscal year $(\$ \mathrm{~K})$

\begin{tabular}{|c|c|c|c|c|c|c|c|}
\hline \multirow[b]{2}{*}{ Action item } & & \multirow[b]{2}{*}{ Total } \\
\hline & 1991 & 1992 & 1993 & 1994 & 1995 & Beyond & \\
\hline 4 & & 700 & & & & & 700 \\
\hline \multicolumn{8}{|l|}{ Status: } \\
\hline \multicolumn{8}{|l|}{ Funded } \\
\hline Requested & & 700 & & & & & \\
\hline New & & & & & & & $\$ 700$ \\
\hline
\end{tabular}

References: Tennessee Rules Chapter 1200-4-1-.05; NPDES Permit No. TN0002941 
Finding No.: $\quad$ SW/CF-5 Lack of NPDES Best Management Practices Program Plan

Finding

Description: MMES-ORNL does not have a finalized NPDES BMP Program Plan as required by 40 CFR 125.104 and the State of Tennessee NPDES Permit.

Code: $\quad$ Compliance

Compliance

Protocol:

Regulations promulgated under the Clean Water Act require all dischargers who use, manufacture, store, handle, or discharge any pollutant listed as toxic or hazardous to prepare a Best Management Practices (BMP) Program Plan to control those activities that may result in significant amounts of those pollutants reaching surface waters. The BMP Program Plan must be submitted as part of a current NPDES application. The existing ORNL NPDES Permit, in effect from April 1986 to March 1991, required the submittal of a BMP Plan within 6 months of the effective date of the permit.

Priority: $\quad$ Energy Systems Risk Weight 354

Tiger Team Action Plan Priority 2

Response: ORNL submitted a BMP Plan to the TDHE on October 31, 1986. TDHE has not commented on the Plan; therefore it has not been finalized. As indicated in the Tiger Team discussion of this finding, the TDHE did not enforce the NPDES requirement for completion of BMP Plans during the term of existing NPDES permits anywhere in the state. In 1989 ORNL noted that the existing BMP Plan document was outdated relative to current ORNL staffing, policies, and facilities; therefore, a program requiring activity- and location-specific BMP Plan was implemented in early 1990. The facility-wide baseline BMP Plan is currently being revised under a subcontract. The subcontract work was completed in December 1990.

This finding had been previously identified by ORNL and documented in the Monthly Environmental Compliance Report. Actions to correct the deficiency had already been implemented as a result of the prior identification.

\section{Root Causes:}

Inadequate policy implementation 
Planned Actions and Schedules:

Item/Description

1. Complete preparation of finalized ORNL BMP Baseline Plan.

2. Submit the BMP Baseline Plan to DOE for transmittal to TDHE.

3. Develop and implement SOPs for NPDES Permit application preparation and submittal. Define requirements and roles/responsibilities.
Completion Date

Complete

Complete

$06 / 91$

Costs:

Type of funds: Overhead

Source of funds: Overhead

\begin{tabular}{|c|c|c|c|c|c|c|c|}
\hline \multirow[b]{2}{*}{ Action item } & \multicolumn{6}{|c|}{ Estimated costs per fiscal year $(\$ K)$} & \multirow[b]{2}{*}{ Total } \\
\hline & 1991 & 1992 & 1993 & 1994 & 1995 & Beyond & \\
\hline All & 25 & & & & & & 25 \\
\hline \multicolumn{8}{|l|}{ Status: } \\
\hline Funded & 25 & & & & & & \\
\hline \multicolumn{8}{|l|}{ Requested } \\
\hline New & & & & & & & $\$ 25$ \\
\hline
\end{tabular}

References: $\quad 40$ CFR 125.104 
Finding No.: SW/CF-6 Deficiencies of Spill Prevention Control and Countermeasure Plan

Finding

Description: The MMES-ORNL combined SPCC and RCRA Contingency Plan contains deficiencies, some of which adversely affect the effectiveness of the spill control program, and is therefore not in confornance with 40 CFR 1123 and best management practice.

Code: $\quad$ Compliance

Compliance Protocol:

Priority: $\quad$ Energy Systems Risk Weight 410

Tiger Team Action Plan Priority 2

Response: A comprehensive survey of all bulk oil storage tanks will be conducted for the purpose of verifying the adequacy of existing diking. All existing dikes will be measured and their volumes calculated. Corrective actions will be taken as necessary to upgrade existing dikes or construct new dikes to meet the requirements of 40 CFR Part 112 for bulk oil storage tanks. The ground-mounted transformers will be reevaluated to determine those facilities that need to be diked; dikes will be constructed for those for which diking is a requirement. The ORNL policy concerning diking of all ground-mounted transformers will be reevaluated and documented during the next scheduled update of EPM-13.0.

The requirement to report to the LEPC will be added to the SPCC Plan during the next revision of the plan.

The amendments/revisions will include incorporation of the results of the ongoing dike survey, i.e., the volumes of all dikes around oil storage tanks will be added to the oil storage locations appendix and the need for additional diking capacity will be noted where necessary. Also, the revisions to the plan will include the requirement to report spills of reportable quantities of hazardous substances that go beyond the facility's boundaries to the local Emergency Planning Committee (LEPC). Furthermore, the plan will be revised to delete the items identified by 
the Tiger Team as "superfluous" to the plan, such as the listing of hazardous substances in the appendix labeled "Oil Storage Locations." Separate lists of petroleum products and hazardous substances will be developed and put in separate appendices so that the SPCC Plan will be more usable.

\section{Root Cause:}

Inadequate policy implementation

\section{Planned Actions and Schedules:}

\section{$\underline{\text { Item/Description }}$}

1. Conduct survey of all bulk oil storage tanks, measure dikes, and calculate required volumes.

2. Evaluate transformers to determine the need for dikes.

3. Modify Environmental Protection Manual (EPM) Procedure 13.0 to include a requirement for listing of secondary containment volume in the Secondary Containment Structure Inspection Report.

4. Amend SPCC Plan.
Completion Date

$06 / 91$

$06 / 91$

09/91

09/91

Costs:

Type of funds: Overhead

Source of funds: Division Administration

Estimated costs per fiscal year $(\$ \mathrm{~K})$

\begin{tabular}{lrrrrrrr}
\cline { 2 - 5 } Action item & 1991 & 1992 & 1993 & 1994 & 1995 & Beyond & Total \\
\hline \multicolumn{1}{c}{ All } & 50 & & & & & & 50 \\
$\begin{array}{l}\text { Status: } \\
\text { Funded } \\
\text { Requested } \\
\text { New }\end{array}$ & 50 & & & & & \\
& & & & & \\
\hline
\end{tabular}

References: $\quad 40$ C.FR Parts 112 and 355

ORNL Environmental Protection Manual, Chapter 13.0 
Finding No.: SW/BMPF-1 Inadequate ORNL Cross-Connections Study

Finding

Description: MMES-ORNL has not conducted a rigorous study at ORNL to identify cross connections or misconnections of storm, process, and sanitary drains as required by current industry practice.

Code: $\quad$ Best Management Practice

Compliance Protocol:

Priority:

Response:
The existence of drain system cross connections or misconnections does not indicate noncompliance; however, these situations can serve as conduits for inappropriate/inadvertent discharges of wastewaters that would represent noncompliance with the Clean Water Act.

\section{Energy Systems Risk Weight 121}

Tiger Team Action Plan Priority 3

This finding has been previously identified by ORNL. ORNL has previously recognized that inappropriate drain connections exist, and has instituted administrative controls in an effort prevent unpermitted discharges via these connections. ORNL is currently conducting an in-house survey to identify all existing information on drain systems including the systems within individual buildings.

\section{Root Causes:}

(1) Inadequate policy implementation; the current industry practice of identifying cross connections has not been implemented at ORNL. (2) Insufficient resources; an unacceptable level of risk may have been taken by ORNL by not providing the resources to preclude unnecessary pollutant discharges to surface waters.

Planned Actions and Schedules:

\section{$\underline{\text { Item/Description }}$}

1. Survey ORNL Divisions and buildings for drain listings, characterizations, activities and substances utilized.

Develop data management system.

2. Initiate comprehensive ORNL outfall and drain system survey to identify and characterize all contributions to ORNL outfalls.

3. Develop corrective action plan and funding request for issues identified in the comprehensive survey.

\section{Completion Date}

$04 / 92$

$06 / 93$

$09 / 93$ 


\section{Casts:}

Type of funds: Overhead

Source of funds: Overhead

Estimated costs per fiscal year (\$K)

\begin{tabular}{rrrrrrrr}
\cline { 2 - 6 } Action item & 1991 & 1992 & 1993 & 1994 & 1995 & Beyond & Total \\
\hline 1 & & & & & & &
\end{tabular}

Status:

Funded

Requested

24

New

Type of funds: ESH Programmatic

Source of funds: ER-AT EC

\begin{tabular}{cccccccc} 
& \multicolumn{6}{c}{ Estimated costs per fiscal year (\$K) } & \\
\cline { 2 - 6 } Action item & 1991 & 1992 & 1993 & 1994 & 1995 & Beyond & Total \\
\hline 2 & \multicolumn{9}{c}{3900} & & & & 3900
\end{tabular}

Status:

Funded

Requested

New

References: None 
Finding No.: SW/BMPF-2 Liquid Radionuclide Releases from ORNL Facilities

Finding Description:

Liquid waste releases from ORNL, including the Non-Radiological Wastewater Treatment Facility (NRWTF) and SWSA 5, are elevating radionuclide concentrations in the receiving streams which is not in accordance with
DOE 54005

Code: $\quad$ Best Management Practice

Compliance

Protocol:

None

Priority: $\quad$ Energy Systems Risk Weight 103

Tiger Team Action Plan Priority 1

Response: $\quad$ NRWTF

The inability of the Process Waste Treatment Plant (PWIP) to remove the cesium-137 present in the process wastewater was identified as a deficiency/concern in the ORNL self-assessment. However, this limitation of the processing capability of the PWTP has been recognized for approximately 4 years. In 1986, the PWTP treatment process was modified in response to a critical need to reduce the volume of liquid low level waste (LLLW) generated by ORNL. The modification resulted in an $80 \%$ decrease in the volume of LLLW generated by the PWTP annually, but adversely affected the capability of the PWTP to remove the cesium-137. Since 1986, development studies have been underway to identify a process to remove the cesium-137 without resulting in an increase in the more hazardous LLLW. Those studies have concluded that a zeolite ion exchange system will remove the cesium-137 while producing a minimum volume of solid secondary waste (spent zeolite resin) for which an approved disposal method exists. During the development period, Waste Operations has used the temporary zeolite column to reduce the cesium-137 concentration in the PWTP effluent when sampling results indicated an elevated concentration in the PWTP influent.

A general plant project will be proposed for fiscal year (FY) 1992 to install a zeolite ion exchange system at the PWTP for removal of cesium-137. The functional requirements document for this system has been prepared and issued to Energy Systems Engineering to prepare the study and estimate and the preliminary
proposal for the project during FY 1991 .

\section{SWSA 5}

Actions required to address the radionuclide releases from SWSA 5 into Melton Branch will include (1) focus on the Melton Branch flood plain in the early phase of the Waste Area Grouping (WAG) 5 and WAG 2 remedial investigations (RI) and take full advantage of ongoing source-term definition studies at SWSA 5; (2) compilation, evaluation, and trending of new and historical surface water 
release data in annual environmental reports; and (3) integration if environmental ALARA goals and objectives into planning and implementing measures to reduce contaminant discharges into surface waters.

The first action will specifically address the cited deficiency in evaluating releases from SWSA 5 seeps into surface waters which flow into Melton Branch. Both the WAG 5 and the WAG 2 RIs are scheduled to begin in FY 1991. In addition, studies of source-term definition will be conducted in FY 1991 and will emphasize storm-event transport and evaluation of the importance of matrix diffusion processes on rates of contaminant releases. Goals are to further identify localized significant sources in SWSA 5 and to provide sufficient characterization for planning effective corrective measures. Data acquired during the RIs and source term definition studies will be factored into the need for and priority of interim corrective measures (ICMs) to reduce or eliminate discharges to surface waters from sources within WAG 5. The ICM prioritization process for FY 1992 funding will include consideration of actions needed to reduce releases from WAG 5.

The second action will more universally address the need for comprehensive review and evaluation of past and current surface water release data. As part of compliance with 10 CFR 834 (Draft), the Energy Systems-ORNL Information Integration and Analysis Group has initiated the evaluation of trends in environmental surveillance and effluent monitoring data. Trends in surface water contaminant characteristics are being investigated with respect to average concentrations, flow-weighted average concentrations, discharge, and discharge relative to flow. Trends will be investigated in different time scales in order to evaluate the impact of averaging upon the recognition of fluctuations in contaminant release rates versus apparent changes in the rate of flux relative to precipitation and discharge. The Energy Systems-ORNL Information Integration and Analysis Group's Standard Operating Procedure "E-SP-005-05, Data Reporting," will be revised in Section 7.1.5 "Trends" to include specific guidance for surface water trend analysis and reporting. Surface water trends analysis will be incorporated into the ORNL portion of the annual Oak Ridge Reservation Annual Environmental Report beginning with the report for 1992.

The third action will address the need to formally integrate environmental ALARA goals and objectives into reducing contaminant releases to surface waters. Beginning with the prioritization process for FY 1992 ICMs, consideration of environmental ALARA goals will formally become a criterion used in the prioritization process. The mechanism by which ALARA will be integrated into the FY 1992 process will be the first of an annual series of baseline design reports for ICMs. These reports will document the scope, cost, and schedule of all ICMs planned for the ORNL site. This first report is scheduled to be released by the end of FY 1991. 


\section{Root Causes:}

(1) NRWTF: inadequate management approach; relying on one process to clispose of LLLW concentrate without foreseeing the loss of that process as a result of regulatory changes has left the Laboratory without an effective means of disposal of LLLW concentrate. (2) SWSA 5: inadequate policy implementation; consideration and implementation of the environmental ALARA principle has been inadequate in planning ICMs. (3) Inadequate policy implementation and inadequate communications; formalization of surface water discharge data analysis, trending, and reporting has been inadequate to integrate the information into environm_..tal restoration activities.

Planned Actions and Schedules:

\section{Item/Description}

\section{NRWTE}

1. Complete the study and estimate and prepare/submit the preliminary proposal to DOE for capital funding of the GPP to install the zeolite ion exchange system at the PWTP.

2. Complete design and construction of the zeolite ion exchange system.

05/94

\section{SWSA 5}

1. Issue the first baseline design repurt for the ICMs incorporating environmental ALARA into the prioritization process.

2. Integrate source-term definition and WAG 5 and WAG 2 remedial investigation data into the annual baseline design report for the ICM prioritization process.

3. Issue the revised standard operating procedure for the Energy Systems-ORNL Information Integration and Analysis Group to formally evaluate and report trends in surface water release data.

4. Issue the first Oak Ridge Reservation Annual Environmental Report incorporating ORNL surface 
Costs: $\quad$ NRWTF

Type of funds: ERWM programmatic

Source of funds: EM-ADS 350 and 366

\begin{tabular}{crrrrrrr} 
& \multicolumn{6}{c}{ Estimated costs per fiscal year (\$K) } & \\
\cline { 2 - 6 } Action item & 1991 & 1992 & 1993 & 1994 & 1995 & Beyond & Total \\
\hline 1 & 110 & & & & & 110 \\
2 & & 45 & 35 & 30 & & 110
\end{tabular}

Status:

Funded

110

Requested

$45 \quad 35 \quad 30$

New

Type of funds: Capital-GPP

Source of funds: EM-ADS 366

Estimated costs per fiscal year (\$K)

\begin{tabular}{|c|c|c|c|c|c|c|c|}
\hline Action item & 1991 & 1992 & 1993 & 1994 & 1995 & Beyond & Total \\
\hline 2 & & 1000 & & & & & 1000 \\
\hline Status: & & & & & & & \\
\hline Funded & & & & & & & \\
\hline Requested & & 1000 & & & & & \\
\hline New & & & & & & & $\$ 1000$ \\
\hline
\end{tabular}


SWSA 5

Type of funds: Overhead

Source of funds: Overhead

\begin{tabular}{|c|c|c|c|c|c|c|c|}
\hline \multirow[b]{2}{*}{ Action item } & \multicolumn{6}{|c|}{ Estimated costs per fiscal year (\$K) } & \multirow[b]{2}{*}{ Total } \\
\hline & 1991 & 1992 & 1993 & 1994 & 1995 & Beyond & \\
\hline 1 & - & & & & & & . \\
\hline 2 & - & & & & & & \\
\hline 3 & & 75 & & & & & 75 \\
\hline 4 & & 75 & & & & & 75 \\
\hline
\end{tabular}

Funded

Requested

New

References: $\quad$ NRWTF

DOE Order 5400.5

Strategy for Management of ORNL Process Wastewater

SWSA 5

DOE Order 5400.5

Environmental Restoration and Waste Management Five-Year Plan 
Finding No.: $\quad$ SW/BMPF-3 Lack of Backflow Prevention Devices

Finding

Description: Backflow prevention devices are not installed in at least five locations in the ORNL water supply system which is not in accordance with best management practice.

Code: $\quad$ Best Management Practice

Compliance

Protocol:

Current industry practice requires the installation of backflow prevention devices to prevent the contamination of potable water systems by the backsiphonage of contaminated water in the event of pressure loss in the water distribution system.

Priority: $\quad$ Energy Systems Risk Weight 6

Tiger Team Action Plan Priority 3

Response: $\quad$ See Finding MA.5-1

Planned Actions and Schedules:

Corrective actions for this finding are addressed in Finding MA.5-1. These actions include the training needed to identify requirements.

Costs: $\quad$ Costs are addressed in Finding MA.5-1.

References: None 
Finding No.: $\quad$ SW/BMPF-4 Unrepaired Leaks from Wastewater Sewer Systems

\section{Finding Description:}

Code:

\section{Compliance}

Protocol:

Priority:

Response:
MMES-OPNL has not repaired some sewer lines at ORNL that are known to be leaking, including low level liquid wastewater (LLLW) lines, noncontaminated wastewater lines, and sanitary sewage lines in accordance with best management practices.

Best Management Practice

None

Energy Jystems Risk Weight 49

Tiger Team Acticn Plan Priority 1

This action plan addresses three activities: (1) rehabilitation of piping within the LLLW system, (2) the installation of flow monitoring equipment within the process waste system, and (3) an upgrade of the sanitary sewer system.

\section{LLLW System}

Projects, both GPPs and Line Items, have either been identified or planned to upgrade the LLLW system at ORNL. A series of 19 GPPs (beginning in FY-1992 and extending through FY 1996) and four Line Items (FY 1991 through FY 1995) are being implemented to upgrade the LLLW collection and transport system. By approximately 2000 , all LLLW lines will be replaced with doubly contained piping or be taken out of service by bottling, trucking, waste reduction, or source treatment. A leak testing program for active lines within the LLLW system will be implemented in accordance with the Federal Facilities Agreement. LLLW systems will be repaired or taken out of service when leaks are discovered. Contaminated soil encountered during reconstruction of the LLLW system will be managed in a manner consistent with the level of contamination present as required by established Energy Systems Health Physics procedures.

\section{Provess Waste System}

It is likely that unidentified leaks exist within the piping systems at ORNL. Rehabilitation of the process waste, sanitary sewer, and storm sewer systems via insitu-lining and point repairs has been conducted over the past several years. Some areas of the process waste system were purposely left unlined to allow collection of groundwater known to be contamiriated (i.e., under Bldg. 3047 and near Bldg. 3517) as an implementation of Best Management Practices. A water balance to identify leaks within the various systems needs to be conducted. Such an effort will require accurate flow rates of the water entering: (1) the plant, (2) the bui.dings where the waste is generated, and (3) each identified subsystem within the sewer system. 
Efforts have been underway since the early 1980 s to identify and eliminate the pathways by which undesirable in-leakage into the process waste system occurs. Upgrading existing flow and radiation monitoring equipment, in addition to the centralized monitoring system, the Waste Operations Control Center, was one of the first steps taken toward determining the location and type of in-leakage. Additionally, an inflow/infiltration study of the process waste system was performed by a subcontractor to provide additional guidance on how to address the problem.

The inflow/infiltration study identified sections of process piping that had cracks, off-set joints, and broken pipes. In situ lining of the process piping was selected as the most cost effective method for rehabilitating the piping. Lining of the process waste system was accomplished primarily under three General Plant Projects (GPP), namely Upgrade 3000 Area Collection System (FY-1985, WBS 3.25), Process Waste System Inflow/Infiltration (FY-1986, WBS 3.18), and Volume Reduction Piping Modifications, PWTP (FY-1985, WBS 3.08), which lined approximately 4100, 3800 , and 2000 feet of process waste piping, respectively. Approximately $\$ 1.15 \mathrm{M}$ of capital funds was spent under the three GPPs mentioned above. Expense funding of approximately $\$ 480 \mathrm{~K}$ was spent in support of these GPPs. In situ lining of process waste piping was also accomplished utilizing non-GPP funding under emergency conditions (i.e., pipe collapsed, pipe in imminent danger of collapsing, etc.).

GPPs to install new flow monitoring equipment in the process waste system are planned: Manhole Monitors - Process Waste, FY 1989, WBS 3.50; Manhole Monitors - Process Waste, 4500, FY 1993, WBS 3.58; and Manhole Monitors Process Waste Phase 2, FY 1993, WBS 3.92. Data collected from these additional flow monitoring stations will be used with data from existing stations to provide a more accurate determination of the amount of unknown infiltration or exfiltration from the process waste system. An evaluation of flow data, both within the process waste system and at the inlet to the generators, will be performed and used to evaluate sealing the manholes within the piping systems would be effective.

\section{Sanitary Sewer System}

Evidence of in-leakage into the sanitary sewer system led to lining approximately $80 \%$ of the piping with diameters 6 in. or greater. The lining was accomplished under two GPPs: Sewer System Infiltration and Inflow (FY 1984), and Sewer System Infiltration Inflow (FY 1985, WBS 3.13). A 30\% reduction in the amount of inflow/infiltration was noted after this work was completed. The capital expenditure for these projects totalled $\$ 828 \mathrm{~K}$, in addition expense funding of $\$ 340 \mathrm{~K}$ for support was spent. Some of the piping identified for rehabilitation under the two sanitary sewer GPPs was deleted because of radioactive contamination and the lack of procedures, safety review, quality assurance, and methods for outside contractors to work in radioactively contaminated areas at the time the projects were underway. The need for Sanitary Sewer Systems improvements has been recognized. A proposed ORNL Line Item, Project No.: 93-ORNL-EX-1, Upgrade Sanitary Sewerage System, ORNL (FY-93) has been submitted and is in the preliminary review process. 
Appropriate corrective actions to address contamination discovered during the upgrade will be developed. The level of remediation required cannot be estimated at this time.

\section{Root Causes:}

LLLW System: insufficient resources. Process Waste System: insufficient resources; currently lack the ability to accurately detect and locate leaks within the system. Sanitary Sewer System: inadequate management approach and policy implementation; the philosophy has been to use a number of GPPs and expense funding in an effort to "make-do" instead of repairing the system using one or two line items.

\section{Planned Actions and Schedules:}

\section{Item/Description}

\section{LLLW System}

1. Identified capital projects and line items to upgrade portions of the waste collection and transfer systems of the LLLW System.

$\frac{\text { WBS }}{3.01}$

3.01

3.02

3.03

3.28

3.31

3.35

3.37

3.45

3.79

3.85

3.96

\section{TITLE}

HFIR LLW Upgrade FY 1992, GPP 3000 Area LLW Upgrade FY 1992, GPP Building 3047 Trucking Station FY 1992, GPP FFA Compliance Work I FY 1992, GPP Bethel Valley FFA Upgrade FY 1994, LI ILLW Treatment Alternatives FY 1993, GPP BVLLW Collection \& Transfer FY 1988, LI MVLLLW Collection \& Transfer FY 1992, LI ORR/BSR LLW Upgrade FY 1992, GPP FFA Compliance Work II FY 1993, GPP 4500 Area LLW Upgrade FY 1992, GPP Bethel Valley FFA II FY 1995, LI

2. Request funding for out-year (beyond FY 1993) GPPs and Line Items.

\section{Completion Date}

\section{Process Waste System}

1. Provide additional flow monitoring capability at five process waste stations in the 4500 area. Each station will be equipped with flow-measuring instrumentation, sampling equipment, telemetry equipment, and an instrument enclosure. WBS 3.50, Manhole Monitors Process Waste, FY 1989, ADS 350. 
2. Provide additional flow-monitoring capability at up to six

$09 / 94$ process waste stations within the Bethel Valley area of ORNL. Each station will be equipped with flowmeasuring instrumentation, sampling equipment, telemetry equipment, and an instrument enclosure.

Manhole Monitors - Process Waste Phase 2, WBS 3.92 and Manhole Monitors - Process Waste 4500, WBS 3.58; FY 1993, ADS 350.

3. Evaluate the need for lining or sealing manholes within the process waste system.

09/95

\section{Sanitary Sewer System}

1. Submit Preliminary Construction Data Sheet for project to upgrade the sanitary sewage collection and disposal system to current standards.

Complete

Costs: $\quad$ Capital and expense funding has been approved for the FY 1989, 1990, and 1991 GPPs. Program planning activities will be accomplished with existing resources. Projects scheduled for FY 1993 and beyond will require receipt of the requested capital and expense funding to meet the goals. Funding sources for upgrading the LLLW System is expected to come from ADS 302, 304, 349, and 378 of the EM program. ADS 350 of the EM program will provide funding for the Process Waste System activities.

\section{LiLW System}

Type of funds: ERWM Programmatic

Source of funds: EM - ADS 302, 304, 378

\begin{tabular}{crrrrrrr} 
& \multicolumn{6}{c}{ Estimated costs per fiscal year (\$K) } & \\
\cline { 2 - 6 } Action item & \multicolumn{1}{c}{1991} & \multicolumn{1}{c}{1992} & 1993 & 1994 & 1995 & Beyond & Total \\
\hline 1 & 5,026 & 6,840 & 16,722 & 11,490 & 8,540 & 16,440 & 65,058 \\
2 & 10 & 10 & 10 & 10 & 10 & 10 & 60
\end{tabular}

Status:

Funded $\quad 5,036$

$\begin{array}{llllll}\text { Requested } & 6,850 & 16,732 & 11,500 & 8,550 & 16,450\end{array}$

New 
Type of funds: Capital-GPP

Source of funds: EM - ADS 378, 349, 350

\begin{tabular}{cccccccc} 
& \multicolumn{6}{c}{ Estimated costs per fiscal year $(\$ \mathrm{~K})$} \\
\cline { 2 - 6 } Action item & 1991 & 1992 & 1993 & 1994 & 1995 & Beyond & Total \\
\hline All & & 6,650 & 3,000 & 4,000 & 4,000 & 4,000 & 21,650 \\
Status: & & & & & & &
\end{tabular}

Funded

$\begin{array}{llllll}\text { Requested } & 6,650 & 3,000 & 4,000 & 4,000 & 4,000\end{array}$

New

Type of funds: Line items

Source of funds: EM ADS 378, 302, 304, 378-AA, 378-AB

Estimated costs per fiscal year (\$K)

\begin{tabular}{lccccccc}
\cline { 2 - 6 } Action item & 1991 & 1992 & 1993 & 1994 & 1995 & Beyond & Total \\
\hline 1 & 7,081 & 4,647 & 15,900 & 26,500 & 39,100 & 35,000 & 128,228 \\
Status: & & & & &
\end{tabular}

Funded 7,081

$\begin{array}{llllll}\text { Requested } & 4,647 & 15,900 & 26,500 & 39,100 & 35,000\end{array}$

New 


\section{Process Waste System}

Type of funds: ERWM Programmatic

Source of funds: EM - ADS 350

Estimated costs per fiscal year (\$K)

\begin{tabular}{crrrrrrr}
\cline { 2 - 5 } Action item & 1991 & 1992 & 1993 & 1994 & 1995 & Beyond & Total \\
\hline 1 & 20 & 40 & 40 & & & 100 \\
2 & & 200 & 100 & 80 & 30 & 410 \\
3 & & & & & 30 & 30
\end{tabular}

Status:

Funded

20

Requested

$240 \quad 140$

$80 \quad 60$

New

Type of funds: Capital-GPP

Source of funds: EM - ADS 350

Estimated costs per fiscal year (\$K)

\begin{tabular}{cccccccc}
\cline { 2 - 6 } Action item & 1991 & 1992 & 1993 & 1994 & 1995 & Beyond & Total \\
\hline 1 & 500 & & & & & & 500 \\
2 & & & 1400 & & & & 1400
\end{tabular}

Status:

Funded

500

Requested

1400

New

$\$ 1900$ 


\section{Sanitary Sewer System}

Type of funds: ESH Programmatic

Source of funds: ER-AT

\begin{tabular}{|c|c|c|c|c|c|c|c|}
\hline \multirow[b]{2}{*}{ Action item } & \multicolumn{6}{|c|}{ Estimated costs per fiscal year $(\$ \mathrm{~K})$} & \multirow[b]{2}{*}{ Total } \\
\hline & 1991 & 1992 & 1993 & 1994 & 1995 & Beyond & \\
\hline 1 & & 100 & 200 & 100 & 100 & 一 & 500 \\
\hline \multicolumn{8}{|l|}{ Status: } \\
\hline \multicolumn{8}{|l|}{ Funded } \\
\hline Requested & & 100 & 200 & 100 & 100 & - & \\
\hline New & & & & & & & $\$ 500$ \\
\hline
\end{tabular}

Type of funds: Line Item

Source of funds: ER

Estimated costs per fiscal year (\$K)

\begin{tabular}{llllllll} 
& \multicolumn{6}{c}{ Estimated costs per fiscal year (\$K) } & \\
\cline { 2 - 6 } Action item & 1991 & 1992 & 1993 & 1994 & 1995 & Beyond & Total \\
\hline 1 & & & 2,000 & 5,000 & 4,000 & 11,000 \\
Status: & & & & & & \\
$\begin{array}{l}\text { Funded } \\
\text { Requested } \\
\text { New }\end{array}$ & - & - & & & & \\
\hline
\end{tabular}

References: $\quad$ LLLW System: ADS 378; ADS 302; ADS 304; ADS 349; Federal Facilities Agreement; Site Specific Plan for the Oak Ridge Reservation, February 1990

Process Waste System: ADS 350; Site Specific Plan for the Oak Ridge

Reservation, February 1990

Sanitary Sewer System: Cost Per Preliminary Construction Data Sheet 
Finding No.: $\quad$ SW/BMPF-5 Lack of Certification of Treatment Plant Operators and Backflow Preventer Repairers

Finding

Description: All MMES-ORNL sewage treatment plant operators and backflow preventer repairers are not certified by the State, as recommended by best management practices.

Code: $\quad$ Best Management Practice

Compliance Protocol:

Priority: $\quad$ Energy Systems Risk Weight 1

Tiger Team Action Plan Priority 4

Response: In accordance with best management practices, supervisors and operators directly responsible for operation of the Sewage Treatment Plant will be state certified to Grade 1 as the state training program permits. Pipefitters involved in repairing backflow preventers will receive state sponsored training on those devices to accomplish state certification as training program vacancies permit.

\section{Root Cause:}

Ambiguous requirements or expectations

Planned Actions and Schedules:

\section{$\underline{\text { Item/Description }}$}

1. Request funding necessary to train, certify, and maintain state certification for steam plant operators and pipefitters.

2. Upon receipt of funding, issue standard operating procedures to establish and implement required certification.

3. Two pipefitters engaged in the repair of backflow preventers will receive state certification each year until all required are certified.

4. One supervisor will receive state certification to operate the Sewage Treatment Plant each year until all are certified.
Completion Date

$06 / 94$

$06 / 96$ 
5. Upon receipt of funding, train, test, and certify four operators/year until all who operate sewage

$12 / 98$ treatment plant are certified.

Costs:

Type of funds: Overhead

Source of funds: Overhead

Estimated costs per fiscal year (\$K)

\begin{tabular}{crrrrrrr}
\cline { 2 - 5 } Action item & 1991 & 1992 & 1993 & 1994 & 1995 & Beyond & Total \\
\hline 1 & - & & & & & & \\
2 & - & & & & & & \\
3 & 2.6 & 2.7 & 2.8 & & & & 8.1 \\
4 & 4 & 4.1 & 4.2 & 4.3 & 4.4 & 4.5 & 25.5 \\
5 & & 60 & 62.7 & 65.5 & 68.5 & 71.6 & 328.3
\end{tabular}

Status:

Funded

6.6

Requested

New

66.8

$69.7 \quad 69.8$

72.9

$76.1^{*} \$ 361.9$

*Estimated annual ongoing cost: $\$ 76.1 \mathrm{~K}$.

References: None 


\subsubsection{Groundwater}

Finding No.: GW/CF-1 Inadequate Hydrogeologic Characterization at SWSA 6

Finding

Description:

Code: $\quad$ Compliance

Compliance Protocol:

Priority: Energy Systems Risk Weight 507

Tiger Team Action Plan Priority 2

Response: $\quad$ SWSA 6 is being closed in accordance with a RCRA closure plan which was approved by the Tennessee Department of Health and Environment (TDHE) in September 1988. The RCRA closure plan specifies a series of activities leading to final closure. These activities follow the RCRA corrective action plan, that is, conducting an RFI and a corrective measures study (CMS) foliowed by design and implementation. The closure plan states that the closure activities will be conducted in general conformance with Comprehensive Environmental Response, Compensation, and Liability Act (CERCLA) guidance although the Environmental 
Protection Agency (EPA) Region IV and TDHE have stated that, as a RCRA closure, site activities need not be conducted in strict accordance with the pending Federal Facility Agreement (FFA). The NCP (40 CFR 300.430) states that "the purpose of the remedial investigation is to collect data necessary to adequately characterize the site for the purpose of developing and evaluating effective remedial alternatives." $40 \mathrm{CFR}$, Part 300, EPA, in the Preamble, stresses "the need to balance the desire for definitive site characterization and alternatives analysis with a bias for initiating response actions or control hazards posed by a site as early as possible." Finally, the newly proposed Subpart S of 40 CFR Part 264 seeks to clarify guidance and allow a streamlining of the remedial facility investigation process. Investigations are to be focused on plausible concerns and conducted in a step-wise fashion, with early screens to determine whether further investigation is necessary. Where an owner/operator proposes a remedy that is effective and protective, it may be appropriate for the regulators to approve the remedy and avoid continued studies that would serve only to delay cleanup. Under this guidance, there is need for a high level of interaction between the permittee, EPA and TDHE to determine the adequacy of an investigation. Since the process is acknowledged as step-wise, and the results of the current site characterization will be submitted to EPA-Region IV and TDHE for evaluation this fiscal year, the best way to address the root cause is to seek clarification from the regulators to resolve ambiguities in how much characterization is enough. This will result directly from review and comment, followed by discussion at the regulatory interface sessions that are held at regular intervals.

\section{Root Cause:}

Ambiguous requirements and expectations

Planned Actions and Schedules:

\section{Item/Description}

1. Submit RFI report to DOE/ORO containing point-bypoint response to the Tiger Team finding for review.

2. Issue RFI containing point-by-point response report to EPA and TDHE for review, comment, and approval, and to EH-5 (DOE Tiger Team Office) for information.

Costs: $\quad$ Preparation of the RFI report will be accomplished with existing Environmental Restoration resources (EW) (ADS OR-363).

References: $\quad$ ORR RCRA/HSWA Permit

40 CFR 300

DOE Order 5400.4

ORNL/RAP/Sub-87/99053/9 \& V1/R1, Closure Plan for Solid Waste Storage Area 6, Volume 1: Closure Plan
Completion Date

$8 / 91$

$9 / 91$ 
Finding No.: GW/CF-2 Inadequate Implementation of Well Purging Procedures at SWSA 6

Finding

Description: Water level measurements and well purging for the SWSA 6 groundwater quality monitoring (GQM) wells are not being implemented in a consistent, reliable manner as required by MMES-ESP-302-2, State of Tennessee Rule 1200-1-11.05, and 40 CFR Part 265, Subpart F, and are resulting in the underestimation of purge volumes.

Code: $\quad$ Compliance

Compliance Protocol:

Energy Systems, the state of Tennessee, and EPA requirements all include provisions that a groundwater sampling program must be implemented utilizing consistent, reliable, and accepted procedures for water level measurements and purging of wells.

Priority: $\quad$ Energy Systems Risk Weight 405

Tiger Team Action Plan Priority 2

Response: Based on a review of well purging records it was determined that the action referenced in this finding in fact only occurred one time and that was during the Tiger Team investigation. The error was due to one "old" reference document describing well depths in SWSA-6 from the ground level instead of the casing level. The day after this finding was recognized memos were issued requiring all files be checked and purged of outdated procedures and protocols. These instructions were followed up by staff inspection of files to ensure that all outdated material had been destroyed. Additional plans are outlined in the actions section in order to ensure that this does not recur.

\section{Root Causes:}

Inadequate communications and inadequate policy implementation

\section{Planned Actions and Schedules:}

\section{$\underline{\text { Item/Description }}$}

1. Issue memo to the Environmental Sampling and Instrumentation Group to purge all files of old outdated materials immediately.

2. Instruct field technicians responsible in all sampling areas, including groundwater, to ensure that all log sheets and associated paperwork are current. This will be accomplished by crosschecking with the Master SOP copies in the Group Leaders office.
Completion Date

Complete

Complete 
3. Inspect group files to ensure all outdated materials have been removed.

Complete

4. Implement an internal audit program (p 3-115 of the Tiger Team Environmental Assessment). The Group

Complete Leader will assign a staff member to develop, oversee, and conduct an internal audit program of field log sheets and associated material every 2 weeks.

5. Conduct internal field surveillances on a monthly basis (normally done quarterly) for the next year in order to ensure that field technicians are properly carrying out all activities in the field per Standard Operating Procedures.

6. Conduct tests covering sampling activities (field and classroom) on a quarterly basis (normally done yearly) for the next year in order to ensure that field technicians are properly carrying out all activities per Standard Operating Procedures.

7. Develop and present a performance-based training Complete module specifically addressing proper use and maintenance of all field log sheets and associated materials. 
Casts:

Type of funds: Overhead

Source of funds: Overhead

Estimated costs per fiscal year (\$K)

\begin{tabular}{|c|c|c|c|c|c|c|c|}
\hline Action item & 1991 & 1992 & 1993 & 1994 & 1995 & Beyond & Total \\
\hline 1 & - & & & & & & \\
\hline 2 & - & & & & & & \\
\hline 3 & - & & & & & & \\
\hline 4 & 1 & * & & & & & 1 \\
\hline 5 & 2 & * & & & & & 2 \\
\hline 6 & 2 & * & & & & & 2 \\
\hline 7 & 5 & * & & & & & 5 \\
\hline
\end{tabular}

Status:

Funded 10

Requested

New

*Estimated annual ongoing cost: $\$ 10 \mathrm{~K}$

References: SOP-EMC-003.025 Collection of RCRA Well Samples 
Firsding No.: GW/BMPF-1 Inadequate Well and Borehole Abandonment

Finding

Description: Many unneeded wells and boreholes at ORNL are not being "abandoned" by MMES-ORNL, which is not in accordance with current industry practice or regulatory agency guidance.

Code: $\quad$ Best Management Practice

Compliance

Protocol:

Priority:

Response:

\section{None}

Energy Systems Risk Weight 50

Tiger Team Action Plan Priority 2
The issue that no uniform, sitewide well and borehole plugging and abandonment (P\&A) program exists for ORNL was identified in the DOE Environmental Survey of 1987, the DOE Oak Ridge Operations Environmental Protection and QA Appraisal of August and September, 1988, and again in the DOE ESH\&QA Appraisal of April, 1990. An organizational basis for a sitewide P\&A program was sug'gested in the draft ORNL Groundwater Protection Program Management Plan (M $a_{j}^{\prime}$ 1990, Sec. 6.9), as a functional responsibility of the Groundwater Protection Program Manager, a position which at that time had not been established. ORNL has now established and filled the position of Groundwater Program Coordinator. The P\&A of unneeded wells and boreholes is an integral part of the WAG closure process, and wells requiring abandonment in and near SWSA 6 current $y$ are being identified by an ORNL interorganizational group under the leadership of the interim GWC.

ORNL will establish and implement a sitewide P\&A program. Tasks identified under planned actions and schedules will also address P\&A for all other ORNL candidate wells except hydrofracture which will be handled separately because of cost and technology required. The need to plug and abandon all wells is not equally urgent because many have been in place for a long time and hydrostatic conditions within and adjacent to such holes are expected to be stabilized. We!ls installed since 1985 have been constructed to high standardis and are likely to have lower priority under the sitewide P\&A program. An additional factor is that 60 percent of the wells in the ORNL area are less than $50 \mathrm{ft}$ deep and only 4 percent are greater than $100 \mathrm{ft}$ deep. There is lower risk of contaminant transfer within a shallow hole than in deeper holes, although a primary concern in regard to shallow holes is that they may be an avenue for entrance of contaminated surface water. The ORNL P\&A program will be carried out in timely fashion, in an orderly, planned sequence, first addressing those wells and boreholes in known contaminated areas that clearly require P\&A. An example category is that of wells in waste areas constructed with continuously-perforated galvanized corrugated pipe, described in Finding GW/BMPF-1. 
The program, as specified in the ORNL Groundwater Protection Program Management Plan (latest revision available end July, 1991), includes a process for identifying candidate wells and boreholes and categories of wells for P\&A; prioritization based on contaminant migration potential within the borehole and from surface inflow of the wells selected; and formulation of a set of P\&A procedures and guidelines applicable to the range of conditions, both of the well and of surrounding subsurface conditions. Responsibility for management, coordination, and continuing administration of the program will be that of the GWC. These current roles and responsibilities are outlined in the ORNL Groundwater Protection Program Management Plan.

Special-case wells and boreholes, such as certain of those referred to in Findings GW/BMPF-1 (Joy Test Well) and GW/BMPF-3 (wells in the vicinity of Hydrofracture Facility No. 4) may require interim corrective measure treatment until decisions are reached as to their ultimate disposition. Special procedures must be developed for these wells, designed to prevent borehole fluid migration and to protect casing integrity from corrosive fluids. Investigation of current status of hydrofracture wells and available technology for ICM and P\&A activities will be initiated in 1992.

\section{Root Causes:}

The need for a sitewide P\&A program was not recognized by management, thus resources were not provided to establish such a program. No organizational responsibility for a sitewide P\&A program was identified. Following earlier audits, action plans for a sitewide P\&A program were not developed, of if developed, were not implemented.

Planned Actions and Schedules:

\section{$\underline{\text { Item/Description }}$}

1. Appoint ORNL Groundwater Coordinator (see Finding GW/BMPF-4, planned actions and schedules).

2. Identify wells and boreholes in WAG 6 that are candidates for P\&A, under leadership of Groundwater Coordinator. Establish a preliminary inventory of wells and boreholes for the X-10 site.

3. Complete planning document for P\&A of WAG 6 wells and boreholes. This document will contain P\&A procedures and will be transmitted to EPA and TDHE to give them the option to comment (per agreement with EPA and TDHE).

4. Review status of hydrofracture wells and the available technology for ICM and P\&A activities.
Completion Date

Complete

Complete

$1 / 92$

$6 / 92$ 
5. Complete planning document for identifying and categorizing non-WAG 6 wells and boreholes requiring $\mathrm{P} \& \mathrm{~A}$ or interim corrective measures (ICM). Develop guidelines for prioritizing on basis of contaminant migration potential. This action does not include hydrofracture wells, which are included in Items 10 and 11.

6. Complete P\&A and ICM procedures and guidelines suited to the range of conditions of ORNL wells and boreholes. This action does not include hydrofracture wells, which are included in Items 10 and 11.

7. Establish an accurate, sitewide well inventory (see Planned Actions and Schedules, Finding GW/BMPF2). This action includes all wells except hydrofracture wells, which are included in Items 10 and 11.

8. Complete P\&A for WAG 6.

9. Identify and prioritize wells and boreholes in/near all WAGs for P\&A; develop schedule for specisc wells and boreholes or groups of wells and boreholes. This action does not include hydrofracture wells, which are included in Items 10 and 11. An agreement has been reached with the EPA and TDHE to formalize this as an interim ROD to be submitted on or before $9 / 93$.

10. Complete P\&A operations at WAGs other than 6 except hydrofracture wells.

11. Conduct ICM cold demonstration, special-case (hydrofracture site) wells.

12. Complete ICMs for hydrofracture injection wells. This includes planning, development of procedures, and implementation.

13. Complete P\&A of the 150 observation wells assoriated with hydrofracture site. This includes planning, development of procedures, and implementation. 


\section{Costs:}

Type of funds: ERWM Programmatic Source of funds: EM-ADS 0332AB (WAG 6)

Estimated costs per fiscal year (\$K)

\begin{tabular}{|c|c|c|c|c|c|c|c|}
\hline Action item & 1991 & 1992 & 1993 & 1994 & 1995 & Beyond & Total \\
\hline 1 & - & & & & & & \\
\hline 2 & 15 & & & & & & 15 \\
\hline 3 & 10 & & & & & & 10 \\
\hline 5 & - & & & & & & \\
\hline 6 & 50 & & & & & & 50 \\
\hline 7 & 50 & & & & & & 50 \\
\hline 8 & 402 & 6390 & & & & & 6792 \\
\hline
\end{tabular}

Status:

Funded

527

Requested

6390

New 
Type of funds: ERWM Programmatic

Source of funds: EM-ADS 0329

Estimated costs per fiscal year (\$K)

\begin{tabular}{crrrrrrr}
\cline { 2 - 5 } Action item & 1991 & 1992 & 1993 & 1994 & 1995 & Beyond $^{*}$ & Total \\
\hline 5 & & 190 & & & & & 190 \\
6 & 90 & & & & & 90 \\
7 & & 120 & 100 & & & & 220 \\
9 & & 50 & 40 & & & & 90 \\
10 & 250 & 0 & 2860 & 3000 & 3000 & 6000 & 15,110
\end{tabular}

Status:

Funded

Requested

$\begin{array}{lllll}450 \quad 3000 & 3000 \quad 3000 & 6000\end{array}$

New

250

$\$ 15,700$

*Through FY 1997.

Type of funds: ERWM Programmatic

Source of funds: EM-ADS 0333

Estimated costs per fiscal year $(\$ K)$

\begin{tabular}{crrrrrrr}
\cline { 2 - 6 } Action item & 1991 & 1992 & 1993 & 1994 & 1995 & Beyond* & Total \\
\hline 4 & & 110 & & & & & 110 \\
11 & & 102 & 1,200 & & & & 1,302 \\
12 & & & & & 1,800 & 19,177 & 20,977 \\
13 & 250 & 2,182 & 1,830 & 3,788 & 4,656 & 2,400 & 15,106
\end{tabular}

Status:

Funded

Requested

New

$\begin{array}{rrrrrr} & 2,284 & 3,030 & 3,788 & 6,456 & 21,577 \\ 250 & 110 & & & & \end{array}$

$\$ 37,495$

*Through FY 1997; FY 1992 required budget as shown, current target is 0.

References: None 
Finding No.: GW/BMPF-2 Inadequate Monitoring Well and Borehole Inventory, Security, and Maintenance

Finding

Description: A significant number of monitoring wells and boreholes at ORNL have been inadequately inventoried, secured, or maintained by ORNL-MMES, which is not in accordance with current industry practice or technical guidance documents.

Code: $\quad$ Best Management Practice

Compliance

Protocol:

Priority:

Response:
None

Energy Systems Risk Weight 50

Tiger Team Action Plan Priority 1

This action plan addresses monitoring well inventory, security, and maintenance of wells and boreholes at ORNL. The need for a monitoring well inventory has been recognized for over a decade. Early compilations of well location, construction and associated observations have been made by the U.S. Geological Survey and by ORNL investigators. In 1986, the Remedial Action Program initiated development of a computerized data base that contained data on locations, construction details, geologic parameters and observations of groundwater variables. At the present time, the data base contains information on over 1500 wells, however there are some gaps in information for wells constructed in earlier decades, and there is some concern that the inventory is incomplete, especially for wells or boreholes constructed in earlier years or in association with limited scope-research activities. Virtually all of the wells that have been constructed for monitoring purposes in recent years were initiated by the Environmental Restoration program or its precursor, and are contained in the data base. The contents of the data base have been documented (Hook et al., 1990) and a guide to the well locations, including maps generated by a Geographical Information System (GIS) program is in preparation. A field inventory of wells in the ORNL Waste Area Grouping (WAG) 6 is planned for FY 1991 to physically check for the existence of wells not in the current inventory and to verify locations of existing wells. This inventory will result in an update of the data base for WAG 6. A more comprehensive plan (GW/BMPF-1 action item 7) will be developed for improving the well inventory for the remaining WAGs and surrounding areas, taking into consideration the planned requirements of the Federal Facilities Agreement, DOE priorities for resource allocation, and the expected schedule for Remedial Investigations (RIs) for ORNL. The responsibility for requesting funding for maintenance of the well inventory data base in future years lies jointly with the ORNL Groundwater Program Coordinator and the Environmental Restoration Program.

Security of wells and boreholes involves procedures designed to assure that control is maintained of the integrity of samples collected and that the well or borehole does not serve as a conduit for transmission of contaminants from one level to another, which could result in contamination of the ground water. For permitted 
facilities, a written schedule for inspection is required, together with a list of items to be inspected. The record of inspections must be kept in an inspection log. The Groundwater Program Coordinator will dcvelop a procedure that includes the written schedule for inspection of wells and boreholes, the items to be checked, and the guideline for maintaining the inspection log. The GWPC will also be responsible for assuring that the inspections are carried out, evaluated, and any needed maintenance is completed.

Maintenance of wells and boreholes will be the joint responsibility of the GWPC and the ER program. The GWPC will develop a plan for well and borehole maintenance that specifies expected performance standards and roles and responsibilities for conducting the maintenance program. This plan will draw upon the well and horehole inventory and inspection activities outlined above. The ER program will carry out surveillance and maintenance tasks.

Coordination of these actions, along with those specified in GW/BMPF-1, GW/BMPF-3, and GW/BMPF-4, is the responsibility of the ORNL Groundwater Coordinator. An integrated approach to these activities is presented in the ORNL Groundwater Protection Program Management Plan (latest revision available end July, 1991).

\section{Root Cause:}

Ambiguous requirements or expectations; unclear expectations resulting in low priority for management action for a centralized groundwater well and borehole management program.

Planned Actions and Schedules:

\section{Item/Description}

1. Appoint an ORNL program coordinator (see Finding GW/BMPF-4).

2. Issue a guide to ORNL well locations. This document will be updated annually from the well and borehole database.

3. Develop a wells and boreholes surveillance and maintenance plan to include preliminary field inventory (see GW/BMPF-1 action item 2) in WAGs and surrounding areas. Provide procedures or guidelines for well security and maintenance inspections, record keeping, and required actions. This plan will include production well evaluation, which will be incorporated into the ORNL Environmental Monitoring Plan.
Completion Date

Complete 
4. Implement, complete, and document execution of the

$9 / 91$ field inventory plan for WAG 6 (See Finding GW/BMPF-1).

5. Implement the wells and boreholes surveillance and $1 / 92$ maintenance plan (Item 3) and document completion of objectives and milestones.

Costs: $\quad$ The position of groundwater program coordinator has been created with existing resources. Program planning activities will require funding of approximately $\$ 50 \mathrm{~K}$, which is currently not budgeted. The field inventory at WAG 6 will require approximately $\$ 50 \mathrm{~K}$, which has been budgeted under Finding GW/BMPF-1, but not yet authorized, through ADS332AB. Reporting requirements are approximately $\$ 40 \mathrm{~K}$, budgeted through $\mathrm{ER}$. Additional funds will be requested for similar inventory verification activities through the Environmental Restoration Program surveillance and maintenance activity for FY 1992-1997 (ADS311AA, see cost table).

Type of funds: Overhead

Source of funds: Energy Systems

Estimated costs per fiscal year (\$K)

\begin{tabular}{|c|c|c|c|c|c|c|c|}
\hline Action item & 1991 & 1992 & 1993 & 1994 & 1995 & Beyond & Total \\
\hline 1 & - & & & & & & \\
\hline 2 & 40 & & & & & & 40 \\
\hline \multicolumn{8}{|l|}{ Status: } \\
\hline \multicolumn{8}{|l|}{ Funded } \\
\hline Requested & 40 & & & & & & \\
\hline New & & & & & & & $\$ 40$ \\
\hline
\end{tabular}


Type of funds: Overhead

Source of funds: Overhead

\begin{tabular}{|c|c|c|c|c|c|c|c|}
\hline \multirow[b]{2}{*}{ Action item } & \multicolumn{6}{|c|}{ Estimated costs per fiscal year $(\$ K)$} & \multirow[b]{2}{*}{ Total } \\
\hline & 1991 & 1992 & 1993 & 1994 & 1995 & Beyond & \\
\hline 3 & 50 & & & & & & 50 \\
\hline \multicolumn{8}{|l|}{ Status: } \\
\hline \multicolumn{8}{|l|}{ Funded } \\
\hline \multicolumn{8}{|l|}{ Requested } \\
\hline New & 50 & & & & & & $\$ 50$ \\
\hline
\end{tabular}

Type of funds: ERWM Programmatic

Source of funds: EM-ADS 311AA

Estimated costs per fiscal year (\$K)

\begin{tabular}{lrrrrrrr}
\cline { 2 - 5 } Action item & 1991 & 1992 & 1993 & 1994 & 1995 & Beyond & Total \\
\hline 5 & & 150 & $*$ & & & & 150 \\
Status: & & & & & & &
\end{tabular}

Funded

Requested

150

New

*Estimated annual ongoing cost: $\$ 350 \mathrm{~K}$.

References: None 
Finding No.: $\quad$ GW/BMPF-3 Cross-Contamination Between Aquifers and Strata

Finding

Description:

Code:

Compliance

Protocol:

Priority:

Response:
Wells, piezometers, and boreholes at ORNL represent conduits for crosscontamination, which is not in accordance with best management practices.

Best Management Practice

None

Energy Systems Risk Weight 50

Tiger Team Action Plan Priority 2

This response pertains to wells associated with hydrofracture, corrugated-pipe wells, and water-supply wells. Most of the issue of cross-contamination via wells as expressed in this finding will be met by the ORNL Well Plugging and Abandonment Program (managed by the GWC), described in the response to Finding GW/BMPF-1, and by the actions described in the response to Finding GW/BMPF-2 relating to well inventory, security, and maintenance (also managed by the GWC).

For planned actions, schedules, and costs associated with cross-contamination between aquifers, see Finding GW/BMPF-1.

With regard to existing uncapped wells, any wells retained for further use at ORNL will be protected as described in the response to Finding GW/BMPF-2. Wells not retained for further use will be candidates for the ORNL P\&A program. Interim maintenance and security measures will be identified for all wells until final resolution is reached.

ORNL will assess the potential of any production wells to induce migration of contaminants, as described in the finding, and will incorporate into its environmental monitoring program a continuing surveillance of potential effects on contaminant movement created by any water supply well in or near known contaminant sources.

Boreholes installed for engineering purposes and never closed will be included in the ORNL well inventory and treated as wells under the P\&A program.

\section{Root Causes:}

Poorly defined roles and responsibilities, insufficient resources, inadequate management approach, and inadequate training. Wells and boreholes have been installed at ORNL by several different organizations, over a period of many years for a wide variety of purposes. There has been no centralized ownership of responsibility for ORNL wells. Organizational responsibility for custody and maintenance of wells and boreholes was not assigned by management. Personnel 
who developed the hydrofracture operation did not adequately recognize the environmental risks associated with the deep wells installed into or through the waste injection zone, or recognize the regulatory implications of issues such as cross-contamination of groundwater. Users of water from the supply well did not fully recognize or investigate the potential for inducing contaminant migration. Funding resources for wells and boreholes management were not allocated.

Planned Actions and Schedules:

See Planned Actions and Schedules in Finding GW/BMPF-1.

Costs: $\quad$ Current funding requirements are included in Finding GW/BMPF-1.

References: None 
Finding No.: GW/BMPF-4 No Custodian for Unused Wells

Finding

Description: MMES-ORNL has not assigned the official responsibility of maintenance, upkeep, closure, and custodianship for unused wells at ORNL to any site organization, which is not in accordance with best management practices.

Code: $\quad$ Best Management Practice

Compliance

Protocol: None

Priority: $\quad$ Energy Systems Risk Weight 50

Tiger Team Action Plan Priority 2

Response: This groundwater finding will be appropriately addressed by the addition of a staff member to serve as ORNL Groundwater Program Coordinator. The coordinator will be currently informed on all ORNL groundwater activities to ensure a comprehensive program without duplications or omissions. The Groundwater Program Coordinator will ensure that wells and boreholes are formally assigned to an appropriate custodian, and the responsibilities of the custodian will be documented of that time. Also ensured by the new position will be effective identification, communication, and recognition of all responsibilities and accountabilities within and among the numerous groundwater activities. Cross reference to other groundwater best management practice findings: GW/BMPF-1, inadequate monitoring well and borehole abandonment; GW/BMPF-2, inadequate monitoring well and borehole inventory, security, and maintenance; GW/BMPF-3, cross-contamination between aquifers and strata; and GW/BMPF-5, inadequate characterization of the hydrogeologic regime.

Root Cause:

Poorly defined roles and responsibilities

Planned Actions and Schedules:

\section{$\underline{\text { Item/Description }}$}

1. Appointment of an interim ORNL Groundwater Program Coordinator. (see Findings GW/BMPF-1, 2, and 5.)

2. Develop approved charter for ORNL Groundwater Program Coordinator position.

3. Hire permanent ORNL Groundwater Program Coordinator.
Completion Date

Complete

Complete

Complete 
4. Complete assignment of custodianship of unused wells.

Casts: No significant costs are associated with Action Items 1 and 2. Estimated annual ongoing costs: \$100K (1.0 FTE).

References: $\quad$ DOE Order 5400.1, General Environmental Protection Program

Immediate Action Directive, Energy Systems Environmental, Health, and Safety Procedure for Groundwater Management Program, ES-ESH-P-1

Immediate Action Directive, Energy Systems Environmental, Health, and Safety Standard for Groundwater Management Program, ES-ESH-17 
Finding No.: GW/BMPF-5 Inadequate Characterization of the Hydrogeologic Regime

\section{Finding Description:}

Code:

Compliance Protocol:

Priority:

Response:
The hydrogeologic regime underlying ORiNL has not been adequately characterized to define aquifer characteristics and boundaries, aquifer communication between geologic formations, vertical and horizontal extent of contamination, and local groundwater flow paths and velocities in accordance with best management practices.

Best Mainagement Practice

DOE Order 5400.4, CERCLA Requirements, and the NCP [40 CFR 300.430(d)] require an adequate hydrologic characterization at inactive waste sites consistent with those required by RCRA [OSWER Directive 9950.1 (RCRA Ground Water Monitoring Technical Enforcement Guidance Document)] for active sites.

Energy Systems Risk Weight 117

Tiger Team Action Plan Priority 2

ORNL has long familiarity with the need for adequate hydrogeologic characterization at proposed, active, and inactive waste sites. A large number of hydrogeologic studies were completed in the period 1951-85, and each of these studies obtained adequate data and information to determine local flow paths and contaminant concentrations. This information was used for corrective actions, wherever and whenever necessary. Beginning in 1985-86, the RAP Program and, later, the Environmental Restoration Program funded studies to obtain the additional hydrogeologic information needed for RI/FS Programs in the WAGs. These studies are continuing and address the hydrogeologic characteristics of subsurface materials in the ORNL area, a determination of the limits of the shallow aquifer, measurement and calculation of the parameter values needed for modeling, the likelihood of deep or offsite flows of groundwater, contaminant source terms, flow paths, and fluxes in the WAGs, and sampling locations for site characterization, risk assessment, closure evaluation, and long-term compliance monitoring. However, because of the extensive area involved, the work is phased by WAG, in a priority ranking established through the Federal Facilities Agreement.

An adequate hydrogeologic characterization of the ORNL area is presently incomplete because the process leading to identification of contamination and evaluation of the need for corrective actions at ORNL is still in the initial stages. A prioritized, phased, scientific approach will be used to obtain the necessary information. It is important, however, that the problems have been identified and that work is underway or planned to correct the most important deficiencies in the understanding of hydrogeologic flow systems. For example, two items mentioned by the Tiger Team are the inadequate understanding of drains, pipe networks, and trench-fill materials in industrial areas and of perched stormflow waters in vegetated areas; the hydrologic importance of both types of features previously 
were findings of research sponsored by Environmental Restoration and other Programs at ORNL. The Tiger Team is correct that additional data are needed on the characteristics of these features and on their relationships to groundwater and surface streams. A study to characterize the industrial area of WAG 1 is presently underway by the RI/FS team. A negotiated schedule for other ORNL work area groupings is contained in the pending rFA. Stormflow research was funded by Envirol'mental Restoration Program in FY 1990, and is being continued under ADS 322 in FY 1991.

Another, broader approach to hydrogeologic characterization o- the Oak Ridge Reservation was begun at ORNL in FY 1990 under the Oak Ridge Reservation Hydrologic and Geologic Studies (ORRHAGS) Program. One goal of this program is an adequate understanding of all flow processes for water on the Oak Ridge Reservation, including flow paths, fluxes, velocities, modeling, and contaminant concentrations in the stormflow and groundwater zones. A summary report, scheduled for release in 1991, will describe all data, parameter values, interpretations, and conclusions from 40 years of hydrogeologic studies, will identify remaining deficiencies in hydrogeologic characterization, and will prioritize these deficiencies. A companion document that focuses on geology will also be issued.Tiger team findings will be considered, but it is presently anticipated that the priority list will differ in some respects from the items listed by the ORNL Tiger Team. It is unlikely, for example, that inadequate delineations of aquifers and zones of contaminations will be priority deficiencies.

There is general agreement among ORNL hydrogeologists that the base of the active groundwater flow system is adequately known; this boundary occurs at depths of about 100-250 ft and is determined by a change in chemical water type and by the absence of tritium and other contaminants in the water. There is also general agreement that the lateral extent of groundwater contamination is in the process of being adequately defined by existing programs for sampling and analysis; there is no need to accelerate this effort. Finally, there is general agreement that geologic contacts are not hydrologic barriers, that three is a single groundwater zone from the water table to the hase of active circulation, and that nearly all groundwater is discharged to a neaiby surface stream. We believe that if the Tiger Team had been given more time to study available data, they would agree with these conclusions, which are based on a convergence of evidence.

Several issues will be given a high priority by the ORRHAGS/hydrogeology report. These deficiencies in hydrogeologic characterization may include (1) surface and groundwater relationships, especially the hydrogeology of riparian zones, (2) the relative importance of matrix diffusion, which may affect groundwater velocities and determine the practicality of groundwater pump-and-treat and other remedial alternatives, (3) the determination of accurate groundwater ages, (4) the use of hydrograph analysis to determine average values of various aquifer parameters such as transmissivity and effective porosity, (5) the use of innovative methods to determine whether contaminant concentrations along the various flow paths are increasing or decreasing, and (6) a determination of the best approach to grour idwater modeling and the data needed for this approach. 
In summary, the determination of an inadequate hydrogeologic characterization by the ORNL Tiger Team is valid. We agree that additional work is needed to support the RI/FS processes of risk analysis and the selection of alternatives for remedial action. However, all specific issues addressed by the Tiger Team have been previously considered and evaluated by the Environmental Restoration Program. The phased RI/FS program to study ORNL waste sites specified in the FFA will implement collection of data to meet deficiencies. The approach is to use ORHSP results and synthesis of information to guide future RI/FS work. Some priority deficiencies will be addressed by Environmental Restoration and other programs through the ORRHAGS project.

\section{Root Causes:}

Insufficient resources-Funding limitations across the DOE system result in phased studies that control the rate of progress. Regulatory requirements for planning and getting approval for remedial investigations constrain the rate of progress that can be made toward hydrogeologic characterization when the scope of the project is of major proportions.

\section{Planned Actions and Schedules:}

\section{Item/Description}

1. Appoint an ORNL groundwater program coordinator (see Findin: GW/BMPF-4).

2. Issue an evaluative report by ORRHAGS investigators on deficiencies of hydrogeologic characterization for the Oak Ridge Reservation (ORR), including ORNL conditions.

3. Issue an evaluative report by ORRHAGS staff on geologic conditions and data gaps for the ORR, including ORNL.

4. Issue an evaluation of model suitability for application to ORNL hyćrogeologic conditions by ORHSP staff.

5. Conduct a workshop between ORHSP staff and staff from other DOE facilities and/or academic institutions to initiate collaboration in modeling and characterizing hyo'rogeology of the ORR.
Completion Date

Compiete

$9 / 91$

9/91

$6 / 92$

6/92 
6. Conduct RI/FS studies, including site-specific grounciwater characterization, in accordan 2 , with the pending Fed... Facilities Agreement between DOE, EPA, and TDHE to provide site characterization of ORNL WAGs. Incorporate conclusions and recommendations from ORHSP evaluations and address all GW/BMPF-5 findings from the Tiger Team point-bypoint response (see Finding GW/CF-1) at the initiation of RI/FS projects and ensure that any deficiencies in earlier RI/FS studies are rectified in subsequent work. This is an ongoing item hecause of the nature of the $\mathrm{RI} / \mathrm{FS}$ process.

Casts: The costs of the groundwater program coordinator have been discussed elsewhere, and are to be met within existing resources. Costs and allocations for Environmental Restoration activities at ORNL are determined by DOE priority rankings, regulatory agreements, and availability of funds. Those specifics are part of the negotiated FFA conditions and cannot be specified here. Costs for activities of ORRHAGS and ORHSP projects will total about $\$ 300 \mathrm{~K}$ in FY 1991, and are in planned budgets, although not all planned funding has been authorized for use. Costs for modeling and characterization activities in FY 1992 and are estimated to be approximately $\$ 450 \mathrm{~K}$. These funds will be requested, but have not been budgeted.

Type of func's: Dverhead

Source of funds: Energy Systems

\begin{tabular}{|c|c|c|c|c|c|c|c|}
\hline \multirow[b]{2}{*}{ Action item } & \multicolumn{6}{|c|}{ Estimated costs per fiscal year (\$K) } & \multirow[b]{2}{*}{ Total } \\
\hline & 1991 & 1992 & 1993 & 1994 & 1995 & Beyond & \\
\hline 1 & - & & & & & & \\
\hline 2 & 75 & & & & & & 75 \\
\hline 3 & 75 & & & & & & 75 \\
\hline 4 & & 100 & & & & & 100 \\
\hline 5 & & 125 & & & & & 125 \\
\hline
\end{tabular}

Status:

Funded

150

Requested 
Type of funds: ERWM Programmatic

Source of funds: EM-ADS 413

Estimated costs per fiscal year (\$K)

\begin{tabular}{crrrrrrr}
\cline { 2 - 6 } Action item & 1991 & 1992 & 1993 & 1994 & 1995 & Beyond & Total \\
\hline 2 & 75 & & & & & & 75 \\
3 & 75 & & & & & & 75 \\
4 & & 100 & & & & & 100 \\
5 & & 125 & & & & & 125
\end{tabular}

Status:

Funded

150

Requested

225

New

Type of funds: ERWM Programmatic

Source of funds: EM-ADS 363, 324 and 325

\begin{tabular}{lccccccc} 
& \multicolumn{6}{c}{ Estimated costs per fiscal year $(\$ K)$} & \\
\cline { 2 - 6 } Action item & 1991 & 1992 & 1993 & 1994 & 1995 & Beyond* & Total \\
\hline 6 & 17,796 & 26,670 & 27,650 & 26,367 & 26,733 & 42,091 & 167,307 \\
$\begin{array}{l}\text { Status: } \\
\text { Funded } \\
\text { Requested }\end{array}$ & 17,796 & & & & & & \\
New & & 26,670 & 27,650 & 26,367 & 26,733 & 42,091 & \\
\hline
\end{tabular}

*Through FY $\mathbf{i} 997$.

References: None 


\subsubsection{Waste Management}

Finding No.: WM/CF-1 Inadequate Operation of Mixed Waste Storage Facilities

Finding

Description: The 7507W and 7654 Mixed Waste Storage Facilities do not meet Tennessee Hazardous Waste Management Rules for segregation of incompatible wastes, and the 7507W Mixed Waste Storage Facility does not meet Tennessee Hazardous Waste Management Rules for site security and does not meet best management practice standards for fire protection/detection, secondary containment, facility location, container locating methods, or aisle space.

Code: $\quad$ Compliance

Compliance Protocol:
Tennessee Hazardous Waste Management Rule 1200-1-11-.05 requires interim status hazardous waste storage facilities to be maintained with adequate site security through either 24-hour surveillance or fencing completely surrounding the facility. This rule also requires incompatible wastes to be segregated.

Best management practices requires that such facilities

1. have a secondary containment that is free of cracks,

2. provide an automatic fire detection or sprinkler system at an unattended hazardous waste storage facility holding flammable waste to ensure prompt emergency response in the event of a fire,

3. be located in clearings away from wooded areas where trees can fall,

4. be able to readily locate individual containers in a storage facility through use of a facility grid map or other means, and

5. provide for adequate aisle space.

Priority: $\quad$ Energy Systems Risk Weight 413

Tiger Team Action Plan Priority 1

Response: The Solid Waste Operations Department (SWOD) in the ORNL Waste Management Operations Section is responsible for the operations conducted in Buildings 7654 and $7507 \mathrm{~W}$ which are both interim status facilities. Building 7654 became operational in 1988 and is used for storage of mixed waste. The majority of the waste consists of bulk scintillation fluids and scintillation vials. In 1981, Building $7507 \mathrm{~W}$ began to be used for the storage of mixed wastes, including oils, scintillation liquids, and corrosive RCRA wastes as well as some nonhazardous radioactive wastes. 
The deficiency cited for Building 7654 has not previously been identified; however, the Energy Systems-ORNL self-assessment identified the findings for $7507 \mathrm{~W}$ and recognized the majority of the best management practice concerns cited.

It has been recognized that Building $7507 \mathrm{~W}$ should be replaced and/or upgraded. Planning for a new facility (Building 7668) was initiated in 1988 and was originally scheduled to be operational in October 1991. Delays may result from NEPA documentation requirements.

Immediate plans call for the reduction of the present inventory of Building $7507 \mathrm{~W}$ by moving approximately 100 drums of oils to another interim status facility. It is anticipated that further reductions of the present inventory can be achieved after the completion of comprehensive waste characterization effort which is scheduled to be completed in FY 1992.

The findings involving segregation of incompatible wastes in Buildings 7654 and $7507 \mathrm{~W}$ will be corrected by segregating the hazardous wastes and placing the different waste types in polyethylene secondary containment. The security finding for Building $7507 \mathrm{~W}$ will be corrected by installing a fence around the area.

The segregation of incompatible wastes into secondary containers will reduce the usable inventory space for Buildings 7654 and $7507 \mathrm{~W}$. Inventories will be reduced as detailed above prior to implementing the incompatible waste segregation corrective action.

The remaining best management practice concerns will be corrected as detailed below in the planned actions.

\section{Root Causes:}

Insufficient resources; lack of funding to provide facilities in a timely manner. Ambiguous requirements or expectations; differences in interpretation of regulatory requirements.

Planned Actions and Schedules:

\section{$\underline{\text { Item/Description }}$}

1. Identify funding from existing programmatic funding to cover corrective action costs.

2. Reduce mixed waste inventories as much as possible.

3. Generators will be notified that mixed waste storage space is limited and that the generation of mixed waste should be minimized.
Completion Date

Complete

Complete

$05 / 91$ 
4. Identify facility needs in annual funding requests

Complete beginning with first ADS submission.

5. Install fence around $7507 \mathrm{~W}$.

$06 / 91$

6. Clear the area around Building $7507 \mathrm{~W}$ to prevent

$06 / 91$ possibility of trees falling on the facility.

7. Segregate incompatible wastes in Buildings 7654 and

$07 / 91$ $7507 \mathrm{~W}$ and place different waste types in polyethylene secondary containment. The polyethylene secondary containment will mitigate the finding relating to cracks in the concrete sink in $7507 \mathrm{~W}$. Building $7507 \mathrm{~W}$ will be replaced with a new facility within the next 2 years.

8. Provide appropriate aisle space between containers.

9. Prepare and implement standard operating procedure

$07 / 91$ for readily identifying location of individual containers in Building $7507 \mathrm{~W}$.

10. Determine most appropriate fire detection system for Building $7507 \mathrm{~W}$ and install system. 
Costs:

Type of funds: ERWM Programmatic

Source of funds: EM-ADS 348

Estimated costs per fiscal year (\$K)

\begin{tabular}{|c|c|c|c|c|c|c|c|}
\hline Action item & 1991 & 1992 & 1993 & 1994 & 1995 & Beyond & Total \\
\hline 1 & - & & & & & & \\
\hline 2 & 5 & & & & & & 5 \\
\hline 3 & - & & & & & & \\
\hline 4 & - & & & & & & \\
\hline 5 & 35 & & & & & & \\
\hline 6 & 2 & & & & & & 35 \\
\hline 7 & 120 & & & & & & 2 \\
\hline 8 & $a$ & & & & & & 120 \\
\hline 9 & 10 & & & & & & 10 \\
\hline 10 & 30 & & & & & & 30 \\
\hline Status: & & & & & & & \\
\hline Funded & 202 & & & & & & \\
\hline Requested & & & & & & & \\
\hline New & & & & & & & $\$ 202$ \\
\hline
\end{tabular}

${ }^{a}$ Will be completed as a part of Action Item 7.

References: Tennessee Hazardous Waste Management Rule 1200-1-11-.05

Tennessee Hazardous Waste Management Rule 1200-1-11-.06

ORNL Self-Assessment Report

40 CFR 265.14

40 CFR 270.72 
Finding No.: WM/CF-2 Improper Operation of Satellite Accumulation Areas at ORNL Facilities at $\mathrm{Y}-12$

Finding

Description: MMES-ORNL does not manage hazardous waste satellite accumulation areas at its Y-12 facilities such that the areas at or near the initial point of accumulation, and the areas contain less than 55 gallons of hazardous waste in accordance with the Tennessee Hazardous Waste Management Rule 1200-1-11-.03.

Code: $\quad$ Compliance

Compliance

Protocol:

Priority: $\quad$ Energy Systems Risk Weight 406

Tiger Team Action Plan Priority 2

Response: ORNL operations at Y-12 satellite accumulation areas are located near the point of generation, and each satellite area is limited to 55 gallons. Satellite accumulation areas which could be moved closer to areas under control of the operators whose processes generate the waste have been moved where possible and where safety issues did not pose problems or concerns. These various satellite areas are established to separate incompatible wastes that cannot be stored together in a 90-day accumulation area or for other safety concerns. Once these ORNL operations at Y-12 research and development laboratory areas purge their systems of these surplus chemicals, many of the satellite areas will be dismantled.

At present, the wastes in these areas are adequately and safely contained in compatible containers of good condition. The TDHE has inspected these areas during previous inspections and has not indicated a problem with the RCRA Waste Management Operations in these various locations.

Root Cause:

Inaccurate policy interpretation

Planned Actions and Schedules:
Completion Date

Complete

1. Review ORNL at Y-12 operations for compliance with TDHE requirements.

2. Verify TDHE requirements met.

Complete

Costs: No additional costs are expected as a result of this review.

References: None 
Finding No.: WM/CF-3 Inadequate Hazardous Waste Accumulation and Minimization at ORNL Biology Division at Y-12 Plant

Finding

Description: Hazardous waste management practices of the MMES-ORNL Biology Division at Y-12 for waste accumulation, minimization, spill control, and training do not conform to the requirements of Tennessee Hazardous Management Rules 1200-1-11-.03 and .05.

Code: $\quad$ Compliance

Compliance

Protocol:

TDHE 1200-1-11-.03, 1200-1-11-.05

Priority: $\quad$ Energy Systems Risk Weight 406

Tiger Team Action Plan Priority 2

Response: The hazardous waste accumulation areas in the ORNL Biology Division have been audited and judged to be in compliance with the requirements of the TDHE. An additional review of the practices including mixed waste handling will be conducted to document full compliance.

The use and accumulation of waste scintillation cocktails in the Biology Division are under review, and non-hazardous cocktails will be substituted wherever possible. Cocktails that are hazardous waste will be segregated to minimize mixed or hazardous waste generation.

The Biology Division complies with the Y-12 Spill Prevention, Control, and Countermeasures Plan and the Y-12 RCRA Contingency Plan for spills, etc.

RCRA-required training will be provided to ensure proper management of hazardous wastes.

\section{Root Causes:}

Inadequate policy implementation and inadequate training

Planned Actions and Schedules:

\section{$\underline{\text { Item/Description }}$}

1. Review and document training requirements for Biology personnel.

2. Review and document use of and management of waste scintillation cocktails as hazardous waste.
Completion Date

Complete

Complete 
3. Develop and distribute waste segregation policy and

Complete action plan.

4. Implement waste segregation action plan.

Complete

5. Review RCRA Contingency Plan and SPCCC Plan and provide spill control equipment as required.

Complete

6. Complete required training.

Complete

7. Review ORNL at Y-12 Hazardous Waste Accumulation Areas for compliance with TDHE requirements.

Complete

Costs: $\quad$ Action Items 1-7 will be accomplished with existing funds.

References: None 
Finding No.: WM/CF-4 Lack of Integrity Assessment of 7860A Hazardous Waste Storage Tank

Finding

Description:

MMES-ORNL has not conducted an integrity assessment of the 7860A interim status hazardous waste storage tank as required by Tennessee Hazardous Waste Management Rule 1200-1-11-.05.

Code: $\quad$ Compliance

Compliance

Protocol:

Tennessee Hazardous Waste Management Rule 1200-1-11-.05

Priority: $\quad$ Energy Systems Risk Weight 409

Tiger Team Action Plan Priority 1

Response: As identified in this finding, Energy Systems-ORNL is delinquent in not having closed the $7860 \mathrm{~A}$ storage tank within one year after having determined it received hazardous waste during its period of operation. In addition, Energy

Systems-ORNL has not conducted an integrity assessment as required to determine the structural soundness of the tank. The planned action to mitigate this deficiency was completed in March 1991 with the removal of the contents of the tank and placement of the RCRA-regulated constituents in a permitted facility.

The root cause of inadequate implementation of regulatory policy at inactive waste sites was previously cited in the self assessment report for the Energy

Systems-ORNL Office of Waste Management and Remedial Action. This problem stems from: (1) inadequate flowdown of regulatory requirements and DOE orders from Energy Systems-ORNL oversight organizations to facility management staff, and (2) the substantial increase in ES\&H-related facility management requirements experienced in recent years. To rectify these problems and thereby the root cause of the finding, the Energy Systems-ORNL Remedial Action Section prepared proposals for the FY 1993 budget submission to acquire its own regulatory expertise to stay abreast of all relevant and appropriate orders and regulations. Once acquired, this additional staff will directly transmit updates, changes, and miscellaneous nuances of ipplicable regulatory and DOE requirements to Remedial Action staff and facility managers.

\section{Root Causes:}

Inadequate policy implementation and inadequate communications; transmittal of regulatory requirements for management of hazardous waste tanks to Energy Systems-ORNL staff responsible for tanks is inadequate. 
Planned Actions and Schedules:

$\underline{\text { Item/Description }}$

1. Remove contents from tank $7860 \mathrm{~A}$.

2. Prepare budget proposals to acquire regulatory expertise staff for the Energy Systems-ORNL Remedial Action Section.

Casts:

Type of funds: ERWM Programmatic

Source of funds: EM ADS 311-AA and 331

\begin{tabular}{|c|c|c|c|c|c|c|c|}
\hline \multirow[b]{2}{*}{ Action item } & \multicolumn{6}{|c|}{ Estimated costs per fiscal year (\$K) } & \multirow[b]{2}{*}{ Total } \\
\hline & 1991 & 1992 & 1993 & 1994 & 1995 & Beyond & \\
\hline 1 & 20 & & & & & & 20 \\
\hline 2 & - & & * & & & & \\
\hline \multicolumn{8}{|l|}{ Status: } \\
\hline Funded & 20 & & & & & & \\
\hline \multicolumn{8}{|l|}{ Requested } \\
\hline New' & & & & & & & $\$ 20$ \\
\hline
\end{tabular}

*Annual ongoing cost will be \$25K per year beginning in FY 1993.

References: Environmental Restoration and Waste Management Five-Year Plan 
Finding No.: WM/CF-5 Inadequate Training for Onsite Hazardous Waste Transporters

Finding

Description:

Code:

Compliance

Protocol:

Priority:

Response:
MMES-ORNL has not provided job-specific hazardous waste training to the laborers and drivers who collect and transport hazardous waste between onsite accumulation areas and onsite storage facilities as required by Tennessee Hazardous Waste Management Rule 1200-1-11-.05(2).

Compliance

Tennessee Hazardous Waste Management Rule 1200-1-11-.05(2) requires facility personnel to complete a program of classroom instruction or on-the-job training that teaches them to perform their duties in a way that ensures the facility's compliance with the requirements of this Rule. This program must include instruction which teaches personnel the hazardous waste management procedures relevant to the positions in which they are employed.

\section{Energy Systems Risk Weight 410}

Tiger Team Action Plan Priority 2

This action plan requires the development and implementation of a procedure for the collection of hazardous waste from accumulation areas and transport of the waste to ORNL centralized storage facilities. The procedure(s) will be incorporated into the "Hazardous Waste Operations Manual," Manual No. WM-SWO-401. This series of procedures governs operations of Hazardous Waste Operations Group of the Office of Waste Management and Remedial Actions which has responsibility for the procedure development and implementation. When the procedure has received final approval, training will be developed and implemented by the Technical Resources and Training Section of the Office of Environmental and Health Protection in accordance with current practices described in the Waste Management Section Training Plan (currently in draft form). This training is to include successful completion of written Procedures-Use Exercises and On-the-Job Training Checklists tailored to the procedure(s). This training strategy ensures that the participant reviews the relevant procedures and performs tasks in accordance with the procedures. Waste Management will ensure that only those Plant and Equipment personnel having successfully completed the procedure-based training are assigned to support the hazardous waste collection and transport operation.

Other than the Hazardous Waste Operations Group Supervisor directing collection and transport operations, a team of two laborers and one driver from the Plant and Equipment (P\&E) Division are involved. A continuing aspect of this action plan requires that requests for P\&E Support by the Hazardous Waste Operations Group be filled only by personnel that have successfully completed the procedure(s)-based training. This responsibility lies with the P\&E Division supervisor supplying the support personnel in cooperation with the P\&E Division Training Coordinator. Currently P\&E support personnel supplied for the collection 
and transport operation have completed the 24 hours of health and safety training for hazardous waste operations and activities; the newly developed training based on the procedure(s) will build upon this training base.

These activities will be reviewed by ongoing hazardous waste activity surveillances.

\section{Root Cause:}

Inadequate policy; lack of Hazardous Waste Operations Group procedise for collection and transport of hazardous waste from accumulation areas to central storage areas.

Planned Actions and Schedules:

\section{Item/Description}

1. Draft procedure(s) for collection/tran:port of hazardous waste from accumulation areas to central storage area.

2. Review and incorporate revisions to procedure(s).

3. Approve final procedure(s).

4. Develop "procedures-use exercises" and "on-the-job training checklists" based on the approved procedure(s).

5. Complete and document training of initially identified Hazardous Waste Operations Group supervisor(s) and Plant and Equipment Division support personnel who perform the hazardous waste collection and transport operation.

\section{Completion Date}

Complete

05/91

$06 / 91$

08/91

$10 / 91$ 
Carts:

Type of funds: ERWM Programmatic

Source of funds: EM-ADS347.348

\begin{tabular}{|c|c|c|c|c|c|c|c|}
\hline \multirow[b]{2}{*}{ Action item } & \multicolumn{6}{|c|}{ Estimated costs per fiscal year (SK) } & \multirow[b]{2}{*}{ Total } \\
\hline & 1991 & 1992 & 1993 & 1994 & 1995 & Beyond & \\
\hline $1-3$ & 9 & & & & & & 9 \\
\hline 4 & 4.5 & & & & & & 4.5 \\
\hline 5 & 9 & & & & & & 9 \\
\hline \multicolumn{8}{|l|}{ Status: } \\
\hline \multicolumn{8}{|l|}{ Funded } \\
\hline \multicolumn{8}{|l|}{ Requested } \\
\hline New & 22.5 & & & & & & $\$ 22.5$ \\
\hline
\end{tabular}

- Estimated annual ongoing cost: \$10K.

References: Tennessee Hazardous Waste Management Rule 1200-1-11-.05(2) Waste Management Section Training Plan (Draft), Oak Ridge National Laboratory,
October 19, 1990 
Finding No.: WM/CF-6 Storage of Land Disposal Restricted Mixed Waste

Finding

Description:

Code: $\quad$ Compliance

Compliance

Protocol:

Prionity:

Response: treatment.
MMES-ORNL is currently storing mixed solvent and corrosive wastes that are subject to LDR requirements for purposes other than accumulating such quantities as necessary to facilitate proper recovery, treatment, or disposal, and is therefore not operating in accordance with the requirements of 40 CFR 268.50.

RCRA requirements found in 40 CFR 268.5, prohibit storage of LDR wastes other than for purposes of accumulating sufficient quantities to facilitate

Energy Systems Risk Weight 406

Tiger Team Action Plan Priority 1

ORNL has recognized and documented this noncompliance in the Office of Waste Management and Remedial Action's Self-Assessment, Sect. 2.4.1. Currently, treatment is unavailable for these mixed wastes, with the exception of some scintillation fluids that meet the waste acceptance criteria of the Quadrex facility in Forida. Thus the wastes are being stored in permitted facilities while capabilities are developing.

ORNL's action plan consists of several elements:

1. Characterization of the waste is planned for FY 1991 to enable ORNL to certify the waste to meet WAC of treatment facilities when they become available.

2. When adequate characterization data is available, wastes will be grouped by treatability, and existing appropriate treatment facilities will be identified and utilized as available. The TSCA Incinerator at the K-25 Plant, expected to begin operation on a large back-log of waste in FY 1991, is the primary facility planned to treat ORNL's mixed waste.

3. Treatment technology development or adaptation will be initrated for waste groupings for which treatment facilities are currently unavailable.

4. Reduction of LDR waste (hazardous scintillation waste) generation at the source will be implemented, as appropriate, by generating divisions. (See also the action plan for WM/BMPF-2.) 
5. An FFCA will continue to be pursued by DOE/ORO and supported through the Energy Systems Central Waste Management Division (CWMD). Extensive supporting data has already been provided, and ORNL has worked with CWMD and HAZWRAP to outline strategic "road maps" for each noncomplian" 'vaste stream.

\section{Root Causes:}

Insufficient resources and regulatory barriers; (1) nationwide unavailability of treatment technology for mixed radioactive wastes and (2) inadequate funding (FY 1991) and staffing (FY 1990) to conduct the characterization on the accelerated schedule deemed appropriate. Although some funding has been provided in FY 1991, characterization cannot be completed without additional funds.

\section{Planned Actions and Schedules:}

\section{Item/Description}

1. Request funding for characterization, treatment development, and treatment.

2. Complete development of technical data package to support RCRA land disposal restrictions FFCA negotiation. (FFCAs [Federal Facilities Compliance Agreements] are negotiated in situations where noncompliances already exist.)

3. DOE/ORO initiate negotiation of "prethirds" FFCA.

4. Issue letter :o scintillation-cocktail-generating divisions requesting source substitution and segregation, as appropriate.

5. Provide technical data in support of negotiation of FFCA concerning RCRA LDR first, second, and thirds mixed waste.

6. Develop schedule for acceptance of ORNL LDR RMW by TSCA Incinerator.

7. Issue letter report on characterization of stored mixed waste (AF WM-8).

8. Issue final report on characterization of stored mixed waste.

\section{Completion Date}

Complete

Complete

Complete

Complete

Complete

06/91

07/91

06/92 
9. Issue initial recommendations on disposition of

$08 / 92$ treatability groupings.

10. Issue report on RMW treatment technology

$09 / 92$ demonstrations.

Costs:

Type of funds: ERWM Programmatic

Source of funds: EM-ADS 349

\begin{tabular}{crrrrrrr} 
& \multicolumn{6}{c}{ Estimated costs per fiscal year (\$K) } & \\
\cline { 2 - 6 } Action item & 1991 & 1992 & 1993 & 1994 & 1995 & Beyond & Total \\
\hline 7 & 240 & & & & & & 240 \\
8 & & 500 & & & & & 500 \\
10 & & 150 & 300 & 300 & 400 & $*$ & 1150
\end{tabular}

Status:

Funded

240

Requested

$650 \quad 300$

300

400

New

*Estimated annual ongoing cost: $\$ 200 \mathrm{~K}$ 
Type of funds: ERWM Programmatic

Source of funds: EM-ADS 350

\begin{tabular}{|c|c|c|c|c|c|c|c|}
\hline \multirow[b]{2}{*}{ Action item } & \multicolumn{6}{|c|}{ Estimated costs per fiscal year ( $\$ \mathbf{K})$} & \multirow[b]{2}{*}{ Total } \\
\hline & 1991 & 1992 & 1993 & 1994 & 1995 & Beyond & \\
\hline 1 & - & & & & & & \\
\hline 2 & - & & & & & & \\
\hline 4 & 5 & & & & & & 5 \\
\hline 5 & 10 & & & & & & 10 \\
\hline 6 & 10 & & & & & & 10 \\
\hline 7 & 275 & & & & & & 275 \\
\hline 8 & & 500 & 350 & 200 & 100 & 50 & 1200 \\
\hline \multicolumn{8}{|l|}{ Status: } \\
\hline Funded & 300 & & & & & & \\
\hline Requested & & 500 & 350 & 200 & 100 & 50 & \\
\hline New & & & & & & & $\$ 1500$ \\
\hline
\end{tabular}

Ref:rences: $\quad$ Letter, C. P. East to T. E. Myrick, "FY 1991 ORNL Corrective Activity and Waste Management Program Prioritized Task Listing," September 5, 1990 
Finding No.: WM/CF-7 Inadequate Storage of Radioactively Contaminated Hazardous Waste Lead

\section{Finding}

Description:

Code:

Compliance

Protocol:

Priority:

Response:
MMES is accumulating and storing radioactively contaminated scrap lead without demonstrating a commitment to a contaminated lead recycling program and without managing it as a hazardous waste subject to RCRA regulation, as required by Tennessee H.azardous Waste Rules 1200-1-11-.01 through .09 and 40 CFR Part 268.

Compliance

Tennessee Rule 1200-1-11 outlines requirements for managing hazardous waste, including storage in a permitted facility. Exception is made for scrap metal and other recyclable materials. ORNL is storing its contaminated lead for decontamination and reuse.

Energy Systems Risk Weight 405

Tiger Team Action Plan Priority 1

In accordance with DOE Order 5400.2A, the Office of Environment, Safetv and Health (EH) was assigned the lead to resolve a number of significant, cross-cutting mixed waste and materials management issues. One of the cross-cutting issues deals with the management of excess or scrap lead. The workgroup has proposed strategies for several different categories of lead. Depending on the category, the lead may or may not be subject to RCRA regulations. The approach taken to address this specific finding is consistent with one of the approaches being developed by the work group.

Once the department's positions and strategies are clarified, discussions will be held with the appropriate regulatory agencies, as necessary. Clear DOE-wide policies and guidance will be prepared.

ORNL's strategy for management of contaminated lead is consistent with the DOE-wide strategy for management of lead and other scrap material. ORNL will document the present inventory of scrap lead. The storage of the scrap will be reviewed to ensure responsible management practices are followed. The ORNL lead management strategy will be documented.

In 1987, HAZWRAP conducted a private sector technology demonstration that successfully decontaminated an assortment of ORNL lead using a high-pressure water system. Secondary wastes were solidified and passed EP toxicity tests. (TCLP tests will need to be done.) ORNL plans to implement a full-scale decontamination campaign during FY 1991, utilizing this proven technology. Funding has been allocated, and contact with several subcontractors has already been made. Markets for the contaminated or recycled lead will be reviewed. 


\section{Root Causes:}

(1) Inadequate oversight; interpretation of requirements that differ from Tiger Team interpretations. (2) Insufficient resources; inadequate funding to implement lead decontamination in prior years.

Planned Actions and Schedules:

\section{$\underline{\text { Item/Description }}$}

1. Prepare an ORNL lead management strategy.

2. Document contaminated le..d inventory.

3. Review storage practices.

4. Issue statement of work for lead decontamination campaign.

5. Initiate lead decontamination.

6. Review potential markets for contaminated and recycled lead.

\section{Completion Date}

Complete

Complete

Complete

$05 / 91$

$08 / 91$

$08 / 91$ 
Costs:

Type of funds: ERWM Programmatic

Source of funds: EM-ADS 349

Estimated costs per fiscal year (\$K)

\begin{tabular}{|c|c|c|c|c|c|c|c|}
\hline Action item & 1991 & 1992 & 1993 & 1994 & 1995 & Beyond & Total \\
\hline 1 & - & & & & & & \\
\hline 2 & - & & & & & & \\
\hline 3 & - & & & & & & \\
\hline 4 & 100 & & & & & & 100 \\
\hline 5 & & * & & & & & \\
\hline 6 & - & & & & & & \\
\hline \multicolumn{8}{|l|}{ Status: } \\
\hline Funded & 100 & & & & & & \\
\hline \multicolumn{8}{|l|}{ Requested } \\
\hline New & & & & & & & $\$ 100$ \\
\hline
\end{tabular}

*Estimated annual ongoing cost: \$150K starting in FY 1992.

References: None. 
Finding No.: WM/CF-8 Inadequate Training Documentation and Procedures for 7507 Hazardous Waste Storage Facility

\section{Finding}

Description: MMES-ORNL has not documented the training for waste management operations in Bldg. 7507 in accordance with Tennessee Hazardous Waste Management Rule 1200-1-11-.05 and has not, as a best management practice, updated the operational procedures to meet new operational conditions at that facility.

Code: $\quad$ Compliance

Compliance Protocol:

Tennessee Hazardous Waste Management Rule 1200-1-11-.05 requires that personnel involved in the operitions of interim status hazardous waste management units be trained in the hazardous waste management procedures necessary to perform their duties and that the training be documented. As a best management practice, documented procedures should be provided for wastehandling activities.

Priority: $\quad$ Energy Systems Risk Weight 5

Tiger Team Action Plan Priority 3

Response: $\quad$ The operations conducted in Building 7507 are assigned to the Hazardous Waste Operations Group (HWOG) in the ORNL Waste Management Operations Section. The HWOG is responsible for the pioper characterization, storage, and disposal of hazardous, mixed, and conventional wastes generated from research, development, and production programs at ORNL. The HWOG operations conducted in Building 7507, as well as other HWOG facilities, are to be conducted in such a manner to ensure compliance with federal, state, DOE, Energy Systems, and ORNL policies, procedures, and regulations. The training requirements and procedi:res detailed in Hazardous Waste Operations Manual WM-SWO-401 are used for meeting existing and anticipated requirements for HWOG activities. Adherence to the training requirements detailed in WM-SWO-401 would have precluded the deficiency cited.

The changes to the facility and operations were communicated verbally to $\mathrm{HW}^{\prime} \mathrm{OG}$ operating personnel.

\section{Root Cause:}

Inadequate management commitment; failure by management to ensure changes in a facility were incorporated in procedures and training. 
Planned Actions and Schedules:

Item/Description

1. Detail operational changes for Building 7507, review with appropriate personnel and document review.

2. Review facility and process control requirements with Solid Waste Operations personnel, including training requirements and document review. (Note: Training requirements are specified in the waste management section training plan and include periodic retraining. Annual regulatory audits help ensure adherence to established procedures.)

3. Revise and issue HWOG procedure to incorporate changes.

4. The need to revise ORNL training programs for providing and documenting regulatory driven or other training on short notice will be reviewed witk the Technical Resources and Training Section of the Office i Environmental and Health Protection.
Completion Date

Complete

Complete

$06 / 91$

$05 / 91$

Costs:

Type of funds: ERWM Programmatic

Source of funds: EM-ADS344

Estimated costs per fiscal year (\$K)

\begin{tabular}{crrrrrrr}
\cline { 2 - 6 } Action item & 1991 & 1992 & 1993 & 1994 & 1995 & Beyond & Total \\
\hline 1 & 1.5 & & & & & & 1.5 \\
2 & 1.5 & & & & & & 1.5 \\
3 & 3 & & & & & & 3 \\
4 & - & & & & & &
\end{tabular}

Status:

Funded

6

Requested

New

References: $\quad$ Tennessee Hazardous Waste Management Rule 1200-1-11-.05 
Finding No.: WM/CF-9 Inadequate Training Records and Inspection Records at the 3001 Storage Canal

\section{Finding}

Description:

Code:

Compliance Protocol:

Priority:

Response:
MMES-ORNL personnel performing RCRA inspections of the 3001 Storage Canal do not have their training documented in accordance with Tennessee Hazardous Waste Management Rule 1200-1-11-.05, and the RCRA inspection record is not complete in accordance with best management practices.

Compliance

Tennessee rule 1200-1-11-.05 requires that personnel involved in the operations of interim status hazardous waste management units be trained to perform their duties and that the training be documented.

40 CFR 265.195(c) requires that facility inspections be documented in the facility operating records.

Energy Systems Risk Weight 405

Tiger Team Action Plan Priority 1

Deficiencies in training and upkeep of training records, and inadequate implementation of regulatory policy at inactive waste sites have been documented in the Environment, Safety and Health and Management and Organization Self Assessment report for the ORNL Office of Waste Management and Remedial Action. To correct these deficiencies, facility-specific RCRA training and documentation will be developed and conducted through the Technical Resources and Training Department in the Office of Environmental Compliance and Health Protection. Retraining is incorporated in the training program. In addition, the Energy Systems-ORNL Remedial Action Section will prepare proposals for the FY 1993 budget submission to acquire additional regulatory compliance staff to ensi:re inspections and surveillances are properly conducted.

In accordance with 40 CFR 265.195(c), the Remedial Action S.ction has documented operating records of facility inspections. However, in keeping with best management practices, documentation will be revised to include personnel sign-offs in the operating records as a requirement.

\section{Root Causes:}

Inadequate policy implementation and inadequate communications; transmittal of regulatory requirements for management of hazardous waste tanks to Energy Systems-ORNL staff responsible for tanks is inadequate. 
Planned Actions and Schedules:

\section{$\underline{\text { Item/Description }}$}

1. Upgrade operating records for the 3001 Storage Canal log to include personnel sign-offs (includes memo informing personnel who perform the daily inspections of this requirement).

2. Conduct and document on-the-job-training of reactor operators performing the daily inspections at the 3001 Storage Canal.

3. Prepare budget proposals to acquire additional staff for regulatory expertise and training oversight for all contaminated sites managed by Energy Systems-ORNL's Remedial Action Section.

4. Develop and implement facility-specific RCRA training module and records management system for all RCRA regulated sites managed by Energy Systems-ORNL's Remedial Action Section.

\section{Completion Date}

$01 / 91$

$01 / 91$

$04 / 91$

$06 / 91$

Costs: Operating records upgrade for additional sign-off, tráning, development, implementation, and budget proposal preparation can be accomplished through Environmental Restoration (EM) funded work under Waste Area Grouping Surveillance and Maintenance - GF (ADS OR 311-AA).

Type of funds: ERWM Programmatic

Source of funds: EM-ADS 311AA

Estimated costs per fiscal year (\$K)

\begin{tabular}{|c|c|c|c|c|c|c|c|}
\hline \multirow[b]{2}{*}{ Action item } & & \multirow[b]{2}{*}{ Total } \\
\hline & 1991 & 1992 & 1993 & 1994 & 1995 & Beyond & \\
\hline 1 & 1 & & & & & & 1 \\
\hline 2 & 1 & & & & & & 1 \\
\hline 3 & 1 & & * & & & & 1 \\
\hline 4 & 25 & & & & & & 25 \\
\hline
\end{tabular}

Status:

Funded 28

Requested

New

*Estimated annual ongoing cost: \$25K per year beginning in FY 1993. 
References: Tennessec Hazardous Waste Management Rule 1200-1-11-.05 40 CFR 265.195(c)

Environmental Reswration and Waste Management Five-Year Plan 
Finding No.: WM/CF-10 Inadequate Leak Detection of Petroleum Underground Storage Tanks

\section{Finding}

Description: MMES-ORNL does not perform daily leak detection by "stick checking" on 8 of approximately 30 nonradioactive petroleum underground storage tanks in accordance with the MMES-ORNL SOP EMC-012-01.

Code: $\quad$ Compliance

Compliance Protocol:

Priority:

Response:

40 CFR 280 defines the requirements for leak detection of underground storage tanks (USTs) that store petroleum products. Deadlines for compliance are determined by specific requirements relating to tank capacity, tank age, etc., and are mandated by this regulation. There are certain types of USTs that are these types are USTs used for storing fuel for the sole purpose of supplying emergency generators. Therefore, all USTs at ORNL that store petroleum levels in USTs to minimize potential environmental liability in the event of a leaking UST.

Energy Systems Risk Weight 5

Tiger Team Action Plan Priority 3

SOP EMC-012-01 was issued in July 1990. Following the issuance of the SOP, a
Completion Date "deferred" from the requirements of leak detection but are included in the requirements for annual fees and potential environmental remediation. Included in products (with the exception of three tanks at the ORNL Fuel Service Station) are deferred from the regulations. As a "best-management" practice and in response to a DOE order, the SOP EMC-012-01 was issued to facilitate monitoring of product letter to the Environmental Protection Officers (EPOs) of each "non-complying" division was sent that specifically listed the tank owners that failed to comply. At that time, the number was thirteen. On November 12,1990, a second letter was sent to the Division EPOs requesting their compliance with the SOP. This time, the number of tank owners that failed to comply was eight. Currently, efforts are underway to resolve discrepancies that exist with these tank owners. This plan proposes final resolution of these discrepancies.

\section{Root Cause:}

Inadequate policy implementation

Planned Actions and Schedules:

\section{$\underline{\text { Item/Description }}$}

1. Identify ORNL organizations responsible for noncompliant USTs.
Complete 
2. Clearly define and document roles and responsibilities (R\&R).

3. Meet with tank owners to discuss specific requirements of the SOP.

4. Initiate quarterly audits of the "stick-check" data for compliance.

5. Complete the training for personnel performing the "stick-checks" with each UST division owner/operator.

Casts: $\quad$ Program planning activities will be accomplished with existing resources. Division activities related to compliance with the SOP will be borne by the respective divisions.

References: $\quad 40$ CFR Part 280, Subtitle I

Technical Regulations of the Tennessee Underground Storage Tank Division, Chapter 1200-1-15-.01 through .07 
Finding No.: $\quad$ WM/CF-11 Inadequate Hazardous Waste Determination of Sanitary Sewage Treatment Plant Sludge

Finding

Description: MMES-ORNL does not subject its sanitary sewage treatment plant sludge to a hazardous waste determination as required by Tennessee Hazardous Waste Rule 1200-1-11-.03.

Code: $\quad$ Compliance

Compliance

Protocol:

Tennessee Hazardous Waste Rule 1200-1-11-.03 requires generators of hazardous waste to perform hazardous waste determinations on all solid waste streams by either performing an analysis or using process knowledge.

Priority: $\quad$ Energy Systems Risk Weight 408

Tiger Team Action Plan Priority 2

Response: $\quad$ Prior to October 1, 1990, ORNL considered the sewage treatment plant sludge to be non-RCRA hazardous waste based on process knowledge. While this determination was not confirmed routinely by analysis, an EP-tox analysis for mercury in 1989 confirmed this parameter to be in accord with the nonhazardous determination and a TCLP analysis of sludge samples taken on November 5, 1990, also confirmed the determination that the sludge is a nonhazardous waste stream.

To provide ongoing assurance that the sewage treatment plant sludge is correctly classified as a nonhazardous waste stream, the following action plan will provide for development and implementation of a sampling and analysis protocol for this waste stream. Characterization of other ORNL solid waste streams is in progress where similar regulatory requirements apply.

\section{Root Cause:}

Inadequate policy implementation

\section{Planned Actions and Schedules:}

\section{Item/Description}

1. Prepare and implement sampling and analysis plan.
Completion Date

$03 / 91$ 


\section{Costs:}

Type of funds: Overhead

Source of funds: Overhead

\begin{tabular}{|c|c|c|c|c|c|c|c|}
\hline \multirow[b]{2}{*}{ Action item } & \multicolumn{6}{|c|}{ Estimated costs per fiscal year (\$K) } & \multirow[b]{2}{*}{ Total } \\
\hline & 1991 & 1992 & 1993 & 1994 & 1995 & Beyond & \\
\hline 1 & 12 & * & & & & & 12 \\
\hline \multicolumn{8}{|l|}{ Status: } \\
\hline Funded & 12 & & & & & & \\
\hline \multicolumn{8}{|l|}{ Requested } \\
\hline New & & & & & & & $\$ 12$ \\
\hline
\end{tabular}

"Estimated annual ongoing cost: \$5K starting in FY 1992.

References: TDHE Hazardous Waste Rule 1200-1-11-.03 
Finding No.: WM/CF-12 Inadequate Daily Inspections of RCRA Facilities on Weekends

Finding Description:

Code: $\quad$ Compliance

Compliance Protocol:

Priority: $\quad$ Energy Systems Risk Weight 405

Tiger Team Action Plan Priority 2

Response: Clarification of the regulatory requirements for facility inspections for Building 7652 and tank $7860 \mathrm{~A}$ and the correction of deficiencies in facility inspections are addressed by this action plan.

To date, verbal clarification of the regulatory interpretation of "daily" inspection requirements for tanks was obtained form T. P. Perry (ESA Central Staff). Perry confirmed that hazardous waste tanks must be inspected each day the tank is storing hazardous waste (i.e, 7 days per week).

Corrective actions will be implemented to ensure that daily (each operating day) inspections are being carried out for tank $7860 \mathrm{~A}$. Standard operating procedures and inspection forms will be revised to reflect the additional inspection requirements for tank systems storing hazardous wastes. Tank operators will receive annual training to ensure the facility's compliance with the RCRA regulations. Compliance staff will review and approve the inspection forms and will confirm that inspections meet the regulato $i ;$ requirements.

The Part B permit for Building 7652 does indicate that daily inspections are to be conducted; however, past verbal communications with TDC staff confirmed their acceptance of normal work week (excluding weekends and holidays) inspections for that permitted unit. To resolve the finding for Building 7652, verbal clarification of the daily inspection requirements for Building 7652 was again obtained from Jacqueline Okoreeh-Baah (TDC) on November 16, 1990.

Okoreeh-Baah indicated that container storage units including Building 7652 need not be inspected each day (i.e., 7 days per week); the state and federal regulations do not require daily inspections of container storage units 
[TN Rule 1200-1-11-.05(9)(e)]. Because TDC recognizes that inspections seven days per week are not required by the regulations, they accept an inspection schedule based on a normal work week for those units including Building $\mathbf{7 6 5 2}$. TDC's acceptance of daily (i.e., normal work week) inspections for container storage areas has been reaffirmed by the September 29,1990, issuance of the final permit for Building 7855, which defines daily as normal work week (see p. 3-6).

Written confirmation of the state's definition of daily inspections for Building 7652 will be obtained form TDHE. ORNL will ask DOE to request that TDHE issue a letter which outlines their position or that they modify the text within the permit to define "daily" for ORNL's permit files for Building 7652.

\section{Root Causes:}

Inadequate policy implementation; poorly defined roles and responsibilities

Planned Actions and Schedules:

\section{$\underline{\text { Item/Description }}$}

1. Request clearly defined inspection requirements under TN Rule 1200-1-11-.05 for container storage facilities from TDHE to verify compliance of Building 7652.

2. Revise standard operating procedures and inspection forms for hazardous waste tank $7860 \mathrm{~A}$ to reflect the additional inspection requirements. Implement the necessary training for facility personnel to ensure that facility will be in compliance and then implement the inspections for tank $7860 \mathrm{~A}$.

3. Verify and document that corrective actions have been fully implemented.

\section{Completion Date}

Complete

Complete

Complete

Costs: $\quad$ Clarification of container storage inspection requirements was accomplished with existing resources at no significant cost.

Revision of standard operating procedures and inspection forms and implementation of the training and actual inspections for tank 7860A were accomplished with existing resources. Verification that corrective actions have been implemented has been accomplished with existing resources.

References: Tennessee Hazardous Waste Storage Rule 1200-1-11-.05(10)(f)

Tennessee Hazardous Waste Permit TN1 890090 003, Hazardous Waste Storage Facility, Building 7652, dated September 26, 1986; revised February 21, 1989 
Memorandum of Conversation between N. S. Dailey and J. Okoreeh-Baah, dated November 16, 1990

Letter from J. H. Swanks to J. L. Radcliffe, "Resubmittal of Oak Ridge National Laboratory's Comments on the Draft Part B Permit for the Transuranic Concrete Cask Storage Unit (Building 7855) and Request for Confirmation of Inspection Requirements for the Hazardous Waste Storage Facility (7652)," dated December 17, 1990

Letter from Larry L. Radcliffe to Dale Ozier, "Resolution of ORNL's Comments on the Draft Part B Permit for the Transuranic Concrete Cask Storage Unit (Bldg. 7855)," dated January 16, 1991. (This letter also requested clarification of "daily" for the 7652 permit.) 
Finding No.: WM/CF-13 Inadequate Characterization of Mixed Waste in Storage

Finding

Description:

Code: Compliance

Compliance

Protocol:

Priority:

Response:
MMES-ORNL has not adequately characterized its stored mixed waste at the Transuranic Retrievable Concrete Cask Burial Ground and at the 7507W LowLevel Mixed Waste Storage Facility to determine whether it is hazardous in accordance with Tennessee Hazardous Waste Rules 1200-1-11-.03 and .05 and the ORNL RCRA Hazardous Waste Analysis Plan, and whether it is restricted from land disposal in accordance with 40 CFR 268.7.

The ORNL RCRA Hazardous Waste Analysis Plan requires stored waste to be characterized to determine if it is hazardous under Tennessee Hazardous Waste Rules 1200-1-11.

Energy Systems Risk Weight 408

Tiger Team Action Plan Priority 2

Remote-Handled Transuranic (RH TRU) Waste

Energy Systems-ORNL is storing 190 casks of RH TRU waste generated before 1980 in the RCRA interim status TRU Retrievable Concrete Cask Burial Ground. None of these casks has received an analysis or characterization. They were generated before Energy Systems-ORNL implemented an approved TRU waste certification program that provides for adequate characterization of newly generated RH TRU waste. Additionally, the RH TRU casks do not have certification data to determine whether they contain hazardous mixed waste or whether the waste is subject to the RCRA Land Disposal Restrictions (LDRs). The information was not developed at the time the casks were filled and put into storage, and the high radiation levels associated with the wastes (i.e., greater than $1000 \mathrm{R} / \mathrm{hr}$ ) and the nature of containment of the casks limit further characterization or analysis from being performed. Energy Systems-ORNL conducted interviews and record reviews to characterize the cask contents and determined that some of the casks contain lead, mercury, and oil. However, Energy Systems-ORNL does not have adequate records to verify which casks contain these wastes, whether the casks also contain small quantities of other hazardous wastes, and whether the wastes are properly stored (i.e., incompatibles are properly segregated).

Because the greatest concern with these mixed wastes is the radiation levels rather than the possible hazardous constituents, during characterization, handling the waste outside a shielded hot cell facility must be minimized. Numerous other technical issues remain to be resolved. Plans are to investigate various approaches to determine the most cost effective means of addressing we finding in a safe manner. Current robotics activities funded by the Office of Technology Development will ultimately contribute pertinent control architectures, 
characterization, and long manipulator reach capabilities to this project. After the technical issues are resolved and feasible options are evaluated, a strategy will be developed to address the finding.

\section{W Waste}

This activity is covered in the response to Finding WM/CF-6 Storage of Land Disposal Restricted Mixed Waste.

ORNL has recognized and documented this noncompliance in the Office of Waste Management and Remedial Action's Self-Assessment, Sect. 2.4.1. Currently, treatment is unavailable for these mixed wastes, with the exception of some scintillation fluids that meet the waste acceptance criteria of the Quadrex facility in Florida. Thus the wastes are being stored in permitted facilities while capabilities are developing.

\section{Root Cause:}

Inadequate management approach

Planned Actions and Schedules:

\section{$\underline{\text { Item/Description }}$}

1. Identify research and development requirements.

2. Based on approved research and development requirements, develop characterization strategy.

3. Conduct development activities.

\section{Completion Date}

$06 / 92$

$08 / 93$

09/97 
Costs:

Type of funds: ERWM Programnatic

Source of funds: EM-ADS 352

\begin{tabular}{cccccccc} 
& \multicolumn{6}{c}{ Estimated costs per fiscal year (\$K) } & \\
\cline { 2 - 6 } Action item & 1991 & 1992 & 1993 & 1994 & 1995 & Beyond & Total \\
\hline All & & 1,000 & 1,000 & 1,000 & 1,500 & 3,500 & 8,000 \\
Status: & & & & & &
\end{tabular}

Funded

$\begin{array}{llllll}\text { Requested } & 1,000 & 1,000 & 1,000 & 1,500 & 3,500\end{array}$

New

References: $\quad$ ORNL RCRA Hazardous Waste Analysis Plan ORNL/TM-11050, RemoteHandled Transuranic Solid Waste Characterization Study 
Finding No.: WM/BMPF-1 Inadequate Assessments of Offsite Vendors Recycling Lead-Acid Batteries and Circuitboards

Finding

Description: MMES-ORNL, does not assess the operations of offsite vendors who recycle RCRA-exempt hazardous recyclable lead-acid batteries and precious-metalcontaining circuitboards, in accordance with best management practices, to ensure that the vendor practices are environmentally sound.

Code: $\quad$ Best Management Practice

Compliance Protocol:

Priority: $\quad$ Energy Systems Risk Weight 5

Tiger Team Action Plan Priority 3

Response: ORNL and Energy Systems have recognized the deficiencies in the off-site vendor surveillance program. Energy Systems Central ES\&H staff will develop a surveillance program to review the recycle and disposal practices of off-site vendors. The review will be periodic, and the results of the review will be documented.

\section{Root Cause:}

Inadequate policy; Energy Systems has not issued a policy requiring surveillance of off-site vendors.

Planned Actions and Schedules:

\section{$\underline{\text { Item/Description }}$}

1. Issue draft Energy Systems vendor surveillance.

2. Initiate off-site vendor surveillance for lead-acid battery and circuitboard recyclers.

Costs: $\quad$ Vendor Surveillance $-\$ 10 \mathrm{~K}$

Existing Energy Systems Central ES\&H funds will be used to accomplish FY 1991 tasks. Ongoing reviews will be incorporated into future Central ES\&H budgat submittals.

\section{References: $\quad$ None}


Finding No.: WM/BMPF-2 Inadequate Waste Minimization Program

\section{Finding Description:}

Code:

Compliance Protocol:

Priority:

Response:
MMES-ORNL does not bave an adequate waste minimization program and therefore is unnecessarily generating hazardous, mixed, and solid wastes, which is not in accordance with best management practices.

Best Management Practice

Tennessee Hazardous Waste Management Rule 1200-1-11-.03, RCRA Section 3002, and DOE Order 5400.3 require waste minimization plans and programs for hazardous and radioactive mixed waste generating facilities. DOE Order 5820.2A requires DOE facilities to establish an auditable waste reduction program for LLW. DOE Order 5400.1 requires preparation of a waste reduction program plan, which must be reviewed annually and updated every three years. In 1985 ORNL issued a letter setting forth hazardous waste minimization as a Laboratory Policy. In addition, in 1986 Energy Systems issued a policy letter calling for implementation of a comprehensive waste minimization program.

\section{Energy Systems Risk Weight 56}

Tiger Team Action Plan Priority 1

ORNL has recognized deficiencies in its Waste Reduction Program, as documented in the Office of Waste Management and Remedial Action's Self Assessment, dated October 1, 1990. The requirements for and benefits of an effective waste reduction program are recognized by waste management staff. ORNL developed its first formal waste reduction plan in 1985, and several revisions have since been issued. The current plan, dated April 1990, outlines an effective waste reduction program that includes waste stream identification, evaluation, and targeting for reduction. However, full implementation of the plan has not occurred due to inadequacies in personnel resources and funding. Partial funding was provided for the continuation of a comprehensive ORNL Waste Reduction Program in FY 1991.

Responsibility for reducing waste generation lies with line management. The general approach taken by this action plan is to charge and empower Division Directors with this responsibility. Each division will be required to develop and imp!ement a plan for evaluating its waste streams, identifying and implementing waste reduction projects, and tracking and reporting progress against quantitative
goals.

A comprehensive ORNL policy procedure for waste reduction will be developed. Waste generation tracking and reporting capabilities will be improved to allow divisions to better monitor their waste reduction progress. Resources will continue to be sought to provide Laboratory-wide leadership, reporting, and evaluation. (See also Findings AX.2.1, p. 3.3.6-7; AX.3.1, p. 3.3.6-9; and RRP.10.2, p. 3.4.13-11.) 


\section{Root Causes:}

(1) Inadequate policy; ORNL has not issued a policy calling for minimization of all types of waste. Only a hazardous waste policy has been issued. (2) Poorly defined roles and responsibilities; DOE-ORO and ORNL have not clearly and formally charged line managers with the responsibility for waste minimization.

(3) Insufficient resources; funding is inadequate to support personnel and activities to implement waste reduction. (4) Inadequate management comonitment; although DOE has declared a policy of waste minimization and established requirements for such in its orders, waste minimization is assigned a priority of 3 (system of 1 through 4) in its budget guidance.

Planned Actions and Schedules:

\section{$\underline{\text { Item/Description }}$}

Completion Date

1. Continue to request funding.

- Request waste minimization funding in draft FY 1993

Complete ADS submission.

- Charge divisions with responsibility for requesting Complete funding for their waste minimization activities (via Task No. 2).

2. Issue letter from ORNL management to Division $05 / 91$ Directors reaffirming commitment to waste minimization and assigning responsibilities and divisional goals and requesting development of divisional waste minimization plans.

3. Conduct first waste-reduction workshop using updated lesson plan.

4. Issue first monthly report by division of generation of all types of waste and progress toward waste minimization goals.

5. Hire waste-reduction coordinator to lead, coordinate, report, and evaluate program.

6. Issue ORNL standard practice procedure for waste minimization.

7. Conduct Energy Systems audit of ORNL waste reduction program. 
8. Revise ORNL waste-reduction plan to reflect divisional roles and plans.

Contingent upon receipt of funding in FY 1992 and assumes no FY 1991 funding.

Costs:

Type of funds: Overhead

Source of funds: Overhead

Estimated costs per fiscal year $(\$ \mathrm{~K})$

\begin{tabular}{crrrrrrrr}
\cline { 2 - 5 } Action item & 1991 & 1992 & 1993 & 1994 & 1995 & Beyond & Total \\
\hline 6 & & 5 & & & & & & 5
\end{tabular}

Status:

Funded

Requested

New 5

Type of funds: ERWM Programmatic

Source of funds: EM-ADS 356

\begin{tabular}{crrrrrrr} 
& \multicolumn{5}{c}{ Estimated costs per fiscal year (\$K) } & \\
\cline { 2 - 6 } Action item & 1991 & 1992 & 1993 & 1994 & 1995 & Beyond & Total \\
\hline 1 & 5 & & & & & & 5 \\
2 & 5 & & & & & \\
5 & & & $*$ & & & & \\
7 & & & & & & \\
8 & & $*$ & & & &
\end{tabular}

Status:

Funded

Requested

New

10

*Estimated annual ongoing cost: $\$ 170 \mathrm{~K}$ starting in FY 1992 (\$5K for Item 8 and $\$ 165 \mathrm{~K}$ for Item 5). 
Type of funds: ERWM Progiammatic

Source of funds: EM-ADS 349

Estimated costs per fiscal year (\$K)

\begin{tabular}{crrrrrrrr}
\cline { 2 - 6 } Action item & 1991 & 1992 & 1993 & 1994 & 1995 & Beyond & Total \\
\hline 4 & 10 & $*$ & & & & & & 10
\end{tabular}

Status:

Funded

Requested

New

${ }^{*}$ Estimated annual ongoing cost: $\$ 165 \mathrm{~K}$.

Type of funds: ERWM Programmatic

Source of funds: EM-ADS 350

Estimated costs per fiscal year (\$K)

\begin{tabular}{crrrrrrrr}
\cline { 2 - 6 } Action item & 1991 & 1992 & 1993 & 1994 & 1995 & Beyond & Total \\
\hline 3 & 10 & $*$ & & & & & & 10
\end{tabular}

Status:

Funded

Requested

New

*Estimated annual ongoing cost: \$10K starting in FY 1992.

References: $\quad$ R. M. Schultz, Waste Reduction Plan for the Oak Ridge National Laboratory, ORNL/TM-11283, April 1990

R. M. Schultz, Waste Reduction Program at Oak Ridge National Laboratory During CY 1989, ORNL/TM-11504, May 1990 


\subsubsection{Toxic and Chemical Materials}

Finding No.: TCM/CF-1 PCB Wastes Stored Longer Than One Year

Finding

Description: MMES-ORNL is storing radioactively contaminated PCB wastes in excess of 1 year in Bldg. 7507W, which is not in accordance with 40 CFR 761.65(a).

Code: $\quad$ Compliance

Compliance Protocol:

Priority: $\quad$ Energy Systems Risk Weight 405

Tiger Team Action Plan Priority 2

Response: This action plan addresses the pruper disposition of one 30-gal and one 55-gal storage drum of radioactively contaminated PCB wastes that have been stored in Building $7507 \mathrm{~W}$ in excess of the one-year regulatory limit. The corrective actions specified will preclude the recurrence of this deficiency.

The deficiency was identified to ORNL compliance personnel by Waste Management Operations personnel in June 1989 and subsequently was identified by Energy Systems-ORNL in the Energy Systems Monthly Environmental Compliance Report for June 1989.

Energy Systems-ORNL requested that this noncompliance be addressed by DOEORO and EPA. A request was submitted by DOE-ORO to FPA Region IV on November 15, 1989, proposing that DOE and EPA enter into an agreement on a schedule to dispose of the stored material. A formal compliance strategy is under development by ORO.

In the interim, a request was made to the K-25 Site for transfer of the two drums to K-25 for storage. The request was denied March 15, 1991. The disposal of the two waste drums will be initiated after the K-25 TSCA incinerator becomes opcrational which is projected to be in 1991 .

Delays have occurred in the transfer of the two waste drums to K-25 as the result of discrepancies in two analyses. A third analysis will be completed to ensure K-25 waste acceptance criteria are met, and the drums will be transferred to K-25.

After completion of the third analysis, the request for storage of the radioactively contaminated PCB wastes at K-25 was denied by K-25 on March 25, 1991. The rejection was based on the concentration of radionuclides present. Negotiations between ORNL and K-25 personnel for storage of the contaminated PCB wastes 
at K-25 will continue. Storage of the two contaminated PCB waste drums at ORNL will continue until approval is recovered to ship them to $\mathrm{K}-25$ for storage.

\section{Root Causes:}

(1) Regulatory barriers; presently, there are no disposal facilities for radioactively contaminated PCB wastes. (2) Inadequate management approach; failure to negotiate a federal facility compliance agreement to address this issue in a timely manner.

Planned Actions and Schedules:

\section{$\underline{\text { Item/Description }}$}

1. Resample the two contaminated $\mathrm{PC}_{\bar{B}}$ waste drums as per $\mathrm{K}-25$ requirements.

2. Energy Systems-ORNL will send a letter to DOE-ORO requesting that this issue and future similar issues be resolved in a timely manner.

3. Complete analyses of the two contaminated PCB waste drums.

4. Request approval for shipment and ship the two contaminated PCB waste drums to $\mathrm{K}-25$; request denied $3 / 15 / 91$.

5. DOE-ORO request negotiation of an FFCA with regulators.
Completion Date

Complete

Complete

Complete

Complete

Complete 
Casts:

Type of funds: ERWM Programmatic

Source of funds: EM-ADS344

\begin{tabular}{|c|c|c|c|c|c|c|c|}
\hline \multirow[b]{2}{*}{ Action item } & \multicolumn{6}{|c|}{ Estimated costs per fiscal year (\$K) } & \multirow[b]{2}{*}{ Total } \\
\hline & 1991 & 1992 & 1993 & 1994 & 1995 & Beyond & \\
\hline 1 & 1.5 & & & & & & 1.5 \\
\hline 2 & - & & & & & & - \\
\hline 3 & 4.5 & & & & & & 4.5 \\
\hline 4 & 1.5 & & & & & & 1.5 \\
\hline 5 & $8.0^{a}$ & & & & & & 8.0 \\
\hline
\end{tabular}

Status:

Funded

20

Requested

New

$\$ 15.5$

${ }^{n}$ Best estimate of staff time required to support DOE-ORO in its negotiations with EPA.

References: $\quad 40$ CFR, Part 761.65(a)

Energy Systems Monthly Environmental Compliance Report, June 1989

Letter from ORNL to DOE-ORO dated July 5, 1989, requesting that DOE and EPA negotiate a consent order to address the noncompliance

DOE-ORO letter to EPA Region IV dated November 15, 1989, proposing that DOE and EPA enter into an agreement on a schedule to dispose of the stored waste 
Finding No.: TCI //CF-2 Deficiencies with the TSCA Assumptions Requirements for Liquid Filled Electrical Equipment

Finding

Description: Some ORNL, transformers which should be assumed to be PCB transformers in accordance with 44 FR 31517, including those in Bldgs. 2018, 1058, and 3025 at ORNL and in Bldg. 9204-3 at Y-12, are not being managed as PCB transformers by MMES-ORNL in accordance with 40 CFR Part 761.

Code: $\quad$ Compliance

Compliance

Protocol:

40 CFR 761

Priority: $\quad$ Energy Systems Risk Weight 410

Tiger Team Action Plan Priority 2

Response: $\quad 40$ CFR 761 requires that any transformer which contains mineral oil but for which there is no information on the concentration of PCBs in the transformer, the concentration of PCBs in the transformer must be assumed to be $50 \mathrm{ppm}$ to 499 ppm. Federal Register $(F R)$ Volume 44, No. 106, May 31, 1079, requires that if a transformer does not have a nameplate to indicate the type of dielectric fluid in it, the transformer must be assumed to be a PCB transformer (500 ppm or higher), unless the dielectric fluid is analyzed and found to contain less than $500 \mathrm{ppm}$ PCB. Transformers at ORNL with no nameplates, no manufacturers information, and no sampling information must be labeled as $>500 \mathrm{ppm}$ and inspected quarterly. If the sampling analyses determine that the PCB contamination is $>500 \mathrm{ppm},>50 \mathrm{ppm}$, or nondetectable, then the transformer should be labeled as explained in Environmental Prutection Manual 4.0-PCB. The ORNL PCB Inventory and Annual Report will be revised to provide the appropriate concentrations for each transformer remaining on site at ORNL. The information for ORNL at Y-12 will be submitted to Y-12 Environmental Compliance for inclusion in the Y-12 PCB Annual Report. PCB requirements are defined in Chapter 4.0 of the Environmental Protection Manual. Training of these requirements to division environmental protection officers and PCB generators was provided in May 1990. PCB issues will be discussed in the ORNL "Hazardous and Mixed Waste Generators Training."

\section{Root Causes:}

Ambiguous requirements or expectations; the previous interpretation of $40 \mathrm{CFR}$ 761 was inadequate in relation to unidentified, untested transformers at ORNL. Under previous policy: assumed to be $50 \mathrm{ppm}$ to $499 \mathrm{ppm}$.

Staffing extra funds to inspect, test, and validate all transformers at ORNL were not appropriated in past years at ORNL. 
Pla.ned Actions and Schedules:

$\underline{\text { Item/Description }}$

1. Prepare letter to each Division Director and Environmental Protection Officer.

2. EPOs update transformer inventory and begin inspections.

3. Initiate sampling for unknown transformers and/or apply labels as necessary.

4. Confirm receipt of sampling results and review inspection logs.

5. Second quarter of inspections by EPOs completed. Environmental Compliance reviews records.

\section{Completion Date}

Complete

$5 / 91$

$6 / 91$

$7 / 91$

9/91

Costs:

Type of funds: Overhead

Source of funds: Division Administration

Estimated costs per fiscal year (\$K)

\begin{tabular}{cccccccc}
\cline { 2 - 6 } Action item & 1991 & 1992 & 1993 & 1994 & 1995 & Beyond & Total \\
\hline 3 & 22 & & & & & & 22 \\
5 & & $*$ & & & & &
\end{tabular}

Status:

Funded

Requested

New

*Annual ongoing costs: $\$ 10 \mathrm{~K}$ for inspections.

References: $\quad 40$ CFR Part 761.3-Definitions

44 FR No. 106 (May 31, 1979) 
Finding No.: TCM/CF-3 Inadequate Labeling of Equipment Containing PCB Capacitors

Finding

Description: MMES-ORNL did not label three electrical equipment units containing large, high-voltage PCB capacitors in Bldg. 4501 and 9201-2, in accordance with 40 CFR 761.40(a)(4) and applicable ORNL and Y-12 MMES procedures.

Code: $\quad$ Compliance

Compliance Protocol:

Prionity:

Response:

\section{Energy Systems Risk Weight 405}

Tiger Team Action Plan Priority 2

40 CFR 761.40(a)(4) requires that equipment which contains large, high-voltage PCB capacitors or a PCB transformer be marked with a PCB label at the time of removal of the equipment from use, if not already marked. Also, Energy Systems Procedure 4.0 requires marking of equipment containing large high-voltage $\mathrm{PCB}$ capacitors or transformers.

The equipment containing PCB capacitors which was observed during audits of Buildings 4501 and 9201-2 has PCB small labels on the capacitors inside the equipment. Each piece of equipment was marked on the outside of the cabinet(s) as the audit was completed for the building.

PCB labeling requirements are defined in Chapter 4.0 of the ORNL Environmental Protection Manual. PCB issues will be discussed in the ORNL "Hazardous and Mixed Waste Generators Training."

\section{Root Causes:}

Ambiguous requirements or expectations-lack of clear instructions to mark the outside of the equipment, instead of (or in addition to) the capacitor(s) inside; also, an interpretation of the regulations was that the equipment must be marked "at the time of removal of the equipment from use . . .;" it was unclear as to the status of the equipment, whether it was in use, on standby, or on reserve.

Planned Actions and Schedules:

\section{Item/Description}

1. Prepare letter to EPOs which clarifies that every piece of equipment containing capacitors (except for small capacitors) must have appropriate sized PCB ML label on the outside.
Completion Date

Complete

Costs: No significant costs are associated with the action listed.

References: $\quad 40$ CFR 761.40(a)(4) and ORNL EPM 4.0 Procedure 
Finding No.: TCM/CF-4 Deficiencies with TSCA Temporary Storage Facility Requirements

Finding

Description: At the Transformer Service Area, Bldg. 9204-3, a drum of PCB waste was not marked with the date the PCB material was placed into the drum as waste, and the arca was not appropriately marked with a large $M_{L}$ mark in accordance with 40 CFR 761.65(c)(1), 40 CFR 761.65(3), and 40 CFR 761.40(a)(10).

Code: $\quad$ Compliance

Compliance Protocol:

Priority: $\quad$ Energy Systems Risk Weight 405

Tiger Team Action Plan Priority 2

Response: A Management Plan for PCBs at the Isotope Enrichment Facility was developed by IEF personnel and submitted to Chemical Technology Division management on November 28, 1990. This plan, among other items, addressed the dating of initial waste storage and the labeling of the PCB storage area. Both of these compliance deficiencies have been corrected and the Management Plan provides for quarterly inspections to ensure future comr ance.

Building $9204-3$ is an ORNL facility located at Y-12. Historically, there have been questions anc problems resulting from this organizational arrangement. The PCB compliance deficiencies are an example of the problems that arise when there are questions over organizational jurisdictions. In some cases, roles, respon sibilities and accountability on issues that involve both organizations are pcorly documented and, to a lesser extent, poorly understood. Recently, a Memorandum of Understanding between ORNL and Y-12 was signed and adopted to try to improve the delineation of roles and responsibilities between these two organizations for ORNL facilities at Y-12. Implementation of the Memorandum of Understanding is in progress; however, since it is a broad agreement between two large organizations, details of implementation remain to be resolved as new situations are encountered.

\section{Root Cause:}

Poorly defined roles, responsibilities, and accountability involving the ORNL/Y-12 interface 
Planned Actions and Schedules:

Item/Description

1. Sign and adapt a memorandum of understanding defining roles and responsibilities of managing ORNL facilities at $\mathrm{Y}-12$.

2. Issue a management plan for PCBs at the Isotope Errichment facility.

\section{Completion Date}

Complete

Complete

Costs: $\quad$ Costs associated with the corrective actions and the development of the PCB Management Plan were less than one full-time equivalent month funded out of the Isotopes Program operating budget.

References: $\quad 40$ CFR 761

Y-12 Plant Procedure 70-905

ORNL Environmental Protection Manual 4.0 
Finding No.: TCM/CF-5 Lack of Hazard Identification Labels for Some Chemical Storage Tanks

Finding

Description: Some aboveground chemical storage tanks, including a total of five tanks located at Bldgs. 5554, 7002, 7740, and Barn "D," do not have hazard identification labels as required by MMES-ORNL EPM-15.0.

Code: $\quad$ Compliance

Compliance

Protocol:

Priority: $\quad$ Energy Systems Risk Weight 10

Tiger Team Action Plan Priority 3

Response: Hazardous material labels have been placed on aboveground chemical storage tanks identified in this finding. However, to facilitate continued compliance with ORNL's hazard identification labeling requirements the environmental tank compliance section over the next 12 months (FY 1991) will conduct random surveys at various AST sites throughout the Laboratory.

\section{Root Cause:}

Inadequate policy implementation

Planned Actions and Schedules:

\section{$\underline{\text { Item/Description }}$}

1. Place labels on tanks

Costs: No significant costs were associated with this action item.
Completion Date

Complete

References: None 
Finding No.: TCM/CF-6 Lack of Secondary Containment for Some Aboveground Hazardous Materials Chemical Storage Tanks/Containers

Finding

Description: Some MMES-ORNL hazardous/tcxic material abovcground storage tanks and containers (drums), and tank truck transfer stations do not have adequate containment structures as required by EPM-13.0.

Code: $\quad$ Compliance

Compliance Protocol:

None

Priority: $\quad$ Energy Systems Risk Weight 9

Tiger Team Action Plan Priority 3

Response: $\quad$ Secondary containment upgrade for truck transfer stations at ORNL's PWTP (Building 3544) is being addressed under a 1992 GPP. The functional requirements for this project have been drafted and are presently in the review cycle. Several internal division assessments for the upgrade of ORNL aboveground storage tanks (AST) with inadequate or no secondary containment has been conducted by respective tank owners. In addition, the lack of secondary containment for truck transfer stations and ASTs were identified and documented in a 1989 tank survey conducted by ORNL's Environmental and Health Protection Division. However, plans to retrofit existing AST systems with adequate secondary containment at Buildings 2522, 3004, 5554, 7002, 7012, and 7702 have been delayed due to lack of funding. Relative to the matter concerning 55-gallon drums, overpacks were provided for those drums needing to be secondarily contained as of November 27, 1990. Therefore, the action to provide secondary containment for 55-gallon drums is closed.

\section{Root Cause:}

Tank systems and truck transfer stations at ORNL which lack secondary containment are structures that began operating in the 1960s. As such, these tanks and truck transfer stations were not mandated in regulations to have secondary containment beyond when they became operational. Plans to upgrade various tanks with secondary containment have been delayed because of lack of funding. 
Planned Actions and Schedules:

$\underline{\text { Item/Description }}$

1. Equip the PWTP with transfer stations that provide a minimum containment volume sufficient to hold the largest tank truck volume which is to be handled.

2. Request funding for secondary containment for ASTs at Buildings 2522, 3004, 7002, 7012, and 7702 in accordance with 40 CFR PT 151 under Hazardous Substance Spill Prevention as well as other relevant DOE, State, and Federal requirements as funding become available.

3. Close ASTs at facilities which are on a "standby" status as an alternative to diking.

4. Add one FTE to the ongoing AST management program to assist in facilitating this long-term compliance of DOE Order 5400.1, Section 5 relative to General Environmental Protection Requirements.

5. Request resources for full implementation of database tracking system for ASTs and other vessels containing liquid regulated substances with a capacity of 55 gallons or greater.
Completion Date

$12 / 92$

$8 / 91$

9/92

$10 / 91$

$12 / 91$

Casts:

Type of funds: Capital

Source of funds: GPP

Estimated costs per fiscal year $(\$ K)$

\begin{tabular}{cccccccc}
\cline { 2 - 6 } Action item & 1991 & 1992 & 1993 & 1994 & 1995 & Beyond & Total \\
\hline 1 & & 1000 & & & & & 1000 \\
2 & & & 750 & & & & 750
\end{tabular}

Status:

Funded

Requested

New

$1000 \quad 750$

$\$ 1750$ 
Type of funds: Overhead

S nurce of funds: Overhead

Estimated costs per fiscal year (\$K)

\begin{tabular}{|c|c|c|c|c|c|c|c|}
\hline \multirow[b]{2}{*}{ Action item } & & \multirow[b]{2}{*}{ Total } \\
\hline & 1991 & 1992 & 1993 & 1994 & 1995 & Beyond & \\
\hline 3 & & 80 & & & & & 80 \\
\hline 4 & & 21 & & & & & 21 \\
\hline 5 & & 5 & & & & & 5 \\
\hline
\end{tabular}

Status:

Funded

Requested

New

References: $\quad$ Clean Water Act of 1972 as amended in 1977

10 CFR Pt. 112-Oil Pollution Prevention

$\because 0$ CFR Pt. 151-Hazardous Substances Spill Prevention

NFPA 30 (1984)-Flammable and Combustible Liquids Code

DOE Order 5400.1, Section 5-General Environmental Protection Program

Requirements (4/7/88)

Energy Systems-ORNL Environmental Protection Manual-13.0 
Finding No.: TCM/CF-7 Deficiency with TSCA Storage for Disposal Monitoring Policy and Storage for Disposal Policy

Finding

Description:

MMES-ORNL has not met the storage for disposal monitoring policy guidelines in accordance with Federal Register, Volume 54, No. 244, December 21, 1989, or the 1-year storage for disposal requirement in accordance with $40 \mathrm{CF} R$ Part 761.65(a) for some of the PCB waste drums in Bldg. 7507.

Code: $\quad$ Compliance

Compliance Protocol:

40 CFR Part 761.65(a) limits the storage of PCB waste to a period of up to one year prior to disposal. Under EPA's existing compliance monitoring policies (Federal Register, Volume 54, No. 244, December 21, 1989), the one-year storage period is allocated between storage at the generator's facility and storage at the commercial disposal facility. Generators of PCB wastes are presumed to be in compliance with the one-year limit on storage if they can demonstrate that the storage period prior to delivery to a disposal facility did not exceed nine months.

Energy Systems-ORNL has not met the storage for disposal monitoring policy guidelines in accordance with Federal Register, Volume 54, No. 244, December 21, 1989 , or the one-year storage fo. disposal requirement in accordance with $40 \mathrm{CFR}$ Part 761.65(a) for some of the PCB waste drums in Building 7507.

Priority: $\quad$ Energy Systems Risk Weight 5

Tiger Team Action Plan Priority 3

Response: The drums of PCB wastes that were generated November 9, 1989, were shipped from ORNL to Rollins Environmental in Texas on November 9, 1990. Part of the drums were incinerated on November 10, 1990, and the remaining drums were incinerated the next week. An Exception Report as required by 40 CFR Part 761.215(d) has been prepared by ORNL which will be submitted to EPA, Region IV in January 1991.

EPM Procedure 4.0-PCBs will be revised to include the February 1990 Federal Register requirements concerning disposal of $\mathrm{PCB}$ wastes, manifesting of wastes, and submittal of Exception Reports. The procedure will encourage the shipment of all PCB wastes (> $50 \mathrm{ppm}$ ) to be shipped off-site to an EPA-TSCA permitted incinerator within nine months of the generation date. PCB issues will be discussed in the ORNL "Hazardous and Mixed Waste Generators Training." 


\section{Root Causes:}

Inadequate policy and inadequate policy implementation; lack of clearly defined ORNL policy for storage and shipment of PCB wastes.

Inadequate oversight and insufficient resources; human factor delays, limited staff time, and laboratory analysis delays which led to the 1-year disposal deadline being missed.

Planned Actions and Schedules:

\section{$\underline{\text { Item/Description }}$}

1. Submit Exception Report to DOE-ORO for submittal to EPA 1/31/91; resubmit to DOE-ORO 3/14/91.

2. Issue revised EPM - 4.0 - PCBs.

3. Initiate quarterly inspections by Environmental Compliance.
$5 / 91$

Completion Date

Complete

$6 / 91$

\section{Costs:}

Type of funds: Overhead

Source of funds: Overhead

Estimated costs per fiscal year (\$K)

\begin{tabular}{|c|c|c|c|c|c|c|c|}
\hline Action item & 1991 & 1992 & 1993 & 1994 & 1995 & Beyond & Total \\
\hline 3 & * & & & & & & \\
\hline \multicolumn{8}{|l|}{ Status: } \\
\hline \multicolumn{8}{|l|}{ Funded } \\
\hline \multicolumn{8}{|l|}{ Requested } \\
\hline New & & & & & & & $\$$ \\
\hline
\end{tabular}

*Estimated annual ongoing cost: $\$ 70 \mathrm{~K}$.

References: None 
Finding No.: $\quad$ TCM/BMPF-1 Pesticides Program Deficiencies

Finding

Descriptior: MMES.ORNL does not adequately label and properly equip pesticide storage areas at Bldgs. 2567 and 0855, Barn "D," and equipment at Barn "D" in complete accordance with 40 CFR 165.10.

Code: $\quad$ Best Management Practice

Compliance

Protocol:

None

Priority: $\quad$ Energy Systems Risk Weight 5

Tiger Team Action Plan Priority 3

Response: $\quad$ Pesticides and herbicides shall be stored in a safe and effective manner to support ongoing field work and programmatic needs. These improvements were accomplished by clarifying the requirements imposed by laws and procedures and implementing these requirements. Labeling requirements for storage areas and training requirements for staff will be incorporated in the ORNL Environmental Protection Manual (EPM).

Root Cause:

Inadequate policy implementation

P'anned Actions and Schedules:

\section{$\underline{\text { Item/Description }}$}

1. Label storage areas and equipment at Bldgs. 2567, 0855, and Barn "D" in accordance with 40 CFR 165.10.

2. Install two $A B C$ type fire extinguishers at Barn $D$.

3. Modify EMP to include procedure defining labeling, training, and monitoring requirements.
Completion Date

Complete

Complete

$09 / 91$

Costs: $\quad$ No significant costs are associated with the actions outlined.

References: None 


\subsubsection{Quality Assurance}

Finding No.: QA/CF-1 Standard Operating Procedures Deficiencies for Some MMES-ORNL Projects

Finding

Description:

Some operating procedures for the ORNL Materials Division, Biological Monitoring and Abatement Program, Pesticides Program, and air and groundwater quality tasks have not been approved (i.e., are drafts) or have not been developed by MMES-ORNL in accordance with the ORNL Quality Assurance Manual and DOE 5700.6B. In addition, 18 of 21 Environmental Protection Manual procedures have not been updated by MMES-ORNL as required by EPM-20.0.

Code: $\quad$ Compliance

Compliance Protocol:

DOE Order 5700.6B invokes ANSI/ASME NQA-1, which requires the development of instructions, procedures, and drawings for activities that affect quality.

Priority: $\quad$ Energy Systems Risk Weight 55

Tiger Team Action Plan Priority 2

Response: The lack of approved procedures to perform important activities consistently at ORNL has been long been recognized as a major concern at ORNL.

A Quality Assurance Bulletin was issued to all ORNL employees on September 10,1990, reminding them that it is their obligation to see that procedures are prepared, reviewed, and approved for all important activities.

The division and program QA specialists will work with their line managers to prepare lists of procedures that need to be developed. These lists will also indicate the persons responsible for developing the procedures and prioritized completion dates. Surveillances will then be conducted to determine adherence to those scheciules.

\section{Root Cause:}

Inadequate policy implementation; management has not fully implemented the policy requiring procedure development. 
Planned Actions and Schedules:

$\underline{\text { Item/Description }}$

1. Instruct $Q A S / Q A C s$ to work with their respective line organizations to develop lists of procedures needed for their important activities.

2. Complete procedures development schedules per Item 1.

3. Perform surveillances to verify adherence to the procedure development schedules.
$7 / 91$

Completion Date

$6 / 91$

$12 / 91$

Costs:

Type of funds: Research Programmatic

Source of funds: ER

Estimated costs per fiscal year $(\$ \mathrm{~K})$

\begin{tabular}{crrrrrrr}
\cline { 2 - 6 } Action item & 1991 & 1992 & 1993 & 1994 & 1995 & Beyond & Total \\
\hline 1 & - & & & & & \\
2 & 2 & $*$ & & & & & 2 \\
3 & 2 & $*$ & & & & & 2
\end{tabular}

Status:

Funded

4

Requested

New

*Estimated annual ongoing cost: $\$ 4 \mathrm{~K}$.

References: ANSI/ASME NQA-1. 
Finding No.: QA/CF-2 Deficiencies with the MMES Environmental Surveillance Procedures Quality Control Program Manual

Finding

Description:

Code: $\quad$ Compliance

Compliance Protocol:

Priority: $\quad$ Energy Systems Risk Weight 5

Tiger Team Action Plan Priority 3

Response: Improvements in the management of the ESPQCP are addressed by this action plan. Energy Systems Central Staff will better define the implementation and revision procedures. Procedures will be reviewed by the Environmental Sampling and Instrumentation Group. Specifically, any procedures that do not follow approved EPA protocols will be submitted for revision. Document control procedures will be improved. Biological Monitoring and Abatement Programassociated SOPs will be written and submitted for inclusion in the ESPQCP, and each ORO site office will be included in the review process.

\section{Root Causes:}

Inadequate oversight, ambiguous requirements or expectations, regulatory barriers, and inadequate policy

Planned Actions and Schedules:

\section{$\underline{\text { Item/Description }}$}

1. Document and distribute to all ES\&H organizations protocols which have been developed for revisions to the ESPQCP.
Completion Date

Complete 
2. Include all DOE Site Offices in the review process for and/or approval will be forwarded Review comments writing.

3. Environmental Sampling and Instrumentation Group will review all procedures in the ESPQCP and specifically submit to Energy Systems Central Staff a procedure for collection of volatile organic samples by the sample container immersion method.

4. Submit any other findings and suggestions for revision to the ESPQCP document to Energy Systems Central Staff.

5. Issue a revised policy for document control of the ESPQCP manual.

6. Develop draft SOPs specifically applying to Biological Monitoring and Abatement Programs for approval by the appropriate ORNL QA representative.

7. Forward SOPs to Energy Systems Central Staff for review and inclusion in the ESPQCP.

Costs: $\quad$ The Action Items will be accomplished with no significant costs.

References: None 
Finding No.: QA/BMPF-1 QAVQC Deficiencies in MMES-ORNL Environmental Sampling Programs

Finding

Description: A few MMES-ORNL environmental sampling activities do not follow such accepted QAVOC practices as collecting blanks and duplicates, field monitoring for pH and conductivity, maintaining chain-of-custody for periphyton samples, and using ink for data entries into analytical logbooks.

Code: $\quad$ Best Management Practice

Compliance Protocol:

Good environmental monitoring practices dictate that standard accepted sampling and analysis protocol be followed to help ensure the generation of defensible analytical data. These practices include: establishing field sampling QC programs, following field monitoring protocols for $\mathrm{pH}$ and conductivity, following chain of custody, and entering all laboratory logbook notations in ink.

Priority: $\quad$ Energy Systems Risk Weight 9

Tiger Team Action Plan Priority 3

Response: A QC program outline was in place at the time of the audit as noted in the discussion. Further development of this program is planned and the lack of a complete program identified in self-assessment. The ESI will provide ESD with appropriate documentation and control procedures and chain of custody for use in their biological monitoring program. Along with these initiatives, the ESI will also perform internal audits on the program, as manager of the BMAP budget, in order to ensure compliance with $\mathrm{QA}$ requirements.

\section{Root Causes:}

Inadequate policy, inadequate communications, inadequate policy implementation, and poorly defined roles and responsibilities

\section{Planned Actions and Schedules:}

\section{Item/Description}

Completion Date

1. Prepare an outline for a complete $\mathrm{QC}$ program for all Complete compliance sampling activities.

2. Inform all field personnel by memo that all entries in Complete BMAP field logbooks must be made in waterproof ink, and $\mathrm{pH}$ and conductivity measurements must be taken in the field.

3. Develop a $\mathrm{QC}$ program plan. 
4. Develop a procedure requiring that field readings for

Complete $\mathrm{pH}$ and conductivity be taken in situ or by grab.

5. Distribute SOP-EMC-003.002 Chain of Custody for use and reference by appropriate staff.

Complete

6. Submit a budget to support the QC program.

$8 / 91$

7. Initiate implemention of the QC program plan.

Costs:

Type of funds: ESH Programmatic

Source of funds: ER-AT EC

Estimated costs per fiscal year (\$K)

\begin{tabular}{cccccccc}
\cline { 2 - 5 } Action item & 1991 & 1992 & 1993 & 1994 & 1995 & Beyond & Total \\
\hline 1 & - & & & & & & \\
2 & - & & & & & & \\
3 & - & & & & & & \\
4 & - & & & & & & \\
5 & - & & & & & & \\
6 & - & & & & & & \\
7 & & & 235 & $*$ & & &
\end{tabular}

Status:

Funded

Requested

New

*Estimated annual ongoing cost: $\$ 235 \mathrm{~K}$.

References: None 


\subsubsection{Radiation}

Finding No.: RAD/CF-1 Inadequate Radiological Dose Assessment

Finding

Description:

The MMES-ORNL dose assessment process has deficiencies in that data documentation and traceability, computer validation, dose calculation assumptions, and dose assessment oversight are not in accordance with DOE 5400.5, 5700.6B, and ANSI/ANS 10.3, 1986.

Code: $\quad$ Compliance

Compliance

Protocol:

Priority:

Response:
DOE Order 5400.5 states, among other things, that doses to members of the public in the vicinity of DOE activities shall be evaluated and documented to demonstrate compliance with the dose limits of the order, that appropriate transport models and dose conversion factors should be used, that calculated doses should be as realistic as practicable, and that parametric values used in the dose calculations should be recorded and should be realistic or, lacking real data, should be conservative.

The draft regulatory guide for 10 CFR 834 restates the requirements of DOE Order 5400.5 and provides additional guidance on some items. Of particular applicability to this finding is that parameter values used in the dose calculations be evaluated and documented.

\section{Energy Systems Risk Weight 55}

Tiger Team Action Plan Priority 2

The deficiencies noted in this finding are attributable to two things: lack of formal procedures and documentation of the rationale for choosing a few parameter values. The lack of formal procedures has been identified in ORNL and Office of Environmental and Health Protection self-assessment reports. Procedure development has begun, and documentation for the identified parameter values will be prepared.

\section{Root Cause:}

Inadequate policy implementation 
Planned Actions and Schedules:

\section{$\underline{\text { Itcm/Description }}$}

1. Reconstruct documentation for generic exposure parameters used in annual surveillance reports.

2. Issue a procedure for "input data management."

3. Issue procedures for performance of dose calculations to demonstrate compliance with DOE Order 5400.5.
Completion Date

7/91

$7 / 91$

$8 / 91$

Costs:

Type of funds: Overhead

Source of funds: Overhead

\begin{tabular}{crrrrrrr} 
& \multicolumn{5}{c}{ Estimated costs per fiscal year (\$K) } \\
\cline { 2 - 6 } Action item & 1991 & 1992 & 1993 & 1994 & 1995 & Beyond & Total \\
\hline 1 & 6 & & & & & & 6 \\
2 & 12 & & & & & & 12 \\
3 & 12 & & & & & 12
\end{tabular}

Funded

Requested

30

New

Estimated annual ongoing cost: $\$ 15 \mathrm{~K}$.

References: None 
Finding No.: RAD/CF-2 Inadequate Radiological Postings

Finding

Description: MMES-ORNL posting of some radioactively contaminated areas, and radiological control boundaries of Solid Waste Storage Area (SWSA) 4, is inconsistent with DOE 5480.11, MMES-ORNL Health Physics Procedure RP 2.3, Revision 1, and OR Radioactive Contamination Control Policy.

Code: $\quad$ Compliance

Compliance Protocol:

Compliance with radioactive contamination posting requirements

Priority: $\quad$ Energy Systems Risk Weight 65

Tiger Team Action Plan Priority 2

Response: The laboratory is in the process of completing the posting requirements as required by the "ORO Contamination Control" implementation plan, dated September 29, 1990. Due to the large areas involved and the replacements that are required because of the weather, posting upgrades at SWSA 4 will be needed. Irregularities in the "zone" and "area" signs will be: corrected. The use of the "General Radiation Hazard Information Sign" is specified in Health Physics procedure RP 2.3, and areas at the SWSAs will continue to use this approved sign to specify and supplement the other radiological postings. Many of the posting discrepancies were corrected as they were identified.

The general SWSA areas and roadways are not contaminated, and the primary control of personnel and vehicle contamination vill continue to be entry and egress controls at the actual contaminated sites vrithin the SWSA boundaries.

The postings of the White Oak area will need to be studied to determine the appropriate use of the "Radiation Hazard Keep Out" signs, as well as the other signs that are presently in use. Agreements with other agencies will be needed on areas that are outside Energy Systems-ORNL control.

A complete survey of the White Oak Creek floodplain and other associated SWSA run-off areas will be completed to determine if any areas, that meet radiological area criteria, are not properly identified.

Additional information is provided in response to SSB/BMPF-1.

Root Causes:

Insufficient resources, inadequate oversight, and inadequate communications 
Planned Actions and Schedules:

Item/Description

1. Complete the posting upgrades around SWSA 4.

2. Post "Radiation Hazard Keep Out" signs at arcas of White Oak Dam and the surrounding areas that are controlled by Energy Systems-ORNL.

3. Request funds to contract a survey of the White Oak floodplain and tributary run-offs leaving the SWSAs.
Completion Date

Complete

$6 / 91$

$8 / 91$

Costs:

Type of funds: Overhead

Source of funds: Overhead

\begin{tabular}{crrrrrrr} 
& \multicolumn{5}{c}{ Estimated costs per fiscal year (\$K) } & \\
\cline { 2 - 6 } Action item & 1991 & 1992 & 1993 & 1994 & 1995 & Beyond & Total \\
\hline 1 & 16 & & & & & & 16 \\
2 & 8 & & & & & & 8
\end{tabular}

Status:

Funded

24

Requested

New

Type of funds: ESH Programmatic

Source of funds: ER-AT RP

Estimated costs per fiscal year (\$K)

\begin{tabular}{|c|c|c|c|c|c|c|c|}
\hline Action item & 1991 & 1992 & 1993 & 1994 & 1995 & Beyond & Total \\
\hline 3 & & & 225 & & & & 225 \\
\hline \multicolumn{8}{|l|}{ Status: } \\
\hline \multicolumn{8}{|l|}{ Funded } \\
\hline \multicolumn{8}{|l|}{ Requested } \\
\hline New & & & 225 & & & & $\$ 225$ \\
\hline
\end{tabular}

References: $\quad$ DOE Order 5480.11, "ORO Contamination Control Policy" 
Finding No.: RAD/CF.3 Unmonitored Decontamination Laundry Discharges

Finding

Des iption: MMES-ORNL inas not adequately cvaluated the discharge of the radioactive component of the iaundry facility effluent to determine if discharges are below 5 tines the DCGs, as required by draft Regulatory Guide for 10 CFR 834 and DOE 5400.5.

Code: $\quad$ Compliance

\section{Compliance}

Protocol:

All liquid effluent streams shall be evaluated and their potential for release of radioactive material assessed. Based on this assessment, decisions shall be made regarding necessary effluent monitoring systems.

Priority: $\quad$ Energy Systems Risk Weight 58

Tiger Team Action Plan Priority 2

Response: $\quad$ Though initially monitored and evaluated for inclusion in the Sewage Treatment Plant wastewater strenm in 1986, the wastewater from the contaminated clothing washer has not been formally evaluated since. Because of this, a new evaluation of this wastestream's components will be made. Using the results of this study, a decision will be made regarding whether the Sewage Treatment System provides for the proper treatment and disposal of this wastestream.

Root Cause:

Inadequate policy implementation

Planned Actions and Schedules:

\section{$\underline{\text { Item/Description }}$}

1. Reevaluate the wastewater stream and the Sewage Treatment Plant's Waste Acceptance Criteria to determine if contaminated washer wastewater can be legally disposed of by this process.

\section{Completion Date}

$10 / 91$ 


\section{Costs:}

Type of funds: ESH Programmatic

Source of funds: ER-AT RP

\begin{tabular}{|c|c|c|c|c|c|c|c|}
\hline \multirow[b]{2}{*}{ Action item } & \multicolumn{6}{|c|}{ Estimated costs per fiscal year $(\$ K)$} & \multirow[b]{2}{*}{ Total } \\
\hline & 1991 & 1992 & 1993 & 1994 & 1995 & Beyond & \\
\hline 1 & & 15 & & & & & 15 \\
\hline \multicolumn{8}{|l|}{ Status: } \\
\hline \multicolumn{8}{|l|}{ Funded } \\
\hline \multicolumn{8}{|l|}{ Requested } \\
\hline New & & 15 & & & & & $\$ 15$ \\
\hline
\end{tabular}

Estimated annual ongoing cost: $\$ 5 \mathrm{~K}$.

References: Draft Regulatory Guide for 10 CFR 834 
Finding No.: RAD/CF-4 Inadequate Calibration of Radiological Monitors

Finding

Description: MMES-ORNL surface water radiological monitors at all monitoring stations are not calibrated in accordance with the Regulatory Guide for 10 CFR 834 (draft) and cannot provide quantitative data for alarm set points and unplanned radiological releases.

Code: $\quad$ Compliance

Compliance

Protocol:

Priority: $\quad$ Energy Systems Risk Weight 55

Tiger Team Action Plan Priority 2

Response: $\quad$ ORNL should establish at stream monitoring sites radiological monitors that are calibrated against known standards. Readout instrumentation in concentration units that correlate directly with regulated discharge limits should also be obtained. It should be pointed out that if possible, all environmental data reported by ORNL (and any accompanying formal actions) are acquired through sampling and analysis; therefore, the importance of this improvement is providing alarm set points. A single system that serves multiple purposes will require establishment of performance criteria, operating procedures, and most probably equipment modifications.

\section{Root Causes:}

Poorly defined roles and responsibilities, inadequate policy, and inadequate policy implementation

Planned Actions and Schedules:
Completion Date

$7 / 91$

1. Issue a project charter and assign duties to a radiological monitoring review committee with participation by responsible ORNL groups including Emergency Response and Waste Operations.

2. Assign a staff member to develop, oversee and conduct an internal audit program of check sheets and associated material for the project and keep all such information in a document file for the life of the project.
Complete 
3. Complete a summary report of committee review of applicable regulations from different sources with common interests to insure all requirements are met.

4. Issue an "approved policy statement" containing specific definitions of responsibilities including funding, training, functional criteria, operational guidelines, matrix support agreements, and referencing the specific data sheets listed in Item 4.

5. Prepare budget and request funding for required upgrades.

6. Modify, upgrade, or replace monitors or system components as determined by the above actions.

7. Issue SOPs for maintenance, calibration, and operation of modified system components.

Costs:

Type of funds: Overhead

Source of funds: Overhead

Estimated costs per fiscal year (\$K)

\begin{tabular}{|c|c|c|c|c|c|c|c|}
\hline Action iten & 1991 & 1992 & 1993 & 1994 & 1995 & Beyond & Total \\
\hline 1 & - & & & & & & \\
\hline 2 & - & & & & & & \\
\hline 3 & - & & & & & & \\
\hline 4 & - & & & & & & \\
\hline 5 & 10 & * & & & & & 10 \\
\hline 6 & - & & & & & & \\
\hline 7 & - & & & & & & \\
\hline
\end{tabular}

Status:

Funded

Requested

New

${ }^{*}$ Estimated annual ongoing cost: $\$ 5 \mathrm{~K}$.

References: None 


\subsubsection{Inactive Waste Sites}

Finding No.: $\quad$ IWS/CF-1 Inadequate Inventory and Identification of Inactive Waste Sites

Finding

Description: OR has not adequately identified all inactive waste sites and does not have formal procedures in place to ensure site identification and reporting of potential inactive waste sites in conformance with its RCRA/HSWA permit and 40 CFR 300.410 and 40 CFR 300.420.

Code: $\quad$ Compliance

Compliance Protocol:

The National Contingency Plan (NCP), 40 CFR 300.410 and 40 CFR 300.420, requires that the lead agency use readily available information to prepare a report that includes a history of waste handling and inventory of potential inactive waste sites.

DOE Order 5400.4, "CERCLA Requirements," states that DOE shall respond to inactive waste sites in compliance with the NCP.

The ORR RCRA/HSWA permit contains a compliance schedule under which DOE must provide the regulatory agencies with a solid waste management unit (SWMU) identification and characterization report, i.e., a RCRA Facility Assessment (RFA) for all SWMUs listed in the attachment to the permit.

Priority: $\quad$ Energy Systems Risk Weight 410

Tiger Team Action Plan Priority 2

Response: $\quad$ Because ORNL's April 1987 RFA was prepared on an accelerated schedule to meet ORR's RCRA/HSWA permit deadline, SWMUs were identified based on readily available information. Since that time additional sites have been identified and further characterization of the new, and existing sites has been accomplished as part of the facility's surveillance and maintenance, environmental compliance and monitoring, and environmental restoration activities. Site characterization will be accomplished mainly via the remedial investigation (RI) process. The schedules for conducting RIs is negotiated with the EPA-Region IV and the TDHE annually as part of the pending Federal Facility Agreement (FFA). Additional sites and/or changes in status of existing sites have been reported to the regulatory agencies and corrective action has been accomplished as required. Reclassification of sites resulting in "no further action" change in regulatory status, i.e., RCRA or CERCLA, and deletion of facilities requiring only decontamination and decommissioning, i.e., not a source of continuing release to the environment, have been negotiated with the regulatory agencies. The lack of a formalized approach, i.e., procedures, for such activities as site identification, preliminary assessment, and determination of regulatory status documentation of agreement has resulted in numerous discrepancies in the site listing. 
The list of sites was revised and baselined in September 1990 incorporating input and review by personnel of the ORNL Office of Environmental Compliance and Documentation (OECD), ORNL Office of Waste Management and Remedial Actions (OWMRA), and the Energy Systems Environmental Restoration Division. This listing of sites provides consistency in site identification and reporting and identifies all major sites requiring, or potentially requiring, remediation at the ORNL. This listing of sites will be incorporated as Appendix $\mathrm{C}$ of the pending FFA. Additional sites of lesser importance may be discovered during such activities as surveillance and maintenance, and remedial investigation but these are expected to be few in number. These sites, as they are discovered, will be reported to the regulatory agencies as required, and corrective actions will be negotiated as part of the pending FFA. It is not considered cost effective to initiate a separate effort at this time to fully investigate the ORNL property to identify any remaining sites. The ORNL OECD will prepare procedures to address roles and responsibilities; site discovery; preliminary assessment/site inspection; regulatory status determination; documentation of regulatory agency interfaces; and site-listing configuration control.

\section{Root Causes:}

Poorly defined roles and responsibilities inadequate policy implementation

Planned Actions and Schedules:

\section{$\underline{\text { Item/Description }}$}

1. Issue plan/schedule for procedures development.

2. Issue approved procedures.

3. Conduct RIs.

4. Baseline site listir.g.
Completion Date

$6 / 91$

9/91

Negotiated annually as part of FFA

Effective date of FFA 
Costs:

Type of funds: ERWM Programmatic

Source of funds: EM-ADS 322

Estimated costs per fiscal year (\$K)

\begin{tabular}{|c|c|c|c|c|c|c|c|}
\hline Action item & 1991 & 1992 & 1993 & 1994 & 1995 & Beyond & Total \\
\hline $1-2$ & 25 & & & & & & 2 \\
\hline
\end{tabular}

Status:

Funded

25

Requested

New

References: $\quad$ ORR RCRA/HSWA Permit

40 CFR 300.410

40 CFR 300.420

DOE Order 5400.4 
Finding No.: IWS/CF-2 Lack of Formal Natural Resources Damage Assessment Notification

\section{Finding}

Description. Formal notification by OR to the Natural Resources Trustees of potential damages to the environment from releases of hazardous substances from inactive waste sites at ORR has not been made, as required by CERCLA Section 104(b) and Executive Order 12580.

Code: $\quad$ Compliance

Compliance Protocol:

DOE, as mandated under Section 107 of CERCLA, serves as the Primary Federal Natural Resource Trustee at DOE facilities and as a CERCLA lead response agency performing environmental restoration actions at DOE facilities, DOE has a dual role.

Pursuant to DOE Order 5400.4, Program Senior Officials are responsible for overseeing implementation of the Natural Resource Trustee provisions of CERCLA, and Heads of Field Organizations are responsible for overseeing response actions under CERCLA. Many of the assessment activities required under the National Resource Damage Assessment (NRDA) are also required by the CERCLA response and/or the federal RCRA corrective action process.

Priority: $\quad$ Energy Systems Risk Weight 495

Tiger Team Action Plan Priority 2

Response: $\quad$ ORO and Energy Systems-ERD are aware of the need for NRDA notification, which was raised in ORO's recent environmental compliance audit at Y-12, as well as in the October 1990 environmental compliance review at ORNL, conducted by Energy Systems corporate staff (IWS-175, in reference to Control Number ORNL-ER-1).

ORR has recently received draft guidance from Headquarters and are actively discussing notification strategies with DOE Headquarters. ORR will use the draft guidance as a baseline in which to implement a standard procedure for the ORO and revise accordingly if Headquarters guidance is substantially modified from the proposed draft.

An NRDA seminar with DOE Headquarters and the Department of the Interior was held in March 1991 in Oak Ridge.

\section{Root Causes:}

Lack of formal guidance from Headquarters and poorly defined roles and responsibilities 
Planned Actions and Schedules:

Item/Description

1. Develop DOE-ORO Environmental Restoration Division roles and responsibilities.

2. Formally notify trustees.

3. Devleop training procedures.
Completion Date

Complete

$5 / 91$

9/91

Costs: $\quad$ This action was accomplished with existing resources.

References: CERCLA Section 104, CERCLA Section 107, Executive Order 12580, DOE Order 5400.4 
Finding No.: IWS/CF-3 Incomplete Distribution of Emergency Planning and Community Rightto-Know Act Reports

Finding

Description:

XSO or OR have not sent the initial list of hazardous chemicals and its first and second updates to the LEPC and jurisdictional fire department; the third update to the SERC, LEPC, and jurisdictional fire department; nor the most recently 1989 EPA Tier I and II forms to the jurisdictional fire department, as required in 40 CFR 370.

Code: $\quad$ Compliance

Compliance Protocol:

Sections 311 and 312 of the Emergency Planning and Community Right-to-Know Act of 1986 (EPCRA or SARA Title III) requires the owner or operator of a facility subject to this act to submit specific emergency planning information "...to the commission, committee, or the fire department having jurisdiction over the facility..." (40 CFR 370, Subpart B).

Priority: $\quad$ Energy Systems Risk Weight 405

Tiger Team Action Plan Priority 2

Response: Due to several DOE reorganizations and the attendant changing of personnel responsible for SARA Title III reporting, the XSO was not able to substantiate that all SARA Title III reports had been transmitted to the appropriate entities since the promulgation of EPCRA, as required in 40 CFR Part 370.

Root Causes:

Poorly defined roles and responsibilities for SARA Title III reporting with DOEORO and inadequate policy implementation as defined in 40 CFR 370

Planned Actions and Schedules:

\section{Item/Description}

1. Transmit the SARA 311 report for 1989 to the ORNL Fire Department.

2. Transmit previous SARA reports to the appropriate commissions, committees, and jurisdictions.

3. Assign XSO responsibilities regarding SARA Title III reporting.

4. Establish filing and distribution system for SARA Title III reports.
Completion Date

Complete

Complete

Complete

Complete 
5. Establish procedure on Environmental Compliance

Complete reporting.

6. Provide SARA Title III training to the XSO

9/91 environmental staff.

Costs: $\quad$ XSO program activities will be accomplished with existing resources.

References: $\quad 40$ CFR Part 370 
Finding No.: IWS/BMPF-1 Informality of Operations in the Environmental Restoration Program

Finding

Description:

OR, XSO, MMES-ERD, and MMES-ORNL have not adequately established formal procedures, lines of communication, and documentation of significant ORR environmental restoration program (ERP) activities and technical review in accordance with best management practice.

Code: $\quad$ Best Management Practice

Compliance Protocol:

Priority:

Response:

\section{None}

Energy Systems Risk Weight 10

Tiger Team Action Plan Priority 3

ORO and Energy Systems-ERD recognize the need for greater formality and procedures, which were identified by Energy Systems-ORNL in its self-assessment, as well as by ORO in several internal audits and in its most recent Performance Evaluation Committee report. The need to formally document day-to-day policy and technical decisions is acknowledged; conscious efforts to maintain record of communication logs, note: to the file, and follow-up letters to each other, as well as the regulators on conversations and other informal direction received, are made.

DOE-ORO is developing a program management plan that will address ER activities at all of its facilities, including ORR and has finalized an internal memorandum of agreement (MOA) between the Assistant Manager for Environmental Restoration and Waste Management and Assistant Manager for Energy Research and Development to coordinate waste management and environmental restoration work at ORNL. Energy Systems-ERD is developing a Remedial Investigation/Feasibility Study Surveillance Plan that will strengthen Energy Systems-ERD's oversight procedures for administering the ERP.

Revised performance criteria are currently under development, including a draft position description for the Technical Oversight and Review Committee chairman. Energy Systems-ERD has also drafted a missions statement for the TORC and operating instructions for document review. Energy Systems-ERD's subcontract team is currently developing procedures to address issue resolution and follow-up.

\section{Root Causes:}

Unclear roles and responsibilities resulting from the recent organizational changes at ORO, Energy Systems-ERD, and Energy Systems-ORNL; inadequate policy implementations; and lack of resources for technical review and oversight from both the regulators and DOE-ORO 
Planned Actions and Schedules:

Item/Description

1. Issue the ORO Environmental/Waste Management program management plan.

2. Finalize the missions statement for the TORC and position description for the TORC chairman.

3. Develop procedures requiring documentation of informal communication and agreements with the regulators.

4. Develop formal procedures for review and approval of ERP documents prior to submittal to DOE Headquarters that will ensure adequate technical oversight by ORO of remedial activities.

5. Issue the ORNL Energy Systems-ERD Remedial Investigation Feasibility Study Surveillance Plan.

6. Develop written procedures for rectifying and tracking issues of concern that are identified by OR.NL Energy Systems-ERD while conducting oversight of subcontractor ERP activities.

Costs: No significant costs are associated with these activities.
Completion Date

Complete

Complete

Complete

Complete

References: None 
Finding No.: IWS/BMPF-2 Proceeding Without Approved Plans

Finding

Description:

Code:

Compliance

Protocol:

Prionity:

Response:
OR and DOB Headquarters are implementing corrective action plans and schedules that have been submitted under ORR's RCRAMHSWA permit without receiving formal regulatory approval from EPA Region IV and TDHE, which is not in accordance with beat management practice.

Best Management Practice

None

Energy Systems Risk Weight 7

Tiger Team Action Plan Priority 3

DOE-ORO and Headquarters are very much aware of the risk associated with implementation of corrective action plans without receiving formal regulatory approval from EPA and TDHE. However, EPA and TDHE have been made aware of our on-going work through regularly scheduled technical working group meetings and have informally supported DOE's initiation of remedial activities.

ORO is under continued pressure by the regulators to proceed with our investigations and not allow the slow approval process to serve as a barrier. We will continue to keep the regulators fully apprised of our on-going investigations being sure to receive verbal approval prior to initiation of any new activities as agreed to in an October 17, 1990, letter to both EPA and TDHE.

DOE-ORO and representatives from the EPA and TDHE agreed that no new field work will proceed without approved work plans. Corrective actions will not occur until the remedial investigation report, feasibility study, and draft remedial action plan are approved.

Root Cause:

Insufficient regulatory resources to ensure timely reviews of DOE plans

Planned Actions and Schedules:

\section{Item/Description}

1. Briefing with both TDHE and EPA will be held prior to initiation of any new investigation. EPA and TDHE must provide verbal concurrence with the proposed actions prior to initiation of work. These briefings will be held on an as needed basis.
Completion Date

Complete 
2. DOE-ORO issue letter to EPA and TDHE to clarify action approval approach.

Costs: $\quad$ Activity accomplished with existing resources.

References: $\quad$ October 17, 1990, letter to EPA and TDHE from DOE ORO-ERD 
Finding No.: IWS/BMPF-3 Lack of Adequate Planning for Federal Facility Agreement Activities

Finding Description:

Contrary to best management practice, ORO has not factored in the costs and additional time associated with preparing NRDAs into any of the FFA negotiations and draft agreements, nor has OR fully analyzed the cost impacts associated with implementing the AIP.

Code: Best Management Practice

Compliance Protocol:

Priority: $\quad$ Energy Systems Risk Weight 42

Tiger Team Action Plan Priority 3

Response: It is true that ORO did not explicitly factor in the costs and additional time associated with preparing NRDAs into any of the FFA negotiations and agreements. However, upon examining what actions are routinely taken in implementing the FFA, such costs are accounted for in budgeting and planning for the RI/FS process including the requirements of the NCP.

While a fully comprehensive analysis of impacts associated with implementing the AIP may not have been performed, ORO was one of the first field offices to recognize that impacts on the M\&O Contractor and Federal staff should be included in the ER budget submissions. A telephone canvass was made to Hanford and Rocky Flats in early 1990 to gather input from DOE staff on the impacts to M\&O and Federal staff caused by execution of the AIPs. This information was passed on to Energy Systems-ERD who later provided DOE-ORO with AIP implementation cost estimates and a draft ADS. ORO and Energy Systems are continuing to refine this cost estimate and to develop a formal AIP implementation plan to define FY 1991 reporting baselines and to meet the FY 1993 Budget submission.

Budgeting and planning for the RI/FS process are addressed in the individual ADSs for site-specific waste area groupings.

\section{Root Cause:}

Ambiguous requirements or expectations 


\section{Planned Actions and Schedules:}

No further action is planned at this time.

Costs: None

References: Energy Systems-ERD AIP implementation cost estimates and draft ADS 
Finding No.: IWS/BMPF-4 Incomplete Evaluation of Continuous Releases

Finding

Description: OR, XSO, and MMES-ORNL have not completed a systematic evaluation of releases to the environment at $O R R$, in accordance with best management practice, to determine whether reporting of continuous releases under $\mathbf{4 0}$ CFR 302.8 is appropriate.

Code: $\quad$ Best Management Practice

Compliance

Protocol:

CERCLA Section 103(f) (2) requires the reporting of non-Federally permitted continuous releases.

Priority: $\quad$ Energy Systems Risk Weight 50

Tiger Team Action Plan Priority 3

Response: ORNL has been directed on two separate occasions to perform an assessment of non-Federally permitted releases to determine if any releases have occurred that were in excess of the Reportable Quantity.

\section{Root Cause:}

Inadequate policy implementation

Planned Actions and Schedules:

Item/Description

1. Issue request to ORNL to perform assessment.

2. Issue directive to ORNL to perform assessment.

3. Develop ORNL action plan for report.
Completion Date

Complete

Complete

Complete

Costs: $\quad$ Action Items 1-3 will be accomplished at no significant costs.

References: $\quad 40$ CFR 302 


\subsubsection{National Environmental Policy Act}

Finding No.: NEPA/CF-1 Inefficient DOE NEPA Implementation Procedures

Finding

Description: Differences among DOE program offices in document format and the number of required concurrences on NEPA-decisional documents are resulting in confusion, lack of NEPA focus, and project delays at ORNL, such that the NEPA process is not always fully implemented in accordance with Council on Environmental Quality regulations and DOE directives.

Code: $\quad$ Compliance

Compliance

Protocol:

Priority: $\quad$ Energy Systems Risk Weight 658

Tiger Team Action Plan Priority 2

Response: The concurrence chains in all programs are built into the DOE system and are therefore difficult to streamline. In ORO, for example, the concurrence chain for a Section D NEPA determination for a project funded by a program that has delegated authority to the manager requires ten signatures. In nondelegated programs, the program secretarial officer (PSO) has his/her own concurrence chain. fnrmst, and submittal procedures.

Once an adequate document has been received from ORNL, processed through the site office, and delivered to ORO, it takes an average of one month to obtain the manager's signature.

Program guidance to standardize NEPA document format and submittal procedures should be provided by EH-25.

Root Cause:

Inadequate policy

Planned Actions and Schedules:

On September 20,1990, ORO met with ORNL staff to discuss NEPA issues and concerns. Additionally, ORNL participated in a NEPA training session on September 25, 1990, and Energy Systems formed a NEPA committee, which met on November 8, 1990, to discuss ways to streamline the NEPA process. Program guidance is provided by DOE-HQ. 


\section{$\underline{\text { Item/Description }}$}

1. Request $\mathrm{HQ}$ guidance on streamlining the NEPA approval and format process.

2. Schedule an ORO/ORNL review of the NEPA process to discuss/agree on specific action items.

3. Train ORO/ORNL staff in NEPA document processing procedures.

4. Issue and implement $O R O$ and site office procedures.

5. Conduct surveillance of NEPA implementation to determine effectiveness of above actions.
Completion Date

Complete

$2 / 91$

$5 / 91$

$6 / 91$

$8 / 91$

Costs: $\quad$ Activities are part of an ongoing improvement effort and will be accomplished with existing resources.

References: None 
Finding No.: NEPA/CF-2 Project Implementation Without Completed NEPA Process

Finding

Description: ORNL removed an underground waste storage tank and modified Bldg. 9401-1 to create an energy technology development laboratory without completing the NEPA process as required by CEQ regulations (40 CFR 1505.1), SEN-15-90, and the DOE NEPA Guidelines (52 FR 47662).

Code: $\quad$ Compliance

Compliance

Protocol:

Priority: $\quad$ Energy Systems Risk Weight 405

Tiger Team Action Plan Priority 2

Response: $\quad$ Adequate NEPA review of all ORNL operations is the responsibility of each ORNL operating organization. The removal of the underground storage tank was completed to meet a scheduled compliance deadline, which could have resulted in fines to ORNL. The building modification was undertaken before the latest DOE NEPA compliance guidance was received.

\section{Root Causes:}

Inadequate policy implementation

Planned Actions and Schedules:

\section{$\underline{\text { Item/Description }}$}

1. Notify all ORNL operating organizations of the requirements for NEPA Complete review of projects.

2. Implement a bi-weekly review with DOE-ORO of projects with regulatory compliance deadlines to ensure timely NEPA review by DOE-ORO.

Costs: No significant costs are associated with the actions outlined.
Completion Date

Complete

Complete 
Finding No.: NEPA/BMPF-1 Lack of Environmental Impact Assessment for ORNL Continuing Operations

Finding

Description:

MMES-ORNL continues to operate without a comprehensive NEPA analysis and is, therefore, not operating in accordance with best management practices.

Code: $\quad$ Best Management Practice

Compliance

Protocol:

Secretary of Energy Notice 15 (SEN-15)

Priority: $\quad$ Energy Systems Risk Weight 10

Tiger Team Action Plan Priority 3

Response: A decision to prepare a sitewide NEPA document for ORNL would involve three or four DOE program offices. ORNL and the other major facilities (Y-12 and $\mathrm{K}-25$ ) on the ORR are operated under a single $\mathrm{M} \& \mathrm{O}$ contract administered by ORO that reports to NE, as specified in SEN-6D-91. Additionally, ER has programmatic and institutional oversight responsibility at ORNL (as per SEN-6D-91). EM has the landlord function for ORNL in FY 1991 and FY 1992, while landlord responsibilities for ORNL will return to ER in FY 1993. NE is the lead PSO with respect to the Field Office, with all three DOE program offices having responsibility for various programmatic activities and functions at the multiprogram laboratory. A portion of the ORNL physical facility also resides at the Y-12 Plant, managed by DP. In addition, several other DOE program offices that support ongoing research at ORNL would need to be informed of any impending action that might affect their mission.

ORO is in the process of considering the issue of a sitewide NEPA review for the ORR as a whole. Any sitewide NEPA reviews for ORNL, the other facilities on the ORR, or the ORR as a whole would need to be coordinated with each other and with the several other large NEPA reviews currently planned or under way, including the EM Programmatic EIS (PEIS) for Environmental Restoration and Waste Management, the DP PEIS for Reconfiguration of the Weapons Complex, and the ORR Environmental Restoration and Waste Management EIS. Because some of the main environmental problems at ORNL (and on the ORR in general) are related to legacy wastes and waste management, completion of the related ORR ER/WM EIS and the larger EM PEIS (before a determination for preparation of a new sitewide NEPA document for either ORNL or the ORR) would permit a new sitewide NEPA document to benefit from these other documents. ORO currently is examining all of these EIS relationships in an effort to make a recommendation to the program offices and to $\mathrm{EH}$ on the best course of action.

A complicating factor in determining the proper course of action on a sitewide NEPA review for ORNL (and for the ORR) is the absence of implementing guidance on the purpose, use, content, and scope of sitewide NEPA documents. 
As a result, the decisions these documents would support and the alternatives they would consider are unclear.

In summary, the feasibility, efficacy, and need for the preparation of a comprehensive NEPA analysis for ORNL are being considered by the affected HQ program offices and ORO. This has a high priority. Such a document will be difficult to plan and coordinate, especially in the absence of implementing guidance that is mutually agreed upon. The ORNL Corrective Action Plan alone cannot resolve all of the difficult issues and unanswered questions related to (1) sitewide reviews for the ORR and other ORR facilities and (2) the development of comprehensive guidance by $\mathrm{EH}$.

\section{Root Causes:}

Inadequate policy and inadequate policy implementation

Planned Actions and Schedules:

\section{$\underline{\text { Item/Description }}$}

PSOs agree on the need and strategy for sitewide NEPA review.

Costs: $\quad$ Costs and schedules for the required documents will be prepared when the scope is defined by DOE policy.

References: SEN-15
Completion Datc

$12 / 91$ 


\subsection{SITEWIDE SAFETY AND HEALTH FINDINGS, RESPONSES, AND PLANNED ACTIONS}




\subsection{SITEWIDE SAFETY AND HEALTH FINDINGS, RESPONSES, AND PLANNED ACTIONS}

\begin{tabular}{|c|c|c|}
\hline Finding discipline & $\begin{array}{c}\text { Finding number } \\
\text { prefix }\end{array}$ & $\begin{array}{l}\text { Section } \\
\text { number }\end{array}$ \\
\hline Organization and Administration & OA & 3.3.1 \\
\hline Quality Verification & QV & 3.3.2 \\
\hline Operations & OP & 3.3.3 \\
\hline Maintenance & MA & 3.3 .4 \\
\hline Training and Certification & $\mathrm{TC}$ & 3.3.5 \\
\hline Auxiliary Systems & $\mathrm{AX}$ & 3.3.6 \\
\hline Emergency Preparedness & $\mathrm{EP}$ & 3.3.7 \\
\hline Technical Support & TS & 3.3.8 \\
\hline Packaging and Transportation & PT & 3.3 .9 \\
\hline Nuclear Criticality Safety & $\mathrm{CS}$ & 3.3 .10 \\
\hline Security/Safety Interface & SS & 3.3 .11 \\
\hline Experimental Activities & EA & 3.3.12 \\
\hline Site/Facility Safety Review & FR & 3.3 .13 \\
\hline Radiological Protection & RP & 3.3.14 \\
\hline Personnel Protection & PP & 3.3.15 \\
\hline Worker Safety and Health Compliance & WS & 3.3 .16 \\
\hline Industrial Hygiene & IH & 3.3.17 \\
\hline Fire Protection & FP & 3.3 .18 \\
\hline Medical Services & MS & 3.3.19 \\
\hline
\end{tabular}




\subsubsection{Organization and Administration}

Finding No.: OA.1-1 Flowdown of ES\&H Policies and Requirements

Finding

Description: The flowdown of policies and requirements from top management to all levels of the organization to implement environmental, health, and safety initiatives is not consistently managed in an effective manner.

Code: $\quad$ Category III

Compliance

Protocol: None

Priority: $\quad$ Energy Systems Risk Weight 69

Tiger Team Action Plan Priority 2

Response: Energy Systems and ORNL recognize the need to effectively manage the flowdown of policies and requirements from top management to all levels of the organization so that the environmental, health, and safety initiatives are consistently implemented. Energy Systems and ORNL will formally assign to executive managers responsibility for functional regulatory and management areas (e.g., industrial hygiene, radiation protection, records management, etc.). These managers will be responsible for developing policies to address new requirements or to initiate corrections to existing shortfalls. Likewise, site and operating managers and personnel, who will report through the above managers, will be identified for the development of necessary site- or organization-specific procedures.

The implementation of the Automated Procedures Requirements Accountability System (APRAS) will provide the method to effectively manage the flowdown of requirements, orders, policies, and procedures. This computer system is accessible to appropriate Energy Systems personnel via mainframe computers. It is intended to identify specific executive managers' responsibilities for regulatory or management areas and to identify specific divisional or lower level managers' responsibilities for implementing procedures. This interactive tracking and commitment system will indicate (via electronic mail) that particular compliance or improvement activities must be performed by designated managers and permit a method to follow up to ensure the actions have been taken. In addition, the system will prompt and ensure that specific policies and procedures are reviewed periodically (e.g., every three years or as specified for a given procedure or policy). Management is currently directly involved with the assignment of responsibilities and actions that must be taken in conjunction with the input of information into APRAS. APRAS will generate follow-up requirements directly to managers or individuals assigned specific responsibilities to ensure that APRAS will work. 


\section{Root Causes:}

Lack of policy, unclear roles and responsibilities, and inadequate management approach

Planned Actions and Schedules:

\section{Item/Description}

1. Develop and implement a policy for the effective dissemination of ES\&H initiatives to all organizational levels. Establish the authority for development and promulgation of ES\&H policy and procedures. (See Finding MF-4.)

2. Place ORNL SPPs in APRAS. (See Finding OA.1-2.)

3. Develop schedule for additional ORNL-specific operating or divisional procedures (such as Industrial Hygiene Department or Laboratory Shift Supervisor's procedures) to be entered into APRAS.

(See Finding OA.1-2.) This activity is intended to ensure that appropriate lower-level operating procedures reflect higher-level directives or mandates.

Costs: $\quad$ Costs are included in Findings MF-4 and OA.1-2.
Completion Date

$5 / 91$

Complete

$12 / 91$

References: None 
Finding No.: OA.1-2 Dissemination of DOE Orders and Other Requirements

\section{Finding}

Description: The process by which DOE Orders and other compliance requirements are disseminated and implemented is not controlled throughout ORNL.

Code: $\quad$ Category III

Compliance

Protocol:

Priority: $\quad$ Energy Systems Risk Weight 58

Tiger Team Action Plan Priority 2

Response: Energy Systems and ORNL recognize the need to control compliance requirements, their dissemination, and their implementation. As a result of a corporate audit finding on lack of command media flowdown, ORNL and Information Resources Division implemented an approach to command media through the Tiger Team Document Control Center. And two new activities now under way are expected to address this need.

The first activity is a management system improvement program, undertaken by Energy Systems, which provide formal assignment of compliance areas to Energy Systems and each of the Energy Systems sites, including ORNL. Executive managers, along with lower-level managers reporting to them, will be assigned responsibility for specific compliance areas. Compliance areas will be divided into functional areas at the Energy Systems level and at the ORNL level. These functions are organized under auditing, business systems, computing telecommunications, and configuration management. (Functional areas include engineering, emergency preparedness, environmental protection, ethics, evaluation, health, human resources, information management, legal, maintenance, policy integration, conduct of operations, policy-standards-procedure sy.iem, public relations, quality assurance, quality control, research and development, safeguards and security, safety, technology transfer, and work for others.) Energy Systems will formally review requirements and promulgate necessary policies; ORNL will also develop necessary site procedures in a similar fashion if necessary. The ORNL functional representatives will be responsible for formal implementation of the regulations as interpreted at the Energy Systems level. The documentation and control of this implementation are addressed in the second activity.

The second activity is the implementation of the Automated Procedures Requirements Accountability System (APRAS). This system provides flow down of requirements, orders, policies, and procedures. It also provides identification of the individual responsible for implementation and will provide notification of responsibility as well as tracking of responsibility for implementation of DOE orders. ORNL is beginning input into this accountability system for ORNL Standard Practice Procedures, and will continue for lower level documents. 
(Reference OA.1-1, which addresses how APRAS will interact with management, which will ensure practical implementation.)

\section{Root Causes:}

Poorly defined roles and responsibilities and inadequate policy implementation

Planned Actions and Schedules:

\section{$\underline{\text { Item/Description }}$}

1. Crganize and introduce the Management System Improvement Program, which specifically defines how Energy Systems and ORNL will review DOE orders and other compliarice requirements and will disseminate and implement internal compliance policies and procedures.

2. Identify and assign responsibilities to specific managers for reviewing compliance areas and how Energy Systems and ORNL will respond to compliance needs. These manigers will identify what policies or procedures need to be written or revised. Initially managers will concentrate on high-priority areas such as environment, safety, or health. [In the future, lower-priority nonES\&H areas will be reviewed.]

3. Develop a schedule for medium- to low-priority nonES\&H functional arear of the Management System Improvement Program (i.e., areas not adiressed under the second action item). This would include identifying specific individuals to review compliance documents that might require creation or revision of Energy Systems or ORNL policies or procedures.

4. Finalize and test APRAS software.

5. Place ORNL SPPs into APRAS.

6. Develop and implement schedule for additional requirements, orders, policies, and procedures to be entered into APRAS for ORNL specific sections or operating areas. (Same action item for UA.1-1 \#3.)
Completion Date

Complete

9/91

$4 / 92$

Complete

Complete 


\section{Costs:}

Type of funds: Overhead

Source of funds: Overhead

\begin{tabular}{crrrrrrr} 
& \multicolumn{5}{c}{ Estimated costs per fiscal year (\$K) } \\
\cline { 2 - 6 } Action item & 1991 & 1992 & 1993 & 1994 & 1995 & Beyond & Total \\
\hline 1 & - & & & & & & \\
2 & - & & & & & & \\
3 & - & & & & & & \\
4 & 1 & $*$ & & & & & \\
5 & 75 & $*$ & & & & & \\
6 & & & & & & & \\
\end{tabular}

Status:

Funded 1

Requested

New 75 $\$ 76$

*Estimated annual ongoing cost: $\$ 8 \mathrm{~K}$.

References: None 
Finding No.: OA.1-3 ES\&H Issues for Construction Management

Finding

Description: The roles and responsibilities for environment, health, and safety to support MKFerguson construction management activities have not been clearly established by the Oak Ridge Operations Office.

Code: $\quad$ Category III

Compliance

Protocol:

Best management practices would indicate that Energy Systems and MK-Ferguson effectively enforce safety requirements unon construction contractors.

Priority: $\quad$ Energy Systems Risk Weight 539

Tiger Team Action Plan Priority 2

Risponse: Verbal instructions were provided to MK-Ferguson and Energy Systems on October 1, 1990, to continue health and safety roles and responsibilities as defined in document DOE/OR-891 until the new three party interface agreement is finalized. These verbal instructions were formalized in letters to MK-Ferguson and Energy Systems on November 17, 1990.

The new interface agreement that clearly defines Energy Systems and MKFerguson roles and responsibilities is now in final draft, and DOE plans to finalize it ty February 17, 1991.

The MK-Ferguson ES\&H Program Plan is being reviewed by DOE and the Contractor Officer's Representative (COR) will assure that Energy Systems interfaces are addressed.

\section{Root Cause:}

Poorly defined roles and responsibilities

Planned Actions and Schedules:

$\underline{\text { Item/Description }}$

1. Complete ES\&H portion of MK-Ferguson-Energy Systems interface document.

2. Complete review of MK-Ferguson ES\&H Program (assure MK-Ferguson-Energy Systems interfaces are addressed).

3. Begin implementation of interface agreements.
Completion Date

Complete

Complete

Complete 
Costs: $\quad$ Completion of activities will be accomplished within the planned MK-Ferguson indirect rate.

References: None 
Finding No.: OA.1-4 Acceptance of ES\&H Requirements at ORNL

Finding

Description: Environment, safety, and health requirements have not been fully integrated into or accepted by divisions acruss ORNL.

Code: $\quad$ Category III

Compliance

Protocol:

None

Priority: $\quad$ Energy Systems Risk Weight 488

Tiger Team Action Plan Priority 2

Response: The level of understanding and acceptance of ES\&H requirements varies from division to division within ORNL. Promulgation of a strong directive to implement and institutionalize ES\&H requirements, development of a comprehensive set of quantifiable ES\&H goals, and an effective set of policies and implementing procedures to communicate ES\&H requirements and expectations would ensure understanding and acceptance at all levels of the organization. See Findings MF-4, MF-7, TC.1-1, OA.1-2, OA.3-1, and OA.1-5.

\section{Root Causes:}

Inadequate policy, inadequate training, and poorly defined roles and responsibilities

Planned Actions and Schedules:

\section{Item/Description}

1. Develop and promulgate a policy with respect to compliance with ES\&H requirements and the authority of ES\&H staff personnel. (See Finding MF-4.)

2. Implement and document training of all personnel (including guests and consultants) on the laboratory policy with respect to compliance with ES\&H requirements, the accountability of Laboratory personnel and the ES\&H goals of the Laboratory and their division. (See Findings MF-7 and TC.1-1.)

3. Develop and implement a program to clearly define the standards and expectations for all ORNL divisions to assess themselves by and the process for performance improvement. (See Finding SA-1.)
Completion Date

$5 / 91$

$6 / 91$

$10 / 91$ 
Costs:

Type of funds: Overhead

Source of funds: Division Administration

\begin{tabular}{|c|c|c|c|c|c|c|c|}
\hline \multirow[b]{2}{*}{ Action item } & \multicolumn{6}{|c|}{ Estimated costs per fiscal year (\$K) } & \multirow[b]{2}{*}{ Total } \\
\hline & 1991 & 1992 & 1993 & 1994 & 1995 & Beyond & \\
\hline 1 & - & & & & & & \\
\hline 2 & $275^{a}$ & & & & & & \\
\hline 3 & - & & & & & & \\
\hline
\end{tabular}

Status:

Funded

Requested

New 275

${ }^{a}$ Requires division to conduct training for each individual or $\sim 5000$ person hours.

References: DOE-EH Radiation Protection TSA 
Finding No.: OA.1-5 Implementing ES\&H Activities

Finding

Description: The roles, responsibilities, and interfaces involving important environment, safety, and health activities are not always well established nor defined.

Code: $\quad$ Category III

Compliance

Protocol: None

Priority: $\quad$ Energy Systems Risk Weight 539

Tiger Team Action Plan Priority 2

Response: This organization and administration finding will be addressed by planned development and implementation of appropriate policies and procedures to establish position, organization, and committee charters which include the desired ES\&H accountabilities and authorities.

Root Causc:

Inadequate management approach

Planned Actions and Schedules:

Item/Description

Completion Date

1. Management System for Roles and Responsibilities (see Finding MF-4).

2. Review and revise as appropriate October 4, 1990, Memorandum of Understanding (MOU) delineating Environmental, Safety, and Health staff responsibilities of ORNL and the Y-12 Plant for ORNL organizations at $\mathrm{Y}-12$.

3. Implement major interface roles between MK-Ferguson and Energy Systems (see Finding OA.1-3).

Costs: $\quad$ Costs are included in Findings MF-4 and MF-7.

References: $\quad$ Memorandum of Understanding-ORNL and Y-12 Responsibilities for ORNL Organizations at Y-12, October 4, 1990

Summary of major interface roles between MK-Ferguson and Energy Systems, Draft 5a, November 27, 1990 
Finding No.: OA.1-6 Consistency of Safety Requirements

Finding

Description: Martin Marietta Energy Systems has not implemented uniform safety requirements at the X-10 Site and Y-12 Plant.

Code: $\quad$ Category III

Compliance Protocol:

Potential for deviations from orders, requirements, regulations, and good practices.

Priority: $\quad$ Energy Systems Risk Weight 65

Tigar Team Action Plan Priority 2

Response: The deficiencies noted in the observations which support this finding indicate lack of consistency between sites (X-10 and Y-12) relative to ES\&H expectations and requirements and lack of clarity regarding roles and responsibility for implementation of ES\&H policies and procedures for X-10 facilities and staff located at the Y-12 site. The Laboratory will address this finding primarily in two ways. The more general address will be implementation of the new Energy Systems-wide management system for roles and responsibilities. The more specific address will be full implementation of the recently established (October 4, 1990) "ORNL and Y-12 Responsibilities for ORNL Organization at Y-12," Memorandum of Understanding (MOU). Because of the newness of this MOU, it had been only recently and perhaps incompletely distributed at the time of the Tiger Team assessment. As a result, the affected staff did not have complete benefit of document familiarity and appropriate training. Implementation of the subject MOU will evolve as necessary to be consistent with roles and responsibilities specified in the new Energy Systems-wide management system for roles and responsibilities. At the appropriate juncture, formal review and revision of the MOU will be considered to ensure appropriate coverage of those specific items that continue to require special treatment in addition to that provided via the new management system for roles and responsibilities.

\section{Root Causes:}

Poorly defined roles and responsibilities and ambiguous requirements or expectations

Planned Actions and Schedules:

Item/Description

Completion Date

1. Management System for Roles and Responsibilities (see Findings OA.1-5, MF-1, and MF-4.

2. Special Attention to Previously Problematic Organizational Interfaces (see Finding OA.1-5). 
Costs: 1. Management System for Roles and Responsibilities (see Finding MF-4).

2. Special Attention to Previously Problematic Organizational Interfaces (see Finding OA.1-5).

References: Memorandum of Understanding-ORNL and Y-12 Responsibilities for ORNL Organizations at Y-12, October 4, 1990 
Finding No.: OA.3-1 ES\&H Goals for ORNL

Finding

Description: ORNL management has not established an integrated set of comprehensive quantifiable environmental, safety, and health goals for all ORNL facilities.

Code: $\quad$ Category III

Compliance

Protocol: None

Priority: $\quad$ Energy Systems Risk Weight 64

Tiger Team Action Plan Priority 2

Response: This deficiency existed primarily because environmental and ALARA concerns are not fully established and formalized as an integral part of the ORNL Safety Action Plans. Efforts under way to resolve this deficiency include establishing a policy that will establish annual ES\&H goals that will be approved by senior management and incorporation of these goals and objectives into personnel performance plans to better identify and reflect progress in crucial areas. Several other action plans support resolution of this deficiency. See Findings AX.5-1, MF-1, and RP.11-1.

\section{Root Causes:}

Inadequate policy, insufficient resources, inadequate management approach, and ambiguous requirements or expectations

Planned Actions and Schedules:

\section{Item/Description}

1. Develop a consistent, ORNL-wide definition of ES\&H goals that stresses specific, positive, attainable and measurable objectives (see Finding MF-1).

2. Establish CY 1991 goals in each ES\&H discipline and issue ORNL goal document to divisions.

3. Division directors establish ES\&H goals for the division.
Completion Date

$5 / 91$

$5 / 91$

$5 / 91$ 
Costs:

Type of funds: Overhead

Source of funds: Overhead

\begin{tabular}{|c|c|c|c|c|c|c|c|}
\hline \multirow[b]{2}{*}{ Action item } & \multicolumn{6}{|c|}{ Estimated costs per fiscal year (SK) } & \multirow[b]{2}{*}{ Total } \\
\hline & 1991 & 1992 & 1993 & 1994 & 1995 & Beyond & \\
\hline 1 & - & & & & & & \\
\hline 2 & 15 & & & & & & 15 \\
\hline 3 & - & & & & & & \\
\hline \multicolumn{8}{|l|}{ Status: } \\
\hline \multicolumn{8}{|l|}{ Funded } \\
\hline \multicolumn{8}{|l|}{ Requested } \\
\hline New & 15 & & & & & & $\$ 15$ \\
\hline
\end{tabular}

Estimated annual ongoing cost: $\$ 15 \mathrm{~K}$.

References: None 
Finding No.: OA.6-1 Requirements for Job Descriptions

Finding

Description: There are neither requirements for job descriptions to be reviewed on a regular basis nor for safety responsibilities to be included in job descriptions.

Code: $\quad$ Category III

Compliance

Protocol:

None

Priority: $\quad$ Energy Systems Risk Weight 58

Tiger Team Action Plan Priority 3

Response: Job descriptions at ORNL are generic and used primarily for bidding and compensation purposes. The Laboratory intends to meet the need for incorporating ES\&H responsibilities in the roles and responsibilities documents, position charters, and performance plans described in response to Finding MF-4.

Root Cause:

Inadequate management approach

Planned Actions and Schedules:

This finding is fully addressed by actions listed in Finding MF-4.

Costs: $\quad$ Costs are included in Finding MF-4.

References: None 
Finding No.: $\quad$ OA.6-2 Employee Appraisal Plan Requirements

Finding

Description:

The Performance Planning and Review system does not require that environment, health, and safety factors be part of the appraisal plan for all employees.

Code: $\quad$ Category III

Compliance

Protocol: None

Priority: $\quad$ Energy Systems Risk Weight 58

Tiger Team Action Plan Priority 3

Response: $\quad$ ES\&H performance is addressed in our present Performance Planning and Review System. The Laboratory intends to address this management concern more formally through the development and implementation of appropriate policies and procedures. The planning aspect will be added.

Root Cause:

Inadequate policy

Planned Actions and Schedules:

This finding is fully addressed by actions in Finding MF-4.

Costs: Costs are included in Finding MF-4.

References: None 
Finding No.: OA.7-1 Centralized System for Safety Document Control

Finding

Description: A centralized system for the rigorous control of important safety documents has not been implemented.

Code: $\quad$ Category III

Compliance

Protocol:

Violation of various directives including DOE orders could result from using outdated safety documents.

Priority: $\quad$ Energy Systems Risk Weight 5

Tiger Team Action Plan Priority 3

Response: Various Energy System and ORNL Quality Program documents define actions and responsibilities required to control documents. However, a comprehensive listing of those documents which require rigorous control does not exist.

Root Cause:

Lack of policy

Planned Actions and Schedules:

$\underline{\text { Item/Description }}$

1. Develop and Implement an ORNL SPP integrating existing Energy Systems and ORNL standards on document control. The SPP should specify
a. the tier of documents for which vigorous control is required,
b. document control procedures,
c. review responsibilities and procedures, and
d. document tracking and retrieval system.

2. Perform a Laboratory-wide audit to ensure all required $2 / 92$ 
Costs:

Type of funds: Overhead

Source of funds: Overhead

Estimated costs per fiscal year (SK)

\begin{tabular}{crrrrrrr}
\cline { 2 - 6 } Action item & 1991 & 1992 & 1993 & 1994 & 1995 & Beyond & Total \\
\hline 1 & 4 & & & & & & 4 \\
2 & & 4 & & & & & 4
\end{tabular}

Status:

Funded

4

Requested

4

New

References: $\quad$ Energy Systems Quality Program Standard ESS 6.1, "Document Control," September 30, 1987; ORNL Quality Assurance Procedure QA-L-6-100, "Document Control," January 31, 1987 
Finding No.: OA 72 Records Storage Factities

Finding

Description:

Reoonds storage facilities at ORNL do not moet the roquirements of DOE 5706.6B. ANSU/ASME NQA-1, and NFPA 232

Code: Calcgory III

Compliance

Protocol:

DOE Order S7(x)GS ANSI ASME NOA-1, and NFPA 232

Prionity: $\quad$ Energy Systems Risk Weight 130

Tiger Team Action Plan Priority 2

Response: Records storage has hing been a concern at ORNL Records storage problems were ciled in the DOE-ORO Multifunctional Appraisal, May 1990, the Martin Marielta Corporate Audit. July 1990, and in other audits prior to those.

It is true that ORNL does not have a single point records storage facility that meets the requirements of NFPA 232. ORNL QA procedure QA-L-17-100 on records storage provides for either single facility storage or dual facility storage as described in Section 4.4.4 of Supplement 175-1 of ANSI/ASME NQA-1. This section allows storage of QA records in dual facilities, and these facilities are not required to meet the requirements of NFPA 232 . While we are not in total compliance with sther single or dual storage points, many of our QA plan records list specific locatious of dual storage points for important records.

The inefficiency of dual storage points and the need for a single point NQA-1 storage area were recognized by the ORNL QA organization and the Energy Systems records personnel. Accordingly, a Performance Improvement Process (PIP) teanı was chartered in April 1989 to study ORNL's records system. One of the team's recommendations was establishment of a central records storage facility for Energy Systems that meets the requirements of NQA-1 and DOE.

As a result of the ORNL PIP team's recommendation, an Energy Systems PIP team was chartered to study the feasibility of a single Energy Systems records facility. Several options are being considered by the team, including a single facility, two facilities, or satellite centers with one and one-half hour fire rated cabinets in sprinkled, alarmed areas. The exact solution and schedule will depend on the team's recommendations, management acceptance of those recommendations, and obtainment of necessary funding. In the interim, ORNL will continue to utilize the dual file point option that is allowed under Supplement 17S-1 of ANSI/ASME NQA-1, Section 4.4.4. 


\section{Root Cause:}

Inadequate policy implementation; all areas of the Laboratory have not consistently implemented policy to utilize either dual record storage or approved single-point NQA-1 storage for designated records.

Planned Actions and Schedules:

\section{$\underline{\text { Item/Description }}$}

1. Complete study of records storage alternatives and make recommendations to Energy Systems Management.

2. Select a storage alternative and develop an implementation schedule to meet the storage requirements.

\section{Completion Date}

$11 / 91$

$1 / 92$

Costs:

Type of funds: Overhead

Source of funds: Overhead

Estimated costs per fiscal year (\$K)

\begin{tabular}{crrrrrrr}
\cline { 2 - 6 } Action itcm & 1991 & 1992 & 1993 & 1994 & 1995 & Beyond & Total \\
\hline 1 & 18 & & & & & 18 \\
2 & & 7 & & & & 7
\end{tabular}

Status:

Funded

Requested

18

New

References: $\quad$ ANSI/ASME NQA-1 
Finding No.: OA.8-1 Supervisory Training in Behavior Observation

\section{Finding}

Description: A formal sitcwide program to train all supervisors in recognition of drug or alcohol use and behavior observation has not been implemented.

Code: $\quad$ Category III

Compliance

Protocol:

None

Priority: $\quad$ Energy Systems Risk Weight 87

Tiger Team Action Plan Priority 2

Response: ORNL has partially addressed this problem in our training on Illegal Drug Use.

We will further address this concern through the completion of supervisory training. See also Finding ROA.8-1.

Root Cause:

Inadequate policy implementation

Planned Actions and Schedules:

\section{Item/Description}

1. Schedule and conduct a 1.5-hour substance abuse awc ness training module and a 1-hour aberrant behavior module for all supervisory and management personnel.
Completion Date

$5 / 91$ 


\section{Casts:}

Type of funds: Overhead

Source of funds: Overhead

Estimated costs per fiscal year $(\$ \mathrm{~K})$

\begin{tabular}{lrrrrrrr}
\cline { 2 - 5 } Action item & 1991 & 1992 & 1993 & 1994 & 1995 & Beyond & Total \\
\hline \multicolumn{1}{c}{1} & 14 & & & & 14 \\
$\begin{array}{l}\text { Status: } \\
\text { Funded } \\
\text { Requested } \\
\text { New }\end{array}$ & 14 & & & & \\
\end{tabular}

Estimated annual ongoing cost: $\$ 6 \mathrm{~K}$.

References: None 


\subsubsection{Quality Verification}

Finding No.: QV.1-1 Resources for QA Functions

Finding

Description: Resources applied to quality assurance functions are less than those required to mect support obligations, which is contrary to the requirements of DOE $5700.6 \mathrm{~B}$, ANSI/ASME NQA-1, and the ORNL Quality Assurance Manual.

Code: $\quad$ Category III

Compliance

Protocol:

Priority:

Response:

\section{DOE Order 5700.6B and ANSI/ASME NQA-1}

Energy Systems Risk Weight 60

Tiger Team Action Plan Priority 2

Similar concerns were expressed by the same auditor during the DOE Headquarters Quality Verification Inspection conducted during May 1990. There was some difference of professional opinion as to how closely the QA Specialists should be involved with the line organization and how much of the QA responsibilities should be discharged by the line organization. There is a general agreement that additional QA staffing is required. Three QA Specialists positions are currently being filled through offers which were temporarily being held up due to reductions in force related to budget uncertainties. An additional QA analyst was added during October from a list of individuals identified for budget-related terminations. Interviews are continuing, and it is anticipated that three additional QA Specialists to replace QA Coordinators in the R\&D divisions (see Finding QV.1-2) and two certified auditors will be added (see Findings QV.1-3 and QV.1-4) by the end of FY 1991. Staffing levels will be reevaluated pricr to submission of the FY 1992 budget, and additional QA Specialist funding will be requested as needed.

ORNL will continue to use existing Quality Assurance Coordinators and provide additional assistance from Quality Assurance Specialists and the ORNL Quality Assurance Program Manager.

Root Cause:

Inadequate policy implementation; adequate quality assurance resources have not been assigned to support full implementation of NQA-1 at ORNL. 
Planned Actions and Schedules:

Item/Description

1. Obtain funding agreement from $R \& D$ divisions for three $\mathrm{QA}$ specialists to replace currently funded $\mathrm{QA}$ coordinators.

2. Evaluate and document needed ORNL QA staffing levels prior to FY 1992 budget submittals.

3. Submit budget requests for any additional $Q A$ specialists identified in Item 2 above.

4. Contingent on approved funding, hire three additional QA specialists for the R\&D divisions per Item 1.

\section{Completion Date}

$07 / 91$

$06 / 91$

$08 / 91$

09/91

Costs:

Type of funds: Research Programmatic

Source of funds: ER

\begin{tabular}{cccccccc} 
& \multicolumn{6}{c}{ Estimated costs per fiscal year $(\$ K)$} & \\
\cline { 2 - 5 } Action item & 1991 & 1992 & 1993 & 1994 & 1995 & Beyond & Total \\
\hline 1 & - & & & & & & \\
2 & - & & & & & & \\
3 & - & & & & & & \\
4 & 85 & $*$ & & & &
\end{tabular}

Status:

Funded

Requested

New

85

${ }^{*}$ Estimated annual ongoing cost: $\$ 255 \mathrm{~K}$.

References: $\quad$ LOE Order 570G.6B, ANSI/ASME NQA-1, ORNL QA Manual 
Finding No.: QV.1-2 Implementation of the QA Program

Finding

Description: Contrary to requirements of DOE 5700.6B and ANSI/ASME NQA-1, many line organizations are not implementing the Quality Assurance program, which results in conflicts of interest situations for Quality Assurance Specialist and Quality Assurance Coordinator personnel.

Code: $\quad$ Category III

Compliance

Protocol:

Priority: $\quad$ Energy Systems Risk Weight 63

Tiger Team Action !lan Priority 2

Response: Potential conflict of interest concerns where line organization personnel function as QA coordinators have long been a concern for the ORNL Quality Department. This concern has been recognized by the DOE Headquarters Quality Verification Inspection, May 1990, as well as earlier audits.

The line organization should play the major role in development of QA plans which define QA actions as a part of their routine activitics (see MF-3). In order to implement an aggressive schedule for development of QA plans, the QA specialists and coordinators have often taken a lead role in their development, even to the point of becoming their authors. This does not mean, however, that the line was not actively involved in every step of the developmeni and review process.

It is ORNL's belief that to be truly effective, a QA specialist or coordinator must be knowledgeable enough of the activities being nerformed to help the line identify the proper controls for their activities. 0 increase the independence of QA verifications, an effort is being made in 1991 for the QA specialists and coordinators to rotate as lead auditors to provide independence for audits within their divisions.

ORNL has been in the process of replacing QA coordinators with full-time QA specialists for the last 5 years. The last step of this process calls for replacement of QA coordinators in the R\&D divisions that do not have a need for full-time specialists by assigning one specialist to two to four divisions. Current plans call for addition of three QA specialists to the ORNL QA staff by the end of FY 1991 to replace the QA coordinators presently being used by the R\&D divisions. This will eliminate any potential conflicts of interest and provide the $R \& D$ divisions with trained quality professionals. A plan must be developed to determine the anticipated grouping of divisions for each specialist and agreement on funding must be obtained from each division. When funding agreement is reached, interviews of qualified individuals will be conducted by the Quality Department 
and key personnel in the R\&D divisions. (Hiring of the three specialists was identified as an action item in Finding QV.1-1).

\section{Root Cause:}

Inadequate management approach

Planned Actions and Schedules:

Item/Description

1. Develop action plan for assignment rotation of $\mathrm{QA}$ Specialists among the R\&D divisions.

2. R\&D QA Specialist positions filled. (See Finding QV.1-1.)

Costs: $\quad$ Costs are reported under Finding QV.1-1.

References: DOE Ordier 5700.6B and ANSI/ASME NQA-1
Completion Date

07/91 
Finding No.: QV.1-3 Quality Department Audit Program

Finding

Description: The Quality Department Audit Program is not in compliance with DOE 5700.6B or the ORNL quality assurance manual in maintaining quality assurance program oversight.

Code: $\quad$ Category III

Compliance

Protocol:

DOE Order 5700.6B

Prionity: $\quad$ Energy Systems Risk Weight 55

Tiger Team Action Plan Priority 2

Response: The number of audits conducted by the ORNL quality audit manager and the division QA specialists and coordinators has been identified as a concern by the DOE Headquarters, Quality Verification Inspection, May 1990, DOE-ORO Multifunctional Appraisal, May 1990, Martin Marietta Corporate Audit, July 1990. and the DOE-ORO quality engineer. Due to the large number of externally conducted audits during 1990 and movement of the quality auditing function from Quality Department to the Office of Operational Readiness and Safety and back to Quality, the number of ORNL QA audits was below those scheduled for 1990.

A quality assurance audit program manager was named, effective December 1, 1990, moving the audit program back from the Office of Operational Readiness and Safety back to the Quality Department. This move places the audit program manager and the QA specialist in the same department, resulting in an organization that will have greater control over the total audit program. Additional budget funds for two full-time auditors will be requested to expand the number of ORNL quality audits performed. As an interim measure, personinel from the ORNL QA staff, division QA specialists, and QA personnel from Energy Systems and the other sites will be used as ORNL auditors to increase the number of audits conducted. All divisions and major programs are required to perform a minimum of one audit per year in addition to three surveillances. These audit and surveillance schedules will be tracked to ensure that they are performed as scheduled.

\section{Root Causes:}

Insufficient resources and inadequate management commitments 
Planned Actions and Schedules:

$\underline{\text { Item/Description }}$

1. Issue ORNL audit and division audit and surveillance schedules for CY 1991.

2. Request budget funding for two auditors.

3. Contingent on approved funding, hire two auditors when budget funding is approved.

4. Conduct a minimum of one ORNL QA audit per each two-month period in CY 1991 (four audits if no full-time auditors are added $t$ s staff).

5. Issue the first quarterly report during CY 1991 on the status of schedule implementation of ORNL and division audits and surveillances.
Completion Date

Complete

Complete

$06 / 91$

$12 / 91$

Complete

Costs:

Type of funds: Overhead

Source of funds: Overhead

Estimated costs per fiscal year (\$K)

\begin{tabular}{cccccccc}
\cline { 2 - 6 } Action item & 1991 & 1992 & 1993 & 1994 & 1995 & Beyond & Total \\
\hline 1 & - & & & & & & \\
2 & - & & & & & & \\
3 & 57 & $*$ & & & & & \\
4 & - & & & & & & \\
5 & - & & & & & \\
Status: & & & & & & \\
Funded & & & & & & \\
Requested & & & & & & \\
New & 57 & & & & & \\
\hline
\end{tabular}

${ }^{*}$ Estimated annual ongoing cost: $\$ 170 \mathrm{~K}$.

I.eferences: $\quad$ DOE Order 5700.6B 
Finding No.: QV.1-4 Frequency of Internal Audits and Surveillances

Finding

Description: Contrary to requirements of DOE 5700.6B, ANSI/ASME NQA-1, and ORNL Quality Assurance Manuals and Plans, internal audits and surveillances are infrequently performed.

Code: $\quad$ Category III

Compliance

Protocol:

DOE Order 5700.6B and ANSI/ASME NQA-1

Priority: $\quad$ Energy Systems Risk Weight 55

Tiger Team Action Plan Priority 2

Response: All ORNL divisions were required to perform a minimum of one audit and three surveillances in 1990 in an attempt to ensure adequate coverage across the Laboratory. While the number of audits and surveillances increased, there were a large number which were not performed due to slippage and attention to external audits such as the Martir Marietta Corporate Audit and the DOE Tiger Team Audit.

Each division has provided tentative audit and surveillance schedules to the ORNL Audit Manager for 1991. Two full time auditors have been requested to work with the division audits and surveillances in addition to supporting the ORNL Audit Manager's schedule. In addition, the Audit Manager will prepare quarterly status reports on implementation of the division audit and surveillance program. This report will be provided to the Deputy Laboratory Director for evaluation of schedule implementation and for appropriate action as needed.

\section{Root Cause:}

Insufficient resources and inadequate management commitment

Planned Actions and Schedules:

This finding is fully addressed by actions listed in Finding QV.1-3.

Casts: $\quad$ See the cost associated with Finding QV.1-3.

References: None 
Finding No.: QV.1-5 QA Plans and Manuals

Finding

Description: Most quality assurance plans and manuals fail to reflect all the requirements of DOE 5700.6B and ANSI/ASME NQA-1.

Code: $\quad$ Category III

Compliance Protocol:

DOE Order 5700.6B and ANSI/ASME NQA-1

Prionity: $\quad$ Energy Systems Risk Weight 56

Tiger Team Action Plan Priority 2

Response: Similar concerns were expressed in the DOE-ORO Multifunctional Appraisal, May 1990, the DOE Headquarters Quality Verification Inspection, May 1990, and the Martin Marietta Corporate Appraisal, July 1990. ORNL's QA Program is based on the premise of graded quality assurance as stated in the Foreword and Quality Assurance Program Basic Requirement of ANSI/ASME NQA-1 that "the Program shall provide control over activities affecting quality to an extent consistent with their importance." The degree of control exercised on bench-scale basic R\&D is not intended to be equivalent to that applied to ORNL's reactors, isotope production, and nuclear waste programs. It is difficult for auditors, many of whom come from the nuclear power industry, to grasp and accept the graded quality assurance concept, especially when viewed in its application to the broad spectrum at ORNL.

Ever-increasing custumer expectations and requirements have created a need to revise some of our QA plans and manuals to invoke more stringent controls. There are currently over 140 QA plans in effect at ORNL. Those plans that are associated with ongoing activities and current projects will be reviewed to assess their reflection of the requirements of DOE Order 5700.6B and ANSI/ASME NQA-1 and revised as necessary. Audits and surveillance will be used to verify implementation of Item 2 below.

Root Cause:

Inadequate policy and inadequate policy implementation 
Planned Actions and Schedules:

Item/Description

1. Review all ORNL QA plans and manuals associated with on-going activities and current projects for inclusion of the appropriate requirements of DOE Order 5700.6B and ANSI/ASME NQA-1 and establish a revision schedule for those prowedures identified as requiring revisions.

2. Issue an SPP that specifies the organizational accountability for training and implementation of QA requirements defined in $\mathrm{QA}$ plans and $\mathrm{QA}$ procedures.

\section{Completion Date}

$12 / 91$

$06 / 91$

Costs:

Type of funds: Overhead

Source of funds: Overhead

\begin{tabular}{crrrrrrr} 
& \multicolumn{6}{c}{ Estimated costs per fiscal year (SK) } & \\
\cline { 2 - 6 } Action item & 1991 & 1992 & 1993 & 1994 & 1995 & Beyond & Total \\
\hline 1 & 60 & 20 & & & & & 80 \\
2 & 15 & & & & & & 15
\end{tabular}

Status:

Funded 75

Requested

New

References: $\quad$ DOE Order 5700.6B and ANSI/ASME NQA-1 
Finding No.: QV.1-6 Effectiveness of Corrective Actions

Finding

Description: Corrective actions have been neither timely nor effective in eliminating the recurrence of problems as required by DOE 5700.6B. (See Concern EP.1-2 and PT. 3-2.)

Code: $\quad$ Category III

Compliance

Protocol:

DOE Order 5700.6B

Priority: $\quad$ Energy Systems Risk Weight 94

Tiger Team Action Plan Priority 2

Response: The lack of a single, effective corrective action tracking system for ORNL was recognized as a concern in the DOE Headquarters Quality Verification Inspection, May 1990, DOE-ORO Multifunctional Appraisal, May 1990, Martin Marietta Corporate Audit, July 1990, and other external audits prior to 1990 . This concern was also recognized in ORNL's self-assessment.

The line organization is responsible under DOE Orders 5700.6B and 5000.3A for determination of root causes of problems and for generating corrective actions to address them. When an investigation team is formed for UORs, QA specialists and coordinators serve as members. All QA specialists will be trained in root cause analysis to ensure the presence of at least one individual on each investigating team with root cause analysis experience.

Effective December 1, 1990, the quality auditing function was returned to the Quality Department. As a part of the reorganization, the Quality Auditing Manager will become a single point for tracking corrective actions, trending problems and concerns, and establishing a lessons-learned system. Once established, a lessons-learned system will allow the line organization to assess its areas for the existence of what could be generic concerns and implement corrective actions to prevent future occurrences.

\section{Root Cause:}

Inadequate management approach 
Planned Actions and Schedules:

Item/Description

Completion Date

1. Consolidate responsibility for corrective action tracking information (completed) (see Finding MF-6).

2. Establish a lessons-learned system (see Finding FR.6-1).

3. Train all QA specialists in root cause analysis.

Costs:

Type of funds: Overhead

Source of funds: Division Administration

Estimated costs per fiscal year (\$K)

\begin{tabular}{crrrrrrr}
\cline { 2 - 5 } Action item & 1991 & 1992 & 1993 & 1994 & 1995 & Beyond & Total \\
\hline 1 & - & & & & & & \\
2 & - & & & & & & \\
3 & & 12 & & & & & 12
\end{tabular}

Status:

Funded

Requested

New

12

$\$ 12$

References: $\quad$ DOE Order 5700.6B 
Finding No.: QV.2-1 Procurement Controls

Finding

Description

Procurement controls are not fully established for the control of purchased material and equipment as required by DOE 5700.6B and ANSI/ASME NQA-1.

Code: $\quad$ Category III

Compliance

Protocol:

DOE Order 5700.6B and ANSI/ASME NQA-1

Priority: $\quad$ Energy Systems Risk Weight 82

Tiger Team Action Plan Priority 2

Response: $\quad$ ESS.4.0, "Procurement Document Control," Section 2.0 Scope states: "The extent of application of this procedure will be dependent on the complexity of the Procurement activity." Section 7.2 of QA-L-4-100 states that requisitions which are marked for "special receiving inspection, Code 2, shall be reviewed..." This approach to determining the need for review based on complexity of the Procurement activity is in keeping with the scope of ESS.4.0, and no additional corrective action is required.

QA-L-5-100 requires QA Specialist review of procurement documents for noncommercial items.

Materials Department operational procedure R-1 Receipt and Inspection outlines a structured and auditable system for receipt, handling, and tracking of ORNL material received. The procedure was approved and implemented on 7-19-90. Materials Department operational procedure SR-2 Receipt and Inspection of Safety Related Items or Components outlines the same system with additional checks and balances, like chain-of-custody requirements for safety related items. The procedure was approved 4-25-90. Procedure implementation is awaiting software development, procurement policy adherence, and warehouse upgrade.

The Materials Department has requested users of stores inventory spare parts to classify each line item as a safety-related item or not. If an item is classified as safety related, the user shall complete the new ORNL stores stock addition form which will identify, if applicable, storage, packaging, shelf life, maintenance requirements, and system description, equipment name, manufacturer, model number, serial number, drawing number, location, and requirements of the vendor. Requests for stores stock addition will not be accepted unless the request is on the new form and complete. The new stores stock addition form requires the requester, if special inspection is required, to identify an inspection plan for each line item.

During the past year, the major emphasis within the Materials Department was focused on the development of a new system for controlling procured materials. This new system would bring ORNL into conformance with the requirements of 
the applicable elements of NQA-1 (elements 4 and 7) and procedures and building quality assurance requirements in those procedures. Due to recent administrative problems, progress on the completion of this system has been halted. The revision and implementation of Department QA Plans are needed to illustrate present quality assurance techniques within the Materials Department. The QA plans will include requirements for a surveillance program.

Due to the limitations of the Materials Management System (MMS), data on vendor performance could not be retrieved from that computer system to assess vendor performance. Using a graded approach, Nonconformance Reports (NCRs) were written for discrepant materials received for special inspection (code 2). Data, including vendor name, item description, and discrepancy, were put into the Energy System Quality Information System (ESQIS) and tracked in an effort to identify trends.

Recently, a software package has been identified which has the capability to interact with the MMS and extract the necessary data to perform trend anaiysis of all discrepant material received at ORNL. This information can also be ussd to develop and maintain a quality costs system for procured material for the Laboratory. These efforts will constitute effective measures to assess the adequacy of ORNL's procurement activities.

\section{Root Causes:}

Poorly defined roles and responsibilities

\section{Planned Actions and Schedules:}

\section{$\underline{\text { Item/Description }}$}

Completion Date

1. Develop software for tracking safety materials data and $09 / 92$ custodianship.

2. Convert Building 7013 to level "A" and "B" storage (see Finding QV.5-1, Item 3).

3. Enclose Building 7060 metal storage shed (see Finding QV.5-1, Item 4).
A. Initiate engineering design
B. Initiate construction

4. Construct level "A" inspection area in Building 7001 (see Finding QV.5-1, Item 5).

5. Review and classify existing stores inventory (see Finding QV.5-1, Item 1). 
6. Revise and issue QA plans to reflect current control mechanism.

7. Upgrade software to implement trend analysis for discrepant material.

8. Develop quality cost system and procedurize.

9. See Finding QV.1-5, Item 2, which addresses QA training for line organizations.

Casts:

Type of funds: Overhead

Source of funds: Overhead

Estimated costs per fiscal year (\$K)

\begin{tabular}{|c|c|c|c|c|c|c|c|}
\hline Action item & 1991 & 1992 & 1993 & 1994 & 1995 & Beyond & Total \\
\hline 1 & 1 & & & & & & 1 \\
\hline 2 & - & & & & & & \\
\hline 3 & - & & & & & & \\
\hline 4 & - & & & & & & \\
\hline 5 & - & & & & & & \\
\hline 6 & 5 & & & & & & 5 \\
\hline 7 & 1 & & & & & & 1 \\
\hline 8 & & 2 & & & & & 2 \\
\hline 9 & - & & & & & & \\
\hline
\end{tabular}

Status:

Funded

Requested

New

2

References: NQA-1, NQA-2, NRC, ORNL QA Manual 
Finding No.: QV.3-1 Implementation of Receiving and Preinstallation Inspections

\section{Finding}

Description: In many instances, provisions for receiving and pre-installation inspections are not implemented as required by ANSI/ASME NQA-1.

Code: $\quad$ Category III

Compliance Protocol:

Priority:

Response:

\section{ANSI/ASME NQA-1}

Energy Systems Risk Weight 59

Tiger Team Action Plan Priority 2

The current system in place to control the performance of receiving inspection is limited to those activities for which the inspection organization is notified. An automatic invocation of receiving inspection has not been formalized although the personnel assigned to stores are performing some inspections without formal direction or methods of control.

Presently the Receiving Inspector, per procedure QA-L-7-100, places a hold tag on goods received requiring inspection. At that point, an inspection plan has not been transmitted to the inspector. Per procedure the inspector contacts his/her supervisor which in turn contacts the customer requesting an inspection plan. The inspection shall not be performed without an inspection plan.

The responsibility for control must be assigned in a top-level document such as an ORNL SPP, the ORNL Accounting Manual, a QA Plan for procurement of commodities, or ORNL QA Procedure QA-L-7-100. The act of issuing the associated procurement documents by F\&M Division will be modified to include notification to the inspection department of the required inspection at receipt. The inspection department, in turn, will generate instruction sheets, including check lists, to perform the inspection and authorize acceptance, rejection, or hold.

For those required items, special inspections are performed under Quality Department IRs. Special high risk areas such as reactors have their own special inspection processes defined and implemented. As part of addressing this issue an Energy Systems audit on receiving inspection has been completed. Surveillances are scheduled to review progress. All of these efforts are designed to improve the system.

\section{Root Causes:}

Inadequate policy implementation and poorly defined roles and responsibilities 
Planned Actions and Schedules:

$\underline{\text { Item/Description }}$

1. Identify controlling documents/manuals/procedures that need revisions.

2. Revise and issue controlling documents (manuals, procedures, etc.) identified in Item 1 above.
Completion Date

$05 / 91$

$12 / 91$

Costs:

Type of funds: Overhead

Source of fur.ds: Overhead

Estimated costs per fiscal year (\$K)

\begin{tabular}{ccccccccc}
\cline { 2 - 6 } Action item & 1991 & 1992 & 1993 & 1994 & 1995 & Beyond & Total \\
\hline 1 & - & & & & & & & \\
2 & & & 7 & & & & & \\
\end{tabular}

Status:

Funded

Requested

New

7

References: QE\&I Procedures Schedules

F\&M Procedures Manual MDR-1, MD-sr-2

Acquisition System Improvement Committee-see A. Kurilik 
Finding No.: QV.4-1 Calibration Facilities

Fisuding

Description: Some calibration facilities are substandard compared to industry metrology standards.

Code: $\quad$ Category III

Compliance

Protocol: Best management practice

Priority: $\quad$ Energy Systems Risk Weight 5

Tiger Team Action Plan Priority 2

Response: During the self-assessment process, I\&C Division reaffirmed the belief held by the Metrology Standards Laboratory manager and staff that the facility was substandard due to environmental control problems. The mission of the Metrology Research and Development Laboratory (MRDL) has been to further measurement science by developing new measurement techniques and to provide traceability of calibration services to National Institute of Standards and Technology (NIST). Present requirements have shifted toward a greater emphasis on providing calibration services to ORNL facilities.

Corrective action, through relocation of some activities, purchase of some additional air-conditioning equipment, and removal of ceramic fiber insulation preceded the arrival of the Tiger Team. The crowded and cramped quarters in the Personal Computer and Process Instrument Maintenance Shop will be relieved in 18 months with the completion of FY 90-91-92 Line Item ORNL 90-R-112, "Measurement and Control Support Facility, ORNL," will add 20,000 $\mathrm{ft}^{2}$ of office, laboratory, and/or shop space to rel:eve present crowded conditions in Building 3500 .

\section{Root Causes:}

Lack of resources and inadequate communications of policy or standards

Planned Actions and Schedules:

\section{Item/Description}

1. Purchase and install equipment needed to make improvements in temperature regulation.

2. Develop a unified quality assurance plan for both field calibration and metrology standards laboratory.
Completion Date

$07 / 91$ 


\section{Costs:}

Type of funds: Capital

Source of funds: GPE

Estimated costs per fiscal year (\$K)

\begin{tabular}{rrrrrrrrr}
\cline { 2 - 5 } Action item & 1991 & 1992 & & $19 / 93$ & 1994 & 1995 & Beyond & Total \\
\hline 1 & 12 & & & & & & &
\end{tabular}

Funded

Requested

New

Type of funds: Overhead

Source of funds: Division Administration

\begin{tabular}{lrlllllll} 
& \multicolumn{5}{c}{ Estimated costs per fiscal year (\$K) } \\
\cline { 2 - 6 } Action item & 1991 & 1992 & 1993 & 1994 & 1995 & Beyond & Total \\
\hline 2 & 5 & & & & & & & 5
\end{tabular}

Funded

Requested

New

5

References: $\quad$ I\&C Division QA Manual Procedure QA-IC-OP8, "Instrumentation and Controls Division Metrology Laboratcry Services" (Draft)

"Long-Range Plan for the Metrology Research and Deyelopment Laboratory," R. L. Anderson and R. K. Adams, 1988

"Recommended Practice Laboratory De: ign," RP-7, July 10, 1986, National Conference of Standards Laboratories Memo, "Major Safety/Facility Deficiencies in Standards Lab," J. O. Hylton,
August 30, 1990 
Finding No.: QV.4-2 Calibrations Program

Finding

Description: The calibrations program does not meet all requirements of DOE 5700.6B, ANSI/ASME NQA-1, and the ORNL Quality Assurance Manual. (See Concern RP.8-1.)

Code: $\quad$ Category III

Compliance

Protocol:

DOE Order 5700.6B and ANSI/ASME NQA-1

Priority: $\quad$ Energy Systems Risk Weight 56

Tiger Team Action Plan Priority 2

Response: Calibration concerns have been expressed as issues in previous audits, irıcluding the DOE Headquarters Quality Verification Inspection, May 1990, the DOE-ORO Multifunctional Appraisal, May 1990, and the Martin Marietta Corporate Audit, July 1990. Ensuring that instruments requiring calibration are identified and properly calibrated was also a concern in ORNL's self-assessment.

In order to clarify the role and responsibility of line management regarding calibration, ORNL QA Procedure QA-L-12-100 was revised in October 1990 to address identification of instrumentation requiring calibration. Section 6.1.2 was added to require project/program mangers to identify and categorize measuring and test equipment. Section 6.1 .8 of this procedure also requires the managers to place their equipment in appropriate calibration programs.

Health Physics Procedure RP-1.6 Section VI requires that health physics instrumentation being specified and procured by the line organizations be reviewed and approved by the Health Physics Instrument Committee. This procedure was issued in February 1990.

Line management now has clear and concise guidance defining their roles and responsibilities in ORNL's calibration program. Procedures QA-L-12-100 and RP-1.6 have been approved by ORNL management and must be followed by the line organizatior.

To ensure that the new requirements of QA-L-12-100 and RP-1.6 are being implemented, surveillances will be conducted by the QA Specialists and Coordinators during 1991 to assess the degree of compliance.

\section{Root Causes:}

Inadequate policy implementation and poorly defined roles and responsibilities 
Planned Actions and Schedules:

Item/Description

1. A letter will be issued to all QA Specialists and Coordinators instructing them to plan a surveill ance during 1991 on the requirements of QA-L-12-100 and RP-1.6.

2. Verify that division/program 1991 surveillance schedules include at least one surveillance on QA-L-12-100 and RP-1.6 requirements.

3. Verify and document that all scheduled surveillances on QA-L-12-100 and RP-1.6 were conducted and analyze the results for the presence of trends.
Completion Date

Complete

Complete

$01 / 92$

\section{Costs:}

Type of funds: Overhead

Source of funds: Overhead

Estimated costs per fisual year (\$K)

\begin{tabular}{cccccccc}
\cline { 2 - 5 } Action item & 1991 & 1992 & 1993 & 1994 & 1995 & Beyond & Total \\
\hline 1 & - & & & & & & \\
2 & - & & & & & & \\
3 & 3 & 3 & & & & & 6
\end{tabular}

Status:

Funded

3

Requested

New

References: $\quad$ ANSI/ASME NQA-1, QA-L-12-100, Rev. 1, and Finding RP-1.6 
Finding No.: QV.5-1 Control of Safety-Related Materials

Finding

Description: Control of safety-related hardware and materials is not accomplished in accordance with requirements of DOE 5700.6B and ANSI/ASME NQA-1. (Sce Concern PT.12-1 and RP.8-1.)

Code: $\quad$ Category III

Compliance

Protocol:

DOE Order 5700.6B and ANSI/ASME NQA-1

Priority: $\quad$ Energy Systems Risk Weight 61

Tiger Team Action Plan Priority 2

Response: Users are currently reviewing spare parts set up in Stores to determine the proper classification (Safety, Insurance, or Critical item). If reviewers want the line item to remain in inventory, they shall evaluate the package and product credibility of each warehouscd item. They also shall complete the new ORNL stores stock addition form which will identify applicable storage, packaging, shelf life, maintenance requirements, system description, equipment name, manufacturer, model number, serial number, drawing number, location, and requirements of the vendor.

Materials Department internal operating procedure MD-SR-2 has been approved for receipt and inspection of safety-related items or components. This procedure identifies handling and document control responsibilities. A chain-of-custody form for safety-related items or components has been designed where each operational employee who has possession must obtain the new custodian's signature before responsibility is relinquished. Materials Department internal operating procedure MD-SR-3 has been approved for warehousing and issuing safety-related items or components. This procedure identifies stores, receiving, withdrawing, inspection, maintenance, and document control responsibilities.

The mechanism for an on-line electronic system has been identified to track part, serial and/or unique identifier number, and purchase order number; storage, packaging, shelf life, and maintenance requirements, and to alert management when maintenance or packaging or shelf life evaluation is to be performed. Software development is included in the Materials Department FY91 budget request.

An engineering study is in its final stage converting Bldg. 7013 to satisfy levels " $A$ " and "B" storage requirements for safety related materials. Engineering is presently developing plans to enclose 7060 metal storage shed to prevent pedigreed metal from being exposed to the elements. Construction cost to convert 7013 and Engineering cost of 7060 design are in the Materials Department FY91 Budget request. Design is complete for a 7001 inspection area which meets storage level "A" requirements under NQA-1/NQA-2 which states that materials shall be segregated and secured in a level "A" facility while in the inspection process. 
Construction cost of level " $A$ " inspection area was in the Materials Department FY91 Budget request.

Root Causes:

Poorly defined roles and responsibilities

Planned Actions and Schedules:

Item/Description

1. Review and classify existing stores inventory.

2. Develop software for tracking safety materials.

3. Convert Building 7013 to level " $A$ " and "B" storage.

4. Enclose Buildirig 7060 metal storage shed.

A. Initiate engineering design.

B. Initiate construction.

5. Construct Level "A" inspection area. See PT-12-1 Item 8.
$09 / 92$

$09 / 93$

Completion Date

$12 / 91$

09/92

09/92

$09 / 92$

Costs:

Type of funds: Overhead

Source of funds: Overhead

\begin{tabular}{|c|c|c|c|c|c|c|c|}
\hline \multirow[b]{2}{*}{ Action item } & \multicolumn{6}{|c|}{ Estimated costs per fiscal year $(\$ K)$} & \multirow[b]{2}{*}{ Total } \\
\hline & 1991 & 1992 & 1993 & 1994 & 1995 & Beyond & \\
\hline 1 & 30 & 12 & & & & & 42 \\
\hline 2 & & 30 & & & & & 30 \\
\hline 3 & & 188 & & & & & 188 \\
\hline 4 & & 75 & & & & & 75 \\
\hline 5 & & & & & & & \\
\hline
\end{tabular}

Status:

Funded

30

Requested

New

References: NQA-1, NQA-2, NRC, 10 CFR Part 50 
Finding No.: QV.6-1 Quality-Related Inspections

Finding

Description: Quality-related inspections are often not performed and are independently verified infrequently at ORNL, which is contrary to the requirements of DOE $5700.6 \mathrm{~B}$ and ANSI/ASME NQA-1. (See Concern MA.5-1 and TS.3-1.)

Code: $\quad$ Category III

Compliance Protocol:

Priority:

Response:
DOE Order 5700.6B and ANS//ASME NQA-1

Energy Systems Risk Weight 62

Tiger Team Action Plan Priority 2

This concern was previously noted in the DOE Headquarters Quality Verification Inspection, May 1990. Inspection concerns are currently broken down into two areas: those items that are fabricated in the ORNL shops and procured items that are inspected by the requisitioner.

Items that are fabricated in the Plant \& Equipment shops must have an associated work request before the item is entered into the shop's backlogs. There is a required entry on the work request forms for indicating whether special QA actions are required or not along with a request for specifying inspection, test, cleanliness, or other requirements.

In addition, Plant \& Equipment Procedure F-1.6 requires that if a QA stanp indicating inspection and other requirements is not on the prints, drawings, or sketch-s furnished by the requestor the supervisor in charge will stamp the instructions provided with a Fabrication Department Work Control Stamp and sign. The customer is then required to fill in needed information on the Work Control Stamp and sign. If there is no personal contact with the customer, the supervisor in charge must fill out the Work Control Stamp. The supervisor in charge is also responsible for signing completed work requests as an indication that all steps have been completed. Surveillance will be conducted by the Plant \& Equipment QA specialist to verify adherence to these requirements by Plant and Equipment personnel.

Procured items requiring special receiving inspection are normally inspected by a qualified inspection group and documented with an inspection report. There are, however, items that are inspected by the requester, and these are not always documented. ORNL's QA procedures on procured items will be reviewed, and an acceptable method of documenting inspections vill be added. Surveillances will be conducted on random special inspection requisitions to verify implementation of the requirements. 


\section{Root Cause:}

Poorly defined roles and responsibilities

Planned Actions and Schedules:

Item/Description

1. The Plant \& Equipment QA specialist will conduct a surveillance of shop-fabricated items to verify implementation of P\&E Procedure F-1.6 by the P\&E organization.

2. ORNL QA Procedure QA-L-7-100 will be reviewed and revised to incorporate requirements defining roles and responsibilities for documentation of requisitioner inspections (see Finding QV.3-1, Item 2).

3. ORNL-level surveillances will be performed on random requisitions requiring requisitioner inspection to verify implementation of the new requirements for inspection documentation by the line organization.
Completion Date

$06 / 91$

$12 / 91$

$03 / 92$

Costs:

Type of funds: Overhead

Source of funds: Overhead

\begin{tabular}{|c|c|c|c|c|c|c|c|}
\hline \multirow[b]{2}{*}{ Action item } & \multicolumn{6}{|c|}{ Estimated costs per fiscal year (\$K) } & \multirow[b]{2}{*}{ Total } \\
\hline & 1991 & 1992 & 1993 & 1994 & 1995 & Beyond & \\
\hline 1 & 4 & & & & & & 4 \\
\hline 2 & 7 & & & & & & 7 \\
\hline 3 & & 2 & & & & & 2 \\
\hline
\end{tabular}

Status:

Funded

11

Requested

New

References: $\quad$ ANSI/ASME NQA-1 
Finding No.: QV.7-1 Special Process Training

Finding

Description: Some special process training does not comply with ANSIASME NQA-1. (See Concern TC.1-2)

Code: $\quad$ Category III

Compliance

Protocol:

ANSI/ASME NQA-1

Priority: $\quad$ Energy Systems Risk Weight 58

Tiger Team Action Plan Priority 2

Response: This concern was previously identified in the DOE Headquarters Quality Verification Inspection, May 1990. An extensive plan was developed in March 1990 for training of all Quality Engineering and Inspection Inspectors. The plan included an aggressive schedule for reviewing and revising 45 inspection procedures. A training schedule for each group of inspectors was also developed, identifying participants, instructors, and completion dates. In the interim period, only those inspectors who have been judged as qualified by their supervision will be allowed to perform inspections until such training can be conducted and documented.

Root Cause:

Inadequate training

Planned Actions and Schectules:

\section{Item/Description}

1. Complete revision of all QE\&I inspection procedures.

2. Revise ACP 14, ACP 15, and NDE 11 to require review of all incorrectly answered questions with the examinee(s).

3. Conduct training to revised procedures and document with examinations (see Items 1 and 2 above).

4. Conduct a surveillance of examination records.

\section{Completion Date}

$06 / 91$

$06 / 91$

09/91

$11 / 91$ 
Costs:

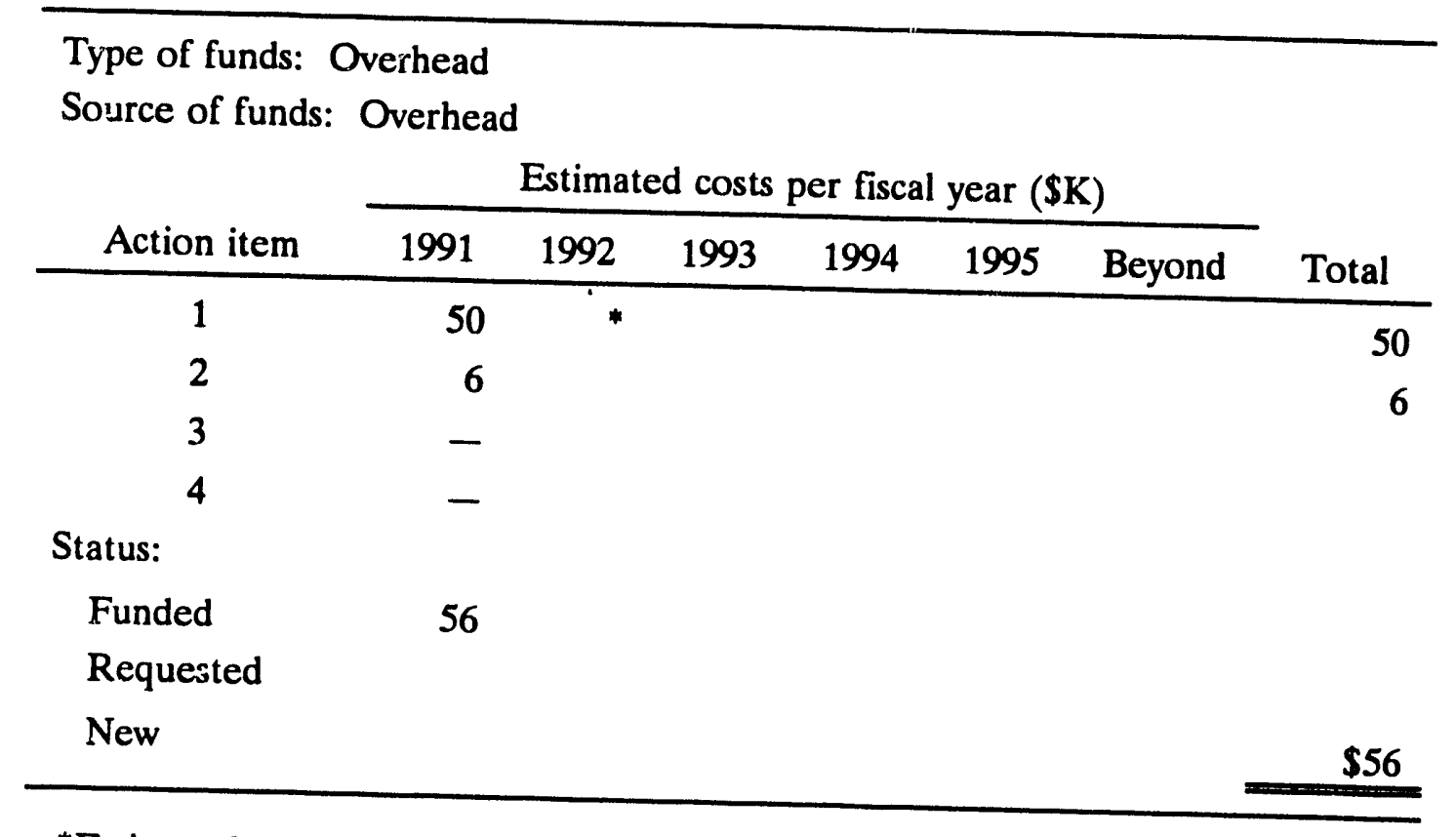

*Estimated annual ongoing costs: $\$ 80 \mathrm{~K}$

References: ANSI/ASME NQA-1 


\subsubsection{Operations}

Finding No.: OP.3-1 ORNL Operating Procedures

Finding

Description- No policy exists at ORNL for standardized format, content, review scope and frequency, and approval of operating procedures, as required by DOE 5480.19.

Code: $\quad$ Category III

Compliance

Protocol:

DOE Order 5480.19

Priority: $\quad$ Energy Systems Risk Weight 55

Tiger Team Action Plan Priority 2

Response: Energy Systems and ORNL agree that no standardized guidance is provided concerning standardized format and content of operating procedures. Energy Systems is developing a writers' guide for Energy Systems and all Energy Systems sites; ORNL is participating in this multisite effort. The guide will address format and content. Administrative procedures will address procedure review and approval.

Root Cause:

Inadequate policy implementation

Planned Actions and Schedules:

\section{Item/Description}

1. Issue draft Energy Systems writers' guide.

2. Issue final version of Energy Systems writers' guide.

3. Develop training module concerning the writing of SOPs and initiate training.

4. Issuc revised SPP to provide guidance and oversight where consistency should be required across ORNL.
Completion Date

$6 / 91$

$12 / 91$

$2 / 92$

$2 / 92$ 


\section{Costs:}

Type of funds: Overhead

Source of funds: Energy Systems

\begin{tabular}{|c|c|c|c|c|c|c|c|}
\hline \multirow[b]{2}{*}{ Action item } & \multicolumn{6}{|c|}{ Estimated costs per fiscal year (\$K) } & \multirow[b]{2}{*}{ Total } \\
\hline & 1991 & 1992 & 1993 & 1994 & 1995 & Beyond & \\
\hline 1 & 11 & & & & & & 11 \\
\hline 2 & & 20 & * & & & & 20 \\
\hline 3 & & 12 & & & & & 12 \\
\hline 4 & & 7 & & & & & 7 \\
\hline
\end{tabular}

Status:

Funded

Requested

New

11

39

*Estimated annual ongoing cost: \$5K starting in FY 1992.

References: None 
Finding No.: OP.4-1 Facility Status Displays

Finding

Description: Facility status displays are not maintained to serve the intended purpose.

Code: $\quad$ Category III

Compliance

Protocol:

DOE Order 5480.19, Chapter II, requires that facility status be understood by operations personnel in order to control and coordinate activities.

DOE Order 5480.19, Chapter VIII, requires supervisory personnel to normally direct operations and maintain system configuration. An accurate system status display is mandatory to fulfill this requirement.

DOE Order 5480.19, Chapter XII, requires supervisory personnel to review the facility status during shift turnover. Maintenance of an accurate status board is necessary to comply with this requirement.

Priority: $\quad$ Energy Systems Risk Weight 56

Tiger Team Action Plan Priority 3

Response: Implementation of the Conduct of Operations philosophy at ORNL will provide a formal, disciplined manner of conducting work, which will enhance safe and efficient management of activities and will foster an overall attitude to achieve excellence. Conduct of Operations will be implemented first through training personnel on the objectives of the program, then through an evaluation of the applicability of the guidelines for each facility operation, followed by the development of an implementation plan. Compensatory actions will be accomplished through a risk-based prioritization of cost/benefit studies of the needed actions. Funding for these programs will depend on the results of the division level applicability evaluation and the priority assigned to the required corrective action.

Maintenance of facility status displays is addressed by this action plan. The fundamental corrective action for this problem is the implementation of Conduct of Operations. The DOE implementation order requires each facility to implement the guidelines of Conduct of Operations in a graded fashion, including those pertaining to facility status boards. The Conduct of Operations implementation plan requires that compensatory actions from the implementation of the guidelines be accomplished in a risk-based manner. This ensures that resources are utilized to address the most serious concern in a prioritized fashion. This plan will ensure that the need for all status boards is identified and requirements for maintaining them are promulgated in an appropriate precedence.

The use and maintenance of status boards is a requirement of the implementation of Conduct of Operations. The need to implement Conduct of Operations was in the ORNL Self-Assessment. 


\section{Root Causes:}

Inadequate policy and inadequate policy implementation

Planned Actions and Schedules:

$\underline{\text { Item/Description }}$

1. Commence ORNL Conduct of Operations training.

2. Develop divisional Conduct of Operations implementation plans.

3. Initiate implementation of Conduct of Operations Compensatory Action plans.

4. Determine facilities requiring status boards based on Conduct of Operations Compensatory Actions.

5. Issue written guidance to operating divisions concerning maintenance of status boards.
Completion Date

Complete

Complete

Complete

$10 / 91$

Costs: $\quad$ Cost of Developing Divisional Action Plan: Based on experience with the pilot plants, approximately 100 hours of senior divisional management time will be required to assess the guidelines and develop the action plan; approximately $\$ 756,000$ will be required.

All costs will be paid by current divisional funding.

Compensatory actions will be accomplished through a risk-based prioritization of cost/benefit studies of the needed actions. Funding for these programs will depend on the divisional findings and the priority. 
Type of funds: Overhead

Source of funds: Division Administration

\begin{tabular}{|c|c|c|c|c|c|c|c|}
\hline \multirow[b]{2}{*}{ Action item } & \multicolumn{6}{|c|}{ Estimated costs per fiscal year (\$K) } & \multirow[b]{2}{*}{ Total } \\
\hline & 1991 & 1992 & 1993 & 1994 & 1995 & Beyond & \\
\hline 1 & - & & & & & & \\
\hline 2 & 756 & & & & & & 756 \\
\hline 3 & - & & & & & & \\
\hline 4 & - & & & & & & \\
\hline 5 & - & & & & & & \\
\hline \multicolumn{8}{|l|}{ Status: } \\
\hline Funded & 756 & & & & & & \\
\hline \multicolumn{8}{|l|}{ Requested } \\
\hline New & & & & & & & $\$ 756$ \\
\hline
\end{tabular}

Estimated annual ongoing costs: dependent on divisional action plans and cost of facility upgrades.

References: $\quad$ DOE Order 5480.19, Conduct of Operations 
Finding No.: OP.4-2 ORNL Lock.cut/Tagout Systems

Finding

Description: The lockout/tagout systems employed at ORNL do not conform to all aspects of the graded performance requirements of DOE 5480.19. (Sce MA.2-1)

Code: $\quad$ Category III

Compliance

Protocol:

DOE Order 5480.19

Priority: $\quad$ Energy Systems Risk Weight 917

Tiger Team Action Plan Priority 2

Response: The need for an improved lockout/tagout procedure was identified in the ORNL self-assessment document. Energy Systems has recognized the need for central guidance to promote uniform compliance with lockout/tagout requirements. A draft procedure consistent with $29 \mathrm{CRF} 1910$ and DOE Order 5480.19 has been issued for comment by Energy Systems. After comments are resolved, the guidance document will be issued as the procedure for lockout/tagout programs at the three Oak Ridge sites. Each site will provide training to fully implement the procedure.

\section{Root Causes:}

Poorly defined roles and responsibilities and inadequate policy implementation

Planned Actions and Schedules:

\section{$\underline{\text { Item/Description }}$}

1. Issue Energy Systems lockout/tagout procedure. (See Finding MA.2-1, Action 1.)

2. Train appropriate Laboratory personnel and implement procedure. (See Finding MA.2-1, Action 2.)

Costs: $\quad$ Costs are included in Finding MA.2-1.

References: $\quad 29$ CFR 1910, DOE Order 5480.19 
Finding No.: OP.7-1 ORNL Shift Overlap

Finding

Description: Not all shifts at ORNL have sufficient required overlap in duty time to permit an effective turnover of duties in conformance with the graded performance requirements of DOE 5480.19.

Code: $\quad$ Category III

Compliance

Protocol:

DOE Order 5480.19

Priority: $\quad$ Encrgy Systems Risk Weight 67

Tiger Team Action Plan Priority 2

Response: Shift turnovers are addressed by this action plan. The fundamental corrective action for this problem is the implementation of Conduct of Operations. The DOE

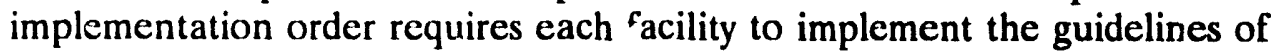
Conduct of Operations in a graded fashion, including those pertaining to shift turnover. The facilities with a need for shift work will be required to include adequate turnover periods.

An adequate turnover is a requirement of the implementation of Conduct of Operations. The need to implement Conduct of Operations and Research was in the ORNL Self-Assessment. The Research Reactors Division has a formalized shift turnover procedure for both supervisors and hourly operators. Waste Management Operations, Analytical Chemistry (REDC), and the Steam Plant require a shift turnover for the supervisors. Negotiations to provide a shift turnover for hourly personnel will be initiated when the affected organizations are identified through implementation of Conduct of Operations.

Root Causes:

Formality and discipline in the conduct of operations and research are inadequate.

Planned Actions and Schedules:

Item/Description

1. See Finding OP.4-1, Items 2 and 3.

2. Determine divisions requiring shift turnover based on Conduct of Operations Compensatory Actions.
Completion Date

$6 / 91$ 
3. Pursue collective bargaining agreement modifications to provide for an acceptable shift turnover to be applied to operations where deemed necessary.

4. Issue written guidance to appropriate operating divisions

$6 / 91$ concerning interim shift turnover practices.

Costs:

Type of funds: Overhead

Source of funds: Division Administration

Estimated costs per fiscal year (\$K)

\begin{tabular}{|c|c|c|c|c|c|c|c|}
\hline Action item & 1991 & 1992 & 1993 & 1994 & 1995 & Beyond & Total \\
\hline 1 & - & & & & & & \\
\hline 2 & - & & & & & & \\
\hline 3 & 5 & * & & & & & 5 \\
\hline 4 & - & & & & & & \\
\hline \multicolumn{8}{|l|}{ Status: } \\
\hline Funded & 5 & & & & & & \\
\hline \multicolumn{8}{|l|}{ Requested } \\
\hline New & & & & & & & $\$ 5$ \\
\hline
\end{tabular}

*Estimated annual ongoing costs: $\$ 115 \mathrm{~K}$ for ongoing shift overlap ( 75 hourly and 25 weekly employees).

The cost of a shift turnover is based on additional work time required of the hourly and nonexempt employee. This cost estimate is for a 15-minute turnover at one and one-half times the normal rate of pay.

References: None 
Finding No.: $\quad$ OP.8-1 Uniformity of Data Collection and Terminology

Finding

Description: Data collection and related communications do not always employ gencrally accepted terminology, thus necessitating specialized additional training and complicating workers' understanding of operational activitics.

Code: $\quad$ Category III

Compliance

Protocol:

Priority: $\quad$ Energy Systems Risk Weight 5

Tiger Team Action Plan Priority 3

Response: Standardized communication and terminology are addressed by this action plan. The fundamental corrective action for this problem is the implementation of Conduct of Operations. Under the DOE implementation order, each facility will apply the guidelines of Conduct of Operations in a graded fashion, including those pertaining to communications. The Conduct of Operations implementation plan requires that compensatory actions from the implement.tion of the guidelines be accomplished in a risk-based manner. This ensures that resources are used to address the most serious concern in a prioritized fashion.

To address the need for common terminology within ORNL, a standard policy will be issued.

The use of clear communications is a requirement of the implementation of Conduct of Operations. The need to implement Conduct of Operations was included in the ORNL Self-Assessment.

\section{Root Causes:}

Inadequate policy and inadequate policy implementation

Planned Actions and Schedules:

\section{$\underline{\text { Item/Description }}$}

1. This finding is fully addressed by the corrective actions identified for Finding OP.4-1, Items 2 and 3.

Costs: See Finding OP.4-1 for Conduct of Operations implementation costs.

References: $\quad$ DOE Order 5480.19, Conduct of Operations 
Finding No.: OP.8-2 Alarm Panel Signal Configuration

Finding

Description:

Not all alarm panels are configured to readily distinguish between signals requiring operator response and signals that merely are indicators of facility status.

Code: $\quad$ Category III

Compliance

Protocol:

DOE Order 5480.19 Chapter II requires alarm and annunciator panels be inspected for abnormal or unusual conditions. Prompt actions are required to be taken if such conditions are noted.

DOE Order 5480.19 Chapter VIII requires the status of alarm and control panels to be readily available. The intent of this requirement is to properly control equipment, satisfying design and operational limits.

Priority: $\quad$ Energy Systems Risk Weight 56

Tiger Team Action Plan Priority 2

Response: Segregation of alarm and status displays are addressed by this action plan. The fundamental corrective action for this problem is the implementation of Conduct of Operatisns. Under the DOE implementation order, each facility will apply the guidelines of Conduct of Operations in a graded fashion, including those pertaining to alarm and status panels. The Conduct of Operations implementation plan requires that compensatory actions from the implementation of the guidelines be accomplished in a risk-based manner. This ensures that resources are used to address the most serious cuncerns in a prioritized fashion. This plan will ensure that the need-appropriate alarm panels are identified and requirements for maintaining them are promulgated in an appropriate precedence

The ability of operators to distinguish between response and status signal is a requirement of implenientation of Conduct of Operations. The need to implement Conduc of Operations was included in the ORNL Self-Assessment.

Root Causcs:

Inadequate policy and inadequate policy implementation 
Planned Actions and Schedules:

\section{Item/Description}

Completion Date

1. See Finding OP.4-1, Items 1-3.

2. Determine and document facilities requiring alarm

$10 / 91$ systems based on Conduct of Operations Compensatory Actions.

3. Request funding for required changes. Establish schedule for implementation based on available resources.

4. Issue written guidance to operating divisions concerning procurement and modification of common alarm panels.

Costs: $\quad$ See Finding OP.4-1 for Conduct of Operations implementation costs.

References: $\quad$ DOE Order 5480.19, Conduct of Operations 
Finding No.: OP.8-3 Uniformity of Labels, Signs, and Signals

Finding

Description: Information is not conveyed in a uniform effective manner to personnel by labeling, signs, and uniformly colored lights.

Code: $\quad$ Category III

Compliance Protocol:

Priority:

Response:
DOE Order 5480.19 Chapter XVIII requires signs to be distinguishable, easy to read, and informative. Color coding for consistency is also recommended. The intent of this requirement is to reduce operator exposure to hazardous materials and to aid in room identification.

29 CFR 1910.145, Appendix A recommends standardized coloring of warning signs, depending upon the threat of personnel hazard.

Energy Systems Risk Weight 56

Tiger Team Action Plan Priority 2

Standardization of labeling, signs, and lights are addressed by this action plan. The deficiency was previously noted in the ORNL Self-Assessment. The fundamental corrective action for this problem is the implementation of Conduct of Operations. The Conduct of Operations implementation plan requires that compensatory actions from the implementation of the guidelines be accomplished in a risk-based manner. This ensures that resources are used to address the most serious concern in a prioritized fashion. To ensure standardization, a Performance Improvement Process committee should analyze the needs of ORNL and set requirements. They should also place the required signs in the AVID system to minimize expense in instituting the new signs.

The use of labeling and posted operator information is a requirement of the implementation of Conduct of Operations. A specific comment on multiple and inconsistent signs was included in the ORNL Self-Assessment. This is also related to Finding RP.3-5.

\section{Root Causes:}

Inadequate policy, inadequate policy implementation, and poorly defined roles and responsibilities 
Planned Actions and Schedules:

Item/Description

1. Appoint a Performance Improvement Process committee to address requirements for posting signs, labels, and lights.

2. See Finding OP.4-1, Items 1-3.

3. Issue a Standard Practice Procedure for the ORNL Safety Manual on installing warning signs, labels, and lights.

4. Place approved standard sign(s) in the AVID system.

5. Complete installation of standardized warning signs, labels, and lights and perform internal self-assessment to verify installation and program effectiveness.

Costs: $\quad$ See Finding OP.4-1 for Conduct of Operations implementation costs.

A commercially available Hazard ID Kit is available from Lab Safety Supply for $\$ 16.00$ per kit in lots of 100 or more. The kit includes one 10-by-10-in. caution sign and 30 smaller color-coded hazard labels. There are approximately 750 laboratory doors that require some degree of hazard posting for an initial estimate of $\$ 12,000$.

Installation of warning lights, such as green safety shower lights and red laser operation lights, would cost approximately $\$ 400$ per installation. 
Type of funds: Overhead

Source of funds: Overhead

Estimated costs per fiscal year (\$K)

\begin{tabular}{|c|c|c|c|c|c|c|c|}
\hline \multirow[b]{2}{*}{ Action item } & & \multirow[b]{2}{*}{ Total } \\
\hline & 1991 & 1992 & 1993 & 1994 & 1995 & Beyond & \\
\hline 1 & 10 & & & & & & 10 \\
\hline 2 & $*$ & & & & & & \\
\hline 3 & & 15 & & & & & 15 \\
\hline 4 & - & & & & & & \\
\hline \multicolumn{8}{|l|}{ Status: } \\
\hline Funded & 10 & & & & & & \\
\hline Requested & & & & & & & \\
\hline New & & 15 & & & & & $\$ 25$ \\
\hline
\end{tabular}

*See Finding OP.4-1.

Type of funds: Overhead

Source of funds: Division Administration

Estimated costs per fiscal year (\$K)

\begin{tabular}{|c|c|c|c|c|c|c|c|}
\hline \multirow[b]{2}{*}{ Action item } & & \multirow[b]{2}{*}{ Total } \\
\hline & 1991 & 1992 & 1993 & 1994 & 1995 & Beyond & \\
\hline 5 & & & 67 & & & & 67 \\
\hline
\end{tabular}

Status:

Funded

Requested

New

References: $\quad$ DOE Order 5480.19, Conduct of Operations, 29 CFR 1910 


\subsubsection{Maintenance}

Finding No.: MA.1-1 Control of Removal of Equipment from Service

\section{Finding}

Description: ORNL has no policy or procedure to control the removal of equipment from service in a manner that ensures continued safety, resulting in a number of potentially hazardous conditions that require identification and correction.

Code: $\quad$ Category III

\section{Compliance}

Protocol:

Maintenance organization and administration should ensure effective implementation and control of maintenance activities.

Priority: $\quad$ Energy Systems Risk Weight 41

Tiger Team Action Plan Priority 3

Response: $\quad$ Although no single document exists which defines all the steps for safe removal of equipment, most information is contained in the numerous procedures already in place (i.e., P\&E Procedure M-1.7, Modifications to Building and Utilities).

\section{Root Causes:}

Inadequate policy and ambiguous requirements or expectations

\section{Planned Actions and Schedules:}

\section{$\underline{\text { Item/Description }}$}

Completion Date

1. Meet with facility managers to discuss expectations and request them to inspect their areas. (See Complete Finding MA.2-2, Items 1 and 3). Items requiring immediate action should be referred to the Rapid Response Team. Work request should be prepared for routine items. Both actions will allow tracking of the corrections.

2. Train P\&E personnel in maintenance fundamentals, stressing excellence of maintenance performance. (See

Complete Finding MA.2-2, Item 4). "Proper Use and Care of Tools" by the Hand Tools Institute was used as the lesson plan and was distributed to all attendees.

3. Issue guidance to all $P \& E$ departments requiring an approved action plan for any job involving long-term removal of equipment from service/retirement in place. 
4. Issue a material deficiency tagging procedure. $\quad 9 / 91$

5. Prepare and issue an ORNL Standard Practice 10/91 Procedure for safe removal of equipment from service or retirement in place.

Costs:

Type of funds: Overhead

Source of funds: Overhead

Estimated costs per fiscal year $(\$ K)$

\begin{tabular}{cccccccc}
\cline { 2 - 5 } Action item & 1991 & 1992 & 1993 & 1994 & 1995 & Beyond & Total \\
\hline 1 & - & & & & & & \\
2 & - & & & & & & \\
3 & - & & & & & & \\
4 & - & & & & & & \\
5 & 1 & & & & & & 1
\end{tabular}

Status:

Funded

1

Requested

New

References: None 
Finding No.: MA.2-1 ORNL Lockout/Tagout System's Effectiveness

Finding

Description: ORNL does not have an effective lockout/tagout system. (See OP.4-2.)

Code: $\quad$ Category III

Compliance

Protocol:

DOE Order 5480.19

Priority: $\quad$ Energy Systems Risk Weight 917

Tiger Team Action Plan Priority 1

Response: The need for an improved lockout/tagout procedure was identified in the ORNL self-assessment document. Energy Systems has recognized the need for central guidance to promote uniform compliance with lockout/tagout requirements at all sites operated by Energy Systems. A draft procedure consistent with 29 CFR 1910 and DOE Order 5480.19 has been issued for comment by Energy Systems. After comments are resolved, the guidance procedure will be issued to become the basis for lockout/tagout programs at each site. Each site will provide training to fully implement the procedure.

See Finding OP.4-2.

Root Causes:

Poorly defined roles and responsibilities and inadequate policy implementation

Planned Actions and Schedules:

\section{$\underline{\text { Item/Description }}$}

1. Energy Systems issue approved lockout/tagout procedure.

2. Train all Laboratory personnel on the procedure.

3. Initiate implementation of procedure.
Completion Date

$5 / 91$

$7 / 91$

$8 / 91$ 
Costs:

\begin{tabular}{|c|c|c|c|c|c|c|c|}
\hline \multicolumn{8}{|c|}{$\begin{array}{l}\text { Type of funds: Overhead } \\
\text { Source of funds: Overhead }\end{array}$} \\
\hline \multirow[b]{2}{*}{ Action item } & \multicolumn{6}{|c|}{ Estimated costs per fiscal year $(\$ \mathrm{~K})$} & \multirow[b]{2}{*}{ Total } \\
\hline & 1991 & 1992 & 1993 & 1994 & 1995 & Beyond & \\
\hline 1 & 12 & & & & & & 12 \\
\hline 2 & 190 & & & & & & 190 \\
\hline 3 & $*$ & & & & & & \\
\hline \multicolumn{8}{|l|}{ Status: } \\
\hline Funded & 202 & & & & & & \\
\hline \multicolumn{8}{|l|}{ Requested } \\
\hline New & & & & & & & $\$ 202$ \\
\hline
\end{tabular}

*Estimated annual ongoing cost: $\$ 66 \mathrm{~K}$.

References: $\quad 29$ CFR 1910, DOE Order 5480.19 
Finding No.: MA.2-2 Conduct of Maintenance

Finding

Description: Plant maintenance personnel do not use fundamental good practices, sometimes resulting in unsafe conditions.

Code: $\quad$ Category II

Compliance

Protocol:

Maintenance should be conducted in a safe and effective manner to support each facility condition and operation on the site.

Priority: $\quad$ Energy Systems Risk Weight 86

Tiger Team Action Plan Priority 2

Response: Improvements in the "Conduct of Maintenance" at the ORNL site are addressed by this action plan. These improvements will be realized by: clarifying roles and responsibilities of facility managers and of maintenance supervision and by training of craft personnel and maintenance supervisors in fundamental good practices.

Root Causes:

Poorly defined roles and responsibilities and inadequate training

Planned Actions and Schedules:

\section{Item/Description}

1. Meet with Division Directors, Facility Managers and P\&E Supervisors to clarify roles and responsibilities and to educate on needs identified by Tiger Team.

2. Issue notice requiring job inspections by $P \& E$ personnel after repair activities (customer orientation).

3. Conduct inspections of selected facilities to identify and correct deficiencies.

4. Train all Plant \& Equipment Division personnel in maintenance fundamentals of quality.

5. Initiate training of appropriate $P \& E$ personnel in Facility Condition Inspection Techniques ("Walk your spaces training").

6. Implement random quality checks of maintenance activities to verify continuing quality.
Completion Date

Complete

Complete

Complete

Complete

$9 / 91$

$6 / 91$ 
7. Define and implement continuing craft specific training activities to ensure quality. (See Finding TC.5-1.)

Sec Finding

1C.5-1

Casts:

Type of funds: Overhead

Source of funds: Overhead

\begin{tabular}{|c|c|c|c|c|c|c|c|c|}
\hline \multirow[b]{2}{*}{ Action item } & \multicolumn{7}{|c|}{ Estimated costs per fiscal year (\$K) } & \multirow[b]{2}{*}{ Total } \\
\hline & 1990 & 1991 & 1992 & 1993 & 1994 & 1995 & Beyond & \\
\hline 1 & & 5 & & & & & & 5 \\
\hline 2 & & - & & & & & & \\
\hline 3 & 529 & 101 & & & & & & 630 \\
\hline 4 & & 20 & & & & & & 20 \\
\hline 5 & & 100 & & & & & & 100 \\
\hline 6 & & 25 & * & & & & & 25 \\
\hline 7 & & - & & & & & & \\
\hline
\end{tabular}

Status:

Funded $\quad 529 \quad 251$

Requested

New

*Estimated annual ongoing cost: $\$ 15 \mathrm{~K}$.

References: None 
Finding No.: MA.5-1 General Facility and Post-Work Inspections

Finding

Description: Systematic, general facility and post-work inspections were not being used to identify all needed corrective actions, resulting in some safety equipment only being identified for corrective maintenance following failure.

Code: $\quad$ Category III

Compliance

Protocol:

Priority: $\quad$ Energy Systems Risk Weight 567

Tiger Team Action Plan Priority 2

Response: Through the years, as maintenance budgets have decreased, various facility inspections have been curtailed. Most significant areas (i.e., roofs, corded equipment, etc.) are still being inspected. The entire process, however, needs to be more formalized with procedures. Manpower resources for implementation of a complete facility inspection program are not available.

P\&E Procedure D-1.9, P\&E Work Request, requires that the supervisor-in-charge perform a post-work inspection of each completed job. This requirement has typically not been emphasized by management. Memos have been issued requesting P\&E supervisors and facility managers to perform post-work inspection of completed jobs.

\section{Root Causes:}

Insufficient resources, ambiguous requirements or expectations, and inadequate management commitment

Planned Actions and Schedules:
Completion Date

Complete

1. Train all $P \& E$ personnel in maintenance fundamentals, stressing excellence of maintenance performance (sce Finding MA.2-2, Item 4).

2. Estimate cost required and request funding for a 
3. Initiate training of appropriate P\&E personnel in Facility Condition Inspection (see Finding MA.2-2, Item 5).

4. Initiate training of Facility Managers in Facility Condition Inspection. ("Walk your spaces training").

5. Initiate comprehensive facility inspection program, including the need for backflow prevention devices and auxiliary systems.

Funding receipt plus 6 months

Costs:

Type of funds: Overhead

Source of funds: Overhead

\begin{tabular}{|c|c|c|c|c|c|c|c|}
\hline \multirow[b]{2}{*}{ Action item } & \multicolumn{6}{|c|}{ Estimated costs per fiscal year (\$K) } & \multirow[b]{2}{*}{ Total } \\
\hline & 1991 & 1992 & 1993 & 1994 & 1995 & Beyond & \\
\hline 1 & - & & & & & & \\
\hline 2 & - & & & & & & \\
\hline 3 & - & & & & & & \\
\hline 4 & 5 & 20 & & & & & 25 \\
\hline 5 & & * & & & & & \\
\hline \multicolumn{8}{|l|}{ Status: } \\
\hline \multicolumn{8}{|l|}{ Funded } \\
\hline \multicolumn{8}{|l|}{ Requested } \\
\hline New & 5 & 20 & & & & & $\$ 25$ \\
\hline
\end{tabular}

*Estimated annual ongoing cost: $\$ \mp j 0 \mathrm{~K}$. Includes Finding AX.1-1.

References: Draft DOE Order 4330.XXX, Maintenance Management Program 
Finding No.: MA.5-2 Identification of Energy Conservation Corrective Actions

Finding

Description: Fundamental assessments of facilities had not been completed to identify energy conservation corrective actions as required in DOE 4330.2C.

Code: $\quad$ Category III

Compliance

Protocol:

DOE Order $4330.2 \mathrm{C}$

Priority: $\quad$ Energy Systems Risk Weight 58

Tiger Team Action Plan Priority 2

Response: Improvements in the assessment of facilities at the ORNL site are addressed by this action plan. These improvements will be realized by clarifying roles and responsibilities of facility managers and by prioritization of energy management activities based on facility inspections, energy intensiveness, and resource allocation.

Root Cáuses:

Poorly defined roles and responsibilities and inadequate resources

Planned Actions and Schedules:

\section{$\underline{\text { Item/Description }}$}

1. Develop initial plan for prioritized rotational assessment of ORNL buildings on a 5-year cycle.

2. Issue ORNL Bulletin asking all employees to identify opportunities for energy conservation.

3. Request funding for assessments and corrective actions.

4. Train facility managers on assessment and identification of energy management opportunities.
Completion Date

9/91

$9 / 91$

$1 / 92$ 


\section{Costs:}

Type of funds: Overhead

Source of funds: Overhead

\begin{tabular}{|c|c|c|c|c|c|c|c|}
\hline \multirow[b]{2}{*}{ Action item } & \multicolumn{6}{|c|}{ Estimáte $\leq$ costs per fiscal year $(\$ K)$} & \multirow[b]{2}{*}{ Total } \\
\hline & 1991 & 1992 & 1993 & 1994 & 1995 & Beyond & \\
\hline 1 & 5 & & & & & & 5 \\
\hline 2 & - & & & & & & \\
\hline 3 & - & & & & & & \\
\hline 4 & & 30 & & & & & 30 \\
\hline \multicolumn{8}{|l|}{ Status: } \\
\hline Funded & 5 & & & & & & \\
\hline \multicolumn{8}{|l|}{ Requested } \\
\hline New & & 30 & & & & & $\$ 35$ \\
\hline
\end{tabular}

Estimated annual ongoing cost: $\$ 200 \mathrm{~K}$.

References: None 
Finding No.: $\quad$ MA.6-1 P\&E Preventive Maintenance Program

Finding

Description: The Plant and Equipment Division preventive maintenance program did not include all components important to safety and reliability and did not address routine equipment preservation. (See AX.1-1 and AX.6-1.)

Code: $\quad$ Category III

Compliance

Protocol:

Priority: $\quad$ Energy Systems Risk Weight 570

Tiger Team Action Plan Priority 2

Response: $\quad$ Adjustments to the PM program are being addressed by this action plan. These adjustments will be realized by: clarifying roles and responsibility for performing $\mathrm{PM}$ on safety valves and relief valves and for ensuring the maintenance is conducted; identifying equipment, piping, valves, and supports which need to be added to the PM program for routine maintenance; and evaluating maintenance practices and developing procedures for maintenasce in these areas. The proposed practice of using operating personnel to adjust par king glands will be reviewed on a case by case basis and will be assigned in accorcance with the Company/Union contract as it pertains to work jurisdiction and assignment of work.

\section{Root Causes:}

Ambiguous requirements or expectations, poorly defined roles and responsibilities, and insufficient resources

Planned Actions and Schedules:

\section{$\underline{\text { Item/Description }}$}

1. Fill PM coordinator position.

2. Issue operating instructions on how to correctly open

3. Perform general area and system inspections to identify

4. Define staffing requirements to implement additional
Complete

$6 / 91$

and close valves.

$8 / 91$ additional equipment, piping, valves, supports, and foundations which need to be added to the PM program.

Completion Date

$\mathrm{PM}$ and system preservation and request funding. 
5. Issue maintenance procedures defining methods of $12 / 91$ preservation for piping, valves, and supports to retard deterioration.

6. Initiate implementation of additional PM.

Funding approval +3 months

Costs:

Type of funds: Overhead

Source of funds: Overhead

Estimated costs per fiscal year $(\$ \mathrm{~K})$

\begin{tabular}{|c|c|c|c|c|c|c|c|}
\hline \multirow[b]{2}{*}{ Action item } & & \multirow[b]{2}{*}{ Total } \\
\hline & 1991 & 1992 & 1993 & 1994 & 1995 & Beyond & \\
\hline 1 & - & & & & & & \\
\hline 2 & - & & & & & & \\
\hline 3 & 10 & & & & & & 10 \\
\hline 4 & - & & & & & & \\
\hline 5 & & 5 & & & & & 5 \\
\hline 6 & & * & & & & & \\
\hline \multicolumn{8}{|l|}{ Status: } \\
\hline Funded & 10 & & & & & & \\
\hline Requested & & 5 & & & & & \\
\hline New & & & & & & & $\$ 15$ \\
\hline
\end{tabular}

${ }^{*}$ Estimated annual ongoing cost: $\$ 250 \mathrm{~K}$.

References: None 
Finding No.: MA.8-1 Appropriate Maintenance Procedures

Finding

Description: Maintenance procedures did not reflect an appropriate level of detail and organization.

Code: $\quad$ Category III

Compliance

Protocol:

Maintenance procedures should be clearly written, accurate, and well organized to ensure an adequate description of the job and the proper sequencing of details.

Priority: $\quad$ Energy Systems Risk Weight 42

Tiger Team Action Plan Priority 3

Response: Improvements in the Plant and Equipment (P\&E) Division procedure generation and revision process are defined by this action plan. The improvements will be realized by reviewing existing procedures and incorporating a working review of the draft prior to publication. Routine review of all active procedures will be as specified in ORNL Quality Assurance Procedures.

\section{Root Cause:}

Ambiguous requirements or expectations

\section{Planned Actions and Schedules:}

\section{$\underline{\text { Item/Description }}$}

1. Issue revised procedure D-1.2, The Preparation of Standard Operating Procedures, to require all procedures to be field tested prior to being published.

2. Issue revised procedure R-3.15, Procedure for Safe Handling and Repair of Contaminated Manipulators.

3. Initiate training of $P \& E$ procedure writers utilizing Energy Systems writers' guide. (See Finding OP.3-1, Action Step 3).
Completion Date

Complete 
Costs:

Type of funds: Overhead

Source of funds: Overhead

\begin{tabular}{|c|c|c|c|c|c|c|c|}
\hline \multirow[b]{2}{*}{ Action item } & \multicolumn{6}{|c|}{ Estimated costs per fiscal year $(\$ K)$} & \multirow[b]{2}{*}{ Total } \\
\hline & 1991 & 1992 & 1993 & 1994 & 1995 & Beyond & \\
\hline 1 & 1 & & & & & & 1 \\
\hline 2 & 3 & & & & & & 3 \\
\hline 3 & & * & & & & & \\
\hline \multicolumn{8}{|l|}{ Status: } \\
\hline Funded & 4 & & & & & & \\
\hline \multicolumn{8}{|l|}{ Requested } \\
\hline New & & & & & & & $\$ 4$ \\
\hline
\end{tabular}

*Estimated annual ongoing cost: $\$ 100 \mathrm{~K}$

References: None 


\subsubsection{Training and Certification}

Finding No.: TC.1-1 Consistency and Administration of Training Programs

Finding

Description: Training programs at the Oak Ridge National Laboratory are not consistent and are not being effectively administered.

Code: $\quad$ Category III

Compliance

Protocol: None

Priority: $\quad$ Energy Systems Risk Weight 358

Tiger Team Action Plan Priority 2

Response: The Training and Development Department has recently been reorganized with the intent to have a central focal point for coordinating training activities to ensure consistency, eliminate redundancy, and achieve programs that are compliance based. The organization concept is in place but lacks procedures that would direct "how to accomplish" the Laboratory training goals. A Training Needs Overview (Assessment) has been initiated to identify (1) training needs for every person, based on over 1200 DOE regulations; and (2) what training is being conducted.

The survey data will allow us to avoid duplication and to administer consistent training. In addition, the size of the Training and Development Department professional staff, consisting of 2-1/2 permanent personnel and one temporary subcontractor, is inconsistent with the department's mandate to achieve effective, centralized coordination and administration of Laboratory training. A review of the objectives has identified a need for four additional HRD staff persons to augment the present staff of $2-1 / 2$ professionals.

Also, see Findings MF-4 and TC.5-1.

The items are categorized chronologically within subject area.

Root Cause:

Inadequate policy implementation and insufficient resources 
Planned Actions and Schedules:

$\underline{\text { Item/Description }}$

1. Prepare a section for the ORNL Standard Practice Procedures Manual on Training Policies and Procedures and establish criteria. Roles and Responsibilities will be outlined to provide clear management guidance.

Examination requirements will be addressed in the above procedure.

2. Identify status of division training manuals and prepare a schedule for completion.

3. Based on DOE regulations (orders, CFRs and other directives), issue a checklist to line management to use in identifying tasks performed by each individual and any existing training addressing each requirement.

4. Prepare training program standards as required for DOE directives.

5. Identify by name baseline requirements and load into TMIS.

6. Schedule the revisions or development of programs and presentation timetable.

7. Issue procedures for systematically upgrading individual training profiles, due to a change in job duties or new requirements, with particular emphasis on ES\&H training programs.

8. Initiate implementation of a Laboratory directive requiring centralized administration of general employee training and retraining for all employees.

9. Produce guest/visitor training video and provide to the Office of Guest and User Interaction, and implement a Laboratory policy requiring administration of guest/user training to all appropriate personnel.

10. Update Instructor Skills Training programs and initiate implementation.

11. Implement procedures to track requalification and retraining requirements for employees prior to expiration.
Completion Date

$10 / 91$

$12 / 91$

Complete

$6 / 91$

$12 / 91$

$3 / 92$

$3 / 92$

Complete

$6 / 91$

$10 / 91$

$2 / 92$ 


\section{Casts:}

Type of funds: ESH Programmatic

Source of funds: ER-AT TC

Estimated costs per fiscal year (\$K)

\begin{tabular}{|c|c|c|c|c|c|c|c|}
\hline Action item & 1991 & 1992 & 1993 & 1994 & 1995 & Beyond & Total \\
\hline 1 & & 14 & & & & & 14 \\
\hline 2 & & 29 & & & & & 29 \\
\hline $3,4,5,6,7$ & 80 & 100 & & & & & 180 \\
\hline 9 & 5 & & & & & & 5 \\
\hline 10 & 116 & & & & & & 116 \\
\hline 11 & & 25 & & & & & 25 \\
\hline
\end{tabular}

Status:

Funded

Requested

New

Estimated annual ongoing cost: Item $2, \$ 4 \mathrm{~K}$; Items 3 through 4 , $\$ 8 \mathrm{~K}$; Item 5, $\$ 10 \mathrm{~K}$; Item 7, \$5K; Item 8, \$8K; Item 9, \$48K; Items 7 and 10, $\$ 8 \mathrm{~K}$.

References: None 
Finding No.: TC.1-2 Adequacy of Training Examinations

Finding

Description: Examinations are not always administered to ensure that trainees understand the course subject matter.

Code: $\quad$ Category III

Compliance

Protocol:

Priority: $\quad$ Energy Systems Risk Weight 37

Tiger Team Action Plan Priority 3

Response: The DOE Environmental, Safety, and Health Performance Objectives and Criteria for Technical Safety Appraisals at DOE Sites and Facilities require a minimum score of $80 \%$ to pass written examinations on the following training programs: GET Radiological Protection, GET Emergency Preparedness, GET Hazardous Materials, GET Criticality Safety, and Radiation Worker. ORNL will prepare an examination policy that will identify the following: programs for which exams should be given; passing standards; and grading, proctoring, and review of exams with employees. This will be a part of Finding TC.1-1, Item 1, Policies and Procedures.

\section{Root Cause:}

Inadequate policy

Planned Actions and Schedules:

This finding is fully addressed by Item 1 of Finding TC.1-1.

Costs: $\quad$ Funded in Finding TC.1-1, Item 1.

References: None 
Finding No.: TC.1-3 Training Staff

\section{Finding}

Description: The training staffs are too small to provide quality training for ORNL, which has numerous hazards.

Code: $\quad$ Category III

Compliance

Protocol: None

Priority: $\quad$ Energy Systems Risk Weight 562

Tiger Team Action Plan Priority 3

Response: $\quad$ ORNL is in noncompliance with a number of training directives from DOE. Adequate training staff must exist to achieve ES\&H and other required training. These deficiencies have been documented. The reduced FY 1991 budget for HRD has resulted in further staffing reductions. A review of the Laboratory-wide staffing needs resulted in an estimate of approximately 50 FTEs needed. The most crucial needs have been estimated at 15 FTEs. The Laboratory needs assessment included in Finding TC.1-1 will enable us to make a more accurate needs request.

This finding is related to Findings TC.1-1, MF-4, TC.5-1, and RTC.5-1.

These staff are required to conduct the compliance training outlined in response to Findings TC.5-1 and RTC.5-1, and other ES\&H training.

\section{Root Cause:}

Insufficient resources

Planned Actions and Schedules:

Item/Description

1. Request funds for $\mathbf{1 5}$ additional training staff.

2. Hire staff commensurate with funds received.
Completion Date

Complete

4 months after funds received 
Casts:

Type of funds: ESH Programmatic

Source of funds: ER-AT TC

Estimated costs per fiscal year (\$K)

\begin{tabular}{lrrrrrrr} 
Action item & 1991 & 1992 & 1993 & 1994 & 1995 & Beyond & Total \\
\hline 2 & & 1035 & 1077 & $*$ & & 2112 \\
$\begin{array}{l}\text { Status: } \\
\text { Funded } \\
\text { Requested } \\
\text { New }\end{array}$ & & & & & & \\
\hline
\end{tabular}

*Estimated annual ongoing cost: \$11i9K starting in FY 1993.

References: None 
Finding No.: TC.5-1 Maintenance Personnel Training Program

Finding

Description: The maintenance training program does not assure that maintenance personnel are qualified to perform their assigned tasks, as required by DOE 5480.5

Section 10.b.(2).

Code: $\quad$ Category III

Compliance

Protocol:

DOE Order 5480.6 and DOE Order 5480.5 Section 10.b.(2)

Priority: $\quad$ Energy Systems Risk Weight 588

Tiger Team Action Plan Priority 2

Response: $\quad$ DOE Order 5480.6 requires selective application of the guidelines provided in ANS 3.1 Draft 1980. The intent of the DOE order and ANS 3.1 is to require formal training and qualification programs for maintenance personnel who support and maintain category " $\mathrm{A}$ " reactor related systems and components. The training programs shall be based on Job/Task Analysis and shall provide the necessary skills, knowledge, and abilities required to perform maintenance on reactor equipment in a safe and efficient manner.

DOE Order 5480.5, "Safety Of Nuclear Facilities" Section 10.b. requires formal training and qualification programs for maintenance personnel who support and maintain nonreactor nuclear facilities systems and components.

A new DOE Order 5480.XX, Draft 3/90, "Personnel Selection Qualification and Training" states that maintenance training programs shall be based on Job/Task Analysis and provide the necessary skills, knowledge, and abilities required to perform maintenance on nuclear-related equipment in a safe and efficient manner.

Maintenance training program deficiencies were identified and documented in "Radiochemical Engineering Development Center, Building 7920, Accreditation Program Initial Self-Evaluation Report" ORNL, August 1, 1990, and "High Flux Isotope Reactor (HFIR) Reactor Maintenance Training Accreditation Program Initial Self-Evaluation Report" ORNL, September 1989.

Skills training has not been provided to personnel assigned to perform maintenance in reactor and nonreactor nuclear facilities in a formal manner to ensure all personnel demonstrate the required skills, knowledge, and abilities. Onthe-job training (OJT) conducted for Plant and Equipment Division and Instrumentation and Controls Division maintenance personnel has not been documented in accordance with current industry standard of excellence. No formal technical skills classroom and/or hands-on laboratory training has been provided for personnel who are assigned to perform maintenance at reactor and nonreactor nuclear facilities. 
Both divisions currently have a minimal dedicated and qualified Technical Training staff adequate at this point in time to identify and plan required training program upgrades and to schedule and coordinate compliance training for 850 Plant and Equipment Division and 150 Instrumentation and Control Division maintenance personnel.

Job/task analyses have not been conducted for maintenance positions at nonreactor nuclear facilities. Job/task analyses must be conducted in accordance with the guidelines provided in DOE EP 095 and must indicate the tasks performed that require the training and or retraining.

See Corrective Action Plan Finding TC.1-3 for staffing necessary to address this concern.

This same finding was also identified by the Reactors TSA tcam as Finding RTC.5-1.

\section{Root Cause:}

Insufficient resources; funding has not been authorized for an adequate staff to analyze, design, develop, conduct, implement, and evaluate Plant and Equipment Division Maintenance Training and Qualification programs.

\section{Planned Actions and Schedules:}

\section{$\underline{\text { Item/Description }}$}

1. Establish Maintenance Training Policy.

2. Request training instructor support to analyze, prepare, conduct, evaluate, schedule, and modify training. Request clerical support for miscellancous administrative duties, typing of lessons, analysis of checklist and job/task, and maintenance of records for 850 Plant and Equipment Division employees and 150 Instrumentation and Controls Division employees. Funding for training staff support has been requested in Finding TC.1-3.

3. Review formal training and qualification programs established at SRP and INEL for good practices and materials that may be used or adapted for use in the development and implementation of Technical Skills Training and Qualification programs.

4. Develop Training Policies and Procedures, Training Program Plans, MOUs, and miscellancous documentation.
Completion Date

$7 / 91$

$7 / 91$

$11 / 91$

$11 / 91$ 
5. Prioritize and conduct job/task analysis for maintenance

$6 / 92$ positions for personnel who support ORNL nonreactor nuclear facilities. Generate reports defining training needs. Initiate design and development of formal classroom and hands-on technical-skills training indicated and required by job/task analysis.

6. Qualify OJT instructors (P\&E and I\&C supervisors, $6 / 92$ Technical Support and selected craft personnel) to develop and conduct formal on-the-job technical skills training.

7. Develop and implement formal OJT checklist and $12 / 92$ performance standards for maintenance personnel who are assign to the reactor and nonreactor nuclear facilities and are to perform maintenance on the systems and compontents regulated by NQA-1. (Maintenance supervisors, technical support personnel, pipe fitters, electricians, millwrights and instrument technicians.)

8. Develop facility-specific access training and systems training. Develop lesson plans and a formal hands-on Laboratory continuing training program for task identified in job/task analysis. Develop maintenance training and personnel-qualification program for reactor and nonreactor nuclear systems and component identified as critical by the facility Safety Analysis Report.

9. Conduct and maintain maintenance training and personnel qualification programs on systems and components identified as critical by the facility Safety Analysis Reports.

Costs: $\quad$ Program activities listed above will be complete as scheduled with manpower/resources identified in Finding TC.1-3. No existing resources are available to complete the activities necessary for compliance.

Estimated costs for these activities exclude REDC and HFIR program upgrade costs, scope, and schedule for Training Program Accreditation.

Training program Accreditation and the required upgrades have been addressed in the Training Program Accreditation Plans, "Radiochemical Engineering Development Center Building 7920, Training Program Accreditation Plan," August 1, 1990; High Flux Isotope Reactor Training Program Accreditation Plan, May 1990. 
References: DOE Order 5480.5 "Safety Of Nuclear Facilities"

DUE Order 5480.6 "Safety Of Department of Energy-Owned Reactors

ANSI/ANS 3.1

Draft 1980, "Selection, Qualification and Training of

Personnel for Nuclear Power Plants"

DOE Order 5480.18 "Accreditation of Performance-Based Training for Category

A Reactors"

DOE Order 5480.XX Draft 3/90 "Personnel Selection, Qualification and Training Requirements" 
Finding No.: TC.7-1 Training Facilities

Finding

Description: Training facilities are not conducive to providing the quality of training required for personnel working at Oak Ridge National Laboratory.

Code: $\quad$ Category III

Compliance

Protocol:

None

Priority: $\quad$ Energy Systems Risk Weight 58

Tiger Team Action Plan Priority 3

Response: $\quad$ Adequate facilities are required to project training in a professional manner and to have an atmosphere conducive to learning. Insufficient facilities send a message that training is not important. Inadequate facilities exist for almost every training organization. The P\&E and I\&C divisions require hands-on training facilities for many crafts. An informal survey was conducted in July 1990 to identify existing training facilities and determine needs. At the present time, only Research Reactors, Chemical Technology, and Environmental Safety \& Health Compliance divisions have any dedicated classroom space. The ES\&H organization has one laboratory for about 32 courses taught. All other classes must be conducted in borrowed space that may or may not be available.

Root Cause:

Insufficient resources

Planned Actions and Schedules:

\section{Item/Description}

1. Identify and dedicate training space as an interim measure until permanent facilities are provided.

2. Conduct preliminary investigation and design.

3. Request funds.

4. Develop specifications and bid documents.
Completion Date

$5 / 91$

$3 / 92$

$5 / 92$

6 months

after

funding

received 
5. Initiate construction of facilities.

12 months after funding received

Casts:

Type of funds: Capital

Source of funds: GPP

Estimated costs per fiscal year (\$K)

\begin{tabular}{|c|c|c|c|c|c|c|c|}
\hline \multirow[b]{2}{*}{ Action item } & & \multirow[b]{2}{*}{ Total } \\
\hline & 1991 & 1992 & 1993 & 1994 & 1995 & Beyond & \\
\hline \multicolumn{8}{|l|}{1} \\
\hline 2 & & 295 & & & & & 295 \\
\hline \multicolumn{8}{|l|}{3} \\
\hline 4 & & 25 & & & & & 25 \\
\hline 5 & & & 2000 & 1000 & 1000 & & 4000 \\
\hline \multicolumn{8}{|l|}{ Status: } \\
\hline \multicolumn{8}{|l|}{ Funded } \\
\hline Requested & & 320 & 2000 & 1000 & 1000 & & \\
\hline New & & & & & & & $\$ 4320$ \\
\hline
\end{tabular}

References: None 
Finding No.: TC.7-2 Storage of Training Records

Finding

Description: Training records required to be stored for extended periods are not being protected from potential damage, deterioration, or loss, as required by ASME NQA-1-1989 and DOE 1324.2A.

Code: $\quad$ Category III

Compliance

Protocol:

ASME NQA-1-1989 and DOE Order 1324.2A

ASME NQA-1-1989, "Quality Assurance Program Requirements for Nuclear Facilities," lists in Appendix 17A-1, Section 3, those records that are to be considered lifetime records. Paragraph 3.6 identifies lifetime records and includes "Training and qualification records for current members of the plant operating staff."

ASME NQA-1-1989, Part II, "Basic Requirements, "Section 17, "Quality Assurance Requirements" requires records to be protected against damage, deterioration or loss.

DOE Order 1324.2A requires the use of ASME NQA-1-1989.

Priority: $\quad$ Energy Systems Risk Weight 58

Tiger Team Action Plan Priority 3

Response: The storage of ORNL training records does not meet ASME NQA-1 requirement. The QA Department is preparing for a study of alternatives of records storage, and will make recommendations.

Root Cause:

Inadequate policy implementation

Planned Actions and Schedules:

\section{$\underline{\text { Item/Description }}$}

1. Evaluate storage alternatives and make recommendations to ORNL management.

2. Request funds for selected method.

3. Implement selected method.
Completion Date

11/91

$12 / 91$

3 months after receipt of funds 


\section{Casts:}

Type of funds: Overhead

Source of funds: Overhead

Estimated costs per fiscal year (\$K)

\begin{tabular}{crrrrrrr}
\cline { 2 - 6 } Action item & 1991 & 1992 & 1993 & 1994 & 1995 & Beyond & Total \\
\hline 1 & & 22 & & & & & 22 \\
2 & - & & & & & & \\
3 & - & 78 & & & & & 78
\end{tabular}

Status:

Funded

Requested

New

100

$\$ 100$

References: None 
Finding No.: TC.10-1 Consistency of Supervisory and Management Training

Finding

Description: The training for persons who oversee the activities of others is not consistently applied.

Code: $\quad$ Category III

Compliance

Protocol: None

Priority: $\quad$ Energy Systems Risk Weight 87

Tiger Team Action Plan Priority 2

Response: ORNL has several supervisory and management-skills training programs as well as a number of compliance programs. The attendance for compliance courses has been good. Renewed efforts will be exerted to ensure that all managers and supervisors attend the appropriate training. Resources are requested in Finding TC.1-3.

The definition of a supervisor is anyone who supervises or oversees the work of one or more individuals. These persons have been identified by divisions/offices and the names loaded into our Training Management Information System. This allows us to schedule for supervisors/managers and identify deficiencies.

Root Cause:

Inadequate policy and insufficient resources

Planned Actions and Schedules:

Item/Description

1. Develop profiles of required classes for managers and supervisors.

2. Review existing programs and initiate revisions or development of required classes.

3. Initiate implementation of training. $2 / 92$

Costs: $\quad$ Included in Findings TC.1-1 and TC.1-3.

References: None 


\subsubsection{Auxiliary Systems}

Finding No.: AX.1-1 Remedial Program for Auxiliary Systems

Finding

Description: ORNL has not established a remedial program for age-related degradation of auxiliary systems.

Code: $\quad$ Category III

Compliance

Protocol:

It is considered a good management practice to address age-related degradation of systems as an element of a comprehensive maintenance program. It is anticipated that the final version of Draft DOE Order 4330.XXX will directly or indirectly include the requirement that the site implementing programs address the aspect of age-related degradation of auxiliary systems. Also, age-related degradation of auxiliary systems is one of the criteria currently considered under the TSA POC.

Priority: $\quad$ Energy Systems Risk Weight 570

Tiger Team Action Plan Priority 2

Response: Currently at ORNL remediation of age-related degradation for auxiliary systems is accomplished piecemeal through the GPP and line item planning process. There has been no official driver for development of a comprehensive maintenance management document to address such issues as age-related degradation of facilities and systems. That driver will be established with the issuance of a DOE Order 4330.XXX, Maintenance Management Program. The order will require that each operating site prepare and implement a comprehensive Maintenance Implementation Plan (MIP). One element of the plan, Facility Material Condition Inspection, will address remediation of age-related degradation of all systems which are important to safe operation. Implementation of DOE Order 4330.XXX will fully address the finding.

\section{Root Cause:}

Inadequate policy; ORNL maintenance policy and standards are not defined.

\section{Planned Actions and Schedules:}

Corrective actions for this finding are addressed in Finding MA.5-1 General Facility and Post-Work Inspections.

Costs: $\quad$ Costs are addressed in Finding MA.5-1.

References: $\quad$ Draft DOE Order 4330.XXX 
Finding No.: AX.1-2 Configuration Control System for Auxiliary Systems

Finding

Description: ORNL has not established a configuration control system for auxiliary systems.

Code: $\quad$ Category III

Compliance

Protocol:

DOE Orders $5480.5,5480.6$, and 5480.19

Priority: $\quad$ Energy Systems Risk Weight 55

Tiger Team Action Plan Priority 2

Response: The planned ORNL configuration-management program will include configuration control for auxiliary systems. The interim management directive on configuration management issued November 1, 1990, included ventilation systems, waste systems, inter-facility alarm systems, and the nuclear-material vault as critical auxiliary systems but did not mention the steam plant, a system identified by the tiger team as critical. Although DOE has not provided consistent guidance on configuration management, from earlier internal and external appraisals and the Laboratory selfassessment, ORNL was aware of inadequacies in configuration control but had not yet specifically identified auxiliary systems as requiring control. Implementation of the configuration-management program will be tied to the plan and schedule for the phased approach in developing facility safety analysis documentation.

\section{Root Causes:}

Inadequate policy and inadequate policy implementation

\section{IRlanned Actions and Schedules:}

\section{$\underline{\text { Item/Description }}$}

1. The Office of Operational Readiness and Safety will revise the list of critical facilities and systems that the interim directive places under configuration management to include critical auxiliary systems.

2. Initiate configuration management training for managers of facilities and systems that are subject to the formal configuration-management program.

3. Appoint a full-time configuration control program manager to provide direction to facilities and divisions in implementing the configuration-management program.

\section{Completion Date}

Complete 
4. Issue an ORNL SPP for configuration management to supersede the interim document issued on November 1 , 1990. The SPP will specify that it is to be phased in as part of the facility safety evaluation process.

Casts:

Type of funds: Overhead

Source of funds: Overhead

\begin{tabular}{crrrrrrr} 
& \multicolumn{5}{c}{ Estimated costs per fiscal year (\$K) } & \\
\cline { 2 - 5 } Action item & 1991 & 1992 & 1993 & 1994 & 1995 & Beyond & Total \\
\hline 1 & - & & & & & \\
2 & $a$ & & & & & \\
3 & 20 & $*$ & & & & 20 \\
4 & $b$ & $b$ & & & &
\end{tabular}

Status:

Funded

Requested

New

*Estimated annual ongoing cost: $\$ 85 \mathrm{~K}$.

${ }^{a}$ See Finding OP.4-1 for conduct of operations training costs.

${ }^{b}$ Included in cost for FTE in Item 3.

References: $\quad$ ORNL Long-range Plan, 1989-95, ORNL 65-25

FWP ERAT850, ORNL Safety and Health -- Regulatory Compliance, June 1, 1990 
Finding No.: AX.2-1 No Implementation of the Waste Minimization Policy

Finding

Description: ORNL has not implemented the 1986 Energy Systems Waste Minimization Policy.

Code: $\quad$ Category III

Compliance

Protocol:

Tennessee Hazardous Waste Management Rule 1200-1-11-.03, RCRA Section 3002 , and DOE Order 5400.3 require waste minimization plans and/or programs for hazardous and radioactive mixed waste generating facilities. DOE

Order 5820.2A requires DOE facilities to establish an auditable waste reduction program for LLW. DOE Order 5400.1 requires preparation of a waste reduction program plan, which must be reviewed annually and updated every three years. In 1985 ORNL issued a letter setting forth hazardous waste minimization as a Laboratory Policy. In addition, in 1986 Energy Systems issued a policy letter calling for implementation of a comprehensive waste minimization program.

Priority: $\quad$ Energy Systems Risk Weight 56

Tiger Team Action Plan Priority 2

Response: $\quad$ See Finding WM/BMPF-2.

Planned Actions and Schedules:

This finding is fully addressed by actions listed in response to Finding WM/BMPF-2.

Costs: $\quad$ Costs associated with this finding are reported under Finding WM/BMPF-2.

References: None 
Finding No.: AX.3-1 Energy Systems Policy Procedure on Waste Management

Finding

Description: The draft Martin Marietta Energy Systems Policy Procedure ESH-18, "Waste Management," does not address goals and operating procedures for minimizing radioactive wastes, and its training objectives do not include making generators of wastes more sensitive to the value of full implementation of the policy.

Code: $\quad$ Category III

Compliance

Protocol: Solid hazardous wastes (including radioactive wastes) should be controlled to minimize the volume generated and handled in a manner that provides safe storage and transportation.

Priority: $\quad$ Energy Systems Risk Weight 6

Tiger Team Action Plan Priority 3

Response: $\quad$ Energy Systems Policy Procedure ESH-18 was issued as a review draft for comments by the installation prior to the issuance of DOE Order 5400.1 which contains detailed guidance on waste minimization requirements. The draft will be revised to incorporate the requirements of that order as well as additional guidance received for implementing DOE Order 5820.2A "Waste Management." The schedule for revision of the draft Energy Systems Policy Procedure ESH-18 "Waste Management" is addressed by this action plan. ESH-18 will be revised to address the comments of the Tiger Team as well as comments received from the installations, other reviewers, and to reflect changes in regulations. In response to the Tiger Team comments, the revision of the waste minimization section of the Policy Procedure will specifically include the addition of direction on the establishment of waste minimization goals, reference to operating procedures as a means of reducing radioactive waste volumes and radioactivity levels of the waste, and the requirements to provide generator/employee awareness training of the overall benefits of waste minimization.

\section{Root Cause:}

Inadequate policy

Planned Actions and Schedules:

$\underline{\text { Item/Description }}$

1. Draft and distribute for review revision to ESH-18 incorporating current DOE requirements.

2. Issue revised ESH-18.

\section{Completion Date}

$6 / 91$

$10 / 91$ 


\section{Casts:}

Type of funds: Overhead

Source of funds: Overhead

\begin{tabular}{cccccccc} 
& \multicolumn{5}{c}{ Estimated costs per fiscal year (\$K) } & \\
\cline { 2 - 6 } Action item & 1991 & 1992 & 1993 & 1994 & 1995 & Beyond & Total \\
\hline $1 \& 2$ & 15 & & & & & & 15
\end{tabular}

Status:

Funded

15

Requested

New

References: None 
Finding No.: AX.4-1 Fissile Material Storage Handling Activities

Finding

Description

Fissile material storage handling activities are not in compliance with DOE 5480.5.

Code:

Category III

Compliance

Protocol:

DOE Order 5480.5

Prionity: $\quad$ Energy Systems Risk Weight 56

Tiger Team Action Plan Priority 2

Response: The fissile materials stored in Building 3033 Annex are contained in either plastic or glass inner containers which are sealed in a metal, carbon steel isotope can. The voids between the inner container and the outer metal container are packed with a sorbent material. This outer isotope can is sealed by double crimping, similar to commercial juice cans. Recent, unanticipated, high-moisture conditions in the storage area of Building 3033 Annex resulted in the rusting of some of these carbon steel isotope cans. The outer metal container, or isotope can, is considered to be the primary container for the fissile material.

Prior to the Tiger Team visit, planning for the disposition of all material which was previously in the Isotope Production and Distribution Program (IPDP) was begun. All of the material in Building 3033 Annex was included in these planning activities, in that it was formerly in the IPDP and is now part of the Isotopes Facilities Shutdown Program (IFSP) at ORNL. This plan will address moving this fissile material to a more stable, long-term storage site. The present plan, as a result of this finding, is to take each package that is now in the $\mathbf{3 0 3 3}$ Annex (that includes both fissile material and other alpha emitters stored in the facility) to disassemble the present isotope cans, to document the integrity of the inner container (either the glass or plastic container), place that inner container into a new metal container and add back the necessary sorbent material, and seal the outer metal container. As a part of this repackaging, an evaluation of a metal container that meets the requirements of DOE Order 5480.5 with respect to corrosion and container integrity will be developed. The final details of this repackaging activity and disposition of all this material is still under development. The material will be moved to either the 3027 Storage Vault or to Building 3019.

Deficiencies in safety documentation (i.e., the references to container integrity and qualification of materials handling) will be addressed in the FSET Phase I for the facilities for which this material will be stored for the long-term. FSET Phase I will not be required for the 3033 Annex because the plan is to remove the fissile and other material from this facility prior to the completion of the FSET Phase I evaluation. 


\section{Root Causes:}

Inadequate policy, inadequate policy implementation, and inadequate training

Planned Actions and Schedules:

\section{Item/Description}

1. Identify appropriate containers that meet the requirements for DOE Order 5480.5 relative to the storage of plutonium and other fissile materials.

2. Issue plans and procedures for repackaging material for shipment to a final storage site.

3. Address requirements of DOE Order 5480.5 in FSET Phase I for 3027 Storage Vault and Building 3019.

4. Repackage all materials and transfer to an appropriate storage site.

5. Using strategies developed and lessons learned from $3033 \mathrm{~A}$ effort, and as required by DOE Order 5480.5 , para. 13.b., repackage fissile material stored in a laboratory or other storage area in a quantity greater than ten grams in containers identified in item \#1 or in other containers that meet fissile material storage requirements. (Quantities of ten grams or less will be treated the same as other high-toxicity alpha-emitters.)

\section{Completion Date}

Complete 


\section{Costs:}

Type of funds: ERWM Programmatic

Source of funds: EM-ADS387

\begin{tabular}{|c|c|c|c|c|c|c|c|}
\hline \multirow[b]{2}{*}{ Action item } & \multicolumn{6}{|c|}{ Estimated costs per fiscal year $(\$ K)$} & \multirow[b]{2}{*}{ Total } \\
\hline & 1991 & 1992 & 1993 & 1994 & 1995 & Beyond & \\
\hline 1 & 3 & & & & & & 3 \\
\hline 2 & 5 & & & & & & 5 \\
\hline 3 & 68 & & & & & & 68 \\
\hline 5 & & 25 & & & & & \\
\hline \multicolumn{8}{|l|}{ Status: } \\
\hline \multicolumn{8}{|l|}{ Funded } \\
\hline Requested & 76 & & & & & & \\
\hline New & & 25 & & & & & $\$ 101$ \\
\hline
\end{tabular}




\section{Type of funds: Overhead}

Source of funds: Overhead

\begin{tabular}{|c|c|c|c|c|c|c|c|}
\hline \multirow[b]{2}{*}{ Action item } & \multicolumn{6}{|c|}{ Estimated costs per fiscal year (\$K) } & \multirow[b]{2}{*}{ Total } \\
\hline & 1991 & 1992 & 1993 & 1994 & 1995 & Beyond & \\
\hline 4 & 4 & & & & & & 4 \\
\hline \multicolumn{8}{|l|}{ Status: } \\
\hline Funded & 4 & & & & & & \\
\hline \multicolumn{8}{|l|}{ Requested } \\
\hline New & & & & & & & $\$ 4$ \\
\hline
\end{tabular}

Type of funds: Research Programmatic

Source of funds: DP-GE

\begin{tabular}{cccccccc} 
& \multicolumn{6}{c}{ Estimated costs per fiscal year $(\$ K)$} \\
\cline { 2 - 6 } Action item & 1991 & 1992 & 1993 & 1994 & 1995 & Beyond & Total \\
\hline 4 & 4 & & & & & & 4
\end{tabular}

Status:

Funded

Requested

New

4

References: $\quad$ JOE Order 5480.5 . 
Finding No.: AX.4-2 Building 3027 Storage Vault Operation

Finding

Description: Contrary to the requirements of DOE 5633.3, ORNL is not in compliance with the need for organizational independence in the operation of Bldg. 3027 Storage Vault.

Code: $\quad$ Category III

Compliance

Protocol:

DOE Order 5633.3

Prionity: $\quad$ Energy Systems Risk Weight 55

Tiger Team Action Plan Priority 2

Response: To resolve the organizational independence issue, external auditors will be exclusively utilized to conduct all requirec' Materials Control and Accountability (MC\&A) audits of the ORNL Central SNM Storage Vault [Material Balance Area (N [BA 06)] which will remain under the management/operational control of the Safeguards and Security Department. New guidance provided by the DOE-ORO MC\&A Branch now allows for the conduct of ruatine and special audits within Energy Systems by use of external auditors. Independent auditing by an organization external to the ORNL Laboratory Protection Division will ensure compliance with the DOE Order 5633.3 rule on organizational independence.

Root ('ause:

Poorly defined roles and responsibilities

Planned Actions and Schedules:

\section{Item/Description}

1. Coordinate with the MC\&A activity at the K-25 Plant on performance of required MC\&A audits of the ORNL Central SNı. Storage Vault (MBA 06) by auditor(s) assigned to K-25.

2. Formalize agreement with $\mathrm{K}-25 \mathrm{MC} \& \mathrm{~A}$ activity for performance of required MC\&A audits of the ORNL Central SNM Storage Vault by auditor(s) assigned to K-25.

\section{Completion Date}

Complete

Complete 
3. Conduct initial external audit of MBA 06 .

Complete

Costs: $\quad$ Additional costs will not accrue to the Laboratory as a result of realignment of audit responsibility for the Storage Vault.

References: $\quad$ DOE Order 5633.3, Chapter 1, paragraph 1.b. 
Finding No.: AX.5-1 Gaseous Effluent Discharges

Finding

Description: ORNL is not in compliance with Martin Marietta Energy Systems as low as reasonably achievable (ALARA) policy and DOE 6430.1A because it is not qualifying or quantifying many gaseous effluent discharges.

Code: $\quad$ Category III

Compliance

Protocol:

DOE Order 6430.1A

Priority: $\quad$ Energy Systems Risk Weight 63

Tiger Team Action Plan Priority 2

Response: The emissions from ORNL facilities are regulated by the Tennessee Department of Health and Environment (TDHE). In response to requests from TDHE to all Energy Systems sites in Tennessee, a plan to demonstrate compliance with established de minimis levels (below concern) is under development. To demonstrate compliance with these requirements, administrative limits on the amounts of materials that can be released from each release point at ORNL will be developed, and documentation will be required of each facility manager.

\section{Root Causes:}

Inadequate policy implementation and ambiguous regulations and expectations

Planned Actions and Schedules:

\section{$\underline{\text { Item/Description }}$}

1. Meet with TDHE regulators to discuss compliance agreement.

2. Document proposed approach to TDHE.

3. Develop emissions inventory.

4. Establish administrative limits for each hazardous and radioactive chemical released from each facility.

5. Perform audits of facility records.
Completion Date

Complete

Complete

$5 / 91$

$7 / 91$

9/91 


\section{Costs:}

Type of funds: Overhead

Source of funds: Overhead

\begin{tabular}{cccccccc} 
& \multicolumn{5}{c}{ Estimated costs per fiscal year (\$K) } \\
\cline { 2 - 5 } Action item & 1991 & 1992 & 1993 & 1994 & 1995 & Beyond & Total \\
\hline 1 & - & & & & & & \\
2 & - & & & & & & \\
3 & 50 & & & & & \\
4 & 30 & & & & & \\
5 & 10 & & & & &
\end{tabular}

Status:

Funded

90

Requested

New

References: None 
Finding No.: AX.6-1 Backup Power Diesel Generators

Finding

Description: Periodic testing of diesel generators supplying backup power for vital systems is not in accordance with recommended standards.

Code: $\quad$ Category III

Compliance

Protocol:

Priority:

Response:
Periodic testing of diesel generators should be done in accordance with recommended standards.

Energy Systems Risk Weight 5

Tiger Team Action Plan Priority 3

This action plan addresses the periodic testing of diesel generators supplying backup power to vital systems. ANSI/IEEE 446-1987 and National Fire Protection Association Standard NFPA-110 contain guidelines for testing emergency and standby power systems. Copies will be obtained, analyzed, and necessary changes made.

\section{Root Cause:}

Inadequate policy implementation; testing procedures were written using manufacturer's recommended test.

Planned Actions and Schedules:

\section{$\underline{\text { Item/Description }}$}

1. Classify diesel generators per instructions in NFPA-110.

2. Issue revised diesel generator testing procedure to comply with ANSI/IEEE 446-1987 and NFPA-110.
Completion Date

$6 / 91$

$9 / 91$ 
Costs:

Type of funds: Overhead

Source of funds: Overhcad

\begin{tabular}{crrrrrrr} 
& \multicolumn{5}{c}{ Estimated costs per fiscal year (\$K) } & \\
\cline { 2 - 6 } Action item & 1991 & 1992 & 1993 & 1994 & 1995 & Beyond & Total \\
\hline 1 & 1 & & & & & & 1 \\
2 & 1.5 & & & & & & 1.5
\end{tabular}

Funded

2.5

Requested

New

References: None 


\subsubsection{Emergency Preparedness}

Finding No.: EP.1-1 Accident Consequence Assessment

Finding

Description: ORNL has not completely analyzed credible accidents as required by DOE 5500.1A nor has it clearly assigned the responsibilities to do so.

Code: $\quad$ Category III

Compliance

Protocol:

DOE Order 5500.1A

Priority: $\quad$ Energy Systems Risk Weight 109

Tiger Team Action Plan Priority 2

Response: $\quad$ Energy Systems has an ongoing SAR update program to conduct Hazard Classification and Qualitative Analysis (Phase I) and Quantitative Accident Analysis (Phase II) of non-reactor nuclear and selected other facilities with significant hazard potential (see Finding FR.2-1). The primary aspect of this program is based on a risk prioritization of activities. This program is designed to address all facilities simultaneously. Additionally, Industrial Hygiene has undertaken a comprehensive facility survey to identify and evaluate all ORNL operations with respect to potential health hazards and OSHA compliance (see Finding IH.2-2). The results of these inquiries will provide information upon which analysis of major credible accidents can be based.

The installation facility safety manager is responsible for analyzing and evaluating potential accidents and their associated risks.

Root Causes:

Poorly defined roles and responsibilities

Planned Actions and Schedules:

$\underline{\text { Item/Description }}$

1. Issue a revised position charter for the emergency preparedness department head to assign the responsibility for coordinating identification and assessment of major credible accidents for emergency planning.

2. Identify the major credible accidents at ORNL. (See Finding EP.1-4.)
Completion Date

Complete

9/91 
3. Prepare prioritized schedule for analysis of the major credible accidents.

4. Analyze major credible accidents for emergency preparedness planning.

\section{Casts:}

Type of funds: ESH Programmatic

Source of funds: ER-AT EP

\begin{tabular}{lrrrrrrr} 
& \multicolumn{5}{c}{ Estimated costs per fiscal year (\$K) } & \\
\cline { 2 - 6 } \multicolumn{1}{c}{ Action item } & 1991 & 1992 & 1993 & 1994 & 1995 & Beyond & Total \\
\hline $\begin{array}{l}\text { Status: } \\
\begin{array}{l}\text { Funded } \\
\text { Requested }\end{array}\end{array}$ & 15 & 5 & 55 & $*$ & & 75 \\
New & 15 & 5 & 55 & & & & \\
\hline
\end{tabular}

*Estimated annual ongoing cost: $\$ 50 \mathrm{~K}$.

References: $\quad$ Energy Systems Comprehensive Emergency Management Plan

Energy Systems Policy Procedures ESH-8 
Finding No.: $\quad$ EP.1-2 Emergency Preparedness Recommendations

Finding

Description: ORNL has no effective system to ensure completion of recommendations in emergency preparedness.

Code: $\quad$ Category III

Compliance

Protocol:

DOE Order 5500.3A (Draft)

Priority: $\quad$ Energy Systems Risk Weight 11

Tiger Team Action Plan Priority 3

Response: The Emergency Response Action Items (ERAI) tracking system which has been used by the Laboratory Shift Superintendent's office is obsolete and not compatible with current equipment. Effective December 1, 1990, the quality auditing function was assigned to the Quality Department. As a part of the reorganization, the Quality auditing managers will become the single point for tracking corrective actions. (See Finding QV.1-6.)

Root Causes:

Inadequate oversight

Planned Actions and Schedules:

Item/Description

1. Implement the Evaluation Data Base System (EDS), the corrective action tracking system prescribed by the Quality Department to ensure completion of emergency preparedness (EP) recommendations.

2. Enter all outstanding EP items to be tracked into the prescribed system.

3. Issue a procedure for entering future items, maintaining contact with persons responsible for action, verifying completion, and closing out of findings.
Completion Date

Complete

Complete

Complete 
Costs:

Type of funds: ESH Programmatic

Source of funds: ER-AT EP

\begin{tabular}{|c|c|c|c|c|c|c|c|}
\hline \multirow[b]{2}{*}{ Action item } & \multicolumn{6}{|c|}{ Estimated costs per fiscal year (\$K) } & \multirow[b]{2}{*}{ Total } \\
\hline & 1991 & 1992 & 1993 & 1994 & 1995 & Beyond & \\
\hline 1 & - & $*$ & & & & & - \\
\hline 2 & 25 & & & & & & 25 \\
\hline 3 & - & & & & & & 20 \\
\hline \multicolumn{8}{|l|}{ Status: } \\
\hline \multicolumn{8}{|l|}{ Funded } \\
\hline \multicolumn{8}{|l|}{ Requested } \\
\hline New & 25 & & & & & & $\$ 25$ \\
\hline
\end{tabular}

*Estimated annual ongoing cost: \$5K.

References: Energy Systems Comprehensive Emergency Management Plan DOE Order 5500.3A (Draft)

DOE Order 5700.6B

Laboratory Protection Division Procedure EP-22. 
Finding No.: EP.1-3 Facility Hazards Information

Finding

Description: The system of building emergency plans and local emergency supervisors in the $\mathrm{X}-10$ Site does not ensure that emergency responders are provided information on hazards in facilities.

Code: $\quad$ Category III

Compliance

Protocol:

Priority: $\quad$ Energy Systems Risk Weight 69

Tiger Team Action Plan Priority 2

Response: Building emergency plans (local emergency manuals) maintained by local emergency supervisors are only one of the means used to provide information to emergency responders on hazards in facilities. Each major facility has its own local emergency squad whose members are aware of the hazards where they work through their individual job assignments, through the hazards communication (HAZCOM) program, and through annual training for their local emergency squad. During regular work hours, Monday - Friday, members of the local emergency squad will meet the site shift emergency squad to direct them to the scene of an incident and advise them of hazards which are present. During off-shifts when most ORNL facilities are unoccupied and their local emergency squads are off-duty, the site shift emergency squad relies on its training (which includes Radiation-Worker Training, Special Access Training for particular facilities, Confined Space Entry Training, and annual Emergency Responder Training), pre-fire plans, and posted warning signs to provide indication of hazards in facilities.

Efforts are underway which will provide emergency responders more definitive information on hazards in facilities. Industrial Hygiene has undertaken a comprehensive facility survey to identify and evaluate all ORNL operations with respect to potential health hazards and OSHA compliance (see Finding IH.2-2). Also, the Energy Systems SAR Update Program is conducting hazard classification and accident analysis of non-reactor nuclear and selected other facilities with significant hazard potential (see Finding FR.2-1). The results of these investigations will provide pertinent information for local facility managers and their local emergency supervisors regarding the need for more documentation in local emergency manuals and the posting of warning signs. This information also may be incorporated into HAZCOM and special access training programs.

Additionally, Energy Systems is developing a computerized Hazardous Materials Inventory System (HMIS), which will provide near real-time accounting of hazardous materials on-site by location and quantity. HMIS is expected to become operational by the end of 1991 . 


\section{Root Causes:}

Inadequate oversight, inadequate management approach, and poorly defined roles and responsibilities

Planned Actions and Schedules:

\section{Item/Description}

1. Revise the annual review page of each local emergency manual to include a block for countersignature by the local emergency supervisor.

2. Conduct training session for local emergency supervisors to review their responsibilities for providing information on hazards in their facilities.

3. Revise the local emergency manual for Building 2525 to spell out the hazards in the plating shop.

4. Issue requirement for Division Environmental, Safety and Health Action Plans to include performance goals and objectives for identifying and documenting a facility's major credible accidents, informing the occupants and, if applicable, restricting access and posting warning signs where significant health and safety hazards exist.

5. Issue a procedure to provide routine and updated output of the HMIS to emergency responders so that they can maintain a state of informed readiness.

\section{Completion Date}

Complete

Complete

Complete

Complete 


\section{Costs:}

Type of funds: Overhead

Source of funds: Overhead

Estimated costs per fiscal year (SK)

\begin{tabular}{|c|c|c|c|c|c|c|c|}
\hline Action item & 1991 & 1992 & 1993 & 1994 & 1995 & Beyond & Total \\
\hline 1 & 2 & & & & & & 2 \\
\hline 2 & 20 & & & & & & 20 \\
\hline 3 & 3 & & & & & & 3 \\
\hline 4 & 5 & & & & & & 5 \\
\hline 5 & & 10 & & & & & 10 \\
\hline
\end{tabular}

Status:

Funded

30

Requested

10

New

Estimated annual ongoing cost: \$10K starting in FY 1993.

References: DOE Order 5500.3A (Draft) 
Finding No.: EP.1-4 Analysis of Emergency Preparedness

Finding

Description.

Code:

Compliance

Protacal.

Priorisy:

Response:
ORNL facilities have not been surveyod to identify potentially suiti wus emergency preparedness deficienciex.

Calcgon. III

DOE Order $5500.3 \mathrm{~A}$ (Draft)

Energy Systems Risk Weight 66

Tiger Team Action Plan Priority 2

As s::eded in EP.1-1 and EP.1-3. Industrial Hygiene has undertaken a comprehensive facility survey to identify and evaluate all ORNL operations with respect to potential health hazards and OSHA compliance (see Finding IH.2-2).

Also. the Energy Systemis SAR Update Program is conducting hazard classification and accident analysis of non-reactor ruclear and selected other facilities with significant hazard potential (see Finding FR.2-1). The results of these studies will provide pertinent information relating to emergency preparedness deficiencies.

Energy Systems has assigned each host site responsibility for emergency preparedness. Ail ORNL facilities at the Y-12 Plant fall under the jurisdiction of the Y-12 emergency preparedness organization and they must abide by $\mathrm{Y}-12$ emergency preparedness policy and procedures. A memorandum of understanding establishes this arrangemerit and is signed by both site managers (see

Finding OA.1-5).

The emergency procedures for Building 9204-3, located at the Y-12 Plant, have been reviewed and accepted by the Y-12 Emergency Preparedness Department. Following the Tiger Team visit there on November $2,19^{\circ} 0$, it was determined that the test button for that building's high level alpha stack alarm is designed to be pressed for one minute before actuating the alarm signal to avoid false alarms due to power surges. When so tested, the audio alarm sounded properly.

For consistency throughout the Y-12 Plant, emergency exit doors are painted red. The "Red Door" concept ensures passage to the outside. Periodic evacuation drills are conducted by Y-12 to ensure that their system wolks. Established practice at the X-10 Site involves maps that identify evacuation route(s) to Local Assembly Points. These maps are maintained in local manuals and are also mounted in conspicuous places for reference during emergencies or evacuation drills. These well-established and well-tested methods have proven to be very effective at the respective sites.

\section{Root Causes:}

Insufficient resources, inadequate oversight, and poorly defined roles and responsibilities 
Planned Actions and Schedules:

\section{$\underline{\text { Item/Description }}$}

1. Conduct evacuation drills for all facilities at the $X-10$ Site to familiarize occupants with the evacuation routes and location of local assembly points.

2. Survey ORNL facilities to further identify potentially scrious emergency preparedness deficiencies.

3. Prioritize identified deficiencies and budget for corrective action (see Finding EP.1-1).

4. Complete correction of deficiencies.

Costs: $\quad$ Cost of actions listed is included in Finding EP.1-1.
Additional funds to be determined following survey completion.

Costs: $\quad$ Cost of actions listed is included in Finding EP.1-1.
Additional funds to be determined following survey completion.

References: $\quad$ DOE Order 5500.3A (Draft)
Completion Date

Complete

Complcte

$9 / 92$

$9 / 93$ 
Finding No.: EP.2-1 Classification of Emergency Events

Finding

Description: The ORNL classification of emergency events is not consistent with DOE 5500.2A, DOE N5500.5, and the State of Tennessee Emergency Plan.

Code: $\quad$ Category III

Compliance

Protocol:

Priority: $\quad$ Energy Systems Risk Weight 56

Tiger Team Action Plan Priority 2

Response: $\quad$ In Chapter 5 of the Energy Systems Comprehensive Emergency Management Plan (CEMP), four levels of event classification are equated to "reduction of the level of safety of the site"; whereas, DOE Order 5500.2A, dated 4-13-88, applies those classifications to "reduction of the level of safety of the facility." The $X-10$ Site Emergency Plan erroneously adopted the CEMP verbiage. Laboratory Shift Superintendent's (LSS) Procedure EP-6 employs the correct terminology.

\section{Root Causes:}

Inadequate policy implementation and ambiguous requirements or expectations

Planned Actions and Schedules:

\section{$\underline{\text { Item/Description }}$}

1. Notify the Enc $\epsilon^{y}$ Systems Central Staff Emergency

Preparedness Manager of the aforementioned discrepancy in the CEMP published by his office.

2. Issue a change to the $X-10$ Site Emergency Plan correcting the event classification section.

3. Revise LSS Procedure EP-6 to acknowledge that emergencies may result from release of hazardous materials that are not radioactive.
Completion Date

Complete

$9 / 91$

$9 / 91$ 
Costs:

Type of funds: ESH Programmatic

Source of funds: ER-AT EP

Estimated costs per fiscal year $(\$ \mathrm{~K})$

\begin{tabular}{lrrrrrrr}
\cline { 2 - 5 } Action item & 1991 & 1992 & 1993 & 1994 & 1995 & Beyond & Total \\
\hline \multicolumn{1}{c}{ All } & 10 & & & & & & 10 \\
$\begin{array}{l}\text { Status } \\
\text { Funded } \\
\text { Requested } \\
\text { New }\end{array}$ & 10 & & & & \\
\end{tabular}

References: $\quad$ DOE Order 5500.2A

DOE Order N5500.5

State of Tennessee Emergency Plan

Energy Systems Comprehensive Emergency Management Plan

ORNL $X-10$ Site Emergency Plan

LSS Procedure EP-6 
Finding No.: $\quad$ EP.2-2 Adequacy of ORNL Emergency Plans

Finding

Description:

ORNL Energency Plans are confusing and incomplete. Implementing procedures have not been prepared as required by DOE 5500.1A.

Code: $\quad$ Category III

Compliance

Protocol:

Priority: $\quad$ Energy Systems Risk Weight 106

Tiger Team Action Plan Priority 2

Response: During FY 1990, the top priority for the new Emergency Preparedness Department was the correction of four Category II concerns from the 1989 Radiation Protection Technical Safety Appraisal. In December 1989, Energy Systems issued the Comprehensive Emergency Management Plan (CEMP), which, for the first time, established corporate standards. The ORNL counterpart to the CEMP, the $X-10$ Site Emergency Plan, was issued in February 1991. The absence of a site-level emergency plan contributed to the confusion cited above.

The field monitoring teams referred to in the site plan were established March 1991 (see Finding EP.5-1). Chapter 8 of the site plan addresses the duties of all ORNL Emergency Operations Center (EOC) cadre positions.

See Finding EP.6-1 for comments concerning protective action guides and site boundaries. In the absence of other criteria, ORNL emergency responders use the U.S. Department of Transportation 1990 Emergency Response Guidebook to Hazardous Materials Incidents (DOT P 5800.5). Additionally, the Laboratory Shift Superintendent (LSS) and the EOC cadre may refer to the American Conference of Governmental Industrial Hygienists (ACGIH) pamphlet 1990-1991 Threshold Limit Values for Chemical Substances and Physical Agents and Biological Exposure Indices and the NIOSH Pocket Guide to Chemical Hazards (DHHS

Pub. No. 85-114). Further, Energy Systems has developed a computerized data base of Material Safety Data Sheets as part of its Hazardous Materials Inventory System (HMIS) (see Finding EP.1-3). Together with consultation with knowledgeable facility experts, these documents provide protective guidance which can be applied on an ad hoc basis.

ORNL currently has 25 emergency preparedness implementing procedures (18 LSS procedures, 3 ORNL standard practice procedures (SPP), and 4 Laboratory Protection Division procedures). Two more SPPs are in draft and will be issued after the review and approval process.

Root Causes:

Ambiguous requirements or expectations 
Planned Actions and Schedules:

Item/Description

1. Issue the $X-10$ Site Emergency Plan.

2. Issue copy of DOT P 5800.5 to each member of site shift emergency squad.

3. Instruct shift emergency squad on use of DOT P 5800.5.

4. Revise EOC cadre position checklists to include review and approval sheet and use of current position titles.
Completion Date

Complete

Complete

Complete

$12 / 91$

Costs:

Type of funds: Overhead

Source of funds: Overhead

Estimated costs per fiscal year $(\$ \mathrm{~K})$

\begin{tabular}{crrrrrrr}
\cline { 2 - 5 } Action item & 1991 & 1992 & 1993 & 1994 & 1995 & Beyond & Total \\
\hline 1 & 18 & & & & 18 \\
2 & 2 & & & & & 2 \\
3 & & & & & & \\
4 & & 20 & & & & \\
& & 20 & & &
\end{tabular}

Status:

Funded

20

Requested

20

New

References: $\quad$ DOE Order 5500.1A

DOT P 5800.5

CEMP

ACGIH: 1990-1991 TLV/BEI

DHHS Pub. No. 85-114 
Finding No.: EP.3-1 Training for Emergency Functions

Finding

Description: Individuals may be called upon in an emergency to perform functions for which they have not been trained.

Code: $\quad$ Category III

Compliance

Protocol:

DOE Order 5500.3A (Draft)

Prionity: $\quad$ Energy Systems Risk Weight 7

Tiger Team Action Plan Priority 3

Response: Historically, individuals were expected to perform emergency duties by virtue of their primary jot assignment. For example, there is one health physics (HP) technician on duty during of:-shifts at ORNL; accordingly, when there has been a radiological emergency during off-shifts, that individual automatically responded as a member of the emergency squad. Similarly, management personnel in Security; Fire Protection; Plant and Equipment; Health and Safety; Hazardous Waste Operations Group; and Reactor Operations have been called upon to serve in the Emergency Operations Center with their qualifications being based on their primary job responsibilities. No formal emergency ce:tification was provided for each emergency function. (See Finding REP.3-1.) However, there has been a robust program of drills and exercises at ORNL which has provided emergency response personnel with practical, on-the-job training. See Finding SA-1 for response on self-assessment.

\section{Root Causes:}

Inadequate training, insufficient resources, and inadequate policy implementation

Planned Actions and Scheciules:

\section{$\underline{\text { Item/Description }}$}

1. Establish a requirement that persons be trained or certified before being placed on the emergency cadre.

2. Notify the Emergency Preparedness Department at the Y-12 Plant about the Tiger Team's comment on training their Plant Shift Superintendents in the hazard of plutonium.

3. Ensure that training/certification records have been established for all EOC cadre members.

4. Verify that all shift emergency squad training records have been brought up to date.
Completion Date

Complete

Complete

Complete

$6 / 91$ 
5. Identify emergency functions for each emergency response group.

6. Issue an Emergency Preparedness Training Plan.

7. Identify training requirements and work with the $6 / 92$ Training Department to develop a program of instruction for each emergency function.

8. Train and zertify each individual responder, as required.

Casts:

Type of funds: Overhead

Source of funds: Overhead

\begin{tabular}{|c|c|c|c|c|c|c|c|}
\hline \multirow[b]{2}{*}{ Action item } & \multicolumn{6}{|c|}{ Estimated costs per fiscal year (\$K) } & \multirow[b]{2}{*}{ Total } \\
\hline & 1991 & 1992 & 1993 & 1994 & 1995 & Beyond & \\
\hline $1-4$ & 10 & & * & & & & 10 \\
\hline $5-6$ & & 50 & & & & & 50 \\
\hline 7 & & 50 & & & & & 50 \\
\hline 8 & & & 100 & & & & 100 \\
\hline
\end{tabular}

Status:

Funded 10

Requested

New 100 100 $\$ 210$

*Estimatud annual ongoing cost: \$100 K starting in FY 1993.

References: DOE Order 5500.3A (Draft) $X-10$ Site Emergency Plan 
Finding No.: $\quad$ EP.3-2 Spill Response Training

\section{Finding}

Description: Emergency squad training in spill response lacks depth and rigor to protect emergency responders and mitigate the consequence of spill accidents.

Code: $\quad$ Category III

Compliance

Protocol:

DOE Order 5500.3A (Draft)

Priority: $\quad$ Energy Systems Risk Weight 7

Tiger Team Action Plan Priority 3

Response: The ORNL Hazardous Waste Operations Group (HWOG) is respc asible for mitigating and cleaning up spills on-site. As part of their qualifications, HWOG personnel have been trained to various hazardous materials certification levels, one of which includes the wearing of total encapsulating suits. Members of the shift emergency squad do not receive the extensive training required for that hazardous materials certification level. The shift emergency squad's duties involve assisting HWOG personnel in donning encapsulating suits, obtaining equipment needed by HWOG personnel, and supporting spill cleanup efforts with minimal cxposure to themselves.

Root Cause:

Inadequate training

Planned Actions and Schedules:

$\underline{\text { Item/Description }}$

1. Change Standard Practice Procedure X-GP-2 to reflect that only personnel trained to the appropriate hazardous materials certification level are permitted to wear total encapsulating suits.

2. Issue procedures clearly defining roles and interfaces for emergency responders in the mitigation of hazardous materials spills.

3. Train emergency squads to cariy out the spill responsibilities to which they are assigned.

Costs: $\quad$ Estimated annual ongoing cost: \$50K starting in FY 1992.

References: $\quad$ DOE Order 5500.3A (Draft)
Completion Date

Complete

$10 / 91$ 
Finding No.: $\quad$ EP.4-1 Emergency Preparedness Exercises

Finding

Description:

Emergency preparedness exercises are not conducted in a manner to ensure proficient response to emergencics.

Code: $\quad$ Category III

Compliance

Protocol:

DCE Order 5500.3A (Draft)

Priority: $\quad$ Energy Systems Risk Weight 7

Tiger Team Action Plan Priority 3

Response: During FY 1990, the Laboratory had three command post exercises (CPXs) in which only the Emergency Operations Center (EOC) was activated and all field responses were simulated and 12 field training exercises (FTXs) in which emergency units actually responded in the field but the EOC was not activated. All these exercises were conducted during regular work hours. No CPX was conducted during off-shift hours and no integrated emergency management exercise (a combined CPX and FTX) was conducted. Further, all the scenarios for the exercises were written in-house.

Dui ing the integrated emergency management exercise conducted for the Tiger Team on October 31, 1990, the fire captain at the scene was well aware of the consequences of drum failure; accordingly, even after the fire was extinguished, using a deluge set, he maintained a water curtain between the ruptured sulfuric acid tank aind the 20 or more 55-gallon drums marked with "bleach" placards, until the Hazardous Waste Operations Group (HWOG) was able to mitigate the spill. These action were witnessed by the Tiger Team Fire Protection Appraiser who found no fault with the tactics used.

The physical location of the emergency preparedness offices in the same building with the Laboratory Shift Superintendents is not as much a factor in compromising the confidentiality of scenarios as is the large number of people who must be told in advance about a full scale exercise. The artificialities of an exercise imposed by safety, security, logistics, staging, communications, and documentation contribute to the problem. Conversely, when advance notification is incomplete, as cited in finding EP.4-2, insufficient involvement and coordination may result.

\section{Root Causes:}

Inadequate oversight and inadequate management approach 
Planned Actions and Schedules:

Item/Description

1. Iis the $X-10$ Site Emergency Plan, require the Emergency Preparedness Department to conduct and critique at least one integrated emergency management exercise annually.

2. In trie $X-10$ Site Emergency Plan, require the Emergency Preparedness Department to conduct and critique an EOC command post exercise off-shift annually.

3. Conduct at least one tabletop session annually for all EOC cadre personnel, primary and alternates, to review lessons learned from drills and exercises.

4. Utilize an outside scenario writer for one exercise annually.

Casts: $\quad$ Estimated annual ongoing cost: $\$ 150 \mathrm{~K}$.

References: DOE Order 5500.3A (Draft)
Completion Date

Complete

Complete

Complete

Complete 
Finding No.: EP.4-2 Drill Planning

Finding

Description: There was insufficient guidance and plant involvement in drill planning.

Code: $\quad$ Category III

Compliance

Protocol:

DOE Order 5500.3A (Draft)

Priority: $\quad$ Energy Systems Risk Weight 6

Tiger Team Action Plan Priority 3

Response: All disciplines with responsibilities for emergency response have not been involved in the planning of drills and exercises, see Finding SA-1 regarding self-assessments.

\section{Root Causes:}

Inadequate oversight and inadequate management approach

Planned Actions and Schedules:

Item/Description

1. In the $X-10$ Site Emergency Plan, describe the functions of controllers and evaluators in drills and exercises.

2. In the $X-10$ Site Emergency Plan, require training session be given for controllers and evaluators before each drill or exercise.

3. In the annual training for emergency responders, explain the methodology for conducting, controlling, evaluating and critiquing drills and exercises.

4. Develop a checklist to ensure involvement from every appropriate group (discipline) when planning drills and exercises.
Completion Date

Complete

Complete

Complete

$6 / 91$ 
Casts:

Type of funds: Overhead

Source of funds: Overhead

Estimated costs per fiscal year (\$K)

\begin{tabular}{crrrrrrr}
\cline { 2 - 6 } Action item & 1991 & 1992 & 1993 & 1994 & 1995 & Beyond & Total \\
\hline $1-3$ & 10 & $*$ & & & & & 10 \\
4 & 5 & & & & & & 5
\end{tabular}

Status:

Funded

Requested

New

*Er.simated annual ongoing cost: \$10K starting in FY 1992.

References: $\quad$ DOE Order 5500.3A (Draft) 
Finding No.: $\quad$ EP.5-1 Emergency Monitoring of Releases

Finding

Description: Emergency field sampling and monitoring of chemical and radiological releases are not provided for as required by DOE 5500.3.

Code: $\quad$ Category II

Compliance

Protocol:

DOE Order 5500.3A (Draft)

Priority: $\quad$ Energy Sysiems Risk Weight 68

Tiger Team Action Plan Priority 2

Response: This action plan addresses formal establishment of field monitoring teams for ORNL. Such teams, consisting of one representative from industrial hygiene, health physics, and environmental monitoring will be dispatched in the event of a release of hazardous or radioactive materials. They will provide real-time data regarding concentrations of the materials. The information collected by these teams will be used by crisis management personnel to formulate appropriate emergency responses.

\section{Root Causes:}

Inadequate policy implementation, inadequate training, and poorly defined roles and responsibilities

Planned Actions and Schedules:

Item/Description

1. Obtain interim mutual aid agreement from the K-25 Site for their field monitoring team to support ORNL if required until establishment of the $\mathrm{X}-10$ site field monitoring teams.

2. Issue a procedure delineating the roles and responsibilities of the $\mathrm{X}-10$ site field monitoring teams.

3. Procure equipment needed to deploy field monitoring teams.

4. Assign coordinators and team members (three teams-primary/alternate for each position).

5. Conduct technical training for field monitoring teams and coordinators (classroom and field training).
Completion Date

Complete

Complete

Complete

Complete

Complete 
6. Conduct an emergency exercise involving field monitoring teams and coordinators.

Costs:

Type of funds: Overhead

Source of funds: Overhead

\begin{tabular}{|c|c|c|c|c|c|c|c|}
\hline \multirow[b]{2}{*}{ Action item } & \multicolumn{6}{|c|}{ Estimated costs per fiscal year (\$K) } & \multirow[b]{2}{*}{ Total } \\
\hline & 1991 & 1992 & 1993 & 1994 & 1995 & Beyond & \\
\hline 1 & - & & & & & & \\
\hline 2 & 10 & & & & & & 10 \\
\hline 3 & 50 & * & & & & & 50 \\
\hline 4 & - & & & & & & \\
\hline 5 & 15 & * & & & & & 15 \\
\hline 6 & 15 & * & & & & & 15 \\
\hline \multicolumn{8}{|l|}{ Status: } \\
\hline Funded & 90 & & & & & & \\
\hline \multicolumn{8}{|l|}{ Requested } \\
\hline New & & & & & & & $\$ 90$ \\
\hline
\end{tabular}

${ }^{*}$ Estimated annual ongoing cost: $\$ 100 \mathrm{~K}$.

References: Energy Systems Comprehensive Emergency Management Plan

DOE Order 5500.3

Laboratory Protection Division Procedures EP-20 
Finding No: EP.5-2 Regional Radiological Event

Finding

Description: DOE Oak Ridge Operations Office has not maintained the capability to respond to a regional radiological event as required by DOE Order $5500.1 \mathrm{~A}$

Code: $\quad$ Category III

Compliance

Protocol:

DOE Order 5500.1A. The regional Radiological Assistance Program (RAP) should be in compliance with DOE orders and directives.

Priority: $\quad$ Energy Systems Risk Weight 56

Tiger Team Action Plan Priority 2

Response: $\quad$ Lack of DOE funding of the regional RAP has been identified and documented. The Deputy Assistant Secretary for Military Applications (DASMA) has now taken the lead role and has identified funding for FY 1991. In FY 1990, ORO funding was provided for upgrade and maintenance of the ORO RAP vehicle. No HQ DOE guidance has been received on the requirements for the RAP program, (i.e. standardized equipment, RAP vehicle requirements, personnel requirements, etc.). An assessment of response requirements, procedure development, and identification of team members is in progress.

As an interim measure until the actions listed below can be accomplished, we will use the radiological assets of the ORNL Field Moritoring Teams (see Finding EP.5-1) for response to RAP emergencies.

Root Cause:

Inadequate resources

Planned Actions and Schedules:

\section{Item/Description}

1. Identify RAP team members and develop primary and alternate team listings.

2. Submit a Field Work Proposal (FWP) requesting FY 1993 funds needed for ORNL to maintain a RAP response capability.

3. Assess the response requirements and determine the capability needed to meet the requirements.

4. Issue RAP procedures and checklists.
Completion Date

Complete

Complete 
5. Develop a RAP training program and conduct initial $6 / 92$ training.

6. Procure identified equipment needed to enhance the emergency response capability.

7. Conduct an exercise of the RAP program to validate procedures.

\section{Costs:}

Type of funds: ESH Programmatic

Source of funds: ER-AT EP

\begin{tabular}{|c|c|c|c|c|c|c|c|}
\hline \multirow[b]{2}{*}{ Action item } & \multicolumn{6}{|c|}{ Estimated costs per fiscal year $(\$ K)$} & \multirow[b]{2}{*}{ Total } \\
\hline & 1991 & 1992 & 1993 & 1994 & 1995 & Beyond & \\
\hline 1 & - & & & & & & \\
\hline 2 & 5 & & & & & & 5 \\
\hline 3 & 5 & & & & & & 5 \\
\hline 4 & & 10 & & & & & 10 \\
\hline 5 & & 100 & & & & & 100 \\
\hline 7 & & & 100 & & & & 100 \\
\hline
\end{tabular}

Status:

Funded

Requested

10

New

110

100

$\$ 220$

Estimated annual ongoing cost: $\$ 150 \mathrm{~K}$. 
Type of funds: Capital

Source of funds: GPE

Estimated costs per fiscal year (\$K)

\begin{tabular}{crrrrrrr}
\cline { 2 - 5 } Action item & 1991 & 1992 & 1993 & 1994 & 1995 & Beyond & Total \\
\hline 6 & & & & & &
\end{tabular}

Status:

Funded

Requested

New

References: DOE Order 5500.1A 
Finding No.: $\quad$ EP.6-1 Protective Action Guides/Emergency Action Levels

Finding

Description: Protective Action Guides are not established for ORNL. Emergency Action Levels exist only for the High Flux Isotope Reactor.

Code: $\quad$ Category III

Compliance

Protocol:

DOE Order 5500.3A (Draft)

Prionity: $\quad$ Energy Systems Risk Weight 56

Tiger Team Action Plin Priority 2

Response: The lack of on-site protective action guides (PAGs) is a problem throughout the entire DOE complex, nation-wide. A DOE-HQ subcommittee on dose assessment has provided no definitive instructions to the field. The EPA radiological guides do not address hazardous chemicals. The American Industrial Hygiene Association's Emergency Response Planning Guidelines (EPRGs) are specific for a very limited number of chemicals and don't begin to approach the thousands of chemicals used at ORNL.

In the absence of other criteria, ORNL emergency responders use the U.S. Department of Transportation's 1990 Emergency Response Guidebook to Hazardous Materials Incidents (DOT P 5800.5). Copies of this document have been issued to ail members of the site shift emergency squad and are used as a text in their annual training.

Additional references available to the Laboratory Shift Superintendent are the American Conference of Governmental Industrial Hygienists (ACGIH) pamphlet 1990-1991 Threshold Limit Values for Chemical Substances and Physical Agents and Biological Exposure Indices and the NIOSH Pocket Guide to Chemical Hazardous (DHHS Pub. No. 85-114).

Neither DOE nor Energy Systems can unilaterally establish PAGs applicable to off-site areas. In December 1990, DOE negotiated am Agreement-In-Principle (AIP) with the State of Tennessee which, when ratified, will provide a formal mechanism by which PAGs for the entire Oak Ridge Federal Reservation (X-10, $\mathrm{Y}-12$, and $\mathrm{K}-25$ ) may be developed in cooperation with the cognizant state agencies.

The boundaries defining the X-10 Site for emergency preparedness purposes are clearly depicted in the $X-10$ Site Emergency Plan and the state of Tennessee Emergency Plan. The most prominent lines of demarcation are the Clinch River along the east, south and west sides and Bear Creek Road along the north side. 


\section{Root Causes:}

Ambiguous requirements or expectations

Planned Actions and Schedules:

\section{Item/Description}

1. In the $X-10$ Site Emergency Plan, define boundaries and stipulate that roadblocks will be used, where necessary, to prohibit and control the movement of traffic through the site during emergencies.

2. Develop interim protective action guides for on-site use.

3. Conduct negotiations with the state of Tennessee regarding off-site protective action guides.

4. Participate in the preparation of protective actions for the most significant radiological hazards for the ORR.

5. Establish emergency action levels after analysis of major credible accidents is completed (see Finding EP.1-1).
Completion Date

Complete

$8 / 91$

$12 / 91$

$3 / 92$

$6 / 92$

\section{Costs:}

Type of funds: Overhead

Source of funds: Overhead

Estimated costs per fiscal year $(\$ \mathrm{~K})$

\begin{tabular}{lrlllllr}
\cline { 2 - 5 } Action item & 1991 & 1992 & 1993 & 1994 & 1995 & Beyond & Total \\
\hline \multicolumn{1}{c}{ All } & 25 & & & & & & 25 \\
$\begin{array}{l}\text { Status: } \\
\text { Funded } \\
\text { Requested } \\
\text { New }\end{array}$ & & & & & \\
\end{tabular}

Estimated annual ongoing cost: $\$ 50 \mathrm{~K}$.

References: $\quad$ Energy Systems Comprehensive Emergency Management Plan

DOE Order 5500.3A (Draft)

DOT P 5800.5

ACGIH - 1990-1991 TLV/BEI

DHHS Pub. 85-114

X-10 Site Emergency Plan

State of Tennessee Emergency Plan 
Finding No.: $\quad$ EP.7-1 Personnel Accountability Systems

Finding

Description: The personnel accountability systems in use at ORNL are incomplete.

Code: $\quad$ Category III

Compliance

Protocol:

DOE Order 5500.3A (Draft)

Priority: $\quad$ Energy Systems Risk Weight 130

Tiger Team Action Plan Priority 2

Response: The 1989 Radiation Protection Program Assessment/Technical Safety Appraisal (RPPA/TSA) of the three Energy Systems Oak Ridge sites (Y-12, K-25, and X-10) cited personnel accountability as a Category II concern. Several actions were taken to correct that deficiency, including the development of a Facility Hazard Gradation Matrix that categorized facilities into five degrees of risk with a corresponding requirement for accountability. The ORNL reactors and about 20 non-reactor nuclear facilities were identified as requiring accountability of personnel within 30 minutes. The remainder of the Laboratory was determined to have an adequate system of ensuring evacuation by use of local emergency squads conducting thorough building searches.

Root Causes:

Inadequate policy implementation and inadequate management approach

Planned Actions and Schedules:

\section{Item/Description}

1. Conduct evacuation drills for all facilities at the $\mathrm{X}-10$ Site to familiarize occupants with the location of local assembly points and to test the effectiveness of building searches conducted by local emergency squads.

2. Perform a comprehensive review of personnel accountability at ORNL.

3. Propose a formal personnel-accountability system and review the system through management channels.

4. Issue a procedure for personnel accountability at ORNL.
Completion Date

Complete

9/91

$10 / 91$

$2 / 92$ 
Costs:

Type of funds: Overhead

Source of funds: Overhead

\begin{tabular}{|c|c|c|c|c|c|c|c|}
\hline \multirow[b]{2}{*}{ Action item } & \multicolumn{6}{|c|}{ Estimated costs per fiscal year (\$K) } & \multirow[b]{2}{*}{ Total } \\
\hline & 1991 & 1992 & 1993 & 1994 & 1995 & Beyond & \\
\hline 1 & 50 & $a$ & & & & & 50 \\
\hline 2 & 10 & & & & & & 10 \\
\hline 3 & & 5 & & & & & 5 \\
\hline 4 & & 15 & & & & & 15 \\
\hline
\end{tabular}

Status:

Funded

60

Requested

New

20

Estimated annual ongoing cost: $\$ 25 \mathrm{~K}$

${ }^{a}$ Additional funds to be determined following review completion.

References: Energy Systems Comprehensive Emergency Management Plan

DOE Order 5500.3A 
Füding No.: EP.7-2 Emergency Notification Systems

Finding

Description: Emergency notification systems at ORNL do not ensure notification of personnel.

Code: $\quad$ Category III

Compliance

Protocol:

DUE Order 5500.3A (Draft)

Priority: $\quad$ Energy Systems Risk Weight 133

Tiger Team Action Plan Priority 2

Response: ORNL's public address system consists of a wired network of more than 2200 loud speakers which provide coverage to $80 \%$ of the site. In April 1991, installation started on a radio-linked, outdoor public warning system, consisting of eleven siren/speakers which will augment the PA system, providing coverage within a two mile radius-the Immediate Notification Zone-surrounding the X-10 site. This new public warning system is scheduled to be operational in July 1991.

\section{Root Causes:}

Insufficient resources and inadequate oversight

Planned Actions and Schedules:

\section{Item/Description}

1. Provide audiotapes of ORNL's current alarm signals to the Training Department for playing during General Employee Training/General Employee Access Training (GET/GEAT) classes.

2. Place radio-linked, outdoor public warning system in service.

3. Issue procedure to require monthly testing of public warning system to determine effectiveness and document performance.

\section{Completion Date}

Complete

$7 / 91$

$7 / 91$ 
Costs:

Type of funds: Overhead

Source of funds: Overhead

Estimated costs per fiscal year (\$K)

\begin{tabular}{crrrrrrr}
\cline { 2 - 6 } Action item & 1991 & 1992 & 1993 & 1994 & 1995 & Beyond & Total \\
\hline 1 & 2 & & & & & & 2 \\
2 & $*$ & & & & & & \\
3 & 8 & & & & & & 8
\end{tabular}

Status:

Funded

Requested

10

New

$\$ 10$

*Estimated annual ongoing cost: \$50K; FY 1990 GPP cost $\$ 410 \mathrm{~K}$.

Additional funds to be determined following determination of required upgrades.

References: Energy Systems Comprehensive Emergency Management Plan DOE Order 5500.3A (Draft) 


\subsubsection{Technical Support}

Finding No.: $\quad$ TS.2-1 Safety Analysis Report Update Program

\section{Finding}

Description:

Code: $\quad$ Category III

Compliance

Protocol:

Priority:

Response:
The Safety Analysis Report Update Program does not call for prioritization of the schedule for Operations Safety Requirements preparation based on relative hazard of the facilities.

Best Management Practice

Energy Systems Risk Weight 311

Tiger Team Action Plan Priority 2

Energy Systems and ORNL have recognized for some time the pressing need to upgrade facility Safety Analysis Report (SAR) and accompanying Operational Safety Requirement (OSR) documents. These upgrades have become necessary due to changes in standards which require a significantly higher degree of tecinnical rigor and completeness in safety documentation (SARs, OSRs, and Technical Specifications) than existed in those documents written in the mid-1980s time frame. A phased-approach SAR Upgrade Program has been developed to determine a risk-based prioritization of the facilities and to schedule the upgrading of the facility safety documentation according to this prioritization. Completion of the document upgrades according to the phased-approach schedule is highly dependent on the identification of resources. To date, the costs associated with Phases 0 and $I$ have been borne by the operating divisions out of their program overheads. However, the resources required for Phase II and III upgrade activity are significantly greater, thus requiring appropriate identification of necessary funding within division operating budgets.

The Safety Analysis Report Upgrade Program was developed to apply a risk prioritization of activities in contrast to a serial approach that would upgrade one (or a small group) of SARs and then update the next (or small group). For facilities that require SARs and OSRs, Phase II of the program develops Quantitative Accident Analysis which includes a facility description, the significant accident analysis and the OSRs. For these facilities, the Safety Analysis Report Update Program Overview and Phase I Implementation Plan (YCSET-1) does not explicitly require a prioritization of facilities based on relative hazard. Section 3.8.4 of the Phase I Program Plan does require the development of an Installation Action Plan for Implementation of Phase II. What this Action Plan entails is presently under development but was expected to contain prioritization of facilities based on relative hazard. When the Action Plan requirements are fully developed, the Section 3.8.4 of the Program Plan will be revised to include the prioritization of the facilities. 


\section{Root Causes:}

Inadequate policy and inadequate resources

Planned Actions and Schedules:

\section{Item/Descrintion}

1. Develop Action Plan requirements and Revise Section 3.8.4 of the Safety Analysis Report Update Program Overview and Phase I Implementation (Y/CSET-1). Include in these requirements a prioritization of facilities based on relative hazard.

2. Issue a letter from the Laboratory Directorate to Division Directors detailing the estimated costs associated with the safety documentation and related configuration management (as-built drawing) upgrades based on the projected hazard screening classification of ORNL facilities. These estimates are for inclusion in division operating budget requests to DOE for FY 1992 and beyond prioritized by hazard screening classification.
Completion Date

Complete

Costs:

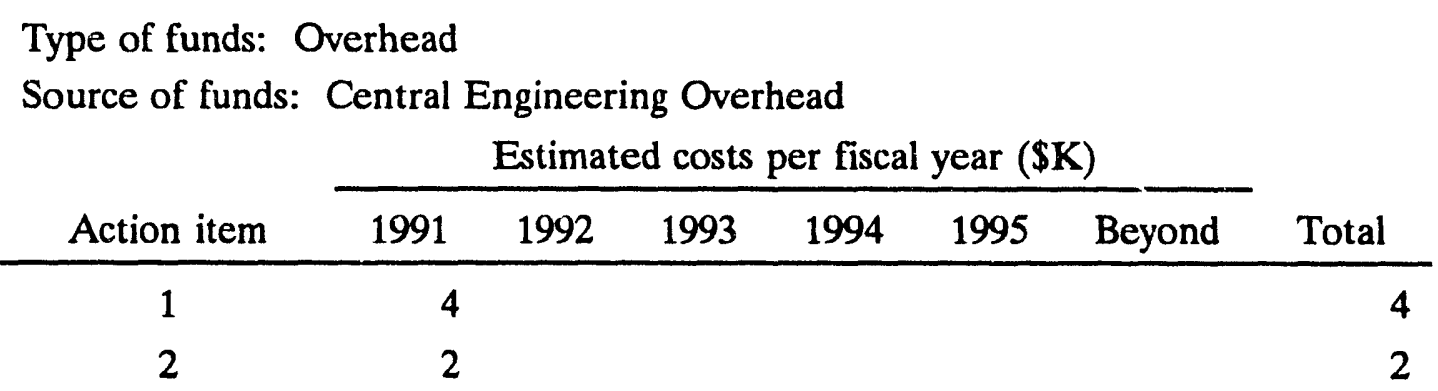

Status:

Funded 6

Requested

New

References: $\quad$ Y/CSET-1 - Safety Analysis Report Update Program Overview and Phase I Implementation 
Finding No.: TS.3-1 Procedures for Low-Cost Facility Modification

Finding

Description: Procedures for low-cost (less than \$2000) modifications of facilities do not require independent evaluation and approval of the proposed change.

Code: $\quad$ Category III

Compliance

Protocol:

Priority: $\quad$ Energy Systens Risk Weight 8

Tiger Team Action Plan Priority 3

Response: The existing SPP on work order system is obsolete. The procedure in use was developed in June 1977 (SPP 38-C). Most of the work orders under \$2000 are for repair work only. However, the concern is that a modification costing less than $\$ 2000$ could have safety implications but is not receiving an independent review. In addition, the $\$ 2000$ threshold was established by the Davis-Bacon Act which says that Energy Systems cannot undertake construction jobs estimated to be more than $\$ 2000$ with Energy Systems craft personnel. These jobs must be referred to the DOE-ORO Davis-Bacon Committee. Therefore, jobs less than $\$ 2000$ are not necessarily reviewed for safety considerations.

\section{Root Causes:}

Inadequate policy, inadequate oversight, and insufficient resources

Planned Actions and Schedules:

\section{$\underline{\text { Item/Description }}$}

1. Issue new work order procedure to incorporate a clear distinction between repairs (without modification) of existing facilities and work that modifies the facility in any way.

2. See Action Item 4 for Finding AX.1-2.
Completion Date

$6 / 91$ 


\section{Costs:}

Type of funds: Overhead

Source of funds: Overhead

\begin{tabular}{|c|c|c|c|c|c|c|c|}
\hline \multirow[b]{2}{*}{ Action item } & \multicolumn{6}{|c|}{ Estimated costs per fiscal year $(\$ \mathrm{~K})$} & \multirow[b]{2}{*}{ Total } \\
\hline & 1991 & 1992 & 1993 & 1994 & 1995 & Beyond & \\
\hline 1 & 15 & & & & & & 15 \\
\hline \multicolumn{8}{|l|}{ Status: } \\
\hline \multicolumn{8}{|l|}{ Funded } \\
\hline \multicolumn{8}{|l|}{ Requested } \\
\hline New & 15 & & & & & & $\$ 15$ \\
\hline
\end{tabular}


Finding No.: TS.3-2 Resource Allocation for Drawing Updates

Finding

Description: In allocating resources to updating of drawings, there is no clear assurance that divisions will receive priority on the basis of need for Safety Analysis Report or Operations Safety Requirements support.

Code: $\quad$ Category III

Compliance Protocol:

Priority:

Response:
Best Management Practice

Energy Systems Risk Weight 311

Tiger Teamı Action Plan Priority 2

The ES\&H Self-Assessment Report, Section 3.7.1, recognizes that the safety analysis process demands that a firm and fixed basis of as-builts exists to perform the required analysis. These as-builts consist of the design drawings for the facility as they reflect field conditions at the time of the analysis, the procedures as they reflect the activities of operation personnel, and other documents that establish the data used in the safety analysis. The SAR Update Program is designed to accommodate these important interfaces by systematically building a body of knowledge that, in turn, earmarks those components and systems requiring configuration management and as-builts.

At present, resources have not been identified to complete the task of as-builts in support of the Safety Analysis Reports other than divisional funding. Funding has previously been requested in ORNL Field Work Proposal for Safety and Health Regulatory Compliance, dated June 1, 1990. Cost estimates for the development of as-built drawings for systems and components requiring configuration management are being refined for inclusion in division operating budget requests. As more information is gained through the SAR Upgrade Program, a more detailed cost estimate will be developed.

The SAR upgrade program is being pursued in three phases. Phase $I$ is a hazard screening analysis that will be completed for all facilities and determines which facilities may require more extensive SAR actions. The schedule and costs for Phases II and III are dependent on the results of the Phase I hazard screening.

\section{Root Causes:}

Inadequate policy and insufficient resources 
Planned Actions and Schedules:

$\underline{\text { Item/Description }}$

Completion Date

1. See Finding TS.2-1, Action Item 2.

2. In conjunction with the SAR Upgrade Program, develop

$6 / 91$ a process to identify those systems, components, equipment, documentation, etc., requiring configuration management and as-built drawing development based on facility hazard screening classification.

3. Complete hazard screening analysis for all facilities with identified funding.

4. Identify funding requirements and scope for Phase II and Phase III of SAR Upgrade Program.

Costs:

Type of funds: Overhead

Source of funds: Overhead

Estimated costs per fiscal year (\$K)

\begin{tabular}{cccccccc}
\cline { 2 - 5 } Action item & 1991 & 1992 & 1993 & 1994 & 1995 & Beyond & Total \\
\hline 2 & 15 & & & & & & \\
\hline
\end{tabular}

Status:

Funded

Requested

New

Type of funds: Research Programmatic

Source of funds: ER

Estimated costs per fiscal year (\$K)

\begin{tabular}{|c|c|c|c|c|c|c|c|}
\hline \multirow[b]{2}{*}{ Action item } & & \multirow[b]{2}{*}{ Total } \\
\hline & 1991 & 1992 & 1993 & 1994 & 1995 & Beyond & \\
\hline 3 & 1609 & & & & & & 1609 \\
\hline \multicolumn{8}{|l|}{ Status: } \\
\hline Funded & 1609 & & & & & & \\
\hline \multicolumn{8}{|l|}{ Requested } \\
\hline New & & & & & & & $\$ 1609$ \\
\hline
\end{tabular}


Type of funds: Research Programmatic

Source of funds: NE

Estimated costs per fiscal year (\$K)

\begin{tabular}{rrrrrrrr}
\cline { 2 - 6 } Action item & 1991 & 1992 & 1993 & 1994 & 1995 & Beyond & Total \\
\hline 3 & 871 & & & & & & 871
\end{tabular}

Status:

Funded

871

Requested

New

$\$ 871$

Type of funds: ERWM Programmatic

Source of funds: EM

Estimated costs per fiscal year (\$K)

\begin{tabular}{llllllll}
\cline { 2 - 6 } Action item & 1991 & 1992 & 1993 & 1994 & 1995 & Beyond & Total \\
\hline 3 & 3366 & & & & & & 3366 \\
Status: & & & & & & & \\
\end{tabular}

Funded

Requested $\quad 3366$

New

$\$ 3366$

References: The Environmental, Safety, and Health Self-Assessment Report

ORNL Field Work Proposal for Safety and Health-Regulatory Compliance, dated June 1, 1990 
Finding No.: TS.4-1 Publication of Unusual Occurrence Reports

Finding

Description: Unusual Occurrence Reports for on-site and off-site occurrences are not sufficiently publicized at ORNL in a summarized, readily comprehended format.

Code: $\quad$ Category III

Compliance

Protocol: None

Priority: $\quad$ Energy Systems Risk Weight 55

Tiger Team Action Plan Priority 2

Response: We are currently in the process of implementing several DOE orders requiring lessons learned. Energy Systems Quality Director is currently revising GP-5.3 to specify an integrated approach for applicability of lessons learned.

Effective December 1,1990 , the quality auditing function was returned to the Quality Department. As a part of the reorganization, the Quality Auditing Manager will become a single point for tracking corrective actions, performing root cause analysis, trending problems and concerns, and establishing a lessons-learned system.

Root Cause:

Inadequate policy; lack of a policy for lessons learned.

Planned Actions and Schedules:

Finding TS.4-1 is fully addressed by actions listed under Finding FR.6-1.

Costs: $\quad$ Costs are reported under Finding FR.6-1.

References: $\quad$ DOE Order 5700.6 and DOE Order 5000.3A 


\subsubsection{Packaging and Transportation}

Finding No.: PT.1-1 Finance and Materials Division Staff

Finding

Description: The Finance and Materials Division is unable to carry out its new responsibilities under the centralized transportation operation due to staff shortages.

Code: $\quad$ Category III

Compliance

Protocol:

Priority:

Response:

\section{None}

Energy Systems Risk Weight 534

Tiger Team Action Plan Priority 2

With the establishment of the Transportation Operations Manager, there was recognition by ORNL management that this function had to be brought up to full staffing with persons trained in the transportation discipline. Finance and Materials Division management identified staffing requirements, and those needs were incorporated into the budget request that was submitted during the summer 1990 budget exercise. Funding has been provided to F\&M Division for two Transportation/Packaging positions and additional requests are pending. However, several packaging and transportation positions remain unfilled.

\section{Root Cause:}

Insufficient resources

Planned Actions and Schedules:

\section{$\underline{\text { Item/Description }}$}

1. Issue position charters and submit in F\&M budget request.

2. Interview and initiate hiring of an on-site transportation specialist.

3. Interview and initiate hiring of a packaging engineer.

4. Resubmit funding request for FY 1992 for additional positions in F\&M budget.
Completion Date

Complete

Complete

$8 / 91$

$8 / 91$ 


\section{Costs:}

Type of funds: Overhead

Source of funds: Overhead

\begin{tabular}{|c|c|c|c|c|c|c|c|}
\hline \multirow[b]{2}{*}{ Action item } & \multicolumn{6}{|c|}{ Estimated costs per fiscal year (\$K) } & \multirow[b]{2}{*}{ Total } \\
\hline & 1991 & 1992 & 1993 & 1994 & 1995 & Beyond & \\
\hline 1 & - & & & & & & \\
\hline 2 & * & & & & & & \\
\hline 3 & ** & & & & & & \\
\hline 4 & - & & & & & & \\
\hline \multicolumn{8}{|l|}{ Status: } \\
\hline \multicolumn{8}{|l|}{ Funded } \\
\hline \multicolumn{8}{|l|}{ Requested } \\
\hline New & & & & & & & $\$$ \\
\hline
\end{tabular}

*Estimated annual ongoing cost: \$133K starting in FY 1991 and $\$ 163 \mathrm{~K}$ starting in FY 1992.

**Already funded in FY 1990 budget.

References: $\quad$ Finance and Materials Division FY 1991 Budget Proposal 
Finding No.: PT.1-2 Packaging and Transportation Procedures

Finding

Description:

Code: $\quad$ Category III

\section{Compliance}

Protocol:

Priority:

Response:
Packaging and transportation procedures do not clearly delineate organizational responsibilities as required by DOE 5480.3A (draft) and DOE N 5480.3.

DOE Order 5480.3A (draft) and DOE Order 5480.3

Energy Systems Risk Weight 93

Tiger Team Action Plan Priority 2

The present ORNL Packaging and Transportation (P\&T) procedures are in a state of change and are incomplete. A lack of procedures was identified as early as 1988 when it was recognized that SPP-65 was too generic for the needs of the evolving P\&T program. As a result of an internal P\&T report, a listing of P\&T procedures were identified and development work was started. However, the writing of SPPs was instituted on a priority basis which has resulted in a lack of coordination between Responsibility Sections for each SPP.

Additionally, the P\&T program at ORNL has been in a dynamic state creating organizational confusion as to responsibilities from one time period to another. Areas such as Waste Operi.sions both at ORNL and at the ORNL facilities at Y-12 have just recently become a part of the centralized P\&T program. The development of the Transportation Operations Committee should assist in resolving controversial responsibility and accountability questions in the future.

This problem was recognized in the Self-Assessment report and the identified resources have been requested in the FY 1991 Budget. Present staffing levels hamper a formalized approach to alleviating this problem, but critical procedural requirements are being addressed.

\section{Root Causes:}

Inadequate policy, insufficient resources, and poorly defined roles and responsibilities 
Planned Actions and Schedules:

$\underline{\text { Item/Description }}$

1. Appoint Transportation Operations Committee.

2. Request funding to implement actions.

3. Review and evaluate previously developed SPPs and issue other P\&T SPPs as needed.

\section{Completion Date}

Complete

Complete

$12 / 91$

Casts:

Type of funds: Overhead

Source of funds: Overhead

\begin{tabular}{|c|c|c|c|c|c|c|c|}
\hline \multirow[b]{2}{*}{ Action item } & \multicolumn{6}{|c|}{ Estimated costs per fiscal year (\$K) } & \multirow[b]{2}{*}{ Total } \\
\hline & 1991 & 1992 & 1993 & 1994 & 1995 & Beyond & \\
\hline 1 & 15 & * & & & & & 15 \\
\hline 2 & $75^{a}$ & * & & & & $b$ & 75 \\
\hline \multicolumn{8}{|l|}{ Status: } \\
\hline \multicolumn{8}{|l|}{ Funded } \\
\hline Requested & 90 & & & & & & \\
\hline New & & & & & & & $\$ 90$ \\
\hline
\end{tabular}

*Estimated annual ongoing cost: \$48K starting in FY 1992.

${ }^{a}$ Subcontractor support to expedite the action.

${ }^{b}$ Hiring of a procedure writer will be an ongoing cost.

References: P\&T PIP Report 
Finding No.: $\quad$ PT.1-3 Packaging and Transportation Procedural Documents

\section{Finding}

Description: Packaging and transportation procedural documents do not meet the requirements of DOE 5480.3 and DOE 5480.3A (draft).

Code: $\quad$ Category III

Compliance

Protocol:

DOE Order 5480.3 and DOE Order 5480.3A (draft)

Priority: $\quad$ Encrgy Systems Risk Weight 93

Tiger Team Action Plan Priority 2

Response: As early as 1988 it was recognized that SPP-65 was too general to suffice for the evolving Packaging and Transportation (P\&T) program. During that year an e'fort was mounted to write SPPs in an expedient manner. However, due to staff shortages, only the most cutical SPPs were written from FY 1988 to FY 1990. This resulted in an incomplete procedural system for the P\&T program. A general procedure was completed, and it (along with some detailed checklists already in place) have sufficed to ensure compliant and safe operations in the interim period.

The 1989 PIP report recommended that an umbrella procedure be written and that a complete set of procedures relating to P\&T be developed. The umbrella SPP was completed; however, it was written after completion of four more critical (and detailed) SPPs. The completion of the remaining SPPs is planned, but the pace is slower than desirable and current P\&T resources do not allow for the dedicated FTE necessary to to a comprehensive job.

Root Cause:

See Finding PT.1-2

Planned Actions and Schedules:

$\underline{\text { Item/Description }}$

Completion Date

See Action 3 in PT.1-2.

Costs: $\quad$ See Costs section for Finding PT.1-2.

References: $\quad$ P\&T PIP Report 
Finding No.: PT.1-4 ORNL Onsite Transportation Manual

Finding

Description: There is no approved Onsite Transportation Manual for the X-10 Site, as required by DOE 5480.3 and DOE $5480.3 \mathrm{~A}$ (draft).

Code: $\quad$ Category III

\section{Compliance}

Protocol:

DOE Order 5480.3 and DOE Order 5480.3A (draft)

Priority: $\quad$ Energy Systems Risk Weight 133

Tiger Team Action Plan Priority 2

Response: $\quad$ Work was started on the Onsite Transportation Manual in FY88 and the manual has since been through four drafts. The fourth draft has undergone a Laboratorywide review.

The onsite issue has been quite controversial throughout DOE, and the ORNL manual has been written with a major assumption. That assumption is that the Energy Systems definition of onsite and offsite is acceptable. DOE-ORO is aware oi this definition and has not objected, however a recent request from DOE-ORO may change/clarify the current definitions. If there are major changes in the definition, the current ORNL Onsite Manual would have to undergo some major revisions and/or the access to the ORNL site would be severely impacted.

After Executive Committee approval and, if requested, DOE-ORO approval, the manual can be implemented.

\section{Root Causes:}

Inadequate policy, insufficient resources, inadequate management commitment

Planned Actions and Schedules:

\section{Item/Description}

1. Present final draft of Onsite Transportation Manual for Executive Committee approval.

Costs: $\quad$ See Costs section for Finding PT.1-1.

References: $\quad$ P\&T PIP Report
Completion Date

06/91 
Finding No.: PT.1-5 Crossover of Packaging and Transportation Responsibilities

Finding

Description: Crossover of packaging and transportation responsibilities between line and staff functions at X-10 could result in conflict of interest and potential degradation of safety.

Code: $\quad$ Category III

Compliance

Protocol: None

Priority: $\quad$ Energy Systems Risk Weight 5

Tiger Team Action Plan Priority 3

Response: The Installation Transportation Safety Manager (ITSM) charter will be revised to define the oversight role of the ITSM. This will eliminate any potential conflict of interest and resultant loss of independence by explicitly defining the interaction between the ITSM and the TOM.

Root Cause:

Poorly defined roles and responsibilities

Planned Actions and Schedules:

\section{$\underline{\text { Item/Description }}$}

1. Issue a revised ITSM charter to clarify the roles and responsibilities and the interaction between the TOM and the ITSM. Review the TOM charter for potential conflict of interest with respect to safety oversight.
Completion Date

Complete

Costs: No significant cost associated with this action.

References: None 
Finding No.: $\quad$ PT.1-6 Transportation Program

\section{Finding}

Description: ORNL has not evaluated whether its present transportation program assures compliance with the new Hazardous Materials Transportation Uniform Safety Act of 1990.

Code: $\quad$ Category III

Compliance

Protocol:

Priority: $\quad$ Energy Systems Risk Weight 45

Tiger Team Action Plan Priority 3

Response: The Hazardous Materials Uniform Safety Act was signed into law in November 1990, and ORNL has conducted an evaluation as to compliance. This action is complete.

Soot Cause:

Ambiguous requirements or expectations

Planned Actions and Schedules:

\section{Item/Description}

1. Evaluate impacts of Hazardous Materials Uniform Safety Act on operations at ORNL.
Completion Date

Complete

Costs: $\quad$ Dependent on evaluation of act impacts on ORNL operations (see Finding PT.1-1).

References: Hazardous Materials Uniform Safety Act 
Finding No.: PT.2-1 Hazardous Materials Transportation Information

Finding

Description: Useful and necessary information on the transportation of hazardous materials is not provided to all ORNL employees who have a need to know.

Code: $\quad$ Category III

Compliance

Protocol:

49 CFR 100-177

Priority: $\quad$ Energy Systems Risk Weight 133

Tiger Team Action Plan Priority 2

Response: The ORNL Training Program has made significant progress in the areas of course development and instruction. However, the recently passed Hazardous Materials Transportation Uniform Safety Act will require more strenuous certification requirements be placed on training programs. In addition, there is no formalized approach to determining training needs for a division/department at ORNL. TOMS has been chartered with ensuring that DOT regulations are enforced and thus is responsible for auditing the training program and ensuring proper training through surveillance. The current training programs have been audited and approved by TOMS; however, surveillance has been nonexistent and guidance has been issued on an as-requested basis.

The lack of a formalized approach has created confusion for divisions as to the amount of training their employees are required to have. In many cases too much training is being given and in some cases not enough proper training is being given.

ORNL became aware of this problem in 1988 and formally addressed it in the 1989 P\&T PIP Report.

Root Cause:

Inadequate communications, ambiguous requirements or expectations, inadequate training

Planned Actions and Schedules:

\section{$\underline{\text { Item/Description }}$}

1. Request divisions to identify personnel who are involved in packaging and transportation activities.
Completion Date

Complete 
2. Prepare a list of all job positions involved in packaging 07/91 and transportation activities.

3. Assess the training needs of these personnel by job

$08 / 91$ requirements utilizing the Transportation Operations Committee (see Finding PT.1-2).

4. Identify required training for each job position. (previously identified in Action 1) and, if necessary, recommend new or enhanced training.

5. Perform initial program self-assessment.

$06 / 92$

Costs: $\quad$ See costs associated with Action 1 in Finding PT.1-2.

References: $\quad$ ES\&H Self-Assessment Report, F\&M Budget FY91, Numerous Transportation Safety Committee Reviews of ORNL P\&T Operations 
Finding No.: PT.3-1 Divisional QA Packaging and Transportation Procedures

Finding

Description:

Divisional Quality Assurance procedures for packaging and transporiation are

Code: $\quad$ Category III

Compliance

Protocol: None

Priority: $\quad$ Energy Systems Risk Weight 5

Tiger Team Action Plan Priority 3

Response: $\quad$ Packaging and transportation related procedures should be reviewed and concurred with by ORNL's transportation staff. The division QA plans for packaging and transportation will be revised to require documented review and by the Transportation Operations Specialist.

\section{Root Cause:}

Inadequate policy implementation and inadequate oversight

Planned Actions and Schedules:

\section{Item/Description}

1. Notify divisions to revise division packaging and transportation QA plans to require review of and concurrence with all packaging and transportation related standard operating procedures by the transportation operations manager and the Transportation Program QAS.

2. Define and initiate a system to ensure review of existing divisional packaging and transportation procedures by the transportation operations manager and Transportation Program QAS against the SPP requirements and/or DOT requirements.

\section{Completion Date}

Complete 


\section{Casts:}

Type of funds: Overhead

Source of funds: Overhead

\begin{tabular}{cccccccc} 
& \multicolumn{5}{c}{ Estimated costs per fiscal year (\$K) } & \\
\cline { 2 - 6 } Action item & 1991 & 1992 & 1993 & 1994 & 1995 & Beyond & Total \\
\hline 1 & 10 & & & & & & 10 \\
2 & & 5 & $*$ & & & & 5
\end{tabular}

Status:

Funded

10

Requested

New

*Estimated annual ongoing cost: $\$ 5 \mathrm{~K}$.

References: $\quad$ ORNL QA procedure QA-L-5-100, "Instructions, Procedures, and Drawings," requires the review of procedures to assure that appropriate quantitative or qualitative acceptance criteria have been specified and that QA requirements have been included. 
Finding No.: $\quad$ PT.3-2 Transportation Program Audits

\section{Finding}

Description: Positive and timcly follow-up and action on all transportation program audits has not been done. See also Concern QV.1-6.

Code: $\quad$ Category III

Compliance

Protocol:

Priority: $\quad$ Energy Systems Risk Weight 30

Tiger Team Action Plan Priority 2

Response: The finding leading to this cuncern resulted from the absence of a documented action plan for the SAIC audit. The audit was a result of a request from DOEORO, for ORNL to perform an internal audit prior to the DOE-HQ review of May 1990. The major finding in the SAIC audit involved the lack of DOT regulatory oversight in the offsite shipments of hazardous wastes. Much discussion was generated by this finding; however, action plans were not documented.

Since the SAIC audit there has been an action plan developed concerning the problems in the offsite shipment of hazardous wastes.

This is the only review for which an action plan was not formally developed in the ORNL P\&T discipline.

Root Causc:

Inadequate policy implementation

Planned Actions and Schedules:
Completion Date

1. Develop necessary action plan(s) for outstanding reviews (see Finding QV.1-6). 


\section{Casts:}

Type of funds: Overhead

Scurce of funds: Overhead

\begin{tabular}{|c|c|c|c|c|c|c|c|}
\hline \multirow[b]{2}{*}{ Action item } & \multicolumn{6}{|c|}{ Estimated costs per fiscal year $(\mathbf{\$ K})$} & \multirow[b]{2}{*}{ Total } \\
\hline & $199 \mathrm{i}$ & 1992 & 1993 & 1994 & 1995 & Beyond & \\
\hline 1 & 9 & & & & & & 9 \\
\hline \multicolumn{8}{|l|}{ Status: } \\
\hline Funded & 9 & & & & & & \\
\hline \multicolumn{8}{|l|}{ Requested } \\
\hline New & & & & & & & $\$ 9$ \\
\hline
\end{tabular}

References: $\quad$ SAIC audit dated 3/30/90 
Finding No.: PT.6-1 Onsite Transport of Waste

\section{Finding}

Description: Radioactive and mixed wastes are being transported onsite at X-10 without specific knowledge of the nature of the contents, as required by 29 CFR 1910.1200.

Code: $\quad$ Category III

Compliance

Protocol: None

Priority: $\quad$ Energy Systems Risk Weight 487

Tiger Team Action Plan Priority 2

Response: After further review of the Tiger Team comments, ORNL has concluded that the Finding relates only to the on-site transport of LLLW. At the time of the Tiger Team Assessment, changes in the transportation system for LLLW were being formulated in anticipation of the signing of the Federal Facilities Agreement (FFA).

During this transition it was recognized that better waste characterization needed to be implemented. As a consequence of these activities, a more defined waste characterization (in compliance with regulatory requirements) will be required by waste acceptance criteria before on-site transfers of LLLW are initiated.

See Finding PT.1-4.

Root Cause:

Inadequate policy 
Planned Actions and Schedules:

$\underline{\text { Item/Description }}$

1. Waste management will issue a waste acceptance criteria which ORNL LLLW Generators will be required to meet.
Completion Date

Complete

Costs: $\quad$ Costs associated with waste acceptance criteria, while believed to be significant, are indeterminable without more in-depth study.

Type of funds: Overhead

Source of funds: Overhead

Estimated costs per fiscal year (\$K)

\begin{tabular}{lrrrrrrr}
\cline { 2 - 5 } Action item & 1991 & 1992 & 1993 & 1994 & 1995 & Beyond & Total \\
\hline 1 & 15 & $*$ & & & & 15 \\
$\begin{array}{l}\text { Status: } \\
\text { Funded } \\
\text { Requested } \\
\text { New }\end{array}$ & 15 & & & & & \\
\end{tabular}

*Estimated annual ongoing costs of $\$ 150 \mathrm{~K}$ to meet the requirements.

References: None 
Finding No.: PT.6-2 Low-Level Waste Bottle Testing

Finding

Description: The absence of full Type A package testing leaves uncertaintics as to the regulatory compliance status of the low-level waste bottles.

Code: $\quad$ Category III

Compliance

Protocol:

DOE Order 5480.3 and Draft DOE Order 5480.3A

Priority: $\quad$ Energy Systems Risk Weight 5

Tiger Team Action Plan Priority 3

Response: The decision to go to Type A packages (bottles) and to test accordingly was driven by the need to comply with the pending Federal Facilities Agreement (FFA). At the time of the Tiger Team Assessment, the transportation modifications to the LLLW system were in a planning stage. The Type A testing of the packages was in progress. At this time, the Type A testing has been completed.

See Finding PT.1-4.

Root Causes:

Inadequate policy 
Planned Actions and Schedules:

\section{Item/Description}

1. Evaluate the need for Type A packaging for low level waste bottles and, if affirmed, ensure it is performed in a compliant manner.
Completion Date

Complete

Costs:

Type of funds: Overhead

Source of funds: Overhead

Estimated costs per fiscal year (\$K)

\begin{tabular}{|c|c|c|c|c|c|c|c|}
\hline Action item & 1991 & 1992 & 1993 & 1994 & 1995 & Beyond & Total \\
\hline 1 & 10 & & & & & & 10 \\
\hline
\end{tabular}

Status:

Funded

10

Requested

New

References: $\quad$ ES\&H Self-Assessment Report, Transportation Safety Committee Review of LLLW Activities 
Finding No.: PT.6-3 Inconsistency of Regulatory Terminology

Finding

Description: Inconsistent and conflicting use of regulatory terminology at the $\mathrm{X}-10$ Site results in a potential for regulatory violations.

Code: $\quad$ Category III

Compliance

Protocol: None

Priority: $\quad$ Energy Systems Risk Weight 40

Tiger Team Action Plan Priority 3

Response: The ORNL Self-Assessment did recognize the problem arising from the lack of a firm definition of on-site and off-site and the problems on the horizon with interpretation of the present DOE definition. However, the problem of having conflicting regulatory terminology commonly used in routine Laboratory activities was not recognized as a problem.

To alleviate the risk of conflicting terminology becoming a safety or compliance problem, it will be necessary to ensure that all official Laboratory documentation use proper regulatory terminology. Additionally, it will be necessary to ensure that all SPPs incorporate proper regulatory terminology.

\section{Root Cause:}

Inadequate communications

Planned Actions and Schedules:

\section{Item/Description}

Completion Date

1. Review and evaluate previously developed SPPs for consistency and accuracy. See Finding PT.1-2.

2. Issue Laboratory directive clarifying use of terminology for documentation purposes.

Costs: $\quad$ Cost are included in Finding PT.1-2.

References: $\quad$ ES\&H Self-Assessment Report 
Finding No.: PT.8-1 Safety Standards for Vehicle Identification

Finding

Description: Vehicles carrying hazardous materials onsite are not always identified in accordance with the safety standards in the Department of Transportation and Department of Energy requirements.

Code: $\quad$ Category III

\section{Compliance}

Protocol:

DOE Order 5480.3 and DOE Order 5480.3A

Priority: $\quad$ Energy Systems Risk Weight 58

Tiger Team Action Plan Priority 2

Response: There are inconsistencies in the manner in which hazard communication is displayed on ORNL onsite transport vehicles. This problem was recognized in the ORNL Self-Assessment as it relates to the Onsite Transportation Operations Manual. This is not a problem with shipments moving offsite. The onsite Transportation Operations Manual when implemented will be specific in terms of hazard communication for onsite vehicles and packages.

See Finding PT.1-4.

Root Cause:

Inadequate policy

Planned Actions and Schedules:

Finding is fully addressed in Finding PT.1-4.

Costs: $\quad$ See costs for Finding PT.1-4.

References: None 
Finding No.: PT.8-2 Unnecessary Transport of Hazardous Materials

Finding

Description: There is unnecessary transportation of hazardous materials onsite at the X-10 Site.

Code: $\quad$ Category III

Compliance

Protocol:

DOE Order 5480.3 and Draft DOE Order 5480.3A

Priority: $\quad$ Energy Systems Risk Weight 87

Tiger Team Action Plan Priority 2

Response: $\quad$ For certain movements of hazardous materials there are unnecessary movements. This practice has evolved from the lack of procedural guidance both from an onsite standpoint and an offsite standpoint.

This concern was not directly addressed in the Self-Assessment Report. Presently none of the existing SPPs address the transfer of material onsite. However, the SPPs do require that this movement be done in accordance with onsite procedures.

This concern can be addressed with an onsite manual describing the safety standards to be used onsite and by revising existing SPPs to require the TOMS and/or RAMSPAC personnel to go to the shipment whenever possible.

See also Findings PT.1-2 and PT.1-4.

Root Cause:

Inadequate policy

Planned Actions and Schedules:

$\underline{\text { Item/Description }}$

Completion Date

1. Issue revised SPPs to ensure unnecessary movements are not executed. See Finding PT.1-2.

Costs: $\quad$ Costs are outlined in Findings PT.1-2 and PT.1-4.

References: None 
Finding No.: PT.8-3 Onsite Transfer of Hazardous Materials

Finding

Description: Onsite transfers of some hazardous materials at the X-10 Site do not meet applicable Department of Transportation standards as specified in DOE N5480.3 and Draft DOE 5480.3A.

Code: $\quad$ Category III

Compliance

Protocol: $\quad$ DOE Order N5480.3 and Draft DOE Order 5480.3A

Priority: $\quad$ Energy Systems Risk Weight 133

Tiger Team Action Plan Priority 2

Response: This concern is recognized at ORNL and is being resolved in the ORNL Onsite Transportation Operations Manual.

See Finding PT.1-4.

Root Cause:

See Finding PT.1-4.

Planned Actions and Schedules:

Actions are outlined in Finding PT.1-4.

Costs: $\quad$ Costs are outlined in Finding PT.1-4.

References: See PT.1-4. 
Finding No.: PT.9-1 Central File for Offsite Shipping Documents

Finding

Description: There is no single central file, either computer or hard copy, of shipping papers for offsite shipments of all hazardous materials.

Code: $\quad$ Category III

Compliance

Protocol: None

Priority: $\quad$ Energy Systems Risk Weight 5

Tiger Team Action Plan Priority 3

Response: This concern has never been identified as a significant problem because both filing systems have been audited and have been found to be sufficient. There also is separate accountability for the records because the waste records are considered to be EPA required documents while the shipping records are considered to be DOT required documents. The retention requirements for the documents differ, thus justifying their being filed under two separate systems.

The wording of the finding indicates that the auditor may be looking for a central file located in one physical location. The opinion of ORNL is that the auditor intended to require accountability for the records. From an accountability standpoint, TOMS would be required to ensure maintenance of DOT type documents per the reguiatory requirements. TOMS should ensure through surveillance and documented procedure that the waste records are maintained in a manner compliant with the DOT regulations.

\section{Root Causes:}

Inadequate oversight and inadequate management approach

Planned Actions and Schedules:

\section{$\underline{\text { Item/Description }}$}

Completion Date

1. Issue revised SPPs to document the TOMS accountability function over all records pertaining to DOT regulations. See Finding PT.1-3.

2. Implement surveillance of all hazardous material offsite $12 / 91$ shipment records pertaining to DOT regulations.

Costs: $\quad$ See costs in Findings PT.1-3 and PT.1-1.

References: None 
Finding No.: $\quad$ PT.9-2 Traffic Hazards on Bethel Valley Road

Finding

Description: Serious, undue, and correctable traffic hazards exist on Bethel Valley Road which is used for ORNL hazardous materials traffic.

Code: $\quad$ Category III

Compliance

Protocol:

None

Priority: $\quad$ Energy Systems Risk Weight 607

Tiger Team Action Plan Priority 1

Response: Transportation hazards exist at both the east and west ends of Bethel Valley Road. Hazardous material shipments between ORNL and Y-12 generally travel east from ORNL through the Bethel Valley Road - Scarboro Road intersection. This intersection has been studied in the past, most recently in 1987, to determine methods to make the intersection safer. Studies of the intersection have included evaluating the other roads intersecting Bethel Valley and Scarboro Roads, since the traffic problem is not limited to the Bethel Valley - Scarboro intersection. The roads involved in the studies are owned and controlled by various parties -

Tennessee Department of Transportation, City of Oak Ridge, DOE and Martin Marietta - so reaching consensus on modifications and identifying funding sources for roadway improvements hasn't been achieved.

The west end of Bethel Valley Road intersects State Highway 95. Although this route doesn't experience as much traffic for shipments between ORNL and Y-12, it is the primary route for shipments between ORNL and K-25. This intersection has a history of accidents, and improvements are needed.

Root Cause:

Inadequate management commitment

Planned Actions and Schedules:

\section{Item/Description}

$$
\text { Bethel Valley Road - East }
$$

1. Review the prior traffic studies conducted for the Bethel Valley Road and Scarboro Road intersection; reassess the cost estimates prepared for each of the studies; and determine if additional studies are needed.
Completion Date

Complete 
2. A working team composed of members from DOE-

$06 / 91$ ORO, Tennessee Department of Transportation, City of Oak Ridge, and Energy Systems will complete a review of the studies and make recommendations for implementation.

3. Request that Tennessee DOT perform a traffic study and recommend changes for the Bethel Valley Road State Route 95 intersection.

4. DOE-ORO, Tennessee DOT, and Energy Systems review the study and recommended changes and determine the method for funding.

5. Submit funding request(s).

$09 / 91$

6. Initiate implementation of recommended changes.

$12 / 92$

Costs: $\quad$ Bethel Valley Road-East will require \$20K in FY 91 funding from the operating overhead budget to support review of the studies, estimating, meetings with DOE-ORO, Tennessee DOT, and preparing funding request documentation. The cost for actual implementation is indeterminable until an acceptable modification is agreed upon and the funding source(s) are determined. Bethel Valley Road-West will require $\$ 5 \mathrm{~K}$. The actual cost for implementation cannot be determined at this time. 
Type of funds: Overhead

Source of funds: Overhead

Estimated costs per fiscal year (\$K)

\begin{tabular}{crrrrrrr} 
Action item & 1991 & 1992 & 1993 & 1994 & 1995 & Beyond & Total \\
\hline $1-2$ & 20 & & & & & & 20 \\
3 & 5 & & & & & & 5 \\
4 & - & & & & & & \\
5 & - & & & & & & \\
6 & & 350 & & & & & 350
\end{tabular}

Status:

Funded

25

Requested

New

'DOE's share of the estimated cost of recommended changes will depend on what cost-sharing agreements, if any, are reached with Tennessee DOT and the City of Oak Ridge.

References: $\quad$ Scarboro Road Traffic Analysis, November 1987, Prepared by EC Design and Technology, Inc. 
Finding No.: PT.12-1 Handling and Storage of Hazardous Materials

Finding

Description:

Code: $\quad$ Category III

Compliance

Protocol:

Priority:

Response:
Present practices regarding handling and storage of hazardous materials, especially incompatible materials, do not conform to regulatory requirements, good practice, or Department of Energy safety policy.

29 CFR 1910

Energy Systems Risk Weight 414

Tiger Team Action Plan Priority 2

Incoming vendor or AVID receipts of hazardous materials are received, staged for immediate delivery or segregated in Bldg. 7001. Technical specification have been developed to procure four portable hazardous materials storage buildings. The four buildings will be located under the $7001 \mathrm{Bldg}$. east overhang. The Chemical Storage buildings will be utilized for segregation and temporary staging receipts of acids, bases, flammable, and toxic materials while awaiting distribution. Energy Systems Procurement is in the process of purchasing these buildings for ORNL. In the interim, pallet racks in Bldg. 7001 will be used for segregating hazardous materials.

Building 7001 is one of our major operational constraints. It was constructed in 1948 and was not designed for receipt, distribution, and warehousing functions. Receiving and distribution work areas are relatively small in size and become very congested. Congestion problems are magnified when forktruck and pedestrian traffic are added. Engineering studies are complete identifying removing a fire wall and the old transportation offices which will increase Receiving Distribution area by 3,000 sq $\mathrm{ft}$. Increased operational space will allow for a safe and systematic system. Construction contracts are in the Materials Department FY91 budget request.

Materials Department operating procedure MD-S-4 Loading and Unloading of Motor Vehicles was approved and implemented 1-25-90. This procedure includes a segregation and separation chart of hazardous materials that is adhered to for staging, segregation, and loading delivery vehicles. Segregation charts have been posted at the hazardous material storage rack in receiving and in the distribution area. Receiving employees have been instructed to stage heavy and/or bulky material on the floor. Interpretation of current DOT regulations transporting less than truck load quantity of hazardous materials necessitates utilizing the segregation chart for single transport vehicle. Distribution employees are continually schooled and supervised for compliance. Materials Department is not in violation of DOT regulations. 
Materials Department operating procedure MD-S-3 Receipt, Handling, and Storage-In-Transit of Hazardous Materials was approved and implemented January 19, 1990. This procedure is a cook book approach for our employees. Employees are continually schooled and supervised for compliance.

In CY90 Materials Department supervisors were trained by the ORNL Training Department on hazardous communications. Supervisors will train their employees to potential hazardous materials which they may encounter in their day to day
duties.

\section{Root Causes:}

Lack of resources, inadequate training, and inadequate policy implementation

\section{Planned Actions and Schedules}

\section{Item/Description}

1. Purchase and install four chemical storage buildings.

2. Construct level " $A$ " inspection area (see Finding QV.5-1).

3. Demolish old Transportation offices.

4. Convert 7001 dry sprinkler system to wet pipe system.

5. Remove 7001 fire wall and relocate offices.

6. Construct exit from mezzanine \#3.

7. Upgrade 7001 electrical.

8. Train employees to site-specific potential hazards.
$9 / 92$

9/92

9/92

$9 / 92$

\section{Completion Date}

9/91

$9 / 92$

\section{9/92}

9/91 
Costs:

Type of funds: Overhead

Source of funds: Overhead

\begin{tabular}{|c|c|c|c|c|c|c|c|}
\hline \multirow[b]{2}{*}{ Action item } & \multicolumn{6}{|c|}{ Estimated costs per fiscal year (\$K) } & \multirow[b]{2}{*}{ Total } \\
\hline & 1991 & 1992 & 1993 & 1994 & 1995 & Beyond & \\
\hline 1 & 100 & & & & & & 100 \\
\hline 2 & & 150 & & & & & 150 \\
\hline 3 & & 125 & & & & & 125 \\
\hline 4 & & 58 & & & & & 58 \\
\hline 5 & & 94 & & & & & 94 \\
\hline 6 & & 50 & & & & & 50 \\
\hline 7 & 75 & 75 & & & & & 150 \\
\hline 8 & 5 & & & & & & 5 \\
\hline
\end{tabular}

Status:

Funded $\quad 100$

Requested

New

180

552

$\$ 732$

References: $\quad 29$ CFR 1910 (Industrial Safety) 
Fünding No.: PT.12-2 Planning of Radioactive Materials Packaging Needs

\section{Finding}

Description: Absence of coordinated planning between ORNL and the Department of Energy on packaging needs has caused delays in shipping radioactive materials, which have
adversely affected operations.

Code: $\quad$ Category III

Compliance

Protocol:

DOE Order 5480.3 and Draft DOE Order 5480.3A

Priority: $\quad$ Energy Systems Risk Weight 8

Tiger Team Action Plan Priority 3

Response: $\quad$ ORNL has four packages in the queue for DOE Certificates of Compliance approval. Those packages have been prioritized, and DOE has been made aware of this prioritization.

A 1988 DOE-ORO audit recommended that ORNL develop a more formalized and organized packaging and transportation program. The reason for this recommendation stemmed from the fact that ORNL had numerous packages in timely renewal status, which was indicative of less than adequate planning for packaging needs. This problem was addressed in the PIP report of 1989, and a recommendation was made for a packaging engineer to be hired by TOMS. To date ORNL has not been successful in hiring the packaging engineer; however, active recruiting has been and is still under way.

\section{Root Causes:}

Inadequate communications, ambiguous requirements or expectations, and poorly defined roles and responsibilities

Planned Actions and Schedules:

\section{Item/Description}

Completion Date

1. Submit letter to DOE-ORO addressing the packaging under timely renewal and packaging which will adversely

Complete impact operations if not available.

2. DOE-ORO submit ORNL letter to DOE-HQ.

3. Submit requisition, interview and select Packaging Engineer for TOMS. 
Costs: $\quad$ See Finding PT.1-1.

References: $\quad$ P\&T PIP report issued 6/29/89; ES\&H Self-Assessment 
Finding No.: PT.12-3 Absence of Onsite Transfer Plan

Finding

Description: The absence of a plan coordinated by the involved divisions for onsite transfers of radioactive and mixed wastes is delaying the implementation of cost-effective waste management practices.

Code: $\quad$ Category III

Compliance

Protocol:

DOE Order 5480.3 and Draft DOE Order 5480.3A

Priority: $\quad$ Energy Systems Risk Weight 5

Tiger Team Action Plan Priority 3

Response: The problem involving onsite transport of radioactive and mixed wastes was recognized in 1988 during a DOE audit which called for the development and implementation of an onsite transportation plan. ORNL set out to develop an onsite plan in 1988 and to date has issued the fourth draft for Laboratory-wide review. Additionally the 1989 P\&T PIP Report called for the development of a Transportation Operations Committee to aid in the solution of complex and controversial problems such as the onsite transfer of radioactive and mixed wastes.

The final draft of the onsite plan will need to address the onsite transfer of radioactive and mixed wastes more specifically. The implementation of the onsite plan and the subsequent development of the Transportation Operations Committee will define the methods of onsite transport. The Committee should provide a mechanism for identifying symptoms of less than adequate waste management practices.

See response to Finding PT.1-4.

\section{Root Cause:}

Inadequate management commitment

Planned Actions and Schedules:

Actions are outlined in Finding PT.1-4.

Costs: $\quad$ Costs are outlined in Finding PT.1-1.

References: Corporate Audit 1990, DOE-HQ Audit 1990 
Finding No.: PT.12-4 Conflicting Contamination Limits

Finding

Description: DOE Headquarters has not resolved the conflicts with regards to the applicable contamination limits.

Code: $\quad$ Category III

Compliance

Protocol:

Priority: $\quad$ Energy Systems Risk Weight 5

Tiger Team Action Plan Priority 3

Response: Although DOE-HQ has been made aware of this conflict, no formal resolution has been forthcoming from DOE-HQ. Consequently, DOE-ORO developed a DOE-ORO Radioactive Material Transportation Contamination Policy that was formally transmitted to ORNL on October 29, 1990. It is intended this DOE-ORO policy remain in effect until such time as DOE-HQ takes action on DOE-ORO's earlier request.

\section{Root Cause:}

Lack of clearly defined DOE-HQ policy in regard to contamination limits for transportation operations 
Planned Actions and Schedules:

\section{Item/Description}

1. DOE-ORO request guidance/clarification from DOE-HQ.

2. While awaiting of DOE-HQ action, DOE-ORO develop and issue policy on transportation contamination.

3. XSO will formally transmit policy to ORNL.

4. XSO provide any changes in the policy (from DOE-HQ) to ORNL.

\section{Completion Date}

Complete

Complete

Complete

As

Necessary

Costs: $\quad$ DOE-ORO and XSO program activities will be accomplished with existing resources.

References: $\quad$ DOE Orders 5480.3 and 5480.11 


\subsubsection{Nuclear Criticality Safety}

Finding No.: CS.1-1 Nuclear Criticality Safety Training Program

Finding

Description: The ORNL Nuclear Criticality Safety Training Program does not meet requirements of DOE 5480.5 pars. 8c and 10.

Code: $\quad$ Category III

Compliance

Protocol:

Priority: $\quad$ Energy Systems Risk Weight 60

Tiger Team Action Plan Priority 2

Response: ORNL will develop and issue a Laboratory procedure requiring fissile-material handlers, fissile material supervisors, and other workers who can affect nuclearcriticality safety to have criticality-safety training in compliance with DOE Order 5480.5, par. 10. The ORNL criticality-safety specialists, with help and advice from the ORNL Training and Development Group within the Human Resources Division, will develop and present criticality-safety courses to all workers authorized to handle fissile material or supervise fissile-material handling within 18 months, while the current criticality safety training program continues in the interim. The Technical Resources and Training Group in the Office of Environmental and Health Protection will maintain documentation of training, testing, and qualification status. Fissile-material handlers and supervisors will be retrained and reexamined for emergency response annually and, as appropriate, biennially for other knowledge related to nuclear-criticality safety. The Laboratory will conduct a biennial management assessment of the training program for criticality safety.

Root Cause:

Inadequate training and inadequate management commitment

Planned Actions and Schedules:

\section{Item/Description}

1. Issue ORNL SPP (or equivalent) to address training requirements of DOE 5480.5, para. 10, and documentation requirements of para. 8c. for fissilematerial workers and supervisors. This same SPP will also address other criticality-safety training at ORNL and
Completion Date

$8 / 91$ 
will identify workers who require NCS training at each level. The SPP will include:

- requirements for testing, documentation and timeliness of documentation;

- stated requirements for passing, including mandatory requirements for correct answers to selected questions;

2. Develop and initiate courses, lesson plans, arsd examinations for fissile-material workers.

3. Complete first training on all upgraded NCS courses. The course upgrades will include:

- revision of criticality-safety training for radiation control officers to include testing;

- a course description and objectives as part of the approval package for each course;

- stated requirements for passing, including mandatory requirements for correct answers to selected questions.

Costs:

Type of funds: Overhead

Source of funds: Overhead

\begin{tabular}{|c|c|c|c|c|c|c|c|}
\hline \multirow[b]{2}{*}{ Action item } & \multicolumn{6}{|c|}{ Estimated costs per fiscal year $(\$ \mathbf{K})$} & \multirow[b]{2}{*}{ Total } \\
\hline & 1991 & 1992 & 1993 & 1994 & 1995 & Beyond & \\
\hline 1 & 15 & & * & & & & 15 \\
\hline 2 & & 40 & & & & & 40 \\
\hline 3 & & & 25 & & & & 25 \\
\hline
\end{tabular}

Status:

Funded

Requested

New

40

*Estimated annual ongoing cost: $\$ 7 \mathrm{~K}$ biennial costs for Item 1 .

References: None 
Finding No.: CS.1-2 ORO Oversight and Support Functions

Finding

Description: The Oak Ridge Operations Office is failing to provide to ORNL all the oversight and support functions required by DOE 5480.5 par. $7 \mathrm{e}$.

Code: $\quad$ Category III

Compliance

Protocol:

DOE Order 5480.5 par. $7 \mathrm{e}$

Priority: $\quad$ Energy Systems Risk Weight 55

Tiger Team Action Plan Priority 2

Response: The Oak Ridge Operations Safety and Health Division has recently reorganized. One entire branch is now devoted to oversight and support of nuclear facility safety. The division has on its staff a Ph.D.-level nuclear physicist with 15 years of applied criticality safety experience for oversight of contractor NCS programs. In addition to providing appraisal and surveillance functions at ORNL, the Safety and Health Division provides direct support to DOE line programs through participation in readiness reviews and investigations, reviews of safety analysis reports (SARs) and operational safety requirements (OSRs), issuance of guidance on SAR and OSR preparation, and review of new designs and proposed configuration changes.

Root Causes:

Inadequate oversight and insufficient resources 
Planned Actions and Schedules:

\section{$\underline{\text { Item/Description }}$}

1. Issue a plan to conduct at least two surveillances per year and to conduct functional appraisals every 2 years.

2. Provide the ORNL site safety representative with the UORs affecting criticality safety from within the DOE Order 5000.3A reporting system.

3. Review ORNL Safety Analysis Reports involving fissile materials as submitted to DOE.

4. Establish a timely renewal program for ORNL Nuclear Safety Reviews (NSRs) for upgrading to current standards. Programs using NSRs with shortcomings that reduce safety will be stopped.

5. Provide the ORNL Criticality Safety Officer with draft reports of surveillances and appraisals within 30 calendar days following the visit.

Costs: No significant costs associated with actions listed.
Completion Date

Complete

Complete

Complete

Complete

Complete

References: None 
Finding No.: CS.1-3 ORNL Criticality Safety Program

\section{Finding \\ Description: The ORNL Criticality Safety Program lacks controls necessary to saticfy requirements of DOE 5480.5 par. 11c.}

Code: $\quad$ Category II

Compliance Protocol:

DOE Order 5480.5 par. 11.c requires that contractor programs involving significant quantities of fissionable materials be governed by written plans and procedures to control the potential of nuclear criticality in the receipt, storage, processing, and shipping of these fissionable materials. The written plans and procedures are to identify the nuclear criticality safety (NCS) controls that were derived from required nuclear criticality safety process analyses.

Priority: $\quad$ Energy Systems Risk Weight 55

Tiger Team Action Plan Priority 2

Response: The performance of process analyses and identification of nuclear criticality safety controls (for inclusion in written plans and procedures that are to govern fissionable material conditions) require initiation by ORNL Line Management as the result of

- their knowledge of where to find procedural and operational guidance in the identification of "significant quantities" of fissionable materials and

- their knowledge of clearly defined policies and procedures outlining the ORNL nuclear criticality safety program controls and personnel responsibilities.

Control deficiencies in the ORNL Nuclear Criticality Safety (NCS) Program have been previously identified in the ORNL Self-Assessment, minor nuclear criticality safety incident reports, Criticality Review Committee appraisals, DOE/ORO audits, ORNL Health and Safety Long-Range Plans, and Energy Systems Criticality Safety Committee audits. Although the action plan in response to the last Energy Systems Criticality Safety Committee audit identifies insufficient personnel and procedural deficiencies affecting program controls, it fails to address operating management awareness and knowledge of regulatory requirements for the possession of significant quantities of fissile materials. Although an existing nuclear materials accountability program identifies locations of significant quantities of fissile materials, this resource does not provide for all the regulatory prerequisites associated with possessing these significant quantities of fissile materials (i.e., criticality safety analyses, training, audits, etc.). Regulatory requirements are embodied in ORNL Health Physics Procedure 2.4; however, a knowledge of its contents and regulatory basis is basic to satisfying the requirements of DOE Order 5480.5 par. 11.c.

ORNL Line Management knowledge, awareness, and control of nuclear criticality safety will be heightened as the result of this immediate action plan which includes 
- Notification to Line Management of compliance expectations as they relate to nuclear criticality safety program controls and

- Line Management compliance reviews for operations involving fissionable materials and definition of program needs.

The completion of the "Planned Actions and Schedule" provides a prompt response for informing Line Management of needed NCS Program controls and provide closure of the implied concern (Root Cause) as evidenced by the supporting findings.

\section{Root Cause:}

Inadequate policy implementation, inadequate management commitment, poorly defined roles and responsibilities, and inadequate personnel resources

Planned Actions and Schedules:

\section{Item/Description}

1. Issue an immediate information notice to applicable Laboratory division directors regarding this TSA concern and requiring immediate review of ORNL Health Physics Procedure RP-2.4, "Fissile Material Safety," for compliance with its requirements (see November 29, 1990, letter from J. H. Swanks, "Fissile Material Safety").

2. Issue memorandum from the Laboratory Director to appropriate line management that outlines regulatory requirements for NCS Program controls and fissile material inventories with a directive for response to Action Item 3 below.

3. Division directors responsible for fissile materials issue report to Laboratory Director (1) on assessment of holdings and planned activities involving significant quantities of fissile materials, (2) on results of compliance reviews of their operations involving fissile materials relative to Action Item 2, and (3) planned action to correct deficiencies identified.

4. Identify one FTE to develop a nuclear criticality safety program manual for Laboratory implementation; manual to define roles and responsibilities with respect to NCS and actions necessary to comply with DOE Order Order 5480.5.
Completion Date

Complete

Complete

$6 / 91$

Complete 
5. Issue revised ORNL Health Physics Manual procedure

RP-2.4 to ensure NCS program controls and develop and implement modified procedures in a distinct ORNL nuclear criticality safety manual that includes necessary NCS program controls in compliance with DOE Order 5480.5.

Costs:

Type of funds: Overhead

Source of funds: Overhead

Estimated costs per fiscal year (\$K)

\begin{tabular}{crrrrrrr}
\cline { 2 - 7 } Action item & 1991 & 1992 & 1993 & 1994 & 1995 & Beyond & Total \\
\hline 1 & - & & & & & & 0 \\
2 & - & & & & & & 0 \\
4 & 50 & $*$ & & & & & 50 \\
5 & & - & & & & &
\end{tabular}

Status:

Funded

Requested

New

*Estimated annual ongoing cost: \$85K starting in FY 1992.

Type of funds: Overhead

Source of funds: Division Administration

Estimated costs per fiscal year (\$K)

\begin{tabular}{crrrrrrr}
\cline { 2 - 6 } Action item & 1991 & 1992 & 1993 & 1994 & 1995 & Beyond & Total \\
\hline 3 & 10 & & & & & & 10
\end{tabular}

Status:

Funded

Requested

New

10

References: $\quad$ DOE Order 5480.5, ANSI/ANS-8.1-1988, ANSI/ANS-8.19-1989 
Finding No.: CS.1-4 Nuclear Criticality Safety Program Documentation

Finding

Description: The ORNL Nuclear Criticality Safety Program does not satisfy nuclear criticality safety documentation requirements of DOE 5480.5 pars. 8a, 8e, 8j, and 11c, and Energy Systems Policy Procedures.

Code: $\quad$ Category III

Compliance

Protocol:

DOE Order 5480.5 , pars. $8 \mathrm{a}, 8 \mathrm{e}, 8 \mathrm{j}$, and $11 \mathrm{c}$

The nuclear criticality safety analysis and review process at the ORNL is generally described in the ORNL Health Physics Manual, procedure number RP-2.4. However, it does not provide in-depth direction:

- for necessary documentation of the identification and control of risks through the application of DOE Order 5481.1B requirements,

- for the execution and documentation of quality assurance program activities as specified in DOE Order 5700.6B,

- for identifying NCS Program records and documents requiring control and traceability, and

- to Line Management for ensuring that written plans and procedures exist for the receiving, inspecting, shipping, processing, and storing of significant quantities of fissile materials

Priority: $\quad$ Energy Systems Risk Weight 55

Tiger Team Action Plan Priority 2

Response: Upgrades to the ORNL NCS Program will address this Concern by replacing the existing ORNL Health Physics Manual procedure RP-2.4 with a comprehensive ORNL Criticality Safety Manual containing specifications for the ORNL Nuclear Criticality Safety Program, which will include a functional role of a Nuclear Criticality Safety Staff.

This corrective action plan (CAP) provides for increasing resources for the purpose of developing procedural requirements for program upgrades, to specify necessary elements for safety analyses, documentation, document traceability and control, facility reviews/audits, training development, and program quality assurance overview. Slightly delayed parallel actions for correcting deficiencies of the stated Findings will be performed as part of the CS.3-1 CAP to reevaluate and document safety analyses in conformance with the program specifications developed in this CAP.

\section{Root Causes:}

Inadequate policy, inadequate policy implementation, and inadequate resources 
Planned Actions and Schedules:

Item/Description

Completion Date

1. Obtain one supplementary staff experienced in nuclear criticality safety (NCS) (see Finding CS.1-3, Item 4).

2. Define new NCS program requirements by adopting and adapting existing NCS procedures from other Energy Systems facilities (see Finding CS.1-3, Item 4).

3. Complete draft of new ORNL NCS Procedure Manual (see Finding CS. 1-3, Item 4).

4. Issue revised NCS Program requirements in an official ORNL Nuclear Criticality Safety Procedure Manual.

5. Issue guidance to staff and line management concerning revised NCS Program requirements.

Costs:

Type of funds: Overhead

Source of funds: Overhead

Estirnated costs per fiscal year (\$K)

\begin{tabular}{crrrrrrr}
\cline { 2 - 5 } Action item & 1991 & 1992 & 1993 & 1994 & 1995 & Beyond & Total \\
\hline 1 & $a$ & & & & & & \\
2 & $a$ & & & & & & \\
3 & $a$ & & & & & & \\
4 & & 10 & & & & 10 \\
5 & & 10 & & & 10
\end{tabular}

Status:

Funded

Requested

New 20

${ }^{a}$ Costs associated with Items 1-3 are covered by Finding CS.1-3, Item 4.

References: None 
Finding No.: CS.3-1 Safety Analysis Documentation Requirements

Finding

Description: The ORNL Nuclear Criticality Safety Program does not satisfy safety analysis documentation requirements of ANSU/ANS-8.1-1983 Sect. 4.1.4 and DOE 5480.5 par. 8a. (Sce also Concern CS.1-4.)

Code: $\quad$ Category III

Compliance Protocol:

Priority: $\quad$ Energy Systems Risk Weight 55

Tiger Team Action Plan Priority 2

Response: This concern is also rooted in the incomplete definition for the specification, performance, audit, oversight, and documentation of the ORNL nuclear criticality safety program and its activities (see Finding CS.1-4) as presented in the Health Physics Manual procedure number RP-2.4.

The corrective action plan (CAP) for Finding CS.1-4 will upgrade the ORNL NCS program and documentation requirements and procedures for nuclear criticality safety and will address the lack of documentation concern. This CAP is provided to increase the necessary resources to upgrade existing safety analyses to meet the NCS program requirements developed by the CAP for Finding CS.1-4.

In event the necessary resources are not obtained, the upgrading of existing nuclear criticality safety analyses will necessarily be completed at a slower rate, extending the completion dates of planned actions 4 and 5 into FY 1996.

\section{Root Causes:}

Inadequate policy implementation and insufficient resources 
Planned Actions and Schedules:

Item/Description

1. Request funding to obtain additional resources to perform nuclear criticality safety (NCS) analysis.

2. Prepare a prioritized schedule fur NCS analysis re-evaluation contingent on approved funding for FY 92 budget.

3. Issue revised NCS requirements (see Finding CS.1-4, Item 4).

4. Contingent upon funding approved for FY 92 budget, re-evaluate and document NCS analyses in accordance with new NCS Procedure Manual being developed in CS. 1-4, Item 4.

5. Perform first annual appraisal of all facilities authorized to possess significant quantities of fissile materials, in accordance with the new NCS Procedure Manual.
Completion Date

$6 / 91$

$10 / 91$

$12 / 92$

Costs:

Type of funds: Overhead

Source of funds: Overhead

Estimated costs per fiscal year (\$K)

\begin{tabular}{crrrrrrr}
\cline { 2 - 6 } Action item & 1991 & 1992 & 1993 & 1994 & 1995 & Beyond & Total \\
\hline 1 & - & & & & & & \\
4 & & 276 & 282 & & & & 558 \\
5 & & & 15 & & & & 15
\end{tabular}

Status:

Funded

Requested

New

References: None 
Finding No.: CS.4-1 Review of Nuclear Criticality Safety Analyses

\section{Finding}

Description: Nuclear criticality safety analyses at ORNL do not always receive a level of review sufficient to satisfy DOE 5480.5 par. 8 a.

Code: $\quad$ Category III

Compliance

Protocol:

DOE Order 5480.5 par. $8 \mathrm{a}$

Priority: $\quad$ Energy Systems Risk Weight 55

Tiger Team Action Plan Priority 2

Response: Operations with significant quantities of fissile material are currently governed by the ORNL Health Physics Procedure (HPP) Manual, Procedure 2.4, "Fissile Material Safety." This procedure is deficient per current DOE regulations.

The upgrade of all criticality analyses identified in Finding CS.3-1 according to the new procedure that will be completed in Finding CS.1-4. The reviews of criticality analyses will be performed independently by knowledgeable criticality staff.

\section{Root Causes:}

Inadequate policy implementation, inadequate oversight, and insufficient resources

Planned Actions and Schedules:

\section{$\underline{\text { Item/Description }}$}

1. Include the requirement of independent review of NCS analyses by knowledgeable NCS staff in the new NCS Procedure Manual being developed in CS.1-4, Item 4.

\section{Completion Date}

Costs: $\quad$ Costs are fully addressed in the action plan for Finding CS.3-1.

References: $\quad$ ANSI/ANS 8.19-1984 Sect. 8 
Finding No.: CS.4-2 Dissemination of Nuclear Criticality Safety Guidance

Finding

Description: Dissemination of nuclear criticality safety guidance at ORNL does not satisfy requirements of ANSI/ANS-8.1-1983 Sect. 4.1.3 and DOE 5480.5 par. 13b(4).

Code: $\quad$ Category III

Compliance Protocol:

Priority:

Response:
ANSI/ANS-8.1-1983 Sect. 4.1.3 and DOE Order 5480.5 par. 13b(4) are being violated.

Energy Systems Risk Weight 55

Tiger Team Action Plan Priority 2

An ORNL-level procedure, Health Physics procedure RP-2.4, requires approved, written procedures for operations with fissile material, but some of the existing operating procedures do not comply with ANSI/ANS 8.1, Section 4.1.3, and some operating personnel do not understand the importance of criticality control parameters. Managers who operate with, store, or transport fissile material will review and appropriately upgrade all procedures with potential impact on criticality safety. The ORNL criticality safety staff will review and concur with the procedures before they receive approval. These procedures will be used for all fissile material operations after the end of December 1991. (Training that will assist the fissile material manager will begin in October 1991. See Finding CS.1-1.)

As an interim measure, criticality safety personnel will visit fissile material storage areas and instruct supervisors on appropriate posting. The criticality safety staff will prepare or revise an ORNL-level procedure to require posting of limits for criticality safety near storage areas as required by DOE Order 5480.5, par. 13.b.(4). Fissile material supervisors will post these areas, and the arcas will be monitored by the criticality safety staff by December 31, 1991 .

\section{Root Causes:}

Inadequate policy implementation and inadequate training

Planned Actions and Schedules:

\section{$\underline{\text { Item/Description }}$}

1. Verify by inspection that interim postings of criticality safety limits in fissile material storage areas are complete as instructed by criticality safety staff per DOE Order 5480.5, par. 13.b(4).
Completion Date

Complete 
2. Include the requirement of posting of storage areas in compliance with DOE Order 5480.5, par. 13.b.(4) in the new NCS Procedure Manual being developed in CS.1-4, Item 4.

3. Initiate upgraded training for fissile material handlers and supervisors. This is part of an action for Finding CS.1-1 and will include guidance on posting areas and appropriate criticality safety parameters for operating procedures.

4. Verify and document that all fissile material operations are conducted using approved written operating procedures that have been reviewed and accepted by the ORNL criticality safety staff.

5. Include the requirement of making a copy of the NCS approval document readily available at the workplace in the new NCS Procedure Manual being developed in CS.1-4, Item 4.

6. Verify by inspection that supervisors have completed posting of NCS limits in storage areas per new NCS Procedures Manual. 


\section{Casts:}

Type of funds: Overhead

Source of funds: Overhead

\begin{tabular}{|c|c|c|c|c|c|c|c|}
\hline \multirow[b]{2}{*}{ Action item } & \multicolumn{6}{|c|}{ Estimated costs per fiscal year (\$K) } & \multirow[b]{2}{*}{ Total } \\
\hline & 1991 & 1992 & 1993 & 1994 & 1995 & Beyond & \\
\hline 1 & - & & & & & & \\
\hline 2 & - & & & & & & \\
\hline 4 & - & 8 & * & & & & 8 \\
\hline 5 & - & & & & & & \\
\hline 6 & - & & & & & & \\
\hline
\end{tabular}

Status:

Funded

Requested

New

8

$\$ 8$

${ }^{*}$ Estimated annual ongoing cost: $\$ 5 \mathrm{~K}$.

References: Internal appraisals by ORNL CRC and Energy Systems Environmental and Safety Activities 
Finding No.: CS.4-3 Nuclear Criticality Safety Remedial Action Plan

Finding

Descripion: The ORNL Nuclear Criticality Safety Program does not have a remedial action plan for incidents that satisfies the requirements of ANSI/ANS-8.1-1983 par. 4.1.5 and DOE 5480.5 par. $8 \mathrm{~g}$.

Code: $\quad$ Category III

Compliance

Protocol:

ANSI/ANS-8.1-1983 par. 4.1.5 and DOE Order 5480.5 par. $8 \mathrm{~g}$

Priority: $\quad$ Energy Systems Risk Weight 55

Tiger Team Action Plan Priority 2

Response: Since October 1, 1990, all ORNL employees adhere to DOE Order 5000.3A, "Occurrence Reporting and Processing of Operations Information." The requirements of the Order are implemented through Energy Systems Policy ?rocedure GP-13 and associated Standards GP-13.1 and GP-13.2. The system in place "to assure remedial action has been implemented" (per DOE Order 5480.5 par. 8.g) is specified in GP-13.2. The data base computer system, ESQIS/ESAMS, provides the systematic tracking, trendirg, and reporting system for Energy Systems Occurrences.

ORNL Standard Practice Procedure, X-ESH-3, "Health Physics Manual (HPP)," assigned the Office of Operational Readiness \& Safety with the responsibility for tracking incident remedial actions (HPP RP2.4, par. E.4). Tracking of criticality incidents has been a simple task as there have only been less than ten incidents in the past five years at ORNL. According to GP-13.2, Line Management has the responsibility to perform corrective actions and verify and validate completion of corrective actions. For those incidents related to criticality safety, the role of the Quality Department is to provide independent assurance that corrective act ions are performed in timely manner. Criticality Safety Section identifies deficiencies in execution of the criticality safety program and provides independent compliance reviews per Criticality Safety SPP.

Root Cause:

Inadequate policy 
Planned Actions and Schedules:

$\underline{\text { Item/Description }}$

1. Issue a new SPP regarding reporting criticality safety incidents per DOE Order 5000.3A. (Part of Finding CS.1-4, Item 4); the SPP will also address the update of criticality incident reporting form.

Sssts: $\quad$ Costs are included in Item 4 for Finding CS.1-3.

References: $\quad$ DOE Order 5000.3A, ANSI/ANS-8.1-1988, Energy Systems Policy Procedure GP-13, and Standards GP-13.1 and GP-13.2, and HPP 2.4 
Finding No.: CS.5-1 Nuclear Criticality Safety Emergency Response Plan

\section{Finding}

Description: The ORNL Nuclear Criticality Safety Program does not include all of the elements of an emergency response plan to satisfy zequirements of ANSIANS-8.1-1983 Sect. 4.1.7 and DOE 5480.5 par. $8 \mathrm{k}$.

Code: $\quad$ Categor' III

Compliance

Protocol: $\quad$ ANSI/ANS-8.1'983 and DOE Order 5480.5

Priority: $\quad$ Energy Systems Risk Weight 55

Tiger Team Action Plan Priority 2

Response: The role of the ORNL Criticality Safety Officer is not specified in an emergency response plan nor is the Criticality Safety Officer listed in an emergency notification roster.

Root Cause:

Poorly defined roles and responsibilities

Planned Actions and Schedules:

\section{$\underline{\text { Item/Description }}$}

1. Specify the role of the Criticality Safety Officer in the X-10 Site Emergency Plan.

2. Include the Criticality Safety Officer on emergency notification rosters.

Costs: None

References: $\quad$ DOE Order 5480.5 and ANSI/ANS-8.1-1983
Completion Date

Complete

Complete 
Finding No.: CS.5-2 Criticality Alarm System Evacuation Drills

Finding

Description: Drills conducted at ORNL in support of criticality alarm system evacuations do not satisfy requirements of ANSI/ANS-8.3-1986 Sect. 7.3 and DOE 5480.5 par. 11c (3)(g).

Code: $\quad$ Category III

Compliance

Protocol:

Priority: $\quad$ Energy Systems Risk Weight 55

Tiger Team Action Plan Priority 2

Response: Annual evacuation drills have been conducted and are documented for Building 3019; however, two other ORNL facilities with criticality alarm systems are the HPRR and the 3027 Vault, neither of which have personnel assigned in residence.

\section{Root Cause:}

Ambiguous requirements or expectations

Planned Actions and Schedules:

\section{$\underline{\text { Item/Description }}$}

1. Document the requirement for an annual preannounced evacuation drill from each facility where a criticiality accident alarm system is required.

Costs: None

References: $\quad$ ANSI/ANS-8.3-1986 and DOE Order 5480.5

ORNL X-10 Site Emergency Plan
Completion Date

Complete 
Finding No.: CS.5-3 Criticality Alarm Systems

Finding

Description: The criticality alarm systems at ORNL do not satisfy requirements of ANSI/ANS-8.3-1986 Sects. 4.3, 4.4.5, 5.4, 6.5, and DOE 5480.5 pars. 11c(3)(g), $11 \mathrm{c}(3)(\mathrm{h})$.

Code: $\quad$ Category III

Compliance

Protocol:

ANSI/ANS-8.3-1986 Sects. 4.3, 4.4.5, 5.4, 6.5, and DOE Order 5480.5 pars. $11 \mathrm{c}(3)(\mathrm{g})$ and $11 \mathrm{c}(3)(\mathrm{h})$

Priority: $\quad$ Energy Systems Risk Weight 58

Tiger Team Action Plan Priority 2

Response: $\quad$ All findings noted in the Tiger Team assessment were already being addressed by an ORNL ad hoc committee on criticality alarms (chaired by W. J. DeRossett). The deficiencies with ANSI/ANS-8.3 are documented in the committee's Action Plan. The cost of the effort to achieve compliance is known. Justifications will be prepared to support the contention that the only ORNL facility requiring a criticality alarm is Bldg. 3019.

Root Cause:

Inadequate resources

Planned Actions and Schedules:

\section{$\underline{\text { Item/Description }}$}

1. Document the justification of not requiring a criticality alarm system at the High Flux Isotope Reactor and Bulk Shielding Reactor per 5480.5 par. 11c3h.

2. Submit a change to SAR for Bldg. 3027 which reflects that no criticality alarm system is required (DOE Order 5480.5 par. $11 \mathrm{c} 3 \mathrm{~h}$ ). Pending approval of this SAR change, an interim waiver will be requested from OORS.
Completion Date

Complete

Complete 
3. Request funding from DOE/Nuclear Fuel Services, Inc., to provide for upgrading of the criticality alarm system in Bldg. 3019. Actions noted in DeRossett's ad hoc committee memorandum including documentation of system placement, maintenance, testing and responsibilities, will be completed nine months after funding is secured. Independent verification of closure will be obtained.

Costs:

Type of funds: Overhead

Source of funds: Overhead

Estimated costs per fiscal year $(\$ \mathrm{~K})$

\begin{tabular}{cccccccc}
\cline { 2 - 6 } Action item & 1991 & 1992 & 1993 & 1994 & 1995 & Beyond & Total \\
\hline 1 & - & & & & & & \\
2 & 2 & & & & & & 2 \\
3 & $*$ & & & & & &
\end{tabular}

Status:

Funded

Requested

New

*Funded by DOE/Nuclear Fuel Services, Inc.

References: $\quad$ DOE Order 5480.5 par. 11c, ANSI/ANS-8.3-1986, Memorandum: W. J. DeRossett to Distribution, November 12, 1990 


\subsubsection{Security/Safety Interface}

Finding No.: $\quad$ SS.1-1 Analyses of Protective Force Equipment

Finding

Description: ORNL does not conduct analyses involving protective force equipment or perform appraisals and audits as required by DOE 5480.16 .

Code: $\quad$ Category III

Compliance

Protocol:

DOE Order 5480.16

Priority: $\quad$ Energy Systems Risk Weight 112

Tiger Team Action Plan Priority 2

Response: This concern is fully addressed by actions outlined in response to Findings RSS.3-1 (Analyses to Determine Appropriate Weapons) and RSS.4-1 (Safety Appraisals and Audits for Firearms).

Planned Actions and Schedules:

See Findings RSS.3-1 and RSS.4-1.

Costs: $\quad$ Costs are included in Findings RSS.3-1 and RSS.4-1.

References: None 


\subsubsection{Experimental Activities}

Finding No.: EA.2-1 ORNL Policy Regarding Independent Safety Reviews

Finding

Description:

There is no Laboratory-wide policy or procedure to address requirements for independent safety review of experiment plans and procedures for low-hazard nuclear and nonnuclear facilities by the Environmental, Safety, and Health Compliance organization.

Code: $\quad$ Category III

Compliance

Protocol:

Priority:

Response:
DOE Orders 5480.1B, 5480.10, 5480.11, and 5483.1A

Energy Systems Risk Weight 69

Tiger Team Action Plan Priority 2

ORNL will prepare and implement a standard-practice procedure (SPP) that will standardize a graded internal (division) review of all experiments and will provide formal guidance to division reviewers for conducting their reviews. The SPP will include guidance on identifying experiments that require independent reviews. An accompanying or included standard will contain guidelines for oversight of lowhazard facilities.

\section{Root Causes:}

Poorly defined roles and responsibilities, inadequate policy, inadequate policy implementation, and inadequate oversight

Planned Actions and Schedules:

\section{Item/Description}

1. Initiate independent surveillance of experiments in nonnuclear facilities at a level of 0.1 FTE using existing staff.

2. Issue an SPP to address experiment review, including requirements for graded internal review and review outside the division. The SPP will define the roles and responsibilities regarding independent safety reviews of experiments.
Completion Date

Complete

$8 / 91$ 
3. Provide a 2- to 4-hour workshop for division safety officers (DSOs and RCOs) to provide guidance on implementation of the SPP.

\section{Costs:}

Type of funds: Overhead

Source of funds: Overhead

\begin{tabular}{crrrrrrr} 
& \multicolumn{5}{c}{ Estimated costs per fiscal year (\$K) } & \\
\cline { 2 - 6 } Action item & 1991 & 1992 & 1993 & 1994 & 1995 & Beyond & Total \\
\hline 1 & 8 & & & & & 8 \\
2 & 15 & & & & & 15 \\
3 & & 2 & & & & 2
\end{tabular}

Status:

Funded

23

Requested

New

2

References: $\quad$ ORNL Long-Range Plan, 1989-95, ORNL 65-25 
Finding No.: EA.3-1 Guidance on Internal Safety Reviews

Finding

Description: ORNL organizations do not uniformly provide guidance for internal safety review and approval of experimental activities.

Code: $\quad$ Category III

Compliance

Protocol:

DOE Orders 5480.1B, 5480.5, 5480.10, and 5483.1A

Priority: $\quad$ Energy Systems Risk Weight 69

Tiger Team Action Plan Priority 2

Response: $\quad$ ORNL will prepare a standard-practice procedure (SPP) that will standardize graded, internal (division) review of all experiments and will provide formal guidance to division reviewers for conducting their reviews. (Refer to Finding EA.2-1.) ORNL management will require divisions to implement the internalreview guidelines. Implementation of this SPP will be evaluated by the independent internal appraisal system (see Finding MF-5).

\section{Root Causes:}

Poorly defined roles and responsibilities, inadequate policy, and inadequate policy implementation

\section{Planned Actions and Schedules:}

This finding is fully addressed by actions outlined in responses to Findings EA.2-1 and MF-5.

Costs: $\quad$ Costs to address this finding are reported under Findings EA.2-1 and MF-5.

References: $\quad$ ORNL Long-Range Plan, 1989-95, ORNL 65-25 
Finding No.: EA.3-2 Documentation of Safety Reviews of Experimental Plans

Finding

Description: Safety reviews of experimental plans and procedures are not always formally documented.

Code: $\quad$ Category III

Compliance

Protocol:

DOE Orders 5480.1B, 5480.10. 5480.11, and 5483.1A

Priority: $\quad$ Energy Systems Risk Weight 55

Tiger Team Action Plan Priority 2

Response: ORNL will prepare a standard-practice procedure (SPP) that will standardize graded, internal (division) review of all experiments and will provide formal guidance to division reviewers for conducting and documenting their reviews. (See Finding EA.2-1.) This SPP will include requirements for formal documentation of review of experiments, experiment plans, and experiment procedures in compliance with ORNL requirements for record management. Implementation of this SPP will be evaluated by the independent internal appraisal system (see Finding MF-5).

Root Causes:

Poorly defined roles and responsibilities, inadequate policy implementation, and inadequate oversight

\section{Planned Actions and Schedules:}

This finding is fully addressed by actions outlined in responses to Findings EA.2-1 and MF-5.

Costs: $\quad$ Costs to implement this finding are reported under Findings EA.2-1 and MF-5.

References: $\quad$ ORNL Long-Range Plan, 1989-95, ORNL 65-25 
Finding No.: EA.4-1 Random Safety Surveillances

Finding

Description: The Office of Operational Readiness and Safety does not routinely conduct random safety surveillances of experimental activities.

Code: $\quad$ Category III

Compliance

Protocol:

DOE Orders 5480.1B, 5480.10, 5480.11, and 5483.1A

Priority: $\quad$ Energy Systems Risk Weight 6

Tiger Team Action Plan Priority 3

Response: The Office of Operational Readiness and Safety is currently performing routine random inspections of experimental activities on a very infrequent basis with approximately 0.1 FTE and will increase oversight and routine random inspections of low-hazard experimental activities to a level of approximately 0.5 FTE beginning in October 1991 after replacement of a staff member. Issuance of an ORNL SPP will follow addressing ORNL and divisional review and oversight of experiments. The SPP will include standards or have standards as an accompanying document. (See Findings EA.2-1 and EA.3-1.)

Root Cause:

Inadequate oversight and insufficient resources

Planned Actions and Schedules:

$\underline{\text { Item/Description }}$

1. Request funds to hire staff member to devote at least half time for oversight and routine random inspections of low-hazard experimental facilities.

2. Contingent on receipt of funding, hire half-time staff member for additional oversight and routine inspections of experimental facilities. Oversight and inspections will follow guidelines from the standard that accompanies the ORNL SPP.
Completion Date

$7 / 91$

$10 / 91$ 


\section{Costs:}

Type of funds: Overhead

Source of funds: Overhead

\begin{tabular}{|c|c|c|c|c|c|c|c|}
\hline \multirow[b]{2}{*}{ Action item } & \multicolumn{6}{|c|}{ Estimated costs per fiscal year (\$K) } & \multirow[b]{2}{*}{ Total } \\
\hline & 1991 & 1992 & 1993 & 1994 & 1995 & Beyond & \\
\hline 2 & & 35 & * & & & & 35 \\
\hline \multicolumn{8}{|l|}{ Status: } \\
\hline \multicolumn{8}{|l|}{ Funded } \\
\hline \multicolumn{8}{|l|}{ Requested } \\
\hline New & & 35 & & & & & $\$ 35$ \\
\hline
\end{tabular}

*Estimated annual ongoing cost: \$37K beginning in FY 1993.

References: $\quad$ ORNL Long-Range Plan, 1989-95, ORNL 6525 


\subsubsection{Site/Facility Safety Review}

Finding No.: FR.1-1 Organization and Implementation of Safety Review System

Finding

Description:

Code:

Compliance

Protocol:
ORNL management has not used good industry practice in organizing and implementing its safety review system.

Category III

DOE Order 5480.5 (9-23-86)-Contractors are required to implement a comprehensive and documented independent review and appraisal system for nonreactor nuclear facilities.

DOE Order 5480.6 (9-23-86)-Contractors are required to implement a comprehensive and documented independent review and appraisal system for all phases of reactor program life.

DOE Order 5482.1B-Contractors are required to implement internal appraisals at the operating level.

Priority: $\quad$ Energy Systems Risk Weight 6

Tiger Team Action Plan Priority 3

Response: The corrective action plan for Finding MF-5 also addresses Findings FR.1-1, FR.3-1, and FR.4-2. These same findings were reported in the DOE-ORO Functional and Multidisciplinary Appraisal, April 1990 (IR-90-04-30-A) and the Martin Marietta Corporate Audit, July 1990 (SAF-14).

Prior to 1990 audit findings, efforts were begun in CY 1990 to enhance the Director's Review Committee (DRC) process. A full-time DRC coordinator was appointed, the process was flow-charted, the existing SPP was revised to reflect the process changes, and quarterly meetings were initiated with the DRC Chairman to discuss improvements and issues. The action plan for Finding MF-5 represents a brief outline of the remaining important steps that must be implemented to fully meet the DOE order requirements. See the response to Finding FR.4-1 for the action plan concerning a strengthened follow-up system.

\section{Root Causes:}

Inadequate management commitment, lack of clear policy, ambiguous requirements or expectations, and inadequate implementation of existing policy

\section{Planned Actions and Schedules:}

This finding is fully addressed by actions outlined in the response to Finding MF-5. 
Cists: The costs to implement this finding are reported under Finding MF-5.

References: $\quad$ DOE Order 5480.5

DOE Order 5480.6

DOE Order 5482.1B

Finding MF-5 
Finding No.: FP.2-1 Revieu of Safety Questions and Topics

Finding Dercription.

ORNL management has not established a system which ensures that all significant safety questions and topics are identifiod and submittod to an appropriate safety committec for revicw. (Soc atso Concern EA.3-1 and PT 3-1.)

Code: Category III

Compliance Protocol.

Section 9.1 of DOE Order 5480.5 requires an internal safety review system that provides an independent determination of whether a proposed activity involves an unreviewed safety question. violation of a Criticality Safety imit, Operational Saíety Requirement (OSR), or any matter for which approval is required.

Section 9.d.(2)(g) of DOE Order 5482.1B requires that the internal appraisal system provide for objective and independent reviews of ES\&H functions for conducting revieus of proposed modifications and experiments, procedures, organization and staffing, operating limits, training, and occurrences.

Priority: $\quad$ Energy Systems Risk Weight 60

Tiger Team Action Plan Priority 2

Response: This actic: plan addresses improvements in the identification of significant safety issues and heir submittal to appropriate safety review committees. The action plan for improvt ment in the review of this finding relates to Findings FR.1-1, EA.2-1, EA.3-1, OA.1-5, PP.3-1, and MF-5.

Correcting the situation which led to this concern requires adequate identification of facility items important to safety. The phased ORNL Safety Documentation Upgrade Program is underway and will accomplish this task by producing updated SARs and OSRs for reactor, nonreactor nuciear, and selected other facilities with significant hazard potential. Refer to Section 3.7, Technical Support, of the ORNL Self-Assessment for more information on this program. A required companion activity to correct the root cause is to implement a configuration management program. This program is to address control, review (including review by the appropriate safety committees), approval, and documentation of changes related to safety. An interim configuration managerient program is underway and a more rigorous and standardized program will be implemented in the long term.

Completion of action items from Findings MF-5 and EA.2-1 along with the action items specified in this corrective action plan will resolve this concern.

Implementation of the processes developed as part of this action plan will be evaluated under the independent internal appraisal system developed in response to Finding MF-5. 


\section{Root Causcs:}

Inadequate policy, inadequate policy implementation, and inadequate management approach

Planned Actions and Schedules:

\section{$\underline{\text { Item/Description }}$}

1. Initiate Interim Configuration Management Program.

2. Develop and initiate implementation of an ORNL SPP for configuration management to supersede the interim document issued on November 1, 1990. The SPP will specify that it is to be phased in as part of the facility safety evaluation process and will address the process to identify those issues which require independent review and approval (see Finding AX.1-2, Item 4).
Completion Date

Complete

$12 / 91$

Casts:

Type of funds: Overhead

Source of funds: Overhead

\begin{tabular}{|c|c|c|c|c|c|c|c|}
\hline \multirow[b]{2}{*}{ Action item } & \multicolumn{6}{|c|}{ Estimated costs per fiscal year (\$K) } & \multirow[b]{2}{*}{ Total } \\
\hline & 1991 & 1992 & 1993 & 1994 & 1995 & Beyond & \\
\hline 1 & - & & & & & & \\
\hline 2 & & 15 & & & & & 15 \\
\hline
\end{tabular}

Status:

Funded

Requested

New

15

References: DOE Order 5480.5

DOE Order 5482.1B

ORNL Self Assessment, Section 3.7, Technical Support

Letter from M. W. Rosenthal of November 1, 1990 "ORNL Interim Configuration Management Program"

"Safety Analysis Report Update Program - Overview and Phase I Implementation," dated October 1990 
Finding No.: FR.3-1 Shortcomings of the Safety Review System

Finding

Description: The present safety review system has not been organized to perform coordinated, multidisciplinary reviews, and the Director's Review Committees do not have proper guidance from management to achieve a high degree of safety.

Code: $\quad$ Category III

Compliance

Protocol.

None

Priority: $\quad$ Energy Systems Risk Weight 63

Tiger Team Action Plan Priority 2

Response: The corrective actions for Finding MF-5 address the upgrade of the Safety Review System.

Root Cause:

Inadequate management approach

Planned Actions and Schedules:

This finding is fully addressed by actions outlined in the response to Finding MF-5.

Costs: $\quad$ See Finding MF-5.

References: $\quad$ Finding MF-5 
Finding No.: FR.4-1 Management Response to Safety Committee Recommendations

Finding

Description: Contrary to DOE 5480.5 and DOE 54821B, ORNL top management has not taken timely acticin on safety committees' recommendations.

Code: $\quad$ Category III

Compliaice Protocol:

DOE Order 5480.5 (9-23-86) 9.a. and c. Management is required to take necessary corrective actions and document such actions in an auditable manner.

DOE Order 5482.1B (9-23-86) 9.c.(4). Appraised organizations shall respond to appraisal recommendations within 30 days.

Priority: $\quad$ Energy Systems Risk Weight 98

Tiger Team Action Plan Priority 2

Response: In the past, the DRC follow-up process has been weak, without a means to escalate critical open and overdue recommendations to the attention of upper management. However, ORNL upper management has been closely involved with the Director Review Committee (DRC) process upgrade in CY 1990. This upgrade includes increased involvement of upper management in the DRC process as well as an improved follow-up system. Implementation of the independent internal review system, as discussed in the corrective actions for Findings MF-5 and MF-6, will verify the adequacy of implementation.

Root Causes:

Inadequate management commitment and inadequate policy

Planned Actions and Schedules:

\section{$\underline{\text { Item/Description }}$}

1. Transmit final review reports to the Laboratory director from the DRC chairmen.

2. Issue interim guidance to DRC Chairmen.

a. Require responsible organizations to respond to the Laboratory Director with corrective actions for DRC recommendations.

b. Quality Department issue monthly reminders and overdue notices regarding DRC recommendation corrective actions.
Completion Date

Complete 
c. OORS present quarterly briefing to the ES\&H coordination committee on DRC review and corrective action status.

Casts:

Type of funds: Overhead

Source of funds: Overhead

Estimated costs per fiscal year (\$K)

\begin{tabular}{cccccccc}
\cline { 2 - 6 } Action item & 1991 & 1992 & 1993 & 1994 & 1995 & Beyond & Total \\
\hline 1 & - & & & & & & \\
2 & 15 & & & & & & 15
\end{tabular}

Status:

Funded

15

Requested

New

References: $\quad$ DOE Orders 5480.5, 5480.6, and 5482.1B

Martin Marietta Corporate Audit July 1990, Findings MNG-01 and MNG-05

DOE Memorandum from the Secretary of Energy, Guidance on Environment, Safety, and Health Self-Assessment, July 31, 1990 
Finding No.: FR.4-2 Annual Facility Appraisals

Finding

Description: The annual facility appraisals have not been independent because those performing appraisals are the same as those performing safety reviews of these facilities.

Code: $\quad$ Category III

Compliance

Protocsl: None

Priority: $\quad$ Energy Systems Risk Weight 1

Tiger Team Action Plan Priority 4

Response: The corrective actions for Finding MF-5 address the annual facility appraisal issue.

Root Cause:

Poorly defined roles and responsibilities

Planned Actions and Schedules:

This finding is fully addressed by actions outlined in the response to Finding MF-5.

Costs: $\quad$ See Finding MF-5.

References: Finding MF-5 
Finding No.: $\quad$ FR.5-1 Triennial Appraisal

Finding

Description: Methods used for the conduct of the triennial appraisal have not provided a comprehensive, independent appraisal of the effectiveness of the overall safety review system at ORNL.

Code: $\quad$ Category III

Compliance

Protocol:

DOE Order 5480.5 (9-23-86) 9.i. Contractor independent review and appraisal system is reviewed by contractor management for adequacy of performance at least every 3 years.

DOE Order 5480.6 (9-23-86) 8.g. (9). Contractor independent review and appraisal system is reviewed by contractor management for adequacy of performance at least every 3 years.

DOE Order 5482.1B (9-23-86) 9.d. (2)(d). Internal appraisals are to be reviewed by management for adequacy of performance every 3 years, or mcre often, as required.

Priority: $\quad$ Energy Systems Risk Weight 5

Tiger Team Action Plan Priority 3

Response: ORNL agrees that the past triennial appraisals were limited in scope, and appraisal team members were not sufficiently independent. Previous findings from the DOEORO Functional Multidisciplinary Appraisal, May 1990, (IR-90-04-30-A and IR-90-04-30-B) address inadequate coverage by the ES\&H internal review program and insufficient review of the appraisal system. Items 3, 5, and 7 in the corrective actions for Finding MF-5 address the methods and conduct of the upgraded triennial appraisal.

Root Cause:

Ambiguous requirements or expectations

Planned Actions and Schedules:

\section{$\underline{\text { Item/Description }}$}

1. Present justification for 1-year extension from DOEORO to delay triennial appraisal based on the following:
a. Tiger Team,
b. other multidisciplinary appraisals, and
c. changes being incorporated.

Completion Date

$8 / 91$ 
2. See Items 3,5 , and 7 of the corrective action for Finding MF-5.

Costs: $\quad$ See the costs associated with Items 3, 5, and 7 of Finding MF-5.

References: $\quad$ DOE Orders 5480.5, 5480.6, and 5482.1B

ESH-20, III. D

DOE-ORO Environment, Safety \& Health and Quality Assurance (ES\&H \& QA) Functional \& Multidisciplinary Appraisal of Oak Ridge National Laboratory 
Finding No.: FR.6-1 Industry Lessons Learned

Finding

Description: ORNL is not fully utilizing an important source of lessons learned in industry in its safety improvement program.

Code: $\quad$ Category III

Compliance

Protocol: None

Priority: $\quad$ Energy Systems Risk Weight 55

Tiger Team Action Plan Priority 2

Response: We are currently in the process of implementing several DOE orders requiring lessons learned. Energy Systems Quality Director is currently revising GP-5.3 to specify an integrated approach for applicability of lessons learned; ORNL Quality staff are participating in the development of ES GP-5.3.

Effective December 1, 1990, the quality auditing function was returned to the Quality Department. As a part of the reorganization, the Quality Auditing Manager (see Finding MF-6) will become a single point for tracking corrective actions, facilitating root cause analysis, trending problems and concerns, and establishing a lessons-learned system. Planned actions listed here also apply to Finding TS.4-1.

Root Cause:

Inadequate resources and inadequate policy; lack of a policy for lessons learned.

Planned Actions and Schedules:

Item/Description

Completion Date

1. Issue appropriate ORNL procedures to implement $7 / 91$ ES GP-5.3.

2. Contingent on approval of funding, hire lessc is-learned $7 / 91$ staff person. 


\section{Casts:}

Type of funds: Overhead

Source of funds: Overhead

\begin{tabular}{|c|c|c|c|c|c|c|c|}
\hline \multirow[b]{2}{*}{ Action item } & \multicolumn{6}{|c|}{ Estimated costs per fiscal year (\$K) } & \multirow[b]{2}{*}{ Total } \\
\hline & 1991 & 1992 & 1993 & 1994 & 1995 & Beyond & \\
\hline 1 & 15 & & & & & & 15 \\
\hline 2 & 21 & * & & & & & 21 \\
\hline \multicolumn{8}{|l|}{ Status: } \\
\hline Funded & 15 & & & & & & \\
\hline \multicolumn{8}{|l|}{ Requested } \\
\hline New & 21 & & & & & & $\$ 36$ \\
\hline
\end{tabular}

*Estimated ongoing annual costs: \$85K starting in FY 1992.

References: DOE Orders 5700.6 and $5000.3 \mathrm{~A}$ 


\subsubsection{Radiological Protection}

Finding No.: RP.1-1 Accomplishing ES\&H Compliance

Firiding

Description: There is little direction in the generation of policy, delegation of responsibilities and authorities, procurement of resources, and management priorities, so that mandatory requirements of DOE 5480.11 are not being met.

Code: $\quad$ Category III

Compliance

Protocol:

DOE Order 5480.11

Priority. $\quad$ Energy Systems Risk Weight 68

Tiger Team Action Plan Priurity 2

Response: $\quad$ ORNL Standard Practice Procedure X-ESH-3, "Health Physics Manual", dated April 16, 1990, authorizes implementation of the ORNL Radiological Protection Program at ORNL's highest management 'evel and assigns management responsibilities for implementing program policies to the Director, Environmental, Safety, and Health Compliance (ESHC). Even through the procedure authorizes the establishment of policy to the ESHC Director, it was found that the Charters of Respcrisibility, Authority, and Accountability for Laboratory and ESHC managers do not specifically address this policy-making delegation.

A "DOE Order 5480.11 Implementation Plan" was approved during March 1990 which identified the plans to obtain compliance with DOE Order 5480.11. This implementation plan identified the cost of implementation and the appropriate methods of obtaining the resources. The implementation plan will be reviewed, revised, and approved by Laboratory and ESHC management to ensure that an aggressive approach to becoming compliant with DOE Order 5480.11 is evident.

The Office of Environmental and Health Proteciion has initiated a surveillance and self-assessment program to ensure that manag ers and supervisors are involved in surveillance of their work areas and the work performed by their employees. A surveillance plan has been developed which specifies surveillance requirements and applicable procedures. A manager or supervisor will be assigned oversight for each surveillance with a Measurements and Assessments Program Professional assisting in the documentation of findings and development of corrective actions. The surveillance and self-assessment program allows for a structure approach for managers and supervisors to determine compliance with procedures and other regulatory compliance, as well as maintaining an effective working relationship with cheir employees.

This concern was previously addressed in the "Radiation Protection Program Appraisal of the Oak Ridge Complex" conducted by DOE during May 1989. 


\section{Root Causes:}

Inadequate poiicy, inadequate management approach, inadequate communications, ambiguous requirements or expectations, and poorly defined roles and responsibilities

\section{Planned Actions and Schedules:}

\section{Item/Description}

1. Review and, if necessary, issue revisions to the Laboratory's DOE Order 5480.11 Implementation Plan dated March 1990 to ensure that adequate resources and funds are requested and plans for obtaining the resources and funds are documented and approved to comply with mandatory requirements. Revised plan will be issued to DOE-ORO.

2. Revise and approve the Charters of Responsibility, Authority, and Accountability for the Environmental, Safety, and Health Compliance (ESHC) Director and the ESHC Office Directors to reflect policy-making authority flowdown for ESHC activities.

Note: The Charters will delineate and assign responsibility to initiate and enforce corrective action.

3. Issue a procedure to ensure that individual Charters of Responsibility, Authority, and Accountability for EHP personnel are reviewed annually to determine accuracy.

4. Issue a standard operating procedure to require EHP management involvement in planned surveillances and assessments for EHP programs and activities in order to provide assurance that managers are aware of area operations.

5. Issue an administrative procedure specifying requirements for incorporation of new and revised procedures into the appropriate training programs.

6. Revise the EHP surveillance plans to assign a responsible oversight manager for each surveillance.

7. Keview the process of funding Radiation Protection activities and provide improvement recommendations to the Environmental, Safety, and Health Coordinatio in Committee for review and implementation of approved recommendations.

\section{Completion Date}

$05 / 91$

$05 / 91$

$06 / 91$

09/91

$09 / 91$ 
Costs:

Type of funds: ESH Programmatic

Source of funds: ER-AT RP

\begin{tabular}{|c|c|c|c|c|c|c|c|}
\hline \multirow[b]{2}{*}{ Action item } & \multicolumn{6}{|c|}{ Estimated costs per fiscal year (\$K) } & \multirow[b]{2}{*}{ Total } \\
\hline & 1991 & 1992 & 1993 & 1994 & 1995 & Beyond & \\
\hline 1 & 23 & & & & & & 23 \\
\hline 2 & 2 & & & & & & 2 \\
\hline 3 & 10 & & & & & & 10 \\
\hline 4 & 10 & & & & & & 10 \\
\hline 5 & 11 & & & & & & 11 \\
\hline 6 & 6 & & & & & & 6 \\
\hline 7 & 4 & & & & & & 4 \\
\hline
\end{tabular}

Status:

Funded

Requested

New

66

References: None 
Finding No.: RP.1-2 ES\&H Compliance Staff

Finding

Description.

Code: $\quad$ Category III

Compliance

Protocol:

Priority:

Response:

None
Neither the Environment and Health Protection office nor the Environment, Safety, and Health Compliance division have a sufficient number of trained and experienced staff to effectively correct identified deficiencies to ensure effective implementation and control of health physics activities.

Energy Systems Risk Weight 95

Tiger Team Action Plan Priority 2

The need for qualified replacements for key personnel was identified during the "DOE Radiation Protection Appraisal of the Oak Ridge Complex" during 1989.

The search for replacements for retiring personnel within the Radiation Protection Section is continuing. Qualified replacements for senior health physicists are not readily available, and recruitment of outside professionals is continuing. ORNL has completed recruitment trips to several institutions with Health Physics programs.

Staffing levels of Radiation Protection personnel are dependent on funding availability from supporting divisions and programs. In some areas of the plant, staffing levels that are directly funded and those that are needed to provide optimum health physics coverage are not in agreement, as is the case in some areas of ORNL's Y-12 facilities. An assessment will be made to determine the appropriate level of staffing to support the $\mathrm{X}-1 \mathrm{U}$ site and ORNL operations at Y-12.

As ORNL proceeds with putting in place policies and procedures to implement the new Energy Systems-wide requirements related to conduct of operations and roles and responsibilities, appropriate procedure inclusions will ensure (1) establishment of baseline ES\&H programs and staffing levels, (2) budget support for the baseline ES\&H programs and staffing, (3) timely communication of staffing needs to staffing organization, (4) ab efficient staffing process to complete requested recruiting and hiring, and (5) self-evaluation of staffing performance and review of baseline goals. (See the RPPA TSA report, Management Issues.)

\section{Root Causes:}

Inadequate training, poorly defined roles and responsibilities, management approach, and inadequate resources 
Planned Actions and Schedules:

\section{Item/Description}

1. Conduct a current Radiation Protection and Radiation Monitoring Needs Assessment to define personnel needs in the ORNL Radiation Protection programs at the Laboratory (X-10 and Y-12 Site Operations).

2. Request funds from divisions, programs, or other sources to obtain resources necessary to support conclusions from the Needs Assessment.

3. Contingent upon availability of funds, hire additional staff identified in the Needs Assessment.

4. Issue a Training and Development Plan for Radiation Protection and Monitoring personnel.

5. Issue a Routine Survey Plan for ORNL Facilities at Y-12 to ensure that survey requirements are in compliance with ORNL Health Physics Procedure RP2.1, "Radiation Surveys," and Standard Operating Procedure 02-50-10, "Radiation Surveillance in Radiological Control Areas."
Completion Date

$0 \varepsilon / 91$

$10 / 91$

$09 / 92$

$09 / 91$

$09 / 91$

\section{Costs:}

Type of funds: ESH Programmatic

Source of funds: ER-AT RP

Estimated costs per fiscal year (\$K)

\begin{tabular}{crrrrrrr}
\cline { 2 - 6 } Action item & 1991 & 1992 & 1993 & 1994 & 1995 & Beyond & Total \\
\hline 1 & 8 & & & & & & 8 \\
4 & 51 & & & & & & 51 \\
5 & 20 & & & & & & 20
\end{tabular}

Status:

Funded

Requested

New 
Type of funds: Overhead

Source of funds: Division Administration

Estimated costs per fiscal year $(\$ K)$

\begin{tabular}{|c|c|c|c|c|c|c|c|}
\hline Action item & 1991 & 1992 & 1993 & 1994 & 1995 & Beyond & Total \\
\hline 3 & & * & & & & & \\
\hline \multicolumn{8}{|l|}{ Status: } \\
\hline \multicolumn{8}{|l|}{ Funded } \\
\hline \multicolumn{8}{|l|}{ Requested } \\
\hline New & & & & & & & $\$ 500$ \\
\hline
\end{tabular}

*Estimated annual ongoing cost: \$500K in FY 1992, and \$1,048K in FY 1993.

References: $\quad$ DOE Order 5480.11 
Finding No.: RP.1-3 Management Oversight

Finding

Description:

Code:

Compliance

Protocol:

Priority:

Response:
There is an absence of oversight at the highest levels of management resulting in a general absence of radiation safety awareness and acceptance of established procedures and practices as required by DOE 5480.11.

Category III

DOE Order 5480.11

Energy Systems Risk Weight 73

Tiger Team Action Plan Priority 2

This concern was previously expressed in the "Radiation Protection Appraisal of the Oak Ridge Complex" conducted by DOE in 1989. Prior to the Tiger Team appraisal, a "DOE Order 5480.11 Implementation Plan" was developed, approved by Laboratory management, and issued to DOE. The implementation of the plan has resulted in a commitment by Laboratory management to become compliant with DOE Order 5480.11. The DOE-ORO "X-10 Site Office" personnel have been proactive in the support and oversight of the implementation plan.

The primary basis of the concern was identified by the observation that the DOE Order 5480.11 was driven through the ESHC organization, and ownership by operating division and program managers was not apparent. Increased management oversight, in the form of walk-throughs and self-surveillances, is needed to ensure compliance, completeness, and consistency with the ORNL Health Physics Procedures Manual procedures. The self-assessment process, as outlined in Finding SA-1, will ensure management involvement in the enforcement of Health Physics requirements.

The new requirements and policies for radiation safety and contamination control require a re-education of a large portion of the Laboratory population. The Technical Resources and Training Section of the Office of Environmental and Health Protection has a well-established and current radiation worker program that introduces new requirements as they are added to approved ORNL Health Physics Manual procedures. There is a need to clarify the requirements for who is required to have the varying levels of training that are presently offered.

The radiation source control program is undergoing program upgrades to comply with the "ORO Radiation Source Control Policy", and increased attention within the Office of Environmental and Health Protection is needed to ensure that progress is made towards compliance. 
Findings associated with the Laboratory's oversight of the accelerators and X-ray machines require restructuring to ensure compliance with appropriate regulatory requirements. The Accelerator and Radiation Sources Review Committee and the $\mathrm{X}$-ray Safety Review Committee responsibilities and activities will be reviewed to determine the appropriate oversight requirements.

The Radiation Source Control Manager has the authority, accountability, and responsibility for the entire source control program at the Laboratory. The source control program is administered across the Laboratory by the Health Physics Procedure RP 2.14, "Radiation Source Control." The Health Physics Manual is an ORNL Standard Practice Procedure. Program compliance will be maintained through the use of the Measurements Assurance surveillance program and an inventories and leak tests performed by a dedicated technician. Program authority may be escalated to the Director of Environmental, Safety, and Health Compliance, if needed.

ORNL will use DOE Order 5000.3A, Attachment 1, as a mechanism to document noncompliance with ORNL's source control procedures, in respect to lack of registration, leaking, or loss of sources in excess of exempt quantities. The submission of an "Occurrence Report" will provide the Source Control Manager access to all the Laboratory's resources for solving program difficulties.

\section{Root Causes:}

Inadequate management commitment, inadequate oversight, and ambiguous requirements or expectations

Planned Actions and Schedules:

\section{Item/Descripition}

1. Review charters and activities of the Accelerator and Radiation Sources Review Committee and the X-Ray Safety Review Committee and approve necessary changes to the charters for effective oversight requirements. (See Finding MF-5.)

2. Hire a Laboratory Radiation Source Control Manager to ensure compliance with the "ORO Radiation Source Control Policy."

3. Review the training status for Laboratory personnel regarding radiation worker training, radiological posting, and radiation source control and make necessary changes to the appropriate training programs.

\section{Completion Date}

Complete

$09 / 91$ 


\section{Costs:}

Type of funds: Overhead

Source of funds: Overhead

\begin{tabular}{cccccccc} 
& \multicolumn{6}{c}{ Estimated costs per fiscal year (\$K) } \\
\cline { 2 - 6 } Action item & 1991 & 1992 & 1993 & 1994 & 1995 & Beyond & Total \\
\hline 1 & - & & & & & & \\
2 & 107 & $*$ & & & & & 107
\end{tabular}

Status:

Funded

107

Requested

New

*Estimated annual ongoing cost: $\$ 160 \mathrm{~K}$.

Type of funds: ESH Programmatic

Source of funds: ER-RP

\begin{tabular}{|c|c|c|c|c|c|c|c|}
\hline \multirow[b]{2}{*}{ Action item } & \multicolumn{6}{|c|}{ Estimated costs per fiscal year (\$K) } & \multirow[b]{2}{*}{ Total } \\
\hline & 1991 & 1992 & 1993 & 1994 & 1995 & Beyond & \\
\hline 3 & 36 & & & & & & 36 \\
\hline \multicolumn{8}{|l|}{ Status: } \\
\hline \multicolumn{8}{|l|}{ Funded } \\
\hline \multicolumn{8}{|l|}{ Requested } \\
\hline New & 36 & & & & & & $\$ 36$ \\
\hline
\end{tabular}

References: $\quad$ DOE Order 5480.11 
Finding No.: RP.3-1 ORNL Posting and Contamination Program

\section{Finding}

Description:

The ORNL posting and contamination program does not meet the intent of DOE 5480.11 as clarified in Draft DOE 5480.11 changes.

Code: $\quad$ Category III

Compliance

Protocol:

Priority:

Response:

\section{Draft DOE Order 5480.11.}

Energy Systems Risk Weight 73

Tiger Team Action Plan Priority 2

DOE-ORO personnel state that the requirements specified in the "Oak Ridge Operations Contamination Control Policy" are in strict compliance with the current DOE Order 5480.11. A goal of the development and implementation of the policy was to provide for the best possible contamination control and radiation surveys with available resources as well as to realize an actual size reduction of the radiological areas within the Controlled Areas at the Laboratory. DOE-ORO involved DOE-EH throughout the development of the policy, and all DOE-EH comments have been incorporated into the policy.

ORNL's approach, under the guidance of the DOE-ORO X-10 Site Office, was to implement the policy in such a manner that the maximum protection for employees, visitors, and the environment is achieved. The approach provides the highest degree of assurance against "track-out" of radioactive materials, given current resource restrictions. An "Oak Ridge Operations Contamination Control Policy Implementation Plan" for ORNL was developed and approved for implementation in August 1990. All of the action items contained in the implementation plan have been completed.

The approach refers to the radiological controls established, not the contamination approach. ORNL, with guidance from ORO, has established Controlled Areas postings at the main fence around the facility and around the other facilities located within the Reservation. The majority of the Laboratory's area within the designated Controlled Area is a Nonradiological Area. Materials that are surveyed for release are routinely stored in nonradiological areas (inside the Controlled Area) while awaiting transport to the salvage holding area. Procedures specify the requirements for release of material into nonradiological areas. Materials released for use inside the Laboratory are surveyed and held to the same rigid contamination and radiation limits as material intended for off-site use.

\section{Root Causes:}

Insufficient resources, inadequate management approach, inadequate oversight, and inadequate policy implementation 
Planned Actions and Schedules:

$\underline{\text { Item/Description }}$

1. Ensure that radiological posting is completed in accordance with the "Oak Ridge Operations Contamination Control Policy Implementation Plan" and document in a letter.

2. Issue revised ORNL Health Physics Manual procedures and the Radiation Protection and Radiation Monitoring Standard Operating Procedures Manual procedures to reflect the approved requirements for contamination control.

3. Review and revise the training programs at ORNL which are used to train personnel in the requirements of the contamination control procedures to reflect the approved requirements. (See Item 5 of Finding RP.1-1, which addresses training.)
Completion Date

Complete

\section{Costs:}

Type of funds: ESH Programmatic

Source of funds: ER-RP

Estimated costs per fiscal year (\$K)

\begin{tabular}{crrrrrrr}
\cline { 2 - 5 } Action item & 1991 & 1992 & 1993 & 1994 & 1995 & Beyond & Total \\
\hline 1 & 5 & & & & & & 5 \\
2 & 23 & & & & & & 23
\end{tabular}

Status:

Funded

Requested

New

References: DOE Order 5480.11, "ORO Contamination Control Policy" 
Füding No.: RP.3-2 Contamination Control Program

Finding

Description: The technical bases and justification of the contamination control program have not been developed by either Oak Ridge or ORNL.

Code: $\quad$ Category III

Compliance

Protocol:

None

Priority: $\quad$ Energy Systems Kisk Wieight 8

Tiger Team Action P'an Priority 3

Response: The ORO Contamination Control Policy is compliant with DOE Order 5480.11. The specific methods of compliance with the policy were developed by ORNL through the ORO Contamination Control Policy Implementation Plan. The DOE/ORO Contamination Control Policy made a significant departure from long-standing ORNL radiation area zoning nomenclature. The policy and the required changes to the zoning requirements were made in October 1990. The policy changed the use of a "Regulated Zone (Area)" from a buffer zone around radiological areas to a low-level contamination area. The zoning change and resulting additional frisking requirements caused some delay in full compliance to the policy. An agreement between ORNL personnel and the ORO Site Representative was made on clarifications to ensure maximum "track-out" control and increased acceptance and understandability to the area postings and controls. The Radiation Protection staff has worked in conjunction with facility managers to ensure the complete implementation of policy requirements. The Measurements Assurance Surveillance Program will continue to monitor and enforce the ORNL Health Physics Manual procedures and the Radiation Protection and Radiation Monitoring Standard Operating Procedures Manual procedures. Emphasis on totalbody frisks will continue on egress from all posted contamination areas and Regulated Areas that have significant transferable contamination.

Further consideration has been given to some of the controls that were initiated for Regulated Areas that are possibly contaminated, but are typically clean. In these areas, procedures and policies will be revised to allow more-professional judgment in establishing survey and protective clothing requirements.

ORNL has requested funding and has proposed Item 1, Finding RP.6-3, for a technical bases document. This document will include a study of current ORNL instruments capabilities and survey techniques used for personnel frisking. Upon conclusion of the study, ORNL will act on the conclusions and recommendations of the report. Results of the study will be accommodated by procurement of fixed contamination monitors in an FY 93 line item request. 


\section{Root Causes:}

Insufficient resources and ambiguous requirements or expectations

Planned Actions and Schedules:

\section{$\underline{\text { Item/Description }}$}

1. Issue revision of the ORNL Health Physics Manual procedures and the Radiation Protection and Radiation Monitoring Standard Operating Procedures Manual procedures to specify the contamination control requirements, based upon the technical bases document.

2. Establish protective measures and surveys required in Regulated Areas and upon exit from these areas, based upon contamination surveys in the area, as well as potential for "track out."

3. Prepare engineering estimate and submit budget request for procurement and installation of truck monitoring station. (See Item 13 of RP.3-6.)

4. Contingent on funding, procure and install truck monitoring station. (See Item 14 of RP.3-6.)

5. Review and revise the radiation worker training to refleci changes in the contamination control program. (See Item 5 of RP.1-1, which addresses training.)
Completion Date

$04 / 92$

$06 / 91$ 


\section{Costs:}

Type of funds: Overhead

Source of funds: Division Administration

\begin{tabular}{lrrrrrrr} 
& \multicolumn{6}{c}{ Estimated costs per fiscal year $(\$ K)$} \\
\cline { 2 - 6 } Action item & 1991 & 1992 & 1993 & 1994 & 1995 & Beyond & Total \\
\hline 1 & & 21 & $*$ & & & & 21
\end{tabular}

Funded

Requested

New

* Estimated annual ongoing cost: $\$ 238 \mathrm{~K}$ in division administration funds for continuous upgrades for procedures and training.

Type of funds: ESH Programmatic

Source of funds: ER-AT RP

\begin{tabular}{crrrrrrr} 
& \multicolumn{5}{c}{ Estimated costs per fiscal year (\$K) } \\
\cline { 2 - 6 } Action item & 1991 & 1992 & 1993 & 1994 & 1995 & Beyond & Total \\
\hline 2 & 32 & & & & & & 32
\end{tabular}

Status:

Funded

Requested

New

References: $\quad$ DOE Order 5480.11, "ORO Contamination Control Policy" INPO Radiological Protection at Nuclear Power Stations 
Finding No.: $\quad$ RP.3-3 Source Control Program

Finding

Description- Compliance with the source control program will be difficult to achieve due to complexity of requirements and dependence on voluntary response.

Code: $\quad$ Category III

Compliance

Protocol:

None

Priority: $\quad$ Energy Systems Risk Weight 16

Tiger 'Team Action Plan Priority 3

Response: ORNL's Radiation Source Control Program has added significantly to the control and accountability of radioactive sources at the Laboratory. On April 10, 1990, the "ORO Radiation Source Control Policy" was issued; it specifies the requirements that ORNL is required to comply with by January i, 1991. Energy Systems has issued ESH-11 to specify requirements for the implementation of the policy.

Office of Environmental and Health Protection personnel are currently initiating a program to become compliant with ESH-11 and the ORO policy. ORNL Health Physics Procedure RP 2.14, "Radioactive Source Control," is currently under revision to specify requirements of the policy. The Radiation Protection and Radiation Monitoring Standard Operating Procedures Manual," Procedure 02-5055, "Surveying Sealed and Unsealed Radioactive Sources," was issued on September 29, 1990, to provide the technical guidance for the leak testing of radioactive sources. In addition, self-paced training program modules are being developed for each individual responsible for radiation source control.

A vacant position for a Radiation Source Control Manager remains to be filled; until the Manager has been hired, the Radiation Protection Section will administer the program. The Radiation Source Control Manager position will provide the inter-divisional oversight required to ensure compliance with the policy. 


\section{Root Causes:}

Inadequate policy implementation, insufficient resources, inadequate oversight, ambiguous requirements or expectations, and poorly defined roles and
responsibilities

Planned Actions and Schedules:

\section{$\underline{\text { Item/Description }}$}

1. Submit the "ORO Radiation Source Control Policy" to DOE-EH for review. (Note: DOE-ORO responsibility.)

2. Hire a Radiation Source Control Manager to provide oversight and ensure that the appropriate procedures are in compliance (see Item 3 of Finding RP.1-3).

3. Review and issue revisions to the ORNL Health Physics Manual procedures and the Radiation Protection and Radiation Monitoring Standard Operating Procedures Manual procedures to reflect the requirements of the policy.

4. Develop and initiate the Radiation Source Control Training Program.

5. Incorporate the requirements of the radiation source control program in the Office of Environmental and Health Proteciion surveillance program to ensure compliance. (See Item 6 of Finding RP.1-1.)
Completion Date

Complete

Complete

$09 / 91$ 


\section{Costs:}

Type of funds: ESH Programmatic

Source of funds: ER-AT RP

Estimated costs per fiscal year $(\$ \mathrm{~K})$

\begin{tabular}{|c|c|c|c|c|c|c|c|}
\hline Action item & 1991 & 1992 & 1993 & 1994 & 1995 & Beyond & Total \\
\hline 1 & 0 & & & & & & 0 \\
\hline 2 & 0 & & & & & & 0 \\
\hline 3 & 17 & & & & & & 17 \\
\hline 4 & 21 & * & & & & & 21 \\
\hline \multicolumn{8}{|l|}{ Status: } \\
\hline \multicolumn{8}{|l|}{ Funded } \\
\hline \multicolumn{8}{|l|}{ Requested } \\
\hline New & 38 & & & & & & $\$ 38$ \\
\hline
\end{tabular}

*Esiimated annual ongoing cost: $\$ 7 \mathrm{~K}$ in division administrative funds.

References: DOE/ORO Radioachive Source Control Policy ESH-11 
Finding No.: RP.3-4 X-Ray Generating Machine Policy and Requirements

Finding

Description:

The ORNL X-ray generating machine policy and requirements are not being applied for ORNL installations in facilities at the Y-12 Plant.

Code: $\quad$ Category III

Compliance

Protocol:

ORNL Health Physics Manual Procedure 2.8 requires fail-safe "X-Ray On" indicators. This procedure also requires that qualified operators shall receive the training course "X-Ray Machine Safety."

Priority: $\quad$ Energy Systems Risk Weight 6

Tiger Team Action Plan Priority 3

Response: $\quad$ Improvements in compliance with HPP 2.8 are addressed by this action plan. ORNL X-ray facilities at Y-12 will comply with ORNL HPP 2.8 by February 1991, or operating divisions will request approval of deviations through submission of plans for providing compensating measures. The X-ray units at all ORNL facilities at Y-12 will receive comprehensive triennial reviews beginning in December 1991.

Root Cause:

Inadequate policy implementation

Planned Actions and Schedules:

\section{$\underline{\text { Item/Description }}$}

1. Appraise all active ORNL $\mathrm{X}$-ray facilities at the $\mathrm{Y}-12$ site.

2. Remove all individuals who have not received the "X-Ray Machine Safety" training course from the list of qualified $X$-ray machine operators.

3. Operators of Biology X-ray facilities with unauthorized lights make the lights fail-safe or provide compensating measures as agreed upon in a formal safety-deviation request and document in a letter to the X-ray Safety Review Committee.

4. Submit review appraisal report to ORNL management.

5. Conduct triennial review of all active $\mathrm{X}$-ray facilities at Y-12 site.

\section{Completion Date}

Complete

Complete

Complete

$10 / 93$ 


\section{Casts:}

Type of funds: Overhead

Source of funds: Overhead

\begin{tabular}{|c|c|c|c|c|c|c|c|}
\hline \multirow[b]{2}{*}{ Action item } & \multicolumn{6}{|c|}{ Estimated costs per fiscal year $(\mathbf{\$ K})$} & \multirow[b]{2}{*}{ Total } \\
\hline & 1991 & 1992 & 1993 & 1994 & 1995 & Beyond & \\
\hline 5 & & * & & & & & \\
\hline \multicolumn{8}{|l|}{ Status: } \\
\hline \multicolumn{8}{|l|}{ Funded } \\
\hline \multicolumn{8}{|l|}{ Requested } \\
\hline New & & & & & & & $\$ *$ \\
\hline
\end{tabular}

*Estimated annual ongoing cost: $\$ 2 \mathrm{~K}$ triennially.

References: None 
Finding No.: RP.3-5 Accelerator Policy Requirements and Oversight

Finding

Description: The ORNL accelerator policy requirements and independent oversight are not consistently applied throughout ORNL.

Code: $\quad$ Category III

Compliance

Protocol:

DOE Order 5482.1B

Priority: $\quad$ Energy Systems Risk Weight 6

Tiger Team Action Plan Priority 3

Response: ORNL will issue requirements for accelerators in the form of an SPP based primarily on ANSI N43.1-1978, ANSI N543-1974, DOE Order 5480.11, and an anticipated DOE order on conduct of operations for accelerators. The requirements will also derive from appropriate elements of the ORNL conduct-ofoperations/research program that does not specifically include accelerators. The management review committee (currently the Accelerators and Radiation Sources Review Committee [ARSRC]) will use formal, management-approved criteria, that are based on the ANSI standards, the two DOE orders, and gond management practices, to improve the current periodic appraisals and to provide better determination whether management and operation of the facilities complies with requirements for safety. The action items to address this finding are described in the corrective action plan for Finding MF.5.

\section{Root Causes:}

Poor!y defined roles and responsibilities, inadequate policy implementation, and inadequate oversight

Planned Actions and Schedules:

\section{$\underline{\text { Item/Description }}$}

Completion Date

1. Prepare draft Laboratory Standard Plant Procedure (SPP) which clearly defines responsibilities for independent internal appraisal functions including Director's Review Committees and the Health Physics Instrument Committee (see Finding MF-5, Item 3).

2. Include accelerator stay-time monitors in survey of personnel protection instruments (see Finding RP.8-2, Item 3).

Costs: $\quad$ Costs are addressed in the corrective action plan for Finding MF-5. 
References: An Environmental, Health, and Safety Self-Assessment of ORNL, November 8, 1990, Chapter 3.12, Site/Facility Safety Review

FWP ERAT850, ORNL Safety and Health-Regulatory Compliance, June 1, 1990 
Finding No.: $\quad$ RP.3-6 Material Clearance

Finding

Description: The material clearance process does not provide complete assurance to prevent materials that are radioactively contaminated from being taken into unrestricted or uncontrolled areas including the public domain as specified by DOE 5480.11 and
DOE 5400.5 .

Code: $\quad$ Category III

Compliance

Protocol:

DOE Orders 5480.11 and 5400.5

Priority: $\quad$ Energy Systems Risk Weight 63

Tiger Team Action Plan Priority 2

Response: This concern was based on several related findings, which said:

- Material Clearance Tags are an uncontrolled stores item.

- Material Clearance Tags are not adequately and legibly filled out.

- Methods and procedures for material excessing, salvage, and sales do not ensure proper radiological monitoring prior to transfer and release of materials.

The concern requires a review of both the material clearance monitoring methods as well as oversight surveillance practices conducted by the Radiation Protection Section supervisors and managers. The planned corrective actions for the concern and related findings will be addressed through an assessment of the risks associated with the potential problems of releasing contaminated materials and the establishment of the necessary procedural control to mitigate the potential release
of contaminated materials.

The Laboratory has placed restrictions and controls on salvage and surplus material. Radiation Protection technicians have been assigned to second-check all salvage material that is generated at a holding area. The holding area is in a Controlled Area. Standard operating procedures have been written to fully detail the material clearance process. The first step in the material release process is an attempt to establish the past history of the material. The standard operating procedures contain requirements to ensure inclusion of all information required in
Chapter 2 of DOE Order 5400.5 .

All materials sent to the salvage holding area have been surveyed completely for release prior to transportation. The numbers of items found on the second checks are statistically negligible; however, all materials found are reported to Radiation Protection Section supervision and to the responsible technician that performed
the survey.

An Energy Systems procedure is being developed for material release surveys. The Energy Systems procedure will not only take into consideration the factors 
required to perform an adequate survey, but also the risk assessment and methodology that is required to ensure the highest degree of safety to the public without requiring all surplus materials be buried in the reservation landfills.

\section{Root Causes:}

Inadequate oversight, inadequate communications, ambiguous requirements or expectations, inadequate training, poorly defined roles and responsibilities, and inadequate policy implementation

Planned Actions and Schedules:

\section{Item/Description}

1. Change the status of the Material Clearance Tags to a controlled stores issue item to ensure that only authorized Radiation Protection Section personnel have access to them.

2. Establish a holding area for salvage materials that are ready for acceptance by K-25 for salvage sales.

3. Issue revised Radiation Protection standard operating procedure requirements to reflect needed changes in survey requirements for salvage materials.

4. Incorporate the requirements of SOP 02-50-80, "Check on the Adequacy of Radiation Surveys," into the Measurements Assurance surveillance program for the Radiation Protection Section. (See Item 6 in Finding RP.1-1.)

5. Conduct training for technicians who are involved in material clearance surveys. This training shall include a lessons-learned approach on how materials are not getting properly identified during initial surveys.

6. Issue a Radiation Safety Bulletin for the plant population to provide insight and guidance for materials that may not be appropriate to be sent to salvage.

7. Make appropriate additions to General Employee Training concerning material clearance policies (see Item 5 in Finding RP.1-1).

8. Issue an Energy Systems Procedure for material survey and release for unrestricted use.
Completion Date

Complete

Complete

Complete

09/91

07/91

09/91

09/91 
9. Prepare and issue Conceptual Design Report (CDR) for Non-Hazardous Materials Clearance Operations (NMCO) Facility.

10. Prepare and submit GPP Budget Request for NMCO Facility.

11. Contingent on funding approval, construct NMCO Facility.

12. Assign additional radiation protection personnel to provide extra surveillance of surplus property.

13. Prepare an engineering estimate and submit budget request for procurement and installation of a truck monitoring station.

14. Procure and install a truck monitoring station.

09/91

Casts:

Type of funds: ESH Programmatic

Source of funds: ER-RP

\begin{tabular}{|c|c|c|c|c|c|c|c|}
\hline \multirow[b]{2}{*}{ Action item } & \multicolumn{6}{|c|}{ Estimated costs per fiscal year (\$K) } & \multirow[b]{2}{*}{ Total } \\
\hline & 1991 & 1992 & 1993 & 1994 & 1995 & Beyond & \\
\hline 1 & 2 & & & & & & 2 \\
\hline 2 & 15 & & & & & & 15 \\
\hline 3 & 17 & & & & & & 17 \\
\hline 5 & 5 & & & & & & 5 \\
\hline 6 & 1 & & & & & & 1 \\
\hline 8 & 5 & & & & & & 5 \\
\hline 9 & 75 & & & & & & 75 \\
\hline 10 & 2 & & & & & & 2 \\
\hline 13 & 15 & & & & & & 15 \\
\hline 14 & & 40 & & & & & 40 \\
\hline
\end{tabular}

Status:

Funded

Requested

New 
Type of funds: Overhead

Source of funds: Overhead

Estimated costs per fiscal year (\$K)

\begin{tabular}{|c|c|c|c|c|c|c|c|}
\hline \multirow[b]{2}{*}{ Action item } & \multirow{2}{*}{\multicolumn{2}{|c|}{1991}} & & \multirow[b]{2}{*}{ Total } \\
\hline & & & 1993 & 1994 & 1995 & Beyond & \\
\hline 12 & 115 & * & & & & & 115 \\
\hline
\end{tabular}

Status:

Funded

Requested

New

*Estimated annual ongoing cost: \$175K starting in FY 1992.

Type of funds: Capital

Source of funds: GPP

Estimated costs per fiscal year (\$K)

\begin{tabular}{cccccccc}
\cline { 2 - 6 } Action item & 1991 & 1992 & 1993 & 1994 & 1995 & Beyond & Total \\
\hline 11 & 1200 & & & & & 1200
\end{tabular}

Status:

Funded

Requested

New

1200

References: $\quad$ DOE Order $\$ 400.5$ 
Finding No.: RP.3-7 Documentation of Radiation Hazards

Finding

Description: Radiation hazards are not adequately documented as required by DOE 5480.11.

Code: $\quad$ Category III

Compliance

Protocol:

DOE Order 5480.11

Priority: $\quad$ Energy Systems Risk Weight 65

Tiger Team Action Plan Priority 2

Response: The Radiation Protection and Radiation Monitoring Standard Operating Procedures Manual has procedures that are sufficient to provide the guidance that is required to fulfill the documentation requirements as set forth in DOE Orders and policies. The uniform execution of the approved procedures, by technicians in the field, is not always sufficient. Increased supervisory oversight, in the form of walk-throughs and self-surveillances, is needed to ensure compliance, completeness, and consistency with the approved standard operating procedures.

Operating division and program personnel who develop internal standard operating procedures having radioactive operations or concerns are required to have a Health Physics review prior to their approval and use. In some cases, Health Physics review is not being performed. A re-emphasis for the requirement of a Health Physics review is needed to ensure adequate documentation of radiation hazards.

The Laboratory has a mature ( $>25$ years) RWP program that is well understood and used by the operating groups. The use and issuing of RWPs by the operating groups is supported by specific training programs presented by the Technical Resource and Training Section.

The ORNL Health Physics Manual is an ORNL level document and is distributed for implementation to all division, office, and program managers. Section 2 of the manual, Radiation and Contamination Control, will be revised to clearly require procedure reviews. The Radiation Protection staff will assist the operating groups to establish the means and methods for the procedure review process.

\section{Root Causes:}

Inadequate oversight, ambiguous requircments or expectations, and inadequate training 
Planned Actions and Schedules:

Item/Description

Completion Date

1. See Finding RP.1-1, Action Item 4.

2. Review and revise routine surveillance plans to ensure proper documentation of surveys that are conducted in the workplace (see Finding R.P.1-1, Item 6).

3. Issue revision to a Health Physics procedure specifying the requirement for a Radiation Protection Section review prior to approval of procedures for operations which have potential health physics hazards.

Casts:

Type of funds: ES\&H Programmatic

Source of funds: ER-AT RP

\begin{tabular}{|c|c|c|c|c|c|c|c|}
\hline \multirow[b]{2}{*}{ Action item } & \multicolumn{6}{|c|}{ Estimated costs per fiscal year (\$K) } & \multirow[b]{2}{*}{ Total } \\
\hline & 1991 & 1952 & 1993 & 1994 & 1995 & Beyond & \\
\hline 3 & 15 & & & & & & 15 \\
\hline \multicolumn{8}{|l|}{ Status: } \\
\hline \multicolumn{8}{|l|}{ Funded } \\
\hline Requested & 15 & & & & & & \\
\hline New & & & & & & & $\$ 15$ \\
\hline
\end{tabular}

References: $\quad$ DOE Order 5480.11

RP SOP 02-50-80. 
Finding No.: RP.5-1 Personnel Nuclear Accident Dosimeters

Finding

Description: ORNL does not use Personnel Nuclear Accident Dosimeters as required by DOE 5480.11 and its draft revision.

Code: $\quad$ Category III

Compliance

Protocol:

DOE Order 5480.11

Priority: $\quad$ Energy Systems Risk Weight 55

Tiger Team Action Plan Priority 2

Response: ORNL does not use Personnel Nuclear Accident Dosimeters as required by DOE Order 5480.11, Section 9.q.3. This concern was previously stated in the Radiation Protection Program Appraisal (RPPA) of the Oak Ridge Complex conducted during 1989.

Following the RPPA, a corrective action plan was approved which specified that a common PNAD be developed for the Oak Ridge, Tennessee, and Paducah, Kentucky, facilities operated by Energy Systems. A PNAD committee was chartered to identify potential PNAD devices and to determine the most-feasible device for use at the Energy System sites. Following a detailed study, the currently used personnel dosimeter was selected for testing to determine its applicability.

Thesults from the measurements of neutron and high gamma doses at accidental levels with the personnel dosimeters, using in-house NIST-traceable sealed gamma and neutron radiation sources, demonstrated the dosimeter's capability as a PNAD. A follow-up test with a fast-burst reactor was conducted in November 1990. The data from the test are currently under evaluation. Preliminary review of the data indicates that the dosimeter is able to respond well to all the conditions tested. Testing of the dosimeters and evaluation of the results have been documented in a technical basis document. A program implementation plan was developed to ensure that the requirements of the DOE Order 5480.11 were met. The PNAD program has been fully implemented at the Laboratory. Continued studies are scheduled to improve the accuracy of the dosimeter readings.

\section{Root Causes:}

Inadequate policy implementation and insufficient resources

Planned Actions and Schedules:

$\underline{\text { Item/Description }}$

1. Complete the technical-basis document for the PNAD program.
Completion Date

Complete 
2. Determine present locations of criticality alarms to ensure that personnel in these areas are monitored by a

Complete PNAD.

3. Develop and issue procedures for processing and issuing PNADs and for field retrieval by Radiation Protection

Complete personnel in the event of an accident.

4. Train Centralized External Dosimetry System personnel and field health physicisis to implement the PNAD procedures.

5. Issue a Radiation Safety Bulletin specifying the new PNAD policy.

Costs:

Type of funds: Overhead

Source of funds: Overhead

\begin{tabular}{|c|c|c|c|c|c|c|c|}
\hline \multirow[b]{2}{*}{ Action item } & \multicolumn{6}{|c|}{ Estimated costs per fiscal year $(\$ K)$} & \multirow[b]{2}{*}{ Total } \\
\hline & 1991 & 1992 & 1993 & 1994 & 1995 & Beyond & \\
\hline 1 & 57 & & & & & & 57 \\
\hline 2 & - & & & & & & \\
\hline 3 & 31 & & & & & & 31 \\
\hline 4 & 14 & & & & & & 14 \\
\hline 5 & 1 & & & & & & 1 \\
\hline
\end{tabular}

Status:

Funded

103

Requested

New

References: $\quad$ DOE Order 5480.11 
Finding No.: $\quad$ RP.5-2, Direct-Reading Dosimeters

Finding

Description: The procedure for use of Direct-Reading Dosimeters is not consistently applied and enforced across ORNL.

Code: $\quad$ Category III

Compliance

Protocol: None

Priority: $\quad$ Energy Systems Risk Weight 10

Tiger Team Action Plan Priority 3

Response: All workers are required to wear a Direct-Reading Dosimeter (DRD) for entry into a Radiation Area and for monitoring dose accumulated on Radiation Work Permits. The dosimetry data are entered into the Health Physics Information Management System (OHIS-an Energy Systems Computer network) for entry into the individual exposure history. The procedural steps required to ensure collection and documentation of DRD data are in need of refinement to more clearly specify the DRD requirements for users and documentation personnel. The DRD is a tool that is used to identify individuals who are approaching administrative dose limits as well as a personnel protection instrument that is readable by the wearer. The dose accumulated on a DRD is retained in the OHIS database, but is not the permanent official occupational exposure that is used to show compliance with DOE Order 5480.11. The accredited external dosimeters are used to determine the official occupational exposure.

Implementation of SOP 02-20-10, "Exposure and Contamination Control," will be verified as part of the ongoing EHP surveillance plan. The procedure addressed the direct-reading dosimeter program.

\section{Root Causes:}

Inadequate oversight, ambiguous requirements or expectations, and inadequate training

Planned Actions and Schedules:

Item/Description

1. Post areas where Direct-Reading Dosimeters are required.
Completion Date

Complete 
2. Revise the ORNL Health Physics Manual procedure

$09 / 91$

RP-3.3, "Personnel Monitoring", and the Radiation

Protection and Radiation Monitoring Standard

Operating Procedure Manual procedure 02-20-10,

"Exposure and Contamination Control", to include detailed instructions concerning the assignment, wearing, reading, and documentation of Direct-Reading

Dosimeters. (See Item 5 of Corrective Action RP.1-1, which addresses training.)

Costs:

Type of funds: Overhead

Source of funds: Overhead

\begin{tabular}{cccccccc} 
& \multicolumn{6}{c}{ Estimated costs per fiscal year (\$: ) } \\
\cline { 2 - 6 } Action item & 1991 & 1992 & 1993 & 1994 & 1995 & Beyond & Total \\
\hline 1 & 6 & & & & & & 6
\end{tabular}

Status:

Funded

6

Requested

New

Type of funds: ES\&H Programmatic

Source of funds: ER-AT RP

Estimated costs per fiscal year (\$K)

\begin{tabular}{|c|c|c|c|c|c|c|c|}
\hline \multirow[b]{2}{*}{ Action item } & \multirow{2}{*}{\multicolumn{2}{|c|}{1992}} & \multirow[b]{2}{*}{1993} & & \multirow[b]{2}{*}{ Total } \\
\hline & & & & 1994 & 1995 & Beyond & \\
\hline
\end{tabular}

Status:

Funded

Requested

New

References: $\quad$ DOE Order 5480.11 
Finding No.: RP.6-1 Air Samples

Finding

Description: The current number and placement of air samplers does not demonstrate compliance with the prospective limit as establisi ed in DOE 5480.11 for operations within the workplace.

Code: $\quad$ Category III

Compliance

Protocol:

DOE Order 5480.11

Priority: $\quad$ Energy Systems Risk Weight 63

Tiger Team Action Plan Priority 2

Response: $\quad$ This finding was previously stated in the Radiation Protection Program Appraisal (RPPA) of the Oak Ridge Complex conducted during 1989. As a result of the finding, a detailed radiological airflow study was conducted in two phases. Phase I identified the location, type, and physical condition of the Laboratory's radiological air monitors. Phase II was a study of the airflows, adequacy of monitors to provide airflow information, and the appropriate placement of the monitors. The location of principal sources of potentially airborne radionuclides were checked with respect to probable worker positions and fixed monitor installations. The study was completed by recommending actions to the Health Physics Instrument Committee for the improvement of airflow measurements. The recommendations have been prioritized, and implementation of approved recommendations will begin during December 1990.

Radiation Protection procedures require that a facility radiological characterization be performed and that facility/area survey plans be developed. These procedures establish criteria for the number and location of air samplers in addition to providing guidance on air-sampling flow rates and sampling intervals, use of appropriate filter media, and dealing with radon daughter interference. Radiation Protection procedures also specify action levels for evaluation of air sampling/monitoring results and for determining necessary exposure controls. The standard operating procedures governing control of exposure to contamination and posting of radiological areas were revised to improve the implementation of these requirements.

Standard operating procedures have been developed to support the calibration of fixed air monitors, based on appropriate technical criteria (including traceability to the NIST), criteria for source checks, electronic checks, and airflow calibrations. Initial calibrations are being established for each monitor. Maintenance records will be combined with calibration records for fixed monitors, as they have been for
portable monitors. 
One ORNL facility at the $\mathrm{Y}-12$ site was not included in the two phases of the airflow study. The facility is in the security exclusion area at the $\mathrm{Y}-12$ site, and appropriate security measures had to be considered prior to allowing subcontract personnel to complete the final study to include the Y-12 facility. The airflow study is currently scheduled for inclusion in the ORNL Radiological Airflow Study document.

The air monitors at the ORNL facilities are old, and some do not meet the monitoring requirements specified in current regulations and standards. A Conceptual Design Report has been completed to support DOE approval of a line item to upgrade all stationary instruments for radiation protection, including air monitors. Pending line item approval, a program of final design, including interconnections and communications interfaces, hardware procurement, and implementation, will be undertaken. This line item will address the deficiencies identified in this finding.

\section{Root Causes:}

Insufficient resources and inadequate oversight

\section{Planned Actions and Schedules:}

\section{Item/Description}

1. Conduct the airflow study of the ORNL facility located in the exclusion area at the $\mathrm{Y}-12$ site.

2. Complete implementation of priority airflow study recommendations.

3. Develop and issue a Conceptual Design Report for stationary instrumentation upgrade.

4. Request funding for the stationary instrumentation upgrade line item project.

5. Review and document instrumentation needs identified during the airflow study which will not be covered by the instrumentation upgrade; if necessary, request additional funding.

6. Contingent on the line item's approval, initiate stationary Health Physics instrument upgrade including air monitors.

\section{Completion Date}

$12 / 91$

$03 / 92$

$09 / 91$

$12 / 91$

03/93

01/93 
Costs:

Type of funds: Overhead

Source of funds: Overhead

\begin{tabular}{|c|c|c|c|c|c|c|c|}
\hline \multirow[b]{2}{*}{ Action item } & \multicolumn{6}{|c|}{ Estimated costs per fiscal year (\$K) } & \multirow[b]{2}{*}{ Total } \\
\hline & 1991 & 1992 & 1993 & 1994 & 1995 & Beyond & \\
\hline 1 & & 23 & & & & & 23 \\
\hline 2 & & 8 & & & & & 8 \\
\hline 3 & 75 & & & & & & 75 \\
\hline 5 & & 15 & & & & & 15 \\
\hline
\end{tabular}

Status:

Funded

75

Requested

New

46

Type of funds: Line Item

Source of funds: ER-AT

\begin{tabular}{llllllll} 
& \multicolumn{5}{c}{ Estimated costs per fiscal year $(\$ \mathrm{~K})$} \\
\cline { 2 - 6 } Action item & 1991 & 1992 & 1993 & 1994 & 1995 & Beyond & Total \\
\hline Status: & & & 18,700 & & & & 18,700
\end{tabular}

Funded

Requested

18,700

New

$\$ 18,700$

References: None 
Finding No.: RP.6-2 Timeliness of Air Sampling Program

Finding

Description: ORNL has not demonstrated the air sampling program will supply the necessary timely data required by the Internal Dosimetry Program Performance standard.

Code: $\quad$ Category III

Compliance

Protocol:

DOE Internal Dosimetry Performance Standard

Priority: $\quad$ Energy Systems Risk Weight 5

Tiger Team Action Plan Priority 3

Response: $\quad$ Radiation Protection and Radiation Monitoring Standard Operating Procedures Manual procedure 02-50-50, "Survey Techniques for Airborne Radioactive Contamination," provides guidance and standardized methods for accurately measuring the presence of airborne radioactivity in the workplace, so that personnel exposures may kept ALARA.

In addition, procedure 02-70-11, "General Counting Techniques," specifies the techniques for measuring radioactivity on smear samples and air filters. The "ORO Contamination Control Policy" specifies mandatory routine monitoring requirements which specify that the turn-around time for the analyses of continuous air samples shall not exceed one week. An exemption of the time limit was requested and approved to allow a ten-working-day time period. The exemption allows an appropriate radon decay period for accurate counting of sample media.

\section{Root Causes:}

Inadequate policy implementation, insufficient resources, and unclear requirements or expectations.

\section{Planned Actions and Schedules:}

\section{Item/Description}

1. Conduct an evaluation of the "Internal Dosimetry Program Performance Standard" against the Radiation Protection and Radiation Monitoring Standard Operating Procedures Manual procedures to determine and document inconsistencies.

2. If inconsistencies exist, revise and issue the affected procedures to ensure compliance with the "Internal Dosimetry Program Performance Standard."
Completion Date

$10 / 92$

$12 / 92$ 
3. Review internal doses to assess adequacy of the air monitoring program and document.

Costs:

Type of funds: ESH Programmatic

Source of funds: ER-AT RP

\begin{tabular}{crrrrrrr} 
& \multicolumn{5}{c}{ Estimated costs per fiscal year (\$K) } & \\
\cline { 2 - 6 } Action item & 1991 & 1992 & 1993 & 1994 & 1995 & Beyond & Total \\
\hline 1 & 23 & & & & & 23 \\
2 & 25 & 8 & & & & 33
\end{tabular}

Status:

Funded

Requested

New

48

8

$\$ 56$

References: $\quad$ DOE Order 5480.11, "Internal Dosimetry Program Performance Standard" 
Finding No.: $\quad$ RP.6-3 Surveying Personnel for Contamination

Finding

Description: There is no technical basis and justification for Department of Energy to require 3-minute frisks in surveying personnel for contamination.

Code: $\quad$ Category III

Compliance

Protocol:

None

Priority: $\quad$ Energy Systems Risk Weight 8

Tiger Team Action Plan Priority 3

Response: ORNL uses a well-established industry standard, three minutes, as its required time to perform an adequate whole-body frisk when using a hand-held pancake GM probe. Three minutes has also been found to be acceptable for alpha monitoring using the ORNL alpha scintillation probe.

Contamination survey results at ORNL are recorded in $\mathrm{dpm} / 100 \mathrm{~cm}^{2}$. Instruments, such as LMAs and LMBs, that are used for frisking give readings in counts per minute (cpm) because of the wide range of isotopes found in Laboratory operations. Identical instructions are posted at each LMA/LMB used for personnel frisking, and in these instructions, any observed count rate above background is to be reported to the health physicist/technician named on the posted notice. This approach is used to give the professional staff the ability to actually determine the true extent of the contamination.

ORNL uses current, proved technology for all personnel survey equipment. The equipment presently in use is capable of detecting low-energy nuclides, such as $\mathrm{C}^{14}$ and $\mathrm{Pm}^{147}$, at a low efficiency value. The Health Physics Instrument Committee will explore emerging technologies in radiation detection, as they become available.

\section{Root Causes:}

Ambiguous requirements or expectations and regulatory barriers

\section{Planned Actions and Schedules:}

\section{Item/Description}

1. Develop a technical-basis document for frisking procedures, for anticontamination clothing requirements, for exit requirements at regulated contamination areas, and for the selection and calibration of monitoring instruments.

\section{Completion Date}

$10 / 91$ 
2. Review and purchase for evaluation commercially available detection equipment for low-energy nuclides, and provide information to the Health Physics Instrument Committee.

3. Revise HPP Procedures to reflect required changes as identified in the Technical Basis Document.

\section{Costs:}

Type of funds: ESH Programmatic

Source of funds: ER-AT RP

Estimated costs per fiscal year (\$K)

\begin{tabular}{crrrrrrr}
\cline { 2 - 6 } Action item & 1991 & 1992 & 1993 & 1994 & 1995 & Beyond & Total \\
\hline 1 & 57 & & & & & & 57 \\
2 & 30 & & & & & & 30 \\
3 & & 70 & & & & & 70
\end{tabular}

Status:

Funded

Requested

New

87

70

References: $\quad$ DOE Order 5480.11

DOE/ORO Contamination Control Policy 
Finding No.: RP.7-1 Internal Radiation Dosimetry Program

Finding

Description: ORNL has not allocated the space and resources to meet DOE 5480.11 effective dose equivalent requirements, the Department of Energy Ir.ternal Dosimetry Standard or to participate in all categories of the Department of Energy Bioassay Laboratory Accreditation Program.

Code: $\quad$ Category III

Compliance

Protocol:

Priority: $\quad$ Energy Systems Risk Weight 58

Tiger Team Action Plan Priority 2

Response: Deficiencies in the ORNL Internal Dosimetry program have been documented in the ORNL self-assessment document, specifically the lack of laboratory space for fume hoods, sinks, and programmable muffle furnaces to ash fecal samples, in order to fully participate in the DOELAP testing and to routinely perform fecal analyses. A full assessment of the ORNL Internal Dosimetry facilities and systems needs to be conducted to ensure that deficiencies to standards and other regulatory criteria are identified and that appropriate corrective actions are identified with funding requested.

The Radiation Exposure Assessment Laboratory (REALab) line item project is planned to correct the deficiencies associated with limited space and resources.

\section{Root Causes:}

Inadequate policy implementation, insufficient resources, inadequate oversight, and ambiguous requirements or expectations

\section{Planned Actions and Schedules:}

\section{$\underline{\text { Item/Description }}$}

1. Assess the ORNL Internal Dosimetry facilities, operations, and personnel against applicable standards, procedures, and regulations and develop an upgrade plan to address deficiencies with corrective actions and funding requirements identified.

2. Following the assessment, review the upgrade plan and request needed funds to correct deficiencies.
Completion Date

$12 / 91$

$2 / 92$ 
3. Issue the Conceptual Design Report for the REALab $2 / 92$ line item project.

4. Request funding for the REALab line item project.

5. Establish a Quality Control Program to support the Internal Dosimetry Program.

6. Issue $\mathrm{QC}$ SOPs.

7. Contingent on approval of the line item funding, initiate 03/93 design/construction of the REALab Facilities.

Costs:

Type of funds: ESH Programmatic Source of funds: ER-AT RP

Estimated costs per fiscal year (\$K)

\begin{tabular}{|c|c|c|c|c|c|c|c|}
\hline Action item & 1991 & 1992 & 1993 & 1994 & 1995 & Beyond & Total \\
\hline 1 & & 80 & & & & & 80 \\
\hline 2 & & - & & & & & \\
\hline 3 & & 285 & & & & & 285 \\
\hline 5 & & 112 & & & & & 112 \\
\hline 6 & & 70 & & & & & 70 \\
\hline
\end{tabular}

Status:

Funded

Requested

New 
Type of funds: Line Item

Source of funds: ER

Estimated costs per fiscal year (\$K)

\begin{tabular}{cccccccc}
\cline { 2 - 6 } Action item & 1991 & 1992 & 1993 & 1994 & 1995 & Beyond & Total \\
\hline 7 & & 41,000 & & & & 41,000
\end{tabular}

Status:

Funded

Requested

New

41,000

$\$ 41,000$

Type of funds: Capital

Source of funds: ER

Estimated costs per fiscal year (\$K)

\begin{tabular}{rrrrrrrr}
\cline { 2 - 6 } Action item & 1991 & 1992 & 1993 & 1994 & 1995 & Beyond & Total \\
\hline 5 & 20 & 325 & & & & & 345
\end{tabular}

Status:

Funded

Requested

New

$20 \quad 325$

References: $\quad$ DOE Order 5480.11

DOE Internal Dosimetry Performance Standard 
Finding No.: RP.7-2 In Vivo Calibrations

Finding

Description: ORNL has not completed all in-vivo calibrations including the lung counter and the wound counter as required by the Department of Energy Internal Dosimetry Performance Standard.

Code: $\quad$ Category III

Compliance

Protocol:

DOE Internal Dosimetry Performance Standard

Priority: $\quad$ Energy Systems Risk Weight 58

Tiger Team Action Plan Priority 2

Response: Deficiencies in the ORNL internal dosimetry program were identified in the ORNL self-assessment document, specifically the need to complete in vivo calibrations. In order to achieve this goal, additional lung and chest plates with a variety of radionuclides will be needed. The lung counting facility also needs to be upgraded. ORNL has only one shielded room; this precludes the use or calibration of the phoswich lung counters while in vivo counts are being conducted. Additional funding is needed to resolve this situation. The wound counter is currently operated by medical personnel and calibratzu by Radiation Protection personnel. The procedures which deal with the calibration and use of this piece of equipment will, in the future, be reviewed by the whole-body counting supervisor and calibrated by RaSCaL personnel.

\section{Root Cause:}

Inadequate resources

Planned Actions and Schedules:

\section{Item/Descriptic:n}

1. Request funds for lung and chest plates and other identified equipment.

2. Purchase lung and chest plates and other identified equipment.
Completion Date

$10 / 91$

$12 / 91$ 
3. Perform an assessment of the Whole-Body Counting Laboratory to applicable standards and regulations and develop a detailed upgrade plan to achieve compliance.

[NOTE: Specific to the identified findings, the upgrade plan would assess: (1) identification of needed additional lung and chest plates and (2) upgrades to equipment, facilities, and procedures.]

4. Review upgrade plan and request funds to implement approved recommendations.

5. Determine calibration adequacy and status of woundcounter operations and issue procedures to ensure compliance to standards.

\section{Casts:}

Type of funds: ESH Programmatic

Source of funds: ER-AT RP

\begin{tabular}{|c|c|c|c|c|c|c|c|}
\hline \multirow[b]{2}{*}{ Action item } & \multicolumn{6}{|c|}{ Estimated costs per fiscal year (\$K) } & \multirow[b]{2}{*}{ Total } \\
\hline & 1991 & 1992 & 1993 & 1994 & 1995 & Beyond & \\
\hline 2 & & 12 & & & & & 12 \\
\hline 3 & & 15 & & & & & 15 \\
\hline 4 & & - & & & & & \\
\hline
\end{tabular}

Status:

Funded

Requested

New 
Type of funds: Overhead

Source of funds: Overhead

\begin{tabular}{lrllllll} 
& \multicolumn{5}{c}{ Estimated costs per fiscal year $(\$ K)$} & \\
\cline { 2 - 6 } Action item & 1991 & 1992 & 1993 & 1994 & 1995 & Beyond & Total \\
\hline 5 & 7 & & & & & & \\
Status:
\end{tabular}

Funded

Requested

New 7

References: $\quad$ DOE Order 5480.11

DOE Internal Dosimetry Ferformance Standard 
Finding No.: RP.8-1 Radiation Protection Instrument Program

Finding

Description: The radiation protection instrument calibration and maintenance program does not meet the American National Standards Institute N323 requirements.

Code: $\quad$ Category III

Compliance

Protocol:

Priority: $\quad$ Energy Systems Risk Weight 61

Tiger Team Action Plan Priority 2

Response: Other findings expressed in the appraisal were:

- There is a lack of maintenance calibration and records procedures to meet ANSI N323.

- Some instruments have not received thorough performance testing.

- Fixed instruments, air monitors, and ORNL's Cutie Pies do not meet ANSI standards.

- Instrument field check procedures, and the sources used to complete them, are not adequate. Sources used are not traceable to laboratory standards.

- Not all monitrons meet ANSI N42.17A and B requirements.

- Lack of funding exists to complete instrument upgrades and completion of the calibration laboratory.

The above were previously addressed in the "Radiation Protection Program Appraisal of the Oak Ridge Complex" (RPPA) conducted by DOE during May 1989.

In response to the RPPA, the following aggressive upgrade programs were initiated to improve the radiation monitoring instruments and the Radiation Standards and Calibration Laboratory.

- Approximately one million dollars have been spent to upgrade the portable instrument program to include procurement of instruments, testing, development of procedures, calibration method development, source procurement, and fixture fabrication for testing.

- Calibration facility upgrades are to include fabrication of a low-scatter radiation facility, source transfer equipment, tracking and holding devices for irradiations, and other state-of-the-art upgrades for calibrations and irradiations.

- The planning, engineering, and specification development of a FY 1993 LineItem project for upgrades of the fixed radiation monitoring instruments.

Although significant improvements have been made and upgrade plans have been developed, the following items have been identified as needing action to meet appropriate standards. 
- Documentation, inventory, and quality control activities.

- Improved energy dependance test capability.

- Environmental condition testing.

- Neutron calibration capability.

- High-range and overload testing capabilities.

Root Causes:

Inadequate policy implementation, insufficient resources, inadequate oversight, inadequate training, and poorly defined roles and responsibilities

Planned Actions and Schedules:

\section{Item/Description}

1. Assess requirements of ANSI N320, ANSI N323, ANSI N42.17A, ANSI N42.17B, and other applicable standards and regulations and document the deficiencies of performance, equipment, and systems at the Laboratory.

2. Develop and approve a facility upgrade plan with funding requirements requested and tentative completion dates established to meet applicable standards and regulations. The upgrade plan would specifically include: (1) necessary procedures and records management for calibrations, irradiations, and source traceability; (2) instrument performance testing requirements; (3) field check sources availability and traceability; (4) monitron system upgrades; (5) cutie pie replacements; and (6) calibration facility and equipment upgrades.

3. Contingent on approval of funding implement upgrade plan outlined in Item 2.
Completion Date

$10 / 91$

$12 / 91$

$03 / 92$ 


\section{Costs:}

Type of funds: ES\&H Programmatic

Source of funds: ER-RP

Estimated costs per fiscal year (\$K)

\begin{tabular}{crrrrrrr}
\cline { 2 - 6 } Action item & 1991 & 1992 & 1993 & 1994 & 1995 & Beyond & Total \\
\hline 1 & 15 & & & & & & 15 \\
2 & & 15 & & & & & 15 \\
3 & & $675^{*}$ & & & & & 675
\end{tabular}

Status:

Funded

Requested

690

New

*Preliminary estimate.

References: DOE/EH 0135

DOE Order 5480.11

ANSI 323-1978 
Finding No.: RP.8-2 Approval of Radiation Protection Instruments

Finding

Description: Many ORNL safety-related radiation protection instruments are purchased and used without the review and approval of Radiation Standards and Calibration Laboratory personnel in developing performance specifications.

Code: $\quad$ Category III

Compliance

Protocol: None

Priority: $\quad$ Energy Systems Risk Weight 56

Tiger Team Action Plan Priority 2

Response: The ORNL Health Physics Instrument Committee (HPIC) has the responsibility to approve radiation detection instruments used for personnel protection. Although the Procurement Division has a system in place to "flag" instrument requisitions, some older instruments have been found that were not on the inventory listing during the appraisal. A need exists for strengthening the computer "flagging" system by adding selected keywords submitted by the HPIC members. Additionally, increased awareness of instrument procurement requirements must be made to Laboratory personnel.

\section{Root Causes:}

Inadequate policy implementation, inadequate management approach, ambiguous requirements or expectations, inadequate training, and poorly defined roles and responsibilities

Planned Actions and Schedules:

\section{$\underline{\text { Item/Description }}$}

1. Issue a Health Physics Bulletin to specify requirements and necessary approvals prior to procurement of radiation detection instruments used for personnel protection.

2. Identify additional key words and include these words in the Procurement Division Computer System. The addition of more flags will strengthen the searching for procurement of radiation instruments, to ensure that approval has been granted.
Completion Date

$08 / 91$ 
3. Perform complete a survey by divisional Radiation $10 / 91$

Control Officers and Radiation Protection personnel to identify instruments (monitrons, truck monitors, $\mathrm{X}$-ray measuring instruments, etc.) to determine those that are not on the inventory listing and are used for personnel protection.

4. Determine the disposition of identified personnel protection instruments to ensure that they function as intended.

Note: The Health Physics Instrument Committee will consider specific instruments on a case-by-case basis to determine disposition.

5. Issue standard operating procedures for the newly identified instruments which include calibration and performance testing.

Costs:

Type of funds: ESH programmatic

Source of funds: ER-AT RP

\begin{tabular}{|c|c|c|c|c|c|c|c|}
\hline \multirow[b]{2}{*}{ Action item } & \multicolumn{6}{|c|}{ Estimated costs per fiscal year $(\$ K)$} & \multirow[b]{2}{*}{ Total } \\
\hline & 1991 & 1992 & 1993 & 1994 & 1995 & Beyond & \\
\hline 1 & 2 & & & & & & 2 \\
\hline 2 & 6 & & & & & & 6 \\
\hline 3 & 33 & & & & & & 33 \\
\hline 4 & 20 & & & & & & 20 \\
\hline 5 & & 225 & & & & & 225 \\
\hline
\end{tabular}

Status:

Funded

Requested

61

New 
Finding No.: RP.8-3 Testing of Safety-Related Instruments

Finding

Description

Testing of safety-related instruments against specifications and periodic calibration and maintenance is not performed in accordance with Department of Energy

Code: $\quad$ Category III

Compliance

Protocol:

DOE Prescribed Standards

Priority: $\quad$ Energy Systems Risk Weight 56

Tiger Team Action Plan Priority 2

Response: The ORNL Health Physics Instrument Committee (HPIC) has the responsibility to approve radiation detection instruments used for personnel protection. The purpose of this review and approval is to ensure that instruments used for personnel protection are adequate as well as manufactured in such a manner that repairs and calibrations are possible. Instruments that are found in use that are not calibrated or approved will be reviewed and determinations made if the instruments meet the standards required to provide the required protection. Calibration and routine performance test procedures will be developed and approved for newly identified radiation protection instruments. Standard Operating
Procedures are existing for currently used instruments.

\section{Root Causes:} Inadequate oversight, inadequate communications, inadequate training, and poorly
defined roles and responsibilities

Planned Actions and Schedules:

This concern is fully addressed by actions outlined in response to Finding RP.8-2.

Costs: None

References: $\quad$ DOE Order 5480.11

DOE/EH 0135 
Finding No.: RP.8-4 High Range Radiation Protection Instrumentation

Finding

Description: Emergency and high-range radiation protection instrumentation does not meet requirements of the American National Standards Institute N320, N323, and N42.17C.

Code: $\quad$ Category III

Compliance

Protocol:

Priority: $\quad$ Energy Systems Risk Weight 58

Tiger Team Action Plan Priority 2

Response: Specific findings identified in the appraisal state that:

- Testing of emergency or high-range radiation protection and safety-related instrumentation is inadequate.

- High-range calibration wells have not been installed to provide capability for calibration of these instruments.

- All monitrons that may become paralyzed in an over-range exposure have not been identified.

Upgrades at the Radiation Standards and Calibration Laboratory (RaSCaL) include the installation of high-range calibration sources and wells and a source transfer system. An Operational Readiness Review will be conducted to determine adequacy and safety of the operation of the new well and source systems, upon completion.

\section{Root Causes:}

Inadequate policy implementation and insufficient resources

Planned Actions and Schedules:
Completion Date

1. See Finding RP.8-1, Item 1 .

2. See Finding RP.8-1, Item 2.

3. Complete installation of high-range wells, source transfer system, and X-ray machine at RaSCaL.

$04 / 92$

4. Complete an Operational Readiness Review of RasCaL. 
Casts:

Type of funds: ESH Programmatic

Source of funds: ER-AT RP

\begin{tabular}{|c|c|c|c|c|c|c|c|}
\hline \multirow[b]{2}{*}{ Action item } & \multicolumn{6}{|c|}{ Estimated costs per fiscal year $(\$ K)$} & \multirow[b]{2}{*}{ Total } \\
\hline & 1991 & 1992 & 1993 & 1994 & 1995 & Beyond & \\
\hline 3 & & $400^{*}$ & & & & & 400 \\
\hline 4 & & 118 & & & & & 118 \\
\hline
\end{tabular}

Funded

Requested

518

New

*Very preliminary estimate

References: $\quad$ DOE Order 5480.11

DOE/EH 0135

ANSI N320

PNL-SA-13346/CONF-840774 
Finding No.: RP.8-5 Radiation Protection Instrumentation Program

Finding

Description: Problems with the operation and logistics of the Radiation Protection Instrumentation Program has led to inefficiency, ponr quality control, and absence of centralized responsibility.

Code: $\quad$ Category III

Compliance

Protocol:

Priority: $\quad$ Energy Systems Risk Weight 5

Tiger Team Action Plan Priority 4

Response: The Radiation Protection Instrumentation Program is described in ORNL Health Physics Procedures Manual procedure RP-1.6, "Health Physics Instruments." The procedure describes the program for providing, maintaining, and calibrating radiation detection instruments for personnel protection in the Laboratory's programs and facilities. Program elements are coordinated through a central Health Physics Instrument Committee (HPIC).

\section{Root Causes:}

Inadequate policy implementation, insufficient resources, inadequate management approach, inadequate oversight, ambiguous requirements or expectations, and poorly defined roles and responsibilities

Planned Actions and Schedules:

\section{Item/Description}

1. An interorganizational team will assess the Radiation Protection Instrumentation Program to address instrument ownership, logistics, quality control, and other requirements as specified in applicable standards and DOE Orders.

2. Initiate implementation of recommendations concerning organization of the Radiation Protection Instrumentation Program.
Completion Date

$01 / 92$ 
Costs:

Type of funds: ES\&H Programmatic

Source of funds: ER-AT RP

\begin{tabular}{cccccccc} 
& \multicolumn{5}{c}{ Estimated costs per fiscal year $(\$ K)$} & \\
\cline { 2 - 6 } Action item & 1991 & 1992 & 1993 & 1994 & 1995 & Beyond & Total \\
\hline 1 & 30 & & & & & & 30 \\
2 & - & & & & & &
\end{tabular}

Status:

Funded

Requested

New

30

References: $\quad$ DOE Order 5480.11

ANSI N320

ANSI N323

ANSI N42.17C 
Finding No.: RP.10-1 Positive Control of Contamination

Finding

Description: Current contamination control, posting practices, policies, and radiation monitoring capabilitics are not conducted or enforced in a manner that ensures positive control of contamination as required by DOE 5480.11 .

Code: $\quad$ Category III

Compliance

Protocol:

DOE Order 5480.11

Priority: $\quad$ Energy Systems Risk Weight 77

Tiger Team Action Plan Priority 2

Response: $\quad$ The Radiation Protection and Radiation Monitoring Standard Operating

Procedures Manual has procedures that are sufficient to comply with the posting and survey requirements specified in DOE Orders and policies. The uniform execution of the approved procedures, by technicians in the field, is not always sufficient. Increased supervisory oversight, in the form of walk-throughs and selfsurveillances, is needed to ensure compliance, completeness, and consistency to the standard operating procedures. Procedure 02-50-80, "Check on the Adequacy of Radiation Surveys," provides a schedule of tours and inspections that are required by all levels of Radiation Protection supervision.

ORNL has developed an implementation plan to provide area postings, required by the "ORO Contamination Control Policy," on September 29, 1990. Significant progress has been made to replace old postings; however, some areas remain to be completed.

Root Causes:

Insufficient resources, ambiguous requirements or expectations, and regulatory barriers

Planned Actions and Schedules:

Item/Description

Completion Date

1. See Finding RP.3-1, Item 3.

2. See Finding RP.1-1, Item 4.

3. Contingent on funding, obtain necessary additional

$01 / 92$ transportation to facilitate surveillance in remote areas. 


\section{Costs:}

Typc of funds: ESH Programmatic

Source of funds: ER-AT RP

\begin{tabular}{lrllllll} 
& \multicolumn{5}{c}{ Estimated costs per fiscal year (\$K) } \\
\cline { 2 - 6 } Action item & 1991 & 1992 & 1993 & 1994 & 1995 & Beyond & Total \\
\hline 3 & & 30 & & & & & 30
\end{tabular}

Funded

Requested

30

New

References: $\quad$ DOE Order 5480.11, "ORO Contamination Control Policy" 
Finding No.: RP.10-2 Consistency of Radiation Protection Policies

Finding

Description: As a result of absence of oversight by both upper management and Oak Ridge Operations, Radiation Protection policies are not uniformly applied across all ORNL facilitics.

Code: $\quad$ Category III

Compliance

Protocol:

Priorify: $\quad$ Energy Systems Risk Weight 73

Tiger Team Action Plan Priority 2

\section{Response: $\quad$ ORNL}

The operating divisions, as well as the radiation protection staff assigned to the Y-12 facilities, have failed to keep pace with the rate of improvement seen in other parts of the Laboratory. Problems that are inherent due to the separation from the bulk of the Laboratory have not been solved by upper-level management.

Radiation Protection Section management focus is needed on the operations of ORNL facilities at the Y-12 plant. The physical separation from the Laboratory has led to the assigned RP staff members losing touch with the changes ongoing at the X-10 site to improve the health and safety programs. Input and review from the RP management are needed to provide guidance into areas where the Y-12 operations are not up to Laboratory standards. A complete review and program, in the form of a Complex Routine Surveillance Plan, shall be established to ensure that all areas of the $\mathrm{Y}-12$ facilities are provided with surficient coverage to provide adequate radiation protection. Increased attention, in the form of surveillances by the Measurements Assurance Section of EHP, shall be scheduled to ensure that Y-12 practices are consistent with those expected elsewhere at other ORNL facilities. See also plan for Finding MF-5.

\section{DOE/ORO}

The need for additional oversight at the site facilities, by the ORO, has been recognized. The approach, recently implemented, to ensure uniform application of Radiation Protection policies shall include the identification of DOE Facility Representatives for all ORNL facilities. Facility representative responsibilities will include scheduled facility inspections to ensure compliance with established radiation protection policies. Uniformity of policy implementation across different facilities will also be reviewed by the X-10 Site Office and during biannual Health Physics appraisals conducted by the ORO Safety and Health Division. 


\section{Root Causes:}

Inadequate policy implementation, insutficient resources: inadequate management commitment, and inadequate management approach

Planned Actions and Schedules:

$$
\text { Item/Description }
$$

Completion Date

ORNL

1. Se $€$ Finding RP.1-2, item 5.

2. See Finding RP.1-2, 1tem 4.

3. Schedule additional surveillance, as part of the existing plan for Radiation Protection, to measure consistence of procedures, postings, and compliance with approved procedures at the ORNL Y-12 facilities (see Finding RP.1-1, item 6).

4. Appoint a Health Physicist, from outside the Y-12 area, to conduct an evaluation of the Pu gloveboxes in 9204-3.

Complete The evaluation shall consider radiological hazards to building personnel, controls presently established, and additional controls and/or radiological surveys needed to reduce the risk associated with this facility until planned D\&D work can be completed. The evaluation shall be forwarded to the Facility Manager, RP Section Head, and the Office Director.

5. Preliminary to permanent closures, cover all glove ports with radiological hazard tape to warn of contamination potential.

\section{DOE/ORO}

6. Identify facility representatives.

Complete

7. Develop a facility inspection schedule.

Complete

8. Initiate routine facility inspections.

Complete

9. Complete $\mathrm{S} \& \mathrm{H}$ training of $\mathrm{X}-10$ Site Office facility representative. 
10. Complete first independent appraisal of ORNL facilities for radiation protection compliance and uniformity of policy implementation across farilities.

Costs: No costs are associated with this finding.

References: $\quad$ DOE Order 5480.11 
Finding No.: RP.10-3 Control of Laundry Wastewater

Finding

Description: Wastewater potentially contaminated with radioactive materials is not being monitored prior to release from the laundry to the sanitary sewer system as required in DOE 5400.5.

Code: $\quad$ Category III

Compliance

Protocol:

DOE Order 5400.5

Priority: $\quad$ Energy Systems Risk Weight 58

Tiger Team Action Plan Priority 2

Response: This action plan validates the internal monitoring processes currently used in the laundry to control the contaminated washer effluent radionuclide concentration. This action, coupled with an annual waste acceptance criteria validation, will ensure that the total annual discharge of radioactive materials to the sanitary sewer system poses a negligible risk to the general public.

\section{Root Causes:}

Insufficient resources

Planned Actions and Schedules:

$\underline{\text { Item/Description }}$

1. Screen all incoming protective clothing to limit possibility of unaccep quantities of contamination.

2. Request funding for hold tank, monitoring equipment, and analytical services.

3. Develop and issue procedure for implementation of the monitoring program for laundry effluent.

4. Evaluate the Decontamination Laundry Wastewater Stream and the Sewage Treatment Plant's Waste Acceptance Criteria to define accep parameters. (See $\mathrm{RAD} / \mathrm{CF}-3$.)

5. If funding is approved, purchase and install equipment.
Completion Date

Complete

Complete

$06 / 91$

03/92

(estimated) 
Costs:

Type of funds: Capital

Source of funds: ER-AT RP

\begin{tabular}{|c|c|c|c|c|c|c|c|}
\hline \multirow[b]{2}{*}{ Action item } & \multicolumn{6}{|c|}{ Estimated costs per fiscal year $(\$ K)$} & \multirow[b]{2}{*}{ Total } \\
\hline & 1991 & 1992 & 1993 & 1994 & 1995 & Beyond & \\
\hline 2 & & - & & & & & \\
\hline 5 & & 250 & & & & & 250 \\
\hline
\end{tabular}

Status:

Funded

Requested

250

New

Type of funds: ES\&H Programmatic

Source of funds: ER-AT RP

Estimated costs per fiscal year (\$K)

\begin{tabular}{|c|c|c|c|c|c|c|c|}
\hline \multirow[b]{2}{*}{ Action item } & & \multirow[b]{2}{*}{ Total } \\
\hline & 1991 & 1992 & 1993 & 1994 & 1995 & Beyond & \\
\hline 1 & - & & & & & & \\
\hline 3 & 7 & $*$ & & & & & 7 \\
\hline 4 & & - & & & & & \\
\hline
\end{tabular}

Status:

Funded

Requested

New

7

*Estimated annual ongoing cost: $\$ 10 \mathrm{~K}$.

References: None 
Finding No.: RP.10-4 Requirements for Laundry Contamination Control

Finding

Description: The ORNL laundry does not fulfill the requirements for physical separation of clean and dirty or contaminated laundry, effective contamination control, and personnel protection.

Code: $\quad$ Category III

\section{Compliance} Protocol:

The ORNL Decontamination Laundry should keep physically separated contaminated laundry and noncontaminated laundry, properly contain contamination, and keep personal exposure within established limits.

Priority: $\quad$ Energy Systems Risk Weight 13

Tiger Team Action Plan Priority 3

Response: $\quad$ Controls are presently established within the Laundry to segregate clothing during the washing process. Separate Regulated Areas and Nonradiological Areas have been established to handle C-Area clothing and khakis, respectively. ORNL, over the course of the next 2 years, will expand the existing facility to allow for complete physical separation of incoming and outgoing laundry at all times.

ORNL Radiation Protection standard operating procedure 02-70-34 and laundry operating procedures do not allow C-Area clothing to leave the facility with detectable contamination. The laundry uses a state-of-the-art, commercially available, plastic scintillator radiation monitor to check each set of C-Area clothing. Laundry handling of washed and monitored C-Area clothing is consistent with ORNL Health Physics Procedures. In addition, ORNL's limits for incoming C-Area laundry is in accordance with very strict limits agreed upon with ORO, based upon effluent considerations.

ORNL will continue to stress the need for a new laundry facility and the line item that this project will require.

\section{Root Causes:}

Insufficient resources

Planned Actions and Schedules:

\section{Item/Description}

1. Evaluate laundry operations to identify interim measures to be taken to improve laundry segregation.

2. Establish controls (procedures, training) necessary to minimize cross contamination possibilities.

\section{Completion Date}

$09 / 91$

09/91 
3. Install new washer/extractor to replace current hot $12 / 91$ washer and hot extractor which will help maintain control of contaminated waste water.

4. Contingent on funding, construct new Decontamination

Laundry Facility (Line Item ORNL-93-KG01-1).

Casts:

Type of funds: Capital

Source of funds: ER-AT

\begin{tabular}{cccccccc} 
& \multicolumn{6}{c}{ Estimated costs per fiscal year (\$K) } & \\
\cline { 2 - 6 } Action item & 1991 & 1992 & 1993 & 1994 & 1995 & Beyond & Total \\
\hline 3 & 40 & & & & & & 40
\end{tabular}

Status:

Funded

Requested

40

New

Type of funds: Line Item

Source of funds: MGPF

Estimated costs per fiscal year (\$K)

\begin{tabular}{cccccccc}
\cline { 2 - 5 } Action item & 1991 & 1992 & 1993 & 1994 & 1995 & Beyond & Total \\
\hline 4 & & & 2600 & 8000 & 5400 & & 16000
\end{tabular}

Status:

Funded

Requested

$2600 \quad 8000 \quad 5400$

New 


\section{Type of funds: ESH Programmatic}

Source of funds: ER-AT RP

\begin{tabular}{lrllllll} 
& \multicolumn{5}{c}{ Estimated costs per fiscal year (\$K) } & \\
\cline { 2 - 6 } Action item & 1991 & 1992 & 1993 & 1994 & 1995 & Beyond & Total \\
\hline 1 & 15 & & & & & & 15 \\
Status: & & & & & & &
\end{tabular}

Funded

Requested

15

New

Type of funds: Overhead

Source of funds: Overhead

Estimated costs per fiscal year (\$K)

\begin{tabular}{rrrrrrrrr} 
Action item & 1991 & 1992 & 1993 & 1994 & 1995 & Beyond & Total \\
\hline 2 & 8 & & & & & & & 8
\end{tabular}

Status:

Funded 8

Requested

New

References: None 
Finding No.: $\quad$ RP.11-1 Management Support for ALARA

Finding

Description: The As Low As Reasonably Achievable (ALARA) program does not have the management support that is required to make the program effective as required by DOE 5480.11 and the DOE ALARA manual.

Code: $\quad$ Category III

Compliance

Protocol:

DOE Order 5480.11 and the DOE ALARA manual

Priority: $\quad$ Energy Systems Risk Weight 63

Tiger Team Action Plan Priority 2

Response: $\quad$ ORNL management supplied adequate funding in FY 1990 to initiate the ALARA Program. While the budget for FY 1991 has not been finalized, ORNL has every intention of continuing the program with adequate funding and with support from the highest levels of Laboratory Management. The Program has been formally chartered by the Laboratory Director, and a steering committee consisting of Division Directors is in place. Laboratory-wide ALARA goals are developed and approved annually by the ALARA Steering Committee. The ALARA Program Manager monitors the progress of the goals and reports quarterly to the Steering Committee. The Laboratory recognizes that additional support in the form of personnel and office space is needed in order for the ALARA Program to fulfill its responsibilities in the field of radiation protection. The lengthy time period for scheduled completion of action item 1 is due to a lack of funding. To fully adopt the ALARA philosophy into operations of the Laboratory, each Division will develop its own ALARA goals that will be tracked by the ALARA manager.

Root Causes:

Insufficient resources and inadequate management commitment

Planned Actions and Schedules:

\section{Item/Description}

1. Complete staffing of the ALARA program ( 2 radiological engineers, 1 computer technician, and 1 clerical person).

2. Issue an ALARA policy through the Laboratory Director's Office to emphasize support and requirements of the ALARA program.

3. Issue a directive to Division Directors to formalize and submit ALARA goals for their Division to the ALARA
Completion Date

$10 / 92$

05/91

05/91 
Program Manager for concurrence. The directive shall state that the ALARA goals will be reflected in the Measures of Performance (MOP) plan.

4. Develop, approve, and issue changes to the Health Physics Manual, Section 6, to include the requirements and guidance for establishing Divisional ALARA goals.

Costs:

Type of funds: ESH Programmatic

Source of funds: ER-AT RP

Estimated costs per fiscal year (\$K)

\begin{tabular}{crrrrrrr}
\cline { 2 - 6 } Action item & 1991 & 1992 & 1993 & 1994 & 1995 & Beyond & Total \\
\hline 2 & 2 & & & & & & 2 \\
3 & 1 & & & & & & 1 \\
4 & & 15 & & & & & 15
\end{tabular}

Status:

Funded

Requested

New

$3 \quad 15$

Type of funds: Overhead

Source of funds: Overhead

Estimated costs per fiscal year (\$K)

\begin{tabular}{crrrrrrr}
\cline { 2 - 5 } Action item & 1991 & 1992 & 1993 & 1994 & 1995 & Beyond & Total \\
\hline 1 & \multicolumn{1}{c}{$510^{*}$} & & & & 510
\end{tabular}

Status:

Funded

Requested

New

${ }^{*}$ Estimated ongoing cost: $\$ 510 \mathrm{~K}$.

References: $\quad$ DOE Order 5480.11 
Finding No.: RP.12-1 Occupational Exposure Records Program

\section{Finding}

Description:

The Occupational Exposure records program is not in compliance with requirements in DOE 5480.11 and ANSI N13.6.

Code: $\quad$ Category III

Compliance

Protocol:

Priority: $\quad$ Energy Systems Risk Weight 65

Tiger Team Action Plan Priority 2

Response: This finding was previously identified in the Radiation Protection Program Appraisal (RPPA) conducted by DOE in 1989.

In response to the RPPA, Energy Systems chartered a management-level task group to evaluate records management requirements. The task group will develop corporate policy and procedures for approval which will provide guidance to ORNL and other Energy System's sites. Concurrent with the task force evaluation, ORNL will evaluate the occupational radiation exposure records system against the requirements of ANSI N13.6, DOE Order 5480.11, and DOE Order 5700.6.

\section{Root Causes:}

Insufficient resources and ambiguous requirements or expectations

Planned Actions and Schedules:

\section{$\underline{\text { Item/Description }}$}

1. Assess the ORNL occupational radiation exposure history records systems and methods against the requirements of ANSI N13.6, DOE Order 5480.11, and DOE Order 5700.6 and develop a comprehensive records management plan.

2. Review the comprehensive records management plan and request funding and resources to implement approved corrective actions.

3. Implement a comprehensive records management plan.

\section{Completion Date}

$12 / 91$ 


\section{Costs:}

Type of funds: ESH Programmatic Source of funds: ER-AT RP

\begin{tabular}{|c|c|c|c|c|c|c|c|}
\hline \multirow[b]{2}{*}{ Action item } & \multicolumn{6}{|c|}{ Estimated costs per fiscal year (\$K) } & \multirow[b]{2}{*}{ Total } \\
\hline & 1991 & 1992 & 1993 & 1994 & 1995 & Beyond & \\
\hline 1 & & 47 & & & & & 47 \\
\hline \multicolumn{8}{|l|}{ Status: } \\
\hline \multicolumn{8}{|l|}{ Funded } \\
\hline \multicolumn{8}{|l|}{ Requested } \\
\hline New & & 47 & & & & & $\$ 47$ \\
\hline
\end{tabular}

References: $\quad$ DOE Order 5480.11

DOE Order 5700.6

ANSI N13.6 
Finding No.: $\quad$ RP.12-2 Reporting of Dosimetry Data

Finding

Description: Dosimetry data are not reported to all staff or visitors as required in DOE 5480.11 and DOE 5484.1.

Code: $\quad$ Category III

Compliance

Protocol:

DOE Orders 5480.11 and 5484.1

Priority: $\quad$ Energy Systems Risk Weight 60

Tiger Team Action Plan Priority 2

Response: $\quad$ DOE Order 5484.1 specifies that all positive internal and external exposures of a visitor must be documented on Form 5484.8 and copies submitted to the visitor's employer or visitor if he/she has no employer and to System Safety Development Center (EG\&G at Idaho) within thirty days after the visit or thirty days after the exposure determination whichever is later. Other specific requirements are stated if exposure limits exceed limits set in DOE Order 5480.11.

A need has been identified to upgrade the current visitor exposure reporting program and to ensure that nonemployee terminations are reported to the occupation radiation exposure reporting personnel in a timely manner by the Human Resource Division personnel.

\section{Root Causes:}

Insufficient resources, inadequate oversight, inadequate communications, and poorly defined roles and responsibilities

Planned Actions and Schedules:

\section{Item/Description}

1. Issue a standard operating procedure for visitor/nonemployee termination reporting methods to ensure required records submittal.
Completion Date

$11 / 91$ 
Casts:

Type of funds: ES\&H Programmatic

Source of funds: ER-AT RP

\begin{tabular}{|c|c|c|c|c|c|c|c|}
\hline \multirow[b]{2}{*}{ Action item } & \multicolumn{6}{|c|}{ Estimated costs per fiscal year (\$K) } & \multirow[b]{2}{*}{ Total } \\
\hline & 1991 & 1992 & 1993 & 1994 & 1995 & Beyond & \\
\hline 1 & 15 & * & & & & & 15 \\
\hline \multicolumn{8}{|l|}{ Status: } \\
\hline \multicolumn{8}{|l|}{ Funded } \\
\hline \multicolumn{8}{|l|}{ Requested } \\
\hline New & 15 & & & & & & $\$ 15$ \\
\hline
\end{tabular}

*Estimated annual ongoing cost: $\$ 5 \mathrm{~K}$ for increased reporting activities to be added to overhead.

References: $\quad$ DOE Order 5480.11

DOE Order 5484.1 
Finding No.: $\quad$ RP.12-3 Control of Occupational Exposure Records

Finding

Description. Occupational Exposure Records are not controlled, maintained, and protected as required in DOE 5480.11, DOE 5484.1, ANSI N13.6, and ANSI/ASME NQA-1.

Code: $\quad$ Category III

Compliance

Protocol:

DOE Orders 5480.11 and 5484.1, ANSI N13.6, and ANSI/ASME NQA-1

Priority: $\quad$ Energy Systems Risk Weight 108

Tiger Team Action Plan Priority 2

Response: ORNL is assessing the requirements of records related to occupational radiation exposure to ensure that the records are systematically generated and maintained consistent with ANSI N13.6 and other applicable standards, procedures, and regulations. Primarily these records include:

- Radiation records related to an individual, (e.g., prior exposure history, bioassay data, dose assessment methodology, personnel dosimetry, results, etc.);

- Radiation records related to status of work areas (e.g., radiation surveys, air sampling results, etc.);

- Records that describe the technical and administrative bases for radiological protection programs (e.g., standards, policies, procedures, methods of dose evaluations, etc.); and

- Records of unusual occurrences, accidents, and incidents (e.g., investigations, corrective action, followup, etc.).

The need to protect exposure data is recognized, and plans are currently being made to correct deficiencies. This finding was previously identified in the Radiation Protection Program Appraisal conducted by DOE in 1989.

\section{Root Causes:}

Insufficient resources and poorly defined roles and rissponsibilitics

Planned Actions and Schedules:

\section{Item/Description}

1. Issue Standard Operating Procedures for the occupational radiation exposure record gencration and retention system.

\section{Completion Date}

$11 / 92$ 
2. Develop comprehensive records management plans for the Dosimetry Data Management Group to ensure that recrids are maintained in accordance with applicable standards, procedures, and orders.

3. Request funding to implement corrective action elements of the comprehensive records management plans.

4. Implement interim methods of record duplication by microfilm copying of exposure data.

5. Appoint a manager of the records management program for radiological protection.

Costs:

Type of funds: ESH Programmatic Source of funds: ER-AT RP

Estimated costs per fiscal year (\$K)

\begin{tabular}{|c|c|c|c|c|c|c|c|}
\hline Action item & 1991 & 1992 & 1993 & 1994 & 1995 & Beyond & Total \\
\hline 1 & & 225 & & & & & 225 \\
\hline 2 & & 30 & & & & & 30 \\
\hline 3 & & - & & & & & \\
\hline 4 & & 127 & $*$ & & & & 127 \\
\hline 5 & & - & & & & & \\
\hline
\end{tabular}

Status:

Funded

Requested

New

*Estimated annual ongoing cost: $\$ 23 \mathrm{~K}$ to maintain microfilming.

References: $\quad$ DOE Order 5480.11

DOE Order 5484.1

ANSI N13.6

A' 'SI/ASME NQA-1 


\subsubsection{Personnel Protection}

Finding No.: PP.1-1 Resources for Workplace Maintenance

Finding

Description: Neither Oak Ridge National Laboratory nor Martin Marietta Energy Systems, Inc., has allocated resources to effectively maintain workplaces free of health and safety concerns.

Code: $\quad$ Category III

Compliance

Protocol:

29 CFR 1910 and 1926 (including the "General Duty Clause") and DOE Orders 5480 and 5483 require the employer to implement an effective personnel protection program.

Priorily: $\quad$ Energy Systems Risk Weight 505

Tiger Team Action Plan Priority 2

Response: This finding was based on several related findings, including those dealing with

- inadequate workplace monitoring and tracking and trending of monitoring and exposure data;

- incomplete corporate-level policies, standards, and procedures;

- vacancies in corporate industrial hygiene staff positions;

- inappropriate assignment of responsibilities for policy development;

- lack of formality in the assignment of responsibilities and authorities for Industrial Hygiene and Industrial Safety;

- lack of procedures for interface between departments;

- lack of quality assurance plans and QA personnel;

- inconsistent interpretation of DOE requirements;

- heavy reliance on the division safety officers for program implementation;

- inadequate position descriptions for DSOs; and

- lack of support for the ORNL safety policy by some division directors.

Many of these findings can be traced to a lack of resources (especially staffing) for Industrial Safety and Indusirial Hygiene. Over the past year, both sections have 
been involved in setting up new programs to ensure personnel protection and to achieve compliance with new regulations. Now that the programs have been developed, impleme:tation will depend on the availability of qualified personnel. Because of the resource restrictions in these sections, both groups have relied in the past on the support of the DSOs. However, the DSOs are often assigned other responsibilities within their own divisions, and these responsibilities sometimes account for as much as $85 \%$ of their time. An in-depth needs assessment dealing with administration of these programs at ORNL is needed (see Corrective Action Items 1 and 2 below). As part of this overall assessment, the proper role of the DSOs will be studied, and recommendations will be made to the division directors.

Many of the corrective action plans contained in this document require the addition of staff to existing ES\&H programs. At ORNL, a shortage of office space already exists. Additional space will be needed to house these new staff members. In addition, more space is needed for such commitments as an expanded records management program. The corrective action shown below (Item 13) is intended to address these office space and storage needs associated with the corrective actions for Findings AX.1-1, CS.3-1, FP.1-1, IH.2-1, IH.5-4, IH.6-1, MS.3-3, OA.7-2, PP.1-1, PP.2-1, PP.2-2, QV.1-1, QV.1-3, QV.4-1, RP.1-3, RP.11-1, RP.12-3, and RP.7-1.

\section{Root Causes:}

Insufficient resources (staffing), poorly defined roles and responsibilities, inadequate policy implementation, and inadequate management commitment

Planned Actions and Schedules:

Item/Description

1. Conduct a needs assessment for administration of the Industrial Safety program at ORNL.

2. Conduct a needs assessment for administration of the Industrial Hygiene program at ORNL.

3. Based on the results of the needs assessment, develop a program management plan for Industrial Safety.

4. Based on the results of the needs assessment, revise the existing program management plan for Industrial Hygiene.

5. Based on the needs assessment and the program management plans, develop position charters for DSOs and submit to the division directors for their comment and approval.
Completion Date

$6 / 91$

$6 / 91$

7/91

9/91

$8 / 91$ 
6. Issue revised guidelines for DSOs to reflect any changes

$9 / 91$ involving DSOs (roles, responsibilities, etc.).

7. Request funding to implement the program management $7 / 91$ plan for Industrial Safety.

8. Request funding to implement the program management $7 / 91$ plan for Industrial Hygiene.

9. Contingent on approval of funding, hire five additional staff members for Industrial Safety. (This number may change, depending on the results of the needs assessment. Additional staffing for Industrial Hygiene is covered in the action items in the IH section of this plan.)

10. Develop additional training modules for DSOs and begin training.

11. Assign members of the Industrial Hygiene and Industrial Safety staffs to serve as liaisons between the two sections on matters of policy and responsibilities.

12. Prepare engineering estimate and submit CDR for office/storage space.

13. Initiate construction of Central Research Support Building. 
Costs:

Type of funds: Overhead

Source of funds: Overhead

\begin{tabular}{|c|c|c|c|c|c|c|c|}
\hline \multirow[b]{2}{*}{ Action item } & \multicolumn{6}{|c|}{ Estimated costs per fiscal year $(\$ \mathrm{~K})$} & \multirow[b]{2}{*}{ Total } \\
\hline & 1991 & 1992 & 1993 & 1994 & 1995 & Beyond & \\
\hline 1 & 6 & & & & & & 6 \\
\hline 2 & 4 & & & & & & 4 \\
\hline 3 & 5 & & & & & & 5 \\
\hline 4 & 2 & & & & & & 2 \\
\hline 5 & 1 & & & & & & 1 \\
\hline 6 & 10 & & & & & & 10 \\
\hline 7 & - & & & & & & - \\
\hline 8 & - & & & & & & - \\
\hline 9 & & * & & & & & \\
\hline 10 & & 8 & & & & & 8 \\
\hline 11 & - & & & & & & \\
\hline 12 & 40 & & & & & & 40 \\
\hline
\end{tabular}

Status:

Funded

Requested

New
28

8

40

*Estimated annual ongoing cost: \$425K starting in FY 1992.

Type of funds: Line Item

Source of funds: ER-AT

\begin{tabular}{|c|c|c|c|c|c|c|c|}
\hline \multirow[b]{2}{*}{ Action item } & \multicolumn{6}{|c|}{ Estimated costs per fiscal year $(\$ K)$} & \multirow[b]{2}{*}{ Total } \\
\hline & 1991 & 1992 & 1993 & 1994 & 1995 & Beyond & \\
\hline 13 & & & 1000 & 3000 & 6000 & 2400 & 12400 \\
\hline \multicolumn{8}{|l|}{ Status: } \\
\hline \multicolumn{8}{|l|}{ Funded } \\
\hline Requested & & & 1000 & 3000 & 6000 & 2400 & \\
\hline New & & & & & & & $\$ 12400$ \\
\hline
\end{tabular}

\subsubsection{5-4}




\section{References: $\quad 29$ CFR 1910 and 29 CFR 1926}

DOE Orders 5480 and 5483

3.3.15-5 
Finding No.: PP.2-1 ORNL Health and Safety Program

Finding

Description: The ORNL Health and Safety Program does not ensure that all work places are free from hazards as required by DOE 5480.4 .

Code: $\quad$ Category III

Compliance

Protocol:

DOE Order 5480.4

Priority: $\quad$ Energy Systems Risk Weight 505

Tiger Team Action Plan Priority 2

Response: The ORNL Safety Manual is recognized as being out of date and not in total compliance with applicable standards due to inadequate resources. Moreover, the tracking of identified hazards has also been hampered by inadequate staff resources. The Industrial Safety Section has recognized the need to update the safety standards, and these needs were discussed in the Section's self-assessment document. All present procedures will be reviewed and revised, as needed, to reflect current DOE, OSHA, and industry consensus requirements. Additional staffing will be required as new procedures are created, as appropriate, to ensure that industrial safety requirements are documented, tracked, and made available to ORNL line management.

\section{Root Causes:}

Inadequate policy, inadequate policy implementation, and insufficient resources

\section{Planned Actions and Schedules:}

\section{$\underline{\text { Item/Description }}$}

1. Evaluate existing procedures and establish priorities for overall procedure upgrade to ensure procedures reflect current DOE, OSHA, and industry consensus requirements.

2. Conduct OSHA compliance inspections, document results, and initiate fixes to ensure workplaces are free from recognized hazards.

3. Request funding to hire an additional staff member.

4. Contingent on approval of funding, hire additional staff to write procedures and to develop hazard-tracking systems.

\section{Completion Date}

Complete 
Costs:

Type of funds: Overhead

Source of funds: Overhead

\begin{tabular}{|c|c|c|c|c|c|c|c|}
\hline \multirow[b]{2}{*}{ Action item } & \multicolumn{6}{|c|}{ Estimated costs per fiscal year (\$K) } & \multirow[b]{2}{*}{ Total } \\
\hline & 1991 & 1992 & 1993 & 1994 & 1995 & Beyond & \\
\hline 1 & - & & & & & & \\
\hline 2 & - & & & & & & \\
\hline 3 & - & & & & & & \\
\hline 4 & & & * & & & & \\
\hline 5 & & 280 & & * & & & \\
\hline \multicolumn{8}{|l|}{ Status: } \\
\hline \multicolumn{8}{|l|}{ Funded } \\
\hline \multicolumn{8}{|l|}{ Requested } \\
\hline New & & 280 & & & & & $\$ 280$ \\
\hline
\end{tabular}

${ }^{*}$ Estimated annual ongoing cost: \$140K starting in FY 1993 and an additional \$315K every other year beginning in FY 1994.

References: $\quad$ DOE Order 5483.1A 
Finding No.: PP.2-2 Workplace Exposure Monitoring and Medical Records

Finding

Description: Workplace exposure monitoring and medical records are not controlled in the manner or are they structured and coordinated to ensure generation of defensible and readily retrievable exposure assessment data required by 29 CFR 1910 Subpart C.

Code: $\quad$ Category III

Compliance

Protocol:

29 CFR 1910 Subpart C

Priority: $\quad$ Energy Systems Risk Weight 415

Tiger Team Action Plan Priority 2

Response: This finding was based on several related findings, including:

- Personnel exposure records are maintained in various locations, which limits the ability to relate a person's exposure to a specific hazard.

- Chain of custody for workplace monitoring samples is not established.

- Budgeted money for Industrial Hygiene, Medical, Industrial Safety, and Radiation Protection improvements to the Occupational Health Information System (OHIS) were eliminated in the FY 1991 budget. This means that the OHIS will operate in a maintenance mode in FY 1991.

- Sample protocols and laboratory and field standard operating procedures are not developed for the evaluation of most hazards.

IHAS, the Industrial Hygiene portion of the Occupational Health Information System (OHIS), will-once it is fully implemented-meet most of the records management needs for employee exposure data. The IH Section is working with the OHIS committee to ensure that the industrial hygiene components of OHIS will receive adequate attention in any upgrade efforts. Any records management needs that are not met by the OHIS upgrade will be considered in the development of the Section's in-house records management system (see action Item 1 below). Funding for OHIS improvements will again be requested for FY 1992. In the meantime, the corrective actions planned to address these findings are listed below.

It is currently possible to manually relate exposures to specific hazards, and this is done routinely; the existing manual process will be automated with full implementation of OHIS/IHAS.

\section{Root Causes:}

Insufficient resources, poorly defined roles and responsibilities, and inadequate policy implementation 
Planned Actions and Schedules:

Item/Description

1. Develop a comprehensive records management system for IH exposure records; this new system will specify a centralized location for storage of all relevant records.

2. Develop and issue a standard operating procedure covering chain-of-custody requirements for workplace monitoring samples.

3. Determine needs for upgrading OHIS to meet records management requirements and submit recommendations to the OHIS committee.

4. Issue standard operating procedures covering field

Completion Date

$6 / 92$

$9 / 91$

$6 / 91$ sampling protocols and laboratory analyses protocols for workplace hazards.

\section{Costs:}

Type of funds: Overhead

Source of funds: Overhead

Estimated costs per fiscal year (\$K)

\begin{tabular}{crrrrrrr}
\cline { 2 - 6 } Action item & 1991 & 1992 & 1993 & 1994 & 1995 & Beyond & Total \\
\hline 2 & 15 & & & & & & 15 \\
3 & 2 & & & & & & 2 \\
4 & 46 & & & & & & 46
\end{tabular}

Status:

Funded

63

Requested

New 
Type of funds: ESH Programmatic

Source of funds: ER-AT IH

\begin{tabular}{|c|c|c|c|c|c|c|c|}
\hline \multirow[b]{2}{*}{ Action item } & \multicolumn{6}{|c|}{ Estimated costs per fiscal year $(\$ \mathrm{~K})$} & \multirow[b]{2}{*}{ Total } \\
\hline & 1991 & 1992 & 1993 & 1994 & 1995 & Beyond & \\
\hline 1 & & 61 & & & & & 61 \\
\hline $\begin{array}{l}\text { Status: } \\
\text { Funded }\end{array}$ & & & & & & & \\
\hline Requested & & 61 & & & & & \\
\hline New & & & & & & & $\$ 61$ \\
\hline
\end{tabular}

References: 29 CFR 1910, Subpart C 
Finding No.: PP.3-1 Health and Safety Concerns

\section{Finding}

Description: ORNL does not have a formal system for identifying and evaluating health and safety concerns for all of its activities.

Code: $\quad$ Category III

\section{Compliance}

Protocol: None

Priority: $\quad$ Energy Systems Risk Weight 68

Tiger Team Action Plan Priority 2

Response: $\quad$ Issues of major environmental, safety, and health (ES\&H) concern (e.g., yttrium-90 production at Building 3038 laboratory, shock-sensitive chemicals at Building 9207, isutope production laboratory shutdown, and removal of cesium and colalt capsules from leaking canal at Building 3001) surface at ORNL, sometimes requiring a rapid and often complicated course of action to correct. At present, no systematic process exists to ensure that the appropriate risk and hazard assessment and independent ES\&H reviews are triggered.

Situations may be further confused by overlapping jurisdictions and responsibilities, both within and external to ORNL.

Unless a systematic process is developed to quickly dispatch clear, responsive authority supported by a team of experts (line management, appropriate ES\&H disciplines, etc.) to develop a course of action based on a comprehensive risk assessment which has early buy-in with DOE, situations such as the long delay in removal of shock-sensitive chemicals from the Biology Division laboratory will continue leaving ORNL and DOE extremely vulnerable.

\section{Root Causes:}

Inadequate policy, inadequate management approach, ambiguous expectations or requirements, and poorly defined roles and responsibilities 
Planned Actions and Schedules:

Item/Description

1. Develop and initiate implementation of a systematic process (including issuance of an SPP) which is initiated by line management to assign clear unambiguous responsibilities and to draw upon the expertise of appropriate ES\&H, QA, security, or other discipline experts to develop a comprehensive course of action for Laboratory activities.
Completion Ditc

$9 / 91$

Process will include methodology to ensure:

a. appropriate safety and environmental assessments are performed,

b. appropriate levels of review and surveillance are conducted,

c. decisions are made at a necessary level to get issues resolved in a saic and timely manner,

d. startup requirements are appropriately defined, and

e. lessons learned are incorporated and deficiencies are corrected.

Costs:

Type of funds: Overhead

Source of funds: Overhead

\begin{tabular}{cccccccc} 
& \multicolumn{5}{c}{ Estimated costs per fiscal year $(\$ K)$} & \\
\cline { 2 - 6 } Action item & 1991 & 1992 & 1993 & 1994 & 1995 & Beyond & Total \\
\hline 1 & 15 & & & & & & 15
\end{tabular}

Status:

Funded

Requested

New

References: None 
Finding No.: PP.3-2 ORNL Construction Oversight Program

\section{Finding}

Description.

Coxde:

\section{Compliance}

Protocol:

Priority:

Response:
The ORNL Construction Oversight Program does not apply an effective system to enforce safety requirements and correct noncompliances.

Category III

DOE Order 5480.9

Energy Systems Risk Weight 68

Tiger Team Action Plan Priority 2

Lack of definition of roles and responsibilities between MK-Ferguson and Energy Systems is the primary problem. Construction activity is in a transition period from the past practices of multiple contracting agencies to the present practice of assigning all construction activity to the construction manager (CM) for his administration. An interface agreement is being developed which will clearly define roles and responsibilities for future construction safety enforcement and oversight. As CM, MK-Ferguson will be responsible for enforcing safety requirements for all construction contractors and for conducting a formalized review of contractor health and safety programs. The $\mathrm{CM}$ will be responsible for tracking all open items and ensuring correction of noncompliances in a timely manner. Energy Systems will monitor and oversee the CM's safety activities and construction projects bringing to the attention of the $\mathrm{CM}$ any noncompliances. In the event of a situation with imminent danger to life or health, Energy Systems will correct the noncompliance immediately and notify the CM.

\section{Root Causes:}

Inadequate policy, inadequate policy implementation, and poorly defined roles and responsibilitics 
Planned Actions und Schedules:

\section{$\underline{\text { Item/Description }}$}

1. Issue an interface document which outlines the roles and responsibilities of environmental, safety, and health personnel of Energy Systems and MK-Ferguson (see Finding OA.1-3).

2. Update and revise the ORNL construction safety standard to reflect current needs (see Finding PP.2-1, Item 4).

3. Develop and implement internal operating procedures for ORNL industrial safety relative to the requirements of the interface document and the ORNL construction safety standard (see Finding PP.2-1, Item 3).
Completion Datc

Complete

Costs:

Type of funds: Overhead

Source of funds: Overhead

Estimated costs per fiscal year (\$K)

\begin{tabular}{cccccccc}
\cline { 2 - 6 } Action item & 1991 & 1992 & 1993 & 1994 & 1995 & Beyond & Total \\
\hline 1 & - & & & & & & \\
2 & 7 & & & & & & 7 \\
3 & - & & & & & &
\end{tabular}

Status:

Funded

7

Requested

New

References: $\quad 29$ CFR 1926

DOE Order 5483.1A 
Finding No.: PP.5.1 Hazard Communication Program Deficiencies

Finding

Description.

The Hazard Communication Program does not fully inform ORNL personncl of industrial hazards as roquirod by 29 CFR 1910.1200 .

Code: Calceon III

Compliance

Protocol.

29 CFR $1910.12(x)$

Prionity: $\quad$ Energy Systems Risk Weight 418

Tige: Team Action Plan Priority 2

Response: This finding was hased on several related findings. which said:

- Aithough a neu hazard communication program has been developed and some ORNL employees. visitors. and visiting scientists have been trained, the program does not contain elements regarding Industrial Safety.

- Employees taking the HAZCOM training course are not formally iested to determine their level of understanding of the program.

- Supervisors are not required to take the basic HAZCOM training program prior to taking the HAZCOM training for supervisors.

On September 12, 1990, the Industrial Hygiene Section issued procedure IHP-01, "ORNL Hazard Communication Program," which was designed to address the requirements of OSHA's HAZCOM regulation. That regulation is specifically targeted at chemical hazards, including the "physical" hazards of chemicals (e.g., flammability, explosiv': potential, instability). The HAZCOM training course and IHP-01 both address uch physical hazards. Awareness of other types of industrial hazards (not related if chemicals) is ensured through other avenues (e.g., divisional safety meetings and Safety Department bulletins).

Corrective actions that have been planned in response to the other findings are listed below.

\section{Root Causcs:}

Inadequate management approach, ambiguous requirements or expectations, and inadequate training 
Planned Actions and Schedules:

$\underline{\text { Item/Description }}$

1. Revise the HAZCOM training courses to include tests that will determine employees' understanding of the program.

2. Issue revised IHP-01 to specify that supervisors must complete the basic HAZCOM course before taking the supervisors' course or combine the general course information with the supervisors' course so that supervisors must take only the one ccurse.
Completion Date

$6 / 91$

$6 / 91$

Casts:

Type of funds: Overhead

Source of funds: Overhead

Estimated costs per fiscal year (\$K)

\begin{tabular}{crrrrrrr}
\cline { 2 - 5 } Action item & 1991 & 1992 & 1993 & 1994 & 1995 & Beyond & Total \\
\hline 1 & 3 & & & & & & 3 \\
2 & 7 & & & & & & 7
\end{tabular}

Status:

Funded

Requested

New

References: $\quad 29$ CFR 1910.1200 
Finding No.: PP.5-2 Health and Safety Program Deficiencies

Finding

Description:

Code:

Compliance

Protocol:

Priority: $\quad$ Energy Systems Risk Weight 13

Tiger Team Action Plan Priority 3

Response: This finding was based on the following:

- Although a documented program exists for the voicing of health and safety concerns of employees to ORNL and DOE management, the tracking system for resolutions of these concerns and the feedback mechanism to the employees are unnecessarily complicated.

The Industrial Safety Standard IS-1.6 outlines the procedure for the employee to follow in submitting a safety suggestion/complaint to their supervisor or industrial safety. This safety standard also documents the tracking system and feedback mechanism that are used in ORNL for an employees safety suggestion/complaint.

ORNL has an ongoing effort to ensure compliance with DOE Order 5483.1A, "Occupational Safety and Health Program for Government-Owned ContractorOperated Facilities." This order requires that ORNL employees be provided with safe and healthful working conditions in accordance with DOE safety and health standards which incorporate OSHA standards. Also, this order specifies the feedback mechanism to the employee when he/she may voice a health and safety complaint to DOE-ORO.

\section{Root Cause:}

Inadequate policy 
Planned Actions and Schedules:

\section{$\underline{\text { Item/Description }}$}

1. Revise and issue Industrial Safety Standard IS-1.5, "Handling Employee Complaints Pertaining to Health and Safety," to include the feedback mechanism as stated in DOE Order 5483.1A.

2. Develop internal procedure to audit tracking system and feedback mechanism quarterly.

Costs: $\quad$ Costs associated with these action items are included in Finding PP.2-1, Item 4.

References: DOE Order 5483.1A

\section{Completion Date}

$3 / 92$

$9 / 92$ 
Finding No.: PP.5-3 Explosives Safety Program

Finding

Description:

The Explosives Safety Program at ORNL is not in compliance with DOE 5480.4 and DOE 5480.3.

Code: $\quad$ Category III

Compliance

Protocol:

DOE Orders 5480.4 and 5480.3

Priority: $\quad$ Energy Systems Risk Weight 133

Tiger Team Action Plan Priority 2

Response: Despite the lack of clearly defined roles and responsibilities governing the program for explosives safety at ORNL, the Industrial Safety Section has continued to pursue a phased approach for compliance with applicable laws, regulations, and DOE orders. This ongoing effort was constrained, in part, due to the perception that support by senior management was limited and confusion over necessary elements of authority and responsibility.

Senior management responded positively as evidenced by approval of a major policy change that restructured the explosives safety program at ORNL. This action signaled a more well-defined and documented effort and provides clear direction for the development and implementation of effective policy for this program. The Industrial Safety Section will aggressively integrate the following planned actions to achieve program compliance while also redirecting staff effort for providing oversight to identify other potential noncompliance issues.

\section{Root Causes:}

Inadequate policy implementation, inadequate training, and poorly defined roles and responsibilities 
Planned Actions and Schedules:

\section{Item/Description}

1. Industrial Safety will obtain and ensure receipt of controlled copies of procedures regarding explosives operations in the Hazardous Waste Operations Manual (WM-SWO-401).

2. Industrial Safety will obtain and verify distribution of additional copies of the DOE Explosives Safety Manual to program managers, division safety officers, line management, and supervisors involved with explosives transportation, use, and storage.

3. ORNL senior management will appoint a coordinator for explosives safety as provided by SPP X-ESH-8.

4. Industrial Safety will revise and reissue the procedure for explosives safety in the ORNL Safety Manual.

5. Technical Resources and Training Section will develop and implement formalized training for employees engaged in explosives handling, transportation, and storage.

\section{Completion Date}

Complete

$5 / 91$

Complete

$7 / 91$

$11 / 91$ 


\section{Costs:}

Type of funds: Overhead

Source of funds: Overhead

Estimated costs per fiscal year (\$K)

\begin{tabular}{crrrrrrr}
\cline { 2 - 5 } Action item & 1991 & 1992 & 1993 & 1994 & 1995 & Beyond & Total \\
\hline 1 & & & & & & & \\
2 & & & & & & & \\
3 & & & & & & & \\
4 & 7 & & & & & & 7 \\
5 & & 10 & $*$ & & & & 10
\end{tabular}

Status:

Funded

7

Requested

New

10

*Estimated annual ongoing cost: $\$ 3 \mathrm{~K}$ for continuing training.

References: $\quad$ DOE Orders 5480.4 and 5480.3 
Finding No.: PP.5-4 Implementation of the Industrial Safety Program

Finding

Description:

As evidenced by the number and severity of work place hazards, the basic concepts of the industrial safety program are not fully implemented by ORNL personnel.

Code: $\quad$ Category III

Compliance

Protocol:

Best management practices indicate that the number and severity of work place hazards should be minimal if the basic concepts of an effective industrial safety
program are fully implemented.

Priority: $\quad$ Energy Systems Risk Weight 5

Tiger Team Action Plan Priority 3

Response: This concern was based on the following related findings:

- Industrial Safety training for ORNL visitors and visiting scientists is not formalized.

- See WS, Section 4.5.1.16.

- The number and severity of workplace hazards ide tified by this appraisal indicate that the basic concept of workplace safety hazard identification by ORNL employees is not fully implemented.

The ORNL Training and Development Depa :ment, Human Resources Division, is in the process of developing safety orientation videotapes for employees, visitors, and visiting scientists which explain basic concepts of ORNL's safety program.

The Technical Resources and Development Section and the Industrial Safety Section developed a training program for supervisors to enable them to identify
workplace hazards.

Root Causes:

Inadequate policy implementation, insufficient resources, and inadequate training 
Planned Actions and Schedules:

Item/Description

1. The ORNL Training and Development Department will complete an orientation videotape for visitors, guests, and facility users (see Finding TC.1-1, Item 7).

2. Division supervision will attend the course, "Industrial Safety for Supervisors," to obtain training in hazards identification (see Finding TC.1-1).

3. Issue an SPP defining ES\&H training requirements for all personnel.
Completion Date

Complete

9/91

$9 / 91$

Casts:

Type of funds: Overhead

Source of funds: Overhead

Estimated costs per fiscal year (\$K)

\begin{tabular}{crrrrrrr}
\cline { 2 - 5 } Action item & 1991 & 1992 & 1993 & 1994 & 1995 & Beyond & Total \\
\hline 3 & 15 & & & & & & 15
\end{tabular}

Status:

Funded

Requested

New

References: $\quad$ DOE Order 5483.1A 


\subsubsection{Worker Safety}

Finding No.: WS.3-1 Control of Asbestos

Finding

Description: ORNL does not control the use of asbestos-containing materials nor do they have a current inventory of the products on site that contain asbestos and therefore does not comply with all requirements of 29 CFR 1910.1001, Asbestos Standard, and 29 CFR 1910.1200, Hazard Communication.

Code: $\quad$ Category III

Compliance

Protocol:

29 CFR 1910.1001 and 29 CFR 1910.1200

Priority: $\quad$ Energy Systems Risk Weight 468

Tiger Team Action Plan Priority 2

Response: This finding was based on several related findings.

- ORNL stores will order products containing asbestos if the Laboratory Director will authorize it by letter or if a substitute is not readily available.

- ORNL has not identified all asbestos containing materials (ACM).

- Areas were noted where ACM are breaking off and causing a housekeeping problem and where they could eventually be reentrained and become an inhalation hazard.

- ORNL acknowledged a considerable amount of asbestos on site.

Over the past year, an extensive survey has been conducted at ORNL to establish an ACM inventory. Over two-thirds of the inventory has already been completed, approximately half of the engineering drawings for the inventory have been finished, and an inventory database has been set up. Additional fundin $::$ be requested for a follow-up survey to ensure that all $\mathrm{ACM}$ areas have $b$.... identified (see Action Item 4 below).

An Asbestos Operations and Management Plan for ORNL is being developed. Once this plan is issued and the IH Section staff members are assigned to provide individual coverage for operating divisions, asbestos issues will receive more detailed attention. The Plan will provide for systematic surveillance and repair of damaged ACM areas. Specific corrective actions planned to address these findings are listed telow. 


\section{Root Causes:}

Inadequate policy implementation

Planned Actions and Schedules:

\section{Item/Description}

1. Issue the ORNL Asbestos O\&M Plan, which will address in detail the policy and management approach for oversight of asbestos activities at ORNL.

2. Issue a procedure concerning review of purchases (including asbestos). (Costs are covered in Finding IH.2-1, Item 4.)

3. Revise the asbestos awareness training to strengthen the guidance concerning procurement of $\mathrm{ACM}$.

4. Request funds to conduct a follow-up survey to ensure that all possible areas involving ACM have been identified.

5. Contingent on funding, iritiate follow-up survi:y.

6. Initiate and document periodic review of Stores inventory for asbestos-containing material.
Completion Date

9/91

$6 / 91$

$5 / 91$

$8 / 91$

$7 / 92$ 
Costs:

Type of funds: Overhead

Source of funds: Overhead

\begin{tabular}{|c|c|c|c|c|c|c|c|}
\hline \multirow[b]{2}{*}{ Action item } & \multicolumn{6}{|c|}{ Estimated costs per fiscal year (\$K) } & \multirow[b]{2}{*}{ Total } \\
\hline & 1991 & 1992 & 1993 & 1994 & 1995 & Beyond & \\
\hline 1 & 77 & & & & & & 77 \\
\hline 2 & & - & & & & & \\
\hline 3 & 8 & & & & & & 8 \\
\hline 4 & - & & & & & & \\
\hline 5 & & 175 & & & & & 175 \\
\hline 6 & & 21 & & & & & 21 \\
\hline
\end{tabular}

Status:

Funded

85

Requested

New

196

$\$ 281$

*Estimated annual ongoing cost: $\$ 21 \mathrm{~K}$.

References: $\quad 29$ CFR 1910.1001

29 CFR 1910.1200 
Finding No.: $\quad$ WS.4-1 Machine Guarding

\section{Finding}

Description: Guarding was not universally available and in place, as specified in 29 CFR 1910 Subpart $O$, for machines and tools with moving and rotating parts.

Code: $\quad$ Category III

Compliance Protocol:

29 CFR 1910 Subpart O. Workplaces should be free of uncontrolled physical hazards and shall be in compliance with DOE-prescribed occupational safety standards.

Priority: $\quad$ Energy Systems Risk Weight 910

Tiger Team Action Plan Priority 2

Response: ORNL recognized the need for proper guarding of machines and equipment in the self-assessment. Beginning in August 1990, the NUS Corporation conducted an Industrial Safety OSHA inspection that included machines and equipment guarding in all the facilities. That inspection has been completed, and each division is correcting noncompliances where funding is available. Also, the Fahrication Department of the Plant and Equipment Division conducted an assessment of all machines and equipment in the machine shops. Most all of the deficiencies have been corrected.

We have a cumprehensive list of guarding deficiencies, and abatement will proceed in direct proportion to funding. A request for funding machine guarding noncompliances was made to DOE in May 1990 (Field Work Proposal "ORNL Safety and Health Regulatory Compliance").

Machine guarding deficiencies have been prioritized with high emphasis placed on employee exposure. Machines that are used frequently have been corrected first. Other pieces of equipment, such as exposed belt pulleys and sprockets on pumps, HVAC systems, or fans, have also been addressed with special emphasis training and safety analysis that is provided to employees on low-risk, unguarded machines. Those that are in traffic areas have been or are being corrected immediately.

\section{Root Cause:}

Inadequate policy, insufficient resources. ambiguous requirements or expectations, inadequate management commitment, and poorly defined roles and responsibilities 
Planned Actions and Schedules:

\section{Item/Description}

1. Issue a Safety Standard on Machine Guarding to include engineering and purchase specifications against OSHA and ANSI machine guarding standards, and organizational roles and responsibilities.

2. Resubmit request for OSHA machine guarding funds with a FWP to DOE.

3. Correct specific machine guarding findings as listed in the Tiger Team assessment (Appendix F) and document in a letter.

4. Develop and initiate implementation of training on 29 CFR 1910 Subpart O for Engineering, Facility Managers, and machinists.

5. Contingent on approval of funding, complete and document installation of machine guarding as identified in comprehensive NUS Corporation and Plant and Equipment Surveys.
Completion Date

$7 / 91$

Complete

$9 / 91$

$12 / 91$

$9 / 94$

Costs:

Type of funds: ESH Programmatic

Source of funds: ER-AT OSHA

Estimated costs per fiscal year (\$K)

\begin{tabular}{crrrrrrr}
\cline { 2 - 5 } Action item & 1991 & 1992 & 1993 & 1994 & 1995 & Beyond & Total \\
\hline 5 & & 405 & 425 & 485 & & & 1315
\end{tabular}

Status:

Funded

Requested

405

425

485

New 
Type of funds: Overhead

Source of funds: Overhead

\begin{tabular}{crrrrrrr} 
& \multicolumn{5}{c}{ Estimated costs per fiscal year (\$K) } & \\
\cline { 2 - 6 } Action item & 1991 & 1992 & 1993 & 1994 & 1995 & Beyond & Total \\
\hline 1 & 15 & & & & & & 15 \\
3 & 150 & & & & & & 150 \\
4 & & 10 & & & & & 10
\end{tabular}

Status:

Funded 165

Requested

New

10 $\$ 175$

References: None 
Fünding No.: WS.4-2 Noncompliances of Building Egress

Finding

Description: Exits were not properly marked, adequately lighted, and not all means of egress are continuous and unobstructed to the nearest exit in accordance with 29 CFR 1910 Subpart E.

Code: $\quad$ Category III

Compliance Protocol:

29 CFR 1910 Subpart E. Workplaces should be free of uncontrolled physical hazards and should be in compliance with DOE-prescribed Occupational Safety Standards.

Priority: $\quad$ Energy Systems Risk Weight 458

Tiger Team Action Plan Priority 2

Response: The ORNL Fire Protection Inspectors conduct monthly building inspections. Inspection criteria includes properly marked, adequately lighted, and clear, unobstructed egress to exits.

Fire Protection Engineering surveys are conducted on all major facilities annually.

A rigorous Plan Review Procedure to address all Fire Protection issues, including means of egress for new and modified facilities, is in place to ensure conformance to the Life Safety Code NFPA 101.

In FY 1991, major ORNL facilities will be inspected as part of the Fire Protection Conformance Plan to comply with Subpait E 29 CFR 1910, General Industry Standards. This inspection will be performed by an outside consulting group to address specific criteria such as emergency lighting, exit signs, and exit travel.

In May 1990, ORNL requested from DOE funding for abatement of Means of Egress noncompliances. Field Work Proposal (ORNL Safety and Health-OSHA Regulatory Compliance) was submitted.

\section{Root Causes:}

Inadequate policy, inadequate policy implementation, insufficient resources, inadequate management approach, inadequate management commitment, and ambiguous requirements and expectations 
Planned Actions and Schedules:

Item/Description

Completion Date

1. See Finding FP.2-3.

2. Resubmit request for OSHA Means of Egress funds with a FWP to DOE.

Complete

3. Prepare an action to plan to correct specific items of

$6 / 91$ noncompliance as listed in Appendix F, Subpart E, Means of Egress, of the Tiger Team Assessment of ORNL.

4. See Finding FP.2-3, Item 1.

5. Contingent upon funding, complete abatement of Priority I noncompliances, as determined by inspections of buildings.

6. Contingent on funding of FWP, complete abatement of remaining noncompliances.

Costs:

Type of funds: ESH Programmatic

Source of funds: ER-AT OSHA

Estimated costs per fiscal year $(\$ \mathrm{~K})$

\begin{tabular}{|c|c|c|c|c|c|c|c|}
\hline Action item & 1991 & 1992 & 1993 & 1994 & 1995 & Beyond & Total \\
\hline 6 & & 625 & 2830 & 3030 & & & 6485 \\
\hline \multicolumn{8}{|l|}{ Status: } \\
\hline \multicolumn{8}{|l|}{ Funded } \\
\hline Requested & & 625 & 2830 & 3030 & & & \\
\hline New & & & & & & & $\$ 6485$ \\
\hline
\end{tabular}


Type of funds: Overhead

Source of funds: Division Administration

\begin{tabular}{|c|c|c|c|c|c|c|c|}
\hline \multirow[b]{2}{*}{ Action item } & \multicolumn{6}{|c|}{ Estimated costs per fiscal year (\$K) } & \multirow[b]{2}{*}{ Total } \\
\hline & 1991 & 1992 & 1993 & 1994 & 1995 & Beyond & \\
\hline 3 & 10 & & & & & & 10 \\
\hline \multicolumn{8}{|l|}{ Status: } \\
\hline Funded & 10 & & & & & & \\
\hline \multicolumn{8}{|l|}{ Requested } \\
\hline New & & & & & & & $\$ 10$ \\
\hline
\end{tabular}

Type of funds: Overhead

Source of funds: Overhead

Estimated costs per fiscal year (\$K)

\begin{tabular}{lrrrrrrr}
\cline { 2 - 5 } Action item & 1991 & 1992 & 1993 & 1994 & 1995 & Beyond & Total \\
\hline \multicolumn{1}{c}{5} & 300 & & & & 300 \\
$\begin{array}{l}\text { Status: } \\
\text { Funded } \\
\text { Requested } \\
\text { New }\end{array}$ & & & & & \\
& 300 & & & \\
\hline
\end{tabular}

References: None 
Finding No.: WS.4-3 Machinery Inspection and Preventative Maintenance

\section{Finding}

Description: The inspection and preventative maintenance program for powered platforms, hoisting and rigging devices, cranes and other powered tools, and machinery was not in compliance with the DOE Hoisting and Rigging Manual and 29 CFR 1910 Subparts $\mathbf{F}$ and $\mathbf{N}$.

Code: $\quad$ Category III

Compliance

Protocol:

Priority: $\quad$ Energy Systems Risk Weight 483

Tiger Team Action Plan Priority 2

Response: Quality Engineering and Inspection hoisting and rigging procedures SSI 220 through SSI 229 will be reviewed for compliance with 29 CFR 1910 Subparts F\&N and the DOE Hoisting and Rigging Manual and revised as necessary. The DOE Hoisting and Rigging Manual and 29 CFR 1910 Subparts F\&N will be listed as references in the procedures to ensure their review and/or revision when the referenced documents are revised in the future. The Quality Engineering and Inspection safety inspectors will be retrained to the revised procedures. Formal sessions on OSHA requirements for hoisting and rigging will be presented to applicable divisions by the Quality Engineering and Inspection group.

\section{Root Causes:}

Inadequate policy implementation and ambiguous requirements or expectations. Flowdown of requirements to ORNL procedures is incomplete or unclear, resulting in incomplete implementation of orders and policies.

\section{Planned Actions and Schedules:}

\section{$\underline{\text { Item/Description }}$}

1. Issue revised QE\&I procedures SSI 220-229 to comply with 29 CFR 1910, Subparts F\&N, and the DOE Hoisting and Rigging Manual and revise as necessary.

2. Retrain safety inspectors to the revised SSI 220-229 procedures.

3. Present formal sessions on OSHA requirements for hoisting and rigging to applicable divisions.

\section{Completion Date}

$12 / 91$ 
Costs:

Type of funds: Overhead

Source of funds: Overhead

\begin{tabular}{|c|c|c|c|c|c|c|c|}
\hline \multirow[b]{2}{*}{ Action item } & \multicolumn{6}{|c|}{ Estimated costs per fiscal year (\$K) } & \multirow[b]{2}{*}{ Total } \\
\hline & 1991 & 1992 & 1993 & 1994 & 1995 & Beyond & \\
\hline 1 & 2 & & & & & & 2 \\
\hline 2 & 2 & & & & & & 2 \\
\hline 3 & 2 & 2 & & & & & 4 \\
\hline \multicolumn{8}{|l|}{ Status: } \\
\hline Funded & 6 & & & & & & \\
\hline Requested & & 2 & & & & & \\
\hline New & & & & & & & $\$ 8$ \\
\hline
\end{tabular}

Estimated annual ongoing cost: $\$ 4 \mathrm{~K}$.

References: 29 CFR 1910, Subparts F\&N, and the DOE Hoisting and Rigging Manual 
Finding No.: $\quad$ WS.4-4 ORNL Electrical Compliance

Finding

Description: Serious electrical noncompliances noted in specific buildings (Bldgs. 2010, 3587, and 3500) and a representative sample of buildings indicate that ORNL does not comply with 29 CFR 1910, Subpart S- Electrical standard.

Code: $\quad$ Category II

Compliance

Protocol:

The electrical system and maintenance program should ensure that workplaces are free of uncontrolled physical hazards and are in compliance with DOE-prescribed
occupational safety standards.

Priority: $\quad$ Energy Systems Risk Weight 935

Tiger Team Action Plan Priority 1

Response: ORNL has over 250 buildings that have been constructed and modified over a period of 47 years. Construction and maintenance practices, as well as electrical codes, standards, and regulations, have changed many times. The Laboratory has not kept pace with the changing regulatory requirements, and this is manifested in the generic electrical deficiencies that have been cited. This problem has been recognized, and several initiatives have been implemented to mitigate the problem. Initiatives that are currently in place include the following.

- An inspection program exists for corded equipment and receptacles. - An OSHA conformance plan has been developed for compliance with
Subpart S.

- OSHA inspections of 46 high-priority buildings for violations of Subpart $S$ have been initiated, and violations have been documented.

- Action plans are in place to correct identified violations.

- A review of engineering standards, technical specifications, and selected procedures is in progress by Science Applications International Corporation (SAIC) to detect any nonconformances to OSHA compliance. SAIC is also preparing training modules for Engineering on OSHA compliance.

- The Facility Manager concept is being implemented to establish accountability.

These initiatives will supplement existing system safeguards that are in place to ensure compliance with regulatory requirements. In addition, increased training will be provided to personnel who have responsibility for electrical maintenance.

The three specific buildings noted in the Tiger Team inspection contained the following deficiencies. 
- Building 2010-Lack of Ground Fault Circuit Interruption (GFCI) in wet or damp areas, unguarded live electrical parts, and unused openings not being effectively closed.

- Building 3587-Unguarded live electrical panels.

- Building 3500-Improper use of a circuit box.

The problems are typical of those found during the ORNL-initiated OSHA-type electrical audit of 46 high-priority buildings that began in May 1990. Limited resour_es have allowed inspections of only high-priority buildings. Building 3500 was included in the 46 high-priority buildings inspected and the deficiencies noted by the Tiger Team were also noted on the previous inspection. Buildings 2010 and 3587 were categorized as medium priority in the ORNL OSHA Electrical Conformance Plan. Limited manpower and funding have allowed only the Priority I issues documented to be abated.

Specific electrical deficiencies (Appendix F) noted in the assessment will be corrected. The remaining ORNL buildings will be inspected as outlined in the ORNL conformance plan for Subpart $S$ and the ORNL OSHA Implementation Plan. Deficiencies are being corrected on a priority basis. All of the Priority I items have been corrected, and corrective action for Priority II items has begun. Budgets and schedules for Priority II and other noncompliances are being developed and resources will be requested.

To ensure that additional serious electrical compliances did not exist at ORNL, a Tiger Team workshop for serious findings meeting was held with all division directors, facility managers, and facility engineers. Pictures of noncompliances that were cited by the Tiger Team were shown; each was returned to those areas to look for similar problems. The quick fix team corrected those items, thus ensuring that similar serious situations did not exist. The remainder of noncompliances arc of a less serious nature, and experienced electricians are correcting these.

\section{Root Causes:}

Inadequate policy implementation, poorly defined roles and responsibilities, lack of management commitment, insufficient resources, and inadequate training

\section{Planned Actions and Schedules:}

\section{Item/Description}

1. Request funding from DOE for abatement of OSHA electrical noncompliances at ORNL.

2. Correct known electrical deficiencies that present an immediate hazard as defined by the Tiger Team.
Completion Date

Complete

Complete 
3. Instruct electrical supervisors concerning importance of job completion. See Finding MA.2-2.

Complete

4. Correct Priority I electrical non-conformances as described in the ORNL electrical inspection findings of

Complete

Near-Term high-priority buildings.

5. Evaluate and prioritize electrical nonconformances listed $6 / 91$ in Appendix $\mathrm{F}$ of the Tiger Team Assessment.

6. Review criteria and clearly define and document roles and responsibilities for post-job inspections to ensure work completion, document and enforce compliance. See Finding MA.2-2.

7. Develop and initiate training on OSHA Subpart S and the National Electric Code (NEC) for electrical maintenance personnel.

8. Issue revised inspection procedure for corded equipment and receptacles to include verification of the proper use of portable cords as described in the National Electric Code (NEC).

9. Resubmit request for OSHA electrical funds with a FWP to DOE.

10. Correct serious OSHA electrical deficiencies in each building at ORNL with a dedicated team of electricians, using specific criteria developed from Lessons Learned of the Tiger Team

11. Contingent on receipt of funding, complete electricalprojects identified above. 
Costs:

Type of funds: Overhead

Source of funds: Overhead

Estimated costs per fiscal year (\$K)

\begin{tabular}{|c|c|c|c|c|c|c|c|}
\hline Action item & 1991 & 1992 & 1993 & 1994 & 1995 & Beyond & Total \\
\hline 1 & - & & & & & & \\
\hline 2 & 80 & & & & & & 80 \\
\hline 3 & - & & & & & & \\
\hline 4 & 60 & & & & & & 60 \\
\hline 5 & 10 & & & & & & 10 \\
\hline 6 & - & & & & & & \\
\hline 7 & 30 & & & & & & 30 \\
\hline 8 & - & & & & & & \\
\hline 10 & 262 & & & & & & 262 \\
\hline
\end{tabular}

Status:

Funded $\quad 442$

Requested

New

Type of funds: ESH Programmatic

Source of funds: ER-AT OSHA

Estimated costs per fiscal year (\$K)

\begin{tabular}{cccccccc}
\cline { 2 - 5 } Action item & 1991 & 1992 & 1993 & 1994 & 1995 & Beyond & Total \\
\hline 11 & & 1350 & 1945 & 2035 & & & 5330
\end{tabular}

Status:

Funded

$\begin{array}{llll}\text { Requested } & 1350 & 1945 & 2035\end{array}$

New

References: $\quad 29$ CFR 1910.303, 304, 305, 307, and 308

National Electric Code

X-03--510; ORNL OSHA Implementation Plan 
Finding No.: $\quad$ WS.4-5 Equipment and Operations Areas

\section{Finding}

Description:

ORNL does not comply in equipment/operations areas with 29 CFR 1910

Subpart Q (Welding, Cutting, and Brazing) and Subpart H (Hazardcus Materials).

Code: $\quad$ Category II

Compliance

Protocol:

29 CFR 1910.253 Section $(e)(3)(C)(2)$ states "Backflow protection shall be provided by an approved device that will prevent oxygen from flowing into the fuel-gas system or fuel from flowing into the oxygen system."

29 CFR 1910.253 Section (d)(2)(iii)(B) states "Unalloyed copper shall not be used for acetylene or acetylenic compounds except in listed equipment."

29 CFR 1910.253 Section (b)(4)(iii) states "Oxygen cylinders in storage shall be separated from fuel-gas cylinders or combustible materials (especially oil or grease), a minimum distance of 20 feet $(6.1 \mathrm{~m})$ or by a noncombustible barricr at least 5 feet $(1.5 \mathrm{~m})$ high having a fire-resistance rating of at least one-half hour."

29 CFR 1910.253 Section (b)(5)(ii)(E) states "Cylinders not having fixed hand wheels shall have keys, handles, or nonadjustable wrenches on valve stems while these cylinders are in service. In multiple cylinder installations only one key or handle is required for each manifold."

29 CFR 1910.253 Section (e)(3)(c)(5)(i) states "Hose for oxy-fuel gas service shall comply with the Specification for Rubber Welding Hose, Compressed Gas Association and Rubber Manufacturers Association."

29 CFR 1910.253 Section (e)(3)(C)(5)(III) states "Hose connections shall comply with the Standard Hose Connection Specification, Compressed Gas Association."

\section{Priority: $\quad$ Energy Systems Risk Weight 935}

Tiger Team Action Plan Priority 1

Response: A meeting was held with facility managers to discuss the noncompliances identified during the review. The managers were informed of the basic requirements for storage, tubing, connections, and valves for compressed gas systems used for welding, cutting, and brazing and were asked to inspect the systems in their areas. They were instructed to correct any noncompliances identified or contact the Safety Department and Plant and Equipment to correct the noncompliances.

\section{Root Causes:}

Inadeq sate policy, inadequate policy implementation, and inadequate communications 
Planned Actions and Schedules:

Item/Description

Completion Date

Immediate

1. Conduct a meeting with all facility managers to

Complete

- inform them of the basic requirements for storage, lubing, connections, and valves for compressed gas systems for welding, brazing, or cutting;

- show pictures of systems which are not in compliance; and

- instruct them to survey their areas to (1) identify where compressed gas systems are used, (2) inspect systems to verify compliance with requircments, and (3) fix any noncompliances which are identified.

2. Issue a memo informing the facility managers (If requirements for compressed gas systems and instruct them not to use compressed gas systems which do not comply with these requirements.

3. Obtain and evaluate torch, hose, and connectors with flashback which are in compliance with the OSHA standard.

4. Establish interim inventory of hoses and flashback devices making them available to the facility managers until the approved items can be made available on the AVID system. Issue guidelines to the facility managers stating that the compressed gas systems must be reviewed and approved by the Safety Department before they can be used.

Complete

Complete

Complete

$\underline{\text { Near Term }}$

5. Modify the Safety for Supervisors Course to address the issues identified in action items 1 and 2 above for use of compressed gas for welding, brazing or cutting.

6. Issue revised Safety Procedure IS-8.8 to reflect current requirements of 29 CFR 1910.253.

7. Make approved torches, hoses, and connectors available on the AVID 2-day delivery system. 
8. Develop and implement a program for routinely inspecting compressed gas systems to verify compliance with revised Safety Procedure IS-8.8. The inspection checklist will include tubing, connector, and storage requirements as well as other critical items identified in IS-8.8.

Costs:

Type of funds: Overhead

Source of funds: Overhead

\begin{tabular}{|c|c|c|c|c|c|c|c|}
\hline \multirow[b]{2}{*}{ Action item } & \multicolumn{6}{|c|}{ Estimated costs per fiscal year $(\$ K)$} & \multirow[b]{2}{*}{ Total } \\
\hline & 1991 & 1992 & 1993 & 1994 & 1995 & Beyond & \\
\hline 1 & 6 & & & & & & 6 \\
\hline 2 & 1 & & & & & & 1 \\
\hline 3 & 4 & & & & & & 4 \\
\hline 4 & 5 & & & & & & 5 \\
\hline 5 & - & & & & & & \\
\hline 6 & 14 & & & & & & 14 \\
\hline 7 & 7 & & & & & & 7 \\
\hline 8 & 42 & * & & & & & 42 \\
\hline
\end{tabular}

Status

Funised

Requested

New

*Estimated annual ongoing cost: $\$ 50 \mathrm{~K}$ division administration.

References: $\quad 20$ CFR 1910.253 
Finding No.: WS.4-6 ORNL Fire Protection

Finding

Description: ORNL docs not comply with 29 CFR 1910 Subpart L: Fire Protection.

Code: $\quad$ Category III

\section{Compliance}

Protocol:

Priority: $\quad$ Energy Systems Rish Weight 409

Tiger Team Action Plan Priority 2

Response: This item was identified in the ORNL Prctective Services 1990 Self-Assessment. A monthly building program is now in place that includes monthly visual checks of fire extinguishers. Tags are not signed for these monthly checks; however, the Building Fire Inspection form includes this check-off provision for the inspector. Fire door operability is also checked monthly during the building inspection and requires check-off by the inspector. Needed fire door repairs are reported immediately to P\&E. Repairs are field verified by fire inspectors.

Portable fire extinguisher annual inspections are currently behind schedule. The schedule slipped due to time demands necessary to meet hydrostatic test data requirements. Available resources were utilized to address the more serious issue of hydrostatic testing.

\section{Root Causes:}

Insufficient resources, inadequate policy implementation, inadequate management commitment, and inadequate management approach

\section{Planned Actions and Schedules:}

\section{$\underline{\text { Item/Description }}$}

1. Fire extinguishers shall be checked and work performed

2. A letter from upper ORNL management, which as required by NFPA/OSHA annually. The backlog of out-of-date fire extinguisher inspections shall receive priority inspections.

Completion Date

$8 / 91$ reiterates ORNL policy regarding fire protection requirements, shall be transmitted to all ORNL employees. 
3. A system shall be developed to report fire protection deficiencies to ORNL managers (see Finding RFP.1-1).

4. The action plan for FP.1-2 shall be referenced for additional resources.

Casts:

Type of funds: Overhead

Source of funds: Overhear

\begin{tabular}{|c|c|c|c|c|c|c|c|}
\hline \multirow[b]{2}{*}{ Action item } & \multicolumn{6}{|c|}{ Estimated costs per fiscal year $(\$ \mathrm{~K})$} & \multirow[b]{2}{*}{ Total } \\
\hline & 1991 & 1992 & 1993 & 1994 & 1995 & Beyond & \\
\hline 1 & 25 & & & & & & 25 \\
\hline \multicolumn{8}{|l|}{ Status: } \\
\hline Funded & 25 & & & & & & \\
\hline \multicolumn{8}{|l|}{ Requested } \\
\hline New & & & & & & & $\$ 25$ \\
\hline
\end{tabular}

References: $\quad 29$ CFR 1910 Subpart L 


\subsubsection{Industrial Hygiene}

Finding No.: IH.2-1 Documentation of Procedures by Industrial Hygiene

Finding

Description: ORNL Industrial Hygiene Department has not documented all procedures as required by DOE 5480.10 .

Code: $\quad$ Category III

Compliance

Protocol:

DOE Order 5480.10

Priority: $\quad$ Energy Systems Risk Weight 68

Tiger Team Action Plan Priority 2

Response: This finding was based on several related findings, which said:

- Although over 250 standard operating procedures have been identified by the Industrial Hygiene Department of ORNL as being required for a safe and healthy work environment, fewer than 20 are in draft form or have been issued.

- Specific quality criteria are not established for data collection and use of data associated with industrial hygiene programs. Chain-of-custody records are not used as part of analytical laboratory practices.

- The IH analytical laboratory turn-around time on some IH samples exceeded two months.

- A complete inventory of beryllium-regulated areas is not maintained, so that there is no record of where all beryllium is used, the equipment used, the location, and the time period the operation was conducted as required in DOE Order 5480.10.

- Substitution of nontoxic chemicals for toxic chemicals has not been integrated into the ORNL IH program and in procurement policies in areas such as cyanide plating, asbestos gasket use, and dioctyl phthalate.

- No implementation schedule has been set for correcting deficiencies in documentation and procedures.

The Industrial Hygiene Section has recognized the need for a comprehensive set of programmatic procedures [Indusirial Hygiene Procedures (IHPs)] as well as standard operating procedures (SOPs). These needs were discussed in the Section's self-assessment document as well as in the Program Management Plan for IH. In May 1990, a professional industrial hygienist was hired to coordinate the production of more than $20 \mathrm{IHPs}$ and at least 250 SOPs. The issuance of the 
Industrial Hygiene Manual, containing the IHPs, was given top priority. After that manual was issued in October 1990, the focus shifted toward production of the SOPs.

Because of the large number of SOPs to be issued over the next three years, the Industrial Hygiene Section has set certain priorities. High-priority procedures are those that have been identified as possibly having a major impact on health and safety and those that are involved with other corrective actions in response to audit/surveillance findings. Medium-priority procedures are those which have significant health risk potential or are associated with programs which are considered high-profile. All other procedures have been assigned low-priority
status.

The corrective actions planned to address these findings are listed below.

\section{Root Causes:}

Insufficient resources, ambiguous requirements or expectations, inadequate training, and poorly defined roles and responsibilities

\section{Planned Actions and Schedules:}

\section{$\underline{\text { Item/Description }}$}

1. Request funds to hire an additional staff member (a procedures writer) in FY 1992.

2. Contingent on approval of funding, hire an additional staff member to write procedures.

3. Evaluate all IH activities to identify and list any additional procedures that are needed, and develop a prioritized schedule for developing SOPs. (Costs are included in Item 4.)

4. Issue all high-priority Industrial Hygiene SOPs

5. Issue all medium-priority Industrial Hygiene SOPs

6. Issue all low-priority Industrial Hygiene SOPs

7. Develop and issue quality assurance plans for field monitoring and laboratory analyses.

8. Develop and issue laboratory analysis SOP that specifies an acceptable turn-around time for sample analysis.

(Costs are included in Item 4 above.)

\section{Completion Date}

$7 / 91$

$8 / 91$

$8 / 91$

$3 / 92$

$10 / 92$

$3 / 93$

Complete

$8 / 91$ 
9. Complete and document a beryllium inventory as part of ther Chemical Carcinogen Control Program.

10. Isstue additional guidelines to Procurement and the operating divisions, specifying that, whenever possible, nontoxic chemicals be substituted for toxic chemicals.

\section{Costs:}

Type of funds: Overhead

Source of funds: Overhead

Estimated costs per fiscal year (\$K)

\begin{tabular}{|c|c|c|c|c|c|c|c|}
\hline Action item & 1991 & 1992 & 1993 & 1994 & 1995 & Beyond & Total \\
\hline 1 & - & & & & & & \\
\hline 2 & 43 & & & & & & 43 \\
\hline 3 & - & & & & & & \\
\hline 4 & 307 & & & * & & & 307 \\
\hline 7 & 15 & & & & & & 15 \\
\hline 8 & - & & & & & & \\
\hline 9 & 11 & & & & & & 11 \\
\hline 10 & 3 & & & & & & 3 \\
\hline
\end{tabular}

Status:

Funded

Requested

New

*Estimated annual ongoing cost: \$91K starting in FY $i 994$. 
Type of funds: ESH Programmatic

Source of funds: ER-AT IH

\begin{tabular}{crrrrrrr} 
& \multicolumn{5}{c}{ Estimated costs per fiscal year (\$K) } \\
\cline { 2 - 6 } Action item & 1991 & 1992 & 1993 & 1994 & 1995 & Beyond & Total \\
\hline 5 & & 852 & & & & & 852 \\
6 & & & 417 & & & & 417
\end{tabular}

Status:

Funded

Requested

New

852

417

$\$ 1269$

References: $\quad$ DOE Order 5480.10 
Finding No.: IH.2-2 Implementation of Industrial Hygiene Reviews

Finding

Description: The policy for implementing Industrial Hygiene reviews is not in place at ORNL.

Code: $\quad$ Category III

Compliance

Protocol:

None

Priority: $\quad$ Energy Systems Risk Weight 90

Tiger Team Action Plan Priority 4

Response: This finding is based on a related finding, which said:

Reviews of proposed activities and modifications to existing operations, including reviews of new or modified processes and chemicals, are not always completed. Interface with Procurement on purchases of new chemicals, asbestos, and other health concerns does not require the endorsement of Industrial Hygiene.

The Industrial Hygiene Section is nearing completion of the first phase of the Comprehensive Facility Survey (CFS), a walk-through effort to identify and evaluate IH concerns throughout ORNL. The results of this initial phase of the CFS will serve as a baseline for future continuing evaluations and assissments.

The IH Section's procedures require the operating divisions to contact Industrial Hygiene whenever a new or modified activity involves the potential for generating hazards. However, Industrial Hygiene recognizes the need for in-depth field surveillance to ensure that the operating divisions are meeting this responsibility. To ensure adequate coverage, one corrective action planned for the first quarter of CY 1991 is the assignment of IH professional staff to particular divisions where they will be responsible for surveillance of those divisions.

The corrective actions planned to correct this finding are listed below.

\section{Root Causes:}

Inadequate policy implementation and poorly defined roles and responsibilities

Planned Actions and Schedules:

\section{$\underline{\text { Item/Description }}$}

1. Revise the Industrial Hygiene Procedure (IHP) as necessary to reflect the items included in Finding PP.3-1, Item 1.
Completion Date

$12 / 91$ 
2. Assign individual Industrial Hygiene Section staff members to provide IH oversight for particular divisions.

3. Issue procedures for conducting IH! reviews of projects/programs. (Costs are covered in Finding IH.2-1, Item 4.)

4. Conduct surveillance and distribute surveillance reports to IH management as well as the management of the operating division(s) involved. Quarterly summary reports will be sent to the director of ES\&H Compliance.

5. Issue a procedure concerning Industrial Hygiene review of purchases (asbestos, respirators, chemical carcinogens, etc.). (Costs are covered in Finding IH.2-1, Item 4.)

Costs:

Type of funds: Overhead

Source of funds: Overhead

Estimated costs for fiscal year (\$K)

\begin{tabular}{|c|c|c|c|c|c|c|c|}
\hline \multirow[b]{2}{*}{ Action item } & & & \multirow[b]{2}{*}{ Total } \\
\hline & 1991 & 1992 & 1993 & 1994 & 1995 & Beyond & \\
\hline 1 & & 2 & & & & & 2 \\
\hline 2 & 11 & & & & & & 11 \\
\hline 4 & 5 & * & & & & & 5 \\
\hline
\end{tabular}

Status:

Funded

Requested

52

New

*Estimated annual ongoing cost: $\$ 18 \mathrm{~K}$.

References: $\quad$ DOE Order 5480.10 
Finding No.: IH.3-1 Personnel Protective Equipment

Finding

Description: ORNL is not in compliance with DOE 5480.10 and 29 CFR 1910.95, .133, .134, .252 , regarding documentation and protective measures in the work place.

Code: $\quad$ Category III

Compliance

Protocol:

Priority:

Response:
DOE Order 5480.10 and 29 CFR $1910.95, .133, .134$, and .252

Energy Systems Risk Weight 458

Tiger Team Action Plan Priority 2

This finding was based on several related findings, which said:

- Periodic inspections and maintenance programs are not performed or established for all mechanical/engineered containment control systems.

- Supervision is not enforcing proper use of personnel protective equipment.

- Eyewash stations were not available in several buildings. Flushing of stations or monitoring for bacteria are not documented or done on a regular basis.

- Identification, evaluation, and control of environmental factors and stresses found in the workplace, including chemical, physical, biological, and ergonomic stresses, are not documented as a means for identifying existing and potential occupational health concerns.

- Documentation, identification, surveillance, and training for all magnetic fields and microwaves are not being completed.

In September and October of 1990, the Industrial Hygiene Section developed a Program Management Plan covering all IH activities at ORNL. As part of the development, IH staff assessed (1) whether their programs were in compliance with existing regulations and DOE Orders and (2) what actions would be needed to achieve compliance. The document includes plans for staffing and near-term action items designed to upgrade the IH programs.

During the first quarter of CY 1991, individual staff members in the Industrial Hygiene Section will be assigned responsibilities for particular operating divisions. These assignments will lead to an increased IH presence "in the field" and thus ensure that many of these findings will be corrected.

As for magnetic fields and microwaves, a staff member was recently added to the IH Section to focus on these areas. Guidelines, procedures, and training programs will be forthcoming. The individual corrective actions planned for these findings are listed below. 


\section{Root Causes:}

Insufficient resources, ambiguous requirements or expectations, poorly defined roles and responsibilities, and inadequate policy implementation

Planned Actions and Schedules:

\section{$\underline{\text { Item/Description }}$}

1. See Finding IH-2.2, Item 2.

2. Evaluate all containment/control systems and add systems, as necessary, to establish an inspection schedule for containment control systems.

3. Conduct field surveillance to ver:iy the adequacy of new SOP for ensuring that supervisors are enforcing proper use of personnel protective equipment, and distribute surveillance reports to supervisors in the field.

4. Conduct a survey to determine the need for additional eyewash stations, document the results, and submit recommendations to the operating divisions.

5. Revise the SPP to document the requirements for flushing or monitoring of eyewash stations. (Costs are included in IH.2-1, Item 4.)

6. Complete and document the results of the Comprehensive Facility Survey.

7. Develop and issue standard operating procedures dealing with the industrial hygiene aspects of magnetic fields and microwaves. (Costs are included in IH.2-1, Item 4.)

8. Develop a training program dealing with the industrial hygiene aspects of magnetic fields and microwaves.

9. Conduct and document a survey of ORNL facilities to establish an inventory of EMF and microwave sources (excluding microwave ovens).
Completion Date

$6 / 91$

Complete

$6 / 91$

$7 / 92$

$6 / 91$

$7 / 92$

$9 / 91$

$9 / 91$ 
Costs:

Type of funds: Overhead

Source of funds: Overhead

Estimated costs per fiscal year (\$K)

\begin{tabular}{|c|c|c|c|c|c|c|c|}
\hline Action item & 1991 & 1992 & 1993 & 1994 & 1995 & Beyond & Total \\
\hline 1 & - & & & & & & \\
\hline 2 & 2 & & & & & & 2 \\
\hline 3 & - & & & & & & \\
\hline 4 & 7 & & & & & & 7 \\
\hline 5 & & - & & & & & \\
\hline 6 & - & & & & & & \\
\hline 7 & & - & & & & & \\
\hline 8 & 7 & & & & & & 7 \\
\hline 9 & - & & & & & & \\
\hline
\end{tabular}

Status:

Funded

16

Requested

New

References: $\quad$ DOE Order 5480.10

29 CFR 1910.95

29 CFR 1910.133

29 CFR 1910.134

29 CFR 1910.252 
Finding No.: IH.4-1 Surveillance of Industrial Hygiene Monitoring

\section{Finding}

Description: Surveillance of industrial hygiene monitoring activities is not performed as required by DOE 5480.10 .

Code: $\quad$ Category III

Compliance

Protocol:

Pricrity: $\quad$ Energy Systems Risk Weight 60

Tiger Team Action Plan Priority 2

Response: This fincing was based on several related findings, which said:

- There is no documented program for periodic monitoring of chemical, physical, and biological stresses to ensure maintenance of satisfactory conditions.

- Quality assurance and quality control programs are not in place for the IH programs at ORNL.

- Line management is not ensuring that all operational areas are being covered by the Comprehensive Facility Survey.

- Management oversight is not present for the formal system that exists for employees to report potential health concerns

- IH professionals are not out in the field overseeing the monitoring activities of technicians or interfacing with line management.

Each ORNL employee has access to Safety Suggestion Forms to report potential health concerns. Management is required to respond to these suggestions.

During the first quarter of CY 1991, individual staff members in the Industrial Hygiene Section will be assigned responsibilities for particular operating divisions. These assignments will lead to an increased $\mathrm{IH}$ presence "in the field" and thus ensure that many of these findings will be corrected.

The finding concerning management oversight for the formal system for reporting health concerns is being addressed as part of the corrective action for PP.5-2. A revision of procedure IS-1.5, "Handling Employee Complaints Pertaining to Health and Safety," will ensure that employee concerns receive appropriate management attention.

The specific actions planned to address these findings are listed below. 


\section{Root Causes:}

Insufficient resources, inadequate oversight, and inadequate policy implementation

Planned Actions and Schedules:

\section{$\underline{\text { Item/Description }}$}

1. Issue procedures related to routine and special monitoring in the workplace. (Costs are covered in Finding IH.2-1, Item 4.)

2. Develop quality assurance plans (which specify quality control measures) for all $\mathrm{IH}$ programs and issue them.

3. Issue summary reports concerning Comprehensive Facility Survey results to each division and request that the management of each division identify any operational areas that were not included in Phase I of the CFS.

4. See Finding IH.2-2, Item 2.

\section{Completion Date}

$9 / 91$

$9 / 92$

$6 / 91$

Costs:

Type of funds: ESH Programmatic

Source of funds: ER-AT IH

Estimated costs per fiscal year $(\$ \mathrm{~K})$

\begin{tabular}{|c|c|c|c|c|c|c|c|}
\hline Action item & 1991 & 1992 & 1993 & 1994 & 1995 & Beyond & Total \\
\hline 2 & & 435 & & & & & 435 \\
\hline \multicolumn{8}{|l|}{ Status: } \\
\hline \multicolumn{8}{|l|}{ Funded } \\
\hline \multicolumn{8}{|l|}{ Requested } \\
\hline New & & 435 & & & & & $\$ 435$ \\
\hline
\end{tabular}

References: $\quad$ DOE Order 5480.10 
Finding No.: IH.5-1 Hearing Conservation Program

Finding

Description:

The ORNL Hearing Conservation Program does not comply with DOE 5480.10 and 29 CFR 1910.95.

Code: $\quad$ Category III

Compliance

Protocol:

DOE Order 5480.10 and 29 CFR 1910.95

Priority: $\quad$ Energy Systems Risk Weight 455

Tiger Team Action Plan Priority 2

Response: This finding was based on several deficiencies identified in the ORNL Hearing Conservation Program:

- Only one person was assigned to fully comply with the needs of the program.

- Although the in-house training and documentation were complete, tests to ensure workers' understanding were not given.

- Transmittal of the Standard Threshoid Shifts from the Medical Department to Industrial Hygiene took longer shan 2 months, thus not allowing prompt evaluation of the work areas.

- Not all areas were identified as requiring hearing protection, and other areas were not posted as to the range of the hazard.

Other findings concerning the Hearing Conservation Program included:

- Employees were observed without hearing protection in areas where protection was required.

- In areas where the standard was exceeded, hearing protection was available but no sign was posted requiring hearing protection.

Industrial Hygiene procedure IHP-04, "IHS Hearing Consurvation Program," was issued on September 12, 1990. IH staff will be conducting surveillance in the field to ensure that employees are fulfilling the requirements of the procedure. The new procedure also specifies that supervisors in the field are responsible for ensuring that their employees wear required hearing protection.

\section{Root Causes:}

Insufficient resources, inadequate oversight, inadequate policy implementation, and poorly defined roles and responsibilities 
Planned Actions and Schedules:

Item/Description

Completion Date

1. See Finding IH.2-2, Item 2.

2. Include written testing as part of the annual training portion of the Hearing Conservation Program.

3. Develop and document a system for ensuring more timely transmittal of STS notifications to permit prompt evaluation of the workplace.

4. Develop a database to serve as an inventory of highnoise sources; these areas are now being identified as part of the Comprehensive Facility Survey.

5. Review all high-noise-source areas and equipment, document the results, and ensure that high-noise sources are posted as required.

6. Conduct field surveillance and document results to ensure compliance with procedural requirements (e.g., the use of hearing protection devices in posted highnoise areas).

7. Review and update the list of Hearing Conservation Program participants and provide any new names to the Health Division. 
Costs:

Type of funds: Overhead

Source of funds: Overhead

Estimated costs per fiscal year $(\$ \mathrm{~K})$

\begin{tabular}{|c|c|c|c|c|c|c|c|}
\hline Action item & 1991 & 1992 & 1993 & 1994 & 1995 & Beyond & Total \\
\hline 2 & 2 & & & & & & 2 \\
\hline 3 & 7 & & & & & & 7 \\
\hline 4 & 7 & & & & & & 7 \\
\hline 5 & & 12 & * & & & & 12 \\
\hline 6 & 1 & * & & & & & 1 \\
\hline 7 & - & & & & & & \\
\hline
\end{tabular}

Status:

Funded

Requested

New

17

12

*Estimated annual ongoing cost: \$13K starting in FY 1993 for Item 5 and $\$ 1 \mathrm{~K}$ starting in FY 1992 for Item 6.

References: $\quad$ DOE Order 5480.10

29 CFR 1910.95 
Finding No.: IH.5-2 Chemical Carcinogen Program

Finding

Description: The Chemical Carcinogen Program does not comply with DOE 5480.10 and 29 CFR 1910 Subpart Z.

Code: $\quad$ Category III

Compliance

Protocol:

Priority: $\quad$ Energy Systems Risk Weight 418

Tiger Team Action Plan Priority 2

Response: This finding was based on several related findings, which said:

- Not all areas have been evaluated, as required by DOE Order 5480.10 , to determine if the use of carcinogens creates a significant potential for occupational exposures.

- An employee was observed without proper personnel protective equipment when potential exposures to a suspected carcinogenic material could occur.

- Pathways of ingestion and skin absorption are not always being considered.

- Although ORNL has a medical surveillance program for carcinogen users, it does not appear to be in place at this time; ORNL does not cover all aspects of the program.

- Laboratory carcinogens are not labeled.

Industrial Hygiene procedure IHP-13, "ORNL Chemical Carcinogen Control Program," was issued on October 8, 1990. The program is still in the initial development phase, and most of these findings will be addressed as the program moves into the actual implementation phase.

Root Causes:

Inadequate oversight and inadequate policy implementation

Planned Actions and Schedules:

\section{$\underline{\text { Item/Description }}$}

1. Complete and issue Standard Operating Procedures for administration of the Chemical Carcinogen Program.

(Costs are covered in Finding IH.2-1, Item 4.)
Completion Date 
2. Provide a copy of the currently existing inventory to individual divisions for their review, validation, and comments.

3. Complete the chemical carcinogen inventory, using division responses and the final data from the Comprehensive Facility Survey.

4. Complete and document the detailed evaluations of any operations currently identified with high or moderate risk potential.

5. Evaluate and document any new operations identified through divisional review of the existing inventory and through the remainder of the baseline CFS.

6. Recommend and verify implementation of additional controls where necessary (as identified by the detailed evaluations).

7. Issue IH guidance covering general rules for handling carcinogens (including guidance on storage, labelling, and minimum controls required for work with carcinogens).

8. Develop and implement a training module dealing with chemical carcinogen work.

9. Provide names to the Health Division of all personnel currently identified as qualifying for medical surveillance. 


\section{Casts:}

Type of funds: Overhead

Source of funds: Overhead

\begin{tabular}{|c|c|c|c|c|c|c|c|}
\hline \multirow[b]{2}{*}{ Action item } & \multicolumn{6}{|c|}{ Estimated costs per fiscal year $(\$ \mathbf{K})$} & \multirow[b]{2}{*}{ Total } \\
\hline & 1991 & 1992 & 1993 & 1994 & 1995 & Beyond & \\
\hline 2 & 2 & & & & & & 2 \\
\hline 3 & 15 & & & & & & 15 \\
\hline 4 & 7 & & & & & & 7 \\
\hline 5 & 7 & & & & & & 7 \\
\hline 6 & 7 & & & & & & 7 \\
\hline 7 & 2 & & & & & & 2 \\
\hline 8 & 7 & & & & & & 7 \\
\hline 9 & - & & & & & & \\
\hline
\end{tabular}

Status:

Funded

40

Requested

New

7

References: $\quad$ DOE Order 5480.10

29 CFR 1910, Subpart Z 
Finding No: IH.5-3 Confined Space Entry

Finding

Description:

Fersonnel are allowed to enter confined working spaces without proper monitoring and training as required by DOE 5480.10 and 29 CFR 1910.146.

Code: $\quad$ Category II

Compliance

Protocol:

29 CFR 1910.146 Section (c)(8) states "Rescue. Ensure that the procedures and equipment necessary to rescue entrants from permit spaces are implemented and provided."

ANSI Z117.1 Section 12.2.1 states "A mechanical device shall be available to retrieve personnel from vertical-type PRCSs greater than five feet in depth."

ANSI Z117.1, Section 13.1 states "Identification. All confined spaces which could be inadvertently entered shall have a sign identifying it as a confined space. Signs shall be maintained in a legible condition. For PRCSs, the sign shall contain a warning that a permit is required before entry."

OSHA 29 CFR 1910.146 Section (g) states "The employer shall ensure that individuals authorizing or in charge of entry receive the appropriate training and perform duties, as follows...."

Priority: $\quad$ Energy Systems Risk Weight 913

Tiger Team Action Plan Priority 0

Response: An interim IH Procedure has been issued specifying requirements related to confined spaces. The Confined Space Inventory is complete and posting has been initiated.

An additional safety harness and tripod have been procured and provided for use by the Emergency Response Organization.

The Confined Space Survey has identified all confined spaces. Posting is now being completed.

A contractor provided general, entrant/attendant, supervisory, instrumentation, and emergency response training in October 1990 and in February 1991. The Office of Environmental and Health Protection Training Section is currently offering general and entrant/attendant training on an on-going basis.

\section{Root Causes:}

Inadequate policy, inadequate policy implementation, and poorly defined roles and responsibilities 
Planned Actions and Schedules:

Item/Description

1. Issue an interim $\mathrm{IH}$ procedure to specify requirements related to confined spaces. (Costs are covered in Finding IH.2-1, Item 4.)

2. Issue standard operating procedure for performing measurements in confined spaces. (Costs are covered in Finding IH.2-1, Item 4.)

3. Conduct a meeting with facility managers to inform them of evaluation, posting, monitoring, and training requirements for control of confined spaces. The managers will be instructed to survey their areas to ensure that confined spaces have been identified and arc posted. (Costs are covered in Finding WS.4-5, Item 1.)

4. Issue a memo to division directors and facility managers informing them of evaluation, posting, monitoring, and training requirements associated with confined spaces. The memo will state that work in confined spaces will not be permitted until an evaluation by the Industrial Hygiene Section has been completed.

5. Complete the Industrial Hygiene Confined Space Inventory.

6. Develop Confined Space Entry training modules for general, entrant/attendant, supervisory, instrumentation, and emergency response. Offer the general and entrant/attendant training on an on-going basis.

7. Identify and assign responsibility to line organizations that require retrieval equipment.

8. Add existing retrieval equipment to Quality Department inspection schedule and perform initial inspection.

9. Execute a memorandum of understanding between Industrial Safety and the Emergency Response Organization to make available retrieval equipment for high-hazard confined space entry jobs.
Completion Date

Complete

Complete

Complete

Complete

Complete

Complete

$5 / 91$

Complete

$5 / 91$ 
10. Implement the Confined Space Entry Training Program, including:

- train the appropriate emergency respc nse personnel;

- identify instructors to provide on-going training modules for supervisory, instrumentation, and emergency response modules;

- issue memo to all divisions specifying confined space entry training criteria; and

- develop standard operating procedure for confined space entry training.

11. Issue revised IHP-14 "ORNL Confined Space Entry

Program" to require

- retrieval equipment be provided by the line organization with guidance from the Safety Department,

- designation of the Industrial Hygiene Section to identify confined spaces and authorize posting of these areas,

- only trained personnel are permitted to work in confined spaces, and

- line organization responsibility for providing retrieval equipment and verifying that personnel are trained before working in confined spaces.

12. Issue guidance to facility managers concerning posting of signs for confined spaces identified during the Confined

Complete Space Inventory.

13. Issue standard operating procedures for the Confined Space Entry Program which require Industrial Hygiene to periodically verify that retrieval equipment is in place and personnel meet applicable training requirements before work is allowed in confined spaces.

14. Assign an Industrial Hygiene staff member to oversee Confined Space Entry program.

Complete 


\section{Casts:}

Type of funds: Overhead

Source of funds: Overhead

\begin{tabular}{|c|c|c|c|c|c|c|c|}
\hline \multirow[b]{2}{*}{ Action item } & \multicolumn{6}{|c|}{ Estimated costs per fiscal year $(\$ K)$} & \multirow[b]{2}{*}{ Total } \\
\hline & 1991 & 1992 & 1993 & 1994 & 1995 & Beyond & \\
\hline 4 & - & & & & & & \\
\hline 5 & 176 & & & & & & 176 \\
\hline 6 & 7 & & & & & & 7 \\
\hline 7 & - & & & & & & \\
\hline 8 & - & & & & & & \\
\hline 9 & - & & & & & & \\
\hline 10 & 43 & * & & & & & 43 \\
\hline 11 & - & & & & & & \\
\hline 12 & - & & & & & & \\
\hline 13 & - & & & & & & \\
\hline 14 & 78 & * & & & & & 78 \\
\hline
\end{tabular}

Status:

Funded 304

Requested

New

"Estimated annual ongoing cost: \$106K starting in FY 1992.

References: $\quad$ DOE Order 5480.10, ANSI Z117.1, and 29 CFR 1910.146 
Finding No.: $\quad$ IH.5-4 Respiratory Protection Program

Finding

Description:

The ORNL respiratory protection program is not in compliance with
DOE 5480.10, ANSI Z88.2, and 29 CFR 1910.134

Code: $\quad$ Category III

Compliance

Protocol:

DOE Order 5480.10, ANSI Z88.2, and 29 CFR 1910.134

Priority: $\quad$ Energy Systems Risk Weight 415

Tiger Team Action Plan Priority 2

Response: This finding was based on several related findings, which said:

- Respirators were stored on shelves stacked on top of each other and in the direct sunlight. Positive control is not exercised in controlling respirators.

- Training as to limitations and proper fit-testing for $3 \mathrm{M}$ dust masks was not provided for the worker.

- An employee's half-face, dual-cartridge respirator was stored inside the work area at the Lead Building.

- A worker was observed donning a respirator without doing a positive and negative fit-test prior to use.

- Industrial Hygiene did not verify, through a field inspection program, whether respirators were being used properly. - Surveillance forms used for field work are not tied back to the supervisor when
misuse of respirators is observed.

- Monitoring of work sites by Industrial Hygiene personnel is not being done prior to issuance of respirators.

- Air-purifying respirators are routinely maintained for emergency use in violation of ANSI Z88.2, which prohibits the use of these devices in an unknown atmosphere.

- A biological laboratory worker and supervisor have not been trained in selection, use, maintenance, and limitations of a respirator. - A worker with a full beard thought a dust mask was adequate for him while he
was mixing a carcinogen. 
The window in the respirator storage room has been covered to protect the equipment from exposure to direct sunlight. Other problems resulting from inadequate storage space are being addressed by plans for a new industrial hygiene office/storage facility (see Finding PP.1-1, Item 13).

The deficiencies leading to several of these findings are already addressed in Respiratory Protection Program procedures. Since the issuance of the procedures, the Respiratory Protection staff has been conducting field surveillances to ensure that workers in the field are complying with requirements. Field surveillance will be stepped up to ensure adherence to procedures, and, if necessary, the training program will be revised to emphasize problem areas. Other specific corrective actions planned to address these findings are listed below.

\section{Root Causes:}

Inadequate training, poorly defined rcles and responsibilities, and inadequate policy implementation

Planned Actions and Schedules:

\section{$\underline{\text { Item/Description }}$}

1. Contingent upon funding, hire one additional person for the Respiratory Protection Program staff.

2. Establish and document a field surveillance schedule and initiate implementation.

3. Review the respiratory protection training module and, if deemed necessary, revise it to strengthen the guidance concerning the use of proper respirators for particular working conditions.

4. Revise the respirator field surveillance form to include the supervisor's name, and revise the procedure to require that surveillance reports be disseminated to the supervisors and the division office.

5. Label emergency cabinets specifying that air-purifying respirators are not to be used in unknown atmospheres.

6. Implement the new training course for supervisors of workers who use respiratory protection; the training will emphasize job-specific respiratory requirements.

7. In a letter to upper management, request a policy decision concerning the storage of non-emergency-type respirators in ORNL emergency cabinets.
Completion Date

$6 / 92$

$5 / 91$

$5 / 91$

$5 / 91$

$5 / 91$

Complete

$7 / 91$ 
Costs:

Type of funds: Overhead

Source of funds: Division Administration

\begin{tabular}{crrrrrrr} 
& \multicolumn{5}{c}{ Estimated costs per fiscal year (\$K) } & \\
\cline { 2 - 6 } Action item & 1991 & 1992 & 1993 & 1994 & 1995 & Bcyond & Total \\
\hline 1 & 7 & $*$ & & & & & 44 \\
2 & - & & & & & & 7 \\
3 & 7 & $*$ & & & & & 7 \\
5 & 7 & & & & & & 7 \\
7 & - & & & & & & -
\end{tabular}

Status:

Funded 21

Requested

New

44

Estimated annual ongoing cost: \$8K for 1992, \$96K starting in 1993.

Type of funds: Overhead

Source of funds: Overhead

\begin{tabular}{lrlllllll} 
& \multicolumn{6}{c}{ Estimated costs per fiscal year $(\$ K)$} & \\
\cline { 2 - 6 } Action item & 1991 & 1992 & 1993 & 1994 & 1995 & Beyond & Total \\
\hline 6 & 28 & $*$ & & & & & 28
\end{tabular}

Funded 28

Requested

New

"Estimated annual ongoing cost: $\$ 31 \mathrm{~K}$

References: $\quad$ DOE Order 5480.10

ANSI Standard Z88.2

29 CFR 1910.134 
Füding No.: IH.5-5 Sanitation and Potable Water Program

Finding

Description:

Code:

Compliance

Protocol:

Priority:

Response:
The ORNL Sanitation and Potable Water Program is not in compliance with DOE 5480.4, DOE 5430.10, ANSI Z4.1-1988, Public Law, Chapter 14, Part I, and the "Tennessee Food Service Establishment Law."

Category III

DOE Order 5480.4; DOE Order 5480.10; ANSI Z4.1-1988; Public Law, Chapter 14, Part I; and the "Tennessee Food Service Establishment Law"

Energy Systems Risk Weight 408

Tiger Team Action Plan Priority 2

This finding was based on several related findings, which said:

- Although the ORNL Industrial Hygiene procedures require inspections and surveillance of eating facilities, vending machines, microwaves, ice machines, and refrigerators, inspections have not been done on a regular basis.

- ORNL found unsanitary conditions in three of five vending machines checked.

- There is no formal inside-building, cross-connection control program with oversight by Industrial Hygiene.

- There were no back-flow valves provided on the potable water side of the syphon hose to prevent contamination of the water system.

In the past, the Industrial Hygiene Section has provided limited, informal coverage for most food sanitation and potable water issues. However, on October 8, 1990, a new IH procedure was issued (IHP-16, "ORNL Program for the Implementation of Health Requirements for Sanitation and Potable Water"). The procedure covers inspection, maintenance, and personnel training activities conducted to ensure compliance with health protection requirements and regulatory standards for sanitation and potable water. Formal administration of the program will be improved once a staff member has been hired to oversee this area.

The finding concerning back-flow valves (in a Biology Division facility at the Y-12 Plant) was corrected immediately. The staff member to be assigned responsibility for potable water will be conducting surveillance to correct any similar deficiencies. Specific corrective actions that will address the other findings are listed below.

Root Causes:

In'ufficient resources and inadequate policy implementation 


\section{Planned Actions and Schedules:}

\section{Item/Description}

1. Assign an Industrial Hygiene staff member to administer the sanitation and potable water program (i.e., conduct inspections, provide surveillance, etc.).

2. Issue standard operating procedures for tasks to be performed as part of the sanitation and potable water program. (Cost covered in Finding IH.2-1, Item 4.)

3. Issue written guidance to the division safety officers and to the Engineering Division concerning control of cross connections inside buildings and the use of back-flow valves or other means to prevent contamination of water systems.
Completion Date

$7 / 91$

$5 / 92$

$12 / 91$

Costs:

Type of funds: Overhead

Source of funds: Overhead

Estimated costs per fiscal year $(\$ \mathrm{~K})$

\begin{tabular}{crrrrrrr}
\cline { 2 - 6 } Action item & 1991 & 1992 & 1993 & 1994 & 1995 & Beyond & Total \\
\hline 1 & 7 & $*$ & & & & & 7 \\
2 & - & & & & & & \\
3 & & 2 & & & & & 2
\end{tabular}

Status:

Funded

7

Requested

2

New

*Estimated ongoing annual cost: $\$ 22 \mathrm{~K}$.

References: $\quad$ DOE Order 5480.4

DOE Order 5480.10

ANSI Standard Z4.1-1988

Public Law, Chapter 14, Part I

Tennessee Food Service Establishment Law 
Finding No.: IH.5-6 Ergonomics Program

Finding

Description: The ORNL Ergonomics Program does not comply with DOE 5480.10.

Code: $\quad$ Category III

Compliance

Protocol:

DOE Order 5480.10

Priority: $\quad$ Energy Systems Risk Weight 80

Tiger Team Action Plan Priority 2

Response: Health problems caused by ergonomic situations have always been a concern of the Health Division. Physicians within the Health Division have taken CME (Continuing Medical Education) courses in Ergonomics. Until recently Health Division activities related to ergonomic problems have been handled on a case by case basis. At the present time, the Health Division is involved in the assessment of a specific ergonomically related problem. Expert consultation has been obtained from Dr. Thomas J. Armstrong, Associate Professor, Industrial and Operations Engineering, The University of Michigan.

Root Cause:

Inadequate resources have not permitted the development of a plant-wide proactive program.

Planned Actions and Schedules:

\section{$\underline{\text { Item/Description }}$}

1. Charter a PIP committee to assess the ORNL Ergonomics Program and to make recommendations for improvement to achieve compliance with DOE Order 5480.10.

2. Assign organizational responsibility for the administration of the Ergonomics Program.

3. Issue ORNL SPP reflecting the recommendations of the PIP committee and the requirements of DOE Order 5480.10.
Completion Date

Complete 
Costs:

Type of funds: Overhead

Source of funds: Overhead

\begin{tabular}{crrrrrrr} 
& \multicolumn{5}{c}{ Estimated costs per fiscal year (\$K) } \\
\cline { 2 - 6 } Action item & 1991 & 1992 & 1993 & 1994 & 1995 & Beyond & Total \\
\hline 1 & 15 & & & & & & 15 \\
2 & $*$ & & & & & & \\
3 & & 15 & & & & 15
\end{tabular}

Status:

Funded

Requested

New 15 15

"Estimated annual ongoing cost: \$44K.

References: $\quad 5480.10$, Contractor Industrial Hygiene Program 
Finding No.: IH.6-1 Handling, Storage, and Labeling of Chemicals

Finding

Description:

Code: $\quad$ Category III

Compliance

Protocol:

Priority:

Response: $\quad$ This finding was based on several related findings, which said:

- Not all initial baseline medical monitoring was conducted for employees who work with regulated substances.

- A complete chemical list for all areas of ORNL does not exist. Only 1 of 31 divisions had completed inventories of chemicals.

- Containers of hazardous chemicals in the workplace are not properly labeled, tagged, or marked.

- Flammable materials were not in an approved storage location; they were observed in wall cabinets and on countertops. Flammable cabinets contained both flammables and nonflammables. Resource Conservation and Recovery Act (RCRA) hazardous waste was stored in laboratory hoods not designed for flammable storage.

- Drinks were placed on benches next to solvents.

- Many employees do not know how to obtain Material Safety Data Sheets for chemicals in their work areas.

Industrial Hygiene procedure IHP-01, "ORNL Hazard Communication Program," was issued on September 12, 1990. The program described in that IHP includes several levels of HAZCOM training: general awareness training for new employees, job-specific training for those who work with hazardous chemicals, and additional training for supervisors and division HAZCOM coordinators.

Once the HAZCOM program is fully implemented with additional staff, training, and surveillance, many of these concerns (e.g., container labelling, storage of flammable chemicals, eating in certain areas) will be corrected. The specific corrective actions planned to address these findings are listed below. 
Root Causes:

Insufficient resources and inadequate policy implementation

Planned Actions and Schedules:

$\underline{\text { Item/Description }}$

1. Initiate hiring of two additional staff members for the Hazardous Materials Management Program (HMMP).

2. Compile a chemical inventory for ORNL divisions, which gives specific locations for all hazardous chemicals.

3. Develop SOPs for HMMP surveillance of areas throughout the Laboratory. (Costs are included in Finding IH.2-1, \#4.)

4. Conduct HMMP surveillance and review surveillance reports to identify any trends of recurring problems, document the results, and, if necessary, revise the HAZCOM training program(s).

5. Issue an informational bulletin emphasizing the importance of MSDSs and how to access them.

6. Review operations involving regulated substances to determine if additional workers need to be added to the medical surveillance program, and, if necessary, provide names to the Health Division.
Completion Date

$5 / 91$

$5 / 91$

$6 / 92$

$2 / 92$

$5 / 91$

$9 / 91$ 
Costs:

Type of funds: Overhead

Source of funds: Overhead

Estimated costs per fiscal year (\$K)

\begin{tabular}{crrrrrrr}
\cline { 2 - 6 } Action item & 1991 & 1992 & 1993 & 1994 & 1995 & Beyond & Total \\
\hline 1 & 85 & $*$ & & & & & 85 \\
2 & 55 & & & & & & 55 \\
3 & - & & & & & & \\
4 & 2 & & & & & & 66 \\
5 & 14 & & & & & & 2 \\
6 & & & & & & 14
\end{tabular}

Status:

Funded

156

Requested

New

66

$\$ 222$

Estimated annual ongoing cost: $\$ 176 \mathrm{~K}$ for Item 1 in 1992 and $\$ 264 \mathrm{~K}$ starting in 1993.

References: $\quad$ DOE Order 5480.10

DOE Order 5480.4

29 CFR 1910.1200

29 CFR 1910.145

29 CFR 1910, Subpart Z 


\subsubsection{Fire Protection}

Finding No.: FP.1-1 Resources of Fire Protection Engineering Section

Finding

Description: The Fire Protection Engineering Section is not provided with adequate resources to carry out its assigned tasks as established by DOE 5480.4, DOE 5480.7, and DOE 6430.1A.

Code: $\quad$ Category III

Compliance Protocol:

DOE Order 5480.4, DOE Order 5480.7, and DOE Order 6430.1A

DOE Order 5480.4 requires the application of the National Fire Codes (NFC) published by the National Fire Protection Association (NFPA) to DOE facilities. DOE Order 5480.7 requires qualified fire protection engineers to review specifications and designs for major construction projects, and to perform scheduled in-depth fire protection engineering surveys and life safety surveys on DOE facilities.

DOE Order 6430.1A contains fire protection requirements for the design and construction of DOE facilities.

Priority: $\quad$ Energy Systems Risk Weight 133

Tiger Team Action Plan Priority 2

Response: The majority of this concern was identified in the ORNL Self-Assessment.

Currently, two fire protection engineers report to the fire department supervisor. The second of the two fire protection engineers was hired in May 1990. This increased staffing by 100 percent in the fire protection engineering group. The impact that the new engineer will have on the groups capability to keep up with design reviews and facility fire protection engineering surveys is not currently apparent. Management approval has been granted to hire an additional fire protection engineer. Evaluation of the current staff's ability to perform the required workload will continue.

Facility operational requirements and experimental requirements are reviewed by Division Safety Officers. Experimental requirements receive additional review by a panel of qualified scientists familiar with the details of the specific experimental arrangements. When requested, fire protection engineer $n$ g expertise is provided to the review groups.

Fire protection recommendations and findings from surveys and appraisals (Factory Mutual, DOE Technical Safety Appraisal, internal reviews, etc.) are prioritized and tracked by ORNL tracking systems. The fire protection section currently has a 
"recommendation tracking" system under development. This PC-based system is called "Evaluation Database System" (EDS) and when complete will provide the mechanism to prioritize, track, and document the status of fire protection recommendations and findings in a centralized management system.

\section{Root Causes:}

Insufficient resources and inadequate management commitment

Planned Actions and Schedules:

\section{Item/Description}

1. Perform "Needs Assessment" to determine how many Fire Protection Engineers are necessary for conducting assigned tasks.

2. Develop a Fire Protection Management Plan for securing the necessary resources.

3. Advise management of the "Needs Assessment" results and the "Fire Protection Management Plan" requirements.

4. Hire additional personnel and secure other resources as directed by management.
Completion Date

$6 / 91$

$8 / 91$

$8 / 91$

$12 / 92$ 
Costs:

Type of funds: Overhead

Source of funds: Overhead

Estimated costs per fiscal year (\$K)

\begin{tabular}{crrrrrrr}
\cline { 2 - 5 } Action item & 1991 & 1992 & 1993 & 1994 & 1995 & Beyond & Total \\
\hline 1 & - & & & & & \\
2 & 2 & & & & & \\
3 & - & & & & & \\
4 & & & $*$ & & & &
\end{tabular}

Status:

Funded

2

Requested

New

$\$ 2$

*Estimated annual ongoing cost: \$60K per fire protection engineer starting in FY 1992.

References: $\quad$ DOE Order 5480.4, DOE Order 548ú.7 
Finding No.: FP.1-2 Fire Department Resources and Work Load

Finding

Description: The Fire Department's allocated resources to carry out mandatory and good practice fire prevention inspections, fire watches, fire protection system inspection and testing, and fire hydrant flow test, as required by mandatory standards listed in DOE 5480.4, are not commensurate with the required work load.

Code: $\quad$ Category III

Compliance

Protocol:

DOE Order 5480.4 requires the National Fire Protection Association codes to be followed. These codes prescribe the inspection, test, and maintenance frequencies of fire protection associated equipment, etc.

Priority: $\quad$ Energy Systems Risk Weight 133

Tiger Team Action Plan Priority 2

Response: This concern was identified in the 1990 ORNL Self-Assessment. Note: One finding leading to this concern indicated that the "Fire Prevention and Protection Policy" published by DOE-ORO does not comply with DOE policy. DOE-ORO will review its policy on Fire Prevention and Protection and make appropriate changes

ORNL upgraded most bargaining unit personnel from drivers/dispatchers to the fire protection inspector classification to allow more flexibility in the assignment of resources. Monthly inspectors of buildings and visual monthly inspections of extinguishers, fire doors, valves, and sprinkler systems, etc. are now being conducted as required.

Only inspection and testing schedules for fixed fire protection systems and fire alarm systems are not being met. Recently increased code requirements, along with continuing lab growth, caused schedule slippage.

\section{Root Causes:}

Insufficient resources, inadequate management commitment, and inadequatc policy
implementation 
Planned Actions and Schedules:

$\underline{\text { Item/Description }}$

1. See action plan for Finding FP.1-1.

2. DOE-ORO review policy, and make appropriate changes.

Costs: $\quad$ See Finding FP.1-1.

References: $\quad$ DOE Order 5480.4
Completion Date

$10 / 91$ 
Finding No.: FP.1-3 Fire Protection of ORNL Facilities at the Y-12 Plant

Finding

Description: ORNL Firc Protection management does not maintain continuous surveillance over ORNL projects or facilities located at the Y-12 Plant as required by DOE 5480.7.

Code: $\quad$ Category III

Compliance

Protocol:

DOE Order 5480.7 requires under section 10.a ". . that strong, tangible evidence be available attesting to existence of continuing sincere interest by management and employees in minimizing losses from fire and related perils." Further, section 10.f.3 relegates this responsibilities to the appraising office.

Priority: $\quad$ Energy Systems Risk Weight 55

Tiger Team Action Plan Priority 2

Response: A review of the findings regarding this concern reveals factual information which includes: (1) a documented, Memorandum of Understanding signed by both site managers is in place which clearly identifies the Y-12 site fire protection engineering and response groups as having responsibility for ORNL facilities at $\mathrm{Y}-12$; (2) the ORNL and Y-12 fire protection managers confer on a regular informal basis, and (3) the Y-12 Fire Protection Engineering Section is staffed by qualified fire protection engineers. Additionally,

- Martin Marietta Energy Systems is the operating contractor for both sites. This is a single contract. This is consistent with the manner in which other ES\&H issues are handled at $\mathrm{Y}-12$.

- Both site managers serve as vice presidents in Energy Systems.

- Facility managers at the Y-12 site report to ORNL upper management.

- Facility managers routinely work with the Y-12 site fire protection organization and understand relationships. Any significant identified problems, concerns, etc. are communicated from Y-12 site facility managers through line management at the ORNL site.

A Fire Protection Engineering appraisal performed in April 1990 for the Advanced Toroidal Facility (Building 9201-2) by a registered Fire Protection Engineer, indicates that the existing level of protection is satisfactory to meet the requirements of DOE Order 5480.7. No additional protection is planned. 
The fire protection engineering (FPE) appraisal of Bldg. 9201-2 was corrected during the factual review process. In August 1990 facility managers and Y-12 FPE conducted meetings and addressed each issue. Organizations were identified that had responsibility, and all issued were assigned. Minor issues were resolved and completed $(-30 \%)$; some major issues were aiready included in line item projects; and others were assigned to programs for funding request.

The PCB-contaminated oils in Bldg. 9204-3, Isotopes Enrichment Facility, were identified in the ORNL Self-Assessment. A management plan to address the issue is in place, and a fire prot ction engineering appraisal of the facility was conducted in November 1990 by an outside consultant. The appraisal addressed a "Credible Fire Loss" (CFL) and a "Maximum Possible Loss" (MPL), as required by DOE Order 5480.7, which involved oil fires. The CFL addressed a fire with the sprinkler system operational. The MPL scenario excluded automatic and manual control.

Root Cause:

Poorly defined roles and responsibilities

Planned Actions and Schedules:

\section{Item/Description}

1. Notify ORNL facility managers at $\mathrm{Y}-12$ and $\mathrm{Y}-12$ Fire Protection Engineering of the specific findings for this concern (Finding FP.1-3) by letter from ORNL management. The purpose of this letter is to refer the findings to the responsible personnel for resolution.

Costs: $\quad$ No significant costs are associated with this action.
Completion Date

Complete

References: $\quad$ DOE Order 5480.7 
Finding No.: FP.1-4 Fire Protection Policies Regarding Improved Risk

Finding Description:

Code:

Compliance Protocol:

Priority:

Response:
The Fire Protection Engineering Section has not developed or implemented comprehensive fire protection policies as mandated by "improved risk" criteria established in DOE 5480.7.

Category III

DOE Order 5480.7 requires a comprehensive fire protection program to be in place sufficient to attain DOE objectives. Requirements include minimizing potential for the occurrence of a fire, establishing the minimum requirements that will provide an acceptable degree of life safety to DOE and contractor personnel, and establishing minimum requirements for the protection of the public from fire in DOE facilities.

\section{Energy Systems Risk Weight 55}

Tiger Team Action Plan Priority 2

This item was identified in the ORNL Protective Services 1990 Self-Assessment. Energy Systems has a written policy procedures manual (8/8/88, number ESH-21) which states that an effective fire protection program shall be in place. This policy procedure flows down to ORNL Standard Practice Procedures (6/6/90, number X-GP-4). Requirements in SOP X-GP-4 include: (1) prohibiting combustible wallboard and combustible acoustical tile or similar materials in the ORNL building program, (2) not allowing rubbish and waste to accumulate in or around buildings, (3) waste and rubbish to te removed from buildings before closing, and (4) inspections that include housekeeping, construction/building materials, smoking and open fires, handling and storage of flammable and combustible liquids and electrical hazards.

Occasionally it is necessary to draw attention to a specific fire protection issue. This is accomplished by publishing an "ORNL Safety Bulletin" which is distributed to the ORNL population. When applicable, specific standards, codes, orders, and laws which govern the specific issue are referenced in the safety bulletin.

Fire protection issues which affect new construction projects and renovaticns are governed by written DOE orders such as 6430.1A (General Design Criteria) and national consensus standards such as the National Fire Codes and applicable building codes. Standard Operating Procedures are in place which require Engineering and Plant and Equipment Divisions to submit construction specifications and design drawings to fire protection engineering for review.

Comprehensive fire protection policies are in place at ORNL, however, the policies, procedures, and other written requirements are not collectively bound in a single dedicated manual. 


\section{Root Cause:}

Ambiguous requirements or expectations

Planned Actions and Schedules:

\section{Item/Description}

1. Review and revise ORNL Standard Practice Procedure X-GP-4 for adequate coverage of fire protection issues.

2. Request the review and revision of Martin Marietta Energy Systems, Inc., Policy Procedure ESH-21 for adequate coverage of fire protection issues.

3. Request the review and revision of the ORNL Operational Safety Organization's SOP X-60-4 for adequate coverage of fire protection issues.

4. Request funds for improving fire protection policies as indicated by the review results.
Completion Date

$7 / 91$

$8 / 91$

$8 / 91$

$1 / 92$

Costs:

Type of funds: Overhead

Source of funds: Overhead

Estimated costs per fiscal year (\$K)

\begin{tabular}{crrrrrrr}
\cline { 2 - 5 } Action item & 1991 & 1992 & 1993 & 1994 & 1995 & Beyond & Total \\
\hline 1 & 7 & & & & & & 7 \\
2 & - & & & & & & \\
3 & - & & & & & & \\
4 & - & & & & & &
\end{tabular}

Status:

Funded

7

Requested

New

References: $\quad$ DOE Order 5480.7 
Finding No.: FP.1-5 Facility Reoccupancy Policy

Finding

Description: ORNL does not have a policy that ensures that reoccupancy of excess or surplus facilities is in compliance with DOE 5480.7, DOE 6430.1A, and other applicable Orders and mandatory National Fire Protection Association standards.

Code: $\quad$ Category III

Compliance

Protocol:

Priority: $\quad$ Energy Systems Risk Weight 105

Tiger Team Action Plan Priority 2

Response: $\quad$ ORNL Fire Department Standard Operating Procedure, FP B-17, Plan Review requires both General Engineering and the Plant and Equipment Division (field engineering) to involve Fire Protection Engineering "... in the review process very early in the planning stage of all planned buildings, modifications and major processes." FP B-17 is approved (signed) by both General and Field Engineering. The reoccupancy of excess or surplus facilities usually requires significant modifications or renovations. These modifications are reviewed in accordance with FP B-17 for compliance with DOE orders. FP B-17 is serving as an interim reoccupancy policy (fire protection) until a formal ORNL policy is issued.

ORNL does not have a separate policy that addressed reoccupancy of older facilities. Plans are in place for older reactor facilities such as the Graphite Reactor, Building 3001, but do not address action to be taken in the "near term" of one year or less. It should be noted that the Graphite Reactor is of "metal or exposed steel beam" construction and that some interior walls, etc. are of combustible construction installed mostly in the 1940's when the reactor was built. Preliminary decommissioning plans were developed under ORO guidance.

An 2.iditional exit has been provided for the basement office module in Building 7503. The additional exit has been provided to meet the intent of the Life
Safety Code.

Root Cause:

Inadequate policy 
Planned Actions and Schedules:

$\underline{\text { Item/Description }}$

1. ORNL Standard Practice Procedure X-GP-4 will be reviewed and revised to include a requirement that fire protection engineering must review the reoccupancy of excess or surplus facilities. (See Action Item 1 in FP.1-4 for costs.)

2. Issue a policy/program for the review of occupancy $12 / 91$ changes in ORNL facilities by Fire Protection Engineering.

Costs:

Type of funds: Overhead

Source of funds: Overhead

Estimated costs per fiscal year (\$K)

\begin{tabular}{cccccccc}
\cline { 2 - 6 } Action item & 1991 & 1992 & 1993 & 1994 & 1995 & Beyond & Total \\
\hline 1 & - & & & & & & \\
2 & 7 & & & & & & \\
\end{tabular}

Status:

Funded

Requested

New

References: $\quad$ DOE Orders 5480.7 and 6430.1A 
Finding No.: FP.1-6 Management's Role in Fire Protection

Finding

Description: Upper ORNL management has not shown a continuing sincere interest in resolving significant fire protection issues as required by DOE 5480.7 and has failed to provide a working communication system to remain informed on such
matters.

Code: $\quad$ Category III

\section{Compliance}

Protocol:

DOE Order 5480.7 requires under section 10.a ". . that strong, tangible evidence be available attesting to existence of continuing sincere interest by management and employees in minimizing losses from fire and related perils." Further, section 10.f.3 relegates this responsibilities to the appraising office.

\section{Priority: $\quad$ Energy Systems Risk Weight 9}

Tiger Team Action Plan Priority 3

Response: $\quad$ Many past actions have demonstrated management's commitment to significant fire protection issues. The following are examples of past actions:

- In 1987 , multiple Factory Mutual recommendations were resolved by $\$ 365 \mathrm{~K}$
GPP funds.

- In 1988, GPP funding of $\sim \$ 200 \mathrm{~K}$ for fire water line extensions and improvements was provided. An additional $\$ 200 \mathrm{~K}$ was provided for a live fire, on-site training tower.

- Also in 1987 and 1988 substantial funds, $\$ 200 \mathrm{~K}+$ was provided for the 1990 , $\$ 3.3$ million fire protection upgrade line item CDR development. The line item was supported and approved.

In 1989

- $\$ 175 \mathrm{~K}$ of GPE funds were used to replace an aging fire pumper with an 1989

- A pilot, 12-hour rotating shift arrangement was supported and all hourly bargaining unit fire department personnel were upgraded to single classification. These actions were noteworthy and provided flexibility for more
work while improving morale.

- A full-time fire training officer slot was authorized and filled. 
- A management initiative on OSHA compliance resulted in 54 people being relocated, facilities improved from a life safety perspective, and new facilities provided; all at substantial cost and effort in support of a fire protection engineering recommendation.

- An additional fire protection engineering position was approved and filled in 1990. Approval has been granted to hire one more fire protection engineer in 1991.

The correction for the unresolved 1973 fire protection issue is included in a 1991 Line Item Project entitled "Fire Protection Upgrade - ORNL." Plans to correct all unresolved fire protection issues will continue to be developed.

The items in Finding \#3 in the Tiger Team report for Concern FP.1-6 are addressed in other ORNL corrective action plans.

There have been some findings by the Tiger Team which seem to reflect a lack of management interest. A system will be initiated to assist in keeping management informed.

\section{Root Causes:}

Inadequate management commitment, inadequate communications, and poorly defined roles and responsibilities

Planned Actions and Schedules:

\section{$\underline{\text { Item/Description }}$}

1. Re-evaluate reporting levels to ensure that fire protection engineering reports to an adequate management level.

2. Issue a procedure to require major fire protection issues to be entered into an ES\&H tracking system. Upper management will routinely review items on this tracking system.

3. Hire an additional fire protection engineer.
Completion Date

$6 / 91$

$3 / 92$

$10 / 91$ 


\section{Costs:}

Type of funds: Overhead

Source of funds: Overhead

\begin{tabular}{|c|c|c|c|c|c|c|c|}
\hline \multirow[b]{2}{*}{ Action item } & \multicolumn{6}{|c|}{ Estimated costs per fiscal year (\$K) } & \multirow[b]{2}{*}{ Total } \\
\hline & 1991 & 1992 & 1993 & 1994 & 1995 & Beyond & \\
\hline 1 & - & & & & & & \\
\hline 2 & & 7 & & & & & 7 \\
\hline 3 & & * & & & & & I \\
\hline \multicolumn{8}{|l|}{ Status: } \\
\hline Funded & & & & & & & \\
\hline Requested & & 7 & & & & & \\
\hline New & & & & & & & $\$ 7$ \\
\hline
\end{tabular}

*Estimated annual ongoing cost \$60K starting in FY 1992.

References: $\quad$ DOE Order 5480.7 
Finding No.: FP.2-1 Egress from Bldg. 4500N, Machinery Space

Finding Description:

Code: $\quad$ Category II

Compliance Protocol:

Priority: $\quad$ Energy Systems Risk Weight 458

Tiger Team Action Plan Priority 2

Response: $\quad$ Significant work has been conducted to provide occupants a clear, unobstructed means of egress path that is separated from machinery areas. Work accomplished to date includes: relocation of people, removal/relocation of machinery, overhead ducts, pipes, etc. were raised to provide 6 feet 8 inches head clearance, physical arrangement of work areas have been altered, and training sessions on egress and changes have been conducted.

Work currently in progress and nearing completion for one protected means of egress include: one-hour rated partitions are being installed, rated fire doors are being installed.

Work currently in progress for a second protected means of egress includes: relocation of equipment, interferences, etc., in preparation for installation of rated fire barriers to provide a second protected means of egress.

Work is in progress and shall be continued until completed. Funding has been committed.

This area is also protected by an automatic sprinkler system. Employee alerting signals (evacuation alarms which are both manually and automatically initiated), emergency lighting, and approved exit signs are in place.

\section{Root Cause:}

Ambiguous requirements or expectations 
Planned Actions and Schedules:

Item/Description

1. Install fire-rated partitions and doors to the northeast stairwell in Wing no. 4 , Bldg. $4500 \mathrm{~N}$.

2. Install fire-rated partition and door to the east center stairwell in Wing no. 4 , Bldg. $4500 \mathrm{~N}$.

3. Install fire-rated partitions and doors, seal penetrations, and complete one-hour rated enclosure to the scuth exit of Wing no. 4 , Bldg. $4500 \mathrm{~N}$.
Completion Date

Complete

Complete

Complete

Costs:

Type of funds: Overhead

Source of funds: Overhead

\begin{tabular}{|c|c|c|c|c|c|c|c|}
\hline \multirow[b]{2}{*}{ Action item } & \multicolumn{6}{|c|}{ Estimated costs per fiscal year (\$K) } & \multirow[b]{2}{*}{ Total } \\
\hline & 1991 & 1992 & 1993 & 1994 & 1995 & Beyond & \\
\hline All & 120 & & & & & & 120 \\
\hline \multicolumn{8}{|l|}{ Status: } \\
\hline Funded & 120 & & & & & & \\
\hline \multicolumn{8}{|l|}{ Requested } \\
\hline New & & & & & & & $\$ 120$ \\
\hline
\end{tabular}

References: $\quad$ DOE Order 5480.4; NFPA 101 
Finding No.: FP.2-2 Egress from Bldg. 4500N, Office Space

Finding

Description: ORNL has not provided a second protected means of egress from all of the office space use areas, Wings 1, 2, \& 3, of the attics of Bldg. 4500N.

Code: $\quad$ Category II

Compliance

Protocol:

NFPA 101, Life Safety Code requires adequate means of egress to ensure safety of occupants from fire related causes.

Priority: $\quad$ Energy Systems Risk Weight 458

Tiger Team Action Plan Priority 2

Response: ORNL does not agree with the Category II level, hazard level, and compliance level assigned to this issue.

This finding had previously been identified and due to the concern of management about the safety of people in the attic spaces, many were relocated, fire-rated partitions were added, training was conducted, 80 percent of combustible fuel loading was removed, and new portable office structures purchased. The concern was identified by an internal self-assessment of OSHA compliance which was initiated by ORNL management.

Plans were in place and work orders written to complete the separation of corridors from equipment areas, e.g., fire doors ordered and openings sealed. The plans were accelerated, given high priority, and completed with the exception of minor technical noncompliance clearance items by November 1990 . The space has been reviewed by the Fire Protection group using NFPA 101 and determined to be in compliance with the standard. We are confident that occupants are not unduly threatened by a fire event and that safe means of egress has been provided.

This area is also protected by an automatic sprinkler system, employee alerting signals (evacuation alarms which are both manually and automatically initiated), emergency lighting, and approved exit signs are in place.

\section{Root Cause:}

Ambiguous requirements or expectations 


\section{Planned Actions and Schedules:}

\section{Item/Description}

Completed before TSA began:

1. Relocate people out of attic in Wing 1, 2, and 3 of the attic in Bldg. $4500 \mathrm{~N}$.

2. Add fire-rated partitions where needed in the attic of Bldg. $4500 \mathrm{~N}$.

3. Train occupants to ensure understanding of means-ofegress modifications.

4. Reduce combustible fuel loading by $80 \%$ in the attic to Bldg. $4500 \mathrm{~N}$.

5. Purchase portable office structures to house evacuees from the attic in Bldg. $4500 \mathrm{~N}$.

6. Install fire doors and seal penetrations. Completed after TSA began.
Complete

Complete

\section{Completion Date}

Complete

Complete

Complete

Complete

\section{Costs:}

Type of funds: Overhead

Source of funds: Overhead

Estimated costs per fiscal year $(\$ \mathrm{~K})$

\begin{tabular}{crrrrrrrr} 
Action item & 1990 & 1991 & 1992 & 1993 & 1994 & 1995 & Beyond & Total \\
\hline 1 & & - & & & & & & \\
2 & & - & & & & & & \\
3 & & - & & & & & & \\
4 & & - & & & & & & \\
5 & 590 & - & & & & & & 590 \\
6 & & 17 & & & & & & 17
\end{tabular}

Status:

Funded

\section{7}

Requested

New 
Finding No.: FP.2-3 Life Safety Code Surveys

\section{Finding \\ Description: \\ Code: \\ Compliance Protocol:}

$\begin{array}{ll}\text { Priority: } & \text { Energy Systems Risk Weight } 133 \\ & \text { Tiger Team Action Plan Priority } 2\end{array}$

Response: This concern was identified in the ORNL Protective Services Department SelfAssessment.

Dedicated life safety surveys were performed at Building $3001,4500 \mathrm{~N}$, and all ORNL Reactor buildings by independent fire protection consulting firms. These surveys were conducted as the result of specific life safety concerns at these facilities. Life safety surveys for other ORNL building are included as part of the internal fire protection engineering survey program.

An inspection criteria has been developed so an independent consulting firm can conduct Life Safety surveys on ORNL facilities. This inspection criteria requires the evaluation of facilities against NFPA-101 and 29 CFR 1910, Subpart E requirements. These surveys are budgeted for FY 1991.

Common path of travel limitations are exceeded in one location in Building 3525. The facility manager is aware of the problem and is investigating methods for providing alternative exits. An additional exit has been provided for basement offices in Building 7503.

Monthly building fire prevention inspections cover only significant life safety issues and are not designed or intended to qualify as an in-depth life safety survey.

DOE Order 5480.4, DOE Order 6430.1A, and ORNL Standard Practice Procedure X-GP-4 identify NFPA standards as mandatory and applicable.

\section{Root Causes:}

Insufficient resources, inadequate policy, and inadequate policy implementation 
Planned Actions and Schedules:

Item/Description

1. Conduct life safety inspections on 39 high-priority ORNL facilities in accordance with existing inspection criteria documentation.

2. Request adequate resources to perform fire protection engineering surveys, which include life safety evaluations. (See Action Items 1, 2, 3 and 4 in Finding FP.1-1.)

3. Issue revised procedures to address Life Safety Code compliance issues.
Completion Date

$6 / 92$

$8 / 91$

$6 / 92$

Costs:

Type of funds: Overhead

Source of funds: Overhead

\begin{tabular}{|c|c|c|c|c|c|c|c|}
\hline \multirow[b]{2}{*}{ Action item } & \multicolumn{6}{|c|}{ Estimated costs per fiscal year (\$K) } & \multirow[b]{2}{*}{ Total } \\
\hline & 1991 & 1992 & 1993 & 1994 & 1995 & Beyond & \\
\hline 1 & 240 & & & & & & 240 \\
\hline 2 & - & & & & & & \\
\hline 3 & - & & & & & & \\
\hline \multicolumn{8}{|l|}{ Status: } \\
\hline $\begin{array}{l}\text { Funded } \\
\text { Requested }\end{array}$ & 240 & & & & & & \\
\hline New & & & & & & & $\$ 240$ \\
\hline
\end{tabular}

References: $\quad$ DOE Order 5480.4; DOE Order 5480.7 
Finding No.: $\quad$ FP.2-4 Action Plans Regarding Life Safety Code Surveys

Finding

Description: ORNL has not developed an action plan to resolve and close out, within a reasonable period, the life safety issues which were identified in Bldg. $3001,4500 \mathrm{~N}$, and 7900 as a result of Life Safety Code surveys.

Code: $\quad$ Category III

Compliance

Protocol:

Priority:

Response: $\quad$ Most life safety improvements have been completed in Building 3001 such as:

(1) the evacuation of occupants above the second floor, (2) installation of illuminated exit signs and emergency lighting, (3) securing certain areas of the building to prevent confusion during evacuations and, (4) marking of aisles and routes to building exits. A local occupant notification system (evacuation horns) has been identified as necessary but is yet to be installed.

Most life safety improvements have been completed in the wing attic spaces of Building 4500N. These improvements include: (1) the evacuation of approximately 56 percent of attic occupants, (2) the installation of one-hour fire-rated partitions and corridor walls to provide a protected means of egress for occupants, (3) improvements to emergency lighting, (4) replacement of non-rated doors in separation walls with fire-rated doors, (5) improvements to illuminated exit sign arrangements, and (6) modifications to eliminate low head clearances such as low HVAC ducts and sprinkler system piping. A fire protection line item is funded for this facility which will significantly improve automatic sprinkler protection.

Recommendations from the Life Safety Code Survey for Building 7900 have been prioritized and entered into the Critical Actions and Requirements Tracking System (CARTS) maintained by the Research Reactors Division. The majority of life safety recommendations for this facility are complete. The conversion of the existing pre-action sprinkler system to a wet-pipe system is planned.

\section{Root Causes:}

Inadequate policy implementation and inadequate management commitment 
Planned Actions and s'chedules:

Item/Description

1. Develon an action plan to resolve and close out life safety issues which were identified in Life Safety Code surveys of Buildings $3001,4500 \mathrm{~N}$, and 7900 .
Completion Date

$6 / 91$

Costs:

Type of funds: Overhead

Source of funds: Overhead

\begin{tabular}{|c|c|c|c|c|c|c|c|}
\hline \multirow[b]{2}{*}{ Action item } & \multicolumn{6}{|c|}{ Estimated costs per fiscal year (\$K) } & \multirow[b]{2}{*}{ Total } \\
\hline & 1991 & 1992 & 1993 & 1994 & 1995 & Beyond & \\
\hline 1 & 5 & & & & & & 5 \\
\hline \multicolumn{8}{|l|}{ Status: } \\
\hline \multicolumn{8}{|l|}{ Funded } \\
\hline \multicolumn{8}{|l|}{ Requested } \\
\hline New & 5 & & & & & & $\$ 5$ \\
\hline
\end{tabular}

References: $\quad$ DOE Order 5480.4 
Finding No.: FP.3-1 ORNL Testing of Fire Equipment

Finding

Description: ORNL has not tested, in accordance with recognized practices, the detection and suppression systems protecting cells and cubicles of Bldg. 7920 to ensure that the devices will function as intended in the event of fire.

Code: $\quad$ Category II

Compliance

Protocol:

DOE Order 5480.4 requires NFPA standards be utilized for testing fire protcction systems.

Priority: $\quad$ Energy Systems Risk Weight 59

Tiger Team Action Plan Priority 2

Response: This action plan addresses the need to test the fire protection system present in the cubicles and cell tank pits of REDC Bldg. 7920.

Root Cause:

Inadequate policy implementation

Planned Actions and Schedules:

\section{Item/Description}

1. Initiate routine periodic visual examination of hot cell cubicles, compensatory to verification of function of fire deteciion and suppression systems in cubicles and cell tank pits.

2. Test fire detection systems in each cubicle and hot cell tank pit. This action involves opening the hot cells and exposing workers to radiation fields. The work will be performed under an ALARA program, but additional worker exposure is unavoidable.

3. Issue an operating procedure requiring evaluation of fire protection adequacy as a result of combustible materials which would be introduced into the cubicles or cell tank pits because of use of new unreviewed processes.

4. Issue an operating procedure for periodic testing of fire detector and suppression system.

5. Request funds from ER and DP for budgets for Item 6 .
Completion Date

Complete

and

continuing

Complete

Complete

$6 / 91$

Complete 
6. Install remotciy testable heat-activated devices (HADs) in cubicles and cells, contingent on increased budgets.

7. Complete necessary piping modifications so full flow testing of cell and cubicle suppression systems can be conducted.

Cists:

Type of funds: Research Progra:..matic

Source of funds: ER

\begin{tabular}{|c|c|c|c|c|c|c|c|}
\hline \multirow[b]{2}{*}{ Action item } & \multicolumn{6}{|c|}{ Estimated costs per fiscal year (\$K) } & \multirow[b]{2}{*}{ Total } \\
\hline & 1991 & 1992 & 1993 & 1994 & 1995 & Beyond & \\
\hline 1 & - & & & & & & \\
\hline 2 & 93 & & & & & & 93 \\
\hline 3 & - & & & & & & \\
\hline 4 & - & & & & & & \\
\hline 5 & & 140 & 210 & & & & 350 \\
\hline 6 & - & & & & & & \\
\hline 7 & 45 & & & & & & 45 \\
\hline
\end{tabular}

Status:

Funded

138

Requested

$140 \quad 210$

New 
Type of funds: Research Programmatic

Source of funds: DP-GE

\begin{tabular}{|c|c|c|c|c|c|c|c|}
\hline \multirow[b]{2}{*}{ Action item } & \multicolumn{6}{|c|}{ Estimated costs per fiscal year $(\$ \mathrm{~K})$} & \multirow[b]{2}{*}{ Total } \\
\hline & 1991 & 1992 & 1993 & 1994 & 1995 & Beyond & \\
\hline 1 & - & & & & & & \\
\hline 2 & 22 & & & & & & 22 \\
\hline 3 & - & & & & & & \\
\hline 4 & - & & & & & & \\
\hline 5 & & 60 & 90 & & & & 150 \\
\hline 6 & - & & & & & & \\
\hline 7 & - & & & & & & \\
\hline \multicolumn{8}{|l|}{ Status: } \\
\hline Funded & 22 & & & & & & \\
\hline Requested & & 60 & 90 & & & & \\
\hline New & & & & & & & $\$ 172$ \\
\hline
\end{tabular}

References: $\quad$ DOE Order 5480.4, Environmental Protection, Safety and Health Protection Standards, February 26, 1985 
Fünding No.: FP.3-2 Adequate Documentation of Fire Study

Finding

Description:

Documentation provided by ORNL and DOE Headquarters does not support the conclusions that a fire originating in the cells or cubicles of Bldg. 7920 at ORNL would not result in the loss of high-efficiency particulate air filters and an unacceptable radiological release.

Code: $\quad$ Category II

Compliance

Protocol:

Priority: $\quad$ Energy Systems Risk Weight 80

Tiger Team Action Plan Priority 2

Response: Previous analyses requested by DOE (Refs. 1-2) and accepted by DOE/ORO indicated that a fire originating in the cells or cubicles of Bldg. 7920 at the ORNL would not result in the loss of high-efficiency particulate air filters. The reviewer felt that this analysis was potentially nonconservative. Particular emphasis was placed by the reviewer on understanding the impact of a fire in the vessel off-gas (VOG) fiberglass reinforced epoxy duct work. An evaluation of the magnitude of radiological release to the environment in the event of a filter failure has not been made. This action plan addresses the need to document an independent expert analysis of the impact of a fire in the vessel off-gas (VOG) ducts of REDC Bldg. 7920.

\section{Root Causes:}

Ambiguous requirements or expectations and inadequate communications

Planned Actions and Schedules:

\section{Item/Description}

1. Review credible accident scenarios in VOG duct work in Bldg. 7920 cells using independent ventilation and fire protection experts.

2. Estimate maximum credible radionuclide release assuming filter failure.

3. Submit to DOE/ORO Fire Protection and DOE/HQ-EH fire scenario(s) and fire modeling methods and maximum release assumptions for concurrence.

\section{Completion Date}

Complete

Complete

$9 / 91$ 
4. Complete fire impact analysis and radiological release impact analysis.

Costs:

Type of funds: Research programmatic

Source of funds: DP-GE

Estimated costs per fiscal year (\$K)

\begin{tabular}{cccccccc}
\cline { 2 - 5 } Action item & 1991 & 1992 & 1993 & 1994 & 1995 & Beyond & Total \\
\hline 1 & - & & & & & \\
2 & - & & & & & \\
3 & - & & & & & & \\
4 & 45 & & & & & & \\
\hline & & & & & & \\
& & & & & &
\end{tabular}

Status:

Funded

Requested

45

New

Type of funds: Research programmatic

Source of funds: ER

Estimated costs per fiscal year (\$K)

\begin{tabular}{crrrrrrr}
\cline { 2 - 5 } Action item & 1991 & 1992 & 1993 & 1994 & 1995 & Beyond & Total \\
\hline 1 & - & & & & & & \\
2 & - & & & & & & \\
3 & - & & & & & & \\
4 & 105 & & & & & & 105
\end{tabular}

Status:

Funded

Requested

105

New 
References: Letter from K. W. Sommerfeld to J. A. Lenhard, "ORNL Fire Protection Appraisal Recommendation 8301, Positive Ventilation Systems," January 20, 1984

Norman J. Alveres, "Analysis of Fire and Smoke Threat to Off-Gas HEPA Filters in a Transuranium Processing Plant," Lawrence Livermore National Laboratory, unpublished, (March 1988) 
Finding No.: FP.3-3 Design Basis Fires Review Program

Finding

Description: ORNL does not have a review program to ensure that design basis fires within nuclear facilities site-wide will not result in an unacceptable release of radioactivity to the environment, as required by DOE 5480.7 .

Code: $\quad$ Category III

Compliance

Protocol:

Priority: $\quad$ Energy Systems Risk Weight 58

Tiger Team Action Plan Priority 2

Response: This concern was identified in the ORNL Protective Services Department SelfAssessment.

The Safety Analysis Report (SAR) for Building 7920 is currently under revision. Fires in cubicles or cells will receive in-depth analysis and the report revised as necessary to reflect conclusions. A major effort is currently active to perform maintenance and testing on the fire protection system for cells and cubicles. Once complete, a detailed operating and testing procedure will be developed for these fire protection systems.

ORNL does not have an on-going hazards analysis program. An on-going fire protection engineering survey program is in place, but surveys are currently behind schedule. Consideration is being given to expanding the engineering survey program so that it qualifies as a fire hazards analysis program.

Fire Protection Engineering does not currently review SARs, when requested, Fire Protection Engineering provides technical expertise to SAR efforts.

Root Causes:

Insufficient resources and inadequate policy

Planned Actions and Schedules:

Item/Description

1. Request the ORNL Operational Safety Organization to review and revise procedures to require Fire Protection Engineering review of Safety Analysis Reports (see Action Item \#3 in FP.1-4).
Completion Date

$8 / 91$ 
2. Expand the Fire Protection Engineering Survey Program to qualify as a Fire Hazards Analysis Program as necessary to comply with DOE Order 5480.7.

Costs:

Type of funds: Overhead

Source of funds: Overhead

\begin{tabular}{|c|c|c|c|c|c|c|c|}
\hline \multirow[b]{2}{*}{ Action item } & \multicolumn{6}{|c|}{ Estimated costs per fiscal year (\$K) } & \multirow[b]{2}{*}{ Total } \\
\hline & 1991 & 1992 & 1993 & 1994 & 1995 & Beyond & \\
\hline 1 & 7 & & & & & & 7 \\
\hline 2 & - & & & & & & \\
\hline \multicolumn{8}{|l|}{ Status: } \\
\hline Funded & 7 & & & & & & \\
\hline \multicolumn{8}{|l|}{ Requester } \\
\hline New & & & & & & & $\$ 7$ \\
\hline
\end{tabular}

References: $\quad$ DOE Order 5480.7 
Finding No.: FP.4-1 Adequacy of Fire Protection

Finding

Description: ORNL has not provided in all facilities the level of fire protection which is designed to ensure that fire will not result in unacceptable programmatic impacts as required by DOE 5480.7 .

Code: $\quad$ Category III

Compliance Protocol:

DOE Order 5480.7 requires that a maximum credible fire not result in the interruption of a vital program for a period longer than that specified by the Program Senior Official.

Priority: $\quad$ Energy Systems Risk Weight 55

Tiger Team Action Plan Priority 2

Response: This concern was identified in the ORNL Protective Services Department SelfAssessment.

A Fire Protection Engineering appraisal performed in April 1990 for the Advanced Toroidal Facility (Building 9201-2) by a registered Fire Protection Engineer, indicates that the existing level of protection is satisfactory to meet the requirements of DOE Order 5480.7. No additional protection is planned.

A Fire Protection Engineering appraisal performed in November 1988 by a fire protection engineering consultant indicates that the level of fire protection in Building 9210 is satisfactory to meet the requirements of DOE Order 5480.7. The building is completely protected with automatic sprinklers and smoke detectors are located in the building air handling systems. No additional protection is planned.

The Cyclotron Room (C-109) in Building 6000 is not provided with automatic fire detection or suppression. Protection for this area will be evaluated and a project initiated to provide necessary protection once the evaluation is complete.

See also Findings FP.1-1 and FP. 1-3.

\section{Root Causes:}

Insufficient resources, inadequate policy, and inadequate management commitment 
Planned Actions and Schedules:

\section{Item/Description}

1. Evaluate and implement fire detection and protection methods for the cyclotron area in Building 6000 .

Request funding to provide protection as determined by the evaluation.

2. Issue revised ORNL Standard Practice Procedure X-GP-4 to include guidance on (1) the performance of fire protection engineering surveys as required by DOE Order 5480.7, (2) identifying facilities requiring fire protection upgrades, and (3) the tracking of necessary fire protection upgrades by ORNL management. (See Action Item \#1 in FP.1-4.)
Completion Date

$1 / 92$

$7 / 91$

Costs:

Type of funds: Research programmatic

Source of funds: ER-KC

\begin{tabular}{|c|c|c|c|c|c|c|c|}
\hline \multirow[b]{2}{*}{ Action item } & \multicolumn{6}{|c|}{ Estimated costs per fiscal year $(\$ K)^{a}$} & \multirow[b]{2}{*}{ Total } \\
\hline & 1991 & 1992 & 1993 & 1994 & 1995 & Beyond & \\
\hline 1 & - & 100 & 400 & & & & 500 \\
\hline 2 & - & & & & & & \\
\hline \multicolumn{8}{|l|}{ Status: } \\
\hline \multicolumn{8}{|l|}{ Funded } \\
\hline \multicolumn{8}{|l|}{ Requested } \\
\hline New & & 100 & 400 & & & & $\$ 500$ \\
\hline
\end{tabular}

${ }^{a}$ Design and installation of protection as determined by the evaluation in Action Item 1 , could cost $\$ 500 \mathrm{~K}$.

References: $\quad$ DOE Order 5480.7 
Finding No.: FP.5-1 Fire Protection Systems in Bldg. 4500N

Finding

Description:

Code:

Compliance

Protocol:

Priority: $\quad$ Energy Systems Risk Weight 60

Tiger Team Action Plan Priority 2

Response: This concern was identified in the ORNL Self-Assessment.

A $\$ 3.3$ million Line Item (Fire Protection Upgrade - ORNL) is in the design stages and will provide automatic sprinkler protection for most of the unsprinklered areas of Building $4500 \mathrm{~N}$. Due to asbestos contamination, the current project is not funded to protect office areas and corridors on the first floor of Wings 1-4 and along the path of ductwork which has a combustible wrapping. ORNL Fire Protection Engineering will provide oversight for this Line Item Project through completion.

Building $4500 \mathrm{~N}$ is divided into seven separate fire areas by 3 -hour fire-rated walls. Openings through these walls are protected by automatic closing fire doors. The above-mentioned \$3.3 million Line Item will install smoke detection in the buildings air handling ducts. No fire doors have been removed in the building.

The majority of doors to wings and stairwells in Building $4500 \mathrm{~N}$ that were previously held open by heat-activated door releases, are now kept closed. A program will be initiated to install smoke-activated door closers on these fire doors.

\section{Root Causes:}

Insufficient resources, inadequate policy, and inadequate management commitment

\section{$\underline{\text { Item/Description }}$}

1. Provide sprinkler systems in unprotected areas of $4500 \mathrm{~N}$ Main and Wing 5. Also upgrade water supply to Building 6000.
Completion Date

9/93 
2. Install smoke detector actuated door closers on second floor fire doors which separate the wings and stairwells from the other parts of Building 4500N.

3. Perform "needs assessment" and develop fire-protection

$8 / 91$ management plan. (See Action Item 1 in Finding FP.1-1.)

4. Revise SPP X-GP-4. (See Action Item 1 in Finding FP.1-4.)

5. Abate asbestos in Wing 1-4 of Building $4500 \mathrm{~N}$ and to 9/96 install automatic sprinklers once asbestos contamination is controlled.

Costs:

Type of funds: Line-Item

Source of funds: MGPF

\begin{tabular}{crrrrrrr} 
& \multicolumn{5}{c}{ Estimated costs per fiscal year $(\$ \mathrm{~K})$} & \\
\cline { 2 - 6 } Action item & 1991 & 1992 & 1993 & 1994 & 1995 & Beyond & Total \\
\hline 1 & 850 & 1660 & 300 & & & & 2810 \\
2 & 10 & & & & & 10 \\
5 & & 190 & & & 1800 & 1990
\end{tabular}

Status:

Funded

850

Requested

$1660 \quad 300$

New

10

190

1800

$\$ 4810$

References: $\quad$ DOE Order 5480.7 
Finding No.: $\quad$ FP.6-1 Physical Fitness Program for Fire Fighters

\section{Finding}

Description: ORNL has not implemented a physical fitness program for fire fighters as required by NFPA 1500 .

Code: $\quad$ Category III

Compliance

Protocol: $\quad$ NFPA 1500

An NFPA 1500 draft implementation and interpretation plan was issued by DOE-HQ.

Priority: $\quad$ Energy Systems Risk Weight 63

Tiger Team Action Plan Priority 2

Response: ORNL has not received direction from DOE which required a physical fitness program be implemented. ORNL received information in 1988 that direction was forthcoming from the Director of DOE medical programs (Neal Goldenberg, EH-321 to David Howard ORO).

The ORNL on-site Health Division currently determines fitness for assigned tasks.

Action is pending contingent on the development of DOE-wide guidance and implementation instructions.

\section{Root Cause:}

Ambiguous requirements or expectations

\section{Planned Actions and Schedules:}

None

Costs: Indeterminable until instruction is received. 
Fünding No.: FP.6-2 Fire Department Staffing Level

Finding

Description: The stating level of the ORNL Fire Department is not commensurate with the
fire risk.

Code: $\quad$ Category III

Compliance

Protocol:

NFPA 1500 , DOE Order 5480.4

Priority: $\quad$ Energy Systems Risk Weight 83

Tiger Team Action Plan Priority 3

Response: $\quad$ The present minimum response to fire incidents is eight personncl (four fully trained fire fighters including one fire officer, three firemen, and four emergency squad members). All are fully trained in the functions they are expected to perform. As funding permits, response forces will be increased to provide five fully trained fire fighters for structural fire fighting and to meet the testing and maintenance requirements in Finding FP.1-2.

\section{Root Causes:}

Ambiguous requirements or expectations and insufficient resources

Planned Actions and Schedules:

\section{Item/Dt ription}

1. Perform a systematic "Needs Assessment" to determine necessary resources for conducting assigned tasks.

2. Utilize the results of the "Needs Assessment" to issue a fire protection management plan for securing the necessary resources.

3. Request funding for additional personnel and other necessary resourccs as justified by the "Needs Assessment." (See Finding FP.1-2.)

4. Additional full-time fire fighters will be added as funding permits to meet staffing needs in the Fire Department for inspection, testing, and maintenance and to provide five fully trained fire fighters on each shift.

\section{Completion Date}

Complete

Complete

$8 / 91$

$12 / 92$ 
Costs:

Type of funds: Orerhead

Source of funds: Oerhead

Estimated costs per fiscal year (SK)

\begin{tabular}{llllllll} 
Action item & 1991 & 1992 & 1993 & 1998 & 1995 & Bcyond & Total \\
\hline 4 & & & & & & \\
Status: & & & & & \\
Funded \\
Requested \\
New
\end{tabular}

-Estimated annual ongoing cost $\$ 250 \mathrm{~K}$ per year. $\$ 50 \mathrm{~K}$ per year for each of the five people required.

References: NFPA 1500 - DOE Implementation Plan 
Finding No.: FP.6-3 Prefire Plans for ORNL Facilities

Finding

Description: Prefire plans for ORNL facilities located at the X-10 Site and Y-12 Plant are not developed in accordance with nationally recognized good practice.

Code: $\quad$ Category III

\section{Compliance}

Protocol:

Priority: $\quad$ Energy Systems Risk Weight 5

Tiger Team Action Plan Priority 3

Response: The ORNL Fire Department maintains up-to-date prefire plans for all major buildings at the X-10 site. The Fire Department rotating shift fire officers are responsible for updating the prefire plans. Basic response information is included in the plans. Preplans are updated every 3 years or sooner if the building is modified.

No recognized standard for prefire plans exists. The International Fire Service Training Association (IFSTA) publishes guidelines concerning prefire plans.

The Y-12 site does not currently have updated prefire plans but have action plans in place to update all prefire plans.

Prefire plans are maintained (X-10 site).

As prefire plans come up for review, appropriate IFSTA ideas shall be incorporated into prefire plans (X-10 site).

\section{Root Causes:}

Ambiguous requirements or expectations and inadequate policy implementation

\section{Planned Actions and Schedules:}

\section{Item/Description}

1. Issue updated prefire plans for $\mathrm{Y}-12$ with full involvement by the uniformed response forces at the $\mathrm{Y}-12$ site.

2. Issue a Fire Department procedure that establishes a prefire schedule and requires revisions in accordance with recognized good practice.

\section{Completion Date}

$11 / 91$

Complete 


\section{Costs:}

Type of funds: Overhead

Source of funds: Overhead

\begin{tabular}{crrrrrrr} 
& \multicolumn{5}{c}{ Estimated costs per fiscal year (\$K) } & \\
\cline { 2 - 6 } Action item & 1991 & 1992 & 1993 & 1994 & 1995 & Beyond & Total \\
\hline 1 & 225 & & & & & & 225 \\
2 & 1 & & & & & & 1
\end{tabular}

Status:

Funded 1

Requested

New

225

$\$ 226$

References: $\quad$ DOE Order 5480.7 
Finding No.: FP.7-1 Fire Protection of Main Computer Centers

\section{Finding}

Description:

Code: $\quad$ Category III

\section{Compliance}

Protocol:

Priority:

Response:
Fire protection in the main computer centers located within Bldg. $4500 \mathrm{~N}$ is not in compliance with DOE/EP-0108.

DOE/EP-U108 contains specific fire protection requirements applicable to essential DOE electronic computer data processing equipment.

Energy Systems Risk Weight 58

Tiger Team Action Plan Priority 2

This concern was partially identified in the ORNL Protective Services Department Self-Assessment.

The non-indicating type control valve which provides sectional control for the under-floor automatic sprinkler system in Room $\Gamma-5$ at Building $4500 \mathrm{~S}$ will be replaced with an indicating (OS\&Y) type. The existing valve is however chained and locked in the open position an indicating type valve can be glanced at to determine if it is shut or open without further investigation (DOE/EP 0108, Para. 402-1a).

The sliding fire door between Rooms 258 and 270 in the Central Computer Center at Building $4500 \mathrm{~N}$ will be modified to close when smoke is detected in the area. The doors currently close when fusible links are exposed to heat and melt (DOE/EP 0108, Para. 204-11).

The side-hinged fire door between Rooms 270 and 294 in the $4500 \mathrm{~N}$ Central Computer Center is a substantially constructed metal door but is not an approved fire door. The existing door will be replaced with an approved fire door (DOE/EP 0108, Para. 204-11).

Plant and Equipment Division has completed an inspection of firewalls in the main computer centers once results are received, a program will be initiated to seal all unsealed penetrations to restore the fire resistive integrity of the walls.

Existing waste containers will be replaced with Underwriters Laboratories (UL) listed waste containers in the main tape storage room (Building $4500 \mathrm{~N}$ ). These containers must be emptied at the end of each shift or at least once each day (DOE/EP 0108, Para. 205-6). 
The "Emergency and Disaster Plan for Computing and Telecommunications Division Computer Operations" will be expanded to include the names of specific qualified contractors who are readily available to conduct computer equipment and information storage system salvage operations. Instructions for contacting each contractor will also be included.

\section{Root Causes:}

Inadequate policy implementation and insufficient resources

\section{Planned Actions and Schedules:}

\section{Item/Description}

Completion Date

1. Replace the non-indicating valve controlling water to under-floor sprinklers in Room T-5 in Building 4500S with an indicating type valve (OS\&Y).

2. Install magnetic hold-open devices, which will release the sliding fire door between Rooms 258 and 270 in the $4500 \mathrm{~N}$ Central Computer Center when area srnoke detectors actuate.

3. Replace the side-hinged door between Rooms 270 and Complete 294 in the $4500 \mathrm{~N}$ Central Computer Center with an approved fire door.

4. Replace standard waste containers in the computer tape storage room with a single, limited-capacity, UL-listed waste receptacle.

5. Issue a revised "Emergency and Disaster Plan for Computing and Telecommunications Division Computer Operations" to include the names of specific qualified contractors who can conduct computer equipment and information storage system salvage operations. 
Costs: $\quad$ Item 1 labor and material costs are estimated at $\$ 1 \mathrm{~K}$. Item 2 labor and material costs are estimated at $\$ 4 \mathrm{~K}$. Item 3 labor and material costs are estimated at $\$ 2 \mathrm{~K}$. Item 4 material cost i.: estimated at $\$ 1 \mathrm{~K}$. Item 5 revision cost is estimated at $\$ 2 \mathrm{~K}$.

Type of funds: Overhead

Source of funds: Overhead

Estimated costs per fiscal year (\$K)

\begin{tabular}{|c|c|c|c|c|c|c|c|}
\hline \multirow[b]{2}{*}{ Action item } & & \multirow[b]{2}{*}{ Total } \\
\hline & 1991 & 1992 & 1993 & 1994 & 1995 & Beyond & \\
\hline All & 10 & & & & & & 10 \\
\hline \multicolumn{8}{|l|}{ Status: } \\
\hline Funded & 1 & & & & & & \\
\hline \multicolumn{8}{|l|}{ Requested } \\
\hline New & 9 & & & & & & $\$ 10$ \\
\hline
\end{tabular}

References: $\quad$ DOE/EP 0108 
Finding No.: FP.7-2 Fire Hazard Analysis and Facility Protection Survey

\section{Finding \\ Description:}

Code: $\quad$ Category III

ORNL does not have a fire hazard analysis or facility fire protection survey program which complies with DOE 5480.7 par. 10.b (2).

\section{Compliance \\ Protocol:}

DOE Order 5480.7 par. 10.b (2) requires regular self-inspections, tests, fire loss potential reviews and appraisals to identify the nature, location, and severity of fire risks (injuries, dollar loss, programmatic interruption, release of toxic and radioactive materials) as well as to determine adequacy of tire los control devices and activities.

\section{Priority: $\quad$ Energy Systems Risk Weight 133}

Tiger Team Action Plan Priority 2

Response: This concern was identified in the ORNL Protective Services Department SelfAssessment.

A Fire Protection Engineering Survey Program is in place at ORNL. However, these surveys are not conducted at the required frequency due to lack of adequate staff. There are key and highly visible facilities that have not been surveyed. Only a small number of surveys are being performed annually compared to the number required on an annual basis. ORNL management is made aware of fire protection deficiencies identified in the Fire Protection Engineering surveys.

The revised DOE Order 5480.7 which is currently in the proposal stages, requires a more detailed approach to fire protection engineering surveys in the form of a fire hazards analysis. If accepted, the new DOE Order 50.7 will require an increased amount of time to be devoted to performing this function.

An additional Fire Protection Engineer was hired in May 1990. The impact that the new engineer will have on the group's capability to perform the required fire protection engineering surveys is not currently apparent. Evaluation of the current staff's ability to perform the required work load will continue. 


\title{
Root Causes:
}

Inadequate policy, insufficient resources, and inadequate management commitment Planned Actions and Schedules:

\author{
$\underline{\text { Item/Description }}$
}

Completion Date

1. See Finding FP.1-1 on needs assessment and Finding FP.4-1 on revision of ORNL Standard Practice Procedure X-GP-4, Fire Prevention and Control.

Costs: $\quad$ See cost sheets on Findings FP.1-1 and FP.1-4.

References: $\quad$ DOE Order 5480.7 
Finding No.: FP.7-3 Fire Protection Oversight

Finding

Description: ORO does not provide a level of fire protection oversight of ORNL as required by DOE 5480.7.

Code: $\quad$ Category III

Compliance

Protocol:

DOE Order 5480.7 entitled Fire Protection requires an "improved risk" level of fire protection sufficient to attain Department of Energy objectives.

Priority: $\quad$ Energy Systems Risk Weight 58

Tiger Team Action Plan Priority 2

Response: We do not concur with this concern. Though the resources committed by ORO to fire protection oversight activities could be improved, the present level of effort on oversight meets DOE Order 5480.7.

DOE Order 5480.7 does not require that fire protection engineering review or approve facility Safety Analysis Reports. The order does not use the term "safety class system."

Experiments are reviewed by an operational safety committee and peers. Fire protection reviews experiments as requested by the committee. There is no information provided that contends the isobutane used in Building 6000 was unsafe.

Though there is inadequate staff both at ORNL and ORO to review safety analysis reports, all operational requirements, and all procedures, most concerns of substance identified by the Tiger Team, had been previously identified by ORO and/or the Contractor prior to the review. Concerns about fire protection at Building 7920 were identified by ORO.

With few exceptions, i.e., when fire protection reviews have been conducted by outside organizations, ORO has performed annual fire protecti in surveys of ORNL.

The following actions are ongoing or were preplanned and support our nonconcurrence with this concern.

\section{Root Causes:}

Ambiguous requirements or expectations 
Planned Actions and Schedules:

Item/Description

1. Conduct fire protection surveillances every 6 months by ORO.

2. Conduct functional appraisals, including a comprehensive Fire Protection review, every 2 years.

3. Conduct ESH\&QA management appraisals at 3-year intervals.

4. Request additional independent oversight from EH; e.g., Factory Mutual review.

5. Request assistance from $\mathrm{EH}$ to resolve professional differences of opinion related to fire protection concerns that may arise from such activities as Tiger Team reviews.

Costs: $\quad$ No additional.

References: None
Completion Date

Ongoing

Ongoing

Start June 1992

March 1992

Ongoing 
Finding No.: FP.7-4 Review of Documents Affecting Fire Protection

Finding

Description:

Safety-related documents affecting fire protection are not always reviewed by Fire Protection Engineering as required by DOE 5480.7.

Code: $\quad$ Category III

Compliance

Protocol:

DOE Order 5480.7

Priority: $\quad$ Energy Systems Risk Weight 58

Tiger Team Action Plan P:iority 2

Response: The Safety Analysis Report Upgrade Program requires the formation of Facility Safety Evaluation Teams (FSETs) for each facility containing unique hazards. The FSETs are responsible for performing the safety documentation for the facility. The FSETs will consist of operatir. personnel from the organization responsible for operating the facility and technical representatives such as Systems Safety Engineering (SSE) personnel, hazard specialists such as fire protection, industrial hygiene, and criticality safety. This inclusion of a fire protection specialist aids in providing proper fire protection input into the preparation of the document.

ORNL SPPs place responsibility for ensuring appropriate internal reviews on the Operating Divisions. The SPP ; will be revised to provide further specific guidance to ensure that appropriate reviews, such as fire protection, are performed. At present no SSE procedure exists to ensure that safety documentation performed by SSE is reviewed by Fire Protection Engineers. SSE will develop an internal operating instruction that will designate fire protection personnel as a standing reviewer for all safety documentation and where fire protection is an important issue. Key reviewers are required to respond to the transmitted safety document even if they have no comments.

\section{Rocit Causes:}

Inadequate policy, inadequate policy implementation, insufficient resources, and ambiguous requirements or expectations

Planned Actions and Schedules:

\section{$\underline{\text { Item/Description }}$}

1. Issue revised SPP X-OS-3 to provide further guidance to operating divisions on internal reviews.
Completion Date

$9 / 91$ 
2. See Finding FP.1-1, which requires the performance of a "Needs Assessment" and the development of a "Fire-Protection Management Plan" for securing necessary resources.

3. See Finding FP.3-3, which addresses the development of a review program requiring Fire Protection Engineering review of safety analysis reports.

Costs: $\quad$ OORS is presently revising SPP-X-OS-3, and no significant addition of funds are required.

Costs associated with this finding are reported under Findings FP.1-1 and FP.3-3.

References: Safety Analysis Report Update Program Overview and Phase I Implementation Plan (Y/CSET-1)

DOE Order 5480.7 
Finding No.: FP.7-5 Fire Water Supply System

Finding

Description: A firc water supply meeting the requirements of DOE 5480.7 and DOE 6430.1A has not been provided, and the fire water system is not being supervised and maintained in accordance with recognized good practice.

Code: $\quad$ Category III

Compliance

Protocol:

DOE Order 5480.7 and DOE Order 6430.1A

Priority: $\quad$ Energy Systems Risk Weight 58

Tiger Team Action Plan Priority 2

Response: $\quad$ Procedure UT-90-020R is in place; it requires a water reserve of $2,000,000$ gallons for fire-fighting purposes should a supply disruption occur.

ORNL has a single water system which provides domestic and fire protection water supplies. The system is gravity fed by reservoirs which are filled by a single 24 -inch line from the water treatment plant at Y-12. On-site reservoirs at ORNL have a 6-million gallon capacity. The stored water is located on two ridges on opposite sides of the plant site, with 3 million gallons stored on each ridge.

All control valves for designated fire protection systems are secured or supervised in accordance with DOE guidelines.

All post indicator valves (PIV) and indicating valves (OS\&Y) in the ORNL water supply system which are not currently supervised will be locked in the open position.

A maintenance program will be initiated to paint PIV's where needed to aid in visibility and to replace glass windows where needed so that position indicaturs are readily visible.

The Fire Department will continue to perform required flow tests of fire hydrants in designated locations on an annual bes:s These tests are compared to previous tests so that changes or possible water supply oustructions can be identitied. The flow tests which are currently conducted are adequate to verify. at the required fire flow for a given area is available. Changes to the current testing arrangement are not planned.

ORNL does not currently have any identified "safety class" fire protection systems that require a water supply to survive a Design Base Earthquake as prescribed by DOE Order 64?0.1A. 


\section{Root Cause:}

Ambiguous requirements or expectations

Planned Actions and Schedules:

\section{Item/Description}

1. Examine ORNL Fire Department Standard Operating Procedure FP B-19 (Water Flow Tests) to ensure recognized good practices are incorporated. Necessary procedural revisions, resulting from the examination, will be included.

2. Secure (lock) all post indicator valves (PIV) and indicating valves that are not currently supervised in the open position.

3. Paint PIVs that need painting to increase visibility, and replace glass windows where necessary so position indicators are readily visible.

4. Install a second water supply to ORNL, upgrade a water reservoir, and improve water-distribution systems. These upgrades and additions to the ORNL water system will be designed in accordance with DOE 6430.1A and will receive the necessary $\mathrm{DBE}$ review.

\section{Completion Date}

$8 / 91$

$6 / 91$

$6 / 92$

$9 / 96$

Costs:

Type of funds: Overhead

Source of funds: Overhead

Estimated costs per fiscal year (\$K)

\begin{tabular}{crrrrrrr}
\cline { 2 - 6 } Action item & 1991 & 1992 & 1993 & 1994 & 1995 & Beyond & Total \\
\hline 1 & 7 & & & & & & 7 \\
2 & - & & & & & & \\
3 & & 30 & & & & & 30
\end{tabular}

Status:

Funded 7

Requested

New 
Type of funds: Line Item

Source of funds: MGPF

Estimated costs per fiscal year (\$K)

\begin{tabular}{|c|c|c|c|c|c|c|c|}
\hline Action item & 1991 & 1992 & 1993 & 1994 & 1995 & Beyond & Total \\
\hline 4 & & & & 5,000 & 10,000 & 7,000 & 22,000 \\
\hline \multicolumn{8}{|l|}{ Status: } \\
\hline \multicolumn{8}{|l|}{ Funded } \\
\hline Requested & & & & 5,000 & 10,000 & 7,000 & \\
\hline New & & & & & & & $\$ 22,000$ \\
\hline
\end{tabular}

References: $\quad$ DOE Order 5480.7 and DOE Order 6430.1A 


\subsubsection{Medical Services}

Finding No.: MS.1-1 Voluntary Health Examination Program

Finding

Description: The frequency of the voluntary health examination program is not in compliance with DOE 5480.8.

Code: $\quad$ Category III

Compliance

Protocol: $\quad$ DOE Order 5480.8

Priority: $\quad$ Energy Systems Risk Weight 55

Tiger Team Action Plan Priority 2

Response: The Health Division suffers from a lack of medical staff and support staff. An increasing number of job classifications and potential exposure situations are required to have annual medical evaluations. To compound the shortage more administrative work (writing procedures and action plans and other activities related to documenting compliance with regulations and requirements) is being required of medical personnel. As a result examinations required by DOE Order 5480.8 are not being conducted at the required frequency.

The Health Division is in the process of recruiting an additional occupational physician. The Health Division is advertising in the Journal of Occupational Medicine and in ACOM Placement Services.

Beyond this addition, recruitment will continue because of the need to replace retiring personnel.

Root Causes:

Inadequate policy implementation and insufficient resources

Planned Actions and Schedules:

\section{Item/Description}

1. Hire an occupational physician to allow implementation of DOE Order 5480.8 .

2. Issue an SPP to specify the required frequency of medical evaluations.
Completion Date

$7 / 91$

$12 / 91$ 


\section{Costs:}

Type of funds: Overhead

Source of funds: Overhead

\begin{tabular}{cccccccc} 
& \multicolumn{6}{c}{ Estimated costs per fiscal year $(\$ K)$} \\
\cline { 2 - 5 } Action item & 1991 & 1992 & 1993 & 1994 & 1995 & Beyond & Total \\
\hline 1 & $*$ & & & & & & \\
2 & - & 15 & & & &
\end{tabular}

Status:

Funded

Requested

New

15

"Estimated annual ongoing costs: $\$ 100 \mathrm{~K}$ starting in FY 1991.

References: $\quad$ DOE Washington Audit of the Occupational Medical (OM) Program at ORNL, September 25, 1989

DOE Order 5480.8, Contractor Occupational Medical Program 
Finding No.: $\quad$ MS.2-1 Medical Division Administrative Assistance

\section{Finding}

Description: Administrative assistance is not available to the Medical Division to complete the administrative policies and procedures.

Code: $\quad$ Category III

Compliance

Protocol: None

Priority: $\quad$ Energy Systems Risk Weight 8

Tiger Team Action Plan Priority 3

Response: $\quad$ The Health Division suffers from a lack of medical staff and support staff. By necessity individuals are being assigned multiple duties. More administrative work is being required of medical personnel especially in the completion of the division's polices, practices and procedures.

\section{Root Cause:}

Insufficient resources

Planned Actions and Schedules:

Item/Description

1. Request funding for administrative assistant.

2. Contingent upon funding, hire an administrative assistant to support necessary work.

3. Issue a plan and schedule to develop necessary policies, practices, and procedures for the Health Division.
Completion Date

$8 / 91$

$12 / 91$

$3 / 92$ 
Costs:

$$
\text { Type of funds: Overhead }
$$

Source of funds: Overhead

\begin{tabular}{|c|c|c|c|c|c|c|c|}
\hline \multirow[b]{2}{*}{ Action item } & \multicolumn{6}{|c|}{ Estimated costs per fiscal year (\$K) } & \multirow[b]{2}{*}{ Total } \\
\hline & 1991 & 1992 & 1993 & 1994 & 1995 & Beyond & \\
\hline 1 & - & & & & & & \\
\hline 2 & & * & & & & & \\
\hline \multicolumn{8}{|l|}{ Status: } \\
\hline \multicolumn{8}{|l|}{ Funded } \\
\hline \multicolumn{8}{|l|}{ Requested } \\
\hline New & & & & & & & 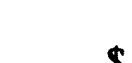 \\
\hline
\end{tabular}

*Estimated annual ongoing cost: \$45K beginning FY 1992.

References: $\quad$ DOE Order 5480.8, Contractor Occupational Medical Program 
Finding No.: $\quad$ MS.3-1 Backup Pulmonary Function Testing Personnel

Finding

Description: The backup personnel performing pulmonary function testing have not received National Institute for Occupational Safety and Health approved training as required by DOE 5480.8 and OSHA.

Code: $\quad$ Category III

Compliance

Protocol:

DOE Order 5480.8 and OSHA standards

Priority: $\quad$ Energy Systems Risk Weight 408

Tiger Team Action Plan Priority 2

Response: The X-ray technician is responsible for X-rays and PFTs performed in the Health Division. Medical technologists have provided assistance in the performance of PFTs. It has been the belief that formal training of the medical technologists in performing PFTs was not necessary because of on-the-job training given to them by the NIOSH trained X-ray technician.

Medical technologists of the Health Division will receive training in a NIOSH approved training program.

Dr. Henry Glindmeyer, Research Associate Professor in the Department of Medicine and Bicmedical Engineering at Tulane University in New Orleans will provide on-site PFT training of medical technologists.

Root Cause:

Inadequate training

Planned Actions and Schedules:

\section{Item/Description}

1. Establish contract for on-site training program for PFT training for ongoing qualification of medical technologists.

2. Conduct NIOSH approved training for PFT personnel.
Completion Date

$8 / 91$

$8 / 91$ 


\section{Costs:}

Type of funds: Overhead

Source of funds: Overhead

\begin{tabular}{|c|c|c|c|c|c|c|c|}
\hline \multirow[b]{2}{*}{ Action item } & \multicolumn{6}{|c|}{ Estimated costs per fiscal year (\$K) } & \multirow[b]{2}{*}{ Total } \\
\hline & 1991 & 1992 & 1993 & 1994 & 1995 & Beyond & \\
\hline 2 & 5 & & & & & & 5 \\
\hline \multicolumn{8}{|l|}{ Status: } \\
\hline \multicolumn{8}{|l|}{ Funded } \\
\hline \multicolumn{8}{|l|}{ Requested } \\
\hline New & 5 & & & & & & $\$ 5$ \\
\hline
\end{tabular}

References: $\quad$ DOE Order 5480.8, Contractor Occupational Medical Program

29 CFR 1926.58 
Finding No.: $\quad$ MS.3-2 Medical Division Space Allocation

Finding

Description: Space allocation is inadequate for proper functioning of the Medical Division.

Code: $\quad$ Category III

Compliance

Protocol: None

Priority: $\quad$ Energy Systems Risk Weight 55

Tiger Team Action Plan Priority 3

Response: The Health Division has insufficient space for maintaining charts, records, and $\mathrm{X}$-ray files and holding staff conferences. There is no office space available for the additional staff boing recruited. There is lack of privacy for patients who are interviewed in the treatment room. The requirements upon and the activities of the Health Division have outgrown its physical resources. Overall ORNL shortage of space has precluded the addition of more space to the Health Division.

There are plans to utilize some of the new decontamination facility (see Finding MS.3-3) as a small amount of storage space. In addition some space would be used temporarily for clerical activity hut would immediately revert to use for triage and decontamination functions when needed.

Root Cause:

Insufficient resources

Pla nned Actions and Schedules:

Item/Description

1. Evaluate spacing requirements and submit recommendations to the Associate Director of Operations.

\section{Completion Date}

Complete

2. Allocate space following approval of recommendations.

Costs: $\quad$ See Finding MS.3-3

References: DOE Washington Audit of the Occupational Medical (OM) Program at ORNL, September 25, 1989

DOE Order 5480.8, Contractor Occupational Medical Program 
Finding No.: MS.3-3 Decontamination Facilities

\section{Finding}

Description:

Code: $\quad$ Category III

Compliance

Protocol:

Priority: $\quad$ Energy Systems Risk Weight 62

Tiger Team Action Plan Priority 2

Response: It has long been recognized that the decontamination facilities of the Health Division are insufficient for properly handling contamination cases especially in the event of multiple patient involvement. A feasibility study was cone in June 1986 resulting in plans to create a facility with triage and decontamination functions.

The title of this project is "Modify ORNL Health Center, Building 4500N." Capital funds (GPP) of $\$ 280,000$ were authorized in FY 1990 for design and the remaining $\$ 920,000$ for construction is contained in the FY 1991 planning base.

For reference, the project is described in Preliminary Proposal 836 which was forwarded to DOE on August 31,1989, requesting approval as a subproject of the Directive CL-577, "ORNL General Plant Projects, Fusion Energy Program," for FY 1990. At that time the total facility was identified as a contingency project. Funds were later identified to do the design in FY 1990.

Currently the design criteria and the necessary documents to bring the architect engineer on board are being prepared. However, no design effort can start until the NEPA documentation is approved.

The first environmental document was submitted to the DOE on August 30, 1989. This document was an Environmental ALARA Memorandum (EAM) and was prior to Secretary Watkin's order SEN 15 . In response to SEN 15 a Project Description Memorandum (PDM), 0991X, was submitted to DOE on April 30, 1990. It was subsequently determined that a different type document would be required and on September 26, 1990, a Request for Categorical Exclusion (CXD), 0741X, was submitted to the DOE. No response has been received.

Plans have been drawn and funds made available to establish an adequate decontamination facility.

\section{Root Causes:}

Insufficient resources and regulatory barriers 
Planned Actions and Schedules:

Item/Description

1. Upon receipt of NEPA documentation approval begin the $\mathrm{AE}$ (architect engineering) phase of design.

2. Construct decontamination facility.
Completion Date

9/91

$12 / 92$

Costs:

Type of funds: Capital

Source of funds: ER-AT

\begin{tabular}{crrrrrrr} 
& \multicolumn{5}{c}{ Estimated costs per fiscal year (\$K) } & \\
\cline { 2 - 6 } Action item & 1991 & 1992 & 1993 & 1994 & 1995 & Beyond & Total \\
\hline 1 & 280 & & & & & & 280 \\
2 & & 920 & & & & & 920
\end{tabular}

Status:

Funded

280

Requested

920

New

References: $\quad$ DOE Washington audit on the Occupational Medical (OM) Program at ORNL, September 25, 1989 

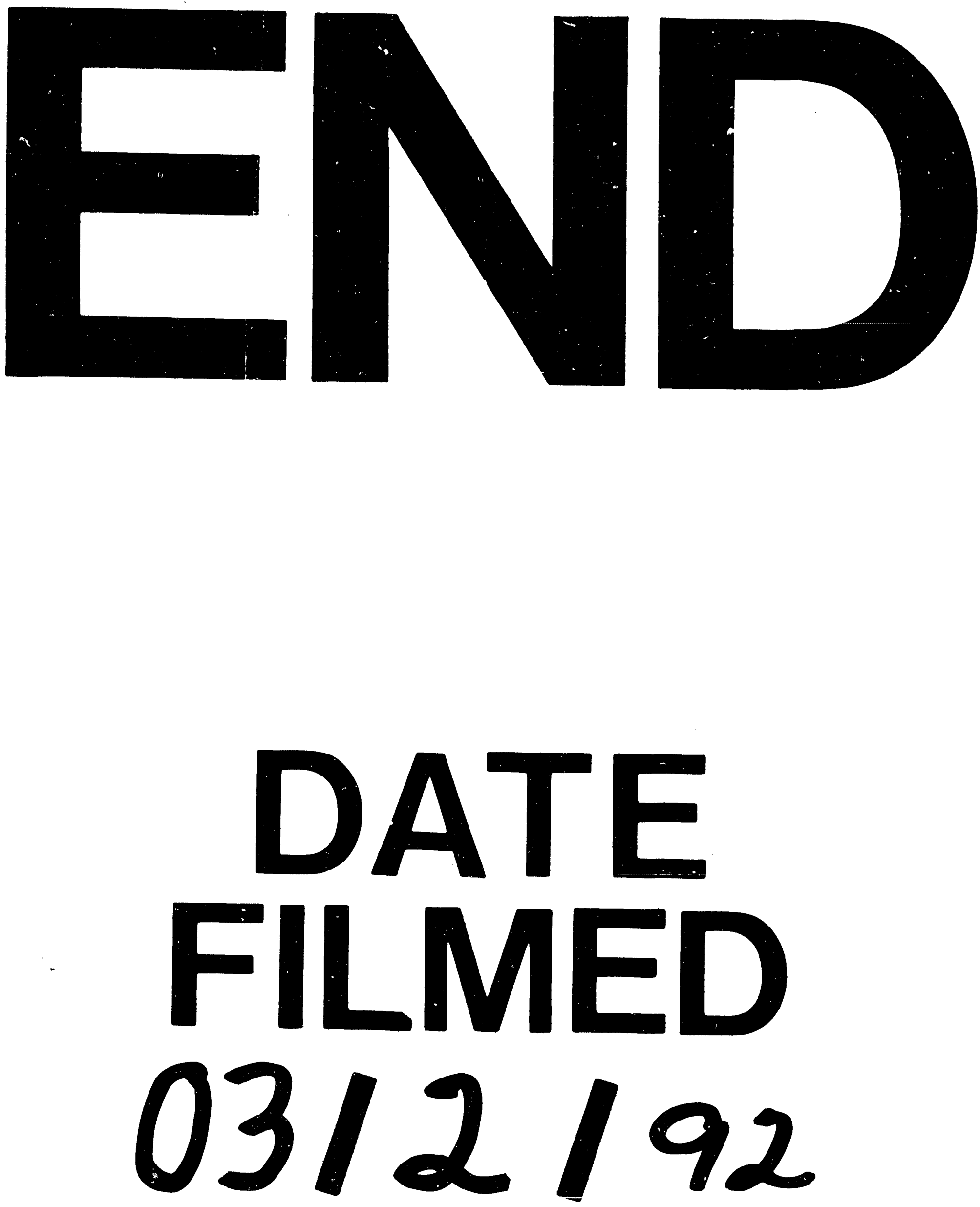